Cochrane Database of Systematic Reviews

\title{
School-based self-management interventions for asthma in children and adolescents: a mixed methods systematic review (Review)
}

Harris K, Kneale D, Lasserson TJ, McDonald VM, Grigg J, Thomas J

Harris K, Kneale D, Lasserson TJ, McDonald VM, Grigg J, Thomas J.

School-based self-management interventions for asthma in children and adolescents: a mixed methods systematic review.

Cochrane Database of Systematic Reviews 2019, Issue 1. Art. No.: CD011651.

DOI: 10.1002/14651858.CD011651.pub2.

www.cochranelibrary.com 
TABLE OF CONTENTS

HEADER 1

ABSTRACT

PLAIN LANGUAGE SUMMARY .....

SUMMARY OF FINDINGS

BACKGROUND

Figure 1.

OBJECTIVES

METHODS

RESULTS

Figure 2.

Figure 3.

Figure 4.

Figure 5.

Figure 6.

Figure 7.

Figure 8.

Figure 9.

Figure 10.

Figure 11.

Figure 12.

Figure 13.

Figure 14.

Figure 15.

Figure 16.

Figure 17.

Figure 18.

Figure 19.

Figure 20.

Figure 21.

Figure 22.

DISCUSSION

AUTHORS' CONCLUSIONS

ACKNOWLEDGEMENTS

REFERENCES

CHARACTERISTICS OF STUDIES

DATA AND ANALYSES

Analysis 1.1. Comparison 1 Effects of school-based asthma interventions vs usual care, Outcome 1 Exacerbations leading to hospitalisation.

Analysis 1.2. Comparison 1 Effects of school-based asthma interventions vs usual care, Outcome 2 Exacerbations leading to emergency department (ED) visits.

Analysis 1.3. Comparison 1 Effects of school-based asthma interventions vs usual care, Outcome 3 Absence from school. ...... Analysis 1.4. Comparison 1 Effects of school-based asthma interventions vs usual care, Outcome 4 Days of restricted activity. . Analysis 1.5. Comparison 1 Effects of school-based asthma interventions vs usual care, Outcome 5 Unplanned visit to hospital or GP due to asthma symptoms.

Analysis 1.6. Comparison 1 Effects of school-based asthma interventions vs usual care, Outcome 6 Experience of daytime and night-time symptoms - daytime symptoms.

Analysis 1.7. Comparison 1 Effects of school-based asthma interventions vs usual care, Outcome 7 Experience of daytime and night-time symptoms - night-time symptoms.

Analysis 1.8. Comparison 1 Effects of school-based asthma interventions vs usual care, Outcome 8 Use of reliever therapies, e.g. beta ${ }_{2}$-agonists.

Analysis 1.9. Comparison 1 Effects of school-based asthma interventions vs usual care, Outcome 9 Corticosteroid dosage and/ or use of add-on therapies (usage of). 
Analysis 1.10. Comparison 1 Effects of school-based asthma interventions vs usual care, Outcome 10 Corticosteroid dosage and/or use of add-on therapies (appropriate usage of).

Analysis 1.11. Comparison 1 Effects of school-based asthma interventions vs usual care, Outcome 11 Health-related quality of life (SMD).

Analysis 1.12. Comparison 1 Effects of school-based asthma interventions vs usual care, Outcome 12 Health-related quality of life (MD).

Analysis 1.13. Comparison 1 Effects of school-based asthma interventions vs usual care, Outcome 13 Withdrawal from the study.

Analysis 2.1. Comparison 2 Effects of school-based asthma interventions vs usual care subgrouped by school type, Outcome 1 Exacerbations leading to emergency department (ED) visits.

Analysis 2.2. Comparison 2 Effects of school-based asthma interventions vs usual care subgrouped by school type, Outcome 2 Absence from school.

Analysis 2.3. Comparison 2 Effects of school-based asthma interventions vs usual care subgrouped by school type, Outcome 3 Withdrawal from the study.

Analysis 3.1. Comparison 3 Effects of school-based asthma interventions vs usual care subgrouped by age of children, Outcome 1 Exacerbations leading to emergency department (ED) visits.

Analysis 3.2. Comparison 3 Effects of school-based asthma interventions vs usual care subgrouped by age of children, Outcome 2 Absence from school.

Analysis 3.3. Comparison 3 Effects of school-based asthma interventions vs usual care subgrouped by age of children, Outcome 3 Withdrawal from the study.

Analysis 4.1. Comparison 4 Effects of school-based asthma interventions vs usual care subgrouped by child socio-economic status (SES), Outcome 1 Exacerbations leading to emergency department (ED) visits.

Analysis 4.2. Comparison 4 Effects of school-based asthma interventions vs usual care subgrouped by child socio-economic status (SES), Outcome 2 Absence from school.

Analysis 4.3. Comparison 4 Effects of school-based asthma interventions vs usual care subgrouped by child socio-economic status (SES), Outcome 3 Withdrawal from the study.

Analysis 5.1. Comparison 5 Effects of school-based asthma interventions vs usual care subgrouped by involvement of school staff in direct delivery of self-management skills to children, Outcome 1 Exacerbations leading to emergency department (ED) visits.

Analysis 5.2. Comparison 5 Effects of school-based asthma interventions vs usual care subgrouped by involvement of school staff in direct delivery of self-management skills to children, Outcome 2 Absence from school.

Analysis 5.3. Comparison 5 Effects of school-based asthma interventions vs usual care subgrouped by involvement of school staff in direct delivery of self-management skills to children, Outcome 3 Withdrawal from the study.

Analysis 6.1. Comparison 6 Effects of school-based asthma interventions vs usual care subgrouped by explicit use of theory, Outcome 1 Exacerbations leading to emergency department (ED) visits.

Analysis 6.2. Comparison 6 Effects of school-based asthma interventions vs usual care subgrouped by explicit use of theory, Outcome 2 Absence from school.

Analysis 6.3. Comparison 6 Effects of school-based asthma interventions vs usual care subgrouped by explicit use of theory, Outcome 3 Withdrawal from the study.

Analysis 7.1. Comparison 7 Effects of school-based asthma interventions vs usual care subgrouped by whether design included active inclusion or participation of parents, Outcome 1 Exacerbations leading to emergency department (ED) visits.

Analysis 7.2. Comparison 7 Effects of school-based asthma interventions vs usual care subgrouped by whether design included active inclusion or participation of parents, Outcome 2 Absence from school.

Analysis 7.3. Comparison 7 Effects of school-based asthma interventions vs usual care subgrouped by whether design included active inclusion or participation of parents, Outcome 3 Withdrawal from the study.

Analysis 8.1. Comparison 8 Effects of school-based asthma interventions vs usual care subgrouped by timing of intervention, Outcome 1 Exacerbations leading to emergency department (ED) visits.

Analysis 8.2. Comparison 8 Effects of school-based asthma interventions vs usual care subgrouped by timing of intervention, Outcome 2 Absence from school.

Analysis 8.3. Comparison 8 Effects of school-based asthma interventions vs usual care subgrouped by timing of intervention, Outcome 3 Withdrawal from the study.

Analysis 9.1. Comparison 9 Effects of school-based asthma interventions vs usual care subgrouped by configuration of conditions, Outcome 1 Exacerbations leading to emergency department (ED) visits.

Analysis 9.2. Comparison 9 Effects of school-based asthma interventions vs usual care subgrouped by configuration of conditions, Outcome 2 Absence from school.

Analysis 9.3. Comparison 9 Effects of school-based asthma interventions vs usual care subgrouped by configuration of conditions, Outcome 3 Withdrawal from the study. 
Analysis 10.1. Comparison 10 Effects of school-based asthma interventions vs usual care subgrouped by number of consistent conditions (use of theory, parental involvement, not in own time), Outcome 1 Exacerbations leading to emergency department (ED) visits.

Analysis 10.2. Comparison 10 Effects of school-based asthma interventions vs usual care subgrouped by number of consistent conditions (use of theory, parental involvement, not in own time), Outcome 2 Absence from school.

Analysis 10.3. Comparison 10 Effects of school-based asthma interventions vs usual care subgrouped by number of consistent conditions (use of theory, parental involvement, not in own time), Outcome 3 Withdrawal from the study.

Analysis 11.1. Comparison 11 Adjunct analyses - impact of Implementation on selected outcomes, Outcome 1 Exacerbations leading to emergency department (ED) visits.

Analysis 11.2. Comparison 11 Adjunct analyses - impact of Implementation on selected outcomes, Outcome 2 Absence from school.

Analysis 12.1. Comparison 12 Effects of school-based asthma interventions vs usual care, including disaggregated effect sizes, Outcome 1 Exacerbations leading to hospitalisation - standardised mean difference.

Analysis 12.2. Comparison 12 Effects of school-based asthma interventions vs usual care, including disaggregated effect sizes, Outcome 2 Exacerbations leading to hospitalisation - odds ratio.

Analysis 12.3. Comparison 12 Effects of school-based asthma interventions vs usual care, including disaggregated effect sizes, Outcome 3 Exacerbations leading to hospitalisation - harmonised effect sizes.

Analysis 12.4. Comparison 12 Effects of school-based asthma interventions vs usual care, including disaggregated effect sizes, Outcome 4 Exacerbations leading to emergency department (ED) visits - standardised mean difference.

Analysis 12.5. Comparison 12 Effects of school-based asthma interventions vs usual care, including disaggregated effect sizes, Outcome 5 Exacerbations leading to emergency department (ED) visits - odds ratio.

Analysis 12.6. Comparison 12 Effects of school-based asthma interventions vs usual care, including disaggregated effect sizes, Outcome 6 Exacerbations leading to emergency department (ED) visits - harmonised effect sizes.

Analysis 12.7. Comparison 12 Effects of school-based asthma interventions vs usual care, including disaggregated effect sizes, Outcome 7 Absence from school - standardised mean difference.

Analysis 12.8. Comparison 12 Effects of school-based asthma interventions vs usual care, including disaggregated effect sizes, Outcome 8 Absence from school - odds ratio.

Analysis 12.9. Comparison 12 Effects of school-based asthma interventions vs usual care, including disaggregated effect sizes, Outcome 9 Absence from school - harmonised effect sizes.

Analysis 12.10. Comparison 12 Effects of school-based asthma interventions vs usual care, including disaggregated effect sizes, Outcome 10 Days of restricted activity - standardised mean difference.

Analysis 12.11. Comparison 12 Effects of school-based asthma interventions vs usual care, including disaggregated effect sizes, Outcome 11 Days of restricted activity - odds ratio.

Analysis 12.12. Comparison 12 Effects of school-based asthma interventions vs usual care, including disaggregated effect sizes, Outcome 12 Days of restricted activity - harmonised effect sizes.

Analysis 12.13. Comparison 12 Effects of school-based asthma interventions vs usual care, including disaggregated effect sizes, Outcome 13 Experience of daytime and night-time symptoms - daytime symptoms - standardised mean difference.

Analysis 12.14. Comparison 12 Effects of school-based asthma interventions vs usual care, including disaggregated effect sizes, Outcome 14 Experience of daytime and night-time symptoms - daytime symptoms - odds ratio.

Analysis 12.15. Comparison 12 Effects of school-based asthma interventions vs usual care, including disaggregated effect sizes, Outcome 15 Experience of daytime and night-time symptoms - daytime symptoms - harmonised effect sizes.

Analysis 12.16. Comparison 12 Effects of school-based asthma interventions vs usual care, including disaggregated effect sizes, Outcome 16 Experience of daytime and night-time symptoms - night-time symptoms - standardised mean difference. ........... Analysis 12.17. Comparison 12 Effects of school-based asthma interventions vs usual care, including disaggregated effect sizes, Outcome 17 Experience of daytime and night-time symptoms - night-time symptoms - odds ratio.

Analysis 12.18. Comparison 12 Effects of school-based asthma interventions vs usual care, including disaggregated effect sizes, Outcome 18 Experience of daytime and night-time symptoms - night-time symptoms - harmonised effect sizes.

Analysis 12.19. Comparison 12 Effects of school-based asthma interventions vs usual care, including disaggregated effect sizes, Outcome 19 Use of reliever therapies, e.g. beta ${ }_{2}$-agonists - odds ratio.

Analysis 12.20. Comparison 12 Effects of school-based asthma interventions vs usual care, including disaggregated effect sizes, Outcome 20 Corticosteroid dosage and/or use of add-on therapies (usage of).

Analysis 12.21. Comparison 12 Effects of school-based asthma interventions vs usual care, including disaggregated effect sizes, Outcome 21 Corticosteroid dosage and/or use of add-on therapies (appropriate usage of).

Analysis 12.22. Comparison 12 Effects of school-based asthma interventions vs usual care, including disaggregated effect sizes, Outcome 22 Health-related quality of life - standardised mean difference.

Analysis 12.23. Comparison 12 Effects of school-based asthma interventions vs usual care, including disaggregated effect sizes, Outcome 23 Health-related quality of life (MD).

School-based self-management interventions for asthma in children and adolescents: a mixed methods systematic review (Review)

Copyright (c) 2019 The Cochrane Collaboration. Published by John Wiley \& Sons, Ltd.

241 
Analysis 12.24. Comparison 12 Effects of school-based asthma interventions vs usual care, including disaggregated effect sizes, Outcome 24 Unplanned visit to hospital or GP due to asthma symptoms - standardised mean difference.

Analysis 12.25. Comparison 12 Effects of school-based asthma interventions vs usual care, including disaggregated effect sizes, Outcome 25 Unplanned visit to hospital or GP due to asthma symptoms - odds ratio.

Analysis 12.26. Comparison 12 Effects of school-based asthma interventions vs usual care, including disaggregated effect sizes, Outcome 26 Unplanned visit to hospital or GP due to asthma symptoms - harmonised effect sizes.

Analysis 12.27. Comparison 12 Effects of school-based asthma interventions vs usual care, including disaggregated effect sizes, Outcome 27 Withdrawal from the study.

ADDITIONAL TABLES

APPENDICES

WHAT'S NEW 
[Intervention Review]

\section{School-based self-management interventions for asthma in children and adolescents: a mixed methods systematic review}

Katherine Harris ${ }^{1}$, Dylan Kneale², Toby J Lasserson ${ }^{3}$, Vanessa M McDonald 4 , Jonathan Grigg ${ }^{1}$, James Thomas ${ }^{2}$

${ }^{1}$ Centre for Child Health, Blizard Institute, Queen Mary University of London, London, UK. 2EPPI-Centre, Social Science Research Unit, UCL Institute of Education, University College London, London, UK. ${ }^{3}$ Editorial \& Methods Department, Cochrane Central Executive, London, UK. ${ }^{4}$ School of Nursing and Midwifery, Priority Reseach Centre for Asthma and Respiratory Disease, The University of Newcastle, Newcastle, Australia

Contact address: Katherine Harris, Centre for Child Health, Blizard Institute, Queen Mary University of London, London, E1 2AT, UK. k.harris@qmul.ac.uk.

Editorial group: Cochrane Airways Group.

Publication status and date: Edited (no change to conclusions), published in Issue 1, 2019.

Citation: Harris K, Kneale D, Lasserson TJ, McDonald VM, Grigg J, Thomas J. School-based self-management interventions for asthma in children and adolescents: a mixed methods systematic review. Cochrane Database of Systematic Reviews 2019, Issue 1. Art. No.: CD011651. DOI: 10.1002/14651858.CD011651.pub2.

Copyright () 2019 The Cochrane Collaboration. Published by John Wiley \& Sons, Ltd.

\section{A B S T R A C T}

\section{Background}

Asthma is a common respiratory condition in children that is characterised by symptoms including wheeze, shortness of breath, chest tightness, and cough. Children with asthma may be able to manage their condition more effectively by improving inhaler technique, and by recognising and responding to symptoms. Schools offer a potentially supportive environment for delivering interventions aimed at improving self-management skills among children. The educational ethos aligns with skill and knowledge acquisition and makes it easier to reach children with asthma who do not regularly engage with primary care. Given the multi-faceted nature of self-management interventions, there is a need to understand the combination of intervention features that are associated with successful delivery of asthma self-management programmes.

\section{Objectives}

This review has two primary objectives.

- To identify the intervention features that are aligned with successful intervention implementation.

- To assess effectiveness of school-based interventions provided to improve asthma self-management among children.

We addressed the first objective by performing qualitative comparative analysis (QCA), a synthesis method described in depth later, of process evaluation studies to identify the combination of intervention components and processes that are aligned with successful intervention implementation.

We pursued the second objective by undertaking meta-analyses of outcomes reported by outcome evaluation studies. We explored the link between how well an intervention is implemented and its effectiveness by using separate models, as well as by undertaking additional subgroup analyses.

\section{Search methods}

We searched the Cochrane Airways Trials Register for randomised studies. To identify eligible process evaluation studies, we searched MEDLINE, Embase, the Cumulative Index to Nursing and Allied Health Literature (CINAHL), PsycINFO, the Cochrane Database of Systematic Reviews (CDSR), Web of Knowledge, the Database of Promoting Health Effectiveness Reviews (DoPHER), the Database of Abstracts of 
Reviews of Effects (DARE), the International Biography of Social Science (IBSS), Bibliomap, Health Technology Assessment (HTA), Applied Social Sciences Index and Abstracts (ASSIA), and Sociological Abstracts (SocAbs). We conducted the latest search on 28 August 2017.

\section{Selection criteria}

Participants were school-aged children with asthma who received the intervention in school. Interventions were eligible if their purpose was to help children improve management of their asthma by increasing knowledge, enhancing skills, or changing behaviour. Studies relevant to our first objective could be based on an experimental or quasi-experimental design and could use qualitative or quantitative methods of data collection. For the second objective we included randomised controlled trials (RCTs) where children were allocated individually or in clusters (e.g. classrooms or schools) to self-management interventions or no intervention control.

\section{Data collection and analysis}

We used qualitative comparative analysis (QCA) to identify intervention features that lead to successful implementation of asthma selfmanagement interventions. We measured implementation success by reviewing reports of attrition, intervention dosage, and treatment adherence, irrespective of effects of the interventions.

To measure the effects of interventions, we combined data from eligible studies for our primary outcomes: admission to hospital, emergency department (ED) visits, absence from school, and days of restricted activity due to asthma symptoms. Secondary outcomes included unplanned visits to healthcare providers, daytime and night-time symptoms, use of reliever therapies, and health-related quality of life as measured by the Asthma Quality of Life Questionnaire (AQLQ).

\section{Main results}

We included 55 studies in the review. Thirty-three studies in 14,174 children provided information for the QCA, and 33 RCTs in 12,623 children measured the effects of interventions. Eleven studies contributed to both the QCA and the analysis of effectiveness. Most studies were conducted in North America in socially disadvantaged populations. High school students were better represented among studies contributing to the QCA than in studies contributing to effectiveness evaluations, which more commonly included younger elementary and junior high school students. The interventions all attempted to improve knowledge of asthma, its triggers, and stressed the importance of regular practitioner review, although there was variation in how they were delivered.

QCA results highlighted the importance of an intervention being theory driven, along with the importance of factors such as parent involvement, child satisfaction, and running the intervention outside the child's own time as drivers of successful implementation.

Compared with no intervention, school-based self-management interventions probably reduce mean hospitalisations by an average of about 0.16 admissions per child over 12 months (SMD $-0.19,95 \% \mathrm{Cl}-0.35$ to $-0.04 ; 1873$ participants; 6 studies, moderate certainty evidence). They may reduce the number of children who visit EDs from $7.5 \%$ to $5.4 \%$ over 12 months (OR $0.70,95 \% \mathrm{Cl} 0.53$ to $0.92 ; 3883$ participants; 13 studies, low certainty evidence), and probably reduce unplanned visits to hospitals or primary care from $26 \%$ to $21 \%$ at 6 to 9 months (OR $0.74,95 \% \mathrm{Cl} 0.60$ to $0.90 ; 3490$ participants; 5 studies, moderate certainty evidence). Self-management interventions probably reduce the number of days of restricted activity by just under half a day over a two-week period (MD 0.38 days $95 \% \mathrm{Cl}-0.41$ to -0.18 ; 1852 participants; 3 studies, moderate certainty evidence). Effects of interventions on school absence are uncertain due to the variation between the results of the studies (MD 0.4 fewer school days missed per year with self-management (-1.25 to $0.45 ; 4609$ participants; 10 studies, low certainty evidence). Evidence is insufficient to show whether the requirement for reliever medications is affected by these interventions (OR 0.52, 95\% Cl 0.15 to 1.81; 437 participants; 2 studies; very low-certainty evidence). Self-management interventions probably improve children's asthma-related quality of life by a small amount (MD 0.36 units higher on the Paediatric AQLQ(95\% CI 0.06 to 0.64; 2587 participants; 7 studies, moderate certainty evidence).

\section{Authors' conclusions}

School-based asthma self-management interventions probably reduce hospital admission and may slightly reduce ED attendance, although their impact on school attendance could not be measured reliably. They may also reduce the number of days where children experience asthma symptoms, and probably lead to small improvements in asthma-related quality of life. Many of the studies tested the intervention in younger children from socially disadvantaged populations. Interventions that had a theoretical framework, engaged parents and were run outside of children's free time were associated with successful implementation.

\section{PLAIN LANGUAGE SUMMARY}

\section{Are asthma self-management interventions effective when delivered in schools for children, and how should they be delivered?}

\section{Background to the question}

Asthma is a common condition among children. Schools are potential sites for developing self-management skills, but evidence that school-based interventions improve asthma control has not been reviewed systematically.

\section{Review question}


We sought to address two questions.

-Which parts of school-based asthma self-management interventions are more likely to make these interventions successful?

-What effect do interventions have on children's asthma control, school attendance, and attendance at GP and hospital settings?

\section{Study characteristics}

We included 55 studies. Thirty three of these studies helped us to gain a better understanding of the best way to deliver an asthma selfmanagement intervention. Thirty three studies helped us to determine whether these interventions are successful in improving children's health and well-being. Eleven studies contributed to both.

\section{Key results}

We included 23 studies in quantitative models measuring children's asthma outcomes (an outcome is something you can measure to find out if an intervention worked). Results show that school-based self-management interventions could improve outcomes such as hospitalisations, emergency department visits, and health-related quality of life. Fewer studies reported improved unplanned medical visits or reduced numbers of days on which children could not do their normal activities. Interventions did not reduce school absences, symptoms, or reliever medication use. The more effective interventions were based on theories about how the intervention might work. Researchers found that including parents in the intervention, making sure children were happy with the intervention, and running the intervention during school hours helped increase fidelity.

\section{Certainty of the evidence}

Studies that measured whether an intervention worked were usually well designed; however sometimes they were difficult to carry out, and some may not have measured outcomes accurately. Reviewers found that some of the studies conducted to understand how an intervention should be delivered were at risk of bias, and certainty of the evidence was generally lower for these studies.

\section{Take-home message}

Evidence suggests that school-based self-management interventions can help children with asthma and can reduce hospital admissions and trips to the emergency department. Study findings suggest that interventions that were based on a theory about how an intervention can be planned and delivered could prove useful in improving children's outcomes, reaching large numbers of children, and keeping dropout rates low, and indicate that those designing interventions should consider factors such as including parents.

This review is current to August 2017. 


\section{SUMMARY OF FINDINGS}

Summary of findings for the main comparison. Effects of school-based asthma interventions compared to usual care for asthma among children and adolescents

\section{Effects of school-based asthma interventions compared to usual care for asthma among children and adolescents}

Patient or population: asthma among children and adolescents

Setting: primary/elementary schools through to high/senior schools

Intervention: effects of school-based asthma interventions

Comparison: usual care

\begin{tabular}{|c|c|c|c|c|c|c|}
\hline \multirow[t]{2}{*}{ Outcomes } & \multicolumn{2}{|c|}{ Anticipated absolute effects ${ }^{\star}(95 \% \mathrm{Cl})$} & \multirow{2}{*}{$\begin{array}{l}\text { Relative effect } \\
(95 \% \mathrm{Cl})\end{array}$} & \multirow{2}{*}{$\begin{array}{l}\text { No. of partici- } \\
\text { pants } \\
\text { (studies) }\end{array}$} & \multirow{2}{*}{$\begin{array}{l}\text { Certainty of } \\
\text { the evidence } \\
\text { (GRADE) }\end{array}$} & \multirow[t]{2}{*}{ Comments } \\
\hline & $\begin{array}{l}\text { Risk with usual } \\
\text { care }\end{array}$ & $\begin{array}{l}\text { Risk with effect of } \\
\text { school-based asthma } \\
\text { interventions }\end{array}$ & & & & \\
\hline \multirow[t]{2}{*}{$\begin{array}{l}\text { Exacerbations leading to } \\
\text { hospitalisation (hospitali- } \\
\text { sations) } \\
\text { assessed with RCT } \\
\text { Follow-up: range } 1 \text { week } \\
\text { to } 12 \text { months }\end{array}$} & & $\begin{array}{l}\text { Mean level of hospital- } \\
\text { isation at post-treat- } \\
\text { ment in the intervention } \\
\text { group was } 0.19 \text { standard } \\
\text { deviations lower than in } \\
\text { the control group }\end{array}$ & \multirow[t]{2}{*}{-} & \multirow[t]{2}{*}{$\begin{array}{l}1873 \\
\text { (6 RCTs) }\end{array}$} & \multirow[t]{2}{*}{$\begin{array}{l}\oplus \oplus \oplus \ominus \\
\text { MODERATE }\end{array}$} & \multirow[t]{2}{*}{$\begin{array}{l}\text { Meta-analysis based on SMD in- } \\
\text { cluding data transformed from OR } \\
\text { (data on median level from Gerald } \\
2006 \text { not included) }\end{array}$} \\
\hline & & ( 0.35 to 0.04 lower) & & & & \\
\hline \multirow{3}{*}{$\begin{array}{l}\text { Asthma symptoms leading } \\
\text { to emergency hospital vis- } \\
\text { its (ED visits) } \\
\text { Follow-up: range } 1 \text { week } \\
\text { to } 12 \text { months }\end{array}$} & \multicolumn{2}{|c|}{ Less than $10 \%$ experience ED visit annually } & \multirow{3}{*}{$\begin{array}{l}\text { OR } 0.70 \\
\text { (0.53 to } 0.92 \text { ) }\end{array}$} & \multirow{3}{*}{$\begin{array}{l}3883 \\
(13 \mathrm{RCTs})\end{array}$} & \multirow{3}{*}{$\begin{array}{l}\oplus \oplus \odot \odot \\
\text { LOWb }\end{array}$} & \multirow{3}{*}{$\begin{array}{l}\text { Data from Gerald } 2006 \text { on median } \\
\text { visits not combined Assumed risk } \\
\text { based on rates over } 12 \text { months } \\
<10 \% \text { based on Horner } 2008 \text {, } \\
\text { McGhan } 2010 \text {, Velsor-Friedrich } \\
2005 \geq 10 \% \text { based on Cicutto } 2013 \text {, } \\
\text { McGhan } 2003\end{array}$} \\
\hline & 75 per 1000 & $\begin{array}{l}54 \text { per } 1000 \\
\text { (41 to } 69)\end{array}$ & & & & \\
\hline & 281 per 1000 & $\begin{array}{l}215 \text { per } 1000 \\
(172 \text { to } 264)\end{array}$ & & & & \\
\hline \multirow{2}{*}{$\begin{array}{l}\text { Unplanned visit to hospi- } \\
\text { tal or GP due to asthma } \\
\text { symptoms (unplanned } \\
\text { medical visits) } \\
\text { Follow-up: range } 1 \text { week }\end{array}$} & \multicolumn{2}{|c|}{ Unplanned visits over 6 to 9 months } & \multirow{2}{*}{$\begin{array}{l}\text { OR } 0.74 \\
(0.60 \text { to } 0.90)\end{array}$} & \multirow{2}{*}{$\begin{array}{l}3283 \\
\text { (5 RCTs) }\end{array}$} & \multirow{2}{*}{$\begin{array}{l}\oplus \oplus \oplus \ominus \\
\text { MODERATEC }\end{array}$} & \multirow{2}{*}{$\begin{array}{l}\text { Unplanned visits over } 6 \text { to } 9 \\
\text { months based on McGhan } 2003 \text {, } \\
\text { Splett 2006; unplanned visits over } \\
12 \text { months based on Cicutto } 2013 \text {, } \\
\text { McGhan } 2010\end{array}$} \\
\hline & 264 per 1000 & $\begin{array}{l}210 \text { per } 1000 \\
\text { (177 to } 244)\end{array}$ & & & & \\
\hline
\end{tabular}

to 12 months

Unplanned visits over 12 months 


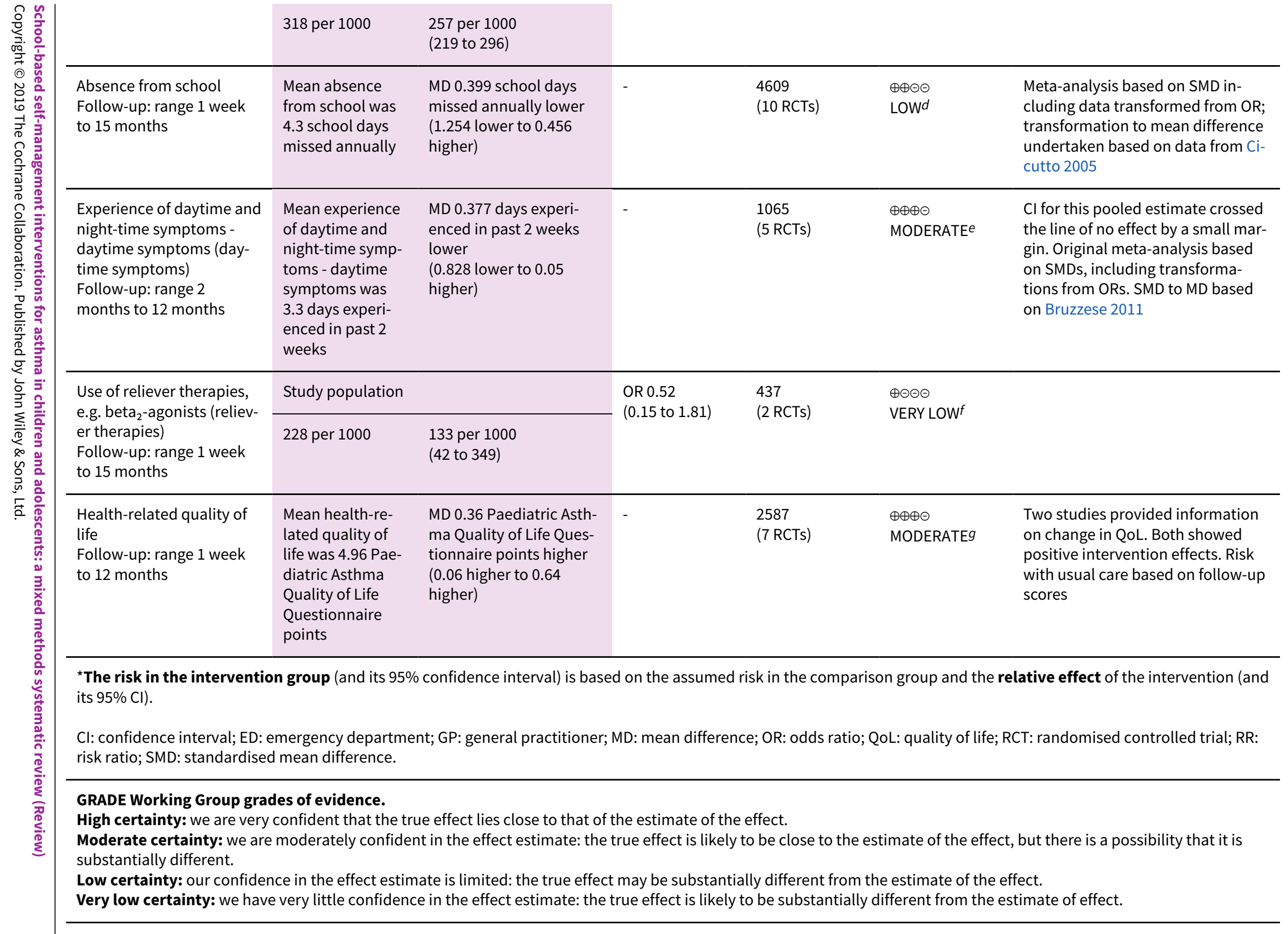

Experience of daytime and daytime symptoms (day-

time symptoms)

Follow-up: range 2

months to 12 month
Study population

133 per 1000

MD 0.36 Paediatric Asth-

uality of Life Ques-

diatric Asthma (0.06 higher to 0.64

Quality of Life higher

Quality of Life

points 
aStudies with high or unclear risk of bias contribute the least to the overall effect size. Hospitalisations may be due to reasons other than asthma (-1 for indirectness).

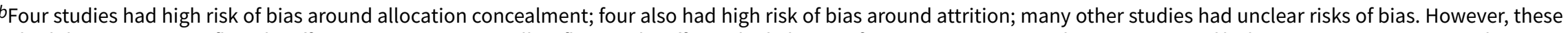
risks did not appear to inflate the effect size nor systematically influence the effect. A high degree of inconsistency was evident, as measured by heterogeneity statistics in the metaanalysis, which was partially explained by subgroup analyses. A large degree of variation was evident in measurement of the outcome, prompting concerns about indirectness; similarly, wide confidence intervals were detected ( 0.53 to 0.95$)$. Study results led to concerns that not all ED visits may be due to asthma (-1 for inconsistency; -1 for indirectness). cNo guarantee that unplanned medical visits were due to asthma (-1 for indirectness).

$d$ School absences could be due to causes other than asthma; heterogeneity statistics suggested a large degree of statistical inconsistency (-1 for indirectness; - 1 for inconsistency).

eHigh risk of bias detected in at least one domain for two out of five studies, which accounted for around a third of the pooled effect size. This included high risk of bias suspected for attrition bias in one study (-1 for risk of bias).

fRisk of bias deemed high for attrition and reporting bias for one of the two studies included in the meta-analysis; very wide confidence interval; although both studies were consistent in the direction of effect, they showed large differences in the magnitude of effect (-1 for risk of bias; - 1 for inconsistency; -1 for imprecision).

gImprecision was deemed to be serious based on the nature of the outcome; five of the seven studies were deemed to have high risk of bias in at least one domain. This included three studies deemed to have high risk of bias for allocation concealment. However, these did not appear to differentially influence the effect size (-1 for imprecision). 


\section{B A C K G R O U N D}

\section{Description of the condition}

Asthma is a chronic respiratory condition characterised by bronchoconstriction, airway inflammation, and mucus hypersecretion leading to variable airflow limitation. Resulting symptoms include wheeze, dyspnoea, cough, and tightness in the chest. No single definitive diagnostic 'test' for asthma is available; instead asthma is diagnosed clinically upon assessment of respiratory symptoms and clinical response to inhaled therapy, and review of evidence of reversible airflow limitation or airway hyper-responsiveness - as in BTS 2016 and Levy 2014 - and elevated exhaled breath nitric oxide - as in NICE 2017. Asthma is the most common chronic disease among children (Neuzil 2000; To 2012), with more than a million children in the UK living with this chronic condition (Asthma UK 2013). Many countries report high prevalence rates of childhood asthma. The International Study of Asthma and Allergy in Children (ISAAC) study, for example, found high prevalence in Australasia and the United Kingdom (Asher 2006). Much of the evidence on non-pharmacological interventions derives from North America, where prevalence is among the highest globally, at $21.5 \%$ and $16.7 \%$ for six- to seven-year-old boys and girls, respectively, and $19.8 \%$ and $23.3 \%$ among children 13 to 14 years of age (Mallol 2013).

In the UK, children from black and white ethnic backgrounds have higher levels of asthma symptoms compared with children from South Asian backgrounds (Netuveli 2005), although substantial variation in the risk of developing asthma has been found within these broad ethnic groups (Kneale 2010). Successful management of asthma among UK children is associated, in part, with social position and socio-economic status. For example, although South Asian children are at lower risk of asthma, they, along with black children, are at higher risk than white children of admission following asthma complications (Netuveli 2005). Indeed a systematic review of socio-economic status and health outcomes found evidence to suggest that the risk of developing asthma is highest among children in the UK from lower-income families (Spencer 2012). Overall, the UK government estimates that a billion pounds is spent annually through the National Health Service (NHS) on treatment and prevention of asthma among adults and children (Department of Health 2012). Thus population-based interventions that improve asthma control have the potential to generate significant savings for the UK NHS

\section{Description of the intervention}

Globally, a large proportion of people with asthma do not receive adequate self-management education and training in primary care, and in England in 2014, more than a quarter of people (adults and children) living with asthma had not undergone an asthma review in the previous 15 months (HSCIC 2014). Moreover, inadequate knowledge of the condition and patient non-adherence with clinician recommendations for asthma treatment (e.g. overuse of long-acting beta ${ }_{2}$-agonists, under-use of inhaled corticosteroids) may contribute towards poor asthma management among children (Piecoro 2001; Walsh 1999).

Children who experience an asthma exacerbation are at risk of hospitalisation and death (Bush 2017). Of the 65,000 hospitalisations for asthma occurring in 2011-2012 in the UK, more than one-third (38\%) occurred in children (aged birth to 14 years); moreover, in an in-depth study of asthma deaths, $14 \%$ (28 of 195) of confirmed deaths from asthma in the UK over a year occurred among children and young people 20 years of age and younger (Levy 2014). Effective self-management of asthma could reduce levels of hospitalisation, which may reduce the financial implications of asthma and improve outcomes for children and adults with asthma, while reducing asthma-related deaths in children.

Living with asthma can impact many other child health and social outcomes, and asthma, particularly severe asthma, is associated with a range of developmental, emotional, and behavioural problems (Blackman 2007). Some studies suggest that children with asthma are disadvantaged in terms of their peer relationships, and other studies report that some children with asthma are bullied (Harris 2017; Wildhaber 2012). Moreover, children with asthma are more likely to limit participation in activities as the result of dyspnoea and other asthma-related symptoms (Van Den Bemt 2011).

Children with asthma tend to have poorer school attendance rates than their peers (Rodriguez 2013). For example, one US study reported that children living with asthma miss an average of 1.5 additional days of school annually compared with their peers, and that increased asthma severity was associated with an increase in the number of days absent from school (Moonie 2006). Furthermore, average school days missed masks large heterogeneity in experience, with some children missing many school days as a result of asthma. A school-based survey, conducted by two members of the review team (KH, JG), assessed current levels of asthma control and school attendance in a sample of 766 children with asthma attending London secondary schools (Harris 2017). Overall, $20.9 \%$ of London school children selfreported at least one school absence due to asthma over a fourweek period. Moreover, children with poor asthma control ( $\mathrm{n}=$ 350) had greater rates of school absence compared to their peers with good asthma control (32.7\% vs $10.9 \%$ ) (Harris 2017). Fowler 1992 found that grade failure is more frequent among children with asthma.

Self-management consists of educating and enabling children to achieve good control over their own asthma symptoms, thereby preventing future exacerbations (Kotses 2010);self-management is viewed as a cornerstone of asthma treatment and care (Bateman 2008; BTS 2016; GINA 2018). Asthma control refers to the degree to which asthma symptoms can be observed and subsequently improved with treatment (GINA 2018). Well-controlled asthma is associated with reduced daytime and night-time symptoms, decreased long-term morbidity, and diminished risk of lifethreatening asthma attacks (Juniper 2006). Asthma control tends to improve with age among children; one study reported excellent or satisfactory control in $38 \%$ of children four to six years of age and in $66 \%$ of children 13 to 16 years of age (Kuehni 2002).

For chronic respiratory diseases, self-management is defined by the British Thoracic Society (BTS) as "the tasks that individuals must undertake to live with chronic conditions, including have the confidence to deal with medical management, role management and emotional management of their conditions" (BTS 2016). For asthma, successful self-management skills include good inhaler technique and ability to recognise and respond to asthma symptoms. Self-management also encourages an alliance between the physician or healthcare professional and the patient for the 
purpose of managing asthma (Kotses 2010). For the purposes of the present review, we have included only self-management studies that provided education on asthma symptoms and their avoidance and management, omitting studies that provided education solely on the nature of asthma.

One main indirect cost of childhood asthma is absence from school, and costs of hospitalisation and of asthma medication drive most of the direct costs of this condition (Bahadori 2009). Although delivery of an asthma self-management intervention in schools has the potential to reduce asthma burden, the effectiveness of this approach across various "proximal" (e.g. improvement in asthma symptoms), "intermediate" (e.g. healthcare usage), and "distal" outcomes (e.g. school achievement) remains unclear (Figure 1). Even when interventions are delivered in similar school settings, several factors can influence success, including variation in treatment settings, study populations, and ways in which schoolbased asthma self-management interventions and intervention components are delivered, in addition to the role of intervention mediators such as changes in school-level policies around asthma or asthma medication (Al Aloola 2014).

Figure 1. Logic model of school-based asthma interventions.

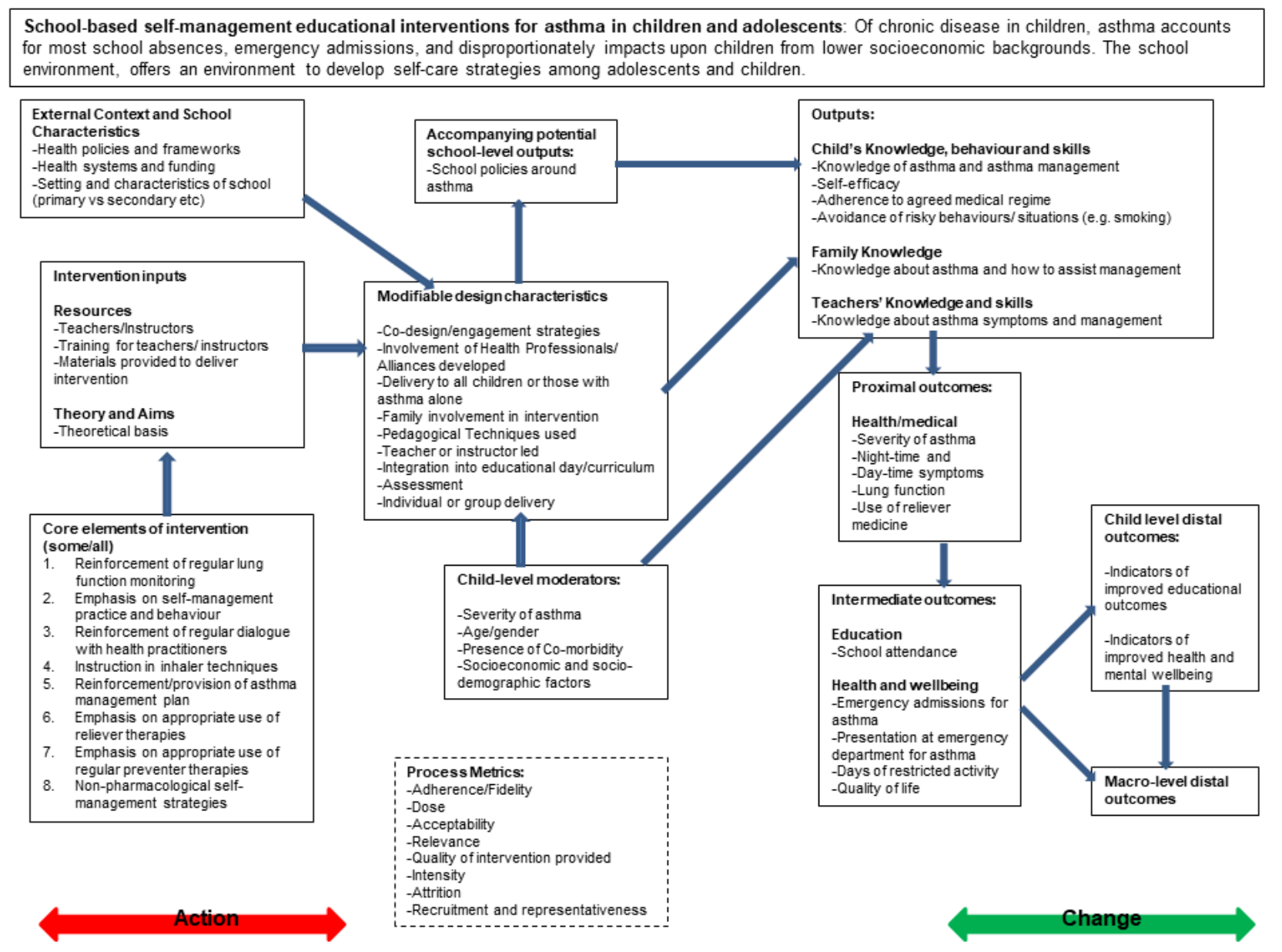

\section{How the intervention might work}

Self-managment works by enabling patients to control their asthma symptoms, thereby preventing future exacerbations and improving their quality of life. Schools are a familiar environment for children's learning, and interventions provided at school have the potential to include large numbers of children with asthma at a single location (Ahmad 2011; Bruzzese 2009; Coffman 2009).

A previous systematic review of self-management interventions delivered in clinic, home, and school environments for children with asthma found that these were positively associated with moderate improvements in lung function, school absenteeism, emergency visits to hospital, and self-efficacy (Guevara 2003).
A separate Cochrane Review reported that targeted selfmanagement interventions can lead to reduced hospital admissions among those at risk of hospitalisation (Boyd 2009). Participants included in both reviews were children from birth to 18 years of age with a diagnosis of asthma. Guevara 2003 excluded children with a pulmonary diagnosis other than asthma. Neither review noted participant comorbidities. Other reviews of selfmanagement interventions for children with asthma suggest that educational interventions delivered to children with asthma can be effective; however, these reviews have considered interventions delivered within schools alongside those delivered in other settings, including the clinic and the home (e.g. Smith 2005; Wolf 2002). Indeed Welsh 2011 points to lack of consensus around the optimal setting for asthma interventions. To date, 
only two systematic reviews have evaluated the evidence for interventions delivered exclusively within schools. These reviews reported a positive impact on school absenteeism but provided less conclusive evidence on the impact on health outcomes such as hospitalisations (Ahmad 2011; Coffman 2009). Notably, both reviews used a narrative approach to synthesis (Ahmad 2011; Coffman 2009). Another review examined outcomes for primary school age children only (Al Aloola 2014).

To date, few reviews have included analyses of accompanying "process-level" measures, such as changes in school policy. Pinnock 2015 is one exception. These review authors explored how asthma self-management interventions should be implemented, although they did not focus on school interventions alone. Nevertheless, based on analysis of two studies conducted in schools, they identified high school turnover and lack of parental involvement as challenges to implementation. Analysis of such process factors would further illuminate the modifiable components of interventions that may be most critical in determining the success (or failure) of interventions, and in mapping out the diverse processes undertaken as part of the intervention.

Systematic reviews of self-management interventions in adults with asthma highlight the importance of gaining a deeper understanding of intervention characteristics and implementation processes. For example, Denford 2013 found that active involvement was associated with greater effect size, but that focus on stress management techniques was potentially counterproductive. Previous studies of self-management in children have focused on child-level moderators. Consequently, the effectiveness of different aspects of school-based interventions for children with asthma is currently unclear.

\section{Background to the methods used in this review}

In this review, we aim to synthesise the evidence for schoolbased interventions by addressing asthma self-management, for the first time, using a mixed methods approach. Mixed methods involves synthesising qualitative and process evaluation evidence, as well as quantitative evidence, in an integrated way. Process evaluation studies explore the implementation, receipt, and setting of an intervention. Although "process" and "qualitative" are often mistakenly used interchangeably, data for process evaluation can be both quantitative and qualitative (Oakley 2006). Process evaluations can be used to develop mechanistic theories around how interventions work, although no universally agreed definition is available for what a process evaluation is and which core components it should include.

Investigators in one study defined a process evaluation as evaluating the quality of the intervention and measuring the disparity between the way in which an intervention was intended to be implemented and the way in which it is actually implemented (Shepherd 2010). This focus on evaluating the processes of delivery and the factors "responsible for successful outcomes, implementation of the intervention, and intervention integrity" is also shared elsewhere (Waters 2006). Meanwhile, UK Medical Research Council guidance on how to conduct process evaluations states that core components of process evaluations include (I) clear description (and evaluation) of implementation and processes of implementation; (ii) clear analysis of the mechanism of impact (participant responses to and interactions with the intervention); and (iii) clear description of context and analysis of how contextual factors affect mechanisms and implementation (Moore 2015).

\section{Qualitative comparative analysis (QCA)}

Although other reviews have set out to apply a mixed methods approach (albeit applied to other health topics) (Hurley 2013; Husk 2016), we sought to review the literature using both metaanalyses of quantitative studies to assess the effectiveness of interventions and qualitative comparative analysis (QCA) to discern the importance of different configurations of intervention features. QCA has its basis in set-theoretic logic, and is a well-placed method for synthesising data from a small number of studies with complex characteristics. This approach aims to uncover the degree of overlap between a set of studies that are successful in their implementation and sets of studies that share different configurations of intervention characteristics. In pursuing the aim described above, we used a logic model to help structure and synthesise review findings (Figure 1), in accordance with the practices described in previous reviews (Glenton 2013).

\section{Logic models}

Logic models are tools that can be used to evaluate the effectiveness of a programme and/or to guide programme planning and implementation (NHS Scotland 2014). The protocol authors developed a logic model to outline some school-based asthma self-management intervention components that may be influential (Figure 1). We developed the logic model from the outcomes to be included in this review, and we worked backwards, theorising the causal chain necessary to lead to these outcomes. We developed the logic model using published literature and systematic reviews, including existing logic models used in studies and policy documents. Use of a logic model assisted us in identifying the types of data that may need to be captured if we are to gain an understanding of intervention components and implementation processes (Kneale 2015). The underlying idea behind a logic model is that a target or final goal is identified, and the preconditions needed to reach this goal are hypothesised as different steps, building up a theorised chain of intervention actions and how they may impact outcomes. The logic model in Figure 1 shows the steps needed to reach the distal (long-term) outcome of improvement in general health, well-being, and educational outcomes among children with asthma; to achieve this long-term outcome, we hypothesise that improvement in more intermediate outcomes such as episodes of healthcare usage and school absences is needed; to achieve improvement in these outcomes, we would expect improvement in asthma symptoms and lung function to be a necessary pre-condition, and, in turn, to improve these, we theorise that children need better knowledge about asthma and improved skill in using inhalers. Changes in children's knowledge and skills follow from exposure to the intervention, although several modifiable intervention design characteristics may cause the intervention to have a differential impact, and may influence the characteristics of children themselves and the context in which the intervention takes place. Each intervention however includes various core elements (reflecting our definition of self-management), as well as a set of resources and theories underlying its delivery. In addition, the logic model recognises that interventions can fail to effect change in children's outcomes because of issues of design or implementation, and a box on 'process metrics' incorporates ways of understanding the success of intervention implementation. 


\section{Why it is important to do this review}

Educational impacts attributable to asthma are larger among children from lower socio-economic groups and/or ethnic minority groups (Milton 2004), with children from ethnic minorities more likely than others to report asthma-related hospitalisations (Netuveli 2005). Such differentials may, in part, reflect the failure of existing intervention models to deliver asthma self-management training equitably to children across socio-demographic groups. Given that the school environment offers a platform by which children from all socio-economic backgrounds can receive the same asthma self-management interventions, delivery of asthma self-management interventions at this level could reduce inequalities in self-management. Indeed, schools were previously identified as effective sites for the delivery of asthma selfmanagement interventions because the school environment is commonly associated with learning of new skills. Schools also provide access to large numbers of children with asthma, including those who do not have a general practitioner (GP) and those who do not regularly attend GP appointments. However, 'school age' (usually five to 18 years old) spans a wide spectrum of child development stages and consequently represents different teaching needs and various responses to self-management interventions. Therefore, an understanding of the processes of implementation (and their success) is essential for the development of mechanistic theories of how and why interventions work that can be understood in the context of the child's characteristics.

In planning the current review, we placed strong emphasis on documenting and understanding the different processes that occur during school-based asthma self-management interventions. We envisaged that this approach would help us to understand the different mechanisms involved and would allow future trialists to evaluate the generalisability of processes and outcomes described and measured. The focus on delivery of interventions to help children self-manage their own chronic condition is encouraged by advisory groups to UK policy-makers. They view the integration of health and educational (and social care) services as critical in improving the quality of life of children with chronic conditions such as asthma, and in reducing differentials in outcomes such as school attendance (Lewis 2012). This systematic review draws on a mixed methods approach, looking at different sets of literature that evaluate intervention implementation and effectiveness, and using different methods to combine this literature. This approach will provide a rich account of school-based asthma interventions by examining whether these interventions are effective in changing children's outcomes and by discerning how they effect change.

\section{OB JECTIVES}

This review has two primary objectives.

- To identify the intervention features that are aligned with successful intervention implementation.

- To assess effectiveness of school-based interventions provided to improve asthma self-management among children.

We addressed the first objective by performing qualitative comparative analysis (QCA), a synthesis method described in depth later, of process evaluation studies to identify the combination of intervention components and processes that are aligned with successful intervention implementation.
We pursued the second objective by undertaking meta-analyses of outcomes reported by outcome evaluation studies. We explored the link between how well an intervention is implemented and its effectiveness by using separate models, as well as by undertaking additional subgroup analyses.

\section{METHODS}

\section{Criteria for considering studies for this review}

\section{Types of studies}

We addressed our first objective (to identify intervention components and processes that are aligned with successful intervention implementation) by exploring process evaluation reports. We pursued the second objective (to assess the effectiveness of school-based interventions for improvement of asthma self-management) by examining outcome evaluation reports (i.e. randomised parallel-group design involving individual or cluster randomisation).

\section{Identifying the intervention components and processes aligned with intervention success in process evaluation studies}

In this review, we identified process evaluations as involving systematic measurements to determine the extent to which a particular programme was implemented, in keeping with the guidance described above. Measures of implementation were focused on fidelity and specifically on attrition, adherence, and dosage. To capture the breadth of evidence about implementation, we identified a process evaluation study as (I) a study that was a self-defined "process evaluation"; or (ii) a study that included the elements of a process evaluation as defined in a section of an outcome evaluation; or (iii) a study in which researchers integrated process evaluation data within an outcome evaluation but provided within the results measures around processes that were detailed and extractable. Studies not self-identified as process evaluation studies must have contained (I) an assessment of core components (implementation, mechanisms, context); (ii) clear research questions guiding the process evaluation; and (iii) use of recognised evaluation methods (described by Moore 2015). We also included studies with a focus on the presence/development of school asthma policies (as represented in the logic model (Figure 1)); we expanded this to include studies measuring broader schoollevel commitment. In this way, use of a logic model explicitly impacted study selection decisions (Kneale 2015).

Previous systematic reviews of process evaluation studies have tended to include only process evaluation studies linked to an outcome evaluation (e.g. Murta 2007). In this review, we have linked included process evaluation studies to randomised controlled trials (RCTs) assessing the effectiveness of the intervention; we have also included trials evaluating the implementation of a variety of study designs, provided they met our other inclusion criteria. This allowed us to use process evaluation data for theory development and testing within a mixed method framework.

\section{Publication date and language}

We imposed criteria around the date on which studies were published to help ensure that the content of self-management interventions was broadly reflective of today's recommendations. Recommendations around the management of asthma in the UK were first developed in 1990 on the basis of articles that 
had appeared in British Medical Journal and Archives of Diseases in Childhood, from 1989 onwards (British Asthma Guidelines 1997); recommendations were developed in the USA around the same time (National Institute of Health 1997). Therefore, we excluded studies that pre-dated the impetus around development of guidelines for the management of asthma, and we included only studies published from 1995 onwards (corresponding with publication of the first Global Initiative for Asthma (GINA) guidelines, which provided a foundation for asthma guidelines globally). We included only studies published in English.

\section{Types of participants}

We included school-aged children and young people (five to 18 years old) with asthma. When the intervention included young people and adults (e.g. when provided in colleges with students 16 to 24 years of age), we intended to include these studies only if most participants were 18 years of age or younger (although we observed no such instance). We also included interventions if they incorporated some components that were delivered to peers, teachers, and/or parents and families, although only when they involved at least partial delivery of the intervention to schoolaged participants with asthma within school environments. We included studies reporting on interventions among children and young people with intermittent or mild to severe or persistent asthma.

We did not impose criteria regarding the types of schools that we included in our scope, as long as schools represented the physical location where intervention participants usually received most of their education.

\section{Types of interventions}

We included asthma self-management interventions delivered at school. Eligible interventions aimed to develop and enhance selfmanagement of asthma among children by achieving the following.

- Increasing knowledge of asthma self-management.

- Enhancing self-management skills.

- Improving self-management behaviours and practice.

Eligible interventions must have included the active transfer of information around at least one of the aspects of asthma selfmanagement outlined below. However, we recognise that for asthma self-management to be effective, a combination of these must be incorporated into the interventions.

- Reinforcement of regular monitoring of lung function.

- Emphasis on the importance of self-management practice and behaviour.

- Development of a partnership/alliance between patient and primary care/healthcare practitioners (including school nursing staff) for the management of asthma.

- Instruction on inhaler techniques.

- Reinforcement/provision of an individualised written asthma management plan.

- Emphasis on the importance and appropriate use of reliever

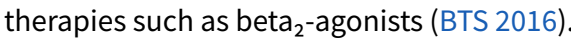

- Emphasis on the importance and appropriate use of regular preventer therapies such as inhaled corticosteroids and combination inhaled corticosteroid and long-acting beta $_{2}$ agonist therapies (BTS 2016).

- Non-pharmacological self-management strategies focused on avoiding or reducing the risk of experiencing asthma or asthma attacks, including lifestyle and behavioural modifications (as set out in BTS 2016).

Interventions that focused only on treating children's asthma in schools, and not on enhancing self-management skills, were not eligible. For example, interventions that provided directly observed therapy but did not seek to actively improve children's selfmanagement skills inside and outside school were not eligible for inclusion. This included studies in which we determined that most of the self-management component of the intervention had not occurred in the school environment. This led to the omission of some studies that otherwise met the inclusion criteria and have been included in previous reviews (e.g. Halterman 2011; Halterman 2012).

Interventions may focus on improving the climate for asthma selfmanagement within schools, for example, by changing school policies around the way that teaching staff may assist in asthma self-management. However, studies that did not also include the development and evaluation of asthma self-management skills and behaviours among children were not eligible. We included self-management interventions if they fit the definition given in the guidelines produced by the British Thoracic Society/Scottish Intercollegiate Guidelines Network, or in the GINA guidelines (BTS 2016; GINA 2018), as described in the Background section. We excluded studies that concentrated on breathing exercise methods (including yoga interventions) if they did not directly focus on the other aspects of self-management listed above.

The intervention could be provided by a trained educator, nurse (including school, practice, or community nurse), doctor or physician, peer, or social worker, and most delivery or access must have been provided on the premises of the school attended by the children. Interventions for which the school setting was not involved in delivery were not eligible for inclusion.

\section{Comparisons}

For outcome evaluation studies, comparison groups were restricted to usual care or to a self-management or health intervention with a focus other than asthma (placebo).

For process evaluation studies, a comparison group could have received another asthma intervention, or the study may not have included a comparison group at all; all process evaluation studies must have included other parameters as described above in terms of study population, study setting, and contents of the asthma intervention.

\section{Types of outcome measures}

\section{Outcomes for meta-analyses}

Our primary outcomes were based on those identified as indicators of good asthma control (BTS 2016), represented as intermediate outcomes in Figure 1. We were also interested in several secondary outcomes (represented as proximal and intermediate outcomes in Figure 1 , as well as a measure of acceptability/implementation in withdrawal from the intervention). 


\section{Primary outcomes}

- Asthma symptoms or exacerbations leading to admission to hospital (children with one or more admissions or high admission rates)

- Asthma symptoms or exacerbations leading to emergency department visits

- Parent-reported absence from school

- Days of restricted activity

\section{Secondary outcomes}

- Unplanned visit to hospital or GP due to asthma symptoms

- Experience of daytime and night-time symptoms ( ${ }^{*}$ these were differentiated from 'any' symptomatology by stating that symptoms occurred either in the daytime or at night-time)

- Lung function (e.g. forced expiratory volume in one second $\left(\mathrm{FEV}_{1}\right)$ in clinic, peak flow at home)

- Use of reliever therapies such as beta ${ }_{2}$-agonists

- Corticosteroid dosage and/or use of add-on therapies (e.g. longacting beta ${ }_{2}$-agonists (LABAs), leukotriene receptor antagonists (LTRAs))

- Health-related quality of life (HRQoL) as measured by a validated questionnaire

- Withdrawal from the study

We extracted data for all points at which the outcomes above were measured and pooled data as appropriate.

\section{Outcomes for qualitative comparative analysis (QCA): defining a successful intervention}

Qualitative comparative analysis (QCA) as used in this review and described in further detail below, is a method of evidence synthesis that enables understanding of which configurations of intervention components and processes trigger successful outcomes. QCA is predicated upon set theory, and in this context essentially involves exploring the degree of overlap between a set of successfully implemented studies and a set of studies with a particular range of intervention components and processes.

A first step in our use of QCA was deciding how 'successful' implementation could be identified. Currently, no approach has been established for categorising the implementation of an intervention as 'successful' or 'not successful' (Schellenberg 2012). We began by examining aspects of intervention implementation that were related to intervention fidelity as well as evidence around attrition, dosage, and adherence. A literature review of implementation scoring methods for public health interventions - Schellenberg 2012 - included one study that examined the implementation of a complex intervention that included a school component (Rosecrans 2008). Study authors used the following criteria: "process indictors for which standards were set, such as fidelity (e.g. \% of minimum foods stocked) or dose received (e.g. $\%$ of family pack cards completed and returned), were assigned to categories of implementation as follows: low (0-49\%), moderate (50-74\%) or high (75-100\%)" (Rosecrans 2008; p75). This $75 \%$ threshold also corresponds with the $25 \%$ attrition rate that is often incorporated within study sample size calculations for public health trials involving children (Berry 2013; Bruzzese 2011; Clark 1986).
A $75 \%$ threshold formed the basis of our coding scheme for the outcome, by which $75 \%$ was used as a cross-over point for a 'high' or 'successful' implementation score. Implementation reflected reports of attrition, dosage, and adherence. For each of these indicators, we set values by using a blend of direct and transformational assignment (see Table 1), whereby we assigned values to qualitative data and then calibrated all data using transformational assignment. This blended approach was necessary to combine qualitative and quantitative data. To derive an outcome variable that reflected intervention implementation more holistically, we aggregated the three separate indicators into a single outcome variable by adding each separate value and calibrating the summed score. This outcome value reflected the mainstay of the analyses and distinguished our successfully implemented intervention set.

\section{Search methods for identification of studies \\ Electronic searches}

We searched the Cochrane Airways Group Specialised Register (see Appendix 1) for trials, using the strategy presented in Appendix 2 , which was developed by the Cochrane Airways Information Specialist (Liz Stovold). We conducted searches in April 2015 and updated them in April 2016. We conducted further searches on 25 August 2017.

We searched the databases below for process evaluations for our qualitative comparative analyses, using the search criteria identified in Appendix 1, although we modified these criteria to account for the different search syntax/parameters used in additional databases (see Appendix 3, Appendix 4, Appendix 5, Appendix 6, and Appendix 7 for example search strategies).

- Database of Promoting Health Effectiveness Reviews (DoPHER).

- Cochrane Database of Systematic Reviews (CDSR).

- Database of Abstracts of Reviews of Effects (DARE).

- The Campbell Library.

- National Institute for Health Research (NIHR) Health Technology Assessment (HTA) Programme website/journals library.

- Health Technology Assessment (HTA) database.

We applied search strategies to a comprehensive search of the following clinical, public health, psychology, and social care databases from 1995 to the present* .

- Allied and Complementary Medicine Database (AMED)

- Applied Social Sciences Index and Abstracts (ASSIA).

- Bibliomap (EPPI-Centre Database of Health Promotion Research).

- ClinicalTrials.gov

- Cochrane Database of Systematic Reviews (CDSR).

- Cochrane Central Register of Controlled Trials (CENTRAL).

- Cumulative Index to Nursing and Allied Health Literature (CINAHL).

- Excerpta Medica dataBASE (EMBASE)

- Health Management Information Consortium (HMIC).

- International Bibliography of the Social Sciences (IBSS).

- National Health Service Economic Evaluation Database (NHS EED).

- Psychlnfo. 
- PubMed.

- Sociological Abstracts (SocAbs).

- Social Policy and Practice (SPP).

- Social Services Abstracts

- Web of Knowledge.

*MEDLINE, the Cumulative Index to Nursing and Allied Health Literature (CINAHL), Embase, the Allied and Complementary Medicine Database (AMED), and PsycINFO are included within the Cochrane Airways Group Specialised Register search.

\section{Searching other resources}

We handsearched Google Scholar, Social Policy Digest (for content up to 2014), and other sources such as the British Thoracic Society and Asthma UK for further studies.

We initially identified integral process evaluations (sibling studies) through backwards and forwards citation searches. As expected, we identified multiple process evaluations for some intervention studies; our strategy also allowed for inclusion of process evaluations without linkage to a trial included for quantitative analyses.

\section{Data collection and analysis}

\section{Selection of studies}

We piloted criteria for title and abstract screening on a random subset of studies for which the review authors who were involved in screening (DK, $\mathrm{KH})$ took part in moderation exercises; we resolved disagreements by discussion and developed a shared understanding of the inclusion criteria. We achieved an agreement rate exceeding $90 \%$ in three consecutive samples before we proceeded to independent screening $(\mathrm{DK}, \mathrm{KH})$. We also employed priority screening (text mining) for independent title and abstract screening (Thomas 2011), after achieving a sufficiently high agreement rate, to locate likely included studies more quickly. However, both review authors (DK, KH) screened all abstracts.

We applied inclusion criteria successively to titles and abstracts, and to full reports. We obtained full-text reports when studies appeared to meet the criteria for title and abstract, or when information was insufficient for a decision. For outcome evaluation studies, screening criteria covered populations (children five to 18 years of age), disease status (asthma), interventions (schoolbased and focused on self-management), comparators (usual care or placebo), study design (randomised controlled trials or cluster randomised controlled trials), date (publication year after 1995), and language (English language). We entered full-text reports into EPPI-Reviewer and reapplied the inclusion criteria (Thomas 2010); we included studies that met these study design criteria (irrespective of the actual outcomes collected). We developed a similar set of inclusion criteria for process evaluation studies covering populations, disease status, interventions, date, and language; additional criteria stipulated that studies must include the core components expected within a process evaluation and must use structured or recognised tools to collect data.

\section{Data extraction and management}

\section{Data management}

We uploaded records identified by searches to the specialist systematic review software EPPI-Reviewer 4 for duplicate stripping and screening (Thomas 2010). This software recorded the bibliographic details of each study considered in the review, the origins of all studies (including search strings), and reasons for their inclusion or exclusion. We first extracted all data into EPPI-Reviewer 4 and later exported them, as appropriate, into other software for synthesis (RevMan 2014; StataCorp 2013; Thiem 2013).

\section{Extraction and management of data from process evaluation studies}

\section{Process evaluation measures - data selection}

\section{Overall approach}

The primary aim of exploring process evaluations using QCA was to identify the combinations of components and processes undertaken for interventions that were associated with successful intervention implementation. QCA is based on set theory, and, in this review, we explored the extent of overlap between a set of studies with successful implementation (our process outcome) and sets of studies that share combinations of different intervention components and processes. We presented extracted intervention components and processes (equivalent to antecedents and referred to as conditions from hereon in, in line with QCA terminology) as modifiable design characteristics in the logic model (Figure 1).

Extracting data and building the data table: initial data reduction and assignment of values

Two review authors (DK, KH) independently extracted the conditions (process evaluation measures) of interest from eligible studies. We developed an extensive data table of information supporting over 90 conditions for each study. These data represented quantitative indicators (showing the level of presence of a condition (e.g. the proportion of children from an ethnic minority recruited into an intervention)); binary indicators (representing whether or not a condition was present (e.g. study authors reported that the asthma curriculum contained information on lung physiology)); or qualitative statements (e.g. when study authors published quotes illustrative of child satisfaction with the intervention). In accordance with guidance provided by Rihoux and De Meur (Rihoux 2009), we developed a set of rules for assigning values to conditions (Table 1 ); these rules reflect a mixture of direct and transformational assignment (we have provided further explanation and an example in Appendix 8).

\section{Reduction of data on conditions}

We extracted more data than any QCA model could support a problem referred to as 'limited diversity in QCA terminology'. Recognising that many of the conditions extracted were binary indicators of constructs related to the same underlying condition, we implemented cluster analyses of linked items (e.g. elements of the curriculum) to create natural groupings and to reduce the number of conditions included in some models (Thomas 2014). We have displayed original and reduced data for these conditions in Table 2. In addition, we used the logic model presented in Figure 1 to guide our analysis, to rationalise and prioritise the conditions entered into models, and to limit the number of conceptually similar conditions that were entered into models.

\section{Reduction of cases}

Although cluster analysis reduced the number of conditions examined, we made the decision to focus on cases (studies) that 
were coded as providing high- or medium-intensity interventions. We did not explicitly mention this in the protocol (therefore it is reported as a deviation), although this approach is congruent with indicators such as attrition and dosage.

\section{Extraction and management of data from outcome evaluation studies (RCTs)}

\section{Outcome measures - data extraction}

Two review authors (DK, KH) independently extracted study characteristics and numerical outcome data from studies meeting the eligibility criteria of the review. In agreement meetings, review authors resolved discrepancies by discussion; we encountered no disagreements that needed resolution through arbitration by senior members of the review team. When we encountered missing data, we recorded these instances and contacted study authors for further information.

\section{Assessment of risk of bias in included studies}

\section{Assessment of risk of bias in included RCTs}

We assessed how the following sources of bias may affect the results of an individual study.

- Sequence generation: we deemed that studies that used a computer-generated allocation procedure, a random number table, or other recognised low-risk means were at low risk of bias (as advised by the Cochrane tool for assessing risk of bias). We deemed that studies that used items such as clinic visit date or date of birth when the order of treatment group assignment was predictable or open to external influence were at high risk of bias. We described studies for which we were unable to ascertain methods of randomisation and allocation as having unclear risk of bias. Given the potential impact of socio-economic imbalance between cluster sites within the same study, we also considered whether study authors had stratified socio-economic variables.

- Allocation concealment: we deemed that studies for which researchers took measures to prevent disclosure of treatment group assignment, such as off-site allocation or allocation by a third party not involved in the study, were at low risk of bias. For cluster randomised studies, an additional consideration was timing of recruitment into the study in relation to assignment.

- Blinding (performance bias and detection bias): we deemed that studies for which investigators took measures to ensure that personnel collecting data were unaware of participants' treatment group assignment were at low risk of bias. However, given the nature of the intervention and the difficulty involved in blinding recipients, a degree of performance bias may have impacted some outcomes, particularly patient-reported outcomes, and this was unavoidable.

- Handling of missing data and attrition: we deemed that studies for which data sets were complete, or for which reasons for missing data were not related to treatment, were at low risk of bias. When attrition rates were particularly high or imbalanced and unexplained, and only an available case set was presented, we deemed that the study was at high risk of bias. We deemed that studies for which study authors did not report the attrition rate separately for treatment and control groups, and for which we were unable to determine satisfactorily the reasons for withdrawal, were at high risk of bias.
- Selective reporting: we restricted assessments of selective reporting to examination of available data related to outcomes included in the 'Summary of findings' table.

- Other bias: we examined baseline imbalances in the characteristics of participants (see also the first point around stratification) for potential bias. We also looked for evidence of contamination between intervention and control groups. We restricted sensitivity analysis to primary outcomes of the review, and we derived overall judgements for each study at the outcome level.

\section{Assessment of risk of bias in included process evaluation studies}

We assessed the quality of process evaluation studies using elements of two tools. The first tool was developed at the EPPICentre to assess the methodological rigour of 'views' studies that aimed to collect information on people's experiences during trials (Harden 2004). This tool considers seven criteria, including (I) whether the study includes an explicit theoretical framework and/ or literature review; (ii) clearly stated aims and objectives; (iii) a clear description of context; (iv) a clear description of the sample and how it was recruited; (v) a clear description of methods used to collect and analyse data; (vi) attempts made to establish the reliability or validity of data analysis; and (vii) inclusion of sufficient original data to mediate between evidence and interpretation. The second tool, which was developed by the EPPI-Centre to assess the quality of process evaluation data (O'Mara-Eves 2013), assesses (I) methods of data collection; (ii) process evaluation participants as described; (iii) timing of the process evaluation with respect to the intervention; (iv) process evaluation data collection methods; (v) process evaluation data analysis methods; (vi) whether findings were supported by data; (vii) breadth and depth of findings; (viii) the extent to which the process evaluation gave privilege to the views of participants; (ix) reliability of findings; and (x) usefulness of process evaluation. As some of these domains overlap, we combined elements from both tools to assess the quality of process measures. This strategy also covers the main domains that had been set out in the Cochrane Qualitative Methods Group guidance that was current at the time (Hannes 2011).

\section{Assessment of bias in conducting the systematic review}

We conducted the review according to the published protocol (Harris 2015), and we reported deviations from it under Differences between protocol and review.

\section{Measures of treatment effect}

\section{Continuous data}

We planned to calculate mean differences (MDs) when continuous data were measured by the same scale or unit; however, this did not occur for most outcomes (one MD model had been constructed to explore quality of life as an outcome). Instead, when similar outcomes were measured by different scales or units, we used standardised mean differences (SMDs) (Hedges' (adjusted) $g$ ).

\section{Dichotomous dato}

For dichotomous data, we calculated odds ratios (ORs), and, when appropriate, we combined results from different trials. 


\section{Ordinal data}

We planned to analyse ordinal outcomes (such as quality of life scales) as continuous variables; when appropriate thresholds were identified, we analysed these as dichotomous variables.

\section{Count data}

We planned to calculate rate ratios for any count data that we encountered that represented the ratio of events experienced between two groups, such as episodes of hospitalisation or absences from school.

\section{Unit of analysis issues \\ Cluster randomised studies}

We included cluster randomised controlled trials in which schools or classes within schools rather than individuals with asthma were the unit of allocation. As variation in response to treatment between clusters may also be influenced by cluster membership, meaning that cluster members' data can no longer be considered independent of one another, we extracted data when study authors had undertaken analysis that properly adjusted for a clustered design. When study authors provided no intracluster correlation coefficient (ICC), we intended to estimate the ICC and the design effect according to methods recommended in Chapter 16 of the Cochrane Handbook for Systematic Reviews of Interventions (Higgins 2011). No study provided a direct estimate of ICC; however, we selected 0.05 based on the ICC estimate used in one of the included papers to calculate the sample size (McCann 2006). We adjusted effect estimates using methods described in Higgins 2011.

\section{Choice of measurement point}

For trials that reported outcomes at multiple time points, such as at post-test with longer follow-up, we extracted all data and combined in meta-analyses the follow-up points most consistently reported among trials.

\section{Dealing with missing data}

When study characteristics and numerical outcome data were missing from studies, we contacted study authors to request missing information. For quantitative aspects of process evaluations, such as satisfaction or participation data, we applied the same procedure. Recording of the 'missingness' of qualitative data in the process evaluations that we include is more oblique, although we recorded instances in which investigators indicated that the data collected were not reported upon as part of the quality assessment.

\section{Assessment of heterogeneity}

We assessed statistical heterogeneity by using the $\mathrm{I}^{2}$ measure (Higgins 2003). We explored possible sources of variation by conducting prespecified sensitivity and subgroup analyses and performing meta-regression analyses. These included those set out in the protocol (Harris 2015), as well those that we developed from QCAs.

We intended to construct random-effects multi-variate metaregression models using STATA, which would allow us to model the impact of different covariates simultaneously after first exploring the impact of these potential effect size study-level moderators in bivariate models. However, a relatively small number of studies (our largest meta-analysis model included 13 studies) meant that we were unable to extend the modelling in this way without compromising the underlying assumptions.

\section{Assessment of reporting biases}

We recorded the number of studies for which we were not able to ascertain the analysis of data related to our primary outcomes. We also recorded the number of studies for which we were not able to extract process measures, and we assessed the breadth and depth of those studies in terms of information on processes. We selected all process evaluation studies conditionally on addressing process-related research questions, although the core process outcomes included within these did not always match our own selected process outcomes (e.g. some studies addressed different recruitment techniques as a central process of interest, although this focus did not match our own focus).

We plotted the distribution of effect sizes for each (outcome) study against study standard errors as a funnel plot for primary outcomes and based our assessment of publication bias on visual inspection (if 10 or more studies contributed to the outcome); we also undertook formal tests for small-study publication bias using Egger's test (Harbord 2009).

\section{Data synthesis}

\section{Data synthesis - adopting a mixed methods approach}

In the first strand of analyses, we explored which intervention features (components and processes) are associated with successful implementation of an intervention. This first strand involved undertaking qualitative comparative analysis (QCA) to uncover which configurations of these features (known as 'conditions' in QCA terminology) are aligned with successful intervention implementation. The QCA served to generate hypotheses about the importance of different intervention components and processes that were tested in meta-analyses (below). Conditions identified through QCA helped us to identify which conditions matter for implementing an intervention, and structuring the meta-analysis helped us to identify their potential impact on the overall effectiveness of interventions. The possibility that hypotheses were generated and tested on the same dataset was avoided due to very little overlap between studies included in the QCA synthesis and studies included in the meta-analyses.

To examine the effectiveness of school-based asthma selfmanagement interventions in improving children's outcomes, we undertook meta-analyses. We performed subgroup analyses based upon results of the QCA described above.

We undertook the synthesis of process evaluations performed before the RCTs were conducted to remain blinded to the possible impact of specific measures. We further examined the link between implementation and effectiveness by estimating whether interventions defined as 'successful' in terms of their implementation were those with greater effect sizes. These analyses focused on a subgroup of studies adopting diverse designs (as outlined below). 
Data synthesis part 1 - using process evaluation studies for qualitative comparative analysis (QCA) of determinant conditions for successful intervention implementation

Qualitative comparative analysis (QCA) is used to identify configurations of conditions associated with successful intervention implementation. QCA takes a study-based approach (accounting for several of the study's observed characteristics simultaneously) rather than a variable-based approach, so that the focus is on different configurations of conditions (Thomas 2014). As this approach is relatively novel to systematic reviews, we have provided further information on the underlying principles and operationalisation of the approach in Appendix 8. The QCA approach used here aimed to generate theories about components 'sufficient' for triggering successful implementation; 'sufficient' relationships signify that an outcome is triggered in the presence of a sufficient condition or a sufficient condition set, but that other pathways to triggering the outcome may also exist. Here the outcome is successful implementation, and conditions are intervention characteristics and processes. In analysing our data, we followed the steps laid out by others (Ragin 2009; Thomas 2014).

- We began by operationalising our data and creating a set of rules on how data should be coded for creating a data table of intervention characteristics (known as 'conditions' in QCA terminology) and the extent to which an intervention was successfully implemented (the outcome in this case). In the section titled Secondary outcomes, we have described the way in which we derived our outcome variable, and in the section titled Data extraction and management, we have described our coding framework for other intervention characteristics of interest. Two review authors (DK, KH) coded data for each study and grouped the information into separate data tables reflecting different domains of an intervention (i.e. conditions): setting and participants (Table 3); recruitment and retention processes (Table 4); curriculum and pedagogical factors (Table 5); modifiable intervention design features (Table 5); and stakeholder involvement (Table 6). We adopted this strategy to avoid 'limited diversity', whereby too many possible combinations of intervention characteristics are unsupported by observed studies.

- We constructed truth tables that move beyond examining individual studies (i.e. one row per study) to examining configurations of conditions. Configurations could be supported by no studies, one study, or multiple studies. Truth tables also show the extent to which a 'set' of studies belonging to a configuration overlap with the outcome set.

- We checked the quality of the truth tables. For each truth table, we considered whether a spread of positive and negative outcomes was triggered; whether configurations were supported by (multiple) cases (especially for configurations triggering a successful outcome); whether some configurations were counterintuitive and whether some conditions showed identical patterns; and whether some conditions occurred too infrequently. Our most important check involved whether we observed contradictory configurations when evidence suggested that configurations triggered positive and negative outcomes. When we were unable to resolve these issues according to guidance provided in Thomas 2014, the analysis progressed no further (see Appendix 8).

- We then implemented Boolean minimisation to identify the most logically simple expression of a 'pathway' to a successful outcome. A pathway in this case represents a configuration of conditions that is observed to sufficiently trigger an outcome. This solution is based on observed configurations of conditions only and is known as a 'complex solution'.

- When we detected logical remainders, we incorporated these into further models as 'intermediate solutions' to simplify the solution and maintain its theoretical coherence (see Appendix 8). For intermediate solutions, review authors (DK, KH) set expectations on whether the conditions entered were likely to lead to success.

- A sixth stage involved interpretation, when review authors considered the plausibility of the solution and determined whether conclusions were consistent with evidence obtained from individual cases. We constructed a consolidated model, using evidence from preceding models. We checked the quality of the overall solution to ensure that it did not trigger negation of the outcome; we also assessed the parameters of fit and the validity of simplifying assumptions.

We constructed all QCA models using $\mathrm{R}$ and a package developed by Thiem and Dusa (Thiem 2013). We have outlined further details of all steps, as well as the background to the method, in Appendix 8.

\section{Data synthesis part 2 - using RCTs for meta-analyses of effectiveness}

We combined data in Review Manager 5.3 (RevMan 2014), and we conducted some analyses and data transformations in STATA (when we encountered cluster randomised trials, we converted our standard errors using EPPI-Reviewer functions (Thomas 2010)). We expected outcomes to be reported as similar units of analysis, although we encountered several variations and used Chinn's formulae for converting effect sizes and standard errors between SMDs and ORs (Chinn 2000), according to direction provided in the Cochrane Handbook for Systematic Reviews of Interventions (Higgins 2011). In addition, although we had originally specified daytime and night-time symptoms as a single outcome, we split this into two separate outcomes to maintain conceptual coherence.

Occasionally, we could not incorporate some data into the metaanalyses because of methodological difficulties in combining these data (including data based on rank (e.g. median)). Other changes and forms of imputation for missingness included the following: (I) basing the effect size for quality of life from Al-Sheyab 2012 on the $P$ value because of uncertainty regarding the effect size derived from point estimates and the precision provided; (ii) basing effect sizes for Cicutto 2013 on approximations of the numbers of participants in control and treatment groups; and (iii) estimating the numbers in treatment and control arms for Clark 2005 (assuming equal distribution of the overall sample size); we also imputed an OR of 0.996 for a value reported as 1.00 for Clark 2005 for ED visits, so we could combine the information from different models.

\section{Data synthesis part 3: adjunct meta-analyses exploring the link between implementation and effectiveness of school-based asthma self-management interventions}

Methods used by review authors for the adjunct meta-analyses followed the same processes as were used for the main metaanalysis (part 2) in terms of the approaches taken in extracting effect sizes and combining data. The difference between analyses is that results of part 3 are based both on RCTs included in the main analyses (part 2) and on studies included in part 1 that allow for calculation of an effect size for school absences and/or emergency department visits. All studies included here must have included a 
control group and must have allowed for calculation of successful implementation, which we defined in the same way as our QCA analysis (part 2), and represented a combined indicator around attrition, adherence, and dosage.

\section{Rating the certainty of the evidence}

The certainty of evidence rating reflects the extent to which we can be confident that results for review outcomes reflect the true effect (Guyatt 2008). We rated the certainty of evidence for our main outcomes using methods developed by the GRADE (Grades of Recommendation, Assessment, Development and Evaluation) Working Group (http://www.gradeworkinggroup.org/publications/ JCE_series.htm). We considered the possible impact of each of the following factors on our outcomes of interest.

- Risk of bias.

- Imprecision.

- Inconsistency.

- Indirectness.

- Publication bias.

We attempted to identify a representative control group risk to illustrate the effects of our meta-analysis results in absolute terms. We tabulated GRADE ratings alongside absolute and relative effects in a 'Summary of findings' (SoF) table for the following outcomes.

- Asthma symptoms or exacerbations leading to admission to hospital.

- Asthma symptoms or exacerbations leading to emergency department (ED) visits.

- Unplanned visit to hospital or GP due to asthma symptoms.

- School absence.

- Experience of daytime symptoms.

- Use of reliever therapies such as beta ${ }_{2}$-agonists.

- (Health-related) quality of life.

We generated the SoF table using the GRADE Guideline Development tool (GDT). We have described elsewhere further analyses undertaken to explore heterogeneity in effect size.

\section{Subgroup analysis and investigation of heterogeneity}

We conducted a statistical test for heterogeneity across subgroups using an $\mathrm{I}^{2}$ statistic. We planned to construct a multi-variate metaregression model based on our results for different outcomes. However, the small number of included studies precluded this possibility. We undertook prespecified subgroup analyses to investigate heterogeneity on the basis of the following characteristics, which are represented in our logic model as child-level, school-level, and contextual moderators, as well as modifiable design characteristics of the intervention itself, which we identified on the basis of QCA.

- Setting: elementary/primary school versus secondary/high school.

- Age: five to 10 years; 11 to 15 years; 16 years and older.

- Socio-economic level: low or mixed/high/unclear.

- Delivery of intervention: healthcare provider (e.g. health educator, school nurse, other healthcare professional) versus other professional (e.g. teacher, mixture) versus other model of delivery (e.g. peer led).
- Other (prespecified): intervention moderators developed from hypotheses generated through syntheses of process evaluation data including whether the intervention was theory driven, whether parents were actively involved, and the timing of the intervention during the school day. We entered these as single conditions and as groups reflecting configurations.

We measured some indicators, such as socio-economic status, very differently, and we used broad groupings based on income, social class, or other indicators of social position, such as having received means tested benefits.

\section{Sensitivity analysis}

We undertook sensitivity analyses on the basis of the following.

- Risk of bias assessment: we included all studies in the primary analysis and restricted included studies to those that were not classed as having high risk of bias for any single domain.

- Fixed-effect modelling.

- Exclusion of cluster study data from outcomes (originally intended when external or imputed ICCs had been used, although this applied to most included cluster RCTs).

We did not plan to apply an equivalent for QCA modelling, although we did conduct robustness checks, including whether solutions predicted negation of the outcome.

We had intended to run sensitivity analyses based on the severity of children's asthma; however, no intervention specifically targeted children at particular levels of asthma severity, and inconsistent and low levels of reporting of asthma severity meant that we did not conduct these analyses. We have reported elsewhere other deviations from the protocol.

\section{RES U LTS}

\section{Description of studies}

We have reported the characteristics of all included studies in the Characteristics of included studies section; Table 7 presents an additional summary of how process evaluations met the review inclusion criteria.

\section{Results of the search}

We performed the first search in April 2015, and an updated search in April 2016. We conducted further searches on 25 August 2017. Two members of the review team (KH, DK) conducted the searches for process evaluation studies (see Figure 2). The Cochrane Airways Information Specialist, Liz Stovold, conducted the searches for outcome evaluation studies (see Figure 3). Review team members (KH, DK) performed initial automated checks for duplication using EPPI-Reviewer software during the data screening and extraction process. After de-duplication, we (KH, DK) screened 29,384 titles and abstracts of potential process evaluation studies, facilitated by text mining, as well as 350 title and abstracts for eligibility as outcome evaluations. Following application of inclusion criteria to review of titles and abstracts, $\mathrm{KH}$ and DK independently assessed the remaining 1066 full-text process evaluation records and 105 full-text outcome evaluation records for eligibility for inclusion. We included 54 papers, from 33 different studies, for further analysis as process evaluation studies, and 44 papers, from 33 different studies, for further analysis as outcome evaluation studies. 
Figure 2. Process evaluation study flow diagram.

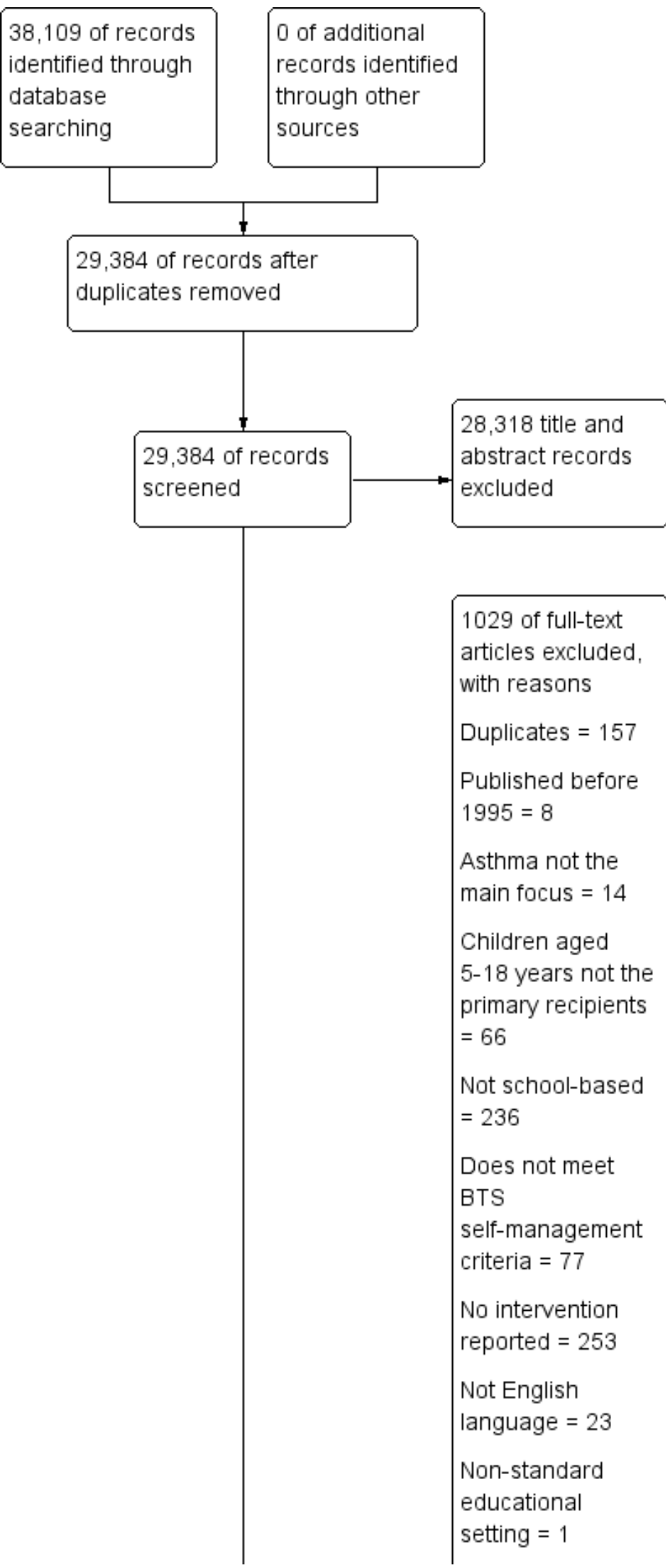


Figure 2. (Continued)

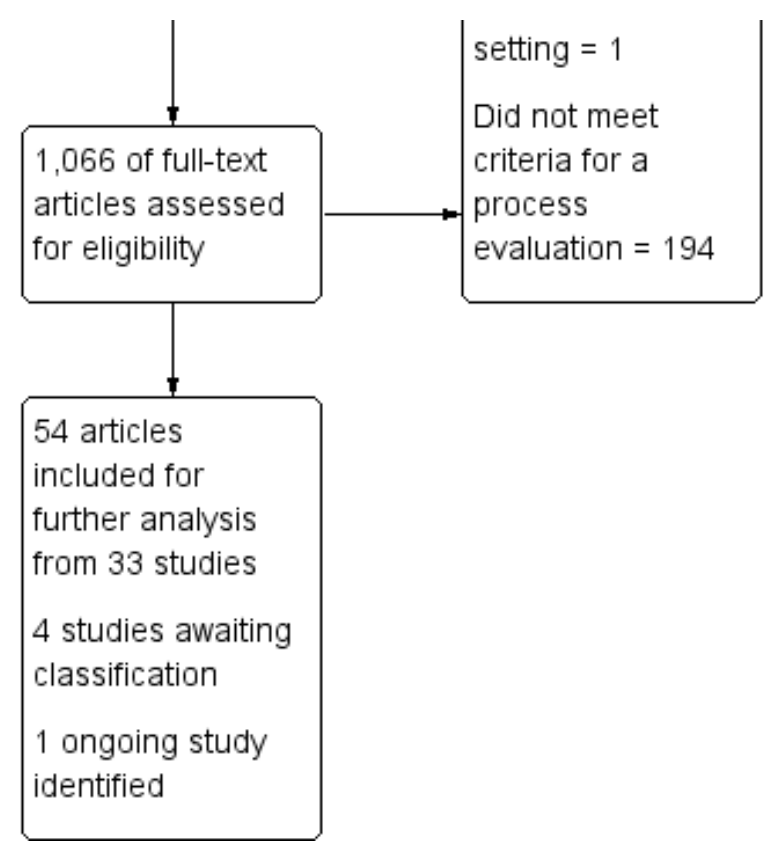


Figure 3. Outcome evaluation study flow diagram.

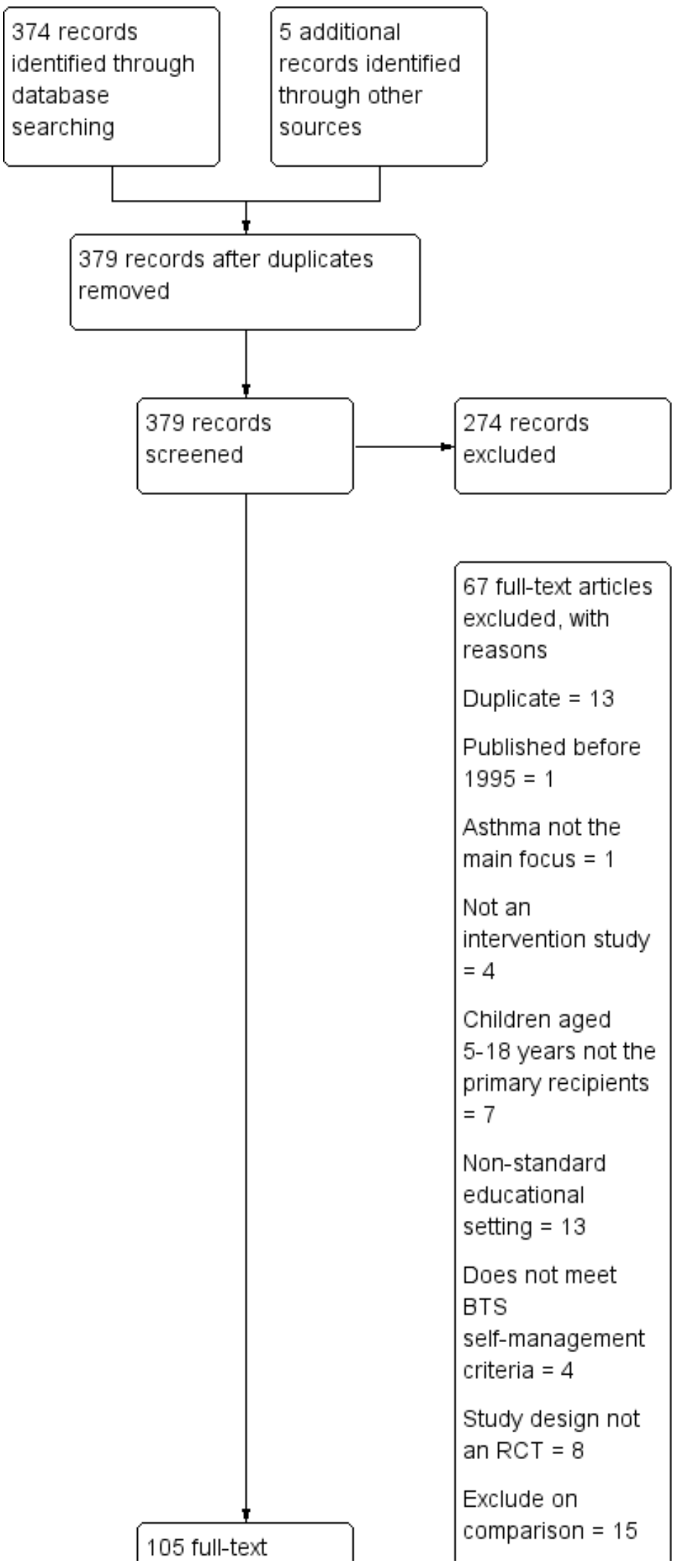


Figure 3. (Continued)

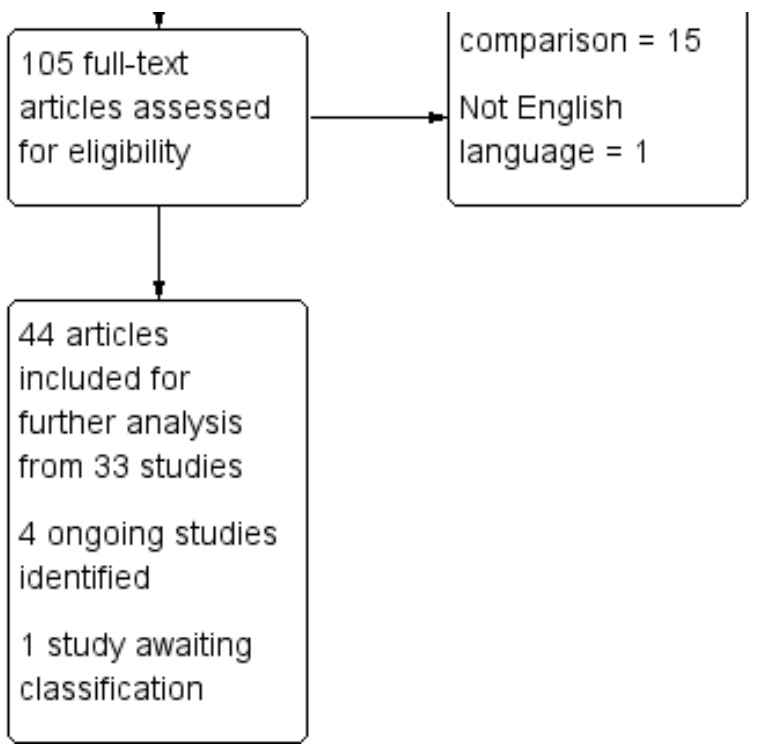

We identified several potential additional sources as ongoing studies ( $n=4$; see Characteristics of ongoing studies) and other studies as awaiting classification ( $n=5$; see Characteristics of studies awaiting classification).

\section{Included studies}

We included in the review 33 process evaluation studies and 33 outcome evaluation studies that met the inclusion criteria. We have described the characteristics of process and outcome evaluation studies separately below. We noted little overlap between the 33 studies included in both sets of studies, with Bruzzese 2004, Bruzzese 2008, Bruzzese 2011, Cicutto 2013, Gerald 2006, Henry 2004, Horner 2015, Howell 2005, Levy 2006, McCann 2006, and Splett $2006(11 / 33)$ common to both sets of studies, although Bruzzese 2004 and McCann 2006 did not contribute data to the meta-analyses.

\section{Characteristics of process evaluation studies}

\section{Study population and intervention characteristics}

Process evaluations of asthma self-management interventions in schools reported on a diversity of intervention models. Nine studies included evaluations of the effectiveness of Open Airways for Schools (OAS) (American Lung Association 2018), or modifications to this programme (see Table 8). OAS consists of six 40-minute sessions, aimed at groups of children aged eight to 11 who learn about different topics including general information about asthma, how to recognise and manage asthma symptoms, and problem-solving and decision-making about asthma medication. Authors of process evaluation studies described other intervention models (e.g. PowerBreathing (Berg 2004); Staying Healthy-Asthma Responsible and Prepared (SHARP; Kintner 2012); Asthma SelfManagement for Adolescents (ASMA; Bruzzese 2004; Bruzzese 2008)), although these were diffuse across studies and were common to no more than two included studies.

Across all studies, investigators taught a diverse curriculum. Although most studies mentioned that the intervention involved developing knowledge and skills around asthma physiology and monitoring and treatment of symptoms, fewer included studies explicitly mentioned that investigators aimed to develop alliances between children/parents and their care provider(s) (Dore-Stites 2007; Gerald 2006; Richmond 2011; Terpstra 2012), and a greater number did involve parents in the intervention in other ways. Most interventions were reliant on trialists, research staff, and others from outside schools to deliver the intervention, although some interventions were primarily delivered, or supported pivotally, by school nurses (Engelke 2013; Langenfeld 2010; Levy 2006; Magzamen 2008; Splett 2006), teachers (Henry 2004; Mujuru 2011; Pike 2011), or children's peers (Magzamen 2008).

Several studies explicitly drew on social cognitive theory (Bruzzese 2008; Bruzzese 2011; Cicutto 2013; Terpstra 2012). Two studies from the same research team drew upon the Health Belief Model (Joseph 2010; Joseph 2013). Other theoretical models featured in only a single study included self-regulation theory (Bruzzese 2004), learning or social learning theory (Berg 2004; Howell 2005), Piaget's pedagogical theory (Crane 2014), Orem's self-care deficit theory (Kouba 2012), attribution theory (Joseph 2013), miscellaneous theoretical concepts that contributed to a theoretical framework (Al-Sheyab 2012a), biopsychosocial theory (Dore-Stites 2007), a transtheoretical model (Joseph 2010), and a functional context model (Lee 2011). A small minority of studies named a theoretical framework that was specific to asthma, with Horner 2015 employing Bruhn's theoretical model of asthma self-management to underpin an intervention (Bruhn 1983), and Kintner 2012 drawing upon an asthma acceptance model (alongside a life course development perspective). These theoretical frameworks also differed in their use and in whether they supported the premise and emphasis of the intervention in a holistic manner, or whether they supported a particular pedagogical technique that was favoured in delivery of the intervention; this distinction was not clear in some studies. Few studies presented a clear logic model or theory of change to describe the underlying conceptual framework (Kneale 2015).

Five studies evaluated implementation of interventions involving delivery of self-management education in part or mainly through electronic games or training provided through computers (Dore- 
Stites 2007; Howell 2005; Joseph 2010; Joseph 2013; Kouba 2012). In two of these interventions (Joseph 2010; Joseph 2013), the information provided was tailored to children based on their input. In total, nine interventions had components that tailored content towards the needs of an individual child through delivery on a oneto-one basis or through delivery of personalised content (Bruzzese 2004; Bruzzese 2008; Howell 2005; Joseph 2010; Joseph 2013; Langenfeld 2010; Spencer 2000; Splett 2006).

Most studies took place in the USA (29/33 studies); several of these US-based studies explicitly mentioned that the intervention took place in an urban or inner city area, or explicitly made reference to the diverse socio-economic or ethnic background of participants (Berg 2004; Bignall 2015; Brasler 2006; Bruzzese 2004; Bruzzese 2010; Bruzzese 2011; Gerald 2006; Joseph 2010; Joseph 2013; Kouba 2012; Levy 2006; Magzamen 2008; Mickel 2016; Pike 2011; Richmond 2011; Splett 2006); in contrast, just two studies specifically explored implementation in rural areas (Horner 2015; Mujuru 2011). Fewer studies took place in high schools (14 studies) than in junior, middle, or elementary/primary schools (see Table 9).

\section{Time of assessment of process outcome measurements}

Twenty-one process evaluation studies collected pre- and posthoc data. Four studies collected post-hoc data only (Al-Sheyab 2012a; Berg 2004; Bruzzese 2004; Richmond 2011). Several studies collected data immediately after the intervention or within three months of cessation of the intervention (Bignall 2015; Bruzzese 2004; Bruzzese 2008; Carpenter 2016; Crane 2014; Gerald 2006; Howell 2005; Jackson 2006; Kintner 2012; Kouba 2012; Magzamen 2008; Mickel 2016; Mujuru 2011; Pike 2011; Spencer 2000; Splett 2006). The longest follow-up data collection period lasted for 12 months post testing (Bruzzese 2011; Cicutto 2013; Horner 2015; Joseph 2010; Joseph 2013; McCann 2006). In a small number of studies, the follow-up duration was unclear (Al-Sheyab 2012a; Dore-Stites 2007; Engelke 2013; Langenfeld 2010; Levy 2006; Richmond 2011; Terpstra 2012).

\section{Measurement of process outcomes}

We included 33 process evaluation studies, most of which adopted a quantitative approach to analyses. Process evaluation elements across these studies included thematic analysis of student perceptions, identification of implementation challenges and facilitators, reach of the intervention, and student satisfaction. We have provided further details of inclusion criteria and process evaluation elements for all process evaluation studies in Table 7 The descriptions below refer to all studies included as process evaluation studies, although we included in QCAs only those that we deemed to be of moderate or high intensity (see section on reduction of cases). Similarly, we transformed the data and ratings described below using direct and indirect transformations (see earlier methods).

\section{Attrition}

A total of 18 studies provided evidence that attrition was low. Five studies showed substantial attrition (Bruzzese 2004; Gerald 2006; Levy 2006; Magzamen 2008; Richmond 2011), with levels of attrition exceeding $20 \%$ and/or reported by trial authors as a substantial challenge.

\section{Adherence to the intervention}

A total of 21 studies reported child adherence. 'Child adherence' broadly referred to the extent to which children followed directions of the intervention, for example, in completing homework assignments, undertaking and completing intervention modules, or completing evaluation instruments. Fourteen studies presented evidence that child adherence with the intervention was good. Six studies highlighted evidence that adherence was not problematic among other stakeholders (Bruzzese 2011; Cicutto 2013; Jackson 2006; Joseph 2013; Kintner 2012; Splett 2006). Child adherence was problematic in eight studies (Brasler 2006; Gerald 2006; Howell 2005; Joseph 2010; Kouba 2012; Magzamen 2008; Richmond 2011; Spencer 2000); these judgements were based on reports from trialists and on reports of completion rates of intervention modules and/or completion of evaluation instruments.

\section{Dosage of intervention received}

'Dosage' broadly referred to the extent to which children received the intervention as intended, for example, in attending the expected number of sessions. This differed from attrition, in that children could have received a low dosage but may have not permanently dropped out; this also differed from adherence, in that children could have received a low dosage but were otherwise adherent. Participants received the intended dose of the intervention in nine studies (Bignall 2015; Bruzzese 2011; Jackson 2006; Joseph 2013; Kintner 2012; McCann 2006; Mickel 2016; Pike 2011; Terpstra 2012). In one study, researchers noted a dose-response relationship (Kouba 2012). Seven studies reported that the intended dose was not achieved (Brasler 2006; Bruzzese 2008; Gerald 2006; Howell 2005; Joseph 2010; Langenfeld 2010; Magzamen 2008), with substantial numbers not receiving the intended intervention. In one study (Gerald 2006), this finding was based on reports of shortening of sessions. In another study, in which parental involvement was an integral component, study authors reported additional problems with dosage received (Bruzzese 2008). One study comparing an individualised intervention model versus a generic intervention model reported that the individualised model had higher levels of dosage, although both models showed relatively low levels of completion of all modules (Joseph 2010).

\section{Combined indicator of 'successful' implementation}

We combined data from process evaluation studies on attrition, adherence, and dosage into a single indicator. We summed scores across the three indicators and calibrated them to fall between zero and one, with 0.5 the point of maximum ambiguity and values over 0.5 indicating partial membership of the successful implementation set, up to a maximum possible value of one, which indicated full membership of the successful implementation set, values under 0.5 indicating more out of than in the set, and a value of 0 indicating full non-membership of the successful implementation set. Eight studies were either fully or strongly within the successful implementation set (Al-Sheyab 2012a; Berg 2004; Bruzzese 2008; Bruzzese 2011; Henry 2004; Joseph 2013; Kintner 2012; Terpstra 2012), and another five studies had scores that were mainly within the successful implementation set (Cicutto 2013; Dore-Stites 2007; Horner 2015; Mujuru 2011; Pike 2011). A further 14 studies provided scores that were ambiguous or low implementation scores (Brasler 2006; Bruzzese 2004; Crane 2014; Engelke 2013; Howell 2005; Gerald 2006; Joseph 2010; Kouba 2012; 
Langenfeld 2010; Lee 2011; Levy 2006; Magzamen 2008; Spencer 2000; Splett 2006).

\section{Characteristics of outcome evaluation studies (RCTs)}

We have included in Table 10 further details of studies that met the criteria for study design, but from which we did not include data in the meta-analysis.

\section{Study population and intervention characteristics}

Most studies took place in the USA (22/33 studies), with fewer taking place in high schools (eight studies) than in junior, middle, or elementary/primary schools (see Table 11). Study reports showed substantial variation in the types of interventions that were trialled, although nine studies included evaluations of the effectiveness of Open Airways for School, or modifications to this programme (see Table 12). Study reports also showed substantial variety in the ways in which asthma self-management interventions were delivered. Children received long programmes of sessions in some interventions, with 16 sessions delivered in two studies (Horner 2008; Horner 2015), and 10 sessions and eight sessions delivered in others (Kintner 2009; Patterson 2005, respectively). In contrast, researchers delivered three interventions in a single group session to children (Gerald 2006; Howell 2005; McCann 2006), although these interventions were supported by other activities including nurse visits or staff training. The number of sessions was not always commensurate with the quantity of content delivered however; for example, the intervention delivered in Atherly 2009 amounted to 4.5 hours of instruction over three sessions, and Horner 2015 delivered 4 hours of content over 16 sessions.

Several studies collected outcome data immediately after the intervention or within three months (Atherly 2009; Bruzzese 2004; Bruzzese 2008; Gerald 2006; Horner 2008; Howell 2005; Kintner 2009; Mosnaim 2011; Patterson 2005; Persaud 1996; Shah 2001; Srof 2012), or they appeared to collect data concurrently with intervention delivery (Splett 2006). The longest period between the end of the intervention and data collection was 36 months in Bartholomew 2006, and 24 months in Clark 2004 and Clark 2010, although for a minority of studies, the length of follow-up was not clear (Levy 2006; Monforte 2012; Pulcini 2007). We included many studies on the basis of study design, although these studies did not contribute to the meta-analyses, as they did not collect data on the outcomes of interest or did not collect these data in an extractable format (see Table 10).

\section{Primary outcomes}

\section{Asthma symptoms or exacerbations leading to admission to hospital}

Six outcome studies provided data on asthma exacerbations leading to admission to hospital that were combined in metaanalyses (Atherly 2009; Bruzzese 2011; Clark 2005; Horner 2008; Horner 2015; Levy 2006). One study collected information on hospitalisations but did not disaggregate the information by treatment status (Bartholomew 2006), and another study provided disaggregated information on median hospitalisations that could not be combined in meta-analyses (Gerald 2006). Two studies assessed hospitalisations using hospital or school medical records (Gerald 2006; Levy 2006); three studies assessed hospitalisations using parent reports (Clark 2005; Horner 2015; Horner 2008); and two studies used child reports (Atherly 2009; Bruzzese 2011). Of the six studies included in the meta-analyses, most collected outcome data on hospitalisations after a substantial period between receipt of the intervention and assessment of the outcome had elapsed (12 months in the case of Bruzzese 2011; Clark 2005; and Horner 2015; and seven months in the case of Horner 2008); less time had elapsed in the case of Atherly 2009 and Levy 2006, in which assessment took place within three months of receipt of the intervention. Studies in which a longer time had elapsed between intervention and assessment tended to be those with a longer exposure time over which the outcome was measured.

\section{Asthma symptoms or exacerbations leading to emergency department visits}

Fifteen outcome evaluation studies collected data on asthma symptoms or exacerbations leading to an emergency department (ED) visit (Atherly 2009; Bartholomew 2006; Bruzzese 2011; Cicutto 2005; Cicutto 2013; Clark 2005; Gerald 2006; Horner 2008; Horner 2015; Howell 2005; Levy 2006; McGhan 2003; McGhan 2010; Persaud 1996; Velsor-Friedrich 2005). However, we did not use data from Bartholomew 2006 because study authors did not disaggregate the data by treatment status, and we could not combine data from Gerald 2006 because of incompatibility in the unit of assessment. Three studies used school or hospital administrative records to assess ED visits, with records provided by the medical hospital (Gerald 2006; Levy 2006; Persaud 1996). Parents were frequently the sources of ED data: one study collected these data using tracking sheets of ED attendance provided by parents (Cicutto 2013); another study collected data through parent interviews (Cicutto 2005); six studies used various parent selfcompletion questionnaires (Clark 2005; Horner 2015; Horner 2008; Howell 2005; McGhan 2003; McGhan 2010), and one specifically used the Usherwood symptom questionnaire (Bartholomew 2006). One study collected data from children's asthma diaries (VelsorFriedrich 2005), and others collected data from children's reports (Atherly 2009; Bruzzese 2011).

Of the 13 studies included in the meta-analyses, most collected outcome data on ED visits after a substantial period had elapsed between receipt of the intervention and assessment of the outcome (12 months in the case of Bruzzese 2011, Cicutto 2005, Cicutto 2013, Clark 2005, Horner 2015, McGhan 2003, McGhan 2010; seven months in the case of Horner 2008; and 20 weeks in the case of Persaud 1996); less time had elapsed in the case of Atherly 2009, Howell 2005, and Levy 2006, which performed assessment within three months of receipt of the intervention. As was the case above, studies in which a longer time had elapsed between intervention and assessment were those with a longer exposure time over which the outcome was measured (see Table 11 for full details).

\section{Absence from school}

Twelve outcome evaluation studies assessed school absence or attendance (Bartholomew 2006; Bruzzese 2011; Cicutto 2005; Cicutto 2013; Clark 2004; Gerald 2006; Gerald 2009; Howell 2005; McGhan 2003; McGhan 2010; Persaud 1996; Splett 2006).

Four studies used administrative school records (Bartholomew 2006; Gerald 2006; Persaud 1996; Splett 2006). One study collected school absenteeism data from parents/guardians using tracking sheets (Cicutto 2013), and five studies used parental interviews or questionnaires (Cicutto 2013; Clark 2004; Howell 2005; McGhan 2003; McGhan 2010). In another study, school staff entered absence data into an intervention tracking system (Gerald 2009). Bruzzese 2011 was the only study that collected self-reported absence data directly from children. 
Bartholomew 2006 did not present disaggregated information, and we will not consider this study further here. Clark 2004 presented information on effectiveness of the intervention in terms of school absence in the form of a risk difference, which was not combined in the meta-analyses, although researchers showed a significant intervention effect in reducing absences at three months and 12 months.

We included data from 10 studies in meta-analysis models. Six of these studies considered long-term impact of the intervention, with follow-up data from nine months or longer collected and included in the meta-analysis (Bruzzese 2011; Cicutto 2005; Cicutto 2013; Gerald 2009; McGhan 2003; McGhan 2010). However, three studies collected follow-up data after three months or sooner (Persaud 1996; Howell 2005; Splett 2006), and one study provided unclear information on this (Gerald 2006). Differences in the exposure period over which absences were considered ranged from a year in three studies - as in Cicutto 2005, Cicutto 2013, and McGhan 2010 - to two weeks in one study - as in Bruzzese 2011. Three studies considered any instance of recorded absence from school (Cicutto 2013; McGhan 2003; McGhan 2010), and the remaining seven studies measured mean number of days of absence or attendance at school. Most studies included in the meta-analysis collected data on any form of absence, with only Gerald 2009 collecting data on absence related to asthma/respiratory illness.

\section{Days of restricted activity}

Three outcome evaluation studies reported days of restricted activity (Bruzzese 2011; Cicutto 2005; Cicutto 2013). One study used parent tracking sheets/diaries to record days of interrupted activity due to asthma (Cicutto 2013), another study used data from parent interviews (Cicutto 2005), and another study collected information directly from children (Bruzzese 2011). We included data from all three studies in the meta-analyses, and all three studies collected data at 12 months' follow-up. Two studies collected data on the mean number of days of restricted activity (Bruzzese 2011; Cicutto 2005), and Cicutto 2013 collected data on any instance of a day of restricted activity.

\section{Secondary outcomes}

\section{Unplanned visit to a hospital or GP due to asthma symptoms}

Five outcome evaluation studies reported on unplanned visits to a hospital or GP due to asthma symptoms (Bruzzese 2011; Cicutto 2013; McGhan 2003; McGhan 2010; Splett 2006). One study recorded unplanned visits using tracking sheets provided to parents (Cicutto 2013); two studies used a parental questionnaire (McGhan 2003; McGhan 2010); one study collected data directly from children (Bruzzese 2011); and a final study collected information on episodic asthma-related visits to a school-based health facility from administrative data (Splett 2006).

We included data from all five studies in the meta-analyses. One study originally collected information on the mean number of unscheduled visits (Bruzzese 2011), and the remaining studies collected information on any instances of unscheduled visits to a medical provider (not captured in hospitalisation or ED utilisation data (above)). All studies collected data after substantial time had elapsed since the intervention began; this extended to nine to 12 months in four studies (Bruzzese 2011; Cicutto 2013; McGhan 2003; McGhan 2010), and in Splett 2006, longitudinal data collection occurred concurrently alongside delivery of the intervention over a period of six months.

\section{Experience of daytime and night-time symptoms}

Nine outcome evaluation studies assessed children's experiences of daytime and night-time symptoms (Atherly 2009; Bruzzese 2008; Bruzzese 2011; Clark 2004; Clark 2010; Howell 2005; McGhan 2003; Shah 2001; Velsor-Friedrich 2005). These studies specifically reported on symptoms occurring during the day or during the night. Data were not combined in meta-analyses for either Clark 2004 or Clark 2010. Clark 2004 collected data on daytime and night-time symptoms as a risk difference, which indicated that the intervention had a positive effect in reducing daytime symptoms for all children but reduced the incidence of night-time symptoms only for children with severe or persistent asthma (yielding a negative effect on night-time symptoms for children with mild asthma). We did not include this in the meta-analyses as it was incompatible with other units of analysis. Meanwhile, Clark 2010 collected information on a change in daytime symptoms, which indicated that the intervention had a positive, but non-statistically significant, impact in terms of a drop in daytime symptoms (an effect size was extractable for one of the treatment arms only, although it was not used in meta-analyses because of statistical and conceptual differences between post-test data and changes in posttest outcome data).

Among the seven studies included in the meta-analysis, five studies reported on the incidence of daytime symptoms (Atherly 2009; Bruzzese 2008; Bruzzese 2011; Shah 2001; Velsor-Friedrich 2005), and in the case of Shah 2001, researchers reported the incidence of daytime symptoms specifically occurring within school; four studies reported on night-time awakenings (Bruzzese 2008; Bruzzese 2011; Howell 2005; McGhan 2003), with two studies reporting on both daytime and night-time symptoms (Bruzzese 2008; Bruzzese 2011). Four studies reported on intervention effects six to 12 months after the intervention (Bruzzese 2011; McGhan 2003; Shah 2001; Velsor-Friedrich 2005), and the remaining three studies included in the meta-analyses information collected from children or parents two to three months post intervention. Similarly, data show a relatively even split between studies reporting on the mean level of asthma symptoms occurring in the daytime/at night-time - Atherly 2009, Bruzzese 2008, Bruzzese 2011, Howell 2005 - and those focused on measuring any reported incidence of daytime/night-time symptoms - McGhan 2003, Shah 2001, and Velsor-Friedrich 2005.

\section{Lung function}

Five outcome evaluation studies assessed lung function (Gerald 2009; Horner 2015; Patterson 2005; Shah 2001; Velsor-Friedrich 2005), although studies measured this in different ways. One study assessed lung function using the peak expiratory flow rate (PEFR) and specifically focused on the occurrence of poor readings (red and yellow readings defined as less than $80 \%$ of best value) (Gerald 2009). A second study measured spirometry by measuring the percentage predicted change in forced expiratory volume in one second $\left(\mathrm{FEV}_{1}\right)$ (Patterson 2005). Shah 2001 reported forced vital capacity (FVC) before use of a bronchodilator. Velsor-Friedrich 2005 measured peak flow increases as a percentage of pretest peak (i.e. change in peak flow); Horner 2015 measured airway inflammation by measuring exhaled nitric oxide as a biomarker of airway inflammation. 
Because of conceptual differences in the outcomes collected, we did not combine these in meta-analyses. Table 13 shows that the individual effects extracted exhibited considerable heterogeneity in the direction and magnitude of effect, confirming that metaanalysis was not desirable due to statistical heterogeneity.

\section{Use of reliever therapies such as beta ${ }_{2}$-agonists}

Four outcome evaluation studies assessed use of reliever therapies (Gerald 2009; McGhan 2003; McGhan 2010; Splett 2006). We combined in meta-analyses two studies that reported on the use of rescue medication and short-acting bronchodilators (SABAs), respectively (Gerald 2009; McGhan 2010). The former captured information on instances when rescue medication was used more than twice a week, and the latter measured any instance in which rescue medication was used; these studies sought to measure longterm intervention effects at 12 months - as in McGhan 2010 - and at 15 months - as in Gerald 2009. The remaining two studies measured appropriate use of reliever medication and access to reliever medication, respectively (McGhan 2003; Splett 2006). Because of conceptual differences in the way in which researchers measured use of reliever therapies, we chose not to meta-analyse this information. We have presented information provided by all four studies in Table 13.

\section{Corticosteroid dosage and/or use of add-on therapies}

Six studies measured corticosteroid usage and dosage (Bruzzese 2011; Horner 2015; Howell 2005; McGhan 2003; McGhan 2010; Splett 2006). One study measured whether children had access to controller medication while visiting the school health office (Splett 2006). Two studies measured whether children were adhering to guidance provided around the correct use of corticosteroid (Horner 2015; Howell 2005), and three studies measured any reported usage of corticosteroid or controller medication (Bruzzese 2011; McGhan 2003; McGhan 2010). We meta-analysed data from these five studies separately, as adherence was deemed to conceptually differ from reports of usage. Horner 2015 and Howell 2005 included information from children at five months and three months, respectively, in meta-analyses of corticosteroid adherence. All three studies in the second meta-analysis on reported instances of corticosteroid or controller medication usage collected information at nine months or 12 months post intervention. We have presented data from all six studies in Table 13.

\section{Health-related quality of life (HRQOL)}

Twelve outcome evaluation studies measured quality of life (AlSheyab 2012; Cicutto 2005; Cicutto 2013; Clark 2010; Henry 2004; Horner 2008; Howell 2005; Kintner 2009; McCann 2006; McGhan 2010; Patterson 2005; Shah 2001). McCann 2006, McGhan 2010, and Clark 2010 did not present data in an extractable format (i.e. described data narratively, did not disaggregate data, or did not include the necessary information to extract an effect size); Patterson 2005 measured change in quality of life; and Shah 2001 measured clinically significant improvements (see Table 13). Among the nine studies that calculated an effect size, eight were based on the Juniper Pediatric Asthma Quality of Life Questionnaire overall quality of life (see Juniper 1996); Al-Sheyab
2012 used an Arabic version of this questionnaire. Kintner 2009 measured quality of life by reviewing responses to the Participation in Life Activities Scale.

We constructed two sets of meta-analyses for a model measuring changes in quality of life. One of these used SMD to calculate effect sizes; this allowed us to incorporate data from Kintner 2009. We meta-analysed change scores to obtain an MD from the data reported in Patterson 2005 and Shah 2001. Therefore data from six studies were common to both models. Several studies measured quality of life within four months of the intervention (Al-Sheyab 2012; Cicutto 2005; Howell 2005; Kintner 2009; Patterson 2005; Shah 2001), two studies collected data at six to seven months after the intervention (Henry 2004; Horner 2008), and one study collected data 12 months after the intervention (Cicutto 2013).

\section{Withdrawal from the study}

Researchers frequently presented withdrawal data, although not always in a format that allowed extraction of data to form an effect size. This often occurred because studies reported overall numbers lost during the study without disaggregating by treatment arm (Cicutto 2013; Velsor-Friedrich 2005), or because studies reported no losses (Persaud 1996). Fourteen studies provided enough data to allow calculation of an effect size (OR) (Al-Sheyab 2012; Bartholomew 2006; Bruzzese 2008; Bruzzese 2011; Cicutto 2005; Gerald 2009; Horner 2008; Horner 2015; Kintner 2009; Levy 2006; McGhan 2003; McGhan 2010; Patterson 2005; Shah 2001). Few studies reported on active withdrawal processes occurring during the intervention; instead investigators reported on failure to collect children's data at follow-up (collected from children and parents). Researchers collected data at different points between intervention and follow-up, including at four months or less (Al-Sheyab 2012; Bruzzese 2008; Patterson 2005; Shah 2001), at six to seven months (Cicutto 2005; Gerald 2009; Horner 2008; McGhan 2010), and at nine to 12 months (Bruzzese 2011; Horner 2015; Kintner 2009; Levy 2006; McGhan 2003). Duration was unclear in one study (Bartholomew 2006).

\section{Excluded studies}

From the title and abstract screening, we excluded 28,318 records because they were clearly outside the remit of the review of process evaluations. Following full-text screening, we excluded another 1029 records, for reasons detailed in the PRISMA diagram (Figure 2).

Based on title and abstract screening, we excluded 274 records as they were outside the remit of the review of outcome evaluation studies. Following full-text screening, we excluded 67 additional records, for reasons detailed in the PRISMA diagram (Figure 3).

\section{Risk of bias in included studies}

We have displayed results of the risk of bias assessment for process and outcome evaluation studies in the risk of bias table and graph. We have presented the agreed judgement of two review authors (DK, KH) regarding the risk of bias for each included study as percentages for each bias item in the risk of bias graph (Figure 4; Figure 5). 


\section{Figure 4. Risk of bias graph: review authors' judgements about each risk of bias item presented as percentages} across all included studies.

Random sequence generation (selection bias)

Allocation concealment (selection bias)

Blinding of participants and personnel (performance bias)

Blinding of outcome assessment (detection bias)

Incomplete outcome data (attrition bias)

Selective reporting (reporting bias)

Other bias

Transparent and clearly stated aims

Explicit theories underpinning and/or literature review

Transparent and clearly stated methods and tools

Selective reporting

Harmful effects

Population and sample described well

Continuous evaluation

Evaluation participation equity and sampling

Design and methods overall approach

Tools and methods of data collection reliable/credible

Tools and methods of data analysis reliable/credible

Performance bias/neutrality/credibility/conformability

Reliability of findings and recommendations

Transferability of findings

Overall risk of bias of process evaluation
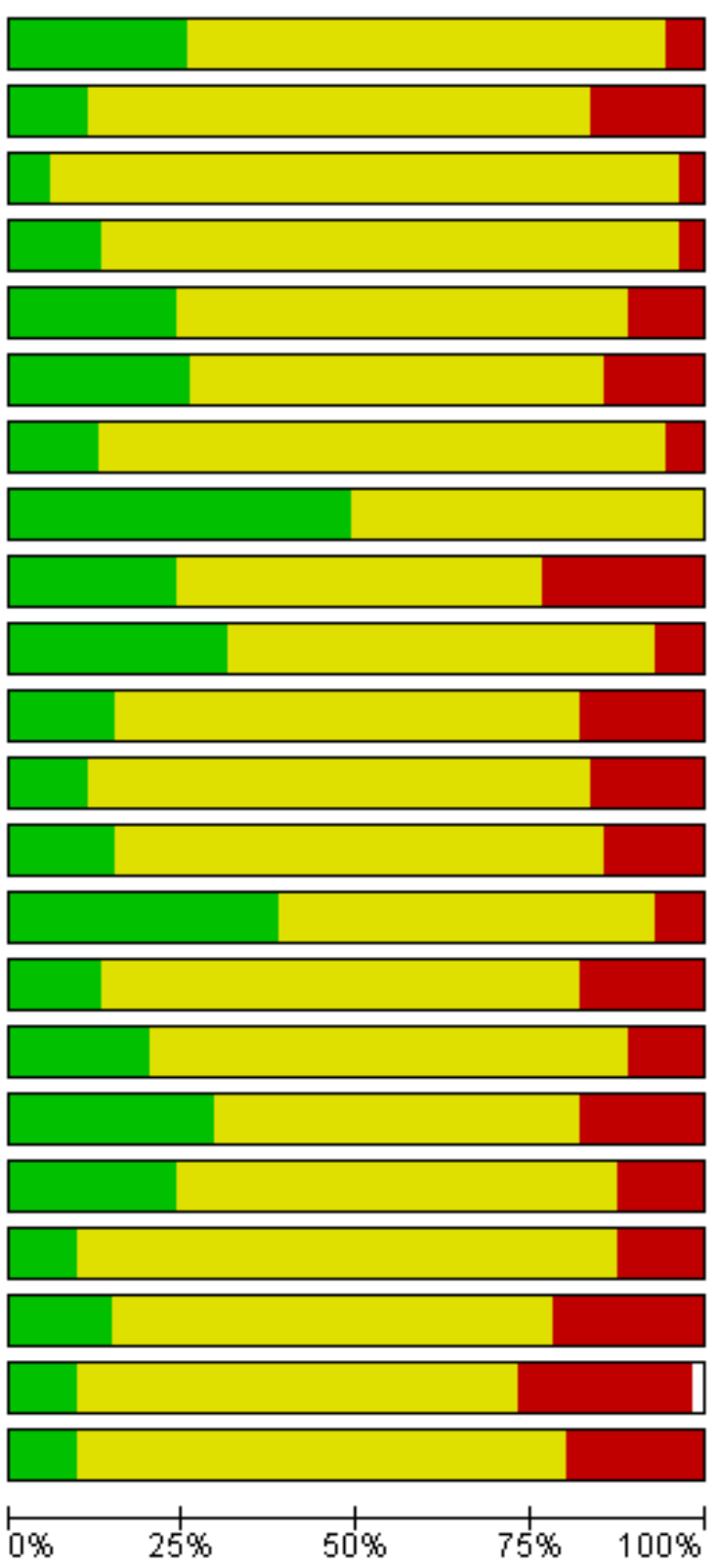

\begin{tabular}{|lllll}
\hline $0 \%$ & $25 \%$ & $50 \%$ & $75 \%$ & $100 \%$
\end{tabular}


Figure 5. Risk of bias summary: review authors' judgements about each risk of bias item for each included study.

\begin{tabular}{|c|c|c|c|c|c|c|c|c|c|c|c|c|c|c|c|c|c|c|c|c|c|c|}
\hline & 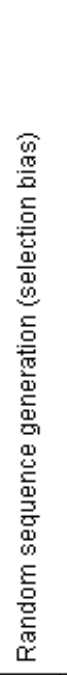 & 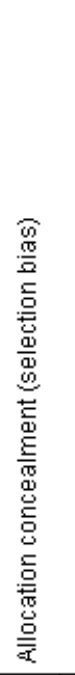 & 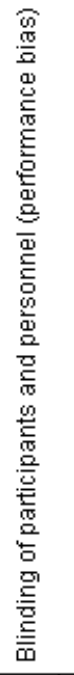 & 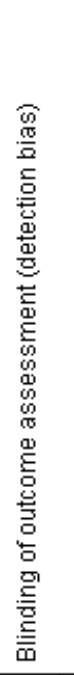 & 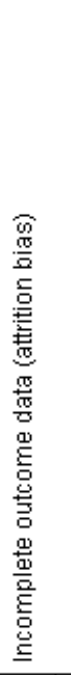 & 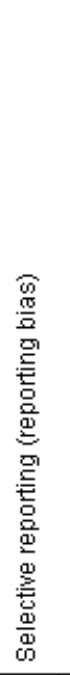 & 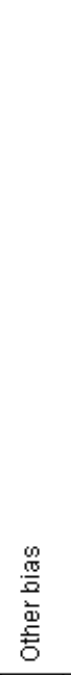 & 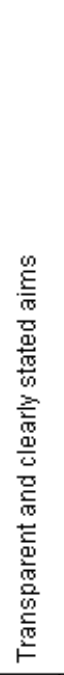 & 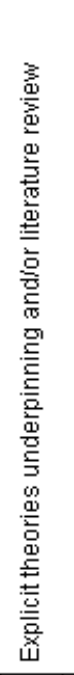 & 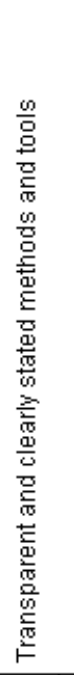 & 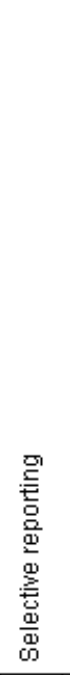 & 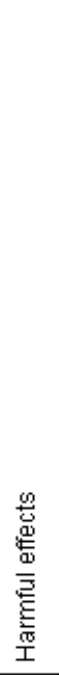 & 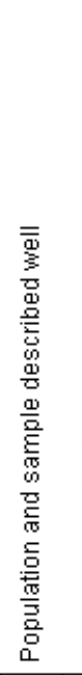 & 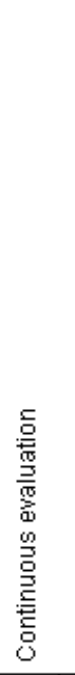 & 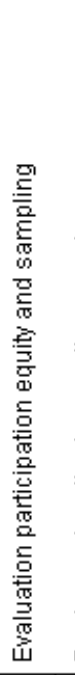 & 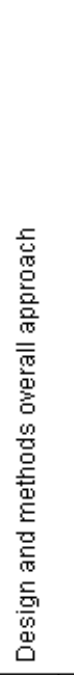 & 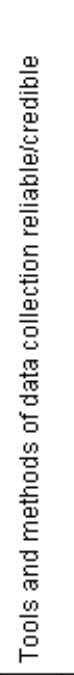 & 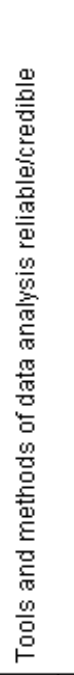 & 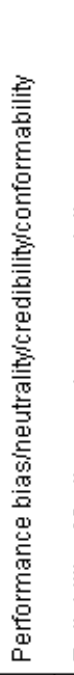 & 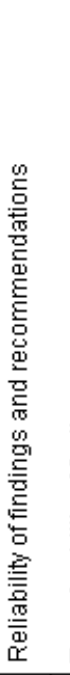 & 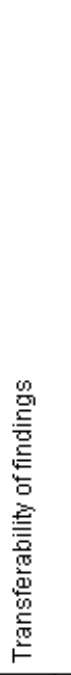 & 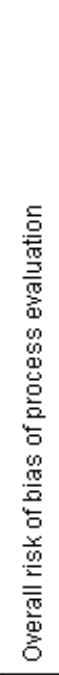 \\
\hline Al-Sheyab 2012 & $\odot$ & $\odot$ & $?$ & $?$ & + & $\odot$ & $?$ & $?$ & $?$ & $?$ & $?$ & $?$ & $?$ & $?$ & $?$ & $?$ & $?$ & $?$ & $?$ & $?$ & $?$ & $?$ \\
\hline Al-Sheyab 2012a & $?$ & $?$ & $?$ & $?$ & $?$ & $?$ & $?$ & $\odot$ & $?$ & $?$ & $\odot$ & $\odot$ & $\odot$ & - & $?$ & $\odot$ & + & $\odot$ & $?$ & $\odot$ & $\ominus$ & e \\
\hline Atherly 2009 & $?$ & $?$ & $?$ & $?$ & $\odot$ & $\odot$ & $\odot$ & $?$ & $?$ & $?$ & $?$ & $?$ & $?$ & $?$ & $?$ & $?$ & $?$ & $?$ & $?$ & $?$ & $?$ & $?$ \\
\hline Bartholomew 2006 & $?$ & $?$ & $?$ & $?$ & $\ominus$ & ○ & $\theta$ & $?$ & $?$ & $?$ & $?$ & $?$ & $?$ & $?$ & $?$ & $?$ & $?$ & $?$ & $?$ & $?$ & $?$ & $?$ \\
\hline Berg 2004 & $?$ & $?$ & $?$ & $?$ & $?$ & $?$ & $?$ & $\odot$ & + & $\odot$ & $\odot$ & $?$ & $?$ & $\odot$ & + & $?$ & + & $\odot$ & $?$ & $\Theta$ & $\ominus$ & $?$ \\
\hline Bignall 2015 & $?$ & $?$ & $?$ & $?$ & $?$ & $?$ & $?$ & $\odot$ & $\theta$ & $?$ & $?$ & $\ominus$ & + & $\odot$ & $\odot$ & ๑ & $?$ & $?$ & $\odot$ & $\odot$ & $\odot$ & - \\
\hline Brasler 2006 & $?$ & $?$ & $?$ & $?$ & $?$ & $?$ & $?$ & $\odot$ & $\theta$ & $\odot$ & $\odot$ & $\odot$ & $?$ & $\odot$ & - & $\ominus$ & $\odot$ & $\odot$ & $\ominus$ & $?$ & $\odot$ & 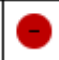 \\
\hline Bruzese 2004 & $?$ & $?$ & $?$ & $?$ & $?$ & $?$ & $?$ & $\odot$ & + & $\odot$ & $?$ & $\odot$ & $?$ & $\ominus$ & $?$ & $?$ & $\odot$ & $\Theta$ & $?$ & $?$ & $?$ & e \\
\hline Bruzzese 2008 & $?$ & $?$ & $?$ & $?$ & $\odot$ & + & $?$ & $\odot$ & + & $?$ & $?$ & $?$ & $\odot$ & $?$ & $\theta$ & $?$ & $\theta$ & + & $?$ & + & 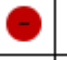 & $?$ \\
\hline Bruzese 2010 & $?$ & $?$ & $?$ & $?$ & $?$ & $?$ & $?$ & + & + & $\odot$ & $\odot$ & $?$ & $\odot$ & + & $\odot$ & + & + & $\odot$ & $\odot$ & $\odot$ & + & + \\
\hline Bruzese 2011 & $\odot$ & $\odot$ & $?$ & ๑) & 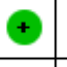 & + & $\odot$ & $?$ & $?$ & $?$ & $?$ & $?$ & $?$ & $?$ & $?$ & $?$ & $?$ & $?$ & $?$ & $?$ & $?$ & $?$ \\
\hline Carpenter 2016 & $?$ & $?$ & $?$ & $?$ & $?$ & $?$ & $?$ & $\odot$ & $\theta$ & $?$ & - & $\Theta$ & $?$ & $?$ & $?$ & $?$ & $?$ & $?$ & 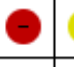 & $?$ & - & - \\
\hline Cicutto 2005 & + & $\odot$ & $?$ & + & + & $?$ & + & $?$ & $?$ & $?$ & $?$ & $?$ & $?$ & $?$ & $?$ & $?$ & $?$ & $?$ & $?$ & $?$ & ? & $?$ \\
\hline Cicutto 2013 & + & + & $?$ & ๑) & $?$ & $?$ & $?$ & + & + & + & $?$ & $?$ & $?$ & + & $\odot$ & $\odot$ & + & $\odot$ & $?$ & $\odot$ & $?$ & $\odot$ \\
\hline Clark 2004 & $\odot$ & $\Theta$ & $?$ & $?$ & $?$ & + & $\odot$ & $?$ & $?$ & $?$ & $?$ & $?$ & $?$ & $?$ & $?$ & $?$ & $?$ & $?$ & $?$ & $?$ & $?$ & $?$ \\
\hline Clark 2005 & $?$ & $?$ & $?$ & $?$ & $?$ & $\ominus$ & $?$ & $?$ & $?$ & $?$ & $?$ & $?$ & $?$ & $?$ & $?$ & $?$ & $?$ & $?$ & $?$ & $?$ & $?$ & $?$ \\
\hline Clark 2010 & $\odot$ & $\odot$ & $?$ & $\ominus$ & $?$ & - & $?$ & $?$ & $?$ & $?$ & $?$ & $?$ & $?$ & $?$ & $?$ & $?$ & $?$ & $?$ & $?$ & $?$ & $?$ & $?$ \\
\hline Crane 2014 & $?$ & $?$ & $?$ & $?$ & $?$ & $?$ & $?$ & $\odot$ & + & + & $\odot$ & $?$ & $?$ & $?$ & $?$ & $?$ & + & $\odot$ & $?$ & $\odot$ & $\ominus$ & $?$ \\
\hline Dore-Stites 2007 & $?$ & $?$ & $?$ & $?$ & $?$ & $?$ & $?$ & $\odot$ & $?$ & ๑ & $?$ & $\odot$ & $?$ & $\odot$ & $\odot$ & $?$ & + & $\odot$ & $?$ & 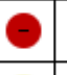 & $\ominus$ & $\theta$ \\
\hline Engelke 2013 & $?$ & $?$ & $?$ & $?$ & $?$ & $?$ & $?$ & $\odot$ & $?$ & $?$ & $\odot$ & 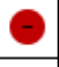 & $\odot$ & $\odot$ & $?$ & 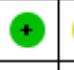 & $?$ & $?$ & $\ominus$ & $?$ & $?$ & $?$ \\
\hline Gerald 2006 & $?$ & $?$ & $?$ & $?$ & $?$ & + & $\odot$ & $?$ & $\ominus$ & - & $?$ & $\odot$ & $\theta$ & $?$ & $\ominus$ & 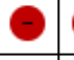 & 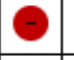 & - & $?$ & $?$ & 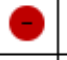 & $?$ \\
\hline Gerald 2009 & + & $\odot$ & $?$ & $?$ & + & $?$ & $?$ & $?$ & $?$ & $?$ & $?$ & $?$ & $?$ & $?$ & $?$ & $?$ & $?$ & $?$ & $?$ & $?$ & $?$ & $?$ \\
\hline Henry 2004 & 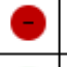 & $?$ & $?$ & $?$ & ? & + & $?$ & $\odot$ & $\theta$ & + & $?$ & $?$ & $\odot$ & $?$ & $?$ & ๑ & + & $\odot$ & $?$ & $?$ & $?$ & $?$ \\
\hline
\end{tabular}


Figure 5. (Continued)

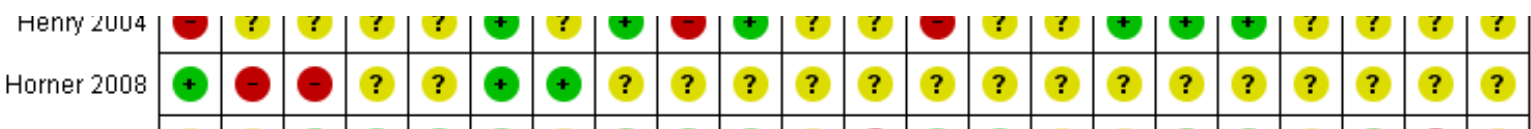

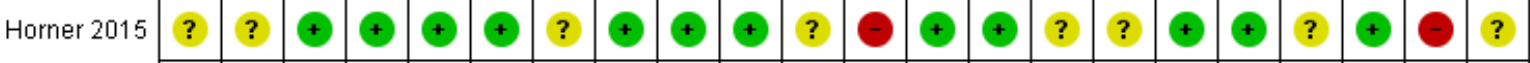

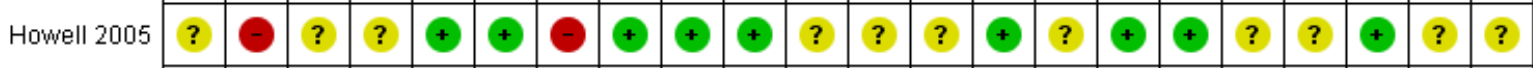

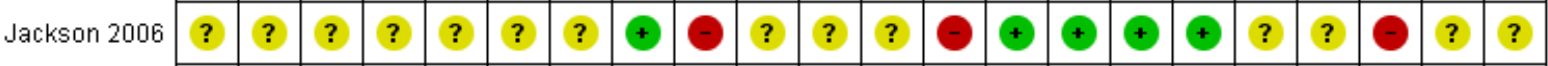

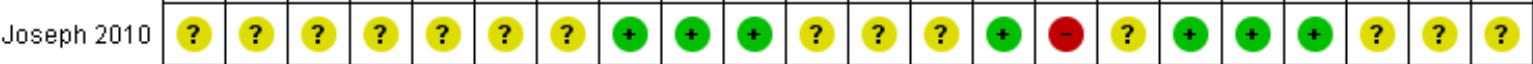

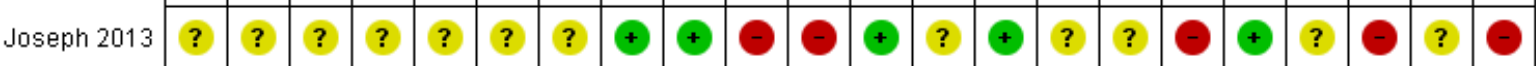

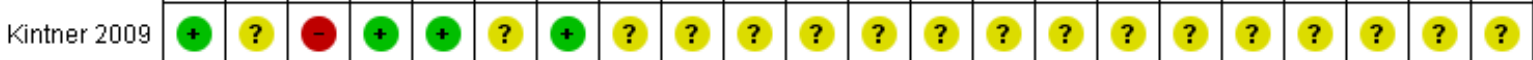

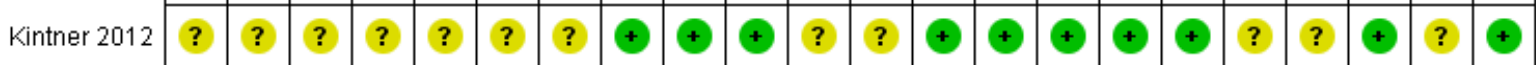

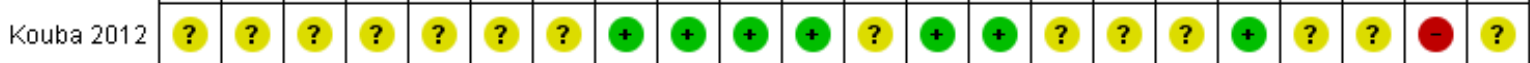

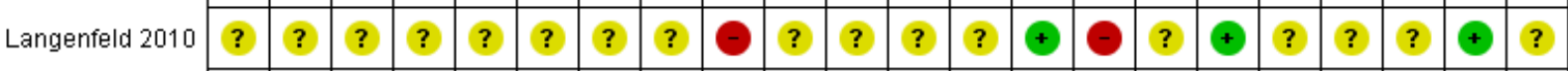

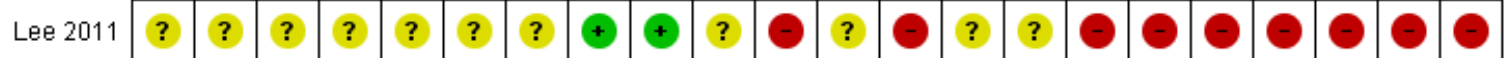

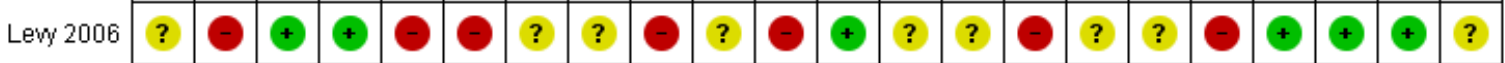

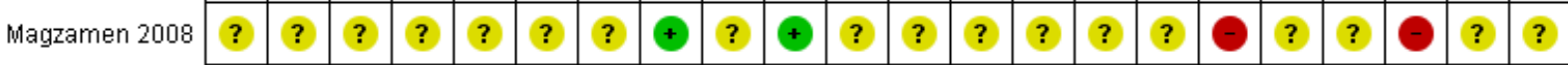

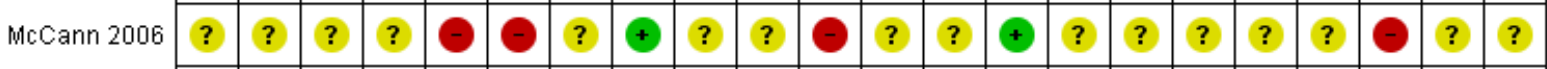

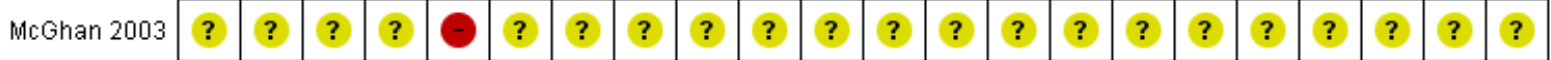

\begin{tabular}{|l|l|l|l|l|l|l|l|l|l|l|l|l|l|l|l|l|l|l|l|l|l|l|} 
McGhan 2010 & $\bullet$ & $?$ & $?$ & $?$ & $\odot$ & $\odot$ & $?$ & $?$ & $?$ & $?$ & $?$ & $?$ & $?$ & $?$ & $?$ & $?$ & $?$ & $?$ & $?$ & $?$ & & $?$ \\
\hline
\end{tabular}

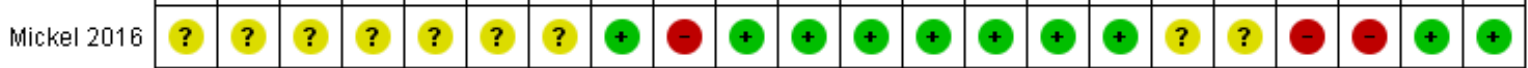

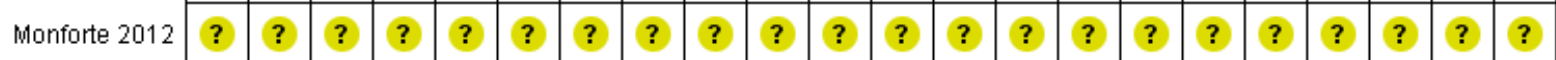

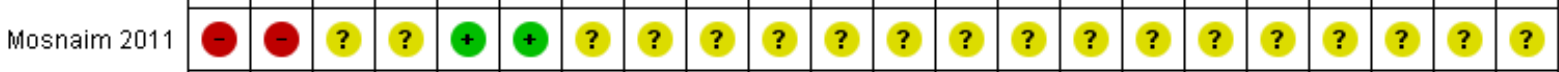

\begin{tabular}{|l|l|l|l|l|l|l|l|l|l|l|l|l|l|l|l|l|l|l|l|l|l|l|} 
Mujuru 2011 & $?$ & $?$ & $?$ & $?$ & $?$ & $?$ & $?$ & $\odot$ & $?$ & $\odot$ & $\odot$ & $\odot$ & $?$ & $\odot$ & $\odot$ & $\odot$ & $\odot$ & $?$ & $?$ & $?$ & $?$ & $\odot$ \\
\hline
\end{tabular}

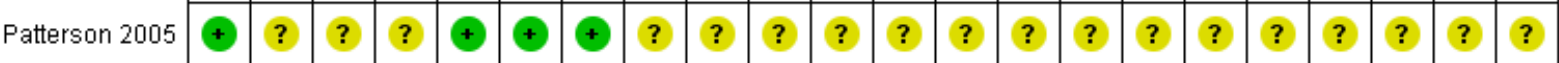

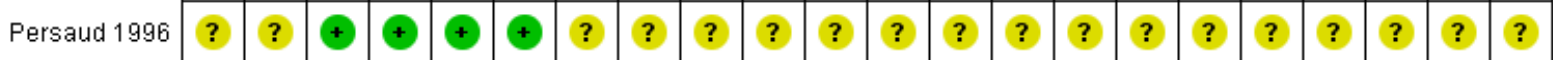

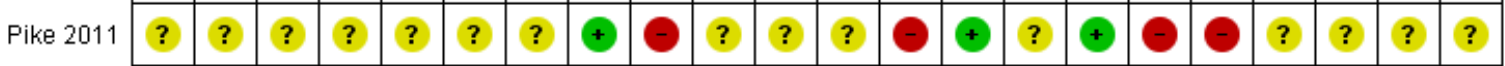

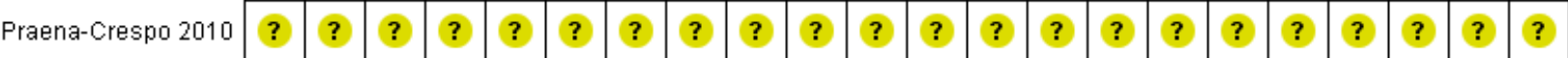

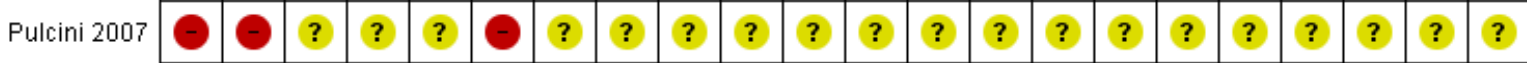

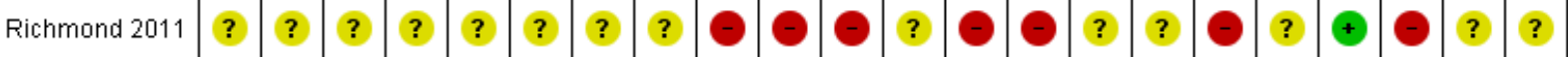

\begin{tabular}{|l|l|l|l|l|l|l|l|l|l|l|l|l|l|l|l|l|l|l|l|l|l|l|} 
Shah 2001 & $\odot$ & $\bullet$ & $?$ & $?$ & $\bullet$ & $?$ & $?$ & $?$ & $?$ & $?$ & $?$ & $?$ & $?$ & $?$ & $?$ & $?$ & $?$ & $?$ & $?$ & $?$ & $?$ & $?$ \\
\hline
\end{tabular}

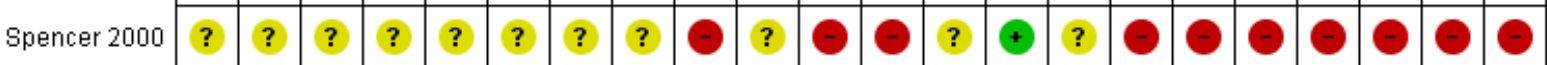

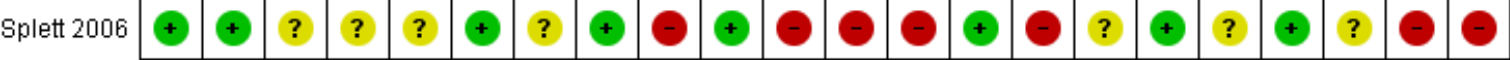

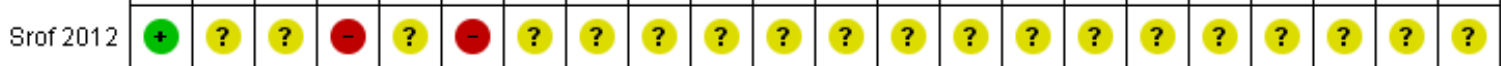

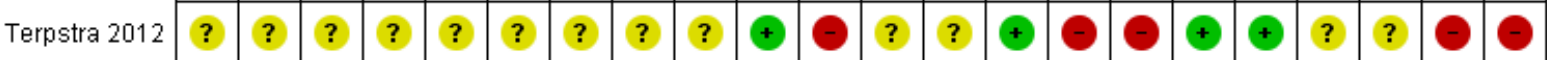

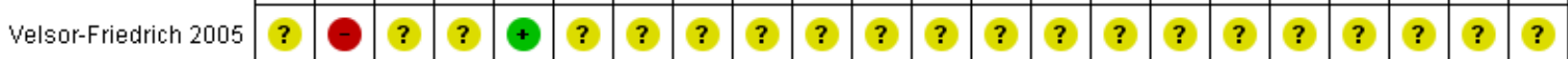

\section{Risk of bias - process evaluation studies}

For process evaluation studies, we assessed risk of bias using a combination of two tools. The first tool was developed at the EPPICentre (Harden 2004) to assess the methodological rigour of 'views' studies; the second tool, which was developed by the EPPI-Centre to assess the quality of process evaluation data (O'Mara-Eves 2013),

We assessed reporting quality across five indicators.

School-based self-management interventions for asthma in children and adolescents: a mixed methods systematic review (Review) 
- Transparent and clearly stated aims (0 high risk of bias, 27 low risk of bias, 6 unclear risk).

- Explicit theories underpinning the intervention (10 high risk of bias, 14 low risk of bias, 9 unclear risk).

- Transparent and clearly stated methods and tools (4 high risk of bias, 17 low risk of bias, 12 unclear risk).

- Selective reporting (10 high risk of bias, 8 low risk of bias, 15 unclear risk).

- Harmful effects (8 high risk of bias, 5 low risk of bias, 20 unclear risk).

We assessed population and selection factors using four indicators.

- Population and sample described well (8 high risk of bias, 8 low risk of bias, 17 unclear risk).

- Continuous evaluation (3 high risk of bias, 8 low risk of bias, 22 unclear risk).

- Evaluation participation equity and sampling ( 9 high risk of bias, 7 low risk of bias, 17 unclear risk).

- Design and methods overall approach (6 high risk of bias, 10 low risk of bias, 16 unclear risk).

We assessed reliability and transferability of findings using two indicators.

- Reliability of findings and recommendations (11 high risk of bias, 8 low risk of bias, 14 unclear risk).

- Transferability of findings (13 high risk of bias, 5 low risk of bias, 15 unclear risk).

Overall, process evaluation studies consisted of 10 high-risk studies, five low-risk studies, and 18 studies at unclear risk.

\section{Risk of bias - outcome evaluation (RCT) studies}

\section{Allocation}

We judged 14 outcome evaluation studies to be at low risk of bias for random sequence generation (Al-Sheyab 2012; Bruzzese 2011; Cicutto 2005; Cicutto 2013; Clark 2004; Clark 2010; Gerald 2009; Horner 2008; Kintner 2009; McGhan 2010; Patterson 2005; Shah 2001; Splett 2006; Srof 2012). We judged three to be at high risk (Henry 2004; Mosnaim 2011; Pulcini 2007). We judged the remainder to be at unclear risk. We judged six of these studies to be at low risk of allocation concealment bias (Bruzzese 2011; Cicutto 2005; Cicutto 2013; Gerald 2009; Shah 2001; Splett 2006). We judged nine studies to be at high risk of allocation concealment bias (Clark 2010; Horner 2008; Howell 2005; Kintner 2009; Levy 2006; McGhan 2010; Mosnaim 2011; Pulcini 2007; Velsor-Friedrich 2005).

\section{Blinding}

We judged three outcome evaluation studies to be at low risk of bias for blinding of participants and personnel (Cicutto 2013; Horner 2015; Levy 2006). We judged two studies to be at high risk of bias for this component (Horner 2008; Kintner 2009). We judged seven outcome evaluation studies to be at low risk for blinding of outcome assessment (Bruzzese 2011; Cicutto 2005; Cicutto 2013; Horner 2015; Kintner 2009; Levy 2006; Persaud 1996). For two outcome evaluation studies, we determined that risk of bias for blinding of outcome assessment was high (Clark 2010; Srof 2012).

\section{Incomplete outcome data}

We judged 13 outcome evaluation studies to be at low risk of bias for incomplete outcome data (Al-Sheyab 2012; Bruzzese 2011; Bruzzese 2008; Cicutto 2005; Gerald 2009; Horner 2015; Howell 2005; Kintner 2009; Mosnaim 2011; Patterson 2005; Persaud 1996; Shah 2001; Velsor-Friedrich 2005). We judged six outcome evaluation studies to be at high risk of bias for incomplete outcome data (Atherly 2009; Bartholomew 2006; Levy 2006; McCann 2006; McGhan 2010; McGhan 2003).

\section{Selective reporting}

We judged 14 outcome evaluation studies to be at low risk of bias for selective reporting (Al-Sheyab 2012; Atherly 2009; Bruzzese 2011; Bruzzese 2008; Clark 2004; Gerald 2006; Henry 2004; Horner 2015; Horner 2008; Howell 2005; Mosnaim 2011; Patterson 2005; Persaud 1996; Splett 2006). We judged eight studies to be at high risk of bias for selective reporting (Bartholomew 2006; Clark 2005; Clark 2010; Levy 2006; McCann 2006; McGhan 2010; Pulcini 2007; Srof 2012).

\section{Other potential sources of bias}

We judged 13 outcome evaluation studies to be at low risk of bias (Al-Sheyab 2012; Atherly 2009; Bruzzese 2008; Bruzzese 2011; Gerald 2009; Gregory 2000; Horner 2008; Kintner 2009; Patterson 2005; Persaud 1996; Shah 2001; Splett 2006; Velsor-Friedrich 2005), along with seven studies at high risk of bias, for missingness (Bartholomew 2006; Bruzzese 2004; Cicutto 2005; Howell 2005; Levy 2006; McGhan 2010; Praena-Crespo 2010).

We judged 15 outcome evaluation studies to be at low risk of bias for baseline imbalance (Bruzzese 2008; Bruzzese 2011; Cicutto 2005; Cicutto 2013; Clark 2004; Gerald 2006; Gerald 2009; Gregory 2000; Horner 2008; Kintner 2009; Levy 2006; McGhan 2010; Splett 2006; Srof 2012; Velsor-Friedrich 2005). We judged six studies to be at high risk for baseline imbalance (Al-Sheyab 2012; Atherly 2009; Clark 2010; Howell 2005; McCann 2006; McGhan 2003).

We judged 27 outcome evaluation studies to be at low risk for contamination (Al-Sheyab 2012; Atherly 2009; Bartholomew 2006; Bruzzese 2011; Cicutto 2005; Cicutto 2013; Clark 2004; Clark 2005; Clark 2010; Gerald 2006; ; Henry 2004; Horner 2008; Horner 2015; Howell 2005; Kintner 2009; Levy 2006; McCann 2006; McGhan 2003; McGhan 2010; Monforte 2012; Mosnaim 2011; Patterson 2005; Praena-Crespo 2010; Pulcini 2007; Shah 2001; Splett 2006; VelsorFriedrich 2005), and we determined that five outcome evaluation studies were at high risk (Bruzzese 2004; Bruzzese 2008; Gerald 2009; Persaud 1996; Srof 2012).

\section{Effects of interventions}

See: Summary of findings for the main comparison Effects of school-based asthma interventions compared to usual care for asthma among children and adolescents

\section{Results of synthesis - part 1: qualitative comparative analysis of determinant conditions for successful intervention implementation}

\section{Descriptive results from process evaluation studies on implementation success}

Across the 27 included studies, review authors identified eight studies as having high implementation scores for our combined outcome (attrition, adherence, dosage) and classified these studies 
as mainly or fully included in a set of studies marked as successfully implemented (Al-Sheyab 2012a; Berg 2004; Bruzzese 2008; Bruzzese 2011; Henry 2004; Joseph 2010; Kintner 2012; Terpstra 2012). In contrast, we identified eight studies as having low implementation success scores and as mainly or entirely outside the successfully implemented set of studies (Brasler 2006; Bruzzese 2004; Gerald 2006; Howell 2005; Kouba 2012; Langenfeld 2010; Magzamen 2008; Spencer 2000). Other studies were more ambiguous regarding their implementation success and had high levels of missing data or conflicting results across indicators.

For many studies reporting lower implementation success, we viewed the difficulty of incorporating an intervention into the busy school curriculum and into children's busy schedules as undermining the intervention (Brasler 2006; Bruzzese 2004; Gerald 2006; Howell 2005; Kouba 2012). Additional factors included difficulties in terms of high staff turnover (Gerald 2006); high child turnover and/or chaotic families (Brasler 2006; Howell 2005); and low motivation among children, particularly in the absence of incentives (Magzamen 2008). Similarly, researchers provided a diverse set of explanations for successful implementation, including high levels of school-level commitment (Henry 2004; Kintner 2012); high levels of child and teacher motivation (AlSheyab 2012a; Berg 2004); and development of group cohesion (Bruzzese 2008), as well as specific intervention design features, including tailoring of messages to children, as in Bruzzese 2011 and Joseph 2010, and additional communications with parents, as in Terpstra 2012.

In the QCA analyses below, we examine factors that could further explain successful implementation by examining which characteristics are shared among studies that were successfully implemented, and whether these differ from studies that were not successfully implemented.

\section{Summary of results from qualitative comparative analysis}

We first explored different domains of implementation separately, before bringing this evidence together in a final model (Table 14). We used this strategy mainly because of the problem of limited diversity, by which observed studies did not support too many possible combinations of intervention characteristics. We found no configurations of characteristics that consistently triggered successful implementation with respect to recruitment and retention, as well as pedagogical factors, although these may be important in other ways for children's outcomes.

In our consolidated model, we prioritised conditions that were included in configurations with high consistency and coverage scores. To facilitate interpretation in the consolidated model, we focused on conditions with a consistent direction. Working from the raw data (Table 15), we created a truth table (Table 16), which showed the extent to which sets of studies with particular configurations of conditions overlapped with a set of studies included in our successful intervention set. Boolean minimisation helped to simplify the solution (Table 17), and we inserted assumptions about logical remainders (configurations with no observed cases) to further simplify the solution (Table 18). After doing this, we observed that four pathways (or configurations of conditions) triggered the outcome, thereby forming our 'solution' (summarised in Table 19).
This solution emphasises the importance of a theory-driven intervention across all settings for successful implementation. Three of these pathways are specific to high schools. Here, the evidence suggests that in addition to the importance of a theorybased intervention, good levels of engagement with parents, high levels of child satisfaction, or running the intervention outside the child's own time can lead to a successfully implemented intervention. A pathway that is not specific to high schools reinforces these findings by showing that being theory-based, fostering high levels of child satisfaction, reporting good levels of parental engagement, and running an intervention outside the child's own time are sufficient conditions for triggering a positive outcome.

As a whole solution, these pathways had a consistency score of 0.862 , suggesting that they were sufficient in triggering the outcome. Interventions that are designed with these sets of characteristics are therefore highly likely to be successfully implemented. We also checked whether any of the configurations described also predicted negation of the outcome, but we found no such evidence. Our coverage score of 0.432 , which is modest, suggests that other pathways can also trigger successful implementation, which may be explained by factors not explored in these models. We were not able to incorporate risk of bias judgements directly into the QCA solution.

Based on results of QCAs, we intended to include the following conditions in meta-analyses, either in the form of subgroup analyses or as covariates in meta-regression. We planned to examine these as binary or ordinal variables in meta-analyses; they reflect the single conditions thought to most commonly trigger a successful outcome.

- Type of school: high school; primary/elementary school; junior/ middle school; other.

- Theory driven: does the study name a theoretical framework that underpins the intervention design or delivery style?

- Parental engagement: did parents engage or participate in the ways they were expected to?

- Child satisfaction: did at least $75 \%$ of children report satisfaction with the intervention, or did study authors report high levels of satisfaction?

- Timing of the intervention: does the intervention interfere with the child's own time (during lunch or after school)?

Due to data constraints, we were not able to explore child satisfaction in meta-analyses, as very few studies captured this information, and we operationalised parental engagement as 'parental involvement' - whether or not parents were actively included in the intervention - for similar reasons. We entered the factors beginning "Theory driven", "Parental engagement", and "Timing of the intervention" into subgroup analyses as configurations of conditions in an attempt to replicate the results of the QCA (above). We further explored the link between implementation and outcomes in the next section.

\section{Results of synthesis - part 2: meta-analyses of effectiveness \\ Primary outcome: asthma symptoms or exacerbations leading to hospitalisation}

We extracted effect sizes from seven studies (Atherly 2009; Bruzzese 2011; Clark 2005; Gerald 2006; Horner 2008; Horner 2015; Levy 
2006), and we analysed the data from six. Evidence showed that school-based asthma self-management interventions were effective in reducing numbers of hospitalisations among children (standardised mean difference (SMD) $-0.19,95 \%$ confidence interval $(\mathrm{Cl})-0.35$ to -0.04 ; participants $=1873$; Figure 6 Analysis 1.1). Effect sizes from all six studies were in the same direction, and $\mathrm{I}^{2}$ and $\mathrm{Q}$ statistic values provided no evidence of statistical heterogeneity. Gerald 2006 presented data on the median number of hospitalisations, which were not compatible with other extracted data, although it is worth noting that the median level of hospitalisation appeared higher for the intervention group than for the control group post intervention.

Figure 6. Forest plot of comparison: 1 School-based asthma interventions vs usual care: outcome: 1.1. Exacerbations leading to hospitalisation.

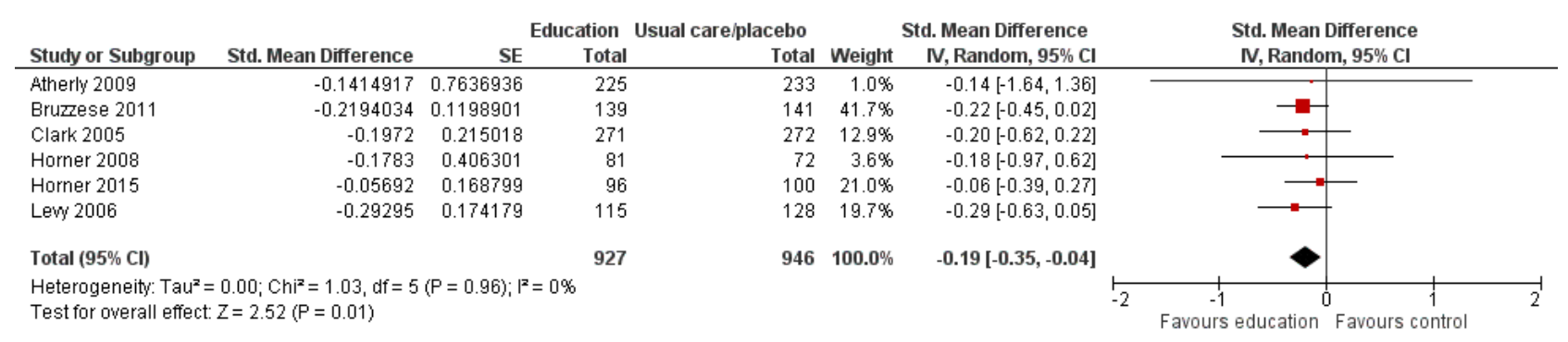

Given that we found no indication of heterogeneity in these models and the likelihood that these analyses would be underpowered, we did not conduct further subgroup analyses. We considered sensitivity analyses, although the small number of studies included in the models precluded a full analysis. All but one of the studies Bruzzese 2011 - reported on cluster randomised trials, and half of the studies originally reported on binary outcomes (Atherly 2009; Clark 2010; Horner 2008), although sensitivity analyses on these factors revealed no significant differences in effect size. Egger's test for publication bias suggested no evidence of publication bias (the $P$ value for the bias coefficient stood at 0.626 ), although the small number of studies meant that the test and observations of the funnel plot (not displayed) were ultimately underpowered.

The small number of included studies precluded a detailed investigation of the way in which risk of bias influenced the effect size for this outcome. However, two of the largest studies, which contributed three-fifths of weighting to the pooled effect size, had low or unclear risk of bias across all domains (Bruzzese 2011; Horner 2015), and in the case of Horner 2015, low risk of bias was seen for each domain, apart from blinding of participants and personnel (unclear risk of bias).

Evidence therefore suggests that school-based asthma selfmanagement interventions do reduce the frequency of asthma symptoms and exacerbations requiring hospitalisation among children, with a high level of consistency in the direction and magnitude of effect.

\section{Primary outcome: asthma symptoms or exacerbations leading to emergency department visits}

We meta-analysed effect sizes from 13 studies and found clear evidence that school-based asthma self-management interventions were effective in reducing the frequency of $E D$ visits (odds ratio (OR) $0.70,95 \% \mathrm{Cl} 0.53$ to 0.92 ; participants $=$ 3883). Gerald 2006 presented data on the median number of hospitalisations, which were not compatible with other extracted data (full details in Table 13), although the median level of ED visits was observed to be slightly lower for the intervention group than for the control group post intervention.

Heterogeneity in the effects of studies was evident, in terms of both magnitude and direction of effect, with three studies having negligible effect sizes (close to one - Atherly 2009; Clark 2005; Horner 2015) and two studies suggesting a negative intervention effect (McGhan 2003; McGhan 2010); this resulted in an $I^{2}$ value of $26 \%$. The number of studies and the level of heterogeneity allowed us to explore potential study characteristics that could help to explain the observed variation.

\section{Subgroup analyses: exacerbations leading to emergency department visits}

Subgroup analyses suggested that the heterogeneity shown in Figure 7 - Analysis 1.2 - was not explained by school type (Figure 8), age (Analysis 3.1), or socio-economic status of children and intervention deliverers involved in the intervention (Analysis 4.1; Analysis 5.1). 
Figure 7. Forest plot of comparison: 1. Effect of school-based asthma interventions vs usual care, outcome: 1.2. Exacerbations leading to emergency department (ED) visits.

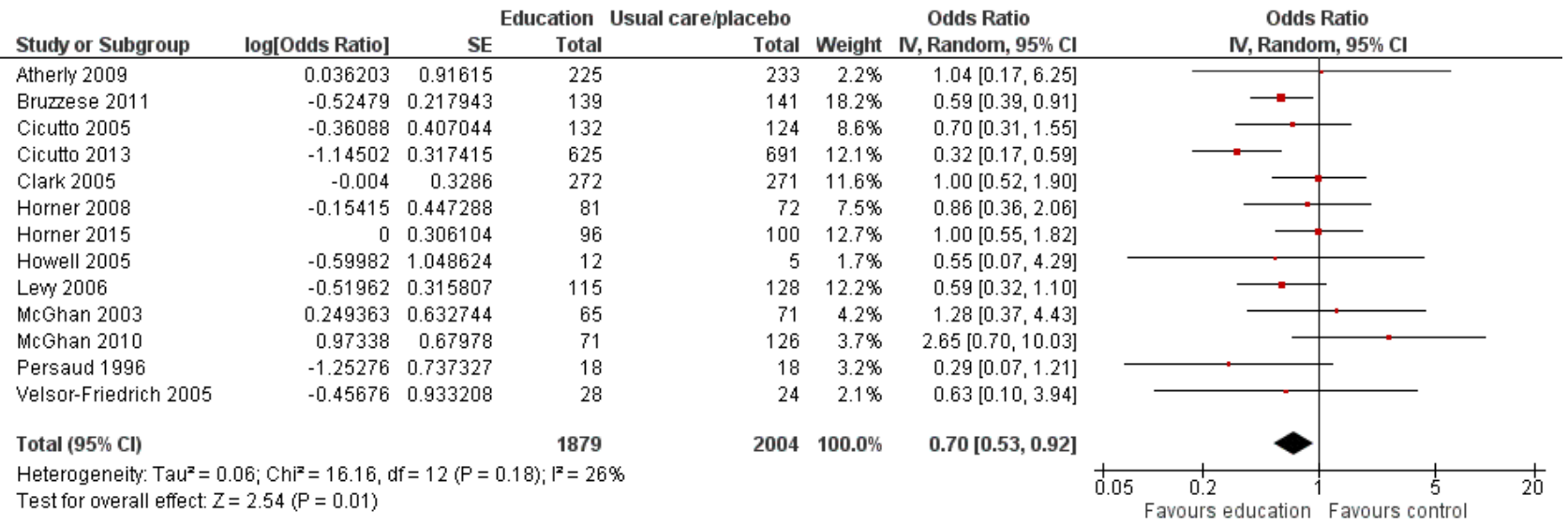

Figure 8. Forest plot of comparison: 2 . Effect of school-based asthma interventions vs usual care subgrouped by school type, outcome: 2.1. Exacerbations leading to emergency department (ED) visits.

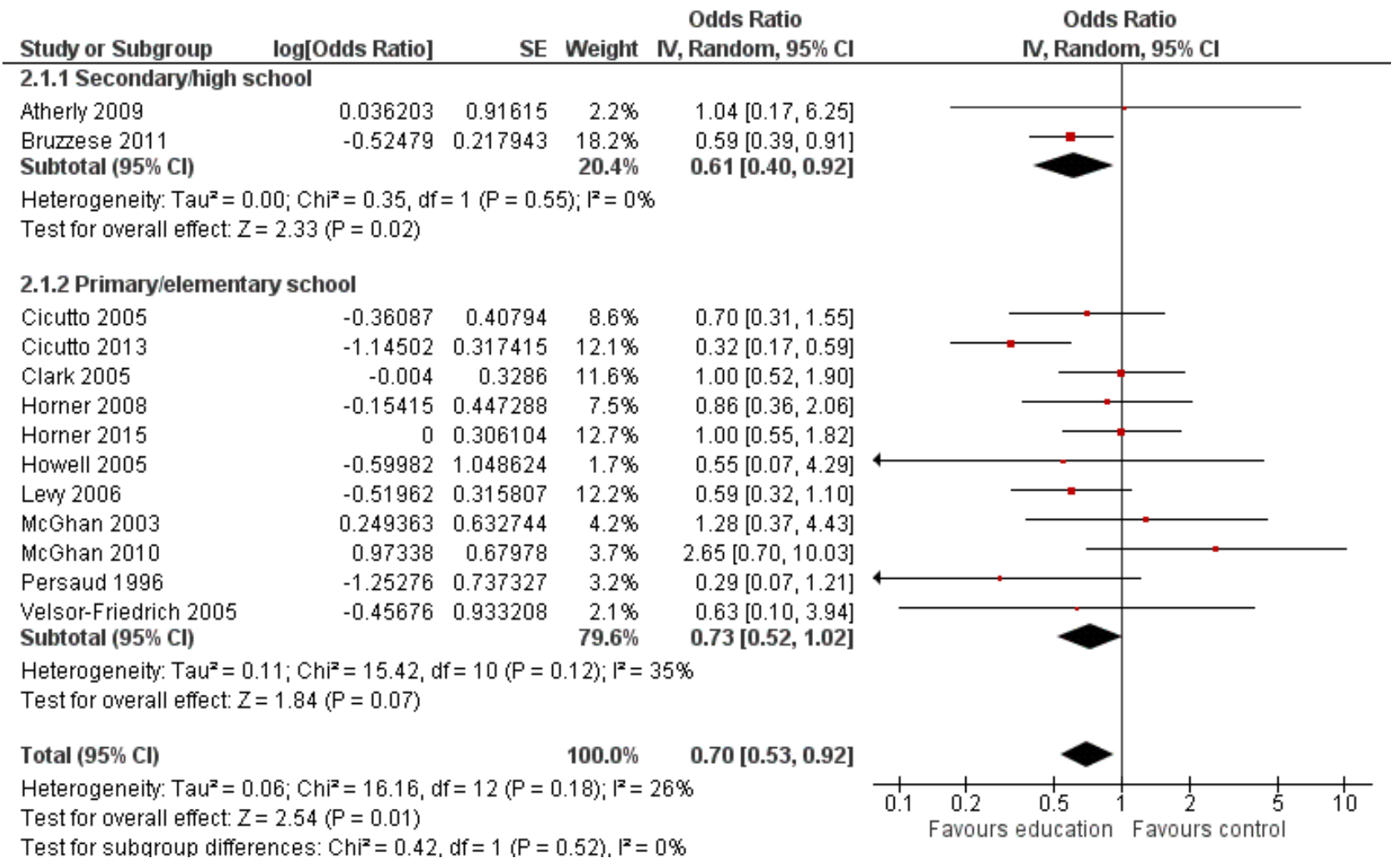

We employed subgroup analyses to examine whether any of the intervention conditions that consistently predicted successful implementation in earlier QCAs, namely, explicit use of theory (Analysis 6.1), inclusion of parents (Analysis 7.1), or timing of the intervention (Analysis 8.1), also helped to explain any of the observed heterogeneity in effect sizes. However, we found no evidence that these factors helped to explain heterogeneity.

We also constructed a variable that attempted to replicate some of the implicants (combinations of intervention characteristics) identified in QCAs that trigger successful implementation; however, results appeared to contradict the findings of earlier analyses. A subgroup of studies that replicated one of the configurations theorised to trigger successful intervention implementation (five studies that were theory driven, did not take place in children's own time, and did not involve school nurses) had inconclusive effect sizes as a group (OR $0.85,95 \% \mathrm{Cl} 0.47$ to 1.52 ); in contrast, a subgroup of studies that did not replicate a configuration were found to trigger successful intervention implementation in the QCAs (OR $0.67,95 \% \mathrm{Cl} 0.47$ to 0.94 ). We also created a variable 
based on a count of intervention characteristics found to trigger successful implementation in our earlier QCAs, and we tested these in subgroup analyses. We constructed a variable reflecting a count of three of the conditions generally found to trigger successful implementation (theory driven, not run in children's own time, and parental engagement (assessed by active involvement of parents)), whereby studies could include zero to three of these 'ingredients'. All studies included in the meta-analyses had incorporated at least one of these conditions, and subgroup analyses suggested that the number of components was inversely related to effect size, with studies with one component (three studies; OR 0.56, 95\% Cl 0.33 to 0.97 ) or two components (seven studies; OR $0.67,95 \% \mathrm{Cl} 0.49$ to 0.94 ) having lower effect sizes than the three studies that included all three components (OR $1.48,95 \% \mathrm{Cl} 0.65$ to 3.40 ); however, the test for differences between subgroups did not suggest that these differences were significant, and moderate heterogeneity remained within one of the subgroups. Among the latter group of studies, two of the three studies evaluated the effectiveness of the RAP (Roaring Adventures of Puff) intervention (McGhan 2003; McGhan 2010). One of these studies provided evidence of a baseline imbalance that could influence the outcome (McGhan 2003), whereby the proportion of intervention group children who had been admitted to an ED was almost ten percentage points higher in the intervention group (23.7\%) than in the control group $(14 \%)$. The second study provided evidence that the mean number of ED visits was higher post intervention in the control group (McGhan 2010), although study authors did not present full data allowing for extraction of the mean number of visits, and the measure used reflected the odds of reporting ED visits.

\section{Sensitivity analyses: exacerbations leading to emergency department} visits

We conducted sensitivity analyses to explore the impact of decisions to transform or combine the data. We detected no differences between effect sizes that were originally measured through binary effect sizes (ORs) and those that were originally measured through continuous measures (SMDs). We detected no differences in whether studies assessed intervention effects at 12 months, four to seven months, or within three months (intervals reflecting the spread of studies). All but two studies - Bruzzese 2011 and Persaud 1996 - had randomised children at the school level (cluster RCTs); little evidence suggested that this distinction explained heterogeneity in effect sizes.

In assessing the impact of study quality on effect sizes, we undertook supplementary analyses using meta-regression in STATA, and, due to the limited number of studies, we combined categories of high and unclear risk when assessing the impact of study quality. We classified none of the studies included in the meta-analysis for ED visits as having high risk of bias for random sequence generation, although we deemed that eight studies were at unclear risk. Results of sensitivity analyses provided moderate evidence that studies had high or unclear risk of selection bias with respect to breaches in allocation concealment with significantly different effect sizes (OR $0.86,95 \% \mathrm{Cl} 0.64$ to 1.16 ), compared to the three studies that we deemed to have low risk of bias (OR 0.51, 95\% $\mathrm{Cl} 0.33$ to 0.78 ). Finally, evidence showed that studies with low risk of bias with respect to collection of outcome data and blinding of collectors were significantly more effective (OR $0.58,95 \% \mathrm{Cl} 0.41$ to 0.81 ) than the seven studies with unclear or high risk of bias (OR $1.04,95 \% \mathrm{Cl} 0.69$ to 1.58$)$. Differences in the risk of bias classification for other domains did not significantly explain heterogeneity in effect sizes between studies. We conducted sensitivity analyses to explore the impact of a random-effects specification on pooled effect size, noting only moderate differences in point estimates between fixed-effect $(\mathrm{OR} 0.68,95 \% \mathrm{Cl} 0.55$ to 0.85$)$ and randomeffects models (OR $0.70,95 \% \mathrm{Cl} 0.53$ to 0.92 ); however, the level of heterogeneity $\left(I^{2}=26 \%\right)$ suggested that studies were not measuring a single common effect size, thereby undermining the fixed-effect assumption (and model results).

Our investigations into the potential impact of publication bias revealed that neither the funnel plot nor Egger's test was indicative of publication bias (the bias coefficient provided weak evidence that smaller studies differed systematically from studies with larger sample sizes).

Evidence therefore suggests that school-based asthma selfmanagement interventions do reduce the frequency of asthma symptoms and exacerbations requiring emergency care among children, although variation in the magnitude and direction of effect was not explained coherently by planned subgroup analyses.

\section{Primary outcome: absences from school}

Ten studies contributed to our meta-analyses of effects of interventions on school absences, although there was uncertainty as to whether school-based self-management interventions had an impact on reducing absences from school (SMD - $0.07,95 \% \mathrm{Cl}-0.22$ to 0.08; participants $=4609$; Analysis 1.3; Figure 9). These studies showed substantial heterogeneity between effect size estimates, with $\mathrm{I}^{2}$ estimated at $70 \%$. Effect sizes from half of the studies included in the meta-analysis indicated that the intervention had a negative impact in slightly or significantly increasing the number of school absences in the intervention group relative to the control group (Gerald 2006; Gerald 2009; Howell 2005; McGhan 2010; Splett 2006). 
Figure 9. Forest plot of comparison: 1. Effect of school-based asthma interventions vs usual care, outcome: 1.3. Absence from school.

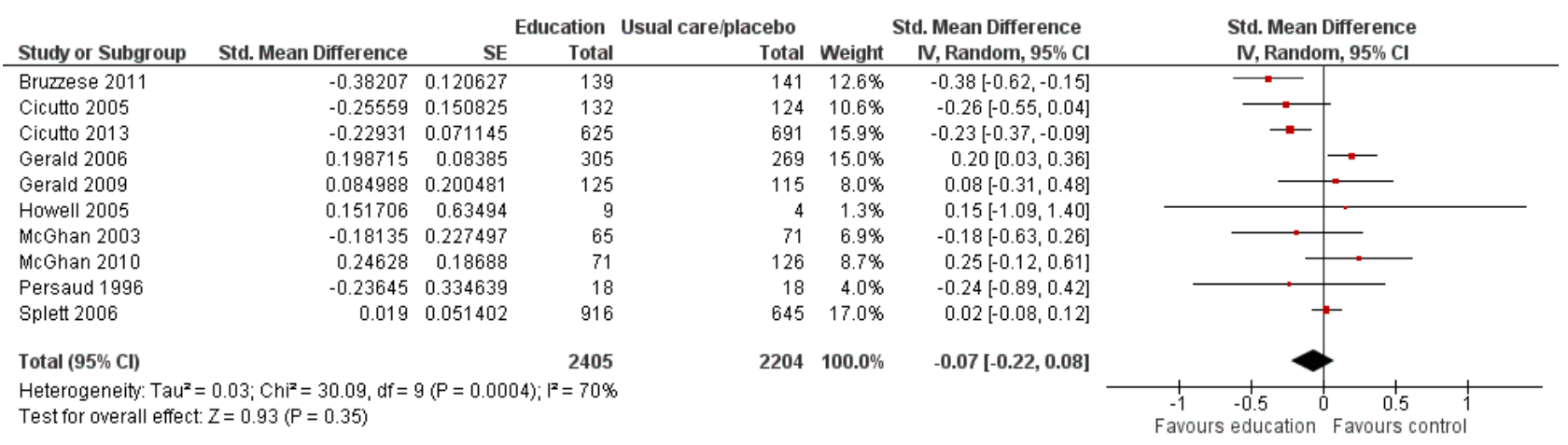

\section{Subgroup analyses: absences from school}

We undertook subgroup analyses to explore studylevel characteristics that could explain this between-study heterogeneity, although it is worth noting that these analyses were likely to be underpowered and to represent indicative factors that could explain observed differences in the direction and magnitude of effect sizes across studies. The only study included in the metaanalyses that focused on high schools (and consequently older children) was highly effective in reducing school absences (SMD $-0.38,95 \% \mathrm{Cl}-0.62$ to -0.15 ) (Bruzzese 2011); this study appeared to drive much of the heterogeneity explained by subgroup analyses examining school type and child age (Figure 10; Analysis 2.2; Analysis 3.2). Studies that included $25 \%$ to $50 \%$ children from lower socio-economic backgrounds were significantly more effective in reducing levels of school absence (SMD $-0.23,95 \% \mathrm{Cl}-0.36$ to -0.09 ; studies $=2$ ) than studies with greater numbers of children from deprived backgrounds (over 50\%) for whom the effect was negligible (SMD $0.01,95 \% \mathrm{Cl}-0.09$ to $0.11 ; 2$ studies) and studies in which less than $25 \%$ of children were from deprived backgrounds or in which this was unclear, where the pooled effect size indicated negligible effect (SMD $-0.02,95 \% \mathrm{Cl}-0.29$ to $0.24 ; 6$ studies). 
Figure 10. Forest plot of comparison: 2. Effect of school-based asthma interventions vs usual care subgrouped by school type, outcome: 2.2. Absence from school.

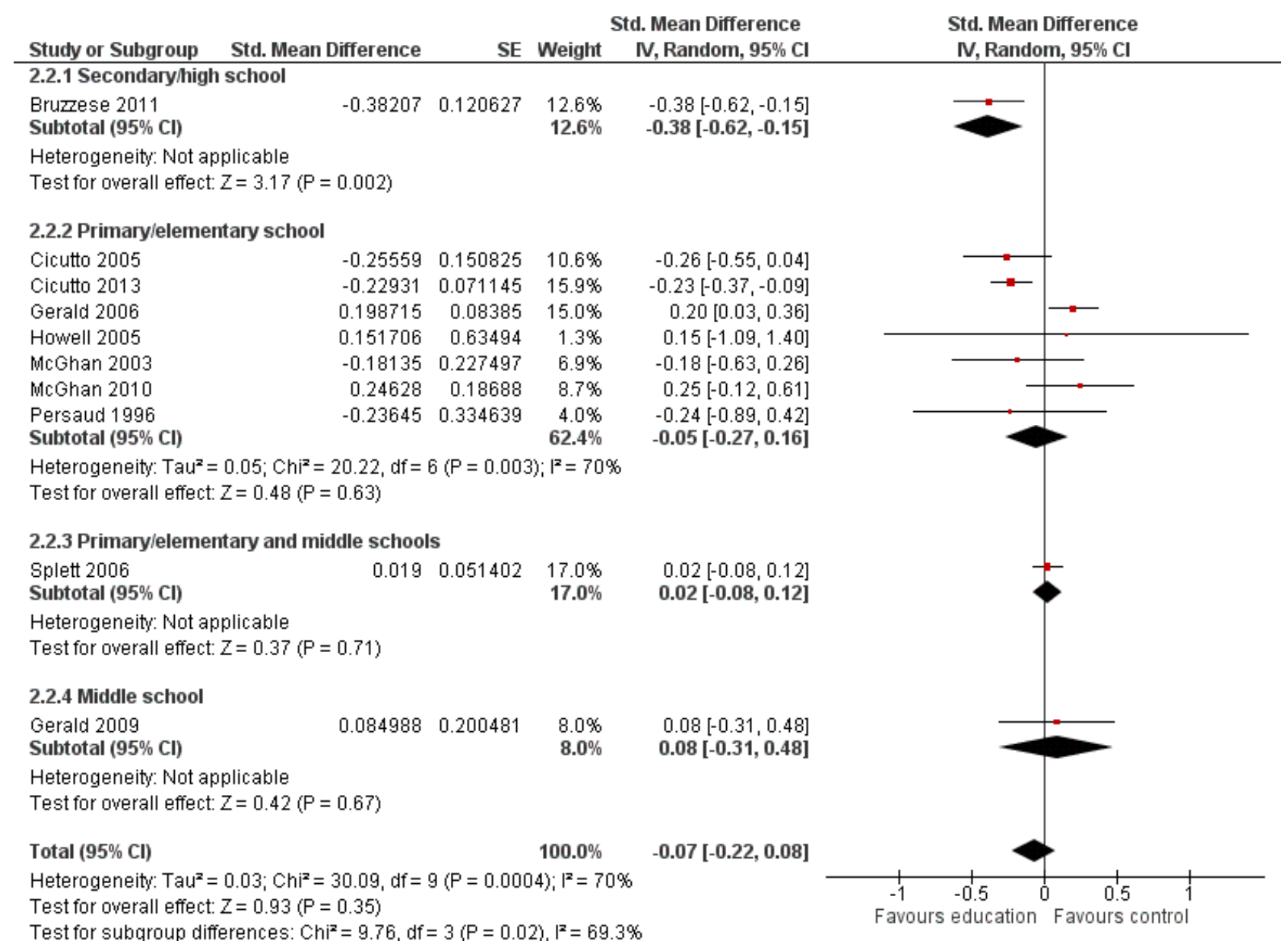

Studies that involved existing school staff (teachers or school nurses) in delivery of the intervention were significantly less effective (SMD $0.08,95 \% \mathrm{Cl}-0.08$ to $0.24 ; 3$ studies) than studies in which the intervention was mainly delivered and facilitated by stakeholders who were external to the school (SMD $-0.17,95 \% \mathrm{Cl}$ -0.32 to $-0.02 ; 7$ studies; Analysis 5.2; Figure 11). Findings of the earlier QCA show that involvement of internal stakeholders within

the school in delivery of the intervention did not always lead to successful intervention implementation, but they also show that involving school staff in intervention delivery may be one of a configuration of conditions that trigger successful implementation, none of which are sufficient alone. Similar processes may occur around their role in reducing the level of school absence. 
Figure 11. Forest plot of comparison: 10. Effect of school-based asthma interventions vs usual care subgrouped by configuration of conditions (il), outcome: 10.3. Absence from school.

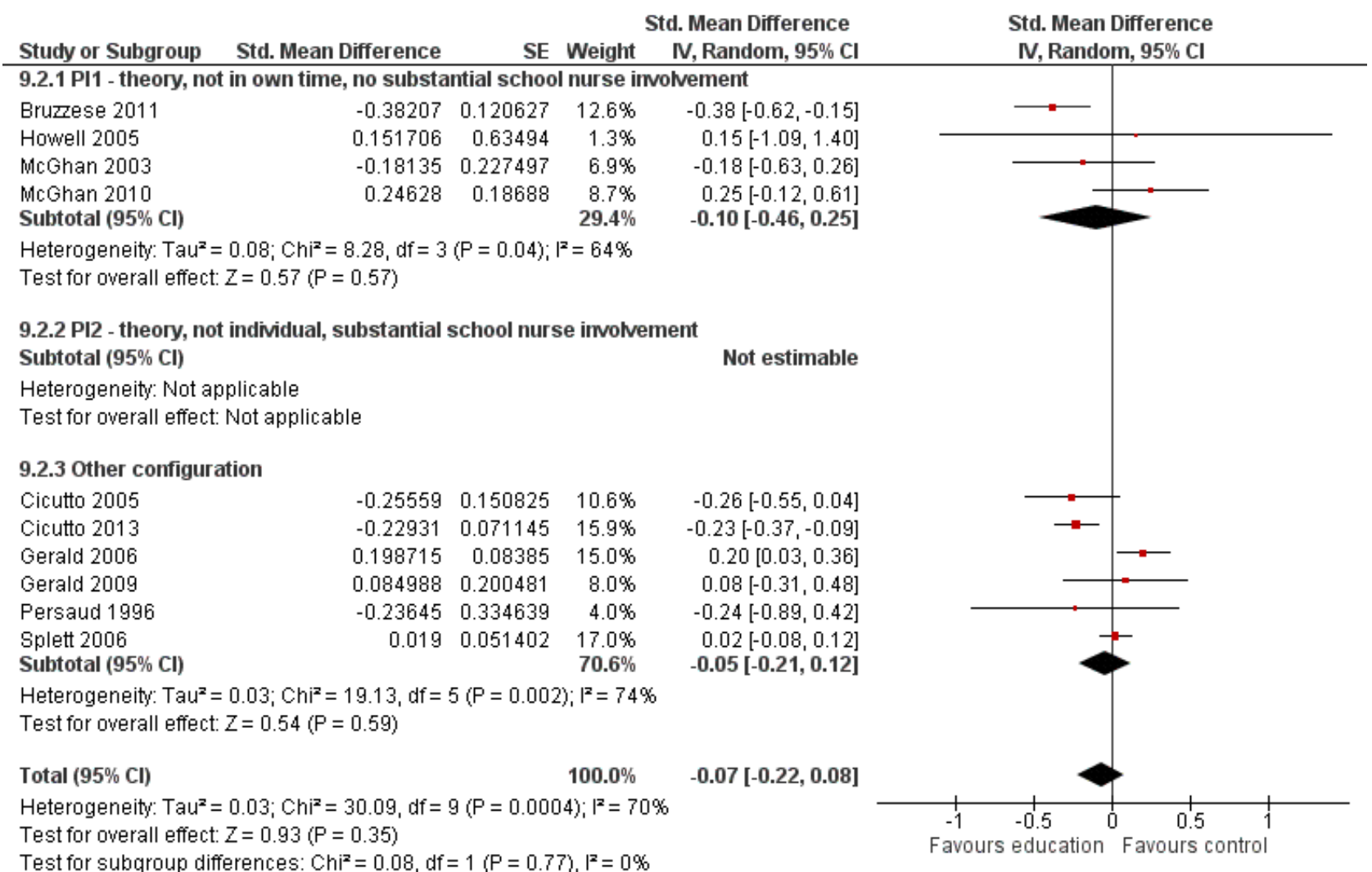

We conducted subgroup analyses involving the conditions and configurations found to be sufficient in earlier QCAs to trigger successful implementation. But these findings did not significantly explain the heterogeneity in effect sizes, with two exceptions. Analysis 8.2 provided evidence that interventions that took place during the child's own time had significantly greater impacts in reducing school absence (SMD $-0.23,95 \% \mathrm{Cl}-0.36$ to $-0.11 ; 2$ studies) than those that took place at another point in the school day (SMD $-0.01,95 \% \mathrm{Cl}-0.18$ to $0.16 ; 8$ studies), although a substantial level of heterogeneity remained among this latter group of studies $\left(I^{2}=62 \%\right)$. We noted strong evidence around the role of theory (Analysis 6.2), whereby studies that reported drawing upon a defined theoretical framework had a significantly more impactful pooled effect size (SMD $-0.20,95 \% \mathrm{Cl}-0.36$ to $-0.04 ; 6$ studies) than studies that did not (SMD $0.08,95 \% \mathrm{Cl}-0.05$ to $0.20 ; 4$ studies). Although moderate levels of heterogeneity remained $\left(\mathrm{I}^{2}=41 \%\right.$ for studies that explicitly drew upon theory and $\mathrm{I}^{2}=28 \%$ for those that did not), and even though interpretation of these results is not straightforward (see discussion), this result indicates that theorydriven studies may achieve better outcomes with respect to this domain.

\section{Sensitivity analyses: absences from school}

We conducted sensitivity analyses to explore whether the following factors, reflecting study design or analytical decisions made during the review process, helped to explain heterogeneity in effect size: (I) transformations were made to the original effect size (conversions between ORs and SMDs; Chinn 2000); (ii) cluster RCT or not; (iii) the data collection period; and (iv) the study's risk of bias. We

found no evidence to suggest that transformations in effect sizes explained heterogeneity, and no evidence indicated that the unit of randomisation (school vs child) explained variation in effect size. The three studies that collected absence data within three months post intervention (or for which the collection date was unclear) did exhibit a weaker effect in reducing school absences (Gerald 2006; Howell 2005; Persaud 1996), with Gerald 2006 and Howell 2005 showing a negative intervention impact, although this was not significantly different from studies that assessed absences over the 12 months post intervention. Little evidence suggests that risk of bias influenced the effect size obtained; however, studies that had taken steps to blind assessment of outcomes and to avoid detection bias had a greater impact in reducing school absences (SMD - 0.27 , $95 \% \mathrm{Cl}-0.38$ to $-0.17 ; 3$ studies) than studies that did not take these steps (SMD $-0.07,95 \% \mathrm{Cl}-0.02$ to $0.16 ; 7$ studies).

We investigated the potential impact of publication bias by examining a funnel plot and the results of Egger's test. These tests did not provide strong evidence that data were impacted by publication bias (the bias coefficient provided weak evidence that smaller studies differed systematically from studies with larger sample sizes). We examined differences between the fixed-effect model and the random-effects model reported above. The fixedeffect model showed that the pooled point estimate remained similar, but with a less conservative confidence interval (SMD - 0.05 , $95 \% \mathrm{Cl}-0.11$ to 0.02 ). However the level of heterogeneity was substantial $\left(I^{2}=70 \%\right)$, which suggested that these studies were not measuring a single common effect size and thereby undermined the fixed-effect assumption (and model results). 
Evidence from the overall pool of studies therefore suggests that school-based asthma self-management interventions did not have an impact in reducing absence from school, although variation in direction and magnitude was substantial. Planned subgroup analyses assisted in identifying particular groups of studies and did, or did not, have a beneficial effect.

\section{Primary outcome: days of restricted activity}

Three studies contributed data to our meta-analysis of the impact of school-based asthma self-management interventions in reducing the number of days of restricted activity that children experienced (Bruzzese 2011; Cicutto 2005; Cicutto 2013). These studies provided evidence that the intervention mode could reduce the number of days of restricted activity experienced (SMD -0.30, $95 \% \mathrm{Cl}-0.41$ to $-0.18 ; 1852$ participants; 3 studies; Analysis 1.4), albeit based on a limited number of studies, two of which evaluated the same intervention design (the Roaring Adventures of Puff) (Cicutto 2005; Cicutto 2013). All three studies provided relatively consistent evidence around the direction and magnitude of effect $\left(I^{2}=0 \%\right)$. Reporting on the results of subgroup analyses is not meaningful in the presence of low heterogeneity and small numbers of studies, and many sensitivity analyses could not be conducted for the same reason, although it is worth noting that we rated none of the included studies as having high risk of bias for any domain assessed for the outcome evaluation risk of bias tool (Figure 12).

Figure 12. Forest plot of comparison: 1. Effect of school-based asthma interventions vs usual care, outcome: 1.4. Days of restricted activity.

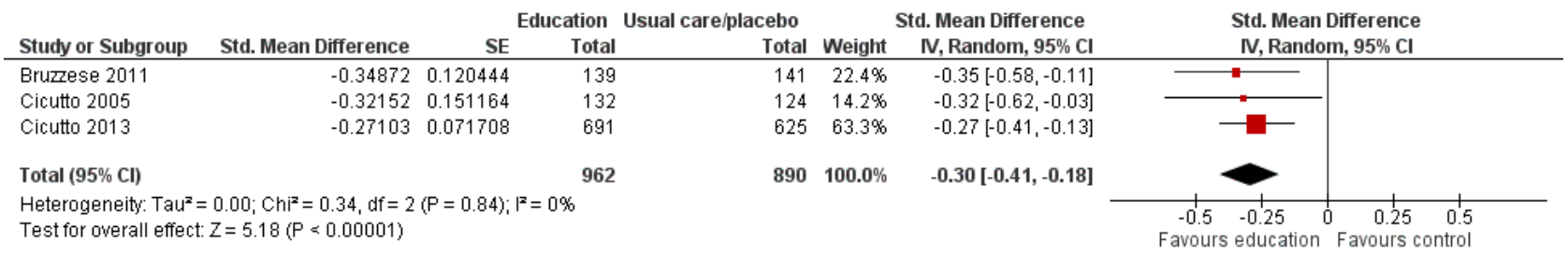

\section{Secondary outcome: unplanned visits to a medical provider}

From a meta-analysis of five studies (Analysis 1.5), evidence shows that school-based asthma self-management interventions did reduce the number of unplanned or unscheduled visits to a medical provider (OR $0.74,95 \% \mathrm{Cl} 0.60$ to $0.90 ; 3490$ participants; 5 studies). Despite inconsistency in the magnitude (and direction) of effect in the case of McGhan 2003, which indicated a small negative intervention impact, the meta-analysis provided little evidence of statistical heterogeneity $\left(I^{2}=0 \%\right)$. As was the case above, the small number of studies and the absence of heterogeneity did not support meaningful investigation of subgroup analyses, nor the opportunity to undertake a full assessment of some of the assumptions made in pooling the data (see Table 13 for further details on the derivation of effect sizes). Similarly, we were not able to assess the potential impact of publication bias. Two studies contributed almost 75\% towards the pooled effect size (Bruzzese 2011; Cicutto 2013), and we rated neither study as having high risk of bias in any domain (Figure 13).

Figure 13. Forest plot of comparison: 1. Effect of school-based asthma interventions vs usual care, outcome: 1.5. Unplanned visit to hospital or GP due to asthma symptoms.

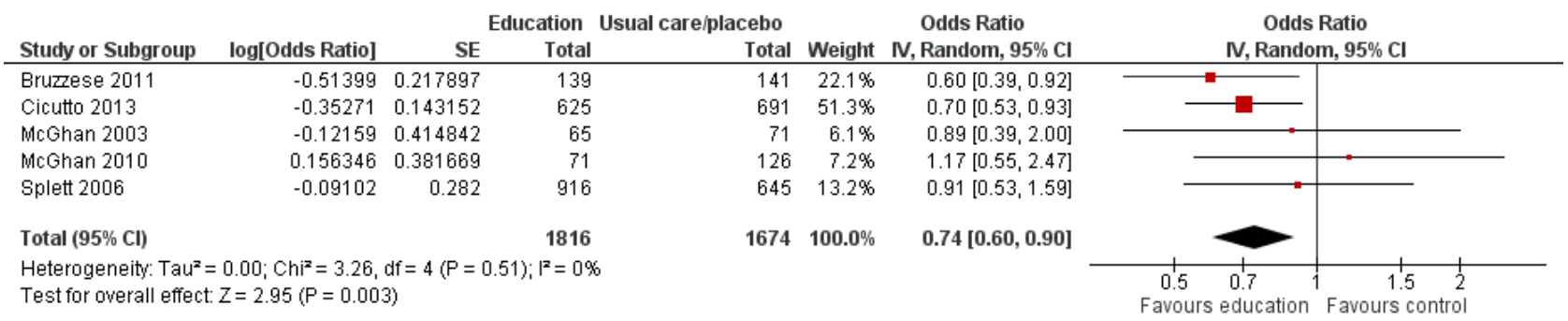

\section{Secondary outcome: experience of daytime and night-time symptoms}

As described in the section on Included studies, trialists adopted different strategies in measuring the impact of interventions on children's daytime and night-time symptoms. We constructed separate models of meta-analysis for studies reporting on daytime symptoms (Analysis 1.6) and night-time symptoms (Analysis 1.7), although some variation remained in the way in which symptom data were collected (Table 13).

Uncertainty surrounded the question of whether school-based selfmanagement interventions reduced the level of daytime symptoms that children experienced (SMD $-0.15,95 \% \mathrm{Cl}-0.32$ to $0.02 ; \mathrm{I}^{2}=$ $0 \% ; 1065$ participants; 5 studies), with the confidence interval just crossing the line of no effect (zero). However, study reports show consistency in the direction of effects (Figure 14). Even greater uncertainty surrounded whether self-management interventions in schools reduced the level of night-time symptoms reported by children in random effects meta-analysis (SMD -0.18, 95\% $\mathrm{Cl}-0.52$ to $0.15 ; \mathrm{I}^{2}=40 \% ; 459$ participants; 4 studies), with two studies providing weak evidence that night-time symptoms actually increased among children receiving school-based asthma self-management interventions. We performed sensitivity analyses using a fixed-effect model, with the pooled effect size across 
the four studies indicating that night-time symptoms decreased (SMD $-0.26,95 \% \mathrm{Cl}-0.46$ to $-0.06 ; 4$ studies), although given the inconsistency in the direction of effect, the underlying assumptions of the fixed-effect model cannot be substantiated, and the randomeffects model may provide a more realistic estimate of intervention effects on night-time symptoms.

Figure 14. Forest plot of comparison: 1. Effect of school-based asthma interventions vs usual care, outcome: 1.6. Experience of daytime and night-time symptoms - daytime symptoms.

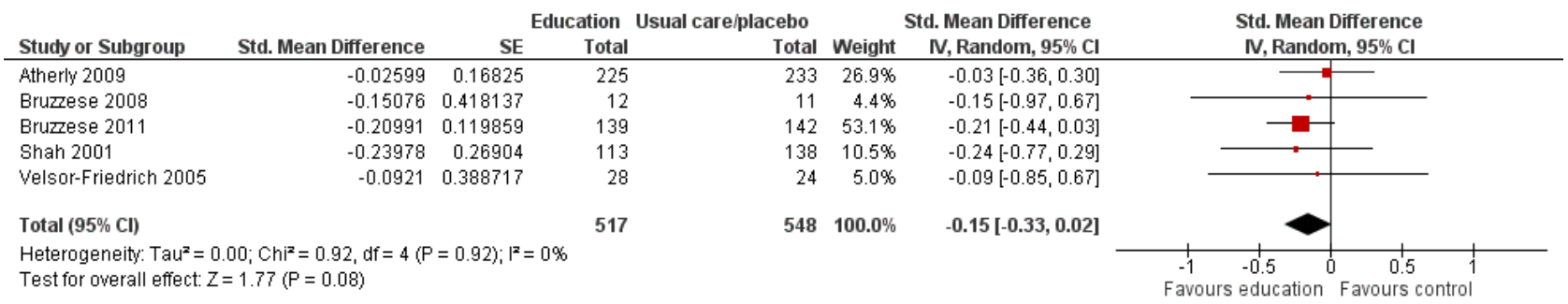

Reporting on the results of subgroup analyses was not meaningful with the few included studies; other sensitivity analyses could not be conducted for the same reason. One study that measured change in daytime symptoms showed a weak effect of the intervention in lowering the level of daytime symptoms (see Table 13) (Clark 2010).

\section{Secondary outcome: lung function}

We extracted outcomes measuring trial impacts on lung function from five studies, although we did not combine these data in metaanalyses due to conceptual (and statistical) heterogeneity. We have presented these outcomes in full in Table 13.

\section{Secondary outcome: use of reliever therapies}

Four studies reported on the use of reliever therapies among children who had received self-management interventions in school (Table 13), and we included effect sizes from two studies with clinical and conceptual equivalence in a random-effects metaanalysis (Figure 15; Analysis 1.8). The pooled result provided uncertain evidence on the impact of the intervention on children's use of reliever therapies (OR $0.52,95 \% \mathrm{Cl} 0.15$ to $1.81 ; 437$ participants; 2 studies). The level of heterogeneity between studies was substantial $\left(I^{2}=68 \%\right)$, although both were somewhat consistent in the direction of effect, indicating lower odds of (frequent) reliever therapy usage. One study had low or unclear risk of bias across all domains considered (Gerald 2009), and we judged McGhan 2010 to have high risk of bias in terms of attrition bias and selective reporting.

Figure 15. Forest plot of comparison: 1. Effect of school-based asthma interventions vs usual care, outcome: 1.8. Use of reliever therapies, e.g. beta ${ }_{2}$-agonists.

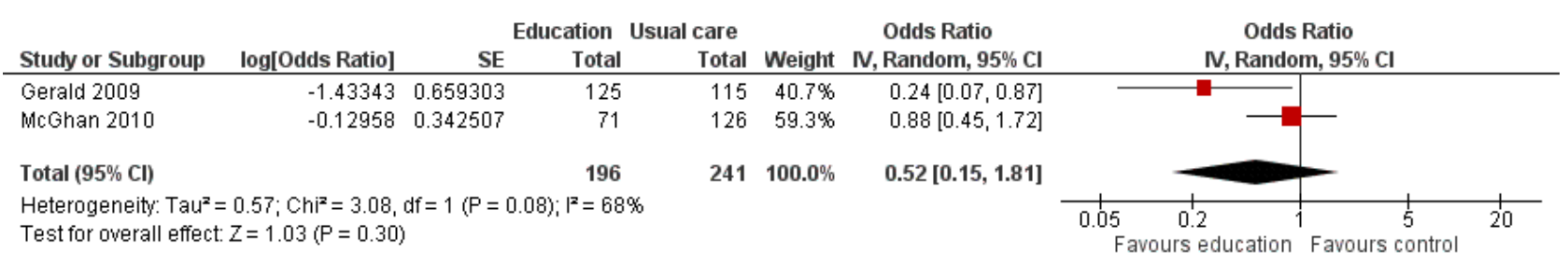

\section{Secondary outcome: corticosteroid dosage and use of add-on therapies}

We found unclear evidence on the impact of interventions on children's use of corticosteroids and add-on therapies (OR 1.25, $95 \% \mathrm{Cl} 0.88$ to $1.77 ; 614$ participants; 3 studies; Figure 16; Analysis 1.9). We noted no evidence of statistical heterogeneity between these study impacts on corticosteroid usage $\left(1^{2}=0 \%\right)$, and as reporting on the results of subgroup analyses is not meaningful with low heterogeneity and few studies, we could not conduct other sensitivity analyses for the same reason. We deemed one study included in the model to have low risk of bias for all domains except blinding of participants and personnel, for which we deemed the risk to be unclear (Bruzzese 2011); we deemed the other two studies to have high risk of bias in one and two domains (McGhan 2003; McGhan 2010), respectively, with both deemed to have high risk of attrition bias from incomplete and unexplained dropouts at outcome data collection. 
Figure 16. Forest plot of comparison: 1. Effect of school-based asthma interventions vs usual care, outcome: 1.9. Corticosteroid dosage and/or use of add-on therapies (usage of).

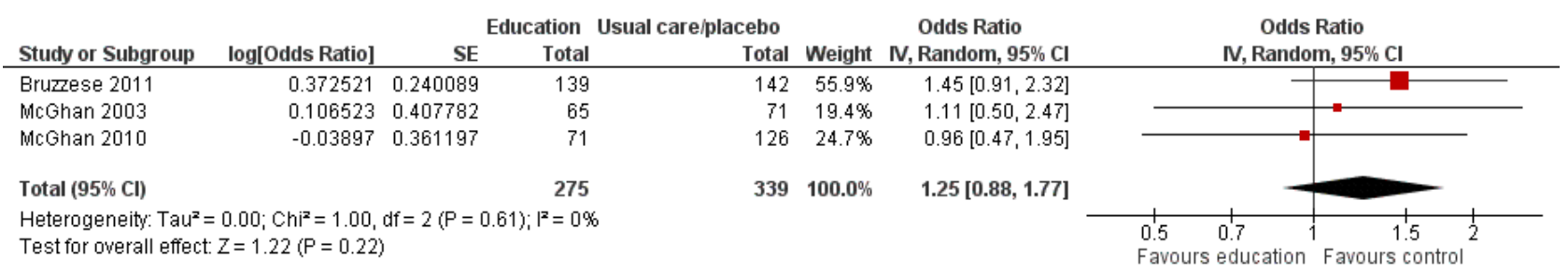

We included two studies reporting appropriate usage of corticosteroids and add-on therapies. Although the direction of findings differed substantially between studies, resulting in considerably high levels of heterogeneity $\left(1^{2}=87 \%\right)$, we did not estimate a pooled effect size (Analysis 1.10).

\section{Secondary outcome: health-related quality of life}

Nine studies provided data on the effectiveness of school-based self-management interventions in improving children's quality of life. Because of conceptual differences in the way in which the outcome was measured, one meta-analysis of seven studies explored intervention impacts on quality of life measures assessed through standardised mean differences using mainly the Paediatric Asthma Quality of Life Questionnaire (PAQLQ) (Figure 17; Analysis
1.11), and provided evidence of effectiveness (SMD 0.27, 95\% Cl 0.18 to $0.36 ; 2587$ participants; 7 studies). This model provided no evidence of statistical heterogeneity in effectiveness $\left(1^{2}=0 \%\right)$, with all studies providing estimates of positive improvements, although these were not all statistically significant in all studies. The low level of heterogeneity and the few included studies meant that conducting subgroup analyses was not appropriate. We deemed that five of the seven studies included in the meta-analysis were at high risk of bias in at least one domain (Al-Sheyab 2012; Henry 2004; Horner 2008; Howell 2005; Kintner 2009), although the two studies with low or unclear risk of bias in all domains contributed over $60 \%$ of the weighted effect size (Cicutto 2005; Cicutto 2013). Explorations of the funnel plot and Egger's test were underpowered, and publication bias could not be adequately tested.

Figure 17. Forest plot of comparison: 1. Effect of school-based asthma interventions vs usual care, outcome: 1.11. Health-related quality of life (SMD).

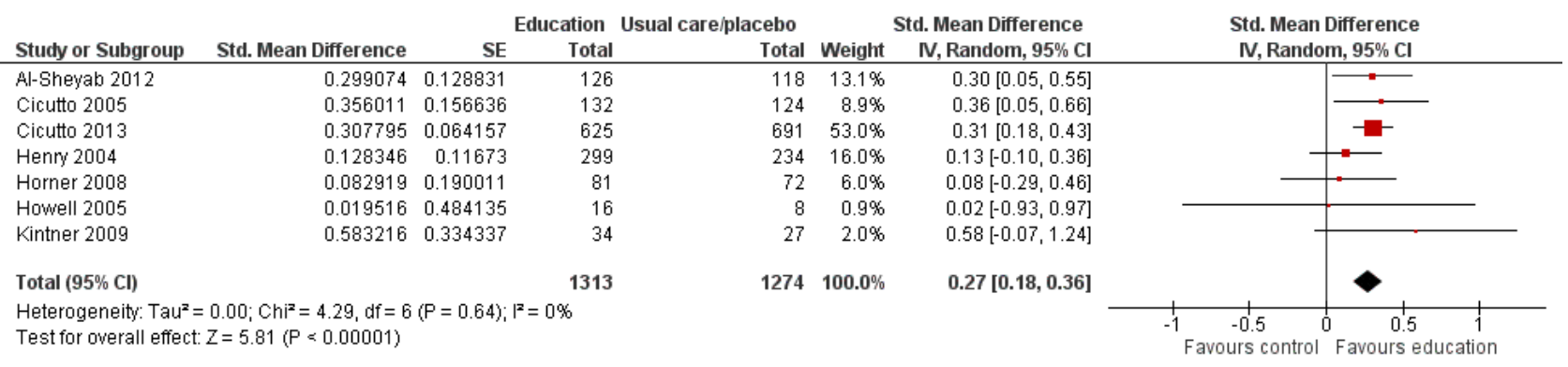

A second meta-analysis involving eight studies also provided evidence that children in intervention groups had higher HRQoL than children in control groups (MD $0.35,95 \% \mathrm{Cl} 0.06$ to 0.64 2950 participants; 8 studies) based on PAQLQ results at follow-up (Analysis 1.12). The mean difference, while again indicating that the impact did not cross the threshold of no effect, fell below 0.5 - the threshold considered to indicate a clinically significant change in HRQoL on this scale. Heterogeneity among studies was considerably high $\left(I^{2}=81 \%\right)$. One study in particular had relatively high levels of baseline imbalance, and a sensitivity analysis removing this value resulted in a lower point estimate but much lower levels of heterogeneity (MD $0.21,95 \% \mathrm{Cl} 0.07$ to 0.36 ; $I^{2}=24 \%$ ) (Al-Sheyab 2012). We included this same study in Analysis 1.11 , although we used different data to obtain an effect size (P value and sample size). We did not further explore heterogeneity because included studies were few and, similarly, explorations of the funnel plot and Egger's test were underpowered; therefore, we could not adequately assess publication bias. We deemed that four of the studies included in Analysis 1.12 were at high risk of bias in at least one domain. A further sensitivity analysis involving constructing a fixed-effect model yielded a similar point estimate (MD $0.32,95 \% \mathrm{Cl} 0.21$ to $0.43 ; \mathrm{I}^{2}=81 \% ; 8$ studies), although the considerably high level of heterogeneity indicates that this is not a suitable analytical framework.

Despite the additional study included in Analysis 1.12, we consider the results from Analysis 1.11 to be more reliable because of the considerably high heterogeneity observed in the model for MD and the insufficient number of studies to fully explore drivers of this heterogeneity.

Therefore, evidence suggests that school-based asthma selfmanagement interventions do improve children's quality of life, although this finding may not reach a point of clinically significant improvement. Although all studies provided an indication of a positive beneficial effect, variation in the size of the effect was substantial. 


\section{Secondary outcome: withdrawal from the study}

Meta-analysis provided no evidence that participation in the intervention was linked to withdrawal from the study (OR 1.14, 95\% $\mathrm{Cl} 0.92$ to 1.43 ; 3442 participants; 13 studies; Figure 18; Analysis 1.13). We detected no substantial statistical heterogeneity $\left(I^{2}=\right.$ $0 \%)$, although some qualitative differences were apparent between studies that reported very low levels of withdrawal among those receiving treatment relative to those in control groups (Bruzzese 2008), and relative to those with very high levels of withdrawal (Kintner 2009; Patterson 2005); in neither case would the level of withdrawal be described as problematic (not exceeding 25\% of participants), and the stark relative effect was driven by very small sample sizes in some studies (Bruzzese 2008; Kintner 2009).

Figure 18. Forest plot of comparison: 1. Effect of school-based asthma interventions vs usual care, outcome: 1.13. Withdrawal from the study.

\begin{tabular}{|c|c|c|c|c|c|c|c|c|c|c|c|}
\hline Study or Subgroup & log[Odds Ratio] & SE & $\begin{array}{c}\text { cation U } \\
\text { Total }\end{array}$ & $\begin{array}{r}\text { Usual care/placebo } \\
\text { Total }\end{array}$ & Weight & $\begin{array}{c}\text { Odds Ratio } \\
\text { IV, Random, } 95 \% \mathrm{Cl}\end{array}$ & & $\begin{array}{r}\text { Odds } \\
\text { IN, Randon }\end{array}$ & $\begin{array}{l}\text { Ratio } \\
\mathrm{m}, 95 \% \mathrm{Cl}\end{array}$ & & \\
\hline Al-Sheyab 2012 & -0.67173 & 1.074323 & 132 & 129 & $1.1 \%$ & $0.51[0.06,4.20]$ & & & & & \\
\hline Bartholomew 2006 & 0.237201 & 0.173008 & 515 & 431 & $42.9 \%$ & $1.27[0.90,1.78]$ & & & 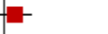 & & \\
\hline Bruzzese 2008 & -1.18199 & 1.683337 & 12 & 12 & $0.5 \%$ & $0.31[0.01,8.31]$ & & & & & \\
\hline Bruzzese 2011 & 0.272668 & 0.278798 & 175 & 170 & $16.5 \%$ & $1.31[0.76,2.27]$ & & & $=-$ & & \\
\hline Cicutto 2005 & 0.58103 & 0.629046 & 132 & 124 & $3.2 \%$ & $1.79[0.52,6.13]$ & & & & & \\
\hline Gerald 2009 & -0.48885 & 0.367885 & 145 & 145 & $9.5 \%$ & $0.61[0.30,1.26]$ & & & & & \\
\hline Horner 2008 & 0.287682 & 0.530694 & 101 & 82 & $4.6 \%$ & $1.33[0.47,3.77]$ & & & & & \\
\hline Horner 2015 & -0.28768 & 0.486469 & 96 & 100 & $5.4 \%$ & $0.75[0.29,1.95]$ & & & - & & \\
\hline Kintner 2009 & 2.607967 & 1.882092 & 38 & 28 & $0.4 \%$ & $13.57[0.34,542.83]$ & & & & & \\
\hline McGhan 2003 & 0.219474 & 0.383454 & 76 & 86 & $8.7 \%$ & $1.25[0.59,2.64]$ & & & & & \\
\hline McGhan 2010 & -0.22186 & 0.511725 & 104 & 162 & $4.9 \%$ & $0.80[0.29,2.18]$ & & & & & \\
\hline Patterson 2005 & 1.736044 & 1.806959 & 83 & 92 & $0.4 \%$ & $5.67[0.16,195.90]$ & & & & & \\
\hline Shah 2001 & 0.295176 & 0.815795 & 124 & 148 & $1.9 \%$ & $1.34[0.27,6.65]$ & & & & & \\
\hline Total $(95 \% \mathrm{Cl})$ & & & 1733 & 1709 & $100.0 \%$ & $1.14[0.92,1.43]$ & & & & & \\
\hline \multicolumn{7}{|c|}{$\begin{array}{l}\text { Heterogeneity: } \operatorname{Tau}^{2}=0.00 ; \mathrm{Ch}^{2}=9.07, \mathrm{df}=12(\mathrm{P}=0.70) ; \mathrm{I}^{2}=0 \% \\
\text { Test for overall effect: } \mathrm{Z}=1.18(\mathrm{P}=0.24)\end{array}$} & 0.01 & $\begin{array}{l}1 \\
0.1 \\
\text { Irs education }\end{array}$ & 1 Favours 1 & $\begin{array}{l}10 \\
\text { control }\end{array}$ & 100 \\
\hline
\end{tabular}

Despite the low level of heterogeneity, we have presented subgroup analyses because of the link between this outcome and the QCAs presented earlier. When we replicated one set of configurations in the subgroup analysis to mirror QCA findings (Analysis 9.3), we found weak/uncertain evidence to suggest that studies that used theory, while avoiding running the intervention in children's own time and having no substantial school staff involvement, were less likely to have children drop out before outcomes were assessed (OR $0.88,95 \% \mathrm{Cl} 0.55$ to 1.40 ; 4 studies) when compared with studies with other configurations of conditions (OR $1.23,95 \% \mathrm{Cl} 0.95$ to 1.58 ; 8 studies). We also found no evidence that withdrawal from the intervention was associated with school type (Figure 19). 
Figure 19. Forest plot of comparison: 2. Effect of school-based asthma interventions vs usual care subgrouped by school type, outcome: 2.3. Withdrawal from the study.

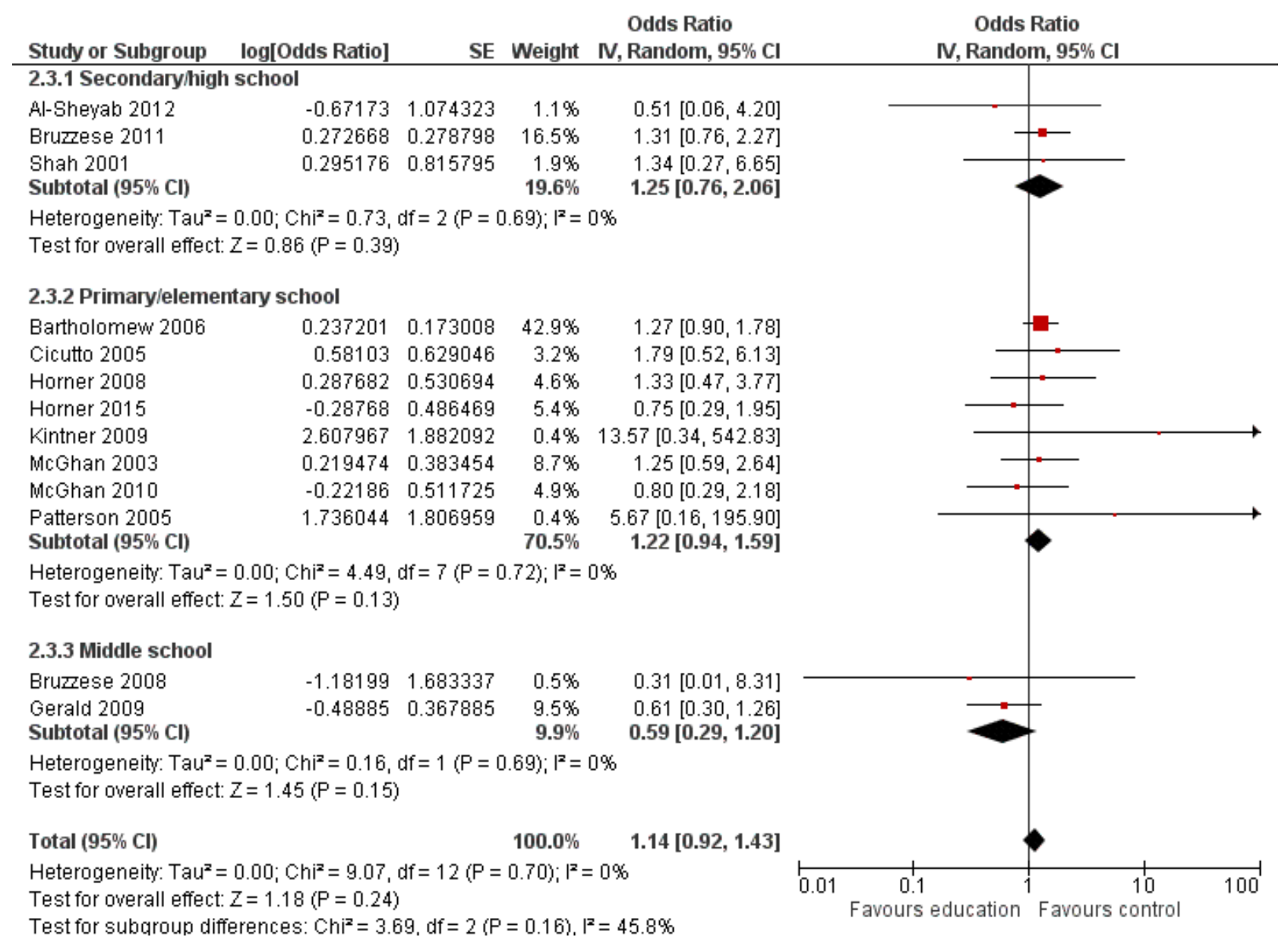

Subgroup analyses seeking to reveal patterns of heterogeneity in the odds of withdrawal did not show that timing of assessment, unit of randomisation (cluster vs individually randomised trials), or risk of bias explained patterns of withdrawal. This included risk of attrition bias assessments, although the meta-analysis explored differential patterns of attrition and did not account for instances in which both intervention and control groups had high levels of attrition (as was the case when risk of attrition bias was assessed). We found no evidence to show that publication bias was an issue in terms of withdrawal data. Because of the low level of statistical heterogeneity, fixed-effect and random-effects specifications for the meta-analyses were equivalent, with one study accounting for $46 \%$ of the weighting; we classified this study as having high risk of bias in three domains and unclear risk of bias in the remaining four domains (Bartholomew 2006).

\section{Results of synthesis - part 3: adjunct meta-analyses exploring the link between implementation and effectiveness of school- based asthma self-management interventions}

We conducted adjunct meta-analyses to explore whether interventions that were deemed successful in terms of implementation were also deemed successful in terms of their effectiveness (see Figure 2), using a subset of studies contained within the process evaluations. For inclusion in these analyses, we considered studies that included a control group; however studies could have employed randomisation or quasi-experimental methods, and control group children could have received an alternative intervention that might have included an asthma component.

Because of conceptual and methodological differences in study design, these studies provide indicative evidence only pertaining to the impact of self-management interventions on children's asthma outcomes, but they help us to establish links between implementation factors and asthma outcomes. Researchers defined successful implementation the same way it was defined in our QCA, and this represented a combined indicator around attrition, adherence, and dosage. We considered two outcomes ED visits and school absences - when we found sufficient studies to form a meta-analysis. Both models included effect sizes from seven studies, with five studies in each appearing in earlier meta-analyses (with studies considered as process and outcome evaluation studies (Bruzzese 2011; Cicutto 2013; Horner 2015; Howell 2005; Levy 2006)), and two studies in each meta-analysis included as process evaluation studies only (Joseph 2010; Joseph 2013).

Meta-analysis of ED visits shows that the included interventions were successful in reducing the number of ED visits (Figure 20; Analysis 11.1 ), but with a high $\mathrm{I}^{2}$ value $(52 \%)$ signalling substantial levels of heterogeneity. Subgroup analyses, based on implementation scores, indicated that studies classified as 
successfully implemented had a greater impact in reducing ED visits (SMD $-0.26,95 \% \mathrm{Cl}-0.48$ to $-0.04 ; 4$ studies) than studies that were not as successful (SMD $-0.09,95 \% \mathrm{Cl}-0.28$ to $0.10 ; 3$ studies), although this difference was not statistically significant ( $P$ value for subgroup differences $=0.26$ ). Meta-analysis of the impact of self-management interventions provided uncertain evidence that these interventions were successful in reducing children's absences from school (SMD $-0.12,95 \% \mathrm{Cl}-0.28$ to $0.04 ; 7$ studies). However, subgroup analyses based on the combined implementation score indicate that studies that were successfully implemented had significantly higher effect sizes (SMD $-0.28,95 \% \mathrm{Cl}-0.39$ to -0.18 ; 3 studies) than those that were not successfully implemented (SMD $0.04,95 \% \mathrm{Cl}-0.09$ to 0.18 ; Figure 21 ).

Figure 20. Forest plot of comparison: 11. Adjunct analyses - impact of Implementation on selected outcomes, outcome: 11.1. Exacerbations leading to emergency department (ED) visits.

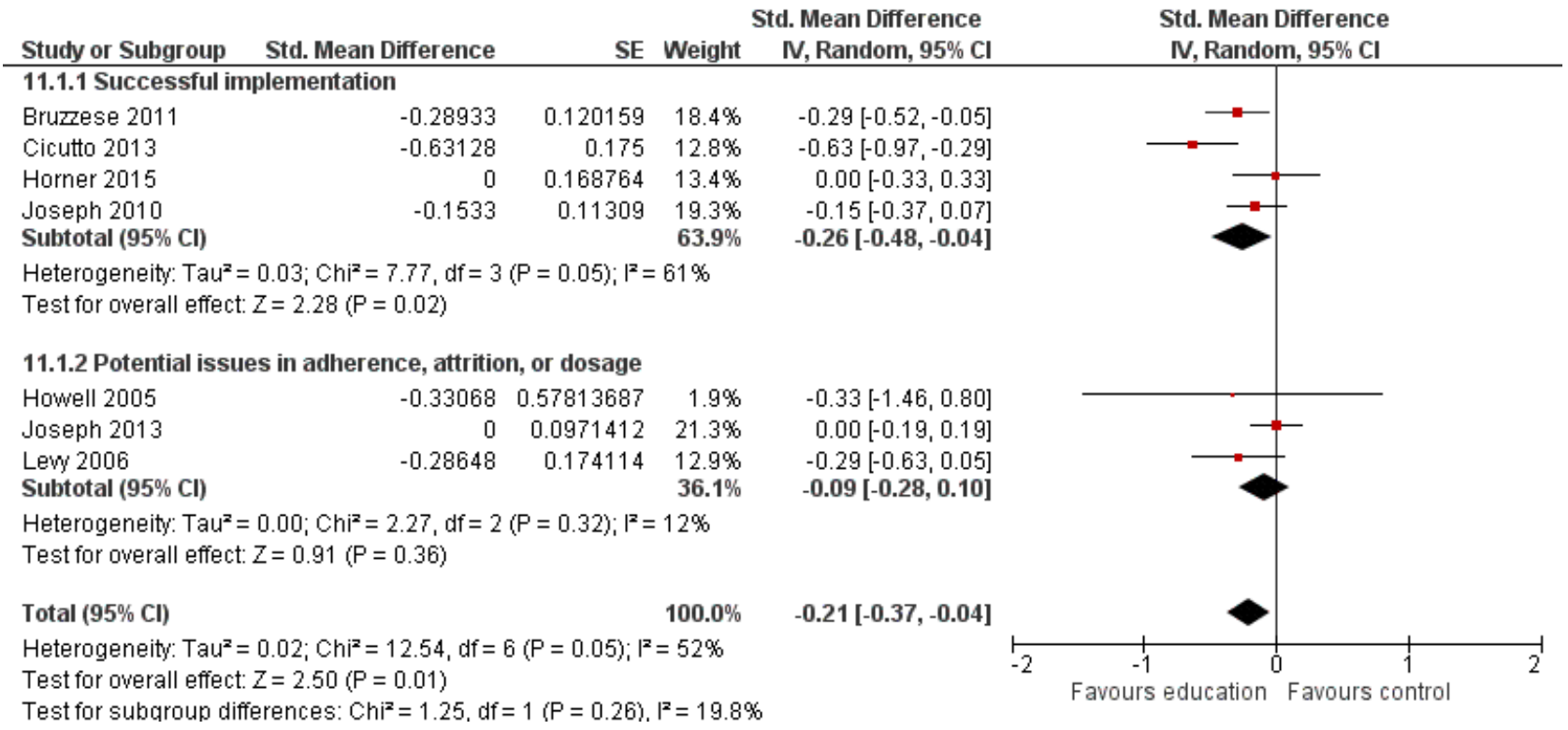

Figure 21. Forest plot of comparison: 11. Adjunct analyses - impact of Implementation on selected outcomes, outcome: 11.2. Absence from school.

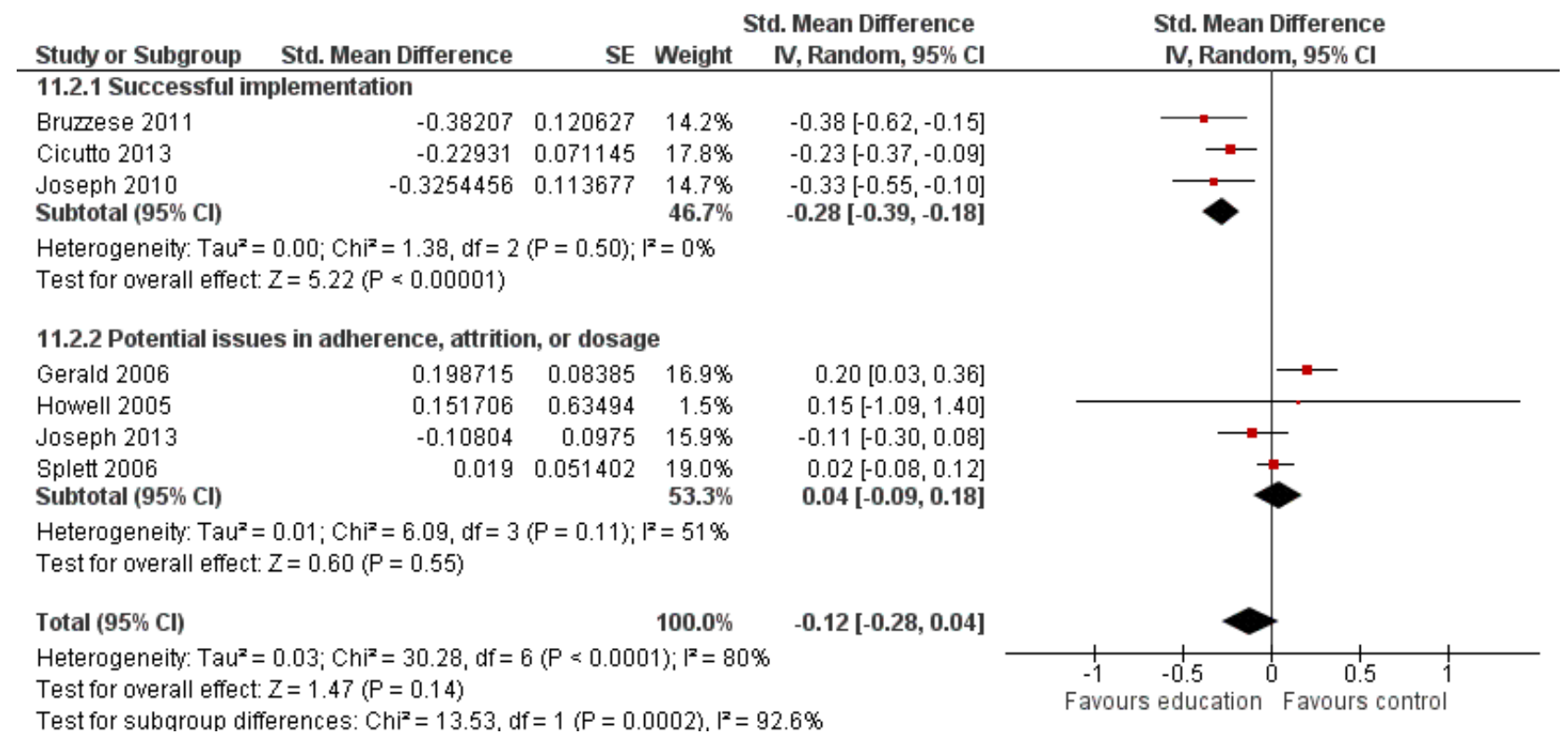

In both models, had the focus been restricted to well-implemented studies only, the conclusions would have changed, and these studies would have provided evidence that school-based asthma self-management interventions were effective in reducing these outcomes. Although restricted to selected outcomes and a subset of studies, these models help to illuminate the links between 
successful implementation and intervention effectiveness, and provide justification for meta-analyses based on earlier QCAs to test emerging hypotheses.

\section{Part 4: update of the logic model}

Figure 22 presents an updated logic model. This is a graphical depiction of synthesised evidence showing that school-based asthma interventions have a positive impact in reducing healthcare usage, improving quality of life (albeit not at a clinically meaningful level), and reducing days of restricted activity (shaded green). These were termed 'intermediate outcomes' in our original model (Figure 1), although some of the pathways through which these improvements are achieved remain poorly understood, particularly around proximal outcomes including lung function and daytime/ night-time symptoms (shaded blue and grey). We found evidence of a link between successful implementation (through results presented in part 3) and improved outcomes, although Figure 22 shows that other factors around the intervention design may directly lead to improvement in 'intermediate' outcomes. Of these, being theory driven is likely to be the most important element leading to successful implementation, and later, successfully improving children's outcomes, although the logic model shows that other conditions are likely to be important in certain circumstances. 
Figure 22.

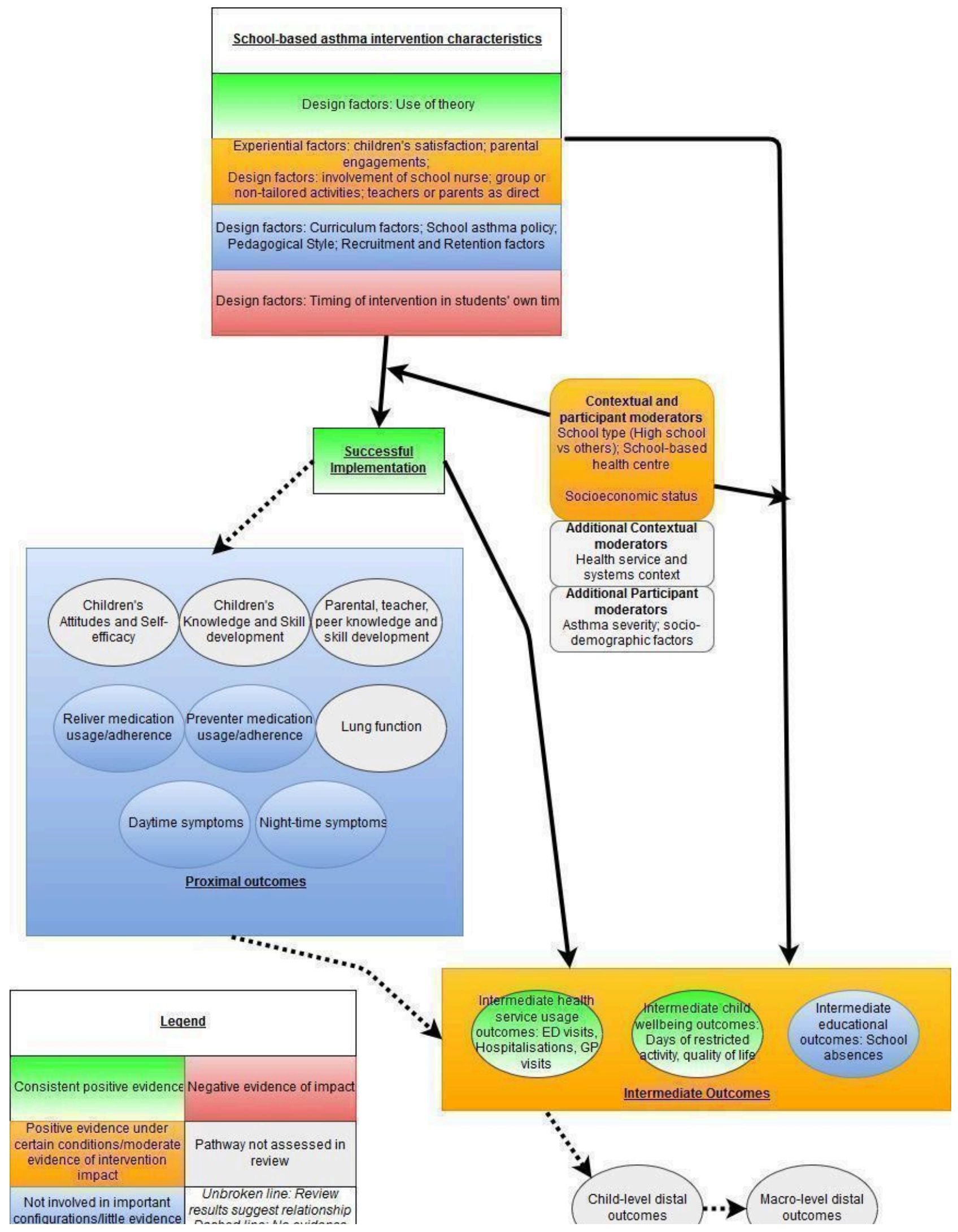




\section{Figure 22. (Continued)}

\begin{tabular}{c|c|}
\hline $\begin{array}{c}\text { Not involved in important } \\
\text { configurations/little evidence } \\
\text { of intervention impact }\end{array}$ & $\begin{array}{c}\text { Unbroken line: Review } \\
\text { results suggest relationship } \\
\text { Dashed line: No evidence } \\
\text { or unassessed }\end{array}$ \\
\hline
\end{tabular}

Use of QCA alongside meta-analysis has helped to disentangle the ways that school-based asthma interventions 'work' to a certain extent. The logic model helps to show the strength of evidence for many parts of the causal chain but also shows gaps in evidence on which future reviewers may focus their efforts (boxes shaded grey in Figure 22), including (I) establishing which proximal outcomes are important elements of the causal chain between intervention and intermediate outcomes; (ii) improving understanding of the role of contextual and participant characteristics; and (iii) examining distal characteristics and stability of improved outcomes.

\section{DISCUSSION}

\section{Summary of main results}

\section{Summary and further description of qualitative comparative analysis (QCA) results}

One of the most consistently positive conditions that appeared in configurations triggering a successfully run intervention was a named theoretical framework described as underpinning the intervention (Table 20). However, a diverse set of theoretical standpoints were represented (see Description of studies), and we are unable to attribute a successful intervention to a single conceptual or theoretical framework. Merely the use of named or explicitly expressed theory, in conjunction with other conditions, led to better implementation. These configurations also included interventions not run in children's own time, good levels of engagement from parents and satisfaction from children reported, and some configurations specific to high schools. It is not clear whether the theories used to underpin an intervention were equally suitable, and we were not able to ascertain how the theoretical framework was used to shape or inform different stages of the intervention.

We found that good levels of engagement from parents and positive experiences among children, in combination with other conditions, were sufficient conditions for a successful intervention. Positive parental engagement reflected high levels of co-operation in providing information to trialists, as noted by study authors (DoreStites 2007; Joseph 2013), or more active forms of engagement, including co-operation with home or school visits (Engelke 2013; Howell 2005), attendance at seminars (Bruzzese 2008), or telephone appointments received from the trialists (Engelke 2013). In contrast, a different set of studies reported difficulties in engaging parents to provide consent (when consent was actively sought) or to assist with data collection (Berg 2004; Gerald 2006; Terpstra 2012); difficulties in participation (Brasler 2006; Cicutto 2013; Kintner 2012; Kouba 2012; Levy 2006); or problems with adherence or behaviour change (Mujuru 2011). Children's satisfaction was found to be a sufficient condition for successful implementation (in combination with other conditions) and was collected in eight included studies, with four studies providing evidence that most children were satisfied through qualitative statements based on children's other stakeholders' perceptions (AlSheyab 2012a; Brasler 2006; Bruzzese 2004; Howell 2005), and four studies providing evidence based on quantitative data (Berg 2004;

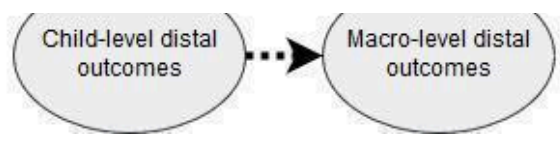

Bruzzese 2008; Dore-Stites 2007; Kintner 2012). None of the studies included in the QCAs reported low levels of child satisfaction, although one study (not included in the QCAs), which provided a low-intensity intervention, did report low levels, with low levels of satisfaction (64\% and 67\%) for some indicators (Jackson 2006).

With respect to school nurse involvement, the presence and involvement of school nurses in interventions appear to be instrumental in triggering a successful intervention under certain conditions when children are not engaged in personalised or tailored interventions. Finally, the timing of interventions was important in triggering successful interventions, with interventions that did not interrupt children's own time triggering successful implementation in two different configurations.

No single condition appeared in isolation as a trigger for successful implementation. This highlights the complexity of triggering a successful intervention, as well as the utility of the QCA approach in capturing complex causal recipes. This finding is further supported by modest levels of coverage of any pathway.

\section{Summary of outcome evaluation results}

Results from meta-analyses show that school-based selfmanagement interventions led to small average improvements in several important outcomes, including hospitalisations (six studies), emergency department (ED) visits (13 studies), and health-related quality of life (seven studies). A smaller number of studies contributed to meta-analyses suggesting positive results for unplanned medical visits (five studies) and days of restricted activity (three studies). Effects on school absences, symptoms, and use of medication were also small, although our certainty for these outcomes was low or very low and confidence intervals included small or no effect. The effect on withdrawals suggested similar levels of attrition between intervention and control conditions.

The original logic model and the updated logic model show that evidence for effectiveness of the intervention was stronger than for urgent care contact and quality of life than for symptoms (Figure 1; Figure 22, respectively). We did not measure distal outcomes (e.g. academic achievement). This is likely a partial reflection of heterogeneity in measurement approach in terms of lung function and daytime and night-time symptoms.

Researchers observed the most prominent intervention impacts for outcomes involving healthcare usage. Although conceptually relatively homogeneous, they were measured in several different ways, prompting us to undertake several transformations to facilitate meta-analysis. The magnitude of effect sizes for hospitalisations, ED visits, and other instances of unplanned healthcare usage was similarly small across all three outcomes when considered in absolute terms. However, this indicates that intervention effect can also reach across children's healthcare pathways to include both primary and secondary episodes of care. In contrast, it was anticipated that a greater effect would be evident for school absences than was apparent in our review. However, heterogeneity was substantial in meta-analyses of school absence 
$\left(1^{2}=70 \%\right)$, and additional subgroup analyses suggest that the way in which the intervention was implemented may have had a substantial impact on this outcome.

Effects of the intervention were relatively consistent across outcomes, with the exception of school absences and ED visits. Most planned investigations of heterogeneity were generally uninformative or inconclusive in explaining variation in our results. Indications suggest that both school type and age of the child may help to explain some between-study heterogeneity in models for school absence, with the intervention exerting a greater impact on older children in high schools, although this result was primarily driven by a single study (Bruzzese 2011). Two studies suggested that interventions with moderate to high numbers of children from lower socio-economic backgrounds (between 25\% and 50\% of children) resulted in fewer school absences for intervention children (Cicutto 2013; McGhan 2003), although the relationship between the proportion of children from a lower socio-economic background and effect size was not linear. We found generally mixed evidence around the impact of including parents. Based on subgroup analyses, interventions that did include parents appeared to confer no additional benefit compared with those that did not. Similarly, meta-analyses provided contrasting evidence as to whether involvement of school nurses had a positive impact on children's outcomes.

\section{Contribution of a mixed methods approach}

The mixed methods approach adopted here allowed us to (I) understand design and implementation processes associated with more successful implementation of school-based selfmanagement interventions; (ii) develop judicious and theorydriven hypotheses for testing in subsequent meta-analyses with covariates that reflected configurations of study conditions as well as single conditions; and (ii) explore the links between successful implementation and intervention outcomes.

Adjunct analyses showed links between intervention implementation and more impactful interventions, although the strength of these relationships differed for Analysis 11.1 and Analysis 11.2. Analysis of ED visits did not rule out differential effects between subgroups. We classified implementation of the intervention as successful in four studies. Study authors reported lower levels of ED visits with the intervention, and this finding was consistent with results for subgroups of studies that classified interventions as not successful. However, the result was inconclusive for studies that did not implement the intervention successfully. In the case of school absence, evidence shows greater impact of studies that were well implemented versus those that were not successfully implemented. This held when we restricted our focus to direct comparison of interventions (five studies) versus usual care.

Meta-analyses based on the findings of earlier QCAs, which assessed the impact of school-based asthma self-management interventions in lowering levels of school absence, also show that individual conditions that were frequently part of configurations that triggered successful intervention implementation explained some of the between-study heterogeneity. Notably, studies that were theory driven had greater impact on reducing school absences than those that were not, with the confidence interval for the subgroup of studies that explicitly used theory clearly within the boundaries of an effective intervention.
Further meta-analyses suggesting that interventions that did not involve existing school staff in a substantial delivery or facilitating role were those that achieved greater levels of impact in lowering school absence. This corresponds with QCA findings that involvement of school staff could be counterproductive in certain configurations. Well-implemented interventions that are supported by theory and can be implemented independently of existing school staff appear to be sufficient for lowering levels of school absence in these analyses.

\section{Translating evidence into practice}

The financial implications of asthma treatment and care for healthcare systems are significant; costs up to $£ 1$ billion per year are reported in the UK. A formal economic evaluation would be needed to determine how the reduction in healthcare use observed in this review impacts the financial burden on healthcare systems incurred by managing asthma. Although a similar reduction in school absence has not been established in this review, subgroup analyses developed on the basis of earlier QCAs identified studylevel characteristics associated with substantial reductions in absence, most notably interventions explicitly using theory.

In terms of the design of interventions, the importance of theory was emphasised in QCA results and was given further limited support by some of the subgroup analyses conducted as part of the meta-analyses. However, it is not clear if the use of theory in interventions is a marker of the quality of the interventions and the experience of researchers, or is more integral to intervention success and provides an anchor for trialists to return to and actively draw upon. Based on QCA results, when trialists take steps to measure levels of child satisfaction (including levels of enjoyment and fulfilment from activities), this is reflected in delivery of a successful intervention. The presence and involvement of school nurses appear to be instrumental in successful implementation of the intervention under certain conditions, particularly when children are not engaged in personalised or tailored interventions.

\section{Overall completeness and applicability of evidence}

Most of the included studies were conducted in the USA, specifically in inner city areas with large numbers of children from ethnic minority backgrounds and/or lower-income households; very few of the included studies came from the UK or Europe. Although we anticipated that broader contextual factors around health policy and access to health care are likely to shape the design and implementation of the intervention (see logic model in Figure 22), we have not synthesised the impact of these contextual factors.

The US focus of studies may have differing implications for the transferability of interventions. The nature of healthcare delivery and the large number of people without adequate healthcare coverage could mean that the intervention has a greater impact in US settings, particularly among lower-income populations with substantial levels of underdiagnosis and low levels of access to appropriate medication plans. Several interventions (e.g. those of Bruzzese 2011 and Gerald 2009) were developed precisely on the basis of this rationale, focusing on low-income groups or ethnic minority groups with inadequate access to health care, and selected schools as the delivery site because of the universality of education (as opposed to health care) in these settings. The implications for transferability could mean that weaker effect sizes are achieved in settings with better healthcare coverage, higher 
rates of diagnosis, and greater equality in access to medication (e.g. in settings such as the UK, where health care is universally free at the point of delivery). In contrast, many of the findings around intervention implementation are likely to be universal across several settings because of the relative universality of the way in which children attend schools, for example, better implementation when the intervention takes place outside children's own time.

Many outcomes with stronger evidence of an intervention effect were those commonly experienced by children with relatively severe asthma. For example, in Atherly 2009, when an intervention was implemented in high schools among children with mild to severe asthma, around 3\% of children had been hospitalised for asthma at baseline, and less than $10 \%$ had visited an ED. Values suggesting that unplanned secondary healthcare utilisation is relatively rare among children with asthma are also observed in prevalence studies, for example, in Harris 2017, which examined asthma patterns in London high schools.

Many of the studies included in the QCA and in the metaanalyses were conducted as cluster randomised controlled trials (RCTs); however few of these studies described the impact of this clustering effect either quantitatively or qualitatively. It is likely that conducting school-level randomisation is an important consideration in terms of the feasibility of the study and serves as a step toward prevention of contamination of treatment impact, although the opportunity to explore implementation and impact of school-level designs is not taken up by many trialists. This means that we are unable to comment on the generalisability of study findings with regards to school cultures.

High schools were better represented among studies included as process evaluations than among those included as outcome evaluations. Whether this is a reflection of the challenge of implementing RCT designs in high schools compared to primary schools was not directly addressed by the studies included in this review, although distinct configurations of conditions that triggered successful interventions were identified in QCAs for high schools and/or older children. Meta-analyses revealed little 'qualitative' impact of conducting interventions in high schools rather than in other types of schools for most outcomes except school absences, although this assertion is based on inclusion of few high schools in subgroup analyses and low heterogeneity for many healthcare usage outcomes.

Many studies did not report on the outcomes specified in the protocol for this review and encountered further issues with the incompatibility of some reported effect sizes. In fact, any of the meta-analyses performed (the largest including 13 studies) provided only a partial account of the total number of studies included. Some models, especially subgroup analyses, may have been effectively underpowered. Future systematic reviewers exploring public health interventions may wish to explicitly include a narrative synthesis of all studies in terms of study design, which may examine both the nature of the intervention, the types of outcomes collected, and the impact of interventions on these outcomes, including graphical representations (Thomson 2013), for a more complete account and understanding of the impact and feasibility of the model.

Finally, because we excluded studies that delivered similar interventions in different settings, we do not know the added value of running an intervention in a school compared with running an intervention in a hospital or community setting. What is clear, however, is that schools provide access to large numbers of children with asthma, including those who do not regularly attend appointments with their medical provider; therefore the school environment can be considered an important third space for delivery of interventions that can improve both children's outcomes and healthcare usage. This review has shown that school-based self-management interventions are effective in improving several outcomes for children with asthma, and that those who design future interventions should consider a number of configurations, including instructor, theory, and time of day, in their design. The outcomes of this review will directly inform the development of a school-based self-management intervention for children with asthma in London secondary schools.

\section{Certainty of the evidence}

The 'Summary of findings' table highlights our reasons for downgrading the certainty of evidence for the main outcomes of interest in this review, with process evaluations considered separately below. We noted issues in the execution of all studies, although the impact of risk of bias differed across outcomes. We deemed that several studies had high or unclear risk of bias, although these results did not appear to inflate the effect size relative to that provided by low-risk studies, and in most cases they did not influence the direction of effect. Studies that we deemed to have unclear or high risk of bias may nevertheless have contributed to decisions to downgrade the certainty of evidence through other factors, including directness of outcome measurements. For example, school absences were measured in a variety of ways, and not all approaches were specific to asthma-related school absences.

We deemed the certainty of evidence to be moderate for four outcomes delineated in the 'Summary of findings' table: hospitalisation, unplanned medical visits, quality of life, and symptoms. Each of these outcomes showed positive intervention effects (or effects that were very close to being classed as positive effects in the case of daytime symptoms). For two of the outcomes reported in the 'Summary of findings' table, we deemed that the certainty of evidence was low (school absences and ED visits), and we found evidence certainty to be very low for a further outcome on medication usage. Again, indirectness and unexplained heterogeneity were the main drivers for downgrading of evidence.

Additional considerations not necessarily captured in the 'Summary of findings' table should be considered when quality of the evidence is examined. First, we decided to include in our analyses some cluster RCTs with relatively low numbers of clusters. Although these studies tended to be comparatively small by their nature and therefore did not contribute greatly to pooled effect sizes, there remains the possibility that the intervention effects are slightly exaggerated compared to those of individually randomised trials or large cluster RCTs (see also the section on bias below). Nevertheless, this risk should be balanced against the potential bias introduced by overlooking information from such (smaller) trials. Similarly, effect sizes were harmonised for most outcomes, with the most substantial transformations involving conversion between standardised mean differences (SMDs) and odds ratios (ORs) to develop a common metric; although this appeared to have minimal impact, and different effect sizes tended to be consistent in direction/impact regardless of original measurement 
(see Analysis 12.1 through to Analysis 12.27), this is further evidence of indirectness in outcome measures, which is an indicator of lowercertainty evidence.

In contrast, we judged the quality of the process evaluation literature to be almost uniformly poor, with many studies having high or unclear risk of bias across several domains. This is likely due to various factors but most plausibly is a reflection of previous lack of guidance around the conduct of process evaluations, as well as difficulty in identifying process evaluations in the literature; there remains a methodological gap in terms of tools to report on and help in identifying process evaluations (as opposed to guidance on conducting process evaluations (Moore 2015)). This review included process evaluation studies that were integrated with outcome evaluation studies, that were presented as separate sections, or that could be considered stand-alone evaluations. The tool used to measure risk of bias in process evaluation studies was an amalgamation of two tools used in reviews of process evaluation studies and resulted in a comprehensive assessment (O'Mara-Eves 2013; Shepherd 2010). We deemed only four studies to have low risk of bias in most domains (Bruzzese 2011; Kintner 2012; Kouba 2012; Mujuru 2011). Of these, only Kintner 2012 could be considered a stand-alone evaluation, with Mujuru 2011 including defined sections evaluating processes, and Bruzzese 2011 and Kouba 2012 presenting process evaluation data that were more integrated. We classified the latter two studies as process evaluations due to their exploration of process-related questions using recognised tools and exploration of context and potential mechanisms. The main weakness of the process evaluation studies included is that they lacked breadth and had considered only a single process of importance in-depth. The impact of these poor quality studies on the QCA is difficult to ascertain, although absence of richer and broader process data may have been a factor as to why we were able to explain only a relatively modest amount of successful implementation via QCA models. A commonly occurring risk of bias among the included process evaluation studies is that the tools and methods of collecting and analysing data were not always deemed to be reliable or credible.

\section{Potential biases in the review process}

Current evidence around the introduction of potential bias through restrictions on publication language is mixed, with some recent studies finding no systematic bias in effect size estimates when languages other than English were excluded (Morrison 2012), although many remain concerned that the results of ineffective trials will be submitted to local (non-English language) journals, leading to the potential for language restrictions and systematic bias (Guyatt 2011). We assessed a potential impact of this restriction by conducting explorations of the impact of publication bias. Imposing a language restriction may also have influenced results of the synthesis of process evaluation data, and may impede the generalisability of results to individuals of non-English speaking cultures, although we were not able to explore the impact of this decision in this review.

\section{We encountered the following limitations in the review process.}

- Potential measurement error: we noted variation in the way in which many outcomes were measured, for example, lung function and school absences. Although no 'gold standard' is available for measuring school absences, lack of continuity across studies may reduce the validity of findings. Further, data for both school attendances and healthcare use may be subject to substantial measurement error, for example, we cannot say for certain that all school absences and healthcare visits that were recorded were specifically due to asthma, or were authorised by either the school or the medical centre. Similarly, measurement error may be a factor with some of the covariates used in subgroup analyses, for example, socioeconomic status (SES) can be measured in different ways through stated household income or evidence of free school meals - although it was not possible to further explore these differences in measurement in the present review.

- Effect size transformations: this review sought to include comprehensive trial data within meta-analytical models, while maintaining construct validity across effect sizes. This often necessitated transforming the data to ensure statistical compatibility, following recommendations within the Cochrane Handbook for Systematic Reviews of Interventions, and undertaking Chinn's transformation (Chinn 2000). Although we have attempted to ensure transparency in fully presenting disaggregated effect sizes alongside those that have been consolidated, and despite sensitivity analyses conducted to ensure the validity of findings, there is potential for these analyses themselves to be confounded, and underlying assumptions around the transformation of effect sizes may not hold with further interrogation. For example, to facilitate transformations, we combined data on SMDs and ORs, although the skewness usually associated with data such as hospitalisations, for example, may not have been fully accounted for in the transformation. This is an important limitation, but it needs to be balanced against research wastage and information lost by excluding studies that use different approaches in measuring outcomes. Encountering such diverse data reinforces our recommendation below for development of a core outcome set.

- Potentially underpowered analyses and treatment of heterogeneity: we included few studies in many of the metaanalysis models, and for random-effects models, the models themselves may have been underpowered (Jackson 2017). In addition, when heterogeneity was encountered, the low number of studies meant either that subgroup analyses were unsuitable, or that the subgroups themselves included few studies. We deemed that planned meta-regression analyses were not suitable for any of the outcomes. Furthermore, unlike many other systematic reviews, we did not present all planned subgroup analyses when we encountered a low number of studies (under 10) and/or a low level of heterogeneity; in this respect, several deviations from the protocol occurred. However, we have greater confidence in the results of subgroup analyses because of our judicious use of these methods.

- Identification of process evaluation studies: identification of process evaluations was a challenge in this review. Although guidance is available to assist trialists in conducting a process evaluation (Moore 2015), this did not necessarily aid in the identification of process evaluation studies from a systematic review perspective. All process evaluation studies included an examination of a given process (or processes) and implementation outcome(s) of interest, as well as their relationship to context (in this case, the immediate context of the schools). However, this group spanned a range of studies - from those that were self-described process evaluations, to those with defined process evaluation sections, to those that included 
process evaluation data embedded within other evaluation data. Although we developed an inclusive strategy around identification of process evaluation studies, there remains the possibility that some trialists may not have considered their own study as fulfilling the remit of a process evaluation. In addition, although guidance for process evaluations states that they can adopt a range of methods for data collection (Moore 2015), unlike other recent reviews (Dickson 2016), many of the studies that we included did not draw upon robust qualitative methods of data collection, which in turn may have limited our understanding of some of the issues surrounding implementation. Consequently, we deemed there remained greater scope within several of the included studies to explore the way in which the school context, and particularly the broader health service context, influenced delivery of the intervention, and we graded much of the process evaluation information as having high risk of bias because of this weakness. This review highlights the need for greater support for review authors in identifying process evaluation studies. In the current review, our original logic model was instrumental in helping to identify the processes and process metrics of interest and informed the selection of studies (Figure 1); in the absence of clearer guidance in this area, the use of logic models may represent an important step in helping review authors to draw criteria around which processes should be considered in a process evaluation.

- Harmful effects: some studies reported negative intervention impacts among children, such as increased levels of ED visits. Such negative effects may reflect the content of selfmanagement information delivered to children, which may, for example, have recommended greater contact with healthcare providers when experiencing exacerbations (although such detail was not reported in studies), in which case an increase in ED visits could be viewed as a positive. A narrative approach to synthesis of outcome evaluations data could lead to a more nuanced understanding.

- Alternative explanations: many other factors might also have influenced review results. For example, although these are school-based asthma self-management interventions, few, if any, of the studies considered seasonality of asthma exacerbations and their relationship within the school year. Another Cochrane Review considered the issue of seasonality and showed that seasonal omalizumab treatment between four and six weeks before children return to school might reduce the number of asthma exacerbations seen in autumn (Pike 2018); however the effect of this on outcomes such as asthma control remains unclear.

- Low number of clusters: some of the cluster RCTs included in this review randomised only a small number of schools. Although it is universally agreed that randomising one cluster per arm would entirely conflate the randomisation/intervention and clustering effect, there is less agreement on the minimum number of clusters needed for a study to qualify as a cluster RCT (one source recommends four clusters per arm). Studies involving a low number of clusters are generally indicative of a small trial and often contribute only sparse data to any one model. Sensitivity analyses for studies with a low number of clusters per arm were conducted (two or three clusters per arm: Al-Sheyab 2012; Howell 2005; Kintner 2012; Shah 2001; Velsor-Friedrich 2005). Results were generally inconclusive and inclusion/exclusion of these studies in models did not qualitatively change results of meta-analyses, with the exception of Al-Sheyab 2012 in one quality of life model. These studies may be particularly prone to baseline imbalances, as well as to issues involving introduction of bias, and their inclusion does represent a potential source of bias.

\section{Agreements and disagreements with other studies or reviews}

This review is one of the first of its kind to employ a mixed study and mixed methods approach to understanding how schoolbased asthma self-management interventions work, and whether they are effective. It is also the first to undertake quantitative synthesis of studies seeking to develop children's asthma selfmanagement skills in the school environment. Direct comparisons are challenging, but a number of similar reviews have focused on different settings, different study designs, or use of different synthesis methods, which allows us to understand results in the context of other evidence.

Pinnock 2015 is one of few reviews that have explored how asthma self-management interventions should be implemented. Review authors focused on a range of settings and age groups and addressed a targeted question around whether interventions primarily targeted at patients, professionals, or the organisation, or explicitly targeting all three levels simultaneously, were differentially effective in changing outcomes, or in changing process measures. They found that complex interventions that explicitly address patient education, professional training, and organisational commitment were associated with improvements in process measures, markers of asthma control, and reduced use of unscheduled health care. Their conclusions that 'individually, the separate components (professional, patient, organisation) of comprehensive self-management support do not appear to be sufficient consistently to improve outcomes in asthma' (p14) are congruent with our own findings from QCA synthesis, which emphasised that no single condition was necessary and sufficient to trigger successful implementation outside a configuration of conditions.

An earlier Cochrane Review explored the effectiveness of selfmanagement education interventions for children aged two to 18 with asthma across a range of settings between 1980 and 2002 (Wolf 2002). Review findings were similar to the findings of this review, with data suggestive of moderate reductions in ED visits and in days of restricted activity. This earlier review also found evidence that self-management education led to a small reduction in school absences, and review authors were able to ascertain a small impact on lung function. It is unclear to what extent the discrepancy in settings, age groups, or inclusion criteria for studies on date would drive the discrepancy in school absence, or another factor. In contrast to the promising results observed for nighttime symptoms in the previous review (Wolf 2002), our review did not find evidence that the intervention made a positive impact, although this was consistent with the findings of a later review that narratively summarised study results (Coffman 2009).

Subsequent reviews include Al Aloola 2014, which focused on primary schools and used a narrative approach to synthesise data. Review authors concluded that most studies were suggestive of positive effects, but as was the case in the present review, they were critical of the measurement of outcomes, which varied greatly among included studies. They also highlighted lack of detail in the descriptions provided for intervention content and 
processes, which is consistent with the outcome evaluations included here. Ahmad 2011 also took a narrative approach to synthesising outcome data from studies that involved school nurses, but nevertheless concluded that results indicated that a decrease in school absences could be expected, but that results for reductions in ED visits and hospital admissions were less certain, in contrast to the results provided here. Coffman 2009 also undertook a narrative descriptive synthesis of the effectiveness of a schoolbased approach, although review authors concluded that there was heterogeneity in the direction and/or magnitude of effect on quality of life, symptom days, night-time symptoms, and school absences, which largely corroborates the findings of the present review. Finally, a more recent review included school-based selfmanagement interventions provided across a diffuse set of studies with regards to design (Carvalho 2016). These review authors also took a narrative approach when synthesising study results, and again showed an overall trend suggestive of heterogeneity in magnitude and direction of effect across a range of outcomes.

This systematic review makes a contribution to the literature by providing the first meta-analyses of asthma self-management interventions focused in schools, and it provides evidence of the effectiveness of this approach in reducing healthcare usage. Methodologically, this is also one of the first Cochrane Reviews to employ a mixed methods approach in synthesising evidence. This mixed methods approach helped to show that although intervention as a whole did not appear to be effective in reducing school absences, interventions that were drawing upon theory were effective in improving school absences.

\section{AUTHORS' CONCLUSIONS}

\section{Implications for practice}

School-based asthma self-management interventions probably reduce hospitalisations and improve symptoms (moderatecertainty evidence), may lower emergency department (ED) attendance (low-certainty evidence), and may decrease children's unplanned and urgent healthcare visits (low-certainty evidence). Their impact on school absence varied between studies (lowcertainty evidence), and probably lead to small improvements in quality of life (moderate-certainty evidence). The effects of these interventions on the requirement for reliever medication are uncertain.

Hospitalisation was reduced by an average of about 0.16 admissions per child over a 12-month period. The proportion of children attending the ED was reduced from 75 per 1000 children to 54 per 1000 children over the course of a year. Similar results were observed for unplanned medical visits. For health policymakers, the results highlight that schools may be an effective location for delivering asthma self-management interventions to potentially large numbers of children, although formal costeffectiveness analysis is needed to determine how reductions in healthcare usage affect financial burden on health systems. Many of the included studies tested the intervention among financially deprived populations, and judging the applicability of the results to more socially diverse populations is difficult.

The mixed methods design of this review has revealed important features of interventions that are of particular interest to educational practitioners and teachers. Variation in school absences may be driven by the results from a subset of explicitly theory-driven interventions that achieved modest decreases. Trialists may wish to take account of this when designing interventions that they intend to evaluate. Our process evaluation shows that when trialists are concerned about the level of child satisfaction (including levels of enjoyment and fulfilment from activities), and when they take steps to measure levels of satisfaction, this is reflected in the delivery of a successfully implemented intervention.

\section{Implications for research}

The evidence presented in this review for school-based asthma selfmanagement interventions varies in degrees of certainty across the outcomes of interest. The updated logic model summarises where evidence has been identified but also highlights where uncertainties remain (Figure 22). In particular, the mechanisms that link participation in a school-based asthma intervention with achievement of these relatively distal outcomes remain undefined. Many analyses of intermediary outcomes provided inconclusive evidence (e.g. analyses reported asthma symptoms (Analysis 1.6; Analysis 1.7); data were insufficient for inclusion in meta-analyses (e.g. lung function data (see Table 13)). In other cases, these outcomes were not included in our protocol. For example, although knowledge was not explicitly measured, we can hypothesise that knowledge and skill development are essential components for changes in self-management and therefore changes in healthcare usage. The current review also did not assess these, and overall, many of the intermediary stages and accompanying changes in healthcare service usage between receipt of the intervention and behaviour change remain unidentified, signalling some of the pathways for future research.

Evaluation of healthcare usage in future studies would help to establish whether the intervention effect transfers to other settings. Researchers providing data on ED visits observed heterogeneity in the magnitude and direction of effect across studies. Research conducted specifically to determine when and how the intervention might increase attendance as observed for a subset of studies would help to explain the variation in direction of effect. For example, although baseline imbalances may be a contributory factor in explaining negative or negligible impacts for some studies implementing the "Roaring Adventures of Puff" manualised intervention in certain settings (McGhan 2003; McGhan 2010), further targeted analyses may reveal the context and mechanisms that explain its effectiveness in others (Cicutto 2005; Cicutto 2013).

This review identified a heterogeneous group of process evaluation studies that were often of low quality and did not present a broad or deep understanding of the processes undertaken and the mechanisms of action reflective of the complexity of the intervention. The quality of the process evaluation literature has been criticised previously (Oakley 2006), and this is relevant when one seeks to understand the causal chains of actions occurring within public health interventions such as school-based asthma self-management interventions. Although guidance on the conduct of process evaluation studies is available (Moore 2015), this review highlights that many trialists do not adequately assess the implementation and context of their interventions. It is notable that only a third of included studies contributed to both sets of syntheses conducted in our review. Enhancing understanding of the barriers preventing conduct and publication of process evaluations is a priority for future research. Systematic reviews 
would benefit from the development of a tool or checklist that can be used to help identify process evaluation studies during screening and/or to better design searches for relevant studies.

The largest meta-analysis includes 13 of the 33 RCTs identified in this review. The need for a more standardised approach to evaluating key asthma outcomes is clear based on this finding. Approaches to developing core outcome sets for clinical trials are increasingly common (Williamson 2012). Some work has been undertaken to consider which domains should be captured in trials involving children with asthma (Sinha 2012). Our review shows that many studies, including those recently published, continue to capture diffuse outcomes that may have little clinical value and/or policy resonance. Further development, refinement, and implementation of a core outcome set for this intervention model would be welcome and would facilitate future reviews, which could include information not only on which domains should be captured, but also on how this information should be measured.

Subgroup analyses suggest that intervention effects were generally consistent across different types of schools (high/senior vs primary/ elementary schools) for outcomes for which we were able to explore differences in effect size. However, further studies within high/senior schools are needed to extend the applicability of the evidence base to children older than those recruited to many of the studies to date. These results should also be considered in light of results from process evaluations, which suggest that the distinction between high/senior school and other types of schools may be important from an implementation perspective, necessitating a modified approach to the design and running of school-based asthma self-management interventions.

Although this review has shown that schools can provide an effective setting for self-management interventions that reduce healthcare usage, we have not been able to explore the optimal setting. This would be a natural direction for future primary research studies and systematic reviews. In addition, although the intervention aim and the setting were the same in all studies included here, interventions have differed substantially. Future reviews should explore whether differences in outcomes are observed across different modes of asthma intervention, and should examine the comparative effectiveness of different programmes (e.g. Open Airways for Schools). Review authors could provide a better understanding of the links between intervention input and more distal outcomes, and this may prove valuable for public health decision-makers. The feasibility of such research is contingent on emergence of a more mature evidence base for this type of intervention in terms of the number of available studies, as well as improvements in collection of standardised outcomes and reporting of processes undertaken and implemented.

\section{ACKNOWLEDGEMENTS}

Thanks to Elizabeth Stovold for helping to design the search strategy. Thanks also to Emma Dennett for comments and ongoing assistance. Thanks to the NIHR Collaborative Leadership in Applied Health Research and Care (CLAHRC) North Thames for continued support.

Chris Cates was the Editor for this review and commented critically on the review.

The Background and Methods sections of this protocol are based on a standard template used by the Cochrane Airways Group.

This project was supported by the National Institute for Health Research (NIHR), via Cochrane Infrastructure funding to the Cochrane Airways Group. The views and opinions expressed therein are those of the review authors and do not necessarily reflect those of the Systematic Reviews Programme, NIHR, NHS, or the Department of Health. 


\section{R E F E R E N C E S}

\section{References to studies included in this review}

\section{Al-Sheyab 2012 \{published data only\}}

* Al-sheyab N, Gallagher R, Crisp J, Shah S. Peer-led education for adolescents with asthma in Jordan: a cluster-randomized controlled trial. Pediatrics 2012;129(1):e106-12.

Al-sheyab NA, Shah S, Gallagher R, Crisp J. Effectiveness of a peer-led education program for adolescents with asthma in Jordanian schools. American Journal of Respiratory and Critical Care Medicine 2010;181:A2253.

\section{Al-Sheyab 2012a \{published data only\}}

Al-Sheyab NA, Gallagher R, Roydhouse JK, Crisp J, Shah S. Feasibility of a peer-led, school-based asthma education programme for adolescents in Jordan. Eastern Mediterranean Health Journal 2012;18(5):468-73.

\section{Atherly 2009 \{published data only\}}

Atherly A, Nurmagambetov T, Williams S, Griffith M. An economic evaluation of the school-based "power breathing" asthma program. Journal of Asthma 2009;46(6):596-9.

\section{Bartholomew 2006 \{published data only\}}

Bartholomew KL, Sockrider M, Abramson SL, Swank PR, Czyzewski DI, Tortolero SR, et al. Partners in school asthma management: evaluation of a self-management program for children with asthma. Journal of School Health 2006;76(6):283-90.

\section{Berg 2004 \{published data only\}}

Berg J, Tichacek MJJ, Theodorakis R. Evaluation of an educational program for adolescents with asthma. Journal of School Nursing 2004;20(1):29-35.

\section{Bignall 2015 \{published data only\}}

Bignall WJR, Luberto CM, Cornette AF, Haj-Hamed M, Cotton S. Breathing retraining for African-American adolescents with asthma: a pilot study of a school-based randomized controlled trial. Journal of Asthma 2015;52(9):889-96.

\section{Brasler 2006 \{published data only\}}

Brasler M, Lewis M. Teens: taking control of asthma. Journal of School Health 2006;76(6):269-72.

\section{Bruzzese 2004 \{published data only\}}

Bruzzese JM, Bonner S, Vincent EJ, Sheares BJ, Mellins RB, Levison MJ, et al. Asthma education: the adolescent experience. Special Issue: Educating and Counseling Children about Physical Health 2004;55(3):396-406.

\section{Bruzzese 2008 \{published data only\}}

Bruzzese JM, Unikel L, Gallagher R, Evans D, Colland V. Feasibility and impact of a school-based intervention for families of urban adolescents with asthma: results from a randomized pilot trial. Family Process 2008;47(1):95-113.
Bruzzese 2010 \{published data only\}

Bruzzese JM, Stepney C, Gallagher R, Wang J, Petkova E, Evans D. Reducing morbidity and urgent healthcare utilization in urban pre-adolescents with asthma: results of a randomized control trial of asthma: it's a family affair. American Journal of Respiratory and Critical Care Medicine 2010;181:A2250.

\section{Bruzzese 2011 \{published data only\}}

Bruzzese JM, Sheares BJ, Vincent EJ, Du Y, Sadeghi H, Levison MJ, et al. Effects of a school-based intervention for urban adolescents with asthma. A controlled trial. American Journal of Respiratory and Critical Care Medicine 2011;183(8):998-1006.

Carpenter 2016 \{published data only\}

Carpenter DM, Alexander DS, Elio A, DeWalt D, Lee C, Sleath BL. Using tailored videos to teach inhaler technique to children with asthma: results from a school nurse-led pilot study. Journal of Pediatric Nursing 2016;31(4):380-9.

Cicutto 2005 \{published data only\}

* Cicutto L, Murphy S, Coutts D, O'Rourke J, Lang G, Chapman C, et al. Breaking the access barrier: evaluating an asthma center's efforts to provide education to children with asthma in schools. Chest 2005;128(4):1928-35.

Cicutto L, Murphy S, Coutts D, O'Rourke J, Lang G, Chapman C, et al. Evaluating an elementary school based asthma education program: effects on quality of life and self efficacy [abstract]. American Thoracic Society 99th International Conference; 2003 May 16-21; Seattle. 2003.

\section{Cicutto 2013 \{published data only\}}

Cicutto L, Conti E, Evans H, Lewis R. Creating asthma-friendly schools: a public health approach. Journal of School Health 2006;76(6):255

* Cicutto L, To T, Murphy S. A randomized controlled trial of a public health nurse-delivered asthma program to elementary schools. Journal of School Health 2013;83(12):876-84.

\section{Clark 2004 \{published data only\}}

* Clark NM, Brown R, Joseph CL, Anderson EW, Liu M, Valerio MA. Effects of a comprehensive school-based asthma program on symptoms, parent management, grades, and absenteeism. Chest 2004;125(5):1674-9.

Petteway RJ, Valerio MA, Patel MR. What about your friends? Exploring asthma-related peer interactions. Journal of Asthma 2011;48(4):393-9.

\section{Clark 2005 \{published data only\}}

* Clark NM, Gong M, Kaciroti N, Yu J, Wu G, Zeng Z, et al. A trial of asthma self-management in Beijing schools. Chronic Illness 2005;1(1):31-8.

Clark NM, Gong MZ, Kaciroti N, Yu J, Zeng Z, Wu G, et al. Effect of self management education on school children with asthma in Beijing, China [abstract]. European Respiratory Society 
13thAnnual Congress; 2003 Sep 28-29; Vienna. 2003; Vol. 22, issue Suppl 45:410s.

\section{Clark 2010 \{published data only\}}

Clark NM, Shah S, Dodge JA, Thomas LJ, Andridge RR, Little RJ, et al. An evaluation of asthma interventions for preteen students. Journal of School Health 2010;80(2):80-7.

\section{Crane 2014 \{published data only\}}

Crane LMM, O'Neal KSS, Honey BLL, Kirkpatrick A. Effectiveness of a modified open airways curriculum. Journal of Asthma 2014;52(5):1-9.

\section{Dore-Stites 2007 \{published data only\}}

Dore-Stites DJ. Evaluation of a School-based Program Targeting Pediatric Asthma Self-Management Skills in an Urban Population [thesis]. Kalamazoo, Michigan: Western Michigan University, 2007.

\section{Engelke 2013 \{published data only\}}

Engelke MK, Swanson M, Guttu M. Process and outcomes of school nurse case management for students with asthma. Journal of School Nursing 2013;30(3):196-205.

\section{Gerald 2006 \{published data only\}}

Gerald LB, Wittich AR, Erwin S, Hains C, Hemstreet MP, Redden D, et al. Outcomes for a comprehensive school-based asthma management program. Journal of School Health 2006;76(6):291-6.

\section{Gerald 2009 \{published data only\}}

Gerald LB, Gerald JK, Gibson L, Patel K, Zhang S, McClure LA. Changes in environmental tobacco smoke exposure and asthma morbidity among urban school children. Chest 2009;135(4):911-6.

Gerald LB, McClure LA, Harrington KF, Mangan JM, Gibson L, Atchison J, et al. Design of the supervised asthma therapy study: implementing an adherence intervention in urban elementary schools. Contemporary Clinical Trials 2008;29(2):304-10.

* Gerald LB, McClure LA, Mangan JM, Harrington KF, Gibson L, Erwin $\mathrm{S}$, et al. Increasing adherence to inhaled steroid therapy among schoolchildren: randomized, controlled trial of school-based supervised asthma therapy. Pediatrics 2009;123(2):466-74.

\section{Henry 2004 \{published data only\}}

Henry RL, Gibson PG, Vimpani GV, Francis JL, Hazell J. Randomized controlled trial of a teacher-led asthma education program. Pediatric Pulmonology 2004;38(6):434-42.

\section{Horner 2008 \{published data only\}}

Horner SD, Brown A. Evaluating the effect of an asthma selfmanagement intervention for rural families. Journal of Asthma 2014;51(2):168-77.

* Horner SD, Fouladi RT. Improvement of rural children's asthma self-management by lay health educators. Journal of School Health 2008;78(9):506-13.
Horner SD, Rew DL, Brown SA. Enhancing asthma management among rural Mexican American, white and African American school-aged children and their parents [Abstract]. American Thoracic Society International Conference; 2007 May 18-23; San Francisco. 2007.

\section{Horner 2015 \{published data only\}}

Horner SD, Brown A, Brown SA, Rew DL. Enhancing asthma self-management in rural school-aged children: a randomized controlled trial. Journal of Rural Health 2015;32(3):260-8.

\section{Howell 2005 \{published data only\}}

Howell KJ. "Quest for the Code": A Study of a Computer Based Education Program for Children With Asthma [dissertation]. Syracuse University (Thesis). Syracuse: Syracuse University Libraries, 2005.

\section{Jackson 2006 \{published data only\}}

Jackson TL, Stensland SL, Todd TJ, Lullo A, Mazan J, Masood AM. Evaluation of a pediatric asthma awareness program. Journal of Asthma 2006;43(4):311-7.

\section{Joseph 2010 \{published data only\}}

Joseph CLM, Baptist AP, Stringer S, Havstad S, Ownby DR, Johnson CC, et al. Identifying students with self-report of asthma and respiratory symptoms in an urban high school setting. Journal of Urban Health 2007;84(1):60-9.

Joseph CLM, Havstad SL, Johnson D, Saltzgaber J, Peterson EL, Resnicow K, et al. Factors associated with nonresponse to a computer-tailored asthma management program for urban adolescents with asthma. Journal of Asthma 2010;47(6):667-73.

Joseph CLM, Stringer S, Ownby DR, Peterson E, Hoerauf S, Gibson-Scipio W, et al. Preliminary results of the Puff City program for urban teens with asthma. Journal of Allergy and Clinical Immunology 2005;115(2):S63.

* Joseph ChLM, Peterson E, Havstad S, Johnson CC, Hoerauf S, Stringer S, et al. A web-based, tailored asthma management program for urban African-American high school students. American Journal of Respiratory and Critical Care Medicine 2007;175(9):888-95.

\section{Joseph 2013 \{published data only\}}

Guglani L, Havstad SL, Johnson CC, Ownby DR, Joseph CLM. Effect of depressive symptoms on asthma intervention in urban teens. Annals of Allergy, Asthma and Immunology 2012;109(4):237-42.

Guglani L, Havstad SL, Ownby DR, Saltzgaber J, Johnson DA, Johnson Christine $\mathrm{C}$, et al. Exploring the impact of elevated depressive symptoms on the ability of a tailored asthma intervention to improve medication adherence among urban adolescents with asthma. Allergy, Asthma and Clinical Immunology 2013;9(1):45.

Guglani LMD, Havstad SL. Usefulness of a home affluence scale administered to urban adolescents with asthma to estimate the family's socioeconomic status. Annals of Epidemiology 2015;30:1e6. 
* Joseph CLM, Ownby DR, Havstad SL, Saltzgaber J, Considine S, Johnson D, et al. Evaluation of a web-based asthma management intervention program for urban teenagers: reaching the hard to reach. Journal of Adolescent Health 2013;52(4):419-26.

Joseph CLM, Saltzgaber J, Havstad SL, Johnson CC, Johnson D, Peterson EL, et al. Comparison of early-, late-, and nonparticipants in a school-based asthma management program for urban high school students. Trials 2011;12(1):141.

Valerio MA, Peterson EL, Wittich AR, Joseph CL. Examining health literacy among urban African-American adolescents with asthma. Journal of Asthma 2016;53(10):1041-7.

\section{Kintner 2009 \{published data only\}}

Kintner EK, Sikorskii A. Randomized clinical trial of a schoolbased academic and counseling program for older school-age students. Nursing Research 2009;58(5):321-31.

\section{Kintner 2012 \{published data only\}}

* Kintner E, Cook G, Allen A, Meeder L, Bumpus J, Lewis K. Feasibility and benefits of a school-based academic and counseling program for older school-age students with asthma. Research in Nursing and Health 2012;35(5):507-17.

Kintner EK, Clary M, Cook GD. Curriculum development for the theory-driven, research guided, school-based Staying Healthy-Asthma Responsible and Prepared Education and Counseling Program. Journal of Allergy and Clinical Immunology 2007;119(1):S285.

\section{Kouba 2012 \{published data only\}}

Kouba J, Velsor-Friedrich B, Militello L, Harrison PR, Becklenberg A, White B, et al. Efficacy of the I Can Control Asthma and Nutrition Now (ICAN) pilot program on health outcomes in high school students with asthma. Journal of School Nursing 2012;29(3):235-47.

\section{Langenfeld 2010 \{published data only\}}

Langenfeld NA, Mast DK, Rasberry CN, Cheung K, Luna P, Buckley R, et al. Strategies for identifying students in need of school-based asthma services: challenges and questions that emerged from a rapid evaluation of a school-based asthma program. Journal of Asthma and Allergy Educators 2010;1(3):109-16.

\section{Lee 2011 \{published data only\}}

Lee E. The Effectiveness of a School-Based Asthma Management Program on Children With Asthma [Thesis]. Minneapolis, Minnesota, USA: Walden University, 2011.

\section{Levy 2006 \{published data only\}}

Levy M, Heffner B, Stewart T, Beeman G. The efficacy of asthma case management in an urban school district in reducing school absences and hospitalizations for asthma. Journal of School Health 2006;76(6):320-4.

\section{Magzamen 2008 \{published data only\}}

Davis A, Brown AS, Edelstein J, Tager IB. Identification and education of adolescents with asthma in an urban school district: results from a large-scale asthma intervention. Journal of Urban Health 2008;85(3):361-74.

Magzamen S, Mortimer KM, Davis A, Tager IB. School-based asthma surveillance: a comparison of student and parental report. Pediatric Allergy and Immunology 2005;16(8):669-78.

* Magzamen S, Patel B, Davis A, Edelstein J, Tager IB. Kickin' Asthma: school-based asthma education in an urban community. Journal of School Health 2008;78(12):655-65.

Patel SB, Hasenbush A, Davis A, Tager I, Magzamen S. Medication use patterns among urban youth participating in school-based asthma education. Journal of Urban Health 2011;88(Suppl 1):73-84.

\section{McCann 2006 \{published data only\}}

* McCann DC, McWhirter J, Coleman H, Calvert M, Warner JO. A controlled trial of a school-based intervention to improve asthma management. European Respiratory Journal 2006;27(5):921-8.

McWhirter J, McCann D, Coleman H, Calvert M, Warner J. Can schools promote the health of children with asthma?. Health Education Research 2008;23(6):917-30.

\section{McGhan 2003 \{published data only\}}

McGhan S, Jhangri G, Wells H, Wong E, Boechler V, Befus D, et al. Results of a controlled study of a school based asthma education program [abstract]. American Journal of Respiratory and Critical Care Medicine 2000;161:A903.

McGhan SL, Wells HM, Befus AD. The "Roaring Adventures of Puff": a childhood asthma education program. Journal of Pediatric Health Care 1998;12(4):191-5.

* McGhan SL, Wong E, Jhangri GS, Wells HM, Michaelchuk DR, Boechler VL, et al. Evaluation of an education program for elementary school children with asthma. Journal of Asthma 2003;40(5):523-33.

\section{McGhan 2010 \{published data only\}}

Mandhane PJ, McGhan SL, Sharpe HM, Wong E, Hessel PA, Befus AD, et al. A child's asthma quality of life rating does not significantly influence management of their asthma. Pediatric Pulmonology 2010;45(2):141-8.

McGhan SL, Wong E, Sharpe HM, Hessel PA, Mandhane P, Boechler VL, et al. A children's asthma education program: Roaring Adventures of Puff (RAP) improves quality of life. Canadian Respiratory Journal 2010;17(2):67-73.

\section{Mickel 2016 \{published data only\}}

Mickel CF, Shanovich KK, Evans MD, Jackson DJ. Evaluation of a school-based asthma education protocol: Iggy and the inhalers. Journal of School Nursing 2016;1:1-9.

Mickel CF, Shanovich KK, Evans MD, Jackson DJ. Evaluation of a school-based asthma education protocol: Iggy and the inhalers. Journal of School Nursing 2017;33(3):189-97. 
Monforte 2012 \{published data only\}

Monforte SE, Gleason M, Covar R, Cicutto L, Szelfer SJ. Reducing health disparities for asthma with a school based asthma education program [Abstract]. Journal of Allergy and Clinical Immunology 2012;129:AB41 [156].

Mosnaim 2011 \{published data only\}

Mosnaim GS, Li H, Damitz M, Sharp LK, Li Z, Talati A, et al. Evaluation of the Fight Asthma Now (FAN) program to improve asthma knowledge in urban youth and teenagers. Annals of Allergy, Asthma and Immunology 2011;107(4):310-6.

Mujuru 2011 \{published data only\}

Mujuru P, Salana H, Kellam N, Howell C. Challenges to childhood asthma intervention delivery in hard-to-reach small rural communities: a school-based approach. Journal of Asthma and Allergy Educators 2011;2(5):225-32.

Patterson 2005 \{published data only\}

* Patterson EE, Brennan MP, Linskey KM, Webb DC, Shields MD, Patterson CC, et al. A cluster randomised intervention trial of asthma clubs to improve quality of life in primary school children: the School Care and Asthma Management Project (SCAMP). Archives of Disease in Childhood 2005;90(8):786-91.

Shields MD, Patterson EE, Brennan MP, Linskey K, Webb D, Patterson CC, et al. A cluster randomised intervention trial of asthma clubs to improve quality of life in primary school children - the school care and asthma management project (SCAMP) [Abstract]. Thorax 2004;59(Suppl II):ii21.

\section{Persaud 1996 \{published data only\}}

Persaud DI, Barnett SE, Weller SC, Baldwin CD, Niebuhr V, McCormick DP. An asthma self-management program for children, including instruction in peak flow monitoring by school nurses. Journal of Asthma 1996;33(1):37-43.

Pike 2011 \{published data only\}

Pike EV, Richmond CM, Hobson A, Kleiss J, Wottowa J, Sterling DA. Development and evaluation of an integrated asthma awareness curriculum for the elementary school classroom. Journal of Urban Health 2011;88(Suppl 1):61-7.

Praena-Crespo 2010 \{published data only\}

Praena-Crespo M, Fernandez-Truan J, Gálvez-González J, Murillo-Fuentes A, Castro-Gómez L, Cenizo-Benjumea J. Randomised controlled trial of educational intervention directed by physical education teachers in high schools. European Journal of Allergy and Clinical Immunology 2010;65:190-1.

\section{Pulcini 2007 \{published data only\}}

Pulcini J, DeSisto MC, Mclntyre CL. An intervention to increase the use of Asthma Action Plans in schools: a MASNRN study. Journal of School Nursing 2007;23(3):170-6.

\section{Richmond 2011 \{published data only\}}

Richmond CM, Hobson A, Pike E, Kleiss J, Wottowa J, Sterling DA. Breathe Your Best for School Success: evaluation of an initiative to enhance asthma action plans in the school setting. Journal of Urban Health 2011;88(Suppl 1):68-72.
Shah 2001 \{published data only\}

Shah S, Peat JK, Mazurski EJ, Wang H, Sindhusake D, Bruce C, et al. Effect of peer led programme for asthma education in adolescents: cluster randomised controlled trial. BMJ 2001;322(7286):583-5.

\section{Spencer 2000 \{published data only\}}

Spencer GA, Atav S, Johnston Y, Harrigan JF. Managing childhood asthma: the effectiveness of the Open Airways for Schools program. Family and Community Health 2000;23(2):20-30.

\section{Splett 2006 \{published data only\}}

Erickson CD, Splett PL, Mullett SS, Jensen C, Belseth SB. The healthy learner model for student chronic condition management - part II: the asthma initiative. Journal of School Nursing 2006;22(6):319-29.

* Splett PL, Erickson CD, Belseth SB, Jensen C. Evaluation and sustainability of the Healthy Learners Asthma Initiative. Journal of School Health 2006;76(6):276-82.

Srof 2012 \{published data only\}

Srof BJ, Velsor-Friedrich B, Penckofer S. The effects of coping skills training among teens with asthma. Western Journal of Nursing Research 2012;34(8):1043-61.

\section{Terpstra 2012 \{published data only\}}

Terpstra JL, Chavez LJ, Ayala GX. An intervention to increase caregiver support for asthma management in middle schoolaged youth. Journal of Asthma 2012;49(3):267-74.

\section{Velsor-Friedrich 2005 \{published data only\}}

Velsor-Friedrich B, Pigott T, Srof B. A practitioner-based asthma intervention program with African American inner-city school children. Journal of Pediatric Health Care 2005;19(3):163-71.

\section{References to studies excluded from this review}

\section{Akasawa 2016 \{published data only\}}

Akasawa A. Asthma management in school. Japanese Journal of Allergology 2016;65(7):901-6.

\section{Al Aloola 2017 \{published data only\}}

Al Aloola NA, Saba M, Nissen L, Alewairdhi HA, Alaloola A, et al. Development and evaluation of a school-based asthma educational program. Journal of Asthma 2017;54(4):419-29.

\section{Alreshidi 2015 \{published data only\}}

Alreshidi N. The impact of a school-based asthma health education programme on quality of life, knowledge and attitudes of Saudi children with asthma. Clinical and Experimental Allergy 2015;45(2):514.

\section{Al-Sheyab 2015 \{published data only\}}

Al-Sheyab N, Alomari M, Shah S, Gallagher R. 'Class smoke-free' pledge impacts on nicotine dependence in male adolescents: a cluster randomized controlled trial. Tropical Medicine and International Health 2015;20:255-6. 
Anderson 2004 \{published data only\}

Anderson ME, Freas MR, Wallace AS, Kempe A, Gelfand EW, Liu AH. Successful school-based intervention for innercity children with persistent asthma. Journal of Asthma 2004;41(4):445-53.

\section{Ando 2016 \{published data only\}}

Ando T, Yamamoto-Hanada K, Nagao M, Fujisawa T, Ohya Y. Combined program with computer-based learning and peer education in early adolescents with asthma: a pilot study. Journal of Allergy and Clinical Immunology 2016;137(2):AB157.

Arnold 2012 \{published data only\}

Arnold RJ, Stingone JA, Claudio L. Computer-assisted schoolbased asthma management: a pilot study. JMIR Research Protocols 2012;1(2):e15.

\section{Arıkan-Ayyıldız 2016 \{published data only\}}

Arıkan-Ayyıldız Z, Işık S, Çağlayan-Sözmen Ş, Ana Ö, Karaman Ö, Uzuner N. Efficacy of asthma education program on asthma control in children with uncontrolled asthma. Turkish Journal of Pediatrics 2016;58(4):383-8.

\section{Augustin 2003 \{published data only\}}

Augustin J. An Intensive Asthma Intervention in a School-Based Clinic [Dissertation]. Chicago, IL: Rush University, 2003:14p.

\section{Becker 2003 \{published data only\}}

Becker AB, Whitters D, Gillespie CA, Filuk SE, McColm JE, Thomas NJ, et al. Impact of a randomized asthma education program on asthma control in children [Abstract]. Journal of Asthma and Clinical Immunology 2003;111:S212.

\section{Bignall 2015a \{published data only\}}

Bignall WJ, Luberto CM, Cornette AF, Haj-Hamed M, Cotton S. Breathing retraining for African-American adolescents with asthma: a pilot study of a school-based randomized controlled trial. Journal of Asthma 2015;52(9):889-96.

\section{Bollinger 2010 \{published data only\}}

Bollinger ME, Morphew T, Mullins CD. The Breathmobile program: a good investment for underserved children with asthma. Annals of Allergy, Asthma and Immunology 2010;105(4):274-81.e1.

\section{Bowen 2013 \{published data only\}}

Bowen F. Asthma education and health outcomes of children aged 8 to 12 years. Clinical Nursing Research 2013;22(2):172-85.

\section{Brooten 2008 \{published data only\}}

Brooten D, Youngblut JM, Royal S, Cohn S, Lobar SL, Hernandez L. Outcomes of an asthma program: Healthy Children, Healthy Homes. Pediatric Nursing 2008;34(6):448-55.

\section{Bruzzese 2001 \{published data only\}}

Bruzzese JM, Markman LB, Appel D, Webber M. An evaluation of Open Airways for Schools: using college students as instructors. Journal of Asthma 2001;38(4):337-42.

\section{Bruzzese 2006 \{published data only\}}

Bruzzese JM, Evans D, Wiesemann S, Pinkett-Heller M, Levison MJ, Du Y, et al. Using school staff to establish a preventive network of care to improve elementary school students' control of asthma. Journal of School Health 2006;76(6):307-12.

\section{Bruzzese 2011a \{published data only\}}

Bruzzese JM, Cespedes A, Sheares BJ, Kingston S, Evans D, Sheares BJ. Feasibility and preliminary outcomes of a schoolbased approach to helping urban ethnic minority adolescents with undiagnosed asthma. Patient Education and Counseling 2011;85(2):290-4

\section{Burgess 2017 \{published data only\}}

Burgess K, Smith B. Efficacy of telemedicine in delivering pediatric asthma education in a rural West Alabama elementary school. Journal of Investigative Medicine 2017;65(2):412-3.

\section{Burkhart 2003 \{published data only\}}

Burkhart PV, Ward HJ. Children's self-reports of characteristics of their asthma episodes. Journal of Asthma 2003;40(8):909-16

Bush 2014 \{published data only\}

Bush JS, Waller JL, Ownby DR, Tingen MS. Do parents influence health literacy and impact asthma self-management in rural Georgia high school students?. Annals of Allergy, Asthma and Immunology 2014;113(5 Suppl 1):A20.

\section{Butz 2005 \{published data only\}}

* Butz A, Pham L, Lewis L, Lewis C, Hill K, Walker J, et al. Rural children with asthma: impact of a parent and child asthma education program. Journal of Asthma 2005;42(10):813-21.

Walker J, Winkelstein M, Land C, Lewis-Boyer L, Quartey R, Pham L, et al. Factors that influence quality of life in rural children with asthma and their parents. Journal of Pediatric Health Care 2008;22(6):343-50.

\section{Carpenter 2016b \{published data only\}}

Carpenter DM, Geryk LL, Sage A, Arrindell C, Sleath BL. Exploring the theoretical pathways through which asthma app features can promote adolescent self-management. Translational Behavioral Medicine 2016;6(4):509-18.

\section{Cheung 2015 \{published data only\}}

Cheung K, Rasberry CN, Dunville RL, Buckley R, Cook D, Daniels B, et al. A multicomponent school-based asthma management program: enhancing connections to clinical care. Journal of School Health 2015;85(2):135-40.

\section{Chini 2011 \{published data only\}}

Chini L, Iannini R, Chianca M, Corrente S, Graziani S, La Rocca M, et al. Happy Air ${ }^{\circledR}$, a successful school-based asthma educational and interventional program for primary school children. Journal of Asthma 2011;48(4):419-26.

Christiansen 1997 \{published data only\}

Christiansen SC, Martin SB, Schleicher NC, Koziol JA, Mathews KP, Zuraw BL. Evaluation of a school-based asthma 
education program for inner-city children. Journal of Allergy and Clinical Immunology 1997;100(5):613-7.

Clark 1986 \{published data only\}

Clark NM, Feldman CH, Evans D, Duzey O, Levison MJ, Wasilewski Y, et al. Managing better: children, parents, and asthma. Patient Education and Counseling 1986;8(1):27-38.

Clark 2003 \{published data only\}

Clark NM. School-based approaches to help pre-teens manage asthma. CRISP (Computer Retrieval of Information on Scientific Projects) 2003;31/01/2008 End date:1-2.

Coté 1997 \{published data only\}

Coté J, Cartier A, Robichaud P, Boutin H, Malo JL, Rouleau M, et al. Influence on asthma morbidity of asthma education programs based on self-management plans following treatment optimization. American Journal of Respiratory and Critical Care Medicine 1997;155(5):1509-14.

De Godoi 2016 \{published data only\}

Molino CG, Romano-Lieber NS, Ribeiro E, de Melo DO. Noncommunicable disease clinical practice guidelines in Brazil: a systematic assessment of methodological quality and transparency. PLoS ONE 2016;11(11):1-15.

\section{de Greef, 2017 \{published data only\}}

de GreefM, Pijnenburg HM, van Hattum MJ, McLeod BD, et al. Parent-professional alliance and outcomes of child, parent, and family treatment: a systematic review. Journal of Child and Family Studies 2017;26(4):961-76.

\section{DePue 2007 \{published data only\}}

DePue JD, McQuaid EL, Koinis-Mitchell D, Camillo C, Alario A, Klein RB. Providence school asthma partnership: school-based asthma program for inner-city families. Journal of Asthma 2007;44(6):449-53.

\section{Eakin 2012 \{published data only\}}

Eakin MN, Rand CS, Bilderback A, Bollinger ME, Butz A, Kandasamy V, et al. Asthma in Head Start children: effects of the Breathmobile program and family communication on asthma outcomes. Journal of Allergy and Clinical Immunology 2012;129(3):664-70

\section{Evans 2001 \{published data only\}}

Evans D, Clark NM, Levison MJ, Levin B, Mellins RB. Can children teach their parents about asthma?. Health Education and Behavior 2001;28(4):500-11.

\section{Fernandes 2006 \{published data only\}}

Fernandes L, Fonseca JA, Costa-pereira A, Delgado L, Martins S, Moreira A, et al. Effect on quality of life of multidisciplinary psycho-educational group interventions: a randomized controlled trial [Abstract]. Journal of Allergy and Clinical Immunology 2006;117:S139.

Francis 2001 \{published data only\}

Francis C. Setting up a school-based clinic to improve adolescent asthma. Community Nurse 2001;7(6):19.

\section{Gardida 2002 \{published data only\}}

Gardida A, Rojas M, Tavera C, Catalan M. Evaluation of an educational program to control asthma in school age children in the Morelos state, Mexico. [Spanish]. Revista del Instituto Nacional de Enfermedades Respiratorias 2002;15(1):27-30.

Gerald 2016 \{published data only\}

Gerald JK, Gerald LB. The unfulfilled promise of school-centered asthma care. Journal of Allergy and Clinical Immunology 2016;4(5):980-1.

\section{Gibson 1998 \{published data only\}}

Gibson PG, Shah S, Mamoon HA. Peer-led asthma education for adolescents: impact evaluation. Journal of Adolescent Health 1998;22(1):66-72.

\section{Grad 2009 \{published data only\}}

Grad R, McClure L, Zhang S, Mangan J, Gibson L, Gerald L. Peak flow measurements in children with asthma: what happens at school?. Journal of Asthma 2009;46(6):535-40.

\section{Greenberg 2010 \{published data only\}}

Greenberg C, Luna P, Simmons G, Huhman M, Merkle S, Robin L, et al. Follow-up of an elementary school intervention for asthma management: do gains last into middle school?. Journal of Asthma 2010;47(5):587-93.

\section{Greer 2009 \{published data only\}}

Greer M, Lin L, Atkinson RK. Using a computer game to teach school-aged children about asthma. Interactive Learning Environments 2017;25(4):431-8.

Greer MZ. Effect of a CD-ROM Game to Teach School-Age Children About Asthma [doctoral thesis]. Tempe, AZ: Arizona State University, 2009.

\section{Gregory 2000 \{published data only\}}

Gregory EK. Empowering students on medication for asthma to be active participants in their care: an exploratory study. Journal of School Nursing 2000;16(1):20-7.

Halterman 2004 \{published data only\}

Halterman JS, Szilagyi PG, Yoos HL, Conn KM, Kaczorowski JM, Holzhauer RJ, et al. Benefits of a school-based asthma treatment program in the absence of secondhand smoke exposure: results of a randomized clinical trial. Archives of Pediatrics and Adolescent Medicine 2004;158(5):460-7.

\section{Halterman 2011 \{published data only\}}

Halterman JS, Riekert K, Bayer A, Fagnano M, Tremblay P, Blaakman S, et al. A pilot study to enhance preventive asthma care among urban adolescents with asthma. Journal of Asthma 2011;48(5):523-30.

\section{Halterman 2011a \{published data only\}}

Blaakman S, Tremblay PJ, Halterman JS, Fagnano M, Borrelli B. Implementation of a community-based secondhand smoke reduction intervention for caregivers of urban children with asthma: process evaluation, successes and challenges. Health Education Research 2012;28(1):141-52. 
* Halterman JS, Szilagyi PG, Fisher SG, Fagnano M, Tremblay P, Conn KM, et al. Randomized controlled trial to improve care for urban children with asthma: results of the school-based asthma therapy trial. Archives of Pediatrics and Adolescent Medicine 2011;165(3):262-8.

Noyes K, Bajorska A, Fisher S, Sauer J, Fagnano M, Halterman JS. Cost-effectiveness of the School-Based Asthma Therapy (SBAT) program. Pediatrics 2013;131(3):e709-17.

\section{Halterman 2012 \{published data only\}}

Halterman JS, Fagnano M, Montes G, Fisher S, Tremblay P, Tajon R, et al. The school-based preventive asthma care trial: results of a pilot study. Journal of Pediatrics 2012;161(6):1109-176.

Halterman JSS, Sauer J, Fagnano M, Montes G, Fisher S, Tremblay P, et al. Working toward a sustainable system of asthma care: development of the School-Based Preventive Asthma Care Technology (SB-PACT) trial. Journal of Asthma 2012;49(4):395-400.

\section{Hemate 2012 \{published data only\}}

Hemate Z, Ghazavi Z, Hasanpor M, Iranpor R, Alidosti M. An examination of the effect of health promotion plan in high school students' on knowledge and performance on peers suffering from asthma in high schools of district 3 in Esfahan, 2010. Journal of Education and Health Promotion 2012;1:33.

\section{Hill 1991 \{published data only\}}

Hill R, Williams J, Britton J, Tattersfield A. Can morbidity associated with untreated asthma in primary school children be reduced? A controlled intervention study. BMJ 1991;303(6811):1169-74.

\section{Horner 1998 \{published data only\}}

Horner SD. Using the Open Airways curriculum to improve selfcare for third grade children with asthma. Journal of School Health 1998;68(8):329-33.

\section{Horner 2003 \{published data only\}}

Horner SD. Enhancing children's and parents' asthma management. CRISP (Computer Retrieval of Information on Scientific Projects) 2003;30/04/2007 end date:1.

\section{Hughes \{published data only\}}

Hughes M, Murphy M. Evaluation of a pilot national online asthma E-learning program for secondary school students*. Issues in Comprehensive Pediatric Nursing 2013;37(2):136-46.

\section{Johnson 2016 \{published data only\}}

Johnson KB, Patterson BL, Ho YX, Chen QX, Nian H, Davison CL, et al. The feasibility of text reminders to improve medication adherence in adolescents with asthma. Journal of the American Medical Informatics Association 2016;23(3):449-55.

\section{Jones 2005 \{published data only\}}

* Jones CA, Clement LT, Hanley-Lopez J, Morphew T, Kwong KYC, Lifson F, et al. The Breathmobile(trademark) Program: structure, implementation, and evolution of a largescale, urban, pediatric asthma disease management program. Disease Management 2005;8(4):205-22.
Jones CA, Clement LT, Morphew T, Kwong KY, Hanley-Lopez J, Lifson F, et al. Achieving and maintaining asthma control in an urban pediatric disease management program: the Breathmobile Program. Journal of Allergy and Clinical Immunology 2007;119(6):1445-53.

\section{Joseph 2004 \{published data only\}}

Joseph V. A study compliance to two alternative drug regimens and the effect of health education on drug compliance in school age children with bronchial asthma. Nursing Journal of India 2004;95(7):153-4.

\section{Joseph 2007 \{published data only\}}

Joseph CL, Peterson E, Havstad S, Johnson CC, Hoerauf S, Stringer S, et al. A web-based, tailored asthma management program for urban African-American high school students. American Journal of Respiratory and Critical Care Medicine 2007;175(9):888-95.

\section{Joseph 2013a \{published data only\}}

Joseph CL, Ownby DR, Havstad SL, Saltzgaber J, Considine S, Johnson D, et al. Evaluation of a web-based asthma management intervention program for urban teenagers: reaching the hard to reach. Journal of Adolescent Health 2013;52(4):419-26.

\section{Kaufman 2011 \{published data only\}}

* Kaufman D, Sauve L, Renaud L. Enhancing learning through an online secondary school educational game. Journal of Educational Computing Research 2011;44(4):409-28.

Kaufman D, Sauve L, Renaud L. The impact of an online educational game on knowledge and attitudes. Proceedings of the 6th International Conference on E-Learning 2011;44:173-82.

\section{Kenny 2016 \{published data only\}}

Kenny P, Dunne M. Asthma Friendly School Award - a new way of engaging schools, students and families in asthma management education. Irish Journal of Medical Science 2016;185:S483.

\section{Khan 2014 \{published data only\}}

Khan R, Maharaj R, Seerattan N, Babwah F. Effectiveness of personalized written asthma action plans in the management of children with partly controlled asthma in Trinidad: a randomized controlled trial. Journal of Tropical Pediatrics 2014;60(1):17-26.

\section{Khoshnavay 2013 \{published data only\}}

Khoshnavay F, Kharazmi RA. The effectiveness of rational emotive behavior therapy (REBT) on the quality of life in asthmatic children. Iranian Journal of Allergy, Asthma and Immunology 2013;12(1):S130.

\section{Kintner 2015 \{published data only\}}

* Kintner E, Cook G, Marti CN, Stoddard D, Gomes M, Harmon P, et al. Comparative effectiveness on cognitive asthma outcomes of the SHARP Academic Asthma Health Education and Counseling Program and a non-academic program. Research in Nursing and Health 2015;38(6):423-35. 
Kintner EK, Cook G, Marti CN, Allen A, Stoddard D, Harmon P, et al. Effectiveness of a school- and community-based academic asthma health education program on use of effective asthma self-care behaviors in older school-age students. Journal for Specialists in Pediatric Nursing 2015;20(1):62-75.

\section{Knight 2005 \{published data only\}}

Knight DD. Expanding Asthma Awareness in Adolescents: A Pilot Investigation [dissertation]. Honolulu, HI: University of Hawaii at Manoa, 2005.

\section{Krishna 2006 \{published data only\}}

Krishna S, Balas EA, Francisco BD, Konig P. Effective and sustainable multimedia education for children with asthma: a randomized controlled trial. Children's Health Care 2006;35(1):75-90.

\section{Lakupoch 2017 \{published data only\}}

Lakupoch K, Manuyakorn W, Preutthipan A, Kamalaporn H. The effectiveness of newly developed written asthma action plan in improvement of asthma outcome in children. Asian Pacific Journal of Allergy and Immunology 2017;1:1-5.

\section{Lewis 2005 \{published data only\}}

Lewis CJ, Thompson RE, Butz AM, Hill KL, Huss K, LewisBowyer LL, et al. Asthma education increases knowledge of rural parents and children with asthma and affects parents' reports of their child's asthma symptoms [Abstract]. Journal of Allergy and Clinical Immunology 2005;115:S132.

\section{Li 2017 \{published data only\}}

Li Z, Leite WL, Thompson LA, Gross HE, Shenkman EA, Reeve BB, et al. Determinants of longitudinal health-related quality-oflife change in children with asthma from low-income families: a report from the PROMIS ${ }^{\circledR}$ Pediatric Asthma Study. Clinical and Experimental Allergy 2017;47(3):383-94.

\section{Liao 2006 \{published data only\}}

Liao O, Morphew T, Amaro S, Galant SP. The Breathmobile: a novel comprehensive school-based mobile asthma care clinic for urban underprivileged children. Journal of School Health 2006;76(6):313-9.

\section{Lin 2017 \{published data only\}}

Lin CH, Zedeck SS, Garcia Lloret MI. UCLA (Long Beach) Breathmobile: improving asthma outcomes in low-income pediatric patients. Journal of Allergy and Clinical Immunology 2017;139(2):AB99.

\section{Lipman 2017 \{published data only\}}

Lipman TH. Using tailored videos to teach inhaler technique to children with asthma: results from a school nurse-led pilot study. MCN. The American Journal of Maternal Child Nursing 2017;42(3):185.

\section{Loman 2017 \{published data only\}}

Loman DG, Kwong CG, Henry LD, Mahl C, Meadows L, Ellis AG. Asthma control and obesity in urban African American children. Journal of Asthma 2017;54(6):578-83.

\section{Louisias 2016 \{published data only\}}

Louisias M, Goldmann D, Phipatanakul W. School nurses' perspectives on barriers to implementing school-based asthma management plans. Journal of Allergy and Clinical Immunology 2016;137(2):AB100.

\section{Lu 2017 \{published data only\}}

Lu KD, Cooper D, Haddad F, Lakes KD, Radom-Aizik S. Four months of a school based exercise intervention improved fitness in normal weight and overweight/obese children with asthma in a minority, low SES population - a pilot study. American Journal of Respiratory and Critical Care Medicine 2017;195:1.

\section{Lurie 2001 \{published data only\}}

Lurie N, Bauer EJ, Brady C. Asthma outcomes at an innercity school-based health center. Journal of School Health 2001;71(1):9-16.

\section{Lwebuga-Mukasa 2002 \{published data only\}}

Lwebuga-Mukasa J, Dunn-Georgiou E. A school-based asthma intervention program in the Buffalo, New York, schools. Journal of School Health 2002;72(1):27-32.

\section{Maa 2010 \{published data only\}}

Maa SH, Chang YC, Chou CL, Ho SC, Sheng TF, MacDonald K, et al. Evaluation of the feasibility of a school-based asthma management programme in Taiwan. Journal of Clinical Nursing 2010;19(17-18):2415-23.

\section{MacPherson 2011 \{published data only\}}

MacPherson A, Snider C, Kakepetum-Schults T, McGhan S. Growing healthy children and youth: an asthma education program. Canadian Respiratory Journal 2011;18:26A.

\section{Mangan 2006 \{published data only\}}

Mangan JMM, Gerald LBB. Asthma agents: monitoring asthma in school. Journal of School Health 2006;76(6):300-2.

\section{Marabini 2002 \{published data only\}}

Marabini A, Brugnami G, Curradi F, Casciola G, Stopponi R, Pettinari $L$, et al. Short-term effectiveness of an asthma educational program: results of a randomized controlled trial. Respiratory Medicine 2002;96(12):993-8.

\section{McClure 2008 \{published data only\}}

McClure LA, Harrington KF, Graham H, Gerald LB. Internet-based monitoring of asthma symptoms, peak flow meter readings, and absence data in a school-based clinical trial. Clinical Trials 2008;5(1):31-7.

\section{McElmurry 1999 \{published data only\}}

McElmurry BJ, Buseh AG, Dublin M. Health education program to control asthma in multiethnic, low-income urban communities: the Chicago Health Corps Asthma Program. Chest 1999;116(4 Suppl 1):198S-9S.

\section{McEwen 1998 \{published data only\}}

McEwen M, Johnson P, Neatherlin J, Millard MW, Lawrence G. School-based management of chronic asthma among inner- 
city African-American schoolchildren in Dallas, Texas. Journal of School Health 1998;68(5):196-201.

\section{McLaughlin 2006 \{published data only\}}

McLaughlin T, Maljanian R, Kornblum R, Clark P, Simpson J, McCormack K. Evaluating the availability and use of asthma action plans for school-based asthma care: a case study in Hartford, Connecticut. Journal of School Health 2006;76(6):325-8.

\section{Meng 2000 \{published data only\}}

Meng A. A school-based asthma clinic: a partnership model for managing childhood asthma. Nurse Practitioner Forum - Current Topics and Communications 2000;11(1):38-47.

\section{Meurer 1999 \{published data only\}}

Meurer JR, McKenzie S, Mischler E, Subichin S, Malloy M, George V. The Awesome Asthma School Days program: educating children, inspiring a community. Journal of School Health 1999;69(2):63-8.

\section{Millard 2003 \{published data only\}}

Millard MW, Johnson PT, McEwen M, Neatherlin J, Lawrence G, Kennerly DK, et al. A randomized controlled trial using the school for anti-inflammatory therapy in asthma. Journal of Asthma 2003;40(7):769-76.

\section{Mitchell 2017 \{published data only\}}

Mitchell DK, McQuaid EL, Fritz GK, Kopel SJ, Seifer R, Esteban CA, et al. Culturally and contextually tailored asthma self-management for urban, Latino middle school students: the Rhode Island-Puerto Rico Asmas program. American Journal of Respiratory and Critical Care Medicine 2017;195:1.

\section{Morphew 2013 \{published data only\}}

* Morphew T, Scott L, Li M, Galant SP, Wong W, Lloret Maria IG, et al. Mobile health care operations and return on investment in predominantly underserved children with asthma: the Breathmobile Program. Population Health Management 2013;16(4):261-9.

Xi SC, Morphew T, Kwong K, Li M, Thobani S, Nichols B, et al. Association between obesity and asthma control in children: the Breathmobile Program. Annals of Allergy, Asthma and Immunology 2015;115(5):A23.

\section{Morphew 2017 \{published data only\}}

Morphew T, Altamirano W, Bassin SL, Galant SP. The Breathmobile improves the asthma medication ratio and decreases emergency department utilization. American Journal of Managed Care 2017;23(4):120-6.

\section{Morton 2017 \{published data only\}}

Morton RW, Elphick HE, Rigby AS, Daw WJ, King DA, Smith LJ, et al. STAAR: a randomised controlled trial of electronic adherence monitoring with reminder alarms and feedback to improve clinical outcomes for children with asthma. Thorax 2017;72(4):347-54.
Mosnaim 2017 \{published data only\}

Mosnaim GS, Akkoyun E, Eng J, Shalowitz MU. Behavioral interventions to improve asthma outcomes: a systematic review of recent publications. Current Opinion in Allergy and Clinical Immunology 2017;17(3):194-200.

\section{NCT00217776 \{published data only\}}

NCT00217776. School-based approaches to help pre-teens manage asthma. clinicaltrials.gov/ct2/show/NCT00217776 (first received 22 September 2005)

\section{Neuharth-Pritchett 2016 \{published data only\}}

Neuharth-Pritchett S, Getch YQ. The effectiveness of a brief asthma education intervention for child care providers and primary school teachers. Early Childhood Education Journal 2016;44(6):555-61.

\section{Nuss 2016 \{published data only\}}

Nuss HJ, Hester LL, Perry MA, Stewart-Briley C, Reagon VM, Collins P. Applying the social ecological model to creating asthma-friendly schools in Louisiana. Journal of School Health 2016;86(3):225-32.

\section{Otim 2015 \{published data only\}}

Otim ME, Jayasinha R, Forbes H, Smita S. Building evidence for peer-led interventions: assessing the cost of the Adolescent Asthma Action program in Australia. Australian Journal of Primary Health 2015;21(4):438-43.

Patel 2007 \{published data only\}

Patel B, Sheridan P, Detjen P, Donnersberger D, Gluck E, Malamut K, et al. Success of a comprehensive school-based asthma intervention on clinical markers and resource utilization for inner-city children with asthma in Chicago: the Mobile C.A.R.E. Foundation's asthma management program. Journal of Asthma 2007;44(2):113-8.

\section{Peers 2017 \{published data only\}}

Peers CB, Mullan A, Chodhari R. Asthma innovation research air. Archives of Disease in Childhood 2017;102:A168.

\section{Pender-Phaneuf 2016 \{published data only\}}

Pender-Phaneuf K. Pediatric asthma management in Massachusetts schools: facilitators and barriers. Nursing Research 2016;65(2):E31-2.

\section{Perry 2000 \{published data only\}}

Perry CS, Toole KA. Impact of school nurse case management on asthma control in school-aged children. Journal of School Health 2000;70(7):303-4.

\section{Petrie 2010 \{published data only\}}

Petrie JL, Segal AR. Clinical pharmacy services provided to asthma patients in a school-based clinic. American Journal of Health-System Pharmacy 2010;67(3):185, 188-9.

\section{Quaranta 2012 \{published data only\}}

Quaranta J, Brown K, Logvis K, Ponticiello D. Using nursing students as open airways facilitators through a community partnership to influence asthma outcomes. Journal of Asthma and Allergy Educators 2012;3(2):56-63. 


\section{Quaranta 2015 \{published data only\}}

Quaranta JE, Spencer GA. Using the health belief model to understand school nurse asthma management. Journal of School Nursing 2015;31(6):430-40.

\section{Rasberry 2014 \{published data only\}}

Rasberry CN, Cheung K, Buckley R, Dunville R, Daniels B, Cook D, et al. Indicators of asthma control among students in a rural, school-based asthma management program. Journal of Asthma 2014;51(8):876-85.

\section{Raun 2017 \{published data only\}}

Raun LH, Campos LA, Stevenson E, Ensor KB, Johnson G, Persse $D$. Analyzing who, when, and where: data for better targeting of resources for school-based asthma interventions. Journal of School Health 2017;87(4):253-61.

Rhee 2012 \{published data only\}

Rhee H, Pesis-Katz I, Xing JP. Cost benefits of a peer-led asthma self-management program for adolescents. Journal of Asthma 2012;49(6):606-13.

\section{Richterová 2016 \{published data only\}}

Richterová J, Richter J. Allergy and asthma at school. Alergie 2016;2016(2):102-8

\section{Rodriguez-Martinez 2017 \{published data only\}}

Rodriguez-Martinez CE, Sossa-Briceno MP, Castro-Rodriguez JA. A cost-effectiveness threshold analysis of a multidisciplinary structured educational intervention in pediatric asthma. Journal of Asthma 2017;1:1-10.

Sabla 2017 \{published data only\}

Sabla GE, McDowell KM, Kercsmar CM, Braun WE. Creating a collaborative and sustainable partnership with a public school to promote peer to peer asthma education. American Thoracic Society International Conference; 2017 May 19-24; Washington. 2017:A4831.

\section{Salisbury 2002 \{published data only\}}

Salisbury C, Francis C, Rogers C, Parry K, Thomas H, Chadwick S, et al. A randomised controlled trial of clinics in secondary schools for adolescents with asthma. British Journal of General Practice 2002;52:988-96.

\section{Scherer 2016 \{published data only\}}

Scherer YK, Foltz-Ramos K, Fabry D, Chao YY. Evaluating simulation methodologies to determine best strategies to maximize student learning. Journal of Professional Nursing 2016;32(5):349-57.

\section{Schlueter 2011 \{published data only\}}

Schlueter DF, Rasberry CN, Buckley R, Mast DK, Cheung K, Luna PJ, et al. Secondhand tobacco smoke exposure among school-aged youth enrolled in school-based asthma management programs: a mixed methods analysis. Journal of Asthma and Allergy Educators 2011;2(4):173-80.

\section{Schneider 1997 \{published data only\}}

Schneider SL, Richard M, Huss K, Huss RW, Thompson LC, Butz AM, et al. Moving health care education into the community. Nursing Management 1997;28(9):40-3.

\section{Schuller 2015 \{published data only\}}

Schuller L, Faulkner G. Providing better asthma care for children in school. Nursing Times 2015;111(40):12-4.

Scott 2006 \{published data only\}

* Scott VL. Self-Management Skills for School-Age Youth With Asthma [PhD thesis]. New York: Hofstra University, 2006.

Scott 2008 \{published data only\}

Scott L, Nichols B, Choi Kwong KY, Morphew T, Jones CA. Longitudinal patterns of predominant asthma disease activity in pediatric patients enrolled in an asthma-specific disease management program. Journal of Asthma 2008;45(6):501-5.

\section{Scott 2011 \{published data only\}}

Scott L, Morphew T, Bollinger ME, Samuelson S, Galant S, Clement $\mathrm{L}$, et al. Achieving and maintaining asthma control in inner-city children. Journal of Allergy and Clinical Immunology 2011;128(1):56-63.

\section{Shanovich 2009 \{published data only\}}

Shanovich KK, Sorkness CA, Wise ME, Pulvermacher AD, Bhattacharya A, Gustafson DH. Internet telehealth for pediatric nurse case management improves asthma control [Abstract]. Journal of Allergy and Clinical Immunology 2009;123(2 Suppl 1):S43.

\section{Sharek 2002 \{published data only\}}

Sharek PJ, Mayer ML, Loewy L, Robinson TN, Shames RS, Umetsu DT, et al. Agreement among measures of asthma status: a prospective study of low-income children with moderate to severe asthma. Pediatrics 2002;110(4):797-804

\section{Shaw 2005 \{published data only\}}

Shaw SF, Marshak HH, Dyjack DT, Neish CM. Effects of a classroom-based asthma education curriculum on asthma knowledge, attitudes, self-efficacy, quality of life, and selfmanagement behaviors among adolescents. American Journal of Health Education 2005;36(3):140-7.

\section{Shaw 2016 \{published data only\}}

Shaw N, Le Souëf P, Turkovic L, McCahon L, Kicic A, Sly PD. Pressurised metered dose inhaler-spacer technique in young children improves with video instruction. European Journal of Pediatrics 2016;175(7):1007-12.

\section{Shegog 2001 \{published data only\}}

Shegog R, Bartholomew LK, Parcel GS, Sockrider MM, Mâsse L, Abramson SL. Impact of a computer-assisted education program on factors related to asthma self-management behavior. Journal of the American Medical Informatics Association 2001;8(1):49-61.

\section{Shelef 2016 \{published data only\}}

Shelef DQ, Rand C, Streisand R, Horn IB, Yadav K, Stewart L, et al. Using stakeholder engagement to develop a patient- 
centered pediatric asthma intervention. Journal of Allergy and Clinical Immunology 2016;138(6):1512-7.

\section{Staudt 2015 \{published data only\}}

Staudt AM, Alamgir H, Long DL, Inscore SC, Wood PR. Developing and implementing a citywide asthma action plan: a community collaborative partnership. Southern Medical Journal 2015;108(12):710-4.

\section{Suwannakeeree 2016 \{published data only\}}

Suwannakeeree P, Deerojanawong J, Prapphal N. Schoolbased educational interventions can significantly improve health outcomes in children with asthma. Journal of the Medical Association of Thailand 2016;99(2):166-74.

\section{Szefler 2016 \{published data only\}}

Szefler SJ. Examining causes of the urban (inner city) asthma epidemic: implementing new management strategies. Allergy and Proceedings 2016;37(1):4-8.

\section{Szefler 2017 \{published data only\}}

Szefler SJ, Cloutier M, Villarreal M, Hollenbach J, Gleason M, Howard $\mathrm{CH}$, et al. Building bridges for asthma care: reducing school absence for children with health disparities. American Journal of Respiratory and Critical Care Medicine 2017;195:A5092.

\section{Tate 2009 \{published data only\}}

Tate ED. Asthma in the Community: Designing Instruction to Help Students Explore Scientific Dilemmas That Impact Their Lives [thesis]. Berkeley, CA: University of California, 2009.

\section{Terpstra 2012a \{published data only\}}

Terpstra JL, Chavez LJ, Ayala GX. An intervention to increase caregiver support for asthma management in middle schoolaged youth. Journal of Asthma 2012;49(3):267-74.

\section{Thornton 2016 \{published data only\}}

Thornton E, Kennedy S, Hayes-Watson C, Krouse RZ, Mitchell H, Cohn RD, et al. Adapting and implementing an evidence-based asthma counseling intervention for resource-poor populations. Journal of Asthma 2016;53(8):825-34.

\section{Urrutia-Pereira 2017 \{published data only\}}

Urrutia-Pereira M, To T, Cruz Á, Solé D. The school as a health promoter for children with asthma: the purpose of an education programme. Allergologia et Immunopathologia 2017;45(1):93-8.

\section{Valery 2007 \{published data only\}}

Valery PC, Masters IB, Clements V, Taylor B, Laifoo Y, Chang AB. $A$ randomised controlled study on education intervention for childhood asthma by the Aboriginal and Torres Strait Islander health workers in Torres Strait region [Abstract]. Medical Journal of Australia 2007;12:A193.

\section{Velsor-Friedrich 2004 \{published data only\}}

Velsor-Friedrich B, Pigott TD, Louloudes A, Velsor-Freidrich B. The effects of a school-based intervention on the self-care and health of African-American inner-city children with asthma. Journal of Pediatric Nursing 2004;19(4):247-56.
Velsor-Friedrich 2012 \{published data only\}

Velsor-Friedrich B, Militello LK, Richards MH, Harrison PR, Gross IM, Romero E, et al. Effects of coping-skills training in low-income urban African-American adolescents with asthma. Journal of Asthma 2012;49:372-9.

\section{Volerman 2017 \{published data only\}}

Volerman A, Hull A, Ignoffo S, Press VG. Overcoming inhaler misuse: looking outside the healthcare setting for assessment and education. American Journal of Respiratory and Critical Care Medicine 2017;195:A3329.

\section{Walter 2016 \{published data only\}}

Walter H, Sadeque-IF, Ulysse R, Castillo D, Fitzpatrick A, Singleton J. Effectiveness of school-based family asthma educational programs on quality of life and asthma exacerbations in asthmatic children aged five to 18: a systematic review. JBI Database of Systematic Reviews and Implementation Reports 2016;14(11):113-38.

Walton 2004 \{published data only\}

Walton I, Harding J, Stewart A, Tunna K. Project for the evaluation of asthma in Tipton schools (PEATS). Quality in Primary Care 2004;12(1):53-8.

Webber 2005 \{published data only\}

Webber MP, Hoxie AM, Odlum M, Oruwariye T, Lo Y, Appel D. Impact of asthma intervention in two elementary schoolbased health centers in the Bronx, New York City. Pediatric Pulmonology 2005;40(6):487-93.

\section{Weng 2007 \{published data only\}}

Weng HC, Yuan BC, Su YT, Perng DS, Chen WH, Lin LJ, et al. Effectiveness of a nurse-led management programme for paediatric asthma in Taiwan. Journal of Paediatrics and Child Health 2007;43(3):134-8.

\section{Wensley 2004 \{published data only\}}

Wensley D, Silverman M. Peak flow monitoring for guided selfmanagement in childhood asthma: a randomized controlled trial. American Journal of Respiratory and Critical Care Medicine 2004;170(6):606-12.

\section{Whitman 1985 \{published data only\}}

Whitman N, West D, Brough FK, Welch M. A study of a self-care rehabilitation program in pediatric asthma. Health Education Quarterly 1985;12(4):333-42.

\section{Willeboordse 2016 \{published data only\}}

Willeboordse M, van de Kant KDG, Tan FE, Mulkens S, Schellings J, et al. A multifactorial weight reduction programme for children with overweight and asthma: a randomized controlled trial. PLoS One 2016;11(6):e0157158.

\section{Wilson 2008 \{published data only\}}

Wilson KD, Kurz RS. Bridging implementation and institutionalization within organizations: proposed employment of continuous quality improvement to further dissemination. Journal of Public Health Management and Practice 2008;14(2):109-16. 


\section{Wyatt 2008 \{published data only\}}

Wyatt TH, Hauenstein EJ. Pilot testing OKAY WITH ASTHMA: an online asthma intervention for school-age children. Journal of School Nursing 2008;24(3):145-50.

\section{Wyatt 2013 \{published data only\}}

Wyatt TH, Li XP, Huang Y, Farmer R, Reed D, Burkhart PV. Developing an interactive story for children with asthma. Nursing Clinics of North America 2013;48(2):271-85.

\section{Yawn 2000 \{published data only\}}

Yawn BP, Algatt-Bergstrom PJ, Yawn RA, Wollan P, Greco M, Gleason M, et al. An in-school CD-ROM asthma education program. Journal of School Health 2000;70(4):153-9.

Yoshida 2011 \{published data only\} Yoshida K, Masuko I, Akada T, Itazawa T, Adachi Y, Aka-Sawa A, et al. The association between asthma symptoms and obesity in adolescents. Journal of Allergy and Clinical Immunology 2011;127(2):AB153.

\section{Young 2001 \{published data only\}}

Young NL, Foster AM, Parkin PC, Reisman J, MacLusky I, Gold M, et al. Assessing the efficacy of a school-based asthma education program for children: a pilot study. Canadian Journal of Public Health-Revue Canadienne De Sante Publique 2001;92(1):30-4.

\section{Zografos 2007 \{published data only\}}

Zografos K, Marshak HH, Dyjack DT, Neis C. The effects of an adolescent asthma education intervention on knowledge, intention, behavior, self-efficacy and self-consciousness. Californian Journal of Health Promotion 2007;8(1):60-71.

\section{References to studies awaiting assessment}

Liptzin 2016a \{published data only\}

Liptzin DR, Gleason MC, Cicutto LC, Cleveland CL, Shocks DJ, White MK, et al. Developing, implementing, and evaluating a school-centered asthma program: step-up asthma program. Journal of Allergy and Clinical Immunology 2016;4(5):972-99.e1.

\section{McCallum 2017 \{published data only\}}

McCallum GB, Chang AB, Wilson CA, Petsky HL, Saunders J, Pizzutto SJ, et al. Feasibility of a peer-led asthma and smoking prevention project in Australian schools with high indigenous youth. Frontiers in Pediatrics 2017;5:33.

\section{Praena-Crespo 2017 \{published data only\}}

Praena-Crespo M, Aquino-Llinares N, Fernández-Truan JC, Castro-Gómez L, Segovia-Ferrera C, GESA network. Asthma education taught by physical education teachers at grade schools: a randomised cluster trial. Allergologia et Immunopathologia 2017;45(4):375-86

\section{Reznik 2016 \{published data only\}}

Reznik M, Ozuah PO. Addressing barriers to physical activity in schoolchildren with asthma: results of a pilot cluster randomized controlled trial. European Journal of Pediatrics 2016;175(11):1604-5.

\section{Warren 2016 \{published data only\}}

Warren CM, Dyer A, Blumenstock J, Gupta RS. Leveraging mobile technology in a school-based participatory asthma intervention: findings from the student media-based asthma research team (SMART) Study. American Journal of Health Education 2016;47(2):59-70.

Yarbrough M, Blumenstock J, Warren C, Dyer A, Wilson J, Smith B, et al. SMART (student media-based asthma research team): engaging adolescents to understand asthma in their communities. Progress in Community Health PartnershipsResearch Education and Action 2016;10(4):523-32.

\section{References to ongoing studies}

Halterman 2017 \{published data only\}

Halterman JS, Tajon R, Tremblay P, Fagnano M, Butz A, Perry TT, et al. Development of school-based asthma management programs in Rochester, New York: presented in honor of $\mathrm{Dr}$ Robert Haggerty. Academic Pediatrics 2017;17(6):595-9.

\section{Lemanske 2016 \{published data only\}}

Lemanske RF, Kakumanu S, Shanovich K, Antos N, Cloutier MM, Mazyck D, et al. Creation and implementation of SAMPRO (TM): a school-based asthma management program. Journal of Allergy and Clinical Immunology 2016;138(3):711-23.

\section{NCT03032744 \{published data only\}}

NCT03032744. Project IMPACT in schools to prevent asthma symptoms [Intervention and management program for asthma control and treatment in schools]. clinicaltrials.gov/show/ NCT03032744 (first received 26 January 2017).

Perry 2015 \{published data only\}

Perry TT, Halterman JS, Brown RH, Hunter CR, Randle SM, Tilford JM, et al. Breath Connection: a school-based telemedicine program for rural children with asthma. Journal of Allergy and Clinical Immunology 2015;135(2 Suppl 1):Abs169.

Phipatanakul 2017 \{published data only\} Phipatanakul W, Koutrakis P, Coull BA, Kang CM, Wolfson JM, Ferguson ST, et al. The school inner-city asthma intervention study: design, rationale, methods, and lessons learned. Contemporary Clinical Trials 2017;60:14-23.

\section{Additional references}

\section{Ahmad 2011}

Ahmad E, Grimes D. The effects of self-management education for school-age children on asthma morbidity: a systematic review. Journal of School Nursing 2011;27(4):282-92.

\section{Al Aloola 2014}

Al Aloola NA, Naik-Panvelkar P, Nissen L, Saini B. Asthma interventions in primary schools - a review. Journal of Asthma 2014;51(8):779-98.

\section{American Lung Association 2018}

American Lung Association. About Open Airways for Schools. http://www.lung.org/lung-health-and-diseases/lung-disease- 
lookup/asthma/asthma-education-advocacy/open-airwaysfor-schools/about-open-airways.html (accessed prior to 12 November 2018).

\section{Asher 2006}

Asher M, Montefort S, Bjorksten B, Lai C, Strachan D, Weiland K, et al. Worldwide trends in the prevalence of symptoms of asthma, allergic rhinoconjunctivitis, and eczema in childhood: ISAAC Phases One and Three repeat multicountry crosssectional surveys. Lancet 2006;368:733-43.

\section{Asthma UK 2013}

Asthma UK. 2 million people unaware they are at risk of an asthma attack. asthma.org.uk/News/2-million-people-unawarethey-are-at-risk-of-an-asthma-attack (accessed 4 February 2015).

\section{Bahadori 2009}

Bahadori K, Doyle-Waters MM, Marra C, Lynd L, Alasaly K, Swiston J, et al. Economic burden of asthma: a systematic review. BMC Pulmonary Medicine 2009;9(1):24.

\section{Bateman 2008}

Bateman ED, Hurd SS, Barnes PJ, Bousquet J, Drazen JM, FitzGerald M, et al. Global strategy for asthma management and prevention: GINA executive summary. European Respiratory Journal 2008;31(1):143-78.

\section{Berry 2013}

Berry DC, Neal M, Hall EG, McMurray RG, Schwartz TA, Skelly AH, et al. Recruitment and retention strategies for a communitybased weight management study for multi-ethnic elementary school children and their parents. Public Health Nursing 2013;30(1):80-6.

\section{Blackman 2007}

Blackman JA, Gurka MJ. Developmental and behavioral comorbidities of asthma in children. Journal of Developmental and Behavioral Pediatrics 2007;28(2):92-9.

\section{Boyd 2009}

Boyd M, Lasserson TJ, McKean MC, Gibson PG, Ducharme FM, Haby M. Interventions for educating children who are at risk of asthma-related emergency department attendance. Cochrane Database of Systematic Reviews 2009, Issue 2. [DOI: 10.1002/14651858.CD001290.pub2]

\section{British Asthma Guidelines 1997}

British Asthma Guidelines Coordinating Committee. British guidelines on asthma management: 1995 review and position statement. Thorax 1997;52(Suppl 1):S1-20.

\section{Bruhn 1983}

Bruhn JG. The application of theory in childhood asthma selfhelp programs. Journal of Allergy and Clinical Immunology 1983;72(5):561-77.

\section{Brunton 2014}

Brunton G, Caird J, Stokes G, Stansfield C, Kneale D, Richardson $\mathrm{M}$, et al. Community engagement for health via coalitions, collaborations and partnerships: a systematic review and meta-analysis. London EPPI-Centre 2014;1:1-548.

\section{Brunton 2014a}

Brunton G, O'Mara-Eves A, Thomas J. The 'active ingredients' for successful community engagement with disadvantaged expectant and new mothers: a qualitative comparative analysis. Journal of Advanced Nursing 2014;70(12):2847-60.

\section{Bruzzese 2009}

Bruzzese JM, Evans D, Kattan M. School-based asthma programs. Journal of Allergy and Clinical Immunology 2009;124(2):195-200.

\section{BTS 2016}

British Thoracic Society. BTS/SIGN Asthma Guideline 2016. brit-thoracic.org.uk/document-library/clinical-information/ asthma/btssign-asthma-guideline-2016/ (accessed before 20 June 2018)

\section{Bush 2017}

Bush A, Griffiths C. Improving treatment of asthma attacks in children. BMJ: British Medical Journal (Online) 2017;359:j5763.

\section{Carvalho 2016}

Carvalho C, Ana C, Barretto C, Laís S, Souza-Machado C de, et al. The impacts of educational asthma interventions in schools: a systematic review of the literature. Canadian Respiratory Journal 2016 Aug 30 [Epub ahead of print]:8476206. [DOI: $10.1155 / 2016 / 8476206]$

\section{Chinn 2000}

Chinn S. A simple method for converting an odds ratio to effect size for use in meta-analysis. Statistics in Medicine 2000;19(22):3127-31.

\section{Coffman 2009}

Coffman JM, Cabana MD, Yelin MH. Do school-based asthma education programs improve self-management and health outcomes?. Pediatrics 2009;124(2):729-42.

\section{Cooper 2005}

Cooper B. Applying Ragin's crisp and fuzzy set QCA to large datasets: social class and educational achievement in the National Child Development Study. Sociological Research Online 2005;10(2):1-20.

\section{Denford 2013}

Denford S, Taylor RD, Campbell JL, Greaves CJ. Effective behavior change techniques in asthma self-care interventions: systematic review and meta-regression. Health Psychology 33;7:577-87.

\section{Department of Health 2012}

Department of Health. NHS atlas of variation in healthcare for people with respiratory disease. Reducing unwarranted variation to increase value and improve quality. September 2012. http://www.rightcare.nhs.uk/index.php/atlas/ respiratorydisease/ (accessed 5 February 2015). 


\section{Dickson 2016}

Dickson K, Melendez-Torres GJ, Fletcher A, Hinds K, Thomas J, Stansfield $C$, et al. How do contextual factors influence implementation and receipt of positive youth development programs addressing substance use and violence? A qualitative meta-synthesis of process evaluations. American Journal of Health Promotion 2016:1-12. [DOI: 10.1177/0890117116670302]

\section{Fowler 1992}

Fowler M, Davenport M, Garg R. School functioning of US children with asthma. Pediatrics 1992;90(6):939-44.

\section{GINA 2018}

Global Initiative for Asthma. 2018 GINA Report, Global Strategy for Asthma Management and Prevention. https:// ginasthma.org/2018-gina-report-global-strategy-for-asthmamanagement-and-prevention/ (accessed before 20 June 2018).

\section{Glenton 2013}

Glenton C, Colvin CJ, Carlsen B, Swartz A, Lewin S, Noyes J, et al. Barriers and facilitators to the implementation of lay health worker programmes to improve access to maternal and child health: qualitative evidence synthesis. Cochrane Database of Systematic Reviews 2013, Issue 10. [DOI: 10.1002/14651858.CD010414.pub2]

\section{Guevara 2003}

Guevara JP, Wolf FM, Grum CM, Clark NM. Effects of educational interventions for self management of asthma in children and adolescents: systematic review and meta-analysis. BMJ 2003;326(7402):1308-9.

\section{Guyatt 2008}

Guyatt GH, Oxman AD, Gunn E, Kunz R, Falck-Ytter Y, AlonsoCoello P, et al. GRADE: what is "quality of evidence" and why is it important to clinicians?. British Medical Journal 2008;336(7650):924-6.

\section{Guyatt 2011}

Guyatt GH, Oxman AD, Montori V, Vist G, Kunz R, Brozek J, et al. GRADE guidelines: 5 . Rating the quality of evidence: publication bias. Journal of Clinical Epidemiology 2011;64(12):1277-82.

\section{Hannes 2011}

Hannes K. Chapter 4. Critical appraisal of qualitative research. In: Noyes J, Booth A, Hannes K, Harden A, Harris J, Lewin $S$, et al (editors). Supplementary Guidance for Inclusion of Qualitative Research in Cochrane Systematic Reviews of Interventions. Version 1 [updated August 2011]. Cochrane Collaboration Qualitative Methods Group, 2011. Critical appraisal of qualitative research. http://cqim.cochrane.org/ supplemental-handbook-guidance (accessed 5 February 2015).

\section{Harbord 2009}

Harbord RM, Harris RJ, Sterne JAC. Updated tests for smallstudy effects in meta-analyses. Stata Journal 2009;9(2):197.

\section{Harden 2004}

Harden AJ, Garcia S, Oliver R, Rees M, Shepherd J, Brunton G, et al. Applying systematic review methods to studies of people's views: an example from public health research. Journal of Epidemiology and Community Health 59;9:794-800.

\section{Harris 2015}

Harris KM, Kneale D, Lasserson TJ, McDonald VM, Grigg J, Thomas J. School-based self management interventions for asthma in children and adolescents: a mixed methods systematic review. Cochrane Database of Systematic Reviews 2015, Issue 4. [DOI: 10.1002/14651858.CD011651]

\section{Harris 2017}

Harris K, Mosler G, Williams SA, Whitehouse A, Raine R, Grigg J. Asthma control in London secondary school children. Journal of Asthma 2017;54:1-8.

\section{Hellström 2001}

Hellström E. Conflict Cultures: Qualitative Comparative Analysis of Environmental Conflicts in Forestry. 2nd Edition. Helsinki, Finland: Finnish Society of Forest Science [and] Finnish Forest Research Institute, 2001.

\section{Higgins 2003}

Higgins J, Thompson SG, Deeks JJ, Altman DG. Measuring inconsistency in meta-analyses. BMJ 2003;327(7414):557-60.

\section{Higgins 2011}

Higgins JPT, Green S (editors). Cochrane Handbook for Systematic Reviews of Interventions Version 5.1 (updated March 2011). The Cochrane Collaboration, 2011. www.cochranehandbook.org.

\section{HSCIC 2014}

Health and Social Care Information Centre. Compendium of population health indicators (CCG Outcome Indicators Dataset). england.nhs.uk/wp-content/uploads/2013/12/ccg-ois-1415tech-guid.pdf (accessed 5 February 2015).

\section{Hurley 2013}

Hurley M, Dickson K, Walsh N, Hauari H, Grant R, Cumming J, et al. Exercise interventions and patient beliefs for people with chronic hip and knee pain: a mixed methods review. Cochrane Database of Systematic Reviews 2013, Issue 12. [DOI: 10.1002/14651858.CD010842]

\section{Husk 2016}

Husk KR, Lovell C, Cooper C, Stahl-Timmins W, Garside R. Participation in environmental enhancement and conservation activities for health and well-being in adults: a review of quantitative and qualitative evidence. Cochrane Database of Systematic Reviews 2016, Issue 5. [DOI: 10.1002/14651858.CD010351.pub2]

\section{Jackson 2017}

Jackson D, Turner R. Power analysis for random-effects metaanalysis. Research Synthesis Methods 2017;8:290-302.

\section{Juniper 1996}

Juniper EF, Guyatt GH, Feeny DH, Ferrie PJ, Griffith LE, Townsend M. Measuring quality of life in children with asthma. Quality of Life Research 1996;5(1):35-46. 


\section{Juniper 2006}

Juniper EF, Bousquet J, Abetz L, Bateman ED. Identifying 'well-controlled' and 'not well-controlled' asthma using the Asthma Control Questionnaire. Respiratory Medicine 2006;100(4):616-21.

\section{Kneale 2010}

Kneale D. Child health. In: Hansen K, Jones E, Joshi H, Budge D editor(s). Millennium Cohort Study: Fourth Survey: A User's Guide to Initial Findings. London: Centre for Longitudinal Studies, Institute of Education, 2010:177-210.

\section{Kneale 2015}

Kneale D, Thomas J, Harris K. Developing and optimising the use of logic models in systematic reviews: exploring practice and good practice in the use of programme theory in reviews. PloS one 2015;10:1-26.

\section{Kotses 2010}

Kotses H, Creer TL. Asthma self-management. Asthma, Health and Society. New York City: Springer, 2010:117-39.

\section{Kuehni 2002}

Kuehni CE, Frey U. Age-related differences in perceived asthma control in childhood: guidelines and reality. European Respiratory Journal 2002;20(4):880-9.

\section{Levy 2014}

Levy M, Andrews R, Buckingham R, Evans H, Francis C, Houston R, et al. Why asthma still kills: the National Review of Asthma Deaths (NRAD). Clinical Effectiveness and Evaluation Unit. London: Royal College of Physicians, 2014.

\section{Lewis 2012}

Lewis I, Lenehan C. Report of the children and young people's health outcomes forum. Report of the Long-term Conditions, Disabilities and Palliative Care Subgroup. London: Department of Health, 2012.

\section{Mallol 2013}

Mallol J, Crane J, von Mutius E, Odhiambo J, Keil U, Stewart A, et al. The International study of asthma and allergies in childhood (ISAAC) phase three: a global synthesis. Allergy and Immunopathology 2013;41(2):73-85.

\section{Miech 2015}

Miech E, Bravata DM, Woodward-Hagg H. Evaluating lean implementation: challenges in developing a research agenda for lean enterprise transformation in healthcare. General Internal Medicine and Geriatrics 2015;1:2172.

\section{Milton 2004}

Milton B, Whitehead M, Holland P, Hamilton V. The social and economic consequences of childhood asthma across the lifecourse: a systematic review. Child 2004;30(6):711-28.

\section{Moonie 2006}

Moonie SA, Sterling DA, Figgs L, Castro M. Asthma status and severity affects missed school days. Journal of School Health 2006;76(1):18-24.

\section{Moore 2015}

Moore GF, Audrey S, Barker M, Bond L, Bonnell C, Hardeman W, et al. Process evaluation of complex interventions: Medical Research Council guidance. BMJ 2015;350:h1258.

\section{Morrison 2012}

Morrison A, Polisena J, Husereau D, Moulton K, Clark M, Fiander M, et al. The effect of English-language restriction on systematic review-based meta-analyses: a systematic review of empirical studies. International Journal of Technology Assessment in Health Care 2012;28(2):138-44.

\section{Murta 2007}

Murta SG, Sanderson K, Oldenburg B. Process evaluation in occupational stress management programs: a systematic review. American Journal of Health Promotion 2007;21(4):248-54.

\section{National Institute of Health 1997}

National Institute of Health. Guidelines for the Diagnosis and Management of Asthma (EPR-3). http://www.nhlbi.nih.gov/ health-pro/guidelines/current/asthma-guidelines (accessed 6 February 2015).

\section{Netuveli 2005}

Netuveli G, Hurwitz B, Levy M, Fletcher M, Barnes G, Durham SR, et al. Ethnic variations in UK asthma frequency, morbidity, and health-service use: a systematic review and meta-analysis. Lancet 2005;365(9456):312-7.

\section{Neuzil 2000}

Neuzil, KM, Wright PF, Mitchel EF Jr, Griffin MR. The burden of influenza illness in children with asthma and other chronic medical conditions. Journal of Pediatrics 2000;137(6):856-64.

\section{NHS Scotland 2014}

NHS Scotland. Logic Models. http://www.healthscotland.com/ scotlands-health/planning/logic-models.aspx 2014.

\section{NICE 2017}

NICE. Asthma: diagnosis, monitoring and chronic asthma management: NICE guideline [NG80]. https://www.nice.org.uk/ guidance/NG80 (accessed prior to 12 November 2018).

\section{O'Mara-Eves 2013}

O'Mara-Eves A, Brunton G, McDaid G, Oliver S, Kavanagh J, Jamal F, et al. Community Engagement to Reduce Inequalities in Health: A Systematic Review, Meta-analysis and Economic Analysis. Southampton (UK): NIHR, 2013.

\section{Oakley 2006}

Oakley A, Strange V, Bonell C, Allen E, Stephenson J. Process evaluation in randomised controlled trials of complex interventions. BMJ (Clinical research ed.) 2006;332(7538):413-6.

\section{Piecoro 2001}

Piecoro LT, Potoski M, Talbert JC, Doherty DE. Asthma prevalence, cost, and adherence with expert guidelines on the utilization of health care services and costs in a state Medicaid population. Health Services Research 2001;36(2):357. 


\section{Pike 2018}

Pike KC, Harris KM, Kneale D. Interventions for autumn exacerbations of asthma in children. Cochrane Database of Systematic Reviews 2018, Issue 3. [DOI: 10.1002/14651858.CD012393]

\section{Pinnock 2015}

Pinnock H, Epiphaniou E, Pearce G, Parke H, Greenhalgh T, Sheikh A, et al. Implementing supported self-management for asthma: a systematic review and suggested hierarchy of evidence of implementation studies. BMC Medicine 2015;13:127.

\section{Ragin 2008}

Ragin CC. Redesigning Social Inquiry: Fuzzy Sets and Beyond. 240th. London, UK: Wiley Online Library, 2008.

\section{Ragin 2009}

Ragin CC. Qualitative comparative analysis using fuzzy sets (fsQCA). In: Rihoux B, Ragin CC editor(s). Configurational Comparative Methods: Qualitative Comparative Analysis (QCA) and Related Techniques. Thousand Oaks, CA: Sage, 2009.

\section{RevMan 2014 [Computer program]}

The Nordic Cochrane Centre, The Cochrane Collaboration. Review Manager (RevMan). Version 5.3. Copenhagen: The Nordic Cochrane Centre, The Cochrane Collaboration, 2014.

\section{Rihoux 2009}

Rihoux BE, De Meur G. Crisp-set qualitative comparative analysis (csQCA). In: Rihoux B, Ragin CC editor(s). Configurational Comparative Methods: Qualitative Comparative Analysis (QCA) and Related Techniques. Thousand Oaks, CA: Sage, 2009.

\section{Rodriguez 2013}

Rodriguez E, Rivera DA, Perlroth D, Becker E, Wang NE, Landau M. School nurses' role in asthma management, school absenteeism, and cost savings: a demonstration project. Journal of School Health 2013;83(12):842-50.

\section{Rosecrans 2008}

Rosecrans AM, Gittelsohn J, Ho LS, Harris SB, Naqshbandi M, Sharma S. Process evaluation of a multi-institutional community-based program for diabetes prevention among First Nations. Health Education Research 2008;23(2):272-86.

\section{Schellenberg 2012}

Schellenberg JA, Bobrova N, Avan BI. Measuring implementation strength: literature review draft report. ideas.Ishtm.ac.uk/wp-content/uploads/2017/08/IDEASMeasuring-implementation-strength-report.pdf (accessed prior to 19 January 2018).

\section{Schneider 2010}

Schneider CQ, Wagemann C. Standards of good practice in qualitative comparative analysis (QCA) and fuzzy-sets. Comparative Sociology 2010;9(3):397-418.

\section{Schwellnus 2013}

Schwellnus G. Eliminating the influence of irrelevant cases on the consistency and coverage of necessary and sufficient conditions in Fuzzy-Set QCA. ecpr.eu/Filestore/ PaperProposal/99d7fbea-b604-4251-847c-f8e33c82cb41.pdf (accessed prior to 19 January 2018).

\section{Shepherd 2010}

Shepherd J, Kavanagh J, Picot J, Cooper K, Harden A, BarnettPage $E$, et al. The effectiveness and cost-effectiveness of behavioural interventions for the prevention of sexually transmitted infections in young people aged 13-19: a systematic review and economic evaluation. Health Technology Assessment 2010;14(7):1-206.

\section{Singh 2012}

Singh HP, Shetty DC, Wadhwan V, Aggarwal P. A quantitative and qualitative comparative analysis of collagen fibers to determine the role of connective tissue stroma on biological behavior of odontogenic cysts: a histochemical study. National Journal of Maxillofacial Surgery 2012;3(1):15.

\section{Sinha 2012}

Sinha IP, Gallagher R, Williamson PR, Smyth RL. Development of a core outcome set for clinical trials in childhood asthma: a survey of clinicians, parents, and young people. Trials 2012;13(1):103.

\section{Smith 2005}

Smith JR, Mugford M, Holland R, Candy B, Noble MJ, Harrison BDW, et al. A systematic review to examine the impact of psycho-educational interventions on health outcomes and costs in adults and children with difficult asthma. Health Technology Assessment 2005;9(23):iii-iv, 1-167.

\section{Spencer 2012}

Spencer N, Thanh TM, Louise S. Low income/socioeconomic status in early childhood and physical health in later childhood/ adolescence: a systematic review. Maternal and Child Health 2012;17(3):424-31.

\section{StataCorp 2013 [Computer program]}

StataCorp. Stata. Version 15. College Station, TX, USA: StataCorp, 2017.

\section{Thiem 2012}

Thiem A, Dusa A. Qualitative Comparative Analysis With R: A User's Guide. 5th Edition. Berlin, Germany: Springer Science \& Business Media, 2012.

\section{Thiem 2013}

Thiem A, Dusa A. QCA: a package for qualitative comparative analysis. The $R$ Journal 2013;5(1):87-97.

\section{Thiem 2015}

Thiem A. Standards of good practice and the methodology of necessary conditions in qualitative comparative analysis: a critical view on Schneider and Wagemann's Theory-Guided/Enhanced Standard Analysis, COMPASSS Working Paper Series 2015-83. www.researchgate.net/ publication/283794175_Standards_of_Good_Practice_and_the_Methodolo \%27s_Theory-GuidedEnhanced_Standard_Analysis (accessed before 19 January 2018). 


\section{Thomas 2010 [Computer program]}

In: Thomas J, Brunton J, Graziosi S (eds). EPPI-Reviewer 4: Software for Research Synthesis. EPPI-Centre Software. Version 4.0. London: Social Science Research Unit, Institute of Education, 2010.

\section{Thomas 2011}

Thomas J, McNaught J, Ananiadou S. Applications of text mining within systematic reviews. Research Synthesis Methods 2011;2(1):1-14.

\section{Thomas 2014}

Thomas J, O'Mara-Eves A, Brunton G. Using qualitative comparative analysis (QCA) in systematic reviews of complex interventions: a worked example. Systematic Reviews 2014;3(1):1-14.

\section{Thomson 2013}

Thomson HJ, Thomas S. The effect direction plot: visual display of non-standardised effects across multiple outcome domains. Research Synthesis Methods 2013;4(1):95-101.

\section{To 2012}

To T, Stanojevic S, Moores G, Gershon AS, Bateman ED, Cruz AA, et al. Global asthma prevalence in adults: findings from the cross-sectional world health survey. BMC Public Health 2012;12(1):204.

\section{Van Den Bemt 2011}

van den Bemt L, Kooijman S, Linssen V, Lucassen P, Muris J, Slabbers $\mathrm{G}$, et al. How does asthma influence the daily life of children? Results of focus group interviews. Health and Quality of Life Outcomes 2011;8(1):5.

\section{Walsh 1999}

Walsh LJ, Wong CA, Cooper S, Guhan AR, Pringle M, Tattersfield AE. Morbidity from asthma in relation to regular treatment: a community based study. Thorax 1999;54(4):296-300.

\section{Waters 2006}

Waters E, Doyle J, Jackson N, Howes F, Brunton G, Oakley A, et al. Evaluating the effectiveness of public health interventions: the role and activities of the Cochrane Collaboration. Journal of Epidemiology and Community Health 2006; Vol. 60, issue 4:285-9.

\section{Welsh 2011}

Welsh E, Hasan M, Li P. Home-based educational interventions for children with asthma. Cochrane Database of Systematic Reviews 2011, Issue 10. [DOI: 10.1002/14651858.CD008469]

\section{Wildhaber 2012}

Wildhaber J, Carroll WD, Brand PLP. Global impact of asthma on children and adolescents' daily lives: the room to breathe survey. Pediatric Pulmonology 2012;47(4):346-57.

\section{Williamson 2012}

Williamson PR, Altman DG, Blazeby JM, Clarke M, Devane D, Gargon E, et al. Developing core outcome sets for clinical trials: issues to consider. Trials 2012;13(1):132.

\section{Wolf 2002}

Wolf FM, Guevara JP, Grum CM, Clark NM, Cates CJ. Educational interventions for asthma in children. Cochrane Database of Systematic Reviews 2002, Issue 4. [DOI: 10.1002/14651858.CD000326]

* Indicates the major publication for the study

\section{CHARACTERISTICS OF STUDIES}

Characteristics of included studies [ordered by study ID]

Methods Included as outcome evaluation

Study design: clustered parallel-group design with schools selected as the unit of randomisation

Setting: study was conducted in 4 public high schools in the Irbid region of northern Jordan - 2 schools randomised to intervention arm and 2 to control arm

Period: dates in which study was conducted - intervention and subsequent data collection - not clear

Participants

Eligible sample frame: 261 pupils found to be eligible

Randomised: 261 pupils randomised at the school level: 132 to the treatment group and 129 to the control group

Completed (intervention): 244 pupils completed the trial

Inclusion criteria: students were eligible for participation in the study if they had reported wheezing in the last 12 months as identified by the Arabic version of the ISAAC written questionnaire; were physical- 
ly and cognitively capable of completing the survey; were able to read and converse in both Arabic and English; and regularly attended school classes

Exclusion criteria: students with other diseases that could affect quality of life measures or who were concurrently involved in another health-related study were not eligible

\section{Baseline characteristics}

Age of children: exact age not given; all children in years 8, 9, and 10 (usually 12 to 15 years old)

Ethnicity: not reported

Socio-economic status: not reported

Gender: 113 female participants (43.3\%); note intervention and control took place in single-sex schools ( 1 of each in treatment and control arms)

Asthma status: 184 students (70.5\%) had a formal asthma diagnosis; 87 students (33.3\%) reported use of reliever medication; and 57 students $(21.8 \%)$ reported use of preventer medication

Interventions

Intervention: bilingual health workers trained peer leaders from year 11 to deliver 3 Triple-A lessons. The content of Triple-A is not described here, but typical topics in Triple-A include basic information on asthma, its triggers, and management; and barriers to optimal asthma management, including risktaking behaviours such as smoking (see earlier description of Triple A provided in Gibson 1998)

Control: not clearly stated (usual care)

Intensity: target asthmatic students received 3 lessons from peer leaders (year 11 students)

Instructor: peers

Theoretical framework: theories involving self-efficacy underpinned the intervention

Parental engagement: not reported

Child satisfaction: not reported

Timing of intervention in school day: not reported

Extractable outcomes were collected for:
Health-related quality of life (measured through the Paediatric Asthma Quality of Life Questionnaire)
Withdrawal

Notes

This intervention tests a model of asthma self-management education developed elsewhere, although modifications to the intervention are not fully described

Considered as a process evaluation but excluded as did not seek to address process evaluation research questions

Funding source: Jordan University of Science and Technology (Irbid, Jordon) and Nursing Council

\section{Risk of bias}

\begin{tabular}{lll}
\hline Bias & Authors' judgement & Support for judgement \\
\hline $\begin{array}{l}\text { Random sequence genera- } \\
\text { tion (selection bias) }\end{array}$ & Low risk & $\begin{array}{l}\text { Low risk - closed-envelope technique used to select initial schools that were } \\
\text { stratified by gender }\end{array}$ \\
\hline $\begin{array}{l}\text { Allocation concealment } \\
\text { (selection bias) }\end{array}$ & High risk & $\begin{array}{l}\text { Closed envelope, although no further details were provided and only a small } \\
\text { number of schools (4) were involved, potentially compromising the conceal- } \\
\text { ment of allocation }\end{array}$ \\
\hline
\end{tabular}


Al-Sheyab 2012 (Continued)

Blinding of participants Unclear risk No further details were given around blinding of personnel and participants and personnel (performance bias)

All outcomes

\begin{tabular}{lll}
\hline $\begin{array}{l}\text { Blinding of outcome as- } \\
\text { sessment (detection bias) } \\
\text { All outcomes }\end{array}$ & Unclear risk & No further details were given around blinding of outcome assessment \\
\hline $\begin{array}{l}\text { Incomplete outcome data } \\
\text { (attrition bias) } \\
\text { All outcomes }\end{array}$ & Low risk & Low levels of attrition, roughly spread across intervention and control arms \\
\hline $\begin{array}{l}\text { Selective reporting (re- } \\
\text { porting bias) }\end{array}$ & Low risk & All stated outcomes were reported upon \\
\hline $\begin{array}{l}\text { Other bias } \\
\text { Unclear risk }\end{array}$ & $\begin{array}{l}\text { Missingness - low risk - low levels of missingness } \\
\text { Baseline imbalance - high risk - differences in asthma reliever therapies at } \\
\text { baseline between groups } \\
\text { Risk of contamination - low - school-based randomisation minimises the po- } \\
\text { tential for contamination between intervention and control groups }\end{array}$
\end{tabular}

Transparent and clearly Unclear risk N/A
stated aims

Explicit theories underpin- Unclear risk N/A
ning and/or literature review

\begin{tabular}{lll}
\hline $\begin{array}{l}\text { Transparent and clearly } \\
\text { stated methods and tools }\end{array}$ & Unclear risk & N/A \\
\hline Selective reporting & Unclear risk & N/A \\
\hline Harmful effects & Unclear risk & N/A \\
\hline $\begin{array}{l}\text { Population and sample } \\
\text { described well }\end{array}$ & Unclear risk & N/A \\
\hline $\begin{array}{l}\text { Continuous evaluation } \\
\text { Evaluation participation } \\
\text { equity and sampling }\end{array}$ & Unclear risk & N/A \\
\hline $\begin{array}{l}\text { Design and methods over- } \\
\text { all approach }\end{array}$ & Unclear risk & N/A \\
\hline $\begin{array}{l}\text { Tools and methods of da- } \\
\text { ta collection reliable/cred- } \\
\text { ible }\end{array}$ & Unclear risk & N/A \\
\hline $\begin{array}{l}\text { Tools and methods of data } \\
\text { analysis reliable/credible }\end{array}$ & Unclear risk & N/A \\
\hline
\end{tabular}


Al-Sheyab 2012 (Continued)

Performance bias/neutral- Unclear risk N/A ity/credibility/conformability

\begin{tabular}{lll}
\hline $\begin{array}{l}\text { Reliability of findings and } \\
\text { recommendations }\end{array}$ & Unclear risk & N/A \\
\hline Transferability of findings & Unclear risk & N/A \\
\hline $\begin{array}{l}\text { Overall risk of bias of } \\
\text { process evaluation }\end{array}$ & Unclear risk & N/A \\
\hline
\end{tabular}

Al-Sheyab 2012a

$\begin{array}{ll}\text { Methods } & \text { Included as process evaluation } \\ & \text { Intervention study design: non-experimental design with post-test only evaluation of feasibility } \\ \text { among intervention groups }\end{array}$

Unit of allocation: N/A

Process evaluation methods: surveys and focus groups among key stakeholders

Setting: a private girl's high school in Jordan
Age of children: students in years 7 to 11 received the intervention delivered by children in years 10
and 11
and 11

Child characteristics (BME/SES): no information

Asthma status: no information

Intervention recipients: children only

Interventions School type: high school (private girl's high school)

Intervention description: study authors report that the Adolescent Asthma Action (Triple A) programme uses a 3-step cascade process from senior to junior students to deliver asthma education and has well-developed resources, including standardised training manuals, educational videos, asthma-related models and devices, and first aid kits. Trained health workers provide initial training for peer leaders and facilitate the steps of the programme. Programme content covers management of asthma exacerbations, resisting pressure to smoke, and asthma medication and triggers. Programme delivery occurs through interactive teaching and learning activities, including role-play and group discussion, all of which are said to be more effective than traditional didactic education for adolescents

Control description: N/A - feasibility study with no control group

Theoretical framework: study authors report that Triple A is grounded in universally applicable theoretical concepts including peer leadership, self-efficacy, and empowerment, suggesting its potential for use in different cultural contexts

Outcomes

Core processes evaluated (child level): the intervention explores child satisfaction in-depth, with the intervention ostensibly implemented as intended

Notes Process evaluation category: stand-alone; core process questions were central

Breadth and depth: neither broad nor deep 
Voice of children given prominence: featured but not sufficiently; unclear about the extent to which children were able to express negative views of the intervention

Funding source: not reported

\section{Risk of bias}

\begin{tabular}{|c|c|c|}
\hline Bias & Authors' judgement & Support for judgement \\
\hline $\begin{array}{l}\text { Random sequence genera- } \\
\text { tion (selection bias) }\end{array}$ & Unclear risk & $\mathrm{N} / \mathrm{A}$ \\
\hline $\begin{array}{l}\text { Allocation concealment } \\
\text { (selection bias) }\end{array}$ & Unclear risk & $\mathrm{N} / \mathrm{A}$ \\
\hline $\begin{array}{l}\text { Blinding of participants } \\
\text { and personnel (perfor- } \\
\text { mance bias) } \\
\text { All outcomes }\end{array}$ & Unclear risk & $\mathrm{N} / \mathrm{A}$ \\
\hline $\begin{array}{l}\text { Blinding of outcome as- } \\
\text { sessment (detection bias) } \\
\text { All outcomes }\end{array}$ & Unclear risk & $\mathrm{N} / \mathrm{A}$ \\
\hline $\begin{array}{l}\text { Incomplete outcome data } \\
\text { (attrition bias) } \\
\text { All outcomes }\end{array}$ & Unclear risk & $\mathrm{N} / \mathrm{A}$ \\
\hline $\begin{array}{l}\text { Selective reporting (re- } \\
\text { porting bias) }\end{array}$ & Unclear risk & $\mathrm{N} / \mathrm{A}$ \\
\hline Other bias & Unclear risk & $\mathrm{N} / \mathrm{A}$ \\
\hline $\begin{array}{l}\text { Transparent and clearly } \\
\text { stated aims }\end{array}$ & Low risk & Study aims were clearly reported \\
\hline $\begin{array}{l}\text { Explicit theories underpin- } \\
\text { ning and/or literature re- } \\
\text { view }\end{array}$ & Unclear risk & $\begin{array}{l}\text { Literature was sufficient to support the direction of the intervention, but a spe- } \\
\text { cific theory was not named to provide evidence of a sound theoretical basis }\end{array}$ \\
\hline $\begin{array}{l}\text { Transparent and clearly } \\
\text { stated methods and tools }\end{array}$ & Unclear risk & $\begin{array}{l}\text { Data collection methods and tools were reported; however data analysis } \\
\text { methods are unclear }\end{array}$ \\
\hline Selective reporting & High risk & $\begin{array}{l}\text { Absence of outcome data (e.g. asthma-related emergencies) directly related to } \\
\text { the aims of the programme }\end{array}$ \\
\hline Harmful effects & High risk & No evidence that any harmful effects were considered \\
\hline $\begin{array}{l}\text { Population and sample } \\
\text { described well }\end{array}$ & High risk & $\begin{array}{l}\text { Difficulty in distinguishing between numbers involved in the intervention and } \\
\text { numbers involved in the process evaluation }\end{array}$ \\
\hline Continuous evaluation & High risk & Data collected only post intervention \\
\hline $\begin{array}{l}\text { Evaluation participation } \\
\text { equity and sampling }\end{array}$ & Unclear risk & $\begin{array}{l}\text { Although the voice of young people was given prominence, it is unclear } \\
\text { whether intervention sessions required school lessons to be moved, and how } \\
\text { teachers felt about this }\end{array}$ \\
\hline
\end{tabular}


Al-Sheyab 2012a (Continued)

Design and methods over- High risk Description of research design and methods was limited, particularly with reall approach gards to the analysis, as study authors stated that this was beyond the scope of the study

$\begin{array}{ll}\begin{array}{l}\text { Tools and methods of da- } \\ \text { ta collection reliable/cred- } \\ \text { ible }\end{array} & \text { Low risk }\end{array}$

Tools and methods of data High risk analysis reliable/credible
Study authors did not report on an analytical framework and did not describe the methods used for data analysis

Performance bias/neutral- Unclear risk Unclear whether this was addressed during the study

ity/credibility/conforma-

bility

\section{Reliability of findings and High risk} recommendations

Transferability of findings High risk

Study authors considered this to be a feasibility study, and the paper suggests that it was conducted successfully. However, the data presented do not support this in all instances
Study authors acknowledged that findings were limited in transferability, as the sample was derived from a single private girl's school, where English was not studied extensively

\begin{tabular}{l}
$\begin{array}{l}\text { Overall risk of bias of } \\
\text { process evaluation }\end{array}$ \\
\hline
\end{tabular}

Atherly 2009

Methods

\section{Included as outcome evaluation}

Study design: clustered parallel-group RCT

Setting: junior high and high schools

Period: 2003-2004 school year

\section{Participants}

Eligible sample frame: not reported

Randomised: numbers for these data are disaggregated. The study included 524 children: 458 children randomised at the school level: 225 to the intervention group; and 233 to the control group

\section{Completed (intervention): 458}

Inclusion criteria: not reported

Exclusion criteria: not reported

\section{Baseline characteristics}

Age of children: mean age, 13.9 in the intervention group; 13.4 in the control group

Ethnicity: not reported

Socio-economic status: not reported

Gender: $46.6 \%$ female in the intervention group; $50.7 \%$ female in the control group

Asthma status: asthmatic children only 
Atherly 2009 (Continued)

Interventions

Intervention: the Power Breathing programme focused on education about asthma, asthma control strategies, and psychosocial concerns

Control: not reported

Intensity: three 90-minute educational sessions

Instructor: teachers and school nurses were involved in the intervention; however their role is unclear

Theoretical framework: not reported

Parental engagement: not reported

Child satisfaction: not reported

Timing of intervention in school day: not reported

\begin{tabular}{|c|c|}
\hline \multirow[t]{4}{*}{ Outcomes } & Extractable outcomes were collected for: \\
\hline & Exacerbations leading to admission to hospital \\
\hline & Asthma symptoms leading to an emergency hospital visit \\
\hline & Experience of daytime and night-time symptoms \\
\hline \multirow[t]{3}{*}{ Notes } & Study presented an economic evaluation of the intervention \\
\hline & $\begin{array}{l}\text { Considered for inclusion as a process evaluation but not deemed to fulfil the criteria of a process evalu- } \\
\text { ation }\end{array}$ \\
\hline & Funding source: not reported \\
\hline
\end{tabular}

\section{Risk of bias}

\begin{tabular}{lll}
\hline Bias & Authors' judgement & Support for judgement \\
\hline $\begin{array}{l}\text { Random sequence genera- } \\
\text { tion (selection bias) }\end{array}$ & Unclear risk & $\begin{array}{l}\text { No detail provided on random assignment procedures } \\
\text { "The schools were then randomly assigned to the intervention or control } \\
\text { group" }\end{array}$ \\
\hline $\begin{array}{l}\text { Allocation concealment } \\
\text { (selection bias) }\end{array}$ & Unclear risk & Not addressed by study authors \\
\hline $\begin{array}{l}\text { Blinding of participants } \\
\text { and personnel (perfor- } \\
\text { mance bias) } \\
\text { All outcomes }\end{array}$ & Unclear risk & Not addressed by study authors \\
\hline $\begin{array}{l}\text { Blinding of outcome as- } \\
\text { sessment (detection bias) }\end{array}$ & Unclear risk & $\begin{array}{l}\text { Not addressed by study authors } \\
\text { All outcomes }\end{array}$ \\
\hline $\begin{array}{l}\text { Incomplete outcome data } \\
\text { (attrition bias) } \\
\text { All outcomes }\end{array}$ & High risk & $\begin{array}{l}\text { Although attrition was relatively low overall, the study did not provide details } \\
\text { of the spread of attrition across arms and was deemed at high risk of bias }\end{array}$ \\
$\begin{array}{l}\text { "The study included 524 adolescents in grades 6-12 from middle and high } \\
\text { schools. Surveys were administered at baseline, immediately postintervention } \\
\text { and three months post-intervention. A total of 458 children completed all sur- } \\
\text { veys, including 225 in the intervention group and 233 in the control group" }\end{array}$ \\
\hline
\end{tabular}

School-based self-management interventions for asthma in children and adolescents: a mixed methods systematic review (Review) 
Atherly 2009 (Continued)

Selective reporting (re- Low risk No evidence of selective reporting
porting bias)

\begin{tabular}{ll}
\hline Other bias $\quad$ High risk & $\begin{array}{l}\text { Missingness - low risk - besides attrition, no additional missing data were re- } \\
\text { ported }\end{array}$
\end{tabular}

Baseline imbalance - high risk - indications showed poorer asthma control at baseline in the control group (e.g. higher level of ED visits)

Risk of contamination - low - randomisation occurred at a school level

\begin{tabular}{|c|c|c|}
\hline $\begin{array}{l}\text { Transparent and clearly } \\
\text { stated aims }\end{array}$ & Unclear risk & $\mathrm{N} / \mathrm{A}$ \\
\hline $\begin{array}{l}\text { Explicit theories underpin- } \\
\text { ning and/or literature re- } \\
\text { view }\end{array}$ & Unclear risk & $\mathrm{N} / \mathrm{A}$ \\
\hline $\begin{array}{l}\text { Transparent and clearly } \\
\text { stated methods and tools }\end{array}$ & Unclear risk & $\mathrm{N} / \mathrm{A}$ \\
\hline Selective reporting & Unclear risk & $\mathrm{N} / \mathrm{A}$ \\
\hline Harmful effects & Unclear risk & $\mathrm{N} / \mathrm{A}$ \\
\hline $\begin{array}{l}\text { Population and sample } \\
\text { described well }\end{array}$ & Unclear risk & $\mathrm{N} / \mathrm{A}$ \\
\hline Continuous evaluation & Unclear risk & $\mathrm{N} / \mathrm{A}$ \\
\hline $\begin{array}{l}\text { Evaluation participation } \\
\text { equity and sampling }\end{array}$ & Unclear risk & $\mathrm{N} / \mathrm{A}$ \\
\hline $\begin{array}{l}\text { Design and methods over- } \\
\text { all approach }\end{array}$ & Unclear risk & $\mathrm{N} / \mathrm{A}$ \\
\hline $\begin{array}{l}\text { Tools and methods of da- } \\
\text { ta collection reliable/cred- } \\
\text { ible }\end{array}$ & Unclear risk & $\mathrm{N} / \mathrm{A}$ \\
\hline $\begin{array}{l}\text { Tools and methods of data } \\
\text { analysis reliable/credible }\end{array}$ & Unclear risk & $\mathrm{N} / \mathrm{A}$ \\
\hline $\begin{array}{l}\text { Performance bias/neutral- } \\
\text { ity/credibility/conforma- } \\
\text { bility }\end{array}$ & Unclear risk & $\mathrm{N} / \mathrm{A}$ \\
\hline $\begin{array}{l}\text { Reliability of findings and } \\
\text { recommendations }\end{array}$ & Unclear risk & $\mathrm{N} / \mathrm{A}$ \\
\hline Transferability of findings & Unclear risk & $\mathrm{N} / \mathrm{A}$ \\
\hline $\begin{array}{l}\text { Overall risk of bias of } \\
\text { process evaluation }\end{array}$ & Unclear risk & $\mathrm{N} / \mathrm{A}$ \\
\hline
\end{tabular}




\section{Methods}

\section{Included as outcome evaluation}

Study design: clustered parallel-group design with schools selected as the unit of randomisation

Setting: study was conducted across inner city elementary schools in Texas, USA. All schools from a single district were invited to participate, with 84 agreeing to participate. 60 schools were selected because two-thirds of students were in receipt of free school meals

Period: study conducted and data collected from 1997 to 2000

Participants

Eligible sample frame: 982 pupils eligible and consented

Randomised: 946 pupils randomised at school level: 515 into treatment group and 431 into control group

Completed (intervention): 503 pupils were available at follow-up; 16 had actively withdrawn, 325 were lost to follow-up, and 102 had graduated

Inclusion criteria: not clearly stated - case detection procedure implemented to discover asthmatic students

Exclusion criteria: not clearly stated at child level (school-level criteria included that schools would have two-thirds of pupils in receipt of free school meals)

\section{Baseline characteristics}

Age of children: based on reports from $88.3 \%$ of pupils: mean age 7.7 years

Ethnicity: based on reports from $88.3 \%$ of pupils: $45 \%$ of children were African American, $51 \%$ Hispanic, $3 \%$ Caucasian, and $1 \%$ from other ethnic groups

Socio-economic status: most students were from households with incomes $<\$ 20,000$ per year; $28 \%$ reported incomes $<\$ 9999$

Gender: based on reports from $88.3 \%$ of pupils: 400 girls ( $47.9 \%)$ and 435 boys $(52.1 \%)$

Asthma status: indicators of asthma severity not provided

Intervention: multi-component intervention involving direct delivery to children, care providers, and parents/guardians. Children received self-management education through the "Watch, Discover, Think, and Act" interactive computer programme, which was based on National Institutes of Health guidelines and pedagogical and self-management theories. Children participated in the intervention for approximately 1 year, during which time they played the computer game and their parents received training materials on managing asthma. School nurses received training to improve communication with community primary care providers and to encourage greater monitoring of children's asthma status. Children with persistent asthma in 15 schools received an enhanced intervention, which involved meetings with a project physician to develop an Asthma Action Plan and receipt of a month's supply of medication, with the plan sent to the child's community primary care provider. Researchers also assessed the quality of the school environment with regards to asthma triggers in intervention schools and communicated these findings with recommendations to schools

Control: not clearly stated (usual care)

Intensity: not clearly described: children were involved in the intervention over the course of a year, and all children completed all levels of the intervention computer programme, although patterns and intensity of usage were not described

Instructor: main standardised instruction provided through a computer programme; other components involved school nurses

Theoretical framework: a logic model was provided and the computer programme was reported as being based on social cognitive theory 
Bartholomew 2006 (Continued)

Parental engagement: not reported directly. Problems reported in gaining consent: "only about $64 \%$ of parents returned case detection surveys, and about half of the families of children with probable asthma agreed to participate in the study"

Child satisfaction: not reported

Timing of intervention in school day: not reported

\begin{tabular}{ll}
\hline Outcomes & Withdrawal from study \\
\hline Notes & $\begin{array}{l}\text { Several primary outcomes of interest were collected but were not disaggregated by treatment status } \\
\text { (exacerbations leading to admission to hospital and absence from school) or were not presented in the } \\
\text { results (asthma symptoms leading to emergency department visits) }\end{array}$ \\
& $\begin{array}{l}\text { Also considered for possible inclusion as a process evaluation - while the study purports to include a } \\
\text { process evaluation section, this was not deemed to include the core components of a process evalua- } \\
\text { tion (i.e. at least a partial focus on implementation outcomes and the relationship with context) }\end{array}$
\end{tabular}

Funding source: National Heart, Lung, and Blood Institute, National Institutes of Health

\section{Risk of bias}

\begin{tabular}{lll} 
Bias & Authors' judgement & Support for judgement \\
\hline $\begin{array}{l}\text { Random sequence genera- } \\
\text { tion (selection bias) }\end{array}$ & Unclear risk & $\begin{array}{l}\text { No information was provided on how randomisation occurred, except schools } \\
\text { were randomly assigned to treatment and control groups }\end{array}$ \\
\hline $\begin{array}{l}\text { Allocation concealment } \\
\text { (selection bias) }\end{array}$ & Unclear risk & $\begin{array}{l}\text { No information was provided on how allocation of schools occurred, except } \\
\text { that schools were randomly assigned to treatment and control groups }\end{array}$ \\
$\begin{array}{l}\text { Also little detail on how schools were additionally allocated to enhanced inter- } \\
\text { vention: in 15 schools, an enhanced intervention allowed children and their } \\
\text { parents to meet with a project physician, develop an asthma action plan, and } \\
\text { receive a 1-month supply of medication; the project physician then followed } \\
\text { up with the child's community physician }\end{array}$
\end{tabular}

\begin{tabular}{|c|c|c|}
\hline $\begin{array}{l}\text { Blinding of participants } \\
\text { and personnel (perfor- } \\
\text { mance bias) }\end{array}$ & Unclear risk & $\begin{array}{l}\text { No measures to limit performance and detection biases were described (not } \\
\text { considered by trial authors) }\end{array}$ \\
\hline
\end{tabular}

mance bias)

All outcomes

\begin{tabular}{lll}
\hline Blinding of outcome as- & Unclear risk & $\begin{array}{l}\text { No measures to blind outcome assessment were described (not considered by } \\
\text { trial authors) }\end{array}$ \\
All outcomes &
\end{tabular}

\begin{tabular}{|c|c|c|}
\hline $\begin{array}{l}\text { Incomplete outcome data } \\
\text { (attrition bias) } \\
\text { All outcomes }\end{array}$ & High risk & $\begin{array}{l}\text { Very high levels of attrition were noted in both outcome and control groups: } \\
43 \% \text { in control and } 49.5 \% \text { in treatment at post test. Some evidence provided } \\
\text { by study authors indicated that "attrition did not create any significant group } \\
\text { differences in the variables measured in the study sample" }\end{array}$ \\
\hline
\end{tabular}

\begin{tabular}{|c|c|c|}
\hline $\begin{array}{l}\text { Selective reporting (re- } \\
\text { porting bias) }\end{array}$ & High risk & $\begin{array}{l}\text { Some outcomes were not reported or results were not disaggregated by inter- } \\
\text { vention and control (e.g. levels of hospitalisation) }\end{array}$ \\
\hline
\end{tabular}

Other bias High risk

\begin{abstract}
Missingness - children were not tracked from school to school because of problems with treatment and control group migration. Around $10 \%$ of children had missing data at baseline or were not considered to have "usable data", and no imputation strategies were described
\end{abstract}

Baseline imbalance - no data on baseline demographic characteristics were given to illuminate the split between control and intervention groups. No dif- 
ferences between groups were found in health status variables, school performance, attendance, or levels of environmental allergens in schools

Risk of contamination - low - randomisation occurred at the school level

\begin{tabular}{|c|c|c|}
\hline $\begin{array}{l}\text { Transparent and clearly } \\
\text { stated aims }\end{array}$ & Unclear risk & $\mathrm{N} / \mathrm{A}$ \\
\hline $\begin{array}{l}\text { Explicit theories underpin- } \\
\text { ning and/or literature re- } \\
\text { view }\end{array}$ & Unclear risk & $\mathrm{N} / \mathrm{A}$ \\
\hline $\begin{array}{l}\text { Transparent and clearly } \\
\text { stated methods and tools }\end{array}$ & Unclear risk & $\mathrm{N} / \mathrm{A}$ \\
\hline Selective reporting & Unclear risk & $\mathrm{N} / \mathrm{A}$ \\
\hline Harmful effects & Unclear risk & $\mathrm{N} / \mathrm{A}$ \\
\hline $\begin{array}{l}\text { Population and sample } \\
\text { described well }\end{array}$ & Unclear risk & $\mathrm{N} / \mathrm{A}$ \\
\hline Continuous evaluation & Unclear risk & $\mathrm{N} / \mathrm{A}$ \\
\hline $\begin{array}{l}\text { Evaluation participation } \\
\text { equity and sampling }\end{array}$ & Unclear risk & $\mathrm{N} / \mathrm{A}$ \\
\hline $\begin{array}{l}\text { Design and methods over- } \\
\text { all approach }\end{array}$ & Unclear risk & $\mathrm{N} / \mathrm{A}$ \\
\hline $\begin{array}{l}\text { Tools and methods of da- } \\
\text { ta collection reliable/cred- } \\
\text { ible }\end{array}$ & Unclear risk & N/A \\
\hline $\begin{array}{l}\text { Tools and methods of data } \\
\text { analysis reliable/credible }\end{array}$ & Unclear risk & $\mathrm{N} / \mathrm{A}$ \\
\hline $\begin{array}{l}\text { Performance bias/neutral- } \\
\text { ity/credibility/conforma- } \\
\text { bility }\end{array}$ & Unclear risk & $\mathrm{N} / \mathrm{A}$ \\
\hline $\begin{array}{l}\text { Reliability of findings and } \\
\text { recommendations }\end{array}$ & Unclear risk & $\mathrm{N} / \mathrm{A}$ \\
\hline Transferability of findings & Unclear risk & $\mathrm{N} / \mathrm{A}$ \\
\hline $\begin{array}{l}\text { Overall risk of bias of } \\
\text { process evaluation }\end{array}$ & Unclear risk & $\mathrm{N} / \mathrm{A}$ \\
\hline
\end{tabular}

Berg 2004

$\begin{array}{ll}\text { Methods } & \text { Included as process evaluation } \\ \text { Intervention study design: quasi-experimental, post test } \\ \text { Unit of allocation: not reported }\end{array}$


Process evaluation methods: descriptive/bivariate (surveys), thematic/grounded theory

\begin{tabular}{ll}
\hline Country: USA \\
Age of children: 15 to 18 years \\
Child characteristics (BME/SES): $46.2 \%$ were of African American ethnicity. The sample was $69.2 \%$ fe- \\
male; SES information was not reported \\
Asthma status: asthmatic only \\
Intervention recipients: children only \\
School type: intervention was implemented in 1 high school \\
Intervention description: the Power Breathing programme consisted of educational sessions in which \\
the children met as a group and were instructed on aspects of asthma management, including triggers, \\
symptoms, and causes
\end{tabular}

Control description: not reported

Theoretical framework: intervention was grounded in social learning theory

Outcomes

Core processes evaluated (child level): attrition and adherence were not problematic; information on dosage was not reported

\section{Notes}

\section{Process evaluation category: integrated}

Breadth and depth: neither broad nor deep

Voice of children given prominence: sufficient coverage

Funding source: intramural grant from the University of California, Los Angeles, School of Nursing

\section{Risk of bias}

\begin{tabular}{lll}
\hline Bias & Authors' judgement & Support for judgement \\
\hline $\begin{array}{l}\text { Random sequence genera- } \\
\text { tion (selection bias) }\end{array}$ & Unclear risk & $\mathrm{N} / \mathrm{A}$ \\
\hline $\begin{array}{l}\text { Allocation concealment } \\
\text { (selection bias) }\end{array}$ & Unclear risk & $\mathrm{N} / \mathrm{A}$ \\
\hline $\begin{array}{l}\text { Blinding of participants } \\
\text { and personnel (perfor- } \\
\text { mance bias) }\end{array}$ & Unclear risk & $\mathrm{N} / \mathrm{A}$ \\
All outcomes & \\
\hline
\end{tabular}

Blinding of outcome as-
sessment (detection bias) $\quad$ Unclear risk N/A

\begin{tabular}{lll}
\hline $\begin{array}{l}\text { Incomplete outcome data } \\
\text { (attrition bias) }\end{array}$ & Unclear risk \\
$\begin{array}{l}\text { All outcomes } \\
\text { Selective reporting (re- } \\
\text { porting bias) }\end{array}$ & Unclear risk & N/A \\
\hline Other bias & Unclear risk & N/A
\end{tabular}


Berg 2004 (Continued)

Transparent and clearly Low risk $\quad$ Study aims were clearly reported
stated aims

\begin{tabular}{|c|c|c|}
\hline $\begin{array}{l}\text { Explicit theories underpin- } \\
\text { ning and/or literature re- } \\
\text { view }\end{array}$ & Low risk & $\begin{array}{l}\text { Theoretical framework guiding the intervention was described as social learn- } \\
\text { ing theory }\end{array}$ \\
\hline $\begin{array}{l}\text { Transparent and clearly } \\
\text { stated methods and tools }\end{array}$ & Low risk & Process of collecting data and the tools used were well described \\
\hline Selective reporting & Low risk & All results of the intervention appear to be documented \\
\hline Harmful effects & Unclear risk & Unclear how much difficulty was involved in engaging with parents \\
\hline $\begin{array}{l}\text { Population and sample } \\
\text { described well }\end{array}$ & Unclear risk & $\begin{array}{l}\text { Population was generally described well; however the severity of asthma } \\
\text { among participants was not reported }\end{array}$ \\
\hline Continuous evaluation & High risk & Data were collected only post intervention \\
\hline $\begin{array}{l}\text { Evaluation participation } \\
\text { equity and sampling }\end{array}$ & Low risk & $\begin{array}{l}\text { All students who participated in the pilot were also included in the process } \\
\text { evaluation }\end{array}$ \\
\hline $\begin{array}{l}\text { Design and methods over- } \\
\text { all approach }\end{array}$ & Unclear risk & Some of the indicators in Table 3 are unconvincing \\
\hline $\begin{array}{l}\text { Tools and methods of da- } \\
\text { ta collection reliable/cred- } \\
\text { ible }\end{array}$ & Low risk & Tools and methods used for data collection were reported on \\
\hline $\begin{array}{l}\text { Tools and methods of data } \\
\text { analysis reliable/credible }\end{array}$ & Low risk & Methods of data analysis were appropriate for the data \\
\hline $\begin{array}{l}\text { Performance bias/neutral- } \\
\text { ity/credibility/conforma- } \\
\text { bility }\end{array}$ & Unclear risk & $\begin{array}{l}\text { Unclear what effect negative cases had on outcomes. Analysis of those who } \\
\text { consented vs those who did not would have been helpful }\end{array}$ \\
\hline $\begin{array}{l}\text { Reliability of findings and } \\
\text { recommendations }\end{array}$ & High risk & $\begin{array}{l}\text { Sample size was too small to allow for too much endorsement of study find- } \\
\text { ings }\end{array}$ \\
\hline Transferability of findings & High risk & $\begin{array}{l}\text { Small sample size limits the transferability of findings. The profile of partici- } \\
\text { pating students is noticeably different from the profile of the school overall }\end{array}$ \\
\hline $\begin{array}{l}\text { Overall risk of bias of } \\
\text { process evaluation }\end{array}$ & Unclear risk & $\begin{array}{l}\text { Not much evidence to determine low risk; however evidence is sufficient for a } \\
\text { process evaluation of a feasibility study }\end{array}$ \\
\hline
\end{tabular}

Bignall 2015

Methods Included as process evaluation

Intervention study design: randomised controlled trial

Unit of allocation: child

Process evaluation methods: descriptive/bivariate (quantitative), descriptive (qualitative)

Participants Setting: single high school in a midwestern city in the USA


Age of children: 12 to 17 years (mean age, 15.47 years)

Child characteristics (BME/SES): African American

Asthma status: asthmatic only

Intervention recipients: children

Intervention description: 2 short instructional sessions for children on relaxation/breathing retraining techniques. Participants completed 2 in-person visits spaced 1 month apart and were given a copy of the script and a CD with breathing retraining techniques to help them practise at home

Control description: participants in the control group received 30 minutes of standard asthma education

Theoretical framework: no specific framework was named (although supporting literature around breathing exercises was provided)

\begin{tabular}{|c|c|c|}
\hline Outcomes & \multicolumn{2}{|c|}{ Core processes evaluated (child level): attrition, dosage, adherence } \\
\hline \multirow[t]{5}{*}{ Notes } & \multicolumn{2}{|c|}{ Process evaluation category: named section(s) on processes included } \\
\hline & \multicolumn{2}{|c|}{ Breadth and depth: neither broad nor deep } \\
\hline & \multicolumn{2}{|c|}{ Voice of children given prominence: featured but not sufficiently } \\
\hline & \multicolumn{2}{|c|}{ Note: not included as outcome evaluation because control received asthma education } \\
\hline & \multicolumn{2}{|c|}{ Funding source: not reported } \\
\hline \multicolumn{3}{|l|}{ Risk of bias } \\
\hline Bias & Authors' judgement & Support for judgement \\
\hline $\begin{array}{l}\text { Random sequence genera- } \\
\text { tion (selection bias) }\end{array}$ & Unclear risk & $\mathrm{N} / \mathrm{A}$ \\
\hline $\begin{array}{l}\text { Allocation concealment } \\
\text { (selection bias) }\end{array}$ & Unclear risk & N/A \\
\hline $\begin{array}{l}\text { Blinding of participants } \\
\text { and personnel (perfor- } \\
\text { mance bias) } \\
\text { All outcomes }\end{array}$ & Unclear risk & $\mathrm{N} / \mathrm{A}$ \\
\hline $\begin{array}{l}\text { Blinding of outcome as- } \\
\text { sessment (detection bias) } \\
\text { All outcomes }\end{array}$ & Unclear risk & N/A \\
\hline $\begin{array}{l}\text { Incomplete outcome data } \\
\text { (attrition bias) } \\
\text { All outcomes }\end{array}$ & Unclear risk & $\mathrm{N} / \mathrm{A}$ \\
\hline $\begin{array}{l}\text { Selective reporting (re- } \\
\text { porting bias) }\end{array}$ & Unclear risk & N/A \\
\hline Other bias & Unclear risk & $\mathrm{N} / \mathrm{A}$ \\
\hline
\end{tabular}


Bignall 2015 (Continued)

Transparent and clearly Low risk Study aims were clearly stated
stated aims

Explicit theories underpin- High risk No named theoretical framework was presented
ning and/or literature re-
view

\begin{tabular}{|c|c|c|}
\hline $\begin{array}{l}\text { Transparent and clearly } \\
\text { stated methods and tools }\end{array}$ & Unclear risk & $\begin{array}{l}\text { Although methods and tools were clearly described, it is unclear who delivered } \\
\text { the intervention. However, tools used and the content of interviews were well } \\
\text { described }\end{array}$ \\
\hline
\end{tabular}

\begin{tabular}{lll}
\hline Selective reporting & Unclear risk & Interviews may have been underreported \\
\hline Harmful effects & High risk & $\begin{array}{l}\text { Not much scope for harmful effects, such as impact of disruption of the inter- } \\
\text { vention, to be studied }\end{array}$ \\
\hline $\begin{array}{l}\text { Population and sample } \\
\text { described well }\end{array}$ & Low risk & The most relevant characteristics of the sample were captured \\
\hline $\begin{array}{l}\text { Continuous evaluation } \\
\begin{array}{l}\text { Evaluation participation } \\
\text { equity and sampling }\end{array}\end{array}$ & Low risk & Data were collected before and after the intervention \\
\hline
\end{tabular}

\begin{tabular}{ll}
\hline $\begin{array}{l}\text { Design and methods over- } \\
\text { all approach }\end{array}$ & Low risk \\
\hline
\end{tabular}

\begin{tabular}{lll}
\hline $\begin{array}{l}\text { Tools and methods of da- } \\
\text { ta collection reliable/cred- } \\
\text { ible }\end{array}$ & Unclear risk & Difficult to establish whether these were reliable, as they were interviews \\
\hline $\begin{array}{l}\text { Tools and methods of data } \\
\text { analysis reliable/credible }\end{array}$ & Unclear risk & $\begin{array}{l}\text { Analysis of quantitative data was comprehensive. However, treatment/analy- } \\
\text { sis of qualitative data was unclear }\end{array}$ \\
\hline $\begin{array}{l}\text { Performance bias/neutral- } \\
\text { ity/credibility/conforma- } \\
\text { bility }\end{array}$ & High risk & $\begin{array}{l}\text { Because of the way in which qualitative interviews were conducted, risk of per- } \\
\text { formance bias was increased }\end{array}$ \\
\hline $\begin{array}{l}\text { Reliability of findings and } \\
\text { recommendations }\end{array}$ & High risk & $\begin{array}{l}\text { Study included a small sample, and target numbers for the study were not } \\
\text { achieved. Presentation of qualitative data was limited }\end{array}$ \\
\hline $\begin{array}{l}\text { Transferability of findings } \\
\text { Ongerall risk of bias of }\end{array}$ & High risk & $\begin{array}{l}\text { Study authors did consider transferability of findings; however analysis of } \\
\text { qualitative data was absent }\end{array}$ \\
\hline $\begin{array}{l}\text { Orisk } \\
\text { process evaluation }\end{array}$ & $\begin{array}{l}\text { Some data were collected well; however treatment of qualitative data reveals } \\
\text { high risk of bias }\end{array}$ \\
\hline
\end{tabular}

Brasler 2006

Methods Included as process evaluation

Intervention study design: single-group pre-post design

Unit of allocation: N/A

Process evaluation methods: descriptive analysis of outcome and process factors 
Brasler 2006 (Continued)

Participants
Setting: conducted in 12 culturally and socio-economically diverse junior and middle schools in school districts in Anchorage, Alaska (AK), and Kansas City (KC) suburbs

Age of children: 11 to 13 years

Child characteristics (BME/SES): $29 \%$ of children in Anchorage and 48\% in Kansas City were African American, Asian, or Native American. An average of $27 \%$ of children across both sites were eligible for free school meals

Asthma status: asthmatic only

Intervention recipients: children only
School type: junior/middle

Intervention description: PBP: "the program, designed for adolescents aged 11-19, covers asthma basics and management, addresses adolescents' social and lifestyle concerns, and encourages them to take control of their asthma". It is a manualised program developed by the Asthma and Allergy Foundation of America

Control description: N/A

Theoretical framework: no single theoretical framework was named

\begin{tabular}{ll}
\hline Outcomes & Core processes evaluated (child level): mainly attrition, dosage, adherence \\
\hline Notes & Process evaluation category: named section \\
& Breadth and depth: breadth not depth - a broad range of processes explored on a superficial level \\
& $\begin{array}{l}\text { Voice of children given prominence: not featured - data collected from children did not allow the } \\
\text { voice of children to be heard }\end{array}$
\end{tabular}

Funding source: not reported

\section{Risk of bias}

\begin{tabular}{|c|c|c|}
\hline Bias & Authors' judgement & Support for judgement \\
\hline $\begin{array}{l}\text { Random sequence genera- } \\
\text { tion (selection bias) }\end{array}$ & Unclear risk & $\mathrm{N} / \mathrm{A}$ \\
\hline $\begin{array}{l}\text { Allocation concealment } \\
\text { (selection bias) }\end{array}$ & Unclear risk & $\mathrm{N} / \mathrm{A}$ \\
\hline $\begin{array}{l}\text { Blinding of participants } \\
\text { and personnel (perfor- } \\
\text { mance bias) } \\
\text { All outcomes }\end{array}$ & Unclear risk & $\mathrm{N} / \mathrm{A}$ \\
\hline $\begin{array}{l}\text { Blinding of outcome as- } \\
\text { sessment (detection bias) } \\
\text { All outcomes }\end{array}$ & Unclear risk & $\mathrm{N} / \mathrm{A}$ \\
\hline $\begin{array}{l}\text { Incomplete outcome data } \\
\text { (attrition bias) } \\
\text { All outcomes }\end{array}$ & Unclear risk & $\mathrm{N} / \mathrm{A}$ \\
\hline $\begin{array}{l}\text { Selective reporting (re- } \\
\text { porting bias) }\end{array}$ & Unclear risk & $\mathrm{N} / \mathrm{A}$ \\
\hline
\end{tabular}

School-based self-management interventions for asthma in children and adolescents: a mixed methods systematic review (Review) 
Brasler 2006 (Continued)

\begin{tabular}{lll} 
Other bias & Unclear risk & N/A \\
\hline $\begin{array}{l}\text { Transparent and clearly } \\
\text { stated aims }\end{array}$ & Low risk & Study aims were clearly stated \\
\hline
\end{tabular}

Explicit theories underpin- High risk $\quad$ No evidence of a named theoretical framework
ning and/or literature re-

view

\begin{tabular}{|c|c|c|}
\hline $\begin{array}{l}\text { Transparent and clearly } \\
\text { stated methods and tools }\end{array}$ & High risk & Most of the content was based on reflective note taking \\
\hline Selective reporting & Low risk & Notes reflected low levels of initial co-operation \\
\hline Harmful effects & Low risk & $\begin{array}{l}\text { Although elements of the intervention did not match the plan, study authors } \\
\text { have discussed these }\end{array}$ \\
\hline $\begin{array}{l}\text { Population and sample } \\
\text { described well }\end{array}$ & Unclear risk & $\begin{array}{l}\text { Some detail was absent, for example, ethnicity and SES data were not well de- } \\
\text { scribed }\end{array}$ \\
\hline Continuous evaluation & Low risk & Data were collected before and after the intervention \\
\hline $\begin{array}{l}\text { Evaluation participation } \\
\text { equity and sampling }\end{array}$ & High risk & $\begin{array}{l}\text { This was a multi-component intervention, but the 'voices' of teachers, nurses, } \\
\text { and parents were absent }\end{array}$ \\
\hline $\begin{array}{l}\text { Design and methods over- } \\
\text { all approach }\end{array}$ & High risk & Tools that were used were unstructured \\
\hline $\begin{array}{l}\text { Tools and methods of da- } \\
\text { ta collection reliable/cred- } \\
\text { ible }\end{array}$ & High risk & Tools used were unstructured, thus reducing reliability \\
\hline $\begin{array}{l}\text { Tools and methods of data } \\
\text { analysis reliable/credible }\end{array}$ & Low risk & Analysis of the data was fair \\
\hline $\begin{array}{l}\text { Performance bias/neutral- } \\
\text { ity/credibility/conforma- } \\
\text { bility }\end{array}$ & High risk & Study authors did not consider performance bias \\
\hline $\begin{array}{l}\text { Reliability of findings and } \\
\text { recommendations }\end{array}$ & Unclear risk & $\begin{array}{l}\text { Tools used to collect these data were not clearly described, although the con- } \\
\text { clusions drawn appear to match the data presented }\end{array}$ \\
\hline Transferability of findings & Low risk & $\begin{array}{l}\text { Process evaluation findings were regarded as transferable; the extent to which } \\
\text { evidence of effectiveness was transferable across sites is unclear }\end{array}$ \\
\hline $\begin{array}{l}\text { Overall risk of bias of } \\
\text { process evaluation }\end{array}$ & High risk & Unstructured data collection methods were used for process evaluation \\
\hline
\end{tabular}

Bruzzese 2004

Methods Included as process evaluation and outcome evaluation

Intervention study design: randomised controlled trial with the child selected as the unit of allocation

Setting: children were recruited from 2 inner city public high schools 
Period: not reported

Participants

Eligible sample frame: 65 students were eligible; 45 were randomised to intervention or control group

Randomised: 45 students were randomised: 23 to the intervention group and 22 to the delayed-treatment control group across the 2 schools

Completed (intervention): $100 \%$ of children in the intervention group received workshop 1; $91 \%$ received workshop 2; $61 \%$ of children in the intervention group received workshop 3, as time did not permit a make-up session

Inclusion criteria: students with persistent asthma symptoms, at least 3 days a week or 3 nights a month

Exclusion criteria: not reported

Baseline characteristics

Age of children: students in 9th and 10th grades

Ethnicity: not reported

Socio-economic status: not reported

Gender: not reported

Asthma status: asthmatic students only

School type: high school

Intervention description: Open Airways for Schools, academic detailing

Control description: usual care/nothing

Theoretical framework: self-regulation theory

Intervention: students received the ASMA programme, in which students were taught how to manage their asthma to prevent symptoms and improve quality of life. One goal of ASMA is to help students incorporate asthma management strategies into their self-identity. Continued medical education was also offered to students' medical providers

Intensity: intervention was delivered over an 8-week period, comprising 3 workshops spaced 2 to 3 weeks apart

Instructor: intervention was delivered by a trained health educator

Parental engagement: not reported

Child satisfaction: most students found the sessions helpful; however a third confirmed that they may or may not participate again

Timing of intervention in school day: every attempt was made to meet with students during their free time

Outcomes Extractable outcomes were collected for: none

Core processes/outcomes evaluated (child level): attrition, adherence

Notes

Process evaluation category: stand-alone

Breadth and depth: neither broad nor deep

Voice of children given prominence: sufficient coverage 
Bruzzese 2004 (Continued)

Funding source: Speakers' fund for public health

\section{Risk of bias}

\begin{tabular}{|c|c|c|}
\hline Bias & Authors' judgement & Support for judgement \\
\hline $\begin{array}{l}\text { Random sequence genera- } \\
\text { tion (selection bias) }\end{array}$ & Unclear risk & $\begin{array}{l}\text { Only detail provided was that students were randomly assigned to treatment } \\
\text { or control groups }\end{array}$ \\
\hline $\begin{array}{l}\text { Allocation concealment } \\
\text { (selection bias) }\end{array}$ & Unclear risk & $\begin{array}{l}\text { No details were provided except that students were randomly assigned to } \\
\text { treatment or control groups }\end{array}$ \\
\hline $\begin{array}{l}\text { Blinding of participants } \\
\text { and personnel (perfor- } \\
\text { mance bias) } \\
\text { All outcomes }\end{array}$ & Unclear risk & Methods used for blinding were not described \\
\hline $\begin{array}{l}\text { Blinding of outcome as- } \\
\text { sessment (detection bias) } \\
\text { All outcomes }\end{array}$ & Unclear risk & Methods used for blinding were not described \\
\hline $\begin{array}{l}\text { Incomplete outcome data } \\
\text { (attrition bias) } \\
\text { All outcomes }\end{array}$ & Unclear risk & $\begin{array}{l}\text { Low levels of post-test outcomes collected: } 14 \text { students who attended group } \\
\text { workshop \#3- } 64 \% \text { of all treatment students - completed an evaluation of the } \\
\text { programme }\end{array}$ \\
\hline $\begin{array}{l}\text { Selective reporting (re- } \\
\text { porting bias) }\end{array}$ & Unclear risk & $\begin{array}{l}\text { No data from the control group were presented. The procedure followed for } \\
\text { control group students is unclear }\end{array}$ \\
\hline Other bias & Unclear risk & $\begin{array}{l}\text { Baseline data were not reported, so we are unable to determine if any imbal- } \\
\text { ances existed at baseline }\end{array}$ \\
\hline $\begin{array}{l}\text { Transparent and clearly } \\
\text { stated aims }\end{array}$ & Low risk & Study aims were clearly stated \\
\hline $\begin{array}{l}\text { Explicit theories underpin- } \\
\text { ning and/or literature re- } \\
\text { view }\end{array}$ & Low risk & Theory guiding the intervention was provided as self-regulation theory \\
\hline $\begin{array}{l}\text { Transparent and clearly } \\
\text { stated methods and tools }\end{array}$ & High risk & Tools used for data collection were inferred, as opposed to being stated \\
\hline Selective reporting & Unclear risk & $\begin{array}{l}\text { Outcomes that study authors intended to measure are unclear; therefore we } \\
\text { are unable to say whether evidence shows selective reporting }\end{array}$ \\
\hline Harmful effects & High risk & No provision was made for harmful effects \\
\hline $\begin{array}{l}\text { Population and sample } \\
\text { described well }\end{array}$ & Unclear risk & $\begin{array}{l}\text { Some aspects of the sample were described in sufficient detail; however no in- } \\
\text { formation was provided on ethnicity, SES, or asthma severity among partici- } \\
\text { pants }\end{array}$ \\
\hline Continuous evaluation & High risk & Study authors did not capture the people who dropped out \\
\hline $\begin{array}{l}\text { Evaluation participation } \\
\text { equity and sampling }\end{array}$ & Unclear risk & Not reported by study authors \\
\hline $\begin{array}{l}\text { Design and methods over- } \\
\text { all approach }\end{array}$ & Unclear risk & Little information was given about the methods used \\
\hline
\end{tabular}


Bruzzese 2004 (Continued)

Tools and methods of da- High risk Small sample size suggests that different approaches to analysis and collecta collection reliable/credtion should have been used

ible

$\begin{array}{ll}\begin{array}{l}\text { Tools and methods of data } \\ \text { analysis reliable/credible }\end{array} & \text { High risk }\end{array}$

\begin{tabular}{lll}
$\begin{array}{l}\text { Performance bias/neutral- } \\
\text { ity/credibility/conforma- } \\
\text { bility }\end{array}$ & Unclear risk & Not reported by study authors \\
\hline $\begin{array}{l}\text { Reliability of findings and } \\
\text { recommendations }\end{array}$ & Unclear risk & Not reported by study authors \\
\hline $\begin{array}{l}\text { Transferability of findings } \\
\text { Onclear risk }\end{array}$ & $\begin{array}{l}\text { Some of the lessons learnt around time-tabling were transferable; however } \\
\text { study authors did not assess transferability }\end{array}$ \\
\hline \begin{tabular}{l} 
Process evaluation \\
\hline
\end{tabular} & High risk & $\begin{array}{l}\text { Points around sampling and absence of continuous evaluation contribute to } \\
\text { high risk of bias }\end{array}$ \\
\hline
\end{tabular}

Methods Included as outcome evaluation and process evaluation

Study design: parallel-group design with families selected as the unit of randomisation

Setting: study recruited children and their families from one middle school in New York City, New York, USA

Period: dates on which study was conducted - intervention and subsequent data collection - not clear; follow-up data collected 2 months post intervention

\section{Participants}

Eligible sample frame: 78 pupils found to be eligible; 24 agreed to participate

Randomised: 24 students randomised (at the student/family level): 12 each in control and intervention groups

Completed (intervention): 12 students and 10 caregivers in the treatment group, and 11 students and 8 caregivers in the control group, completed immediate follow-up

Inclusion criteria: after completing a case detection survey, eligible students were identified as having had "an asthma diagnosis from a medical provider, and over the past 12 months exhibited asthma symptoms an average of three times per month and used asthma medication"

Exclusion criteria: families were excluded if "(1) the child had a co-morbid disease that affects lung functioning or highly specialized developmental or learning needs, (2) the child and/or the caregiver did not speak English, or (3) the caregiver and child did not live together"

\section{Baseline characteristics}

Age of children: mean age, 12.8 years (grades 6 to 8 )

Ethnicity: child ethnicity described as Hispanic (41\%); White, not of Hispanic origin (17\%); African American, not of Hispanic origin (8\%); and other (34\%)

Socio-economic status: employment status of participating caregivers (but socio-economic circumstances of family) presented. Unemployed (8\%); employed part-time (21\%); employed full-time (71\%). Data on highest educational level completed by caregiver were also presented, with $66 \%$ having postcompulsory education 
Gender: males accounted for 13 of the child participants (54\%), and females for 11 of the participants (46\%)

Asthma status: direct information on severity not presented

Interventions

School type: junior/middle

Intervention description: OAS, ASMA, caregiver education

Control description: usual care/nothing

Theoretical framework: social cognitive theory and cognitive-behavioural therapy

Intervention: implemented "Asthma: It's a Family Affair!" intervention. "Intervention students received six group sessions on prevention and management of asthma. Lesson topics included: (1) information and feelings about asthma; (2) asthma medication; (3) prevention and management of asthma symptoms; (4) problem-solving and coping with negative feelings about asthma; and (5) communicating about asthma to peers and teachers, relaxation exercises, and healthy behaviours, including smoking refusal skills and avoiding exposure to secondhand smoke". The sixth session included a comprehensive review designed to reinforce key messages and to enhance students' confidence in managing their asthma. Curriculum was based on existing materials from Coping with Asthma at Home and at School, OAS, and ASMA. Caregivers participated in five 90-minute group sessions held once per week intended to support the child's autonomy and development of asthma self-management skills

Control: no treatment (usual care)

Intensity: children received 6 lessons on a weekly basis

Instructor: children's sessions were delivered by a developmental psychologist

Theoretical framework: integration of 4 psychological theories: social cognition theory, cognitive-behavioural theory, and 2 forms of family systems theory (parenting styles and behavioural family systems theory)

Parental engagement: low attrition among caregivers and high levels of satisfaction

Child satisfaction: high levels of satisfaction: "All students reported that the intervention gave them a better understanding of asthma, and $91 \%$ reported that the handouts helped them understand the topics discussed. Many reported that the best aspect of the program was 'talking about my asthma'"

Timing of intervention in school day: not reported

Outcomes Extractable outcomes were collected for:

Experience of daytime and night-time symptoms

Withdrawal from the study

Core processes/outcomes evaluated (child level): attrition, dosage, adherence

Notes Process evaluation category: named section

Breadth and depth: neither broad nor deep

Voice of children given prominence: featured but not sufficiently

Funding source: not reported

\section{Risk of bias}

Bias Authors' judgement Support for judgement


Bruzzese 2008 (Continued)

Random sequence genera- Unclear risk No information on how randomisation occurred
tion (selection bias)

$\begin{aligned} & \text { Allocation concealment } \\ & \text { (selection bias) }\end{aligned}$
Unclear risk No information on how allocation was concealed

Blinding of participants Unclear risk No information; blinding was not assessed by study authors
and personnel (perfor-
mance bias)
All outcomes

\begin{tabular}{lll}
\hline Blinding of outcome as- & Unclear risk & No information; blinding was not assessed by study authors \\
sessment (detection bias)
\end{tabular}

All outcomes

Incomplete outcome data Low risk Few participants dropped out (1 out of 12 students in the control group)
(attrition bias)
All outcomes

\begin{tabular}{lll}
\hline $\begin{array}{l}\text { Selective reporting (re- } \\
\text { porting bias) }\end{array}$ & Low risk & No evidence of selective reporting \\
\hline Other bias & Unclear risk & Missingness - low risk - no evidence of missing indicators \\
& $\begin{array}{l}\text { Baseline imbalance - low risk - no evidence of systematic differences in base- } \\
\text { line characteristics }\end{array}$ \\
& Contamination - high - randomisation was by family
\end{tabular}

\begin{tabular}{|c|c|c|}
\hline $\begin{array}{l}\text { Transparent and clearly } \\
\text { stated aims }\end{array}$ & Low risk & Study aims were clearly stated \\
\hline $\begin{array}{l}\text { Explicit theories underpin- } \\
\text { ning and/or literature re- } \\
\text { view }\end{array}$ & Low risk & $\begin{array}{l}\text { Intervention development was guided by social cognitive theory and cogni- } \\
\text { tive-behavioural therapy }\end{array}$ \\
\hline $\begin{array}{l}\text { Transparent and clearly } \\
\text { stated methods and tools }\end{array}$ & Unclear risk & Medium bias - tools were poorly described \\
\hline Selective reporting & Unclear risk & $\begin{array}{l}\text { As data collection tools were not that well stated, it is difficult to assess } \\
\text { whether results show any evidence of selective reporting }\end{array}$ \\
\hline Harmful effects & Unclear risk & Not considered by trial authors \\
\hline $\begin{array}{l}\text { Population and sample } \\
\text { described well }\end{array}$ & Low risk & Population and sample were well described \\
\hline Continuous evaluation & Unclear risk & $\begin{array}{l}\text { Data were collected before and after the intervention; however the level of } \\
\text { participation was low }\end{array}$ \\
\hline $\begin{array}{l}\text { Evaluation participation } \\
\text { equity and sampling }\end{array}$ & High risk & Little evidence of evaluation from children \\
\hline $\begin{array}{l}\text { Design and methods over- } \\
\text { all approach }\end{array}$ & Unclear risk & Unclear how data were collected \\
\hline
\end{tabular}


Bruzzese 2008 (Continued)

Tools and methods of da- High risk Unclear whether tools used had been validated ta collection reliable/credible

Tools and methods of data Low risk
analysis reliable/credible

Performance bias/neutral- Unclear risk Unclear whether this was given consideration
ity/credibility/conforma-
bility

\begin{tabular}{lll}
\hline $\begin{array}{l}\text { Reliability of findings and } \\
\text { recommendations }\end{array}$ & Low risk & Reliability of findings clear; conclusions justified \\
\hline Transferability of findings & High risk & No consideration given to transferability of findings \\
\hline $\begin{array}{l}\text { Overall risk of bias of } \\
\text { process evaluation }\end{array}$ & Unclear risk & Medium bias \\
\hline
\end{tabular}

Bruzzese 2010

\begin{tabular}{|c|c|}
\hline \multirow[t]{4}{*}{ Methods } & Included as outcome evaluation and process evaluation \\
\hline & Intervention study design: $\mathrm{RCT}$, parallel group, randomised at the child level \\
\hline & Setting: 25 public schools in New York City \\
\hline & Period: intervention was conducted over 4 years \\
\hline
\end{tabular}

Participants

Eligible sample frame: 393 students were eligible; 288 completed the 12-month follow-up

Randomised: 393 students were randomised; numbers of students in intervention and control groups not reported

Completed (intervention): 288 students completed the 12-month follow-up

Inclusion criteria: students with persistent asthma and their caregivers

Exclusion criteria: not reported

\section{Baseline characteristics}

Age of children: 14 to 16

Child characteristics (BME/SES): $45.51 \%$ Hispanic; $75 \%$ of students on free school meals

Asthma status: asthmatic only

Intervention recipients: children only

Intervention description: ASMA, academic detailing

Control description: usual care/nothing

Theoretical framework: social cognitive theory 
Intervention: a family affair programme. Students in the intervention group attended 6 workshops to empower them to manage their asthma; their parents attended training group workshops to teach childrearing skills that support their child's growing autonomy and need to manage their asthma

Intensity: children attended 6 workshops, and parents/caregivers attended 5

Instructor: not reported

Parental engagement: not reported

Child satisfaction: not reported

Timing of intervention in school day: not reported

\begin{tabular}{ll} 
Outcomes & Extractable outcomes were collected for: \\
& Withdrawal \\
Core processes evaluated (child level): attrition, dosage, adherence \\
\hline Potes & Process evaluation category: integrated \\
Breadth and depth: neither broad nor deep \\
Voice of children given prominence: featured but not sufficiently \\
Funding source: National Heart, Lung, and Blood Institute; NYC Speakers' Fund
\end{tabular}

\section{Risk of bias}

\begin{tabular}{|c|c|c|}
\hline Bias & Authors' judgement & Support for judgement \\
\hline $\begin{array}{l}\text { Random sequence genera- } \\
\text { tion (selection bias) }\end{array}$ & Unclear risk & Not reported by study authors \\
\hline $\begin{array}{l}\text { Allocation concealment } \\
\text { (selection bias) }\end{array}$ & Unclear risk & Not reported by study authors \\
\hline $\begin{array}{l}\text { Blinding of participants } \\
\text { and personnel (perfor- } \\
\text { mance bias) } \\
\text { All outcomes }\end{array}$ & Unclear risk & Abstract only; no information on blinding presented \\
\hline $\begin{array}{l}\text { Blinding of outcome as- } \\
\text { sessment (detection bias) } \\
\text { All outcomes }\end{array}$ & Unclear risk & Abstract only; no information on blinding presented \\
\hline $\begin{array}{l}\text { Incomplete outcome data } \\
\text { (attrition bias) } \\
\text { All outcomes }\end{array}$ & Unclear risk & Abstract only; no information on blinding presented \\
\hline $\begin{array}{l}\text { Selective reporting (re- } \\
\text { porting bias) }\end{array}$ & Unclear risk & Abstract only; no information on blinding presented \\
\hline Other bias & Unclear risk & Abstract only; no information on blinding presented \\
\hline $\begin{array}{l}\text { Transparent and clearly } \\
\text { stated aims }\end{array}$ & Low risk & Study aims were clearly stated \\
\hline
\end{tabular}


Bruzzese 2010 (Continued)

Explicit theories underpin- Low risk Social cognitive theory guided development of the intervention ning and/or literature review

\begin{tabular}{lll}
\hline $\begin{array}{l}\text { Transparent and clearly } \\
\text { stated methods and tools }\end{array}$ & Low risk & Methods and tools used were clearly described \\
\hline Selective reporting & Low risk & All collected data were reported on \\
\hline Harmful effects & Unclear risk & Medium bias - some harmful effects around differential reach can be inferred \\
\hline $\begin{array}{l}\text { Population and sample } \\
\text { described well }\end{array}$ & Low risk & Sufficient data were included to provide a depiction of context \\
\hline $\begin{array}{l}\text { Continuous evaluation } \\
\text { Evaluation participation } \\
\text { equity and sampling }\end{array}$ & Low risk & Two rounds of follow-up were described for outcomes \\
\hline
\end{tabular}

Design and methods over- Low risk Administrative and survey data were collected at multiple points
all approach

\begin{tabular}{|c|c|c|}
\hline $\begin{array}{l}\text { Tools and methods of da- } \\
\text { ta collection reliable/cred- } \\
\text { ible }\end{array}$ & Low risk & Validated tools were used \\
\hline $\begin{array}{l}\text { Tools and methods of data } \\
\text { analysis reliable/credible }\end{array}$ & Low risk & $\begin{array}{l}\text { Credible analysis - zip regression modelling was employed to address skew- } \\
\text { ness }\end{array}$ \\
\hline $\begin{array}{l}\text { Performance bias/neutral- } \\
\text { ity/credibility/conforma- } \\
\text { bility }\end{array}$ & Low risk & Blinding was explicitly mentioned \\
\hline $\begin{array}{l}\text { Reliability of findings and } \\
\text { recommendations }\end{array}$ & Low risk & No issues were reported \\
\hline \multirow[t]{2}{*}{ Transferability of findings } & Low risk & $\begin{array}{l}\text { Study authors have considered this; however their findings have comparative- } \\
\text { ly low transferability }\end{array}$ \\
\hline & & $\begin{array}{l}\text { "We are also unable to extrapolate study results to other populations of high } \\
\text { school students with asthma (e.g. white suburban adolescents with mild asth- } \\
\text { ma) because we limited enrolment to minority youth with moderate to severe } \\
\text { persistent asthma" }\end{array}$ \\
\hline
\end{tabular}

Methods Included as outcome evaluation

Intervention study design: randomised controlled trial parallel group

Setting: conducted at 5 participating high schools in New York, USA

Period: study enrolment took place over 4 consecutive school years from 2001 to 2004 
Bruzzese 2011 (Continued)

Participants
Eligible sample frame: 261 pupils found to be eligible

Randomised: 345 students randomised: 175 to intervention group and 170 to control group

Completed (intervention): 139 (79.4\%) in the intervention group completed follow-up, as did 142 $(83.5 \%)$ in the control group

Inclusion criteria: 9th and 10th graders with moderate to severe persistent asthma who were taking medication prescribed by a medical provider in the last 12 months

Exclusion criteria: none stated

Baseline characteristics

Age of children: mean age, 15.10 years

Ethnicity: 45.5\% Hispanic/Latino/a or Hispanic American; 37.7\% African American/African or Caribbean American/Caribbean; $11.6 \%$ mixed ethnicity; $5.2 \%$ other ethnicity

Socio-economic status: not reported

Gender: $29.6 \%$ male; $70.4 \%$ female

Asthma status: $68.70 \%$ moderate persistent asthma, $31.30 \%$ severe persistent asthma. No information on SES

Interventions

Intervention: ASMA consisted of 2 components: (I) an 8-week intensive programme for students, and (ii) academic detailing for adolescents' medical providers. Student intervention consisted of three 45to 60-minute group sessions, and individual tailored coaching sessions held at least once per week for 5 weeks. Sessions were delivered by trained health educators during the school day. Students were taught asthma management skills and ways to cope with asthma, and were encouraged to see their medical provider for clinical evaluation and treatment (see Bruzzese 2004 for a full outline of ASMA content)

Control: wait-list control (usual care)

Intensity: three 45- to 60-minute group sessions for children over 8 weeks and individual tailored coaching sessions once a week for 5 weeks

Instructor: health educators

Theoretical framework: ASMA described as grounded in social cognitive theory

Parental engagement: not reported

Child satisfaction: not reported

Timing: unclear but at some point during the school day

Outcomes

Exacerbations leading to hospital admission

Asthma symptoms leading to emergency hospital visits

Absence from school

Days of restricted activity

Unplanned GP or hospital visit due to asthma

Experience of daytime and night-time symptoms

Corticosteroid dosage

School-based self-management interventions for asthma in children and adolescents: a mixed methods systematic review (Review) 
Bruzzese 2011 (Continued)

Withdrawal

Notes $\quad$ Funding source: National Heart, Lung, and Blood Institute; NYC Speakers' Fund

\section{Risk of bias}

\begin{tabular}{|c|c|c|}
\hline Bias & Authors' judgement & Support for judgement \\
\hline $\begin{array}{l}\text { Random sequence genera- } \\
\text { tion (selection bias) }\end{array}$ & Low risk & $\begin{array}{l}\text { Study authors reported: "Within each stratum, we randomised students to } \\
\text { control or intervention using computerized randomisation lists generated in } \\
\text { advance by the data manager who concealed them until randomisation" }\end{array}$ \\
\hline $\begin{array}{l}\text { Allocation concealment } \\
\text { (selection bias) }\end{array}$ & Low risk & $\begin{array}{l}\text { Randomisation lists were generated in advance by the data manager, who } \\
\text { concealed them until randomisation }\end{array}$ \\
\hline $\begin{array}{l}\text { Blinding of participants } \\
\text { and personnel (perfor- } \\
\text { mance bias) } \\
\text { All outcomes }\end{array}$ & Unclear risk & $\begin{array}{l}\text { Interviewers were blind to group assignment. Whether participants were blind- } \\
\text { ed is unclear }\end{array}$ \\
\hline $\begin{array}{l}\text { Blinding of outcome as- } \\
\text { sessment (detection bias) } \\
\text { All outcomes }\end{array}$ & Low risk & Interviewers were blinded \\
\hline $\begin{array}{l}\text { Incomplete outcome data } \\
\text { (attrition bias) } \\
\text { All outcomes }\end{array}$ & Low risk & $\begin{array}{l}\text { No differences were noted between the } 2 \text { groups - incomplete data were un- } \\
\text { likely to affect outcomes }\end{array}$ \\
\hline $\begin{array}{l}\text { Selective reporting (re- } \\
\text { porting bias) }\end{array}$ & Low risk & All outcomes were reported \\
\hline \multirow[t]{3}{*}{ Other bias } & Low risk & $\begin{array}{l}\text { Missingness - low risk - appears that more participants who did not drop out } \\
\text { submitted their data }\end{array}$ \\
\hline & & $\begin{array}{l}\text { Baseline imbalance - low risk - intervention and control groups were relatively } \\
\text { evenly matched in characteristics }\end{array}$ \\
\hline & & $\begin{array}{l}\text { Risk of contamination - low - informal interviews with control participants re- } \\
\text { garding their contact with other students in the programme suggest that cont- } \\
\text { amination did not occur }\end{array}$ \\
\hline $\begin{array}{l}\text { Transparent and clearly } \\
\text { stated aims }\end{array}$ & Unclear risk & $\mathrm{N} / \mathrm{A}$ \\
\hline $\begin{array}{l}\text { Explicit theories underpin- } \\
\text { ning and/or literature re- } \\
\text { view }\end{array}$ & Unclear risk & $\mathrm{N} / \mathrm{A}$ \\
\hline $\begin{array}{l}\text { Transparent and clearly } \\
\text { stated methods and tools }\end{array}$ & Unclear risk & $\mathrm{N} / \mathrm{A}$ \\
\hline Selective reporting & Unclear risk & $\mathrm{N} / \mathrm{A}$ \\
\hline Harmful effects & Unclear risk & $\mathrm{N} / \mathrm{A}$ \\
\hline $\begin{array}{l}\text { Population and sample } \\
\text { described well }\end{array}$ & Unclear risk & $\mathrm{N} / \mathrm{A}$ \\
\hline
\end{tabular}


Bruzzese 2011 (Continued)

\begin{tabular}{|c|c|c|}
\hline Continuous evaluation & Unclear risk & $\mathrm{N} / \mathrm{A}$ \\
\hline $\begin{array}{l}\text { Evaluation participation } \\
\text { equity and sampling }\end{array}$ & Unclear risk & $\mathrm{N} / \mathrm{A}$ \\
\hline $\begin{array}{l}\text { Design and methods over- } \\
\text { all approach }\end{array}$ & Unclear risk & $\mathrm{N} / \mathrm{A}$ \\
\hline $\begin{array}{l}\text { Tools and methods of da- } \\
\text { ta collection reliable/cred- } \\
\text { ible }\end{array}$ & Unclear risk & $\mathrm{N} / \mathrm{A}$ \\
\hline $\begin{array}{l}\text { Tools and methods of data } \\
\text { analysis reliable/credible }\end{array}$ & Unclear risk & $\mathrm{N} / \mathrm{A}$ \\
\hline $\begin{array}{l}\text { Performance bias/neutral- } \\
\text { ity/credibility/conforma- } \\
\text { bility }\end{array}$ & Unclear risk & $\mathrm{N} / \mathrm{A}$ \\
\hline $\begin{array}{l}\text { Reliability of findings and } \\
\text { recommendations }\end{array}$ & Unclear risk & $\mathrm{N} / \mathrm{A}$ \\
\hline Transferability of findings & Unclear risk & $\mathrm{N} / \mathrm{A}$ \\
\hline $\begin{array}{l}\text { Overall risk of bias of } \\
\text { process evaluation }\end{array}$ & Unclear risk & $\mathrm{N} / \mathrm{A}$ \\
\hline
\end{tabular}

Methods Included as process evaluation

Intervention study design: study design was quasi-experimental, with pre-post follow-up and no control

Unit of allocation: N/A

Process evaluation methods: descriptive/bivariate

Participants Country: USA

Age of children: 25 children 7 to 17 years old were recruited

Child characteristics (BME/SES): sample comprised $72 \%$ non-Hispanic white children

Asthma status: asthmatic children only

Intervention recipients: children and nurses

School type: intervention was delivered at 7 different schools, consisting of high schools, junior/middle schools, and primary/elementary schools

Intervention description: the intervention consisted of 2 sessions for children. Children were asked to watch a tailored video and to demonstrate their inhaler technique before and after the video. One month later, at the second session, children demonstrated their inhaler technique again to the school nurse and were allowed to watch the video again

Control description: N/A 
Carpenter 2016 (Continued)

Theoretical framework: not reported

\begin{tabular}{ll}
\hline Outcomes & Core processes evaluated (child level): adherence \\
\hline Notes & Process evaluation category: named section \\
Breadth and depth: neither broad nor deep \\
Voice of children given prominence: sufficient coverage \\
Funding source: not reported
\end{tabular}

\section{Risk of bias}

\begin{tabular}{|c|c|c|}
\hline Bias & Authors' judgement & Support for judgement \\
\hline $\begin{array}{l}\text { Random sequence genera- } \\
\text { tion (selection bias) }\end{array}$ & Unclear risk & N/A \\
\hline $\begin{array}{l}\text { Allocation concealment } \\
\text { (selection bias) }\end{array}$ & Unclear risk & N/A \\
\hline $\begin{array}{l}\text { Blinding of participants } \\
\text { and personnel (perfor- } \\
\text { mance bias) } \\
\text { All outcomes }\end{array}$ & Unclear risk & $\mathrm{N} / \mathrm{A}$ \\
\hline $\begin{array}{l}\text { Blinding of outcome as- } \\
\text { sessment (detection bias) } \\
\text { All outcomes }\end{array}$ & Unclear risk & $\mathrm{N} / \mathrm{A}$ \\
\hline $\begin{array}{l}\text { Incomplete outcome data } \\
\text { (attrition bias) } \\
\text { All outcomes }\end{array}$ & Unclear risk & $\mathrm{N} / \mathrm{A}$ \\
\hline
\end{tabular}

\begin{tabular}{l}
$\begin{array}{l}\text { Selective reporting (re- } \\
\text { porting bias) }\end{array}$ \\
\hline
\end{tabular}

\begin{tabular}{lll}
\hline Other bias & Unclear risk & N/A \\
\hline $\begin{array}{l}\text { Transparent and clearly } \\
\text { stated aims }\end{array}$ & Low risk & Study aims were clearly stated \\
\hline
\end{tabular}

\begin{tabular}{|c|c|c|}
\hline $\begin{array}{l}\text { Explicit theories underpin- } \\
\text { ning and/or literature re- } \\
\text { view }\end{array}$ & High risk & No theoretical framework was presented to inform the intervention \\
\hline $\begin{array}{l}\text { Transparent and clearly } \\
\text { stated methods and tools }\end{array}$ & Unclear risk & Medium bias - content and conduct of the focus group were not presented \\
\hline Selective reporting & High risk & $\begin{array}{l}\text { Study authors provided some example quotations; however it is unclear how } \\
\text { themes were derived }\end{array}$ \\
\hline Harmful effects & High risk & $\begin{array}{l}\text { The premise of the intervention could be considered harmful, as the informa- } \\
\text { tion that was collected should be provided only by demographically equiva- } \\
\text { lent people. However, this was not explored in depth }\end{array}$ \\
\hline
\end{tabular}

\begin{tabular}{|c|c|c|}
\hline $\begin{array}{l}\text { Population and sample } \\
\text { described well }\end{array}$ & Unclear risk & $\begin{array}{l}\text { The reach of this study is unclear; very little information on the sample was } \\
\text { presented }\end{array}$ \\
\hline
\end{tabular}


Carpenter 2016 (Continued)

\begin{tabular}{lll} 
Continuous evaluation & Unclear risk & Not reported by study authors \\
\hline $\begin{array}{l}\text { Evaluation participation } \\
\text { equity and sampling }\end{array}$ & Unclear risk & $\begin{array}{l}\text { Some information was collected from different actors; however information } \\
\text { was not collected to the same degree of robustness as for other participants }\end{array}$ \\
\hline $\begin{array}{l}\text { Design and methods over- } \\
\text { all approach }\end{array}$ & Unclear risk & $\begin{array}{l}\text { Medium bias - premise of the intervention is suspect, and approach needs to } \\
\text { be grounded in providing information around a need - but this info is lacking }\end{array}$ \\
\hline $\begin{array}{l}\text { Tools and methods of da- } \\
\text { ta collection reliable/cred- } \\
\text { ible }\end{array}$ & Unclear risk & $\begin{array}{l}\text { The focus of the intervention is not reflected strongly enough in the data col- } \\
\text { lected }\end{array}$ \\
\hline $\begin{array}{l}\text { Tools and methods of data } \\
\text { analysis reliable/credible }\end{array}$ & Unclear risk & $\begin{array}{l}\text { No information was provided on how data were collected in the focus group, } \\
\text { so whether data analysis methods used were appropriate is not clear }\end{array}$ \\
\hline $\begin{array}{l}\text { Performance bias/neutral- } \\
\text { ity/credibility/conforma- } \\
\text { bility }\end{array}$ & High risk & $\begin{array}{l}\text { No evidence indicates whether concerns were addressed during the data col- } \\
\text { lection process }\end{array}$ \\
\hline $\begin{array}{l}\text { Reliability of findings and } \\
\text { recommendations }\end{array}$ & Unclear risk & \begin{tabular}{l} 
Although data were presented, how data were collected remains unclear \\
\hline $\begin{array}{l}\text { Transferability of findings } \\
\text { High risk }\end{array}$
\end{tabular}
\end{tabular}

Overall risk of bias of High risk The premise of the intervention was not explored

process evaluation

\section{Cicutto 2005}

\section{Methods}

\section{Included as outcome evaluation}

Study design: clustered parallel-group design with schools selected as the unit of randomisation

Setting: study was conducted across 26 elementary schools in a suburb of Toronto (Canada)

Period: dates on which the study was conducted - including intervention and subsequent data collection - not clear; intervention and data collection was conducted over the period of a year

\section{Participants}

Eligible sample frame: 40 elementary schools were randomly selected from a pool of 147 potential elementary schools for inclusion in the Toronto area. Parents and children were invited to participate, and eligibility was assessed (see below). Based on the information returned, 26 schools had sufficient numbers of pupils (more than 7 pupils per school) to allow the trial to go ahead. In total, 297 eligible pupils were identified across the 26 schools

Randomised: 256 pupils randomised at the school level: 132 to the treatment group and 124 to the control group

Completed (intervention): 248 pupils remained at the 6-month data collection point: 130 treatment group children and 118 control group children. 239 children completed the intervention: 121 in the treatment and 118 in the control group.

Inclusion criteria: students were eligible for participation if they were reported as (I) having physician-diagnosed asthma, (ii) having used an asthma medication (i.e. bronchodilator and/or anti-inflammatory agents) for breathing difficulties, and (iii) having experienced asthma symptoms 3 or more times in the past year. Students had to meet all 3 criteria to be eligible 
Exclusion criteria: children were excluded from the study if they had a second (major) chronic illness with a pulmonary component (e.g. cystic fibrosis)

\section{Baseline characteristics}

Age of children: mean age, 8.6 years across treatment and control groups

Ethnicity: not reported

Socio-economic status: indicative evidence that none of the children were from low-income families: "The average income of the parent/guardian who participated in the study was approximately $\$ 53,000.00$ (Canadian dollars) with a range of $\$ 20,500.00$ to $\$ 200,000.00$. In Canada, low-income families earn $\$ 19,000.00$ per year"

Gender: treatment group: $58.3 \%$ male, $41.7 \%$ female; control group: 59.6\% male, $40.4 \%$ female

Asthma status: At baseline, according to parental report: Treatment group: $68.2 \%$ of children had mild asthma, $20.4 \%$ had moderate asthma, $5.4 \%$ had severe asthma; Control group: $69.5 \%$ of children had mild asthma; $23.4 \%$ had moderate asthma; $7.3 \%$ had severe asthma

Interventions

Intervention: children in treatment group received "Roaring Adventures of Puff" (RAP) intervention. This consisted of 6 sessions that included: "(1) getting to know each other, goal setting, use of a peak flowmeter, and diary monitoring; (2) trigger identification, control, and avoidance, and basic pathophysiology; (3) medications and the proper use of inhalers; (4) symptom recognition and action plan use; (5) lifestyle, exercise, and managing an asthma episode; and (6) sharing asthma information with teachers and parents". Parents were invited to attend the final session and were encouraged to take part in assisting with children's homework

Control: usual care

Intensity: children attended 6 sessions 50 to 60 minutes in length that were held once a week over 6 consecutive weeks

Instructor: asthma educator

Theoretical framework: theories involving social cognitive theory and self-regulation underpinned the intervention

Parental engagement: reported as low $-<20 \%$ of children had a parent who attended the final session

Child satisfaction: not reported

Timing of intervention in school day: sessions took place over the lunch period

Extractable outcomes were collected for:
Exacerbations leading to hospital admission
Asthma symptoms leading to emergency hospital visits
Absence from school
Days of restricted activity

Funding source: Change Foundation 
Cicutto 2005 (Continued)

$\begin{array}{ll}\begin{array}{l}\text { Random sequence genera- } \\ \text { tion (selection bias) }\end{array} & \text { Low risk } \\ \end{array}$
tion (selection bias) programme

\begin{tabular}{|c|c|c|}
\hline $\begin{array}{l}\text { Allocation concealment } \\
\text { (selection bias) }\end{array}$ & Low risk & $\begin{array}{l}\text { sation was centrally controlled by a computerised randomisation } \\
\text { me }\end{array}$ \\
\hline
\end{tabular}

Blinding of participants Unclear risk Not addressed by study authors

and personnel (perfor-

mance bias)

All outcomes

\begin{tabular}{|c|c|c|}
\hline $\begin{array}{l}\text { Blinding of outcome as- } \\
\text { sessment (detection bias) } \\
\text { All outcomes }\end{array}$ & Low risk & Assessors and participants were blinded to group assignment \\
\hline $\begin{array}{l}\text { Incomplete outcome data } \\
\text { (attrition bias) }\end{array}$ & Low risk & $\begin{array}{l}\text { Low levels of attrition/dropout with outcome data collected from } 93 \% \text { of stu- } \\
\text { dents, who were randomised }\end{array}$ \\
\hline
\end{tabular}

All outcomes

\begin{tabular}{|c|c|c|}
\hline $\begin{array}{l}\text { Selective reporting (re- } \\
\text { porting bias) }\end{array}$ & Unclear risk & $\begin{array}{l}\text { Not all outcomes were fully reported, but they were described narratively in } \\
\text { some places }\end{array}$ \\
\hline
\end{tabular}

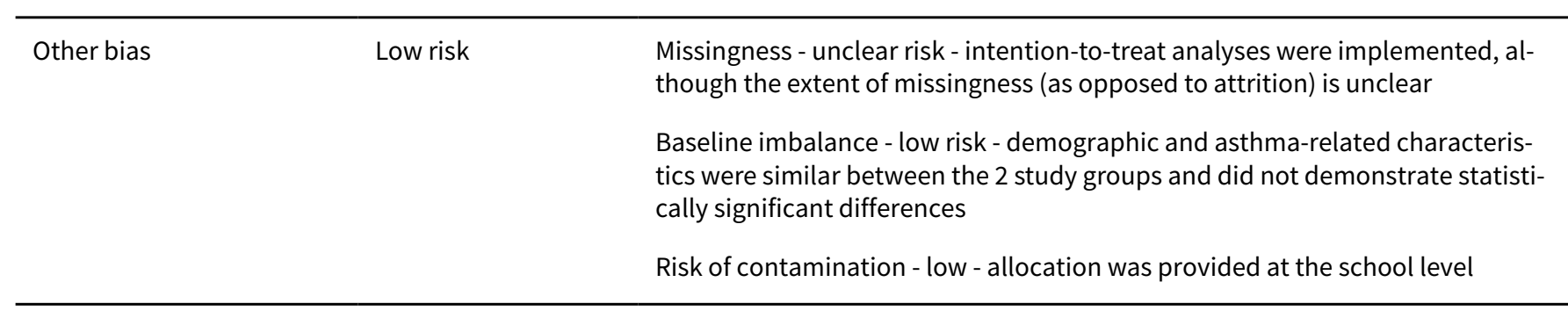

\begin{tabular}{lll}
\hline $\begin{array}{l}\text { Transparent and clearly } \\
\text { stated aims }\end{array}$ & Unclear risk & \\
\hline $\begin{array}{l}\text { Explicit theories underpin- } \\
\text { ning and/or literature re- } \\
\text { view }\end{array}$ & Unclear risk & N/A \\
\hline $\begin{array}{l}\text { Transparent and clearly } \\
\text { stated methods and tools }\end{array}$ & Unclear risk & N/A \\
\hline Selective reporting & Unclear risk & N/A \\
\hline $\begin{array}{l}\text { Harmful effects } \\
\text { Population and sample } \\
\text { described well }\end{array}$ & Unclear risk & N/A \\
\hline $\begin{array}{l}\text { Continuous evaluation } \\
\text { Evaluation participation } \\
\text { equity and sampling }\end{array}$ & Unclear risk & N/A \\
\hline $\begin{array}{l}\text { Design and methods over- } \\
\text { all approach }\end{array}$ & Unclear risk & N/A \\
\hline
\end{tabular}


Cicutto 2005 (Continued)

Tools and methods of da- Unclear risk N/A ta collection reliable/cred-

ible

$\begin{aligned} & \text { Tools and methods of data } \\ & \text { analysis reliable/credible }\end{aligned}$
Unclear risk N/A

Performance bias/neutral- Unclear risk
ity/credibility/conforma-
bility

\begin{tabular}{lll}
\hline $\begin{array}{l}\text { Reliability of findings and } \\
\text { recommendations }\end{array}$ & Unclear risk & N/A \\
\hline Transferability of findings & Unclear risk & N/A \\
\hline $\begin{array}{l}\text { Overall risk of bias of } \\
\text { process evaluation }\end{array}$ & Unclear risk & N/A \\
\hline
\end{tabular}

Cicutto 2013

Included as process evaluation and outcome evaluation
Intervention study design: cluster RCT
Setting: 170 primary/elementary schools from 5 public health units across Ontario, Canada
Period: not reported

Participants

Eligible sample frame: 180 schools were eligible and 170 schools were randomised ( 85 in each arm). 2502 families were eligible and 1316 were enrolled

Randomised: 1316 participants were enrolled

Completed (intervention): 1172 completed the study; 144 children withdrew, representing an 11\% withdrawal rate

Inclusion criteria: parental report of physician-diagnosed asthma, use of asthma medications, asthma symptoms at least 3 times in the past year, enrolment in grades 1 through 5 at school, ability to speak English, no other chronic conditions that could mimic asthma

Exclusion criteria: not reported

\section{Baseline characteristics}

Age of children: 8 years old

Ethnicity: not reported

Socio-economic status: $26.4 \%$ of children had a mother who did not have a high school diploma

Gender: the sample comprised $57.4 \%$ males; $58.4 \%$ of control children and $56.6 \%$ of intervention children were male

Asthma status: asthmatic only

Interventions

School type: primary/elementary

Intervention description: Roaring Adventures of Puff 
Cicutto 2013 (Continued)

Control description: usual care/nothing

Theoretical framework: social cognitive theory

Intervention: workshops included goal setting and self-monitoring, trigger identification, control and avoidance, basic pathophysiology, medication and proper inhaler use, symptom recognition and the asthma action plan, lifestyle and exercise, managing an asthma exacerbation, and showcasing learning with teachers and parents. The intervention used interactive techniques to educate the children. Makeup sessions were available for those who missed a class

Intensity: children attended six 45-minute sessions

Instructor: public health nurses

Parental engagement: difficulty in getting parents/guardians involved

Child satisfaction: not reported

Timing of intervention in school day: lunchtime

Extractable outcomes collected for:
Asthma symptoms leading to emergency hospital visits
Parent-reported absence from school
Days of restricted activity
Unplanned visit to hospital or GP due to asthma symptoms
Health-related quality of life
Withdrawal

Core processes evaluated (child level): attrition

Notes Process evaluation category: stand-alone and Integrated (2 papers)

Breadth and depth: breadth - not depth

Voice of children given prominence: not featured

Funding source: Ontario Ministry of Health and Long Term Care

\section{Risk of bias}

\begin{tabular}{lll}
\hline Bias & Authors' judgement & Support for judgement \\
\hline $\begin{array}{l}\text { Random sequence genera- } \\
\text { tion (selection bias) }\end{array}$ & Low risk & $\begin{array}{l}\text { Random sequence: of the } 180 \text { schools, } 170 \text { schools with the largest numbers } \\
\text { of students with asthma were randomised to intervention or control groups } \\
\text { through a computer-generated table of random numbers }\end{array}$ \\
\hline $\begin{array}{l}\text { Allocation concealment } \\
\text { (selection bias) }\end{array}$ & Low risk & $\begin{array}{l}\text { Central allocation indicated: of the } 180 \text { schools, } 170 \text { schools with the largest } \\
\text { numbers of students with asthma were randomised to intervention or control } \\
\text { groups through a computer-generated table of random numbers }\end{array}$ \\
\hline $\begin{array}{l}\text { Blinding of participants } \\
\text { and personnel (perfor- } \\
\text { mance bias) }\end{array}$ & Unclear risk & $\begin{array}{l}\text { Not clear whether measures were taken and whether this would have influ- } \\
\text { enced the outcome }\end{array}$ \\
\hline $\begin{array}{l}\text { Blinding of outcome as- } \\
\text { sessment (detection bias) }\end{array}$ & Low risk & $\begin{array}{l}\text { Study authors reported: "Data collectors were blinded to group assignment. } \\
\text { A post survey of data collectors revealed that blinding was successful. Da- }\end{array}$ \\
\hline
\end{tabular}


Cicutto 2013 (Continued)

All outcomes ta collectors became unblinded to group assignment for $9 \%$ of participating families, which occurred during the data collection interviews with parents/guardians"

$\begin{array}{lll}\begin{array}{l}\text { Incomplete outcome data } \\ \text { (attrition bias) }\end{array} & \text { Unclear risk } & \begin{array}{l}\text { Attrition data were presented, although data were not disaggregated by treat } \\ \text { ment status }\end{array}\end{array}$

All outcomes ment status

\section{Selective reporting (re- Unclear risk $\quad$ Absence of clear numbers hinders interpretation of some data} porting bias)

\begin{tabular}{|c|c|c|}
\hline \multirow[t]{2}{*}{ Other bias } & Unclear risk & $\begin{array}{l}\text { Missingness - high risk - evidence of unexplained missing data in Table } 2 \\
\text { Baseline imbalance - low risk - study authors reported: "Randomisation was } \\
\text { successful in that comparison of baseline variables for the control and exper- } \\
\text { imental groups suggested they were similar or balanced before the interven- } \\
\text { tion" }\end{array}$ \\
\hline & & $\begin{array}{l}\text { Risk of contamination - low - unit of randomisation was at the school level, re- } \\
\text { ducing the threat of contamination }\end{array}$ \\
\hline
\end{tabular}

Transparent and clearly Low risk Study aims were clearly stated
stated aims

\begin{tabular}{|c|c|c|}
\hline $\begin{array}{l}\text { Explicit theories underpin- } \\
\text { ning and/or literature re- } \\
\text { view }\end{array}$ & Low risk & Intervention development was informed by social cognitive theory \\
\hline $\begin{array}{l}\text { Transparent and clearly } \\
\text { stated methods and tools }\end{array}$ & Low risk & Tools and methods were clearly reported \\
\hline Selective reporting & Unclear risk & Unclear how this was addressed \\
\hline Harmful effects & Unclear risk & $\begin{array}{l}\text { Study authors did report on low parental engagement; however they offered } \\
\text { no explanation as to why all treatment schools did not adopt the policy }\end{array}$ \\
\hline $\begin{array}{l}\text { Population and sample } \\
\text { described well }\end{array}$ & Unclear risk & Individual schools were not described sufficiently \\
\hline Continuous evaluation & Low risk & $\begin{array}{l}\text { Policy development was monitored early on; } 2 \text { sets of data were collected after } \\
\text { baseline }\end{array}$ \\
\hline
\end{tabular}

\begin{tabular}{lll}
\hline $\begin{array}{l}\text { Evaluation participation } \\
\text { equity and sampling }\end{array}$ & High risk & Stakeholders were not directly involved \\
\hline $\begin{array}{l}\text { Design and methods over- } \\
\text { all approach }\end{array}$ & Low risk & Pre-post assessment data were collected \\
\hline $\begin{array}{l}\text { Tools and methods of da- } \\
\text { ta collection reliable/cred- } \\
\text { ible }\end{array}$ & Low risk & Study authors used validated data collection tools \\
\hline $\begin{array}{l}\text { Tools and methods of data } \\
\text { analysis reliable/credible }\end{array}$ & Low risk & Data analysis methods used were suitable for the data \\
\hline $\begin{array}{l}\text { Performance bias/neutral- } \\
\text { ity/credibility/conforma- } \\
\text { bility }\end{array}$ & Unclear risk & Blinding was attempted but was not always successful \\
\hline
\end{tabular}


Cicutto 2013 (Continued)

Reliability of findings and Low risk Clearly reported by study authors. Findings appear to be reliable recommendations

\begin{tabular}{lll}
\hline Transferability of findings & Unclear risk & Not explored \\
\hline $\begin{array}{l}\text { Overall risk of bias of } \\
\text { process evaluation }\end{array}$ & Low risk & $\begin{array}{l}\text { Important indicators, for example, design methods and continuous evalua- } \\
\text { tion, were at low risk }\end{array}$ \\
\hline
\end{tabular}

\section{Clark 2004}

Methods

\section{Included as outcome evaluation}

Study design: clustered parallel-group design with schools selected as the unit of randomisation

Setting: study was conducted at 14 public high schools in Detroit, Michigan, USA. Seven schools (416 children) were assigned to the treatment arm, and 7 schools ( 419 children) to the control group

Period: dates on which study was conducted - intervention and subsequent data collection - were not clear; follow-up data were collected 2 years after baseline

Participants

Eligible sample frame: following a case detection survey, 1217 children were identified as eligible and 835 parents provided consent for their child to participate

Randomised: 835 pupils randomised at the school level: 416 to the treatment group and 419 to the control group

Completed (intervention): unclear; 674 parents participated in follow-up survey 2 years later, but this was not disaggregated and completion figures were not provided

Inclusion criteria: students were eligible for participation in the study if they had: "(1) a physician's diagnosis of asthma and active symptoms, or a diagnosis and received a prescription for asthma medications in the previous year; or (2) no physician's diagnosis, but reported presence of three or more of seven asthma symptoms in the past year, or reported either of two exercise-related asthma symptoms with frequency of three times or more, in the past year"

Exclusion criteria: no additional exclusion criteria reported

\section{Baseline characteristics}

Age of children: all children were in grades 2 through 5; 93\% were between 7 years and 10 years of age

Ethnicity: $98 \%$ of children were African American

Socio-economic status: schools were located in areas of high poverty. Almost half of students (45\%) were from families with annual income under $\$ 15,000$

Gender: no gender breakdown was provided

Asthma status: 236 students had mild persistent asthma (28.3\%), 128 had moderate asthma (15.3\%), and 40 had severe asthma (4.7\%)

Interventions

\section{Intervention}

This was a comprehensive programme of asthma self-management targeted at children, caregivers, and the wider school: "The program elements were as follows: (1) OAS disease management training for children adapted to local needs (for example, related to smoking among elementary school-aged children), which included handouts and homework assignments involving parents; (2) 'Environmental Detective', two classroom sessions for classmates to enhance their understanding of factors that may influence respiratory health in general, and to help them develop empathy for children with asthma in particular; (3) orientation to asthma and control strategies for school principals and counsellors; (4) 
Clark 2004 (Continued)

briefings and building walk-throughs for custodial personnel regarding potential environmental triggers to asthma symptoms and practical means of remediation; (5) school fairs for children and their caretakers, including asthma care question-and-answer sessions for the adults; (6) written communication on behalf of the family with the child's clinician providing information about the school program, encouraging completion of an asthma action plan for the child, and requesting provision of a copy to the school"

Control: wait-list control (usual care)

Intensity: target students received OAS training (which usually consists of $6^{*} 60$-minute sessions) as well as 2 additional classroom sessions through the 'environmental detective' component

Instructor: not reported

Theoretical framework: not explicitly described

Parental engagement: not reported in detail, although elements of the programme reported as having been "completed with reasonable success", except the element that involved written communication with the child's physician

Child satisfaction: not reported

Timing of intervention in school day: not reported

\begin{tabular}{ll}
\hline Outcomes & $\begin{array}{l}\text { Selected outcomes were extracted but were not combined in meta-analyses due to incompatibility of } \\
\text { unit of analysis (risk difference) between this study and others }\end{array}$ \\
\hline Notes & $\begin{array}{l}\text { Absence from school and experience of daytime and night-time symptoms were collected in the study, } \\
\text { but sample sizes disaggregated by study arm that could allow for extraction and inclusion in meta- } \\
\text { analysis were not included }\end{array}$
\end{tabular}

Funding source: not reported

\section{Risk of bias}

\begin{tabular}{lll}
\hline Bias & Authors' judgement & Support for judgement \\
\hline $\begin{array}{l}\text { Random sequence genera- } \\
\text { tion (selection bias) }\end{array}$ & Low risk & $\begin{array}{l}\text { Schools were randomly assigned via a random numbers table to receive the } \\
\text { programme (7 schools and 416 children) or to be assigned to a wait-list control } \\
\text { group (7 schools and 419 children) }\end{array}$ \\
\hline $\begin{array}{l}\text { Allocation concealment } \\
\text { (selection bias) }\end{array}$ & High risk & $\begin{array}{l}\text { Use of pre-defined random number table potentially compromised allocation } \\
\text { concealment }\end{array}$ \\
\hline $\begin{array}{l}\text { Blinding of participants } \\
\text { and personnel (perfor- } \\
\text { mance bias) } \\
\text { All outcomes }\end{array}$ & Unclear risk & $\begin{array}{l}\text { No information was provided on blinding; this was not addressed by study au- } \\
\text { thors }\end{array}$ \\
\hline
\end{tabular}

\begin{tabular}{|c|c|c|}
\hline $\begin{array}{l}\text { Blinding of outcome as- } \\
\text { sessment (detection bias) }\end{array}$ & Unclear risk & $\begin{array}{l}\text { No information was provided on blinding; this was not addressed by study au- } \\
\text { thors }\end{array}$ \\
\hline
\end{tabular}

All outcomes

Incomplete outcome data $\quad$ Unclear risk Unclear - full information on attrition was not presented
(attrition bias)

\begin{tabular}{ll}
\hline $\begin{array}{l}\text { Selective reporting (re- } \\
\text { porting bias) }\end{array}$ & Low risk \\
& $\begin{array}{l}\text { No evidence indicated selective reporting (although the unit of analysis was in- } \\
\text { compatible for the meta-analytical framework implemented) }\end{array}$
\end{tabular}

Other bias Low risk Missingness - unclear - information on missing data was not provided

School-based self-management interventions for asthma in children and adolescents: a mixed methods systematic review (Review) 
Clark 2004 (Continued)

Baseline imbalance - low risk - no differences in characteristics of intervention and control groups were described

Risk of contamination - low - schools were the unit of randomisation, providing low risk of contamination

\begin{tabular}{|c|c|c|}
\hline $\begin{array}{l}\text { Transparent and clearly } \\
\text { stated aims }\end{array}$ & Unclear risk & $\mathrm{N} / \mathrm{A}$ \\
\hline $\begin{array}{l}\text { Explicit theories underpin- } \\
\text { ning and/or literature re- } \\
\text { view }\end{array}$ & Unclear risk & $\mathrm{N} / \mathrm{A}$ \\
\hline $\begin{array}{l}\text { Transparent and clearly } \\
\text { stated methods and tools }\end{array}$ & Unclear risk & $\mathrm{N} / \mathrm{A}$ \\
\hline Selective reporting & Unclear risk & $\mathrm{N} / \mathrm{A}$ \\
\hline Harmful effects & Unclear risk & $\mathrm{N} / \mathrm{A}$ \\
\hline $\begin{array}{l}\text { Population and sample } \\
\text { described well }\end{array}$ & Unclear risk & $\mathrm{N} / \mathrm{A}$ \\
\hline Continuous evaluation & Unclear risk & $\mathrm{N} / \mathrm{A}$ \\
\hline $\begin{array}{l}\text { Evaluation participation } \\
\text { equity and sampling }\end{array}$ & Unclear risk & $\mathrm{N} / \mathrm{A}$ \\
\hline
\end{tabular}

Design and methods over- Unclear risk N/A

all approach

\begin{tabular}{lll}
\hline $\begin{array}{l}\text { Tools and methods of da- } \\
\text { ta collection reliable/cred- } \\
\text { ible }\end{array}$ & Unclear risk & N/A \\
\hline $\begin{array}{l}\text { Tools and methods of data } \\
\text { analysis reliable/credible }\end{array}$ & Unclear risk & N/A \\
\hline $\begin{array}{l}\text { Performance bias/neutral- } \\
\text { ity/credibility/conforma- } \\
\text { bility }\end{array}$ & Unclear risk & N/A \\
\hline $\begin{array}{l}\text { Reliability of findings and } \\
\text { recommendations }\end{array}$ & Unclear risk & N/A \\
\hline $\begin{array}{l}\text { Transferability of findings } \\
\text { Unclear risk }\end{array}$ & N/A \\
\hline $\begin{array}{l}\text { Overall risk of bias of } \\
\text { process evaluation }\end{array}$ & Unclear risk & N/A \\
\hline
\end{tabular}

Clark 2005

Methods Included as outcome evaluation

Intervention study design: clustered parallel-group randomised controlled trial with schools selected as the unit of randomisation; "schools similar in size and student body within the same district were randomly assigned to either the intervention or control group" 
Setting: study was conducted at 21 elementary schools in 1 agricultural area and 1 industrial area of Beijing, China

Period: not clear

Length: dates on which study was conducted - intervention and subsequent data collection - not clear

Participants
Eligible sample frame: 9040 parents of children surveyed in case detection survey, with 8724 returning a questionnaire, revealing 639 children with a diagnosis of asthma

Randomised: 639 children randomised according to their school (note numbers of children in intervention and control groups were not provided)

Completed (intervention): unclear; 543 parents of children returned follow-up questionnaire a year after start (note numbers of children in intervention and control groups were not provided)

Inclusion criteria: children eligible if they had "(1) three or more of seven asthma symptoms reported in the past year; (2) one or more of two exercise symptoms reported three times or more in the past year; and (3) a physician's diagnosis of asthma, with any symptoms reported or medication prescribed in the past year"

Exclusion criteria: not directly reported

\section{Baseline characteristics}

Age of children: all 7 to 11 years of age

Ethnicity: not reported

Socio-economic status: not reported

Gender: not reported

Asthma status: mixed severity: "29\% of the children were classified as having mild (20\%) or moderate persistent asthma (9\%) and $71 \%$ as having mild intermittent asthma. No severe persistent asthma was noted"
Interventions

Intervention: based on Open Airways for Schools (OAS) model. The OAS programme manual and teaching materials were translated into Chinese and adapted for specifics related to the Beijing idiom. Some changes were made, but researchers reported that the substantial focus of the programme was not modified. Topics covered included "basic information/feelings about asthma, recognising and managing asthma symptoms, solving problems with medicines, deciding severity of symptoms, finding and controlling asthma triggers, staying healthy and doing well at school"

Control: not clearly stated (usual care)

Intensity: 5 sessions over a 5-week period, each lasting approximately 25 minutes. Sessions were split according to children's age/grade

Instructor: teachers provided the programme in schools and were trained in advance

Theoretical Framework: social cognitive theory, especially principles of self-regulation

Parental engagement: no info

Child satisfaction: no info

Timing: children met for the programme at the end of the class day

Outcomes

Exacerbations leading to hospital admission

Asthma symptoms leading to emergency hospital visits 
Clark 2005 (Continued)

Notes

Unclear how many participants were included in intervention and control groups

Quality of life and withdrawal data collected but not extractable from the information presented

Funding source: Thrasher Fund Award

\section{Risk of bias}

\begin{tabular}{|c|c|c|}
\hline Bias & Authors' judgement & Support for judgement \\
\hline $\begin{array}{l}\text { Random sequence genera- } \\
\text { tion (selection bias) }\end{array}$ & Unclear risk & No additional information was provided to support a judgement \\
\hline $\begin{array}{l}\text { Allocation concealment } \\
\text { (selection bias) }\end{array}$ & Unclear risk & No additional information was provided to support a judgement \\
\hline $\begin{array}{l}\text { Blinding of participants } \\
\text { and personnel (perfor- } \\
\text { mance bias) } \\
\text { All outcomes }\end{array}$ & Unclear risk & No additional information was provided to support a judgement \\
\hline $\begin{array}{l}\text { Blinding of outcome as- } \\
\text { sessment (detection bias) } \\
\text { All outcomes }\end{array}$ & Unclear risk & No additional information was provided to support a judgement \\
\hline $\begin{array}{l}\text { Incomplete outcome data } \\
\text { (attrition bias) } \\
\text { All outcomes }\end{array}$ & Unclear risk & $\begin{array}{l}\text { No information was provided to support a judgement. Around } 100 \text { children } \\
\text { dropped out of the intervention (although the spread of these children across } \\
\text { treatment arms remains unclear) }\end{array}$ \\
\hline $\begin{array}{l}\text { Selective reporting (re- } \\
\text { porting bias) }\end{array}$ & High risk & Many outcomes could not be extracted in full for meta-analyses \\
\hline Other bias & Unclear risk & $\begin{array}{l}\text { Missingness - unclear risk - additional reports of missingness were not de- } \\
\text { scribed by study authors } \\
\text { Baseline imbalance - unclear risk - this was not addressed by study authors } \\
\text { Risk of contamination - low - schools were the unit of randomisation, lowering } \\
\text { the risk of contamination }\end{array}$ \\
\hline $\begin{array}{l}\text { Transparent and clearly } \\
\text { stated aims }\end{array}$ & Unclear risk & $\mathrm{N} / \mathrm{A}$ \\
\hline $\begin{array}{l}\text { Explicit theories underpin- } \\
\text { ning and/or literature re- } \\
\text { view }\end{array}$ & Unclear risk & $\mathrm{N} / \mathrm{A}$ \\
\hline $\begin{array}{l}\text { Transparent and clearly } \\
\text { stated methods and tools }\end{array}$ & Unclear risk & $\mathrm{N} / \mathrm{A}$ \\
\hline Selective reporting & Unclear risk & $\mathrm{N} / \mathrm{A}$ \\
\hline Harmful effects & Unclear risk & $\mathrm{N} / \mathrm{A}$ \\
\hline $\begin{array}{l}\text { Population and sample } \\
\text { described well }\end{array}$ & Unclear risk & N/A \\
\hline Continuous evaluation & Unclear risk & $\mathrm{N} / \mathrm{A}$ \\
\hline
\end{tabular}


Clark 2005 (Continued)

\begin{tabular}{lll}
$\begin{array}{l}\text { Evaluation participation } \\
\text { equity and sampling }\end{array}$ & Unclear risk & N/A \\
\hline $\begin{array}{l}\text { Design and methods over- } \\
\text { all approach }\end{array}$ & Unclear risk & N/A \\
\hline $\begin{array}{l}\text { Tools and methods of da- } \\
\text { ta collection reliable/cred- } \\
\text { ible }\end{array}$ & Unclear risk & \\
\hline $\begin{array}{l}\text { Tools and methods of data } \\
\text { analysis reliable/credible }\end{array}$ & Unclear risk & N/A \\
\hline $\begin{array}{l}\text { Performance bias/neutral- } \\
\text { ity/credibility/conforma- } \\
\text { bility }\end{array}$ & Unclear risk & N/A \\
\hline $\begin{array}{l}\text { Reliability of findings and } \\
\text { recommendations }\end{array}$ & Unclear risk & N/A \\
\hline $\begin{array}{l}\text { Transferability of findings } \\
\text { Overall risk of bias of } \\
\text { process evaluation }\end{array}$ & Unclear risk & N/A \\
\hline
\end{tabular}

Clark 2010

Methods Included as outcome evaluation

Study design: clustered parallel-group design with schools selected as the unit of randomisation

Setting: study was conducted at 19 middle schools in Detroit, Michigan, USA. Seven schools were randomised to one of the treatment arms, 6 schools to another treatment arm, and 6 schools to a control arm

Period: this was a 5-year study with a 2-year enrolment period starting in 2003

Eligible sample frame: through a case detection survey, 1292 students were identified as probably having asthma (and eligible for the intervention)

Randomised: students randomised at the school level - 468 pupils in one treatment arm, 416 in a second treatment arm, and 408 in a control arm

Completed (intervention): data from 921 pupils were collected at 12-month follow-up; disaggregated numbers by trial arm were not reported

Inclusion criteria: students were eligible for participation in the study if they met the "definition of probable asthma as well as levels of severity based on NAEPP guidelines"

Exclusion criteria: no further criteria reported

\section{Baseline characteristics}

Age of children: mean age, 11.6 years across all 3 trial arms

Ethnicity: $93 \%$ of children involved in the study were African American; this varied between $90 \%$ and $98 \%$ across trial arms 
Socio-economic status: a large proportion of children were from low-income households - $48 \%$ of all children (44\% to $50 \%$ across different arms) were from households with annual income under $\$ 15,000$ per annum

Gender: $48 \%$ of children were female (ranging from $46 \%$ to $50 \%$ across different trial arms)

Asthma status: indicators of asthma severity at baseline included $58 \%$ of children (caregivers) reporting obtaining prescriptions for asthma medications in the past year and $52 \%$ reporting persistent nighttime asthma

Interventions

Intervention: trial included 2 treatment arms and 1 control arm. Both treatment arms tested the effectiveness of different forms of Open Airways for Schools (OAS): Treatment arm 1: adapted form of OAS delivered as a 6- to 7-lesson curriculum including interactive problem-solving activities appropriate for groups of pre-teens. Topics included (I) basic information and feelings about asthma; (ii) facts about asthma medicines and their use (emphasising partnership with the physician); (iii) how to make good decisions about activities; (iv) how to manage an asthma attack at home or school, deciding when to go to the doctor, and making the doctor visit more effective; (v) how to keep yourself healthy, including smoking avoidance; and (vi) personal characteristics, actions, and environmental factors that lead to successful asthma control. Groups of pre-teens met during school hours for approximately $1 \frac{1}{2}$ hours each week for 6 weeks. Take-home assignments and handout materials for parents were provided at each session

Treatment arm 2: included the adapted OAS above and a peer education component. Peer leaders were sought from the general population of eighth grade students and were trained by project staff to provide 3 asthma awareness lessons to seventh grade students. Two to three peer leaders were trained as a team. Peer leaders developed skits and game shows as part of training to impart an important message about asthma. Teams of peer leaders taught the 3 asthma awareness lessons to seventh grade students. Participants discussed a video, played games demonstrating and testing asthma knowledge, and discussed barriers to self-management. Finally, younger students voted on key messages to communicate to sixth grade schoolmates. In step 3, with help from peer leaders, project staff, and a teacher, seventh grade students translated asthma messages into skits, songs, and dramas, and performed these for an assembly of sixth grade students. All 3 steps focused on enabling students to understand and support their classmates with asthma

Control: treatment not described (usual care)

Intensity: students in treatment group 1 received 6 lessons; students in treatment group 2 received the same 6 lessons and additional input that differed depending on school grade

Instructor: OAS sessions were led by graduate students and community leaders who were trained in programme methods and approach; in treatment arm 2, some components may have been delivered directly by peers

Theoretical framework: not explicitly discussed

Parental engagement: not reported

Child satisfaction: not reported

Timing of intervention in school day: described as during school day (not after school), but exact timing not reported

Outcomes Data on daytime and night-time symptoms were extracted

Notes

Health-related quality of life and experience of daytime and night-time symptoms were collected, but data needed for extraction and inclusion in meta-analysis were not presented

Considered as a process evaluation: did not include implementation research questions nor in-depth process or contextual information (did not meet the criteria for a process evaluation)

Funding source: not reported 
Clark 2010 (Continued)

\section{Risk of bias}

\begin{tabular}{lll}
\hline Bias & Authors' judgement & Support for judgement \\
\hline $\begin{array}{l}\text { Random sequence genera- } \\
\text { tion (selection bias) }\end{array}$ & Low risk & $\begin{array}{l}\text { Schools were stratified by geographical location and disease prevalence to en- } \\
\text { sure homogeneity across groups. Within each of the resulting } 4 \text { strata, schools } \\
\text { were randomised via a table of random numbers }\end{array}$
\end{tabular}

\begin{tabular}{|c|c|c|}
\hline $\begin{array}{l}\text { Allocation concealment } \\
\text { (selection bias) }\end{array}$ & High risk & $\begin{array}{l}\text { Potential for concealment to be breached through open random numbers ta- } \\
\text { ble }\end{array}$ \\
\hline
\end{tabular}

Blinding of participants Unclear risk Unclear which measures were taken to ensure blinding of participants
and personnel (perfor-
mance bias)
All outcomes

\begin{tabular}{|c|c|c|}
\hline $\begin{array}{l}\text { Blinding of outcome as- } \\
\text { sessment (detection bias) } \\
\text { All outcomes }\end{array}$ & High risk & $\begin{array}{l}\text { Likely any blinding of interviewers would have been breached through da- } \\
\text { ta collection methods: data were collected from school records and through } \\
\text { face-to-face interviews with students at each school }\end{array}$ \\
\hline
\end{tabular}

\begin{tabular}{ll}
\hline $\begin{array}{l}\text { Incomplete outcome data } \\
\text { (attrition bias) }\end{array}$ & $\begin{array}{l}\text { Missing data and attrition did occur - but impacts were said to have been at- } \\
\text { tenuated through multiple imputations }\end{array}$ \\
All outcomes &
\end{tabular}

\begin{tabular}{|c|c|c|}
\hline $\begin{array}{l}\text { Selective reporting (re- } \\
\text { porting bias) }\end{array}$ & High risk & $\begin{array}{l}\text { Risk of selective reporting was high, as outcomes were not reported in full and } \\
\text { we were not able to extract several outcomes of interest }\end{array}$ \\
\hline \multirow[t]{3}{*}{ Other bias } & Unclear risk & $\begin{array}{l}\text { Missingness - unclear risk - missingness was present, but multiple imputations } \\
\text { were implemented and impact is unknown }\end{array}$ \\
\hline & & $\begin{array}{l}\text { Baseline imbalance - high risk - at baseline, despite randomisation, one inter- } \\
\text { vention arm had lower grades than controls. This may impact responsiveness } \\
\text { to the intervention }\end{array}$ \\
\hline & & Risk of contamination - low - schools were the unit of analysis \\
\hline
\end{tabular}

\begin{tabular}{|c|c|c|}
\hline $\begin{array}{l}\text { Transparent and clearly } \\
\text { stated aims }\end{array}$ & Unclear risk & $\mathrm{N} / \mathrm{A}$ \\
\hline $\begin{array}{l}\text { Explicit theories underpin- } \\
\text { ning and/or literature re- } \\
\text { view }\end{array}$ & Unclear risk & $\mathrm{N} / \mathrm{A}$ \\
\hline $\begin{array}{l}\text { Transparent and clearly } \\
\text { stated methods and tools }\end{array}$ & Unclear risk & $\mathrm{N} / \mathrm{A}$ \\
\hline Selective reporting & Unclear risk & $\mathrm{N} / \mathrm{A}$ \\
\hline Harmful effects & Unclear risk & $\mathrm{N} / \mathrm{A}$ \\
\hline $\begin{array}{l}\text { Population and sample } \\
\text { described well }\end{array}$ & Unclear risk & $\mathrm{N} / \mathrm{A}$ \\
\hline Continuous evaluation & Unclear risk & $\mathrm{N} / \mathrm{A}$ \\
\hline $\begin{array}{l}\text { Evaluation participation } \\
\text { equity and sampling }\end{array}$ & Unclear risk & $\mathrm{N} / \mathrm{A}$ \\
\hline
\end{tabular}


Clark 2010 (Continued)

\begin{tabular}{lll}
$\begin{array}{l}\text { Design and methods over- } \\
\text { all approach }\end{array}$ & Unclear risk & N/A \\
\hline $\begin{array}{l}\text { Tools and methods of da- } \\
\text { ta collection reliable/cred- } \\
\text { ible }\end{array}$ & Unclear risk & N/A \\
\hline $\begin{array}{l}\text { Tools and methods of data } \\
\text { analysis reliable/credible }\end{array}$ & Unclear risk & N/A \\
\hline $\begin{array}{l}\text { Performance bias/neutral- } \\
\text { ity/credibility/conforma- } \\
\text { bility }\end{array}$ & Unclear risk & \\
\hline $\begin{array}{l}\text { Reliability of findings and } \\
\text { recommendations }\end{array}$ & Unclear risk & N/A \\
\hline $\begin{array}{l}\text { Transferability of findings } \\
\text { Unclear risk }\end{array}$ & N/A \\
\hline $\begin{array}{l}\text { Overall risk of bias of } \\
\text { process evaluation }\end{array}$ & Unclear risk & N/A \\
\hline
\end{tabular}

Crane 2014

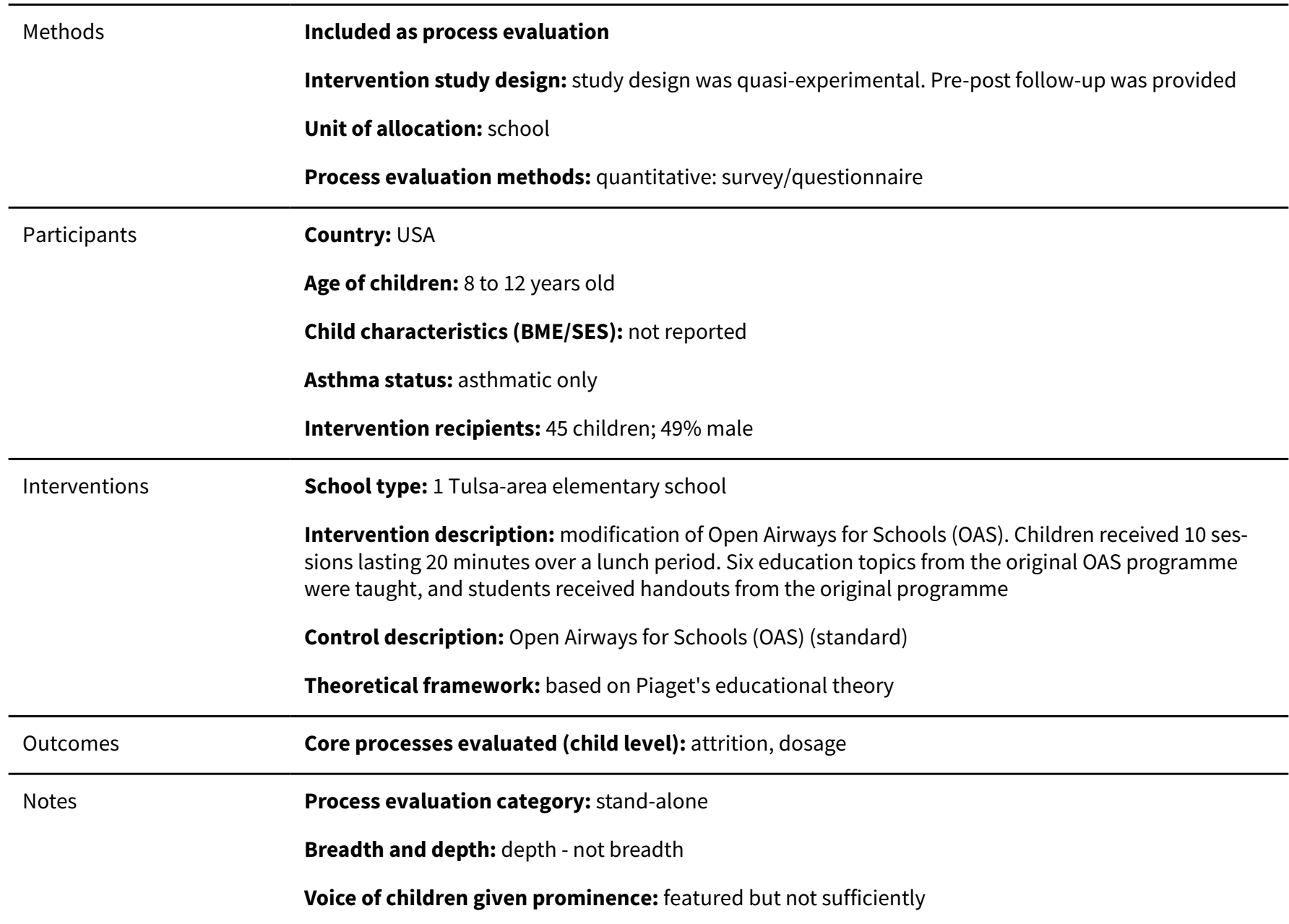


Funding source: not reported

\section{Risk of bias}

\begin{tabular}{|c|c|c|}
\hline Bias & Authors' judgement & Support for judgement \\
\hline $\begin{array}{l}\text { Random sequence genera- } \\
\text { tion (selection bias) }\end{array}$ & Unclear risk & $\mathrm{N} / \mathrm{A}$ \\
\hline $\begin{array}{l}\text { Allocation concealment } \\
\text { (selection bias) }\end{array}$ & Unclear risk & $\mathrm{N} / \mathrm{A}$ \\
\hline $\begin{array}{l}\text { Blinding of participants } \\
\text { and personnel (perfor- } \\
\text { mance bias) } \\
\text { All outcomes }\end{array}$ & Unclear risk & $\mathrm{N} / \mathrm{A}$ \\
\hline $\begin{array}{l}\text { Blinding of outcome as- } \\
\text { sessment (detection bias) } \\
\text { All outcomes }\end{array}$ & Unclear risk & $\mathrm{N} / \mathrm{A}$ \\
\hline $\begin{array}{l}\text { Incomplete outcome data } \\
\text { (attrition bias) } \\
\text { All outcomes }\end{array}$ & Unclear risk & $\mathrm{N} / \mathrm{A}$ \\
\hline $\begin{array}{l}\text { Selective reporting (re- } \\
\text { porting bias) }\end{array}$ & Unclear risk & $\mathrm{N} / \mathrm{A}$ \\
\hline Other bias & Unclear risk & $\mathrm{N} / \mathrm{A}$ \\
\hline $\begin{array}{l}\text { Transparent and clearly } \\
\text { stated aims }\end{array}$ & Low risk & Study aims were clearly stated \\
\hline
\end{tabular}

Explicit theories underpin- Low risk Based on the educational theory of Jean Piaget
ning and/or literature review

\begin{tabular}{lll}
\hline $\begin{array}{l}\text { Transparent and clearly } \\
\text { stated methods and tools }\end{array}$ & Low risk & Methods and tools were well described \\
\hline Selective reporting & Low risk & What was purported to be measured was included in the report \\
\hline Harmful effects & Unclear risk & $\begin{array}{l}\text { Harmful effects were discussed, for example, time-tabling issues and conflicts. } \\
\text { However, harmful effects were not collected by structured means }\end{array}$ \\
\hline $\begin{array}{l}\text { Population and sample } \\
\text { described well }\end{array}$ & Unclear risk & Information around asthma burden and ethnicity was not collected \\
\hline $\begin{array}{l}\text { Continuous evaluation } \\
\text { Evaluation participation } \\
\text { equity and sampling }\end{array}$ & Unclear risk & Only 1 drop out was reported; however relevant data were not collected \\
\hline $\begin{array}{l}\text { Design and methods over- } \\
\text { all approach }\end{array}$ & Unclear risk & Not all stakeholders were included in the evaluation \\
\hline
\end{tabular}


Crane 2014 (Continued)

Tools and methods of da- Low risk All data collection methods and tools were reliable ta collection reliable/credible

Tools and methods of data Low risk Data analysis methods were credible for the data
analysis reliable/credible

\begin{tabular}{ll}
\hline $\begin{array}{l}\text { Performance bias/neutral- } \\
\text { ity/credibility/conforma- }\end{array}$ & Unclear risk
\end{tabular}
ity/credibility/conformability

Reliability of findings and Low risk Findings of the process evaluation were sufficiently supported by the data
recommendations

\begin{tabular}{lll}
\hline Transferability of findings & High risk & Small sample size makes it difficult for findings to be transferable \\
\hline $\begin{array}{l}\text { Overall risk of bias of } \\
\text { process evaluation }\end{array}$ & Unclear risk & $\begin{array}{l}\text { The narrow confines of the focus probably account for medium risk when } \\
\text { viewed as a 'process evaluation' }\end{array}$ \\
\hline
\end{tabular}

Dore-Stites 2007

$\begin{array}{ll}\text { Methods } & \text { Included as process evaluation } \\ & \text { Intervention study design: quasi-experimental single-group intervention examining change pre-post } \\ \text { intervention }\end{array}$

Unit of allocation: N/A

Process evaluation methods: descriptive/bivariate (hypothesis testing)

Setting: 5 elementary schools in the Detroit area, USA
Age of children: 5 to 10 years (mean age, 9.1 years)
Child characteristics (BME/SES): 39\% African American, $14 \%$ Hispanic, and $18 \%$ mixed ethnicity chil-
dren; $34.6 \%$ were from low-income families (< USD20,000 per annum)

Asthma status: asthmatic only

Intervention recipients: children and parents

Interventions School type: primary/elementary

Intervention description: treatment consisted of 3 components: a computer-based educational game (Asthma: Quest for the Code), home activities, and caregiver information. The computer game included modules on: Lung Physiology; Symptom Recognition; Trigger Recognition; Peak Flow Meter Usage; Appropriate Use of Long-Term and Reliever Medication; Correct Usage of Common Asthma Medication; and Effect of Asthma Medications on Lungs. The study author reports: "The self-directed activities spanned from 10 to 20 minutes per session and were presented to participants individually during their school day. Modules were embedded within a larger, multiphase game and advancement to the next level occurred contingent upon answering quiz questions correctly. Children completed one or two modules per session dependent upon progression through each section and academic schedule. At a minimum, an individual participant could complete all activities in Asthma: Quest for the Code in approximately 1.25 hours although typically children utilised the CD-ROM for approximately 20 minutes once per week over the course of eight to nine weeks for an approximate total time of 2.5 hours in instruction"

Control description: N/A 
Dore-Stites 2007 (Continued)

Theoretical framework: not 1 overarching theory utilised, but the study draws upon several theoreti-

cal standpoints

\begin{tabular}{ll}
\hline Outcomes Core processes evaluated (child level): attrition \\
\hline
\end{tabular}

Process evaluation category: integrated
Breadth and depth: neither broad nor deep
Voice of children given prominence: sufficient coverage
Funding source: Blue Cross Blue Shield Foundation

Risk of bias

\begin{tabular}{|c|c|c|}
\hline Bias & Authors' judgement & Support for judgement \\
\hline $\begin{array}{l}\text { Random sequence genera- } \\
\text { tion (selection bias) }\end{array}$ & Unclear risk & $\mathrm{N} / \mathrm{A}$ \\
\hline $\begin{array}{l}\text { Allocation concealment } \\
\text { (selection bias) }\end{array}$ & Unclear risk & $\mathrm{N} / \mathrm{A}$ \\
\hline $\begin{array}{l}\text { Blinding of participants } \\
\text { and personnel (perfor- } \\
\text { mance bias) } \\
\text { All outcomes }\end{array}$ & Unclear risk & $\mathrm{N} / \mathrm{A}$ \\
\hline $\begin{array}{l}\text { Blinding of outcome as- } \\
\text { sessment (detection bias) } \\
\text { All outcomes }\end{array}$ & Unclear risk & N/A \\
\hline $\begin{array}{l}\text { Incomplete outcome data } \\
\text { (attrition bias) } \\
\text { All outcomes }\end{array}$ & Unclear risk & $\mathrm{N} / \mathrm{A}$ \\
\hline $\begin{array}{l}\text { Selective reporting (re- } \\
\text { porting bias) }\end{array}$ & Unclear risk & $\mathrm{N} / \mathrm{A}$ \\
\hline Other bias & Unclear risk & $\mathrm{N} / \mathrm{A}$ \\
\hline $\begin{array}{l}\text { Transparent and clearly } \\
\text { stated aims }\end{array}$ & Low risk & Study aims were clearly stated and were related to exploring feasibility \\
\hline $\begin{array}{l}\text { Explicit theories underpin- } \\
\text { ning and/or literature re- } \\
\text { view }\end{array}$ & Unclear risk & $\begin{array}{l}\text { Sufficent literature was provided to support the study, drawing upon several } \\
\text { theoretical standpoints }\end{array}$ \\
\hline $\begin{array}{l}\text { Transparent and clearly } \\
\text { stated methods and tools }\end{array}$ & Low risk & Methods and tools were clearly stated \\
\hline Selective reporting & Unclear risk & $\begin{array}{l}\text { Parental engagement was a key component of the study but was deemed to } \\
\text { have not been reported in full }\end{array}$ \\
\hline Harmful effects & High risk & $\begin{array}{l}\text { Not fully addressed - implementation data on the support needed to get chil- } \\
\text { dren to play the game were lacking }\end{array}$ \\
\hline $\begin{array}{l}\text { Population and sample } \\
\text { described well }\end{array}$ & Unclear risk & Caregiver demographics were not reported in full \\
\hline
\end{tabular}


Dore-Stites 2007 (Continued)

\begin{tabular}{lll} 
Continuous evaluation & Low risk & A pre-post design was utilised \\
\hline $\begin{array}{l}\text { Evaluation participation } \\
\text { equity and sampling }\end{array}$ & Low risk & $\begin{array}{l}\text { All participants involved in the programme had the opportunity to participate } \\
\text { in the evaluation }\end{array}$
\end{tabular}

Design and methods over- Unclear risk This was reported; however not much information was provided
all approach

\begin{tabular}{lll}
\hline $\begin{array}{l}\text { Tools and methods of da- } \\
\text { ta collection reliable/cred- } \\
\text { ible }\end{array}$ & Low risk & Tools that were used were well described and were recognised tools \\
\hline $\begin{array}{l}\text { Tools and methods of data } \\
\text { analysis reliable/credible }\end{array}$ & Low risk & $\begin{array}{l}\text { Analytical plan seems to be appropriate for the data; however it is unclear how } \\
\text { (or if) the hierarchical nature of the data was accounted for }\end{array}$ \\
\hline $\begin{array}{l}\text { Performance bias/neutral- } \\
\text { ity/credibility/conforma- } \\
\text { bility }\end{array}$ & Unclear risk & Not addressed by the study author \\
\hline $\begin{array}{l}\text { Reliability of findings and } \\
\text { recommendations }\end{array}$ & High risk & The study sample was very small, reducing the reliability of study findings \\
\hline $\begin{array}{l}\text { Transferability of findings } \\
\text { High risk }\end{array}$ & High risk & $\begin{array}{l}\text { Transferability of findings is unclear; however given the small sample size, } \\
\text { transferability of study findings should be limited }\end{array}$ \\
\hline $\begin{array}{l}\text { Overall risk of bias of } \\
\text { process evaluation }\end{array}$ & $\begin{array}{l}\text { Data regarding dosage and adherence are limited. Moreover, the small sample } \\
\text { size compromised the study as a whole }\end{array}$ \\
\hline
\end{tabular}

Engelke 2013

$\begin{array}{ll}\text { Methods } & \text { Included as process evaluation } \\ \text { Intervention study design: quasi-experimental, pre-post, no control } \\ \text { Unit of allocation: N/A } \\ \text { Process evaluation methods: bivariate analysis }\end{array}$

Participants

Country: USA

Age of children: 143 children in grades 1 to 12

Child characteristics (BME/SES): $40.6 \%$ of children were Caucasian; $50.3 \%$ were male; $63.6 \%$ received Medicaid

Asthma status: asthmatic only

Intervention recipients: children, teachers, parents, nurses

School type: 303 schools, including junior/middle, primary/elementary, and high schools, from 24 school districts participated

Intervention description: interventions were divided into 5 categories, including direct care, student education/counselling, parent/family education, teacher/staff education, and healthcare co-ordination. After initial assessment, the nurse chose an individual goal for each student

Control description: N/A 
Engelke 2013 (Continued)

Theoretical framework: case management theory

\begin{tabular}{ll}
\hline Outcomes & Core processes evaluated (child level): not reported \\
\hline Notes & Process evaluation category: named section \\
& Breadth and depth: depth - not breadth \\
& Voice of children given prominence: not featured \\
& Funding source: Kate B. Reynolds Charitable Trust
\end{tabular}

\section{Risk of bias}

\begin{tabular}{|c|c|c|}
\hline Bias & Authors' judgement & Support for judgement \\
\hline $\begin{array}{l}\text { Random sequence genera- } \\
\text { tion (selection bias) }\end{array}$ & Unclear risk & $\mathrm{N} / \mathrm{A}$ \\
\hline $\begin{array}{l}\text { Allocation concealment } \\
\text { (selection bias) }\end{array}$ & Unclear risk & $\mathrm{N} / \mathrm{A}$ \\
\hline $\begin{array}{l}\text { Blinding of participants } \\
\text { and personnel (perfor- } \\
\text { mance bias) } \\
\text { All outcomes }\end{array}$ & Unclear risk & $\mathrm{N} / \mathrm{A}$ \\
\hline $\begin{array}{l}\text { Blinding of outcome as- } \\
\text { sessment (detection bias) } \\
\text { All outcomes }\end{array}$ & Unclear risk & $\mathrm{N} / \mathrm{A}$ \\
\hline $\begin{array}{l}\text { Incomplete outcome data } \\
\text { (attrition bias) } \\
\text { All outcomes }\end{array}$ & Unclear risk & $\mathrm{N} / \mathrm{A}$ \\
\hline $\begin{array}{l}\text { Selective reporting (re- } \\
\text { porting bias) }\end{array}$ & Unclear risk & $\mathrm{N} / \mathrm{A}$ \\
\hline Other bias & Unclear risk & $\mathrm{N} / \mathrm{A}$ \\
\hline $\begin{array}{l}\text { Transparent and clearly } \\
\text { stated aims }\end{array}$ & Low risk & Study aims were clearly stated \\
\hline $\begin{array}{l}\text { Explicit theories underpin- } \\
\text { ning and/or literature re- } \\
\text { view }\end{array}$ & Unclear risk & $\begin{array}{l}\text { Literature around case management was provided; however the actual theory } \\
\text { remains unclear }\end{array}$ \\
\hline $\begin{array}{l}\text { Transparent and clearly } \\
\text { stated methods and tools }\end{array}$ & Unclear risk & Instruments used are not entirely clear, for example, scoring and analysis tools \\
\hline Selective reporting & Low risk & All outcomes were reported on satisfactorily \\
\hline Harmful effects & High risk & $\begin{array}{l}\text { Any negative effects of the intervention on the nurse workload were not ad- } \\
\text { dressed }\end{array}$ \\
\hline $\begin{array}{l}\text { Population and sample } \\
\text { described well }\end{array}$ & Low risk & Study population was adequately described \\
\hline
\end{tabular}


Engelke 2013 (Continued)

\begin{tabular}{lll} 
Continuous evaluation & Low risk & Pre-post assessment findings were reported \\
\hline $\begin{array}{l}\text { Evaluation participation } \\
\text { equity and sampling }\end{array}$ & Unclear risk & Voice of the children was not included in the evaluation \\
\hline
\end{tabular}

Design and methods over- Low risk Parent and nurse reports were collected twice

all approach

\begin{tabular}{lll}
\hline $\begin{array}{l}\text { Tools and methods of da- } \\
\text { ta collection reliable/cred- } \\
\text { ible }\end{array}$ & Unclear risk & It is unclear if the analytical method or the tool itself was reliable \\
\hline $\begin{array}{l}\text { Tools and methods of data } \\
\text { analysis reliable/credible }\end{array}$ & Unclear risk & It is unclear if the analytical method or the tool itself was reliable \\
\hline $\begin{array}{l}\text { Performance bias/neutral- } \\
\text { ity/credibility/conforma- } \\
\text { bility }\end{array}$ & High risk & No steps were taken to reduce this \\
\hline $\begin{array}{l}\text { Reliability of findings and } \\
\text { recommendations }\end{array}$ & Unclear risk & Multi-variate analysis was not used \\
\hline $\begin{array}{l}\text { Transferability of findings } \\
\text { Unclear risk }\end{array}$ & $\begin{array}{l}\text { School factors are unclear, for example, what kinds of schools were used and } \\
\text { what types of findings were obtained }\end{array}$ \\
\hline $\begin{array}{l}\text { Overall risk of bias of } \\
\text { process evaluation }\end{array}$ & Unclear risk & $\begin{array}{l}\text { Because many classifications were unclear, it is difficult to categorise this trial; } \\
\text { however as a process evaluation, this is not a bad study }\end{array}$ \\
\hline
\end{tabular}

\section{Gerald 2006}

Methods

\section{Included as outcome evaluation and process evaluation}

Study design: parallel-group design. The study was split across cohorts, and schools within cohorts were randomised

Setting: the study was conducted at 54 elementary schools in Birmingham, Alabama, USA

Period: the intervention was implemented over 3 years, with 1 cohort receiving the intervention each year

\section{Participants}

Eligible sample frame: 736 were enrolled into the study

Randomised: 736 pupils enrolled, but unclear how many were included in each arm

Completed (intervention): not reported

Inclusion criteria: children identified by the case detection procedure as having previously diagnosed asthma or suspected asthma

Exclusion criteria: not reported

\section{Baseline characteristics}

Age of children: elementary school age - grades 1 to 4 (even split)

Ethnicity: $97 \%$ black ethnicity

Socio-economic status: not reported. 
Gerald 2006 (Continued)

Gender: $56 \%$ and $52 \%$ of participants were male in intervention and control groups, respectively

Asthma status: not reported

Interventions

Intervention: the intervention consisted of 3 separate educational programmes and medical management for children with asthma: asthma education for school faculty and staff (Managing Asthma: A Guide for Schools), for the general student body (Asthma Awareness: A Curriculum for the Elementary School Classroom), and for students with asthma (Open Airways for Schools). Education was provided for school faculty and staff at an in-service meeting. Asthma awareness classes were provided to all children at each elementary school. Content was modified slightly for children younger than age 8

Control: delayed intervention, but usual care at the time of data collection

Intensity: the Open Airways for Schools programme consists of six 40-minute sessions

Instructor: in the first cohort, teachers were trained to deliver sessions. In the second cohort, classes were taught by study personnel

Theoretical framework: not reported

Parental engagement: parental satisfaction was low. Parents either did not attend scheduled visits or did not return completed questionnaires

Child satisfaction: not reported

Timing of intervention in school day: during physical education periods

Outcomes

Core processes evaluated: attrition, dosage, adherence

Extractable outcomes were collected for:

Exacerbations leading to admission to hospital

Asthma symptoms leading to an emergency hospital visit

Parent-reported absence from school
Process evaluation category: named section(s)/integrated within the study

Breadth and depth: neither broad nor deep

Voice of children given prominence: not featured directly

Funding source: National Heart, Lung, and Blood Institute

\section{Risk of bias}

\begin{tabular}{lll}
\hline Bias & Authors' judgement & Support for judgement \\
\hline $\begin{array}{l}\text { Random sequence genera- } \\
\text { tion (selection bias) }\end{array}$ & Unclear risk & How schools were randomised was not described \\
\hline $\begin{array}{l}\text { Allocation concealment } \\
\text { (selection bias) }\end{array}$ & Unclear risk & Not addressed by study authors \\
\hline $\begin{array}{l}\text { Blinding of participants } \\
\text { and personnel (perfor- } \\
\text { mance bias) }\end{array}$ & Unclear risk & Not addressed by study authors \\
All outcomes & & \\
\hline
\end{tabular}

$\begin{array}{lll}\text { Blinding of outcome as- } & \text { Unclear risk } & \text { Not addressed by study authors } \\ \text { sessment (detection bias) }\end{array}$


Gerald 2006 (Continued)

All outcomes

\begin{tabular}{|c|c|}
\hline Incomplete outcome data & Unclear risk \\
\hline
\end{tabular}

(attrition bias)

All outcomes

\begin{tabular}{|c|c|c|}
\hline $\begin{array}{l}\text { Selective reporting (re- } \\
\text { porting bias) }\end{array}$ & Low risk & No evidence shows selective reporting \\
\hline \multirow[t]{3}{*}{ Other bias } & Low risk & $\begin{array}{l}\text { Misssingness - unclear risk - unclear the extent to which missing data were due } \\
\text { to attrition or to additional survey non-response }\end{array}$ \\
\hline & & $\begin{array}{l}\text { Baseline imbalance - low risk - both arms were balanced on characteristics of } \\
\text { importance }\end{array}$ \\
\hline & & Risk of contamination - low - schools were the unit of randomisation \\
\hline
\end{tabular}

Transparent and clearly $\quad$ Unclear risk $\quad$ Medium bias - study aims are not explicitly clear
stated aims

\begin{tabular}{|c|c|c|}
\hline $\begin{array}{l}\text { Explicit theories underpin- } \\
\text { ning and/or literature re- } \\
\text { view }\end{array}$ & High risk & No theoretical framework was presented \\
\hline $\begin{array}{l}\text { Transparent and clearly } \\
\text { stated methods and tools }\end{array}$ & Low risk & Methods were clearly reported \\
\hline Selective reporting & Unclear risk & $\begin{array}{l}\text { Many aspects did not match the plan, although the tools used to collect this in- } \\
\text { formation were not entirely structured }\end{array}$ \\
\hline Harmful effects & Low risk & Difficulty in maintaining fidelity was discussed \\
\hline $\begin{array}{l}\text { Population and sample } \\
\text { described well }\end{array}$ & High risk & Unclear how many dropped out of intervention and treatment groups \\
\hline Continuous evaluation & Unclear risk & Medium bias - use of administrative records lowered the risk of bias \\
\hline $\begin{array}{l}\text { Evaluation participation } \\
\text { equity and sampling }\end{array}$ & High risk & $\begin{array}{l}\text { The voices of children, parents, and teachers were not included in the evalua- } \\
\text { tion }\end{array}$ \\
\hline $\begin{array}{l}\text { Design and methods over- } \\
\text { all approach }\end{array}$ & High risk & Many of the methods used did not capture key process data \\
\hline $\begin{array}{l}\text { Tools and methods of da- } \\
\text { ta collection reliable/cred- } \\
\text { ible }\end{array}$ & High risk & Study authors reported on the high level of measurement error \\
\hline $\begin{array}{l}\text { Tools and methods of data } \\
\text { analysis reliable/credible }\end{array}$ & High risk & No adjustment was made for missing data \\
\hline $\begin{array}{l}\text { Performance bias/neutral- } \\
\text { ity/credibility/conforma- } \\
\text { bility }\end{array}$ & Unclear risk & The degree of neutrality is not clear \\
\hline $\begin{array}{l}\text { Reliability of findings and } \\
\text { recommendations }\end{array}$ & Unclear risk & A lot of variation in implementation is evident \\
\hline
\end{tabular}


Gerald 2006 (Continued) $\begin{array}{ll}\text { Transferability of findings } \quad \text { High risk } & \begin{array}{l}\text { The quality of the study is not high enough to support the claim that this style } \\ \text { of intervention does work in inner city schools }\end{array}\end{array}$

Overall risk of bias of Unclear risk Use of unstructured tools to collect process data means that the risk of bias is

process evaluation not entirely clear

\section{Included as outcome evaluation}

Study design: parallel-group design, with a 2-group randomised longitudinal design, randomised by the child

\section{Setting: USA}

Period: baseline data collection occurred from October 2005 to December 2006. Children were randomised in January 2006. The study comprised a longitudinal design with 15-month follow-up. Follow-up data were collected from January 2006 to December 2006

Participants

Eligible sample frame: 290 children were randomised

Randomised: 290 children were randomised - 145 in each arm

Completed (intervention): 240 (83\%)

Inclusion criteria: children were eligible if they had physician-diagnosed persistent asthma requiring daily controller medication, they attended one of the 36 participating schools, and they were able to use a dry powder inhaler and a peak flow meter

Exclusion criteria: not reported

\section{Baseline characteristics}

Age of children: mean age, 11.0 years

Ethnicity: $91 \%$ black ethnicity

Socio-economic status: not reported.

Gender: $57 \%$ male; $43 \%$ female in total

Asthma status: mixed levels of severity - 15\% mild persistent asthma, 79\% moderate persistent asthma, $6 \%$ severe persistent asthma

Intervention: children were given 20 minutes of asthma education, including discussion about avoidance of triggers. Children in the supervised therapy group also received supervision from study staff each day on the use of inhaled corticosteroids. If a child was observed using the inhaler incorrectly, staff provided education with the aid of a placebo inhaler

Control: usual care

Intensity: a single education session for 20 minutes; multiple supervisions for the intervention group

Instructor: study personnel

Theoretical framework: not reported

Parental engagement: not reported

Child satisfaction: not reported 
Gerald 2009 (Continued)

Timing of intervention in school day: not reported

Outcomes Extractable outcomes were collected for:

Parent-reported absence from school

Lung function

Use of reliever therapies

Withdrawal

Notes

This paper did not provide much detail; however the study was previously reported in a separate paper (Gerald 2009)

Considered for process evaluation: implementation data were not considered to have been collected via structured or recognised tools

Funding source: not reported

\section{Risk of bias}

\begin{tabular}{lll}
\hline Bias & Authors' judgement & Support for judgement \\
\hline $\begin{array}{ll}\text { Random sequence genera- } \\
\text { tion (selection bias) }\end{array}$ & Low risk & $\begin{array}{l}\text { Study authors reported: "a random sequence of treatment codes, stratified by } \\
\text { school system, was generated using the SAS System (Version 9.1, Cary, North } \\
\text { Carolina) by the statistician" }\end{array}$ \\
& &
\end{tabular}

\begin{tabular}{ll}
\hline $\begin{array}{l}\text { Allocation concealment } \\
\text { (selection bias) }\end{array}$ & Low risk \\
& $\begin{array}{l}\text { Centrally generated: study authors reported: "a random sequence of treat- } \\
\text { ment codes, stratified by school system, was generated using the SAS System } \\
\text { (Version 9.1, Cary, North Carolina) by the statistician" }\end{array}$
\end{tabular}

Blinding of participants $\quad$ Unclear risk No measures were described as implemented around blinding
and personnel (perfor-
mance bias)

\begin{tabular}{|c|c|c|}
\hline $\begin{array}{l}\text { Blinding of outcome as- } \\
\text { sessment (detection bias) } \\
\text { All outcomes }\end{array}$ & Unclear risk & No measures were described as implemented around blinding \\
\hline $\begin{array}{l}\text { Incomplete outcome data } \\
\text { (attrition bias) } \\
\text { All outcomes }\end{array}$ & Low risk & $\begin{array}{l}\text { Study authors described: " } 79.3 \% \text { completion rate in the control group and } \\
86 \% \text { completion rate in the intervention group. Reasons and details provided" }\end{array}$ \\
\hline $\begin{array}{l}\text { Selective reporting (re- } \\
\text { porting bias) }\end{array}$ & Unclear risk & No evidence shows selective reporting \\
\hline Other bias & Unclear risk & $\begin{array}{l}\text { Missingness - low risk - all those who were followed up submitted information } \\
\text { Baseline imbalance - low risk - no significant differences between groups were } \\
\text { found in baseline demographic characteristics or asthma symptom } \\
\text { Risk of contamination - high - children were the unit of randomisation; chil- } \\
\text { dren with different treatment allocations were present in the same school }\end{array}$ \\
\hline $\begin{array}{l}\text { Transparent and clearly } \\
\text { stated aims }\end{array}$ & Unclear risk & $\mathrm{N} / \mathrm{A}$ \\
\hline $\begin{array}{l}\text { Explicit theories underpin- } \\
\text { ning and/or literature re- } \\
\text { view }\end{array}$ & Unclear risk & $\mathrm{N} / \mathrm{A}$ \\
\hline
\end{tabular}


Gerald 2009 (Continued)

\begin{tabular}{|c|c|c|}
\hline $\begin{array}{l}\text { Transparent and clearly } \\
\text { stated methods and tools }\end{array}$ & Unclear risk & $\mathrm{N} / \mathrm{A}$ \\
\hline Selective reporting & Unclear risk & N/A \\
\hline Harmful effects & Unclear risk & $\mathrm{N} / \mathrm{A}$ \\
\hline $\begin{array}{l}\text { Population and sample } \\
\text { described well }\end{array}$ & Unclear risk & $\mathrm{N} / \mathrm{A}$ \\
\hline Continuous evaluation & Unclear risk & $\mathrm{N} / \mathrm{A}$ \\
\hline $\begin{array}{l}\text { Evaluation participation } \\
\text { equity and sampling }\end{array}$ & Unclear risk & $\mathrm{N} / \mathrm{A}$ \\
\hline $\begin{array}{l}\text { Design and methods over- } \\
\text { all approach }\end{array}$ & Unclear risk & $\mathrm{N} / \mathrm{A}$ \\
\hline $\begin{array}{l}\text { Tools and methods of da- } \\
\text { ta collection reliable/cred- } \\
\text { ible }\end{array}$ & Unclear risk & $\mathrm{N} / \mathrm{A}$ \\
\hline $\begin{array}{l}\text { Tools and methods of data } \\
\text { analysis reliable/credible }\end{array}$ & Unclear risk & $\mathrm{N} / \mathrm{A}$ \\
\hline $\begin{array}{l}\text { Performance bias/neutral- } \\
\text { ity/credibility/conforma- } \\
\text { bility }\end{array}$ & Unclear risk & $\mathrm{N} / \mathrm{A}$ \\
\hline $\begin{array}{l}\text { Reliability of findings and } \\
\text { recommendations }\end{array}$ & Unclear risk & $\mathrm{N} / \mathrm{A}$ \\
\hline Transferability of findings & Unclear risk & $\mathrm{N} / \mathrm{A}$ \\
\hline $\begin{array}{l}\text { Overall risk of bias of } \\
\text { process evaluation }\end{array}$ & Unclear risk & N/A \\
\hline
\end{tabular}

Henry 2004

Methods Included as outcome evaluation and process evaluation

Study design: clustered parallel-group design

Setting: secondary schools in Newcastle, New South Wales, Australia

Period: baseline data were collected between February and March 1993. Follow-up data were collected between August and October 1993. Follow-up questionnaires were sent to the heads of participating schools in 1999

Process evaluation methods: descriptive/bivariate

Participants

Eligible sample frame: 33 schools were eligible for participation, with a total of 4475 year 8 students, $23 \%$ of whom had current asthma. In total, $76.7 \%$ of all eligible students and $82.7 \%$ of students recruited into th initial phase contributed to data analysis

Randomised: not reported 
Inclusion criteria: not reported

Exclusion criteria: not reported

\section{Baseline characteristics}

Age of children: year 8 students were eligible; adolescents aged 13 to 14 years were targeted

Ethnicity: numbers were not reported; however schools included a predominantly Caucasian population

Socio-economic status: not reported.

Gender: males represented $52.4 \%$ of intervention students with matched data and $52.9 \%$ of control adolescents

Asthma status: not reported

Intervention: intervention schools received a 3-lesson package about asthma designed to be taught within the Personal Development/Health/Physical Education (PD/H/PE) strand of the school curriculum. Each school was invited to send a delegate to learn the curriculum and was provided with the Living With Asthma teaching kit

Control: usual care

Intensity: 3 lessons; however the duration of these lessons was not reported

Instructor: in some schools, teachers who attended the training seminar delivered the lessons; in other schools, teachers trained their colleagues

Theoretical framework: not reported

Parental engagement: not reported

Child satisfaction: not reported

Timing of intervention in school day: taught within the Personal Development/Health/Physical Education strand of the school curriculum

Core processes evaluated (child level): adherence (long-term)
Extractable outcomes were collected for:
Health-related quality of life (HRQOL)

Notes

Process evaluation category: integrated

Breadth and depth: depth - not breadth

Voice of children given prominence: featured but not sufficiently

Funding source: not reported

\section{Risk of bias}

\begin{tabular}{lll}
\hline Bias & Authors' judgement & Support for judgement \\
\hline $\begin{array}{l}\text { Random sequence genera- } \\
\text { tion (selection bias) }\end{array}$ & High risk & $\begin{array}{l}\text { Method of stratification is unclear: schools were randomised to control or } \\
\text { intervention, with an attempt to obtain similar demographic mixes in the } 2 \\
\text { groups }\end{array}$
\end{tabular}


Henry 2004 (Continued)

\begin{tabular}{lll}
$\begin{array}{l}\text { Allocation concealment } \\
\text { (selection bias) }\end{array}$ & Unclear risk & Not addressed by study authors \\
\hline $\begin{array}{l}\text { Blinding of participants } \\
\text { and personnel (perfor- } \\
\text { mance bias) }\end{array}$ & Unclear risk & Not addressed by study authors \\
All outcomes & &
\end{tabular}

\begin{tabular}{lll}
\hline Blinding of outcome as- & Unclear risk & Not addressed by study authors \\
sessment (detection bias) &
\end{tabular}

All outcomes

Incomplete outcome data Unclear risk $\quad$ Attrition data for asthmatic children were not provided in full
(attrition bias)

All outcomes

\begin{tabular}{lll}
\hline $\begin{array}{l}\text { Selective reporting (re- } \\
\text { porting bias) }\end{array}$ & Low risk & All data were reported by study authors \\
\hline Other bias & Unclear risk & $\begin{array}{l}\text { Missingness - unclear risk - missing data are apparent with no explanation pro- } \\
\text { vided } \\
\text { Baseline imbalance - unclear risk - this was not addressed by study authors } \\
\text { Risk of contamination - low - risk of contamination was low due to the study } \\
\text { design }\end{array}$ \\
\hline $\begin{array}{l}\text { Transparent and clearly } \\
\text { stated aims }\end{array}$ & Low risk & Study aims were clearly stated \\
\hline
\end{tabular}

\begin{tabular}{|c|c|c|}
\hline $\begin{array}{l}\text { Explicit theories underpin- } \\
\text { ning and/or literature re- } \\
\text { view }\end{array}$ & High risk & No theoretical framework and very little supporting literature were provided \\
\hline $\begin{array}{l}\text { Transparent and clearly } \\
\text { stated methods and tools }\end{array}$ & Low risk & Study aims were clearly stated \\
\hline Selective reporting & Unclear risk & $\begin{array}{l}\text { Medium bias - problems with linking surveys were experienced (pre-post inter- } \\
\text { vention) }\end{array}$ \\
\hline Harmful effects & Unclear risk & $\begin{array}{l}\text { How this was addressed is unclear. In particular, this might not have been ben- } \\
\text { eficial for many children }\end{array}$ \\
\hline $\begin{array}{l}\text { Population and sample } \\
\text { described well }\end{array}$ & High risk & Level of baseline imbalance was not reported \\
\hline Continuous evaluation & Unclear risk & $\begin{array}{l}\text { Pre-post assessment data were used for the majority, but an element of con- } \\
\text { tinuous evaluation was included in the school policy analysis }\end{array}$ \\
\hline $\begin{array}{l}\text { Evaluation participation } \\
\text { equity and sampling }\end{array}$ & Unclear risk & Moderate evidence shows that the voice of children was reflected adequately \\
\hline $\begin{array}{l}\text { Design and methods over- } \\
\text { all approach }\end{array}$ & Low risk & Design and methods were appropriate for this study \\
\hline $\begin{array}{l}\text { Tools and methods of da- } \\
\text { ta collection reliable/cred- } \\
\text { ible }\end{array}$ & Low risk & Data collection tools used were credible and reliable \\
\hline
\end{tabular}


Henry 2004 (Continued)

Tools and methods of data Low risk Analysis of quantitative data was reliable analysis reliable/credible

Performance bias/neutral- Unclear risk No evidence shows how confidentiality was maintained

ity/credibility/conforma-

bility

\begin{tabular}{lll}
\hline $\begin{array}{l}\text { Reliability of findings and } \\
\text { recommendations }\end{array}$ & Unclear risk & Level of baseline imbalance is unclear \\
\hline Transferability of findings & Unclear risk & Transferability was not assessed by study authors \\
\hline $\begin{array}{l}\text { Overall risk of bias of } \\
\text { process evaluation }\end{array}$ & Unclear risk & $\begin{array}{l}\text { Some aspects of study design and study characteristics were not explained ful- } \\
\text { ly }\end{array}$ \\
\hline
\end{tabular}

Horner 2008

Methods Included as outcome evaluation

Study design: clustered parallel-group design with schools selected as the unit of randomisation

Setting: elementary schools in the USA; 10 treatment and 8 attention control schools

Period: each of the participating school districts participated for 1 academic year, and the whole project spanned 2003 to 2006 academic years. Analysis occurred over 12 weeks of the academic year from study enrolment to 6 weeks post intervention

Randomised: 183 pupils were randomised: 101 into treatment group and 82 into control group

Completed (intervention): 163 pupils completed the trial

Inclusion criteria: students were eligible for participation in the study if they had doctor-diagnosed asthma, had experienced asthma symptoms in the previous 12 months, had no other significant comorbidity that would preclude participation in classes, spoke either English or Spanish, and were enrolled in grades 2 to 5

Exclusion criteria: not reported

\section{Baseline characteristics}

Age of children: mean age, 8.78 years

Ethnicity: within the sample, 47\% were Mexican American, 30\% white, 22\% African American, and 1\% other

Socio-economic status: not reported

Gender: 108 male; 75 female

Asthma status: not reported

Control: the attention-control group mirrored the treatment group and received education on health promotion topics appropriate for children

Intensity: 16 sessions, each for 15 minutes 
Horner 2008 (Continued)

Instructor: 18 lay health educators

Theoretical framework: the asthma health education model that informed the study was adapted from Bruhn's theoretical model of asthma management

Parental engagement: not reported

Child satisfaction: not reported

Timing of intervention in school day: lunch breaks

\begin{tabular}{ll}
\hline Outcomes & Extractable outcomes were collected for: \\
& Hospitalisation \\
& Withdrawal \\
\hline Notes & Funding source: not reported
\end{tabular}

\section{Risk of bias}

\begin{tabular}{|c|c|c|}
\hline Bias & Authors' judgement & Support for judgement \\
\hline $\begin{array}{l}\text { Random sequence genera- } \\
\text { tion (selection bias) }\end{array}$ & Low risk & $\begin{array}{l}\text { Study authors reported: "schools were randomised through a simple coin toss } \\
\text { at a summer meeting held with elementary school principals" }\end{array}$ \\
\hline $\begin{array}{l}\text { Allocation concealment } \\
\text { (selection bias) }\end{array}$ & High risk & Risk that concealment of allocation was breached \\
\hline $\begin{array}{l}\text { Blinding of participants } \\
\text { and personnel (perfor- } \\
\text { mance bias) } \\
\text { All outcomes }\end{array}$ & High risk & $\begin{array}{l}\text { Study authors reported: "Because the participants could not be blinded to } \\
\text { their treatment condition, this information was disclosed to parents during } \\
\text { this first telephone call" }\end{array}$ \\
\hline $\begin{array}{l}\text { Blinding of outcome as- } \\
\text { sessment (detection bias) } \\
\text { All outcomes }\end{array}$ & Unclear risk & Not assessed by study authors \\
\hline $\begin{array}{l}\text { Incomplete outcome data } \\
\text { (attrition bias) } \\
\text { All outcomes }\end{array}$ & Unclear risk & $\begin{array}{l}\text { Missing outcome data roughly balanced across intervention and control } \\
\text { groups, with similar reasons for missingness and study authors reporting: } \\
\text { "Comparing baseline scores of those who dropped out of the study with those } \\
\text { who were retained (i.e. completers) showed no significant differences in terms } \\
\text { of demographic or study variables" }\end{array}$ \\
\hline $\begin{array}{l}\text { Selective reporting (re- } \\
\text { porting bias) }\end{array}$ & Low risk & No evidence of selective reporting \\
\hline \multirow[t]{3}{*}{ Other bias } & Low risk & $\begin{array}{l}\text { Missingness - low risk - strategies implemented for missingness (which ac- } \\
\text { counted for less than } 10 \% \text { of the sample) described as follows: "Missing items } \\
\text { were handled by substituting the participant's mean score for the missed item } \\
\text { in those cases where fewer than } 10 \% \text { of the items were missing for a scale. In } \\
\text { this study, there were no instances of more than } 10 \% \text { of missed items for a } \\
\text { scale" }\end{array}$ \\
\hline & & $\begin{array}{l}\text { Baseline imbalance - low risk - comparing groups at baseline on study mea- } \\
\text { sures revealed no significant differences between groups except for asthma } \\
\text { severity, which was greater in the treatment group }\end{array}$ \\
\hline & & $\begin{array}{l}\text { Risk of contamination - low - schools were the unit of randomisation, lowering } \\
\text { the potential for contamination }\end{array}$ \\
\hline
\end{tabular}


Horner 2008 (Continued)

Transparent and clearly $\quad$ Unclear risk
stated aims

Explicit theories underpin- $\quad$ Unclear risk
ning and/or literature re-

view

\begin{tabular}{|c|c|c|}
\hline $\begin{array}{l}\text { Transparent and clearly } \\
\text { stated methods and tools }\end{array}$ & Unclear risk & $\mathrm{N} / \mathrm{A}$ \\
\hline Selective reporting & Unclear risk & $\mathrm{N} / \mathrm{A}$ \\
\hline Harmful effects & Unclear risk & $\mathrm{N} / \mathrm{A}$ \\
\hline $\begin{array}{l}\text { Population and sample } \\
\text { described well }\end{array}$ & Unclear risk & $\mathrm{N} / \mathrm{A}$ \\
\hline Continuous evaluation & Unclear risk & $\mathrm{N} / \mathrm{A}$ \\
\hline $\begin{array}{l}\text { Evaluation participation } \\
\text { equity and sampling }\end{array}$ & Unclear risk & $\mathrm{N} / \mathrm{A}$ \\
\hline $\begin{array}{l}\text { Design and methods over- } \\
\text { all approach }\end{array}$ & Unclear risk & $\mathrm{N} / \mathrm{A}$ \\
\hline $\begin{array}{l}\text { Tools and methods of da- } \\
\text { ta collection reliable/cred- } \\
\text { ible }\end{array}$ & Unclear risk & $\mathrm{N} / \mathrm{A}$ \\
\hline $\begin{array}{l}\text { Tools and methods of data } \\
\text { analysis reliable/credible }\end{array}$ & Unclear risk & $\mathrm{N} / \mathrm{A}$ \\
\hline $\begin{array}{l}\text { Performance bias/neutral- } \\
\text { ity/credibility/conforma- } \\
\text { bility }\end{array}$ & Unclear risk & $\mathrm{N} / \mathrm{A}$ \\
\hline $\begin{array}{l}\text { Reliability of findings and } \\
\text { recommendations }\end{array}$ & Unclear risk & $\mathrm{N} / \mathrm{A}$ \\
\hline Transferability of findings & Unclear risk & $\mathrm{N} / \mathrm{A}$ \\
\hline $\begin{array}{l}\text { Overall risk of bias of } \\
\text { process evaluation }\end{array}$ & Unclear risk & $\mathrm{N} / \mathrm{A}$ \\
\hline
\end{tabular}

\section{Methods Included as outcome evaluation and process evaluation}

Intervention study design: cluster parallel-group design, with schools selected as unit of randomisation, using a stratified design according to school characteristics

Setting: 33 elementary schools in 5 rural Texas, USA, districts participated in the study

Period: dates on which study was conducted - intervention and subsequent data collection - not clear; data were collected over a 12-month period 
Horner 2015 (Continued)

Participants
Eligible sample frame: a total of 292 children were enrolled

Randomised: a total of 292 children were enrolled and were randomised to 1 of 3 groups; information from 2 groups of interest provided here. 96 children were randomised to the school-based intervention group and 100 children to the attention control group

Completed (intervention): 84 children completed the intervention and attention control

Inclusion criteria: children were eligible if they had doctor-diagnosed asthma, had experienced asthma symptoms in the previous 12 months, and could speak either English or Spanish

Exclusion criteria: no significant comorbidity that would preclude participation in classes

\section{Baseline characteristics}

Age of children (based on completers): mean age, 8.8 years

Ethnicity (based on completers): treatment: 22.9\% white, 55.2\% Hispanic, 21.9\% African American; control: $11.3 \%$ white, $60.8 \%$ Hispanic, $27.8 \%$ African American

Socio-economic status (based on completers): 31.2\% defined as lower SES group in intervention group and $29.8 \%$ in control group

Gender (based on completers): treatment: $55.2 \%$ male, $44.6 \%$ female; control: $76.0 \%$ male, $24.0 \%$ female

Asthma status: mixed levels of asthma severity; similar levels between treatment and control groups when measured on the Severity of Chronic Asthma Scale

Interventions

Intervention: the "Asthma Plan for Kids" curriculum, specifically designed for children in rural areas, was provided to the intervention group as group instruction. Topics within the curriculum included: "(1) identifying lung function, asthma warning signs, symptoms, and triggers; (2) learning skills to manage symptoms, including peak expiratory flow score interpretation, communication with adults, medication use, and inhaler technique; (3) evaluating asthma symptoms and the effectiveness of management; and (4) discussing how to safely keep active during physical activity and sports"

Control: an equivalent attention control was provided. This mirrored the structure of the school-based intervention but differed in providing a non-asthma-based curriculum. The content consisted of topics on general health information identified by school nurses as useful, including handwashing, nutrition, brushing teeth, and exercising

Intensity: 15-minute lessons spread over 16 sessions that took place within a 5-week period

Instructor: trained lay health educators

Theoretical framework: Bruhn's theoretical model of asthma self-management

Parental engagement: not reported

Child satisfaction: not reported

Timing: sessions held during lunchtime (15-minute blocks)

\section{Quantitative outcomes}

Extractable outcomes were collected for:

Exacerbations leading to hospital admission

Asthma symptoms leading to emergency hospital visits

Withdrawal

Core processes evaluated (child level): attrition, adherence 
Horner 2015 (Continued)

Notes
This study compared the treatment (a school-based intervention) group vs an attention control group; also compared the school-based intervention vs the same intervention provided at an asthma day camp (although these data are not extracted here)

Data were collected on office visits, but unclear whether office visits were restricted to community primary care providers or included specialist consultations

\section{Additional measures of process evaluation quality}

Process evaluation category: integrated within outcome evaluation

Breadth and depth: neither broad nor deep

Voice of children given prominence: featured but not sufficiently

Funding source: National Institutes of Health; Nursing Research; National Heart, Lung, and Blood Institute

\section{Risk of bias}

\begin{tabular}{lll}
\hline Bias & Authors' judgement & Support for judgement \\
\hline $\begin{array}{l}\text { Random sequence genera- } \\
\text { tion (selection bias) }\end{array}$ & Unclear risk & $\begin{array}{l}\text { Not addressed in full by study authors: actual method of randomisation un- } \\
\text { clear }\end{array}$ \\
\hline $\begin{array}{l}\text { Allocation concealment } \\
\text { (selection bias) }\end{array}$ & Unclear risk & Not addressed by study authors \\
\hline $\begin{array}{l}\text { Blinding of participants } \\
\text { and personnel (perfor- } \\
\text { mance bias) }\end{array}$ & Low risk & Evidence shows that steps were taken to ensure blinding \\
All outcomes & \\
\hline $\begin{array}{l}\text { Blinding of outcome as- } \\
\text { sessment (detection bias) } \\
\text { All outcomes }\end{array}$ & Low risk & $\begin{array}{l}\text { Study authors reported: "Data were collected 4 times over the 12-month study } \\
\text { during home visits, scheduled at times convenient to the families, by RAs who } \\
\text { were blind to group assignment" }\end{array}$ \\
\hline $\begin{array}{l}\text { Incomplete outcome data } \\
\text { (attrition bias) } \\
\text { All outcomes }\end{array}$ & Low risk & $\begin{array}{l}\text { Study authors reported: "A total of 292 children were enrolled and 257 com- } \\
\text { pleted the 12-month study (87.7\% retention). There were no significant base- } \\
\text { line differences in age, gender, race/ethnicity, SES, language spoken by par- } \\
\text { ents, or asthma severity between the children who completed the study and } \\
\text { those who dropped out" }\end{array}$ \\
\hline
\end{tabular}

Selective reporting (re- Low risk All collected outcomes apparently reported in full
porting bias)
porting bias)

\begin{tabular}{lll}
\hline Other bias & Unclear risk & $\begin{array}{l}\text { Missingness - unclear risk - no evidence of additional missing data } \\
\text { Baseline imbalance - unclear risk - children in the control group were more } \\
\text { likely to be male; the impact that this could have on study outcomes is unclear } \\
\text { Risk of contamination - low - schools were the unit of randomisation, lowering } \\
\text { the risk of contamination }\end{array}$ \\
\hline $\begin{array}{l}\text { Transparent and clearly } \\
\text { stated aims }\end{array}$ & Low risk & Study aims were clearly stated \\
\hline $\begin{array}{l}\text { Explicit theories underpin- } \\
\text { ning and/or literature re- } \\
\text { view }\end{array}$ & Low risk & $\begin{array}{l}\text { Intervention was guided by Bruhn's theoretical model of asthma self-manage- } \\
\text { ment }\end{array}$
\end{tabular}


Horner 2015 (Continued)

\begin{tabular}{|c|c|c|}
\hline $\begin{array}{l}\text { Transparent and clearly } \\
\text { stated methods and tools }\end{array}$ & Low risk & Study methods were clearly stated \\
\hline Selective reporting & Unclear risk & A limited set of data was presented for the process evaluation \\
\hline Harmful effects & High risk & Only a few negative outcomes were considered \\
\hline $\begin{array}{l}\text { Population and sample } \\
\text { described well }\end{array}$ & Low risk & Study sample was well described \\
\hline Continuous evaluation & Low risk & Data were collected at several time points \\
\hline $\begin{array}{l}\text { Evaluation participation } \\
\text { equity and sampling }\end{array}$ & Unclear risk & Not many stakeholders were involved in the evaluation \\
\hline $\begin{array}{l}\text { Design and methods over- } \\
\text { all approach }\end{array}$ & Unclear risk & $\begin{array}{l}\text { This is unclear, as risk of bias for outcome evaluation was low; however risk of } \\
\text { bias for process evaluation was high }\end{array}$ \\
\hline $\begin{array}{l}\text { Tools and methods of da- } \\
\text { ta collection reliable/cred- } \\
\text { ible }\end{array}$ & Low risk & Tools used for data collection were credible \\
\hline $\begin{array}{l}\text { Tools and methods of data } \\
\text { analysis reliable/credible }\end{array}$ & Low risk & Data analysis methods were appropriate for the data \\
\hline
\end{tabular}

Performance bias/neutral- Unclear risk Credible findings focused mainly on outcomes

ity/credibility/conforma-

bility

\begin{tabular}{lll}
\hline $\begin{array}{l}\text { Reliability of findings and } \\
\text { recommendations }\end{array}$ & Low risk & Findings are transparent \\
\hline Transferability of findings & High risk & $\begin{array}{l}\text { Some factors around transferability were assessed, for example, reach and at- } \\
\text { trition; however no other factors were assessed }\end{array}$ \\
\hline $\begin{array}{l}\text { Overall risk of bias of } \\
\text { process evaluation }\end{array}$ & Unclear risk & $\begin{array}{l}\text { As an outcome evaluation, few concerns are evident; as a process evaluation, } \\
\text { this study is questionable }\end{array}$ \\
\hline
\end{tabular}

Howell 2005

Methods Included as outcome evaluation and process evaluation

Study design: clustered parallel-group design with schools selected as the unit of randomisation

Setting: 4 elementary schools in Syracuse City, New York, USA

Period: conducted December 2003 to January 2004; subsequent data collected through September 2004

Process evaluation methods: descriptive/bivariate

Participants

Eligible sample frame: 40 families found to be eligible and 30 consented, with 5 others unable to participate before randomisation

Randomised: 25 families (children and caregiver), with 16 selected into the intervention group and 9 into the control group 
Inclusion criteria: target child was between 8 and 11 years of age and was in third to fifth grade with a diagnosis of asthma and prescribed daily medications for asthma as reported by nurse and/or parent

Exclusion criteria: coexisting chronic illness that required daily medication (e.g. insulin-dependent diabetes)

\section{Baseline characteristics}

Age of children: mean age, 9 years 7 months; range, 8 to 11 years

Ethnicity: $75 \%$ of children were African American

Socio-economic status: unclear

Gender: 63\% male

Asthma status: all children had a record of asthma, although study authors did not describe asthma severity

Intervention description: children in the intervention condition received four 30-minute sessions on the "Quest for the Code" computer game at their school. The computer game included modules on Lung Physiology; Symptom Recognition; Trigger Recognition; Peak Flow Meter Usage; Appropriate Use of Long-Term and Reliever Medication; Correct Usage of Common Asthma Medication; and Effect of Asthma Medications on Lungs. The child's primary caregiver participated in a medication routine interview in the home and received a 1-time home visit at which a medication routine plan was developed

Control description: usual care/no additional intervention

Theoretical framework: learning theory principles and behaviour modification

\begin{tabular}{|c|c|c|}
\hline Outcomes & \multicolumn{2}{|c|}{ Core processes evaluated (child level): attrition, dosage, adherence } \\
\hline \multirow[t]{4}{*}{ Notes } & \multicolumn{2}{|c|}{ Process evaluation category: named section } \\
\hline & \multicolumn{2}{|c|}{ Breadth and depth: neither broad nor deep } \\
\hline & \multicolumn{2}{|c|}{ Voice of children given prominence: the voice of children was featured but not sufficiently } \\
\hline & \multicolumn{2}{|c|}{ Funding source: not reported } \\
\hline \multicolumn{3}{|l|}{ Risk of bias } \\
\hline Bias & Authors' judgement & Support for judgement \\
\hline $\begin{array}{l}\text { Random sequence genera- } \\
\text { tion (selection bias) }\end{array}$ & Unclear risk & $\begin{array}{l}\text { Information not given: this study was a pretest, intervention, post-test, fol- } \\
\text { low-up (PPF) RCT with random assignment based on school site }\end{array}$ \\
\hline $\begin{array}{l}\text { Allocation concealment } \\
\text { (selection bias) }\end{array}$ & High risk & $\begin{array}{l}\text { Information not given; few students were randomised and concealment may } \\
\text { have been breached }\end{array}$ \\
\hline $\begin{array}{l}\text { Blinding of participants } \\
\text { and personnel (perfor- } \\
\text { mance bias) } \\
\text { All outcomes }\end{array}$ & Unclear risk & Not addressed in the study \\
\hline
\end{tabular}

\begin{tabular}{ll}
\hline Blinding of outcome as- & Unclear risk Not addressed in the study \\
sessment (detection bias) & \\
All outcomes &
\end{tabular}


Howell 2005 (Continued)
Incomplete outcome data Low risk
Low risk - low levels of attrition are reported in the study
(attrition bias)

All outcomes

\begin{tabular}{lll}
\hline $\begin{array}{l}\text { Selective reporting (re- } \\
\text { porting bias) }\end{array}$ & Low risk & No evidence of selective reporting \\
\hline Other bias & High risk & $\begin{array}{l}\text { Missingness - high risk - small sample and evidence of missingness for some in- } \\
\text { dicators } \\
\text { Baseline imbalance - high risk - described in the introduction as imbalanced }\end{array}$ \\
& Risk of contamination - low - randomisation occurred at a school level \\
\hline
\end{tabular}

Transparent and clearly Low risk Study aims were clearly stated
stated aims

stated aims

$\begin{array}{ll}\text { Explicit theories underpin- } & \text { Low risk } \\ \text { ning and/or literature re- } & \begin{array}{l}\text { Intervention was informed by learning theory principles and behaviour modifi- } \\ \text { cation }\end{array}\end{array}$
view

\begin{tabular}{|c|c|c|}
\hline $\begin{array}{l}\text { Transparent and clearly } \\
\text { stated methods and tools }\end{array}$ & Low risk & $\begin{array}{l}\text { Study included a broad-ranging description of tools implemented at different } \\
\text { time points }\end{array}$ \\
\hline
\end{tabular}

\begin{tabular}{|c|c|c|}
\hline Selective reporting & Unclear risk & $\begin{array}{l}\text { Some statements from parents are impenetrable; other statements describe } \\
\text { kids enjoying the intervention but are not entirely clear on how this feedback } \\
\text { was obtained }\end{array}$ \\
\hline Harmful effects & Unclear risk & Study methods do not highlight the possibility of negative effects \\
\hline $\begin{array}{l}\text { Population and sample } \\
\text { described well }\end{array}$ & Unclear risk & Only basic demographic information was included \\
\hline Continuous evaluation & Low risk & Not continuous; however 3 time points were considered \\
\hline $\begin{array}{l}\text { Evaluation participation } \\
\text { equity and sampling }\end{array}$ & Unclear risk & Information was collected from parents and children only \\
\hline
\end{tabular}

Design and methods over- Low risk This was an RCT but with dedicated attention to feasibility
all approach

Tools and methods of da- Low risk $\quad$ Validated measures were used
ta collection reliable/cred-
ible

Tools and methods of data
analysis reliable/crediblear risk

\begin{tabular}{lll}
\hline $\begin{array}{l}\text { Performance bias/neutral- } \\
\text { ity/credibility/conforma- } \\
\text { bility }\end{array}$ & Unclear risk & Process was limited \\
\hline $\begin{array}{l}\text { Reliability of findings and } \\
\text { recommendations }\end{array}$ & Low risk & How the data relate to study findings is clear \\
\hline $\begin{array}{l}\text { Transferability of findings } \\
\text { Overall risk of bias of }\end{array}$ & Unclear risk & Study sample was too small, so transferability is limited \\
\hline \hline
\end{tabular}

School-based self-management interventions for asthma in children and adolescents: a mixed methods systematic review (Review) 
Jackson 2006

Included as process evaluation
Intervention study design: quasi-experimental design with pre-post follow-up and no control group
Unit of allocation: N/A
Process evaluation methods: descriptive/bivariate analysis methods

Country: USA
Age of children: this study recruited 943 third grade students, aged 8 to 9 years
Child characteristics (BME/SES): not reported
Asthma status: asthmatic and non-asthmatic children
Intervention recipients: children only

Interventions

School type: public and private elementary schools in Chicago

Intervention description: children completed a 1-hour asthma education programme entitled "The lion who couldn't roar". Teachers were encouraged to attend the workshop

\section{Control description: N/A}

Theoretical framework: not reported

\begin{tabular}{ll}
\hline Outcomes & Core processes evaluated (child level): attrition, dosage, adherence \\
\hline Notes & Process evaluation category: integrated \\
& Breadth and depth: breadth - not depth \\
& Voice of children given prominence: sufficient coverage \\
& Funding source: Midwestern University and Majestic Steel Erections \\
\hline
\end{tabular}

\section{Risk of bias}

\begin{tabular}{|c|c|c|}
\hline Bias & Authors' judgement & Support for judgement \\
\hline $\begin{array}{l}\text { Random sequence genera- } \\
\text { tion (selection bias) }\end{array}$ & Unclear risk & $\mathrm{N} / \mathrm{A}$ \\
\hline $\begin{array}{l}\text { Allocation concealment } \\
\text { (selection bias) }\end{array}$ & Unclear risk & $\mathrm{N} / \mathrm{A}$ \\
\hline $\begin{array}{l}\text { Blinding of participants } \\
\text { and personnel (perfor- } \\
\text { mance bias) } \\
\text { All outcomes }\end{array}$ & Unclear risk & $\mathrm{N} / \mathrm{A}$ \\
\hline $\begin{array}{l}\text { Blinding of outcome as- } \\
\text { sessment (detection bias) } \\
\text { All outcomes }\end{array}$ & Unclear risk & $\mathrm{N} / \mathrm{A}$ \\
\hline $\begin{array}{l}\text { Incomplete outcome data } \\
\text { (attrition bias) } \\
\text { All outcomes }\end{array}$ & Unclear risk & $\mathrm{N} / \mathrm{A}$ \\
\hline
\end{tabular}


Jackson 2006 (Continued)

\begin{tabular}{lll}
$\begin{array}{l}\text { Selective reporting (re- } \\
\text { porting bias) }\end{array}$ & Unclear risk & N/A \\
\hline Other bias & Unclear risk & N/A \\
\hline $\begin{array}{l}\text { Transparent and clearly } \\
\text { stated aims }\end{array}$ & Low risk & Study aims were clearly stated \\
\hline
\end{tabular}

Explicit theories underpin- High risk No evidence of a theoretical framework guiding intervention development
ning and/or literature re-
view

\begin{tabular}{|c|c|c|}
\hline $\begin{array}{l}\text { Transparent and clearly } \\
\text { stated methods and tools }\end{array}$ & Unclear risk & Some detail provided; however information is limited \\
\hline Selective reporting & Unclear risk & $\begin{array}{l}\text { Unclear whether selective reporting was considered, but most aspects of the } \\
\text { data collected were presented; they are not entirely interpretable }\end{array}$ \\
\hline Harmful effects & Unclear risk & $\begin{array}{l}\text { Most aspects of the data collected were presented; they are not entirely inter- } \\
\text { pretable }\end{array}$ \\
\hline $\begin{array}{l}\text { Population and sample } \\
\text { described well }\end{array}$ & High risk & No demographic information about the children was provided \\
\hline Continuous evaluation & Low risk & Appropriate for intensity of the intervention \\
\hline $\begin{array}{l}\text { Evaluation participation } \\
\text { equity and sampling }\end{array}$ & Low risk & Relevant stakeholders were included in the evaluation \\
\hline $\begin{array}{l}\text { Design and methods over- } \\
\text { all approach }\end{array}$ & Low risk & Appropriate for intensity of the intervention \\
\hline $\begin{array}{l}\text { Tools and methods of da- } \\
\text { ta collection reliable/cred- } \\
\text { ible }\end{array}$ & Low risk & Tools used were appropriate and well described \\
\hline $\begin{array}{l}\text { Tools and methods of data } \\
\text { analysis reliable/credible }\end{array}$ & Unclear risk & Presentation of data collection methods and analysis is difficult to follow \\
\hline $\begin{array}{l}\text { Performance bias/neutral- } \\
\text { ity/credibility/conforma- } \\
\text { bility }\end{array}$ & Unclear risk & Data are unclear \\
\hline $\begin{array}{l}\text { Reliability of findings and } \\
\text { recommendations }\end{array}$ & High risk & No evidence from the intervention data that findings are reliable \\
\hline Transferability of findings & Unclear risk & No exploration of how the impact of study findings differed by school \\
\hline $\begin{array}{l}\text { Overall risk of bias of } \\
\text { process evaluation }\end{array}$ & Unclear risk & Data are unclear \\
\hline
\end{tabular}

$\begin{array}{ll}\text { Methods } & \text { Included as process evaluation } \\ & \text { Intervention study design: parallel-group randomised controlled trial }\end{array}$


Unit of allocation: child

Process evaluation methods: survey based, including multi-variate analyses of outcomes

Setting: 6 high schools in Detroit, Michigan, USA
Age of children: mean age, 15.3 years
Child characteristics (BME/SES): $97 \%$ of students were African American; $52 \%$ were eligible for federal
school lunch programmes
Asthma status: asthmatic only; severity unclear
Intervention recipients: children only
School type: high schools
Intervention description: tailored computer programme (Puff City): the web-based programme fo-
cuses on 3 core behaviours, namely, controller medication adherence, rescue inhaler availability, and
smoking cessation/reduction, and consists of 4 consecutive educational computer sessions that make
use of both normative ("compared with other students") and ipsative ("compared with your last ses-
sion") feedback. Messages are voiced over to accommodate low literacy. Participant-specific informa-
tion necessary for tailoring is obtained at baseline and during the 4 sessions.

Control description: students randomised to the control group were directed to existing generic asthma websites

Theoretical framework: not 1 single framework was named, but theories around content were mentioned

\begin{tabular}{|c|c|c|}
\hline Outcomes & \multicolumn{2}{|c|}{ Core processes evaluated (child level): attrition, dosage, adherence } \\
\hline \multirow[t]{5}{*}{ Notes } & \multicolumn{2}{|c|}{ Process evaluation category: stand-alone and integrated ( 2 papers) } \\
\hline & \multicolumn{2}{|c|}{ Breadth and depth: breadth and depth } \\
\hline & \multicolumn{2}{|c|}{ Voice of children given prominence: featured but not sufficiently } \\
\hline & \multicolumn{2}{|c|}{$\begin{array}{l}\text { Note: study is not included as an outcome evaluation because the comparison group received asthma } \\
\text { education (this study evaluated the added impact of providing tailored messaging) }\end{array}$} \\
\hline & \multicolumn{2}{|c|}{ Funding source: National Heart, Lung, and Blood Institute, National Institutes of Health } \\
\hline \multicolumn{3}{|l|}{ Risk of bias } \\
\hline Bias & Authors' judgement & Support for judgement \\
\hline $\begin{array}{l}\text { Random sequence genera- } \\
\text { tion (selection bias) }\end{array}$ & Unclear risk & N/A \\
\hline $\begin{array}{l}\text { Allocation concealment } \\
\text { (selection bias) }\end{array}$ & Unclear risk & N/A \\
\hline $\begin{array}{l}\text { Blinding of participants } \\
\text { and personnel (perfor- } \\
\text { mance bias) } \\
\text { All outcomes }\end{array}$ & Unclear risk & $\mathrm{N} / \mathrm{A}$ \\
\hline $\begin{array}{l}\text { Blinding of outcome as- } \\
\text { sessment (detection bias) } \\
\text { All outcomes }\end{array}$ & Unclear risk & N/A \\
\hline
\end{tabular}


Joseph 2010 (Continued)

Incomplete outcome data Unclear risk N/A
(attrition bias)

All outcomes

\begin{tabular}{lll}
\hline $\begin{array}{l}\text { Selective reporting (re- } \\
\text { porting bias) }\end{array}$ & Unclear risk & N/A \\
\hline Other bias & Unclear risk & N/A \\
\hline $\begin{array}{l}\text { Transparent and clearly } \\
\text { stated aims }\end{array}$ & Low risk & Each paper includes clearly stated aims \\
\hline $\begin{array}{l}\text { Explicit theories underpin- } \\
\text { ning and/or literature re- } \\
\text { view }\end{array}$ & Low risk & $\begin{array}{l}\text { Not a single theory, but some aspects of learning are grounded in pedagogical } \\
\text { techniques }\end{array}$ \\
\hline $\begin{array}{l}\text { Transparent and clearly } \\
\text { stated methods and tools }\end{array}$ & Low risk & \begin{tabular}{l} 
All methods were clearly stated \\
\hline $\begin{array}{l}\text { Selective reporting } \\
\text { Unclear risk }\end{array}$
\end{tabular} \\
& $\begin{array}{l}\text { Data were apparently collected at different time points, but only follow-up da- } \\
\text { ta were presented. Otherwise no evidence indicates that data collected were } \\
\text { not presented }\end{array}$
\end{tabular}

\begin{tabular}{ll}
\hline Harmful effects & Unclear risk \\
& $\begin{array}{l}\text { Some consideration of negative factors in the design - e.g. access to the refer- } \\
\text { ral co-ordinator. However, whether the analysis fully accounts for this is un- } \\
\text { clear }\end{array}$
\end{tabular}

\begin{tabular}{|c|c|c|}
\hline $\begin{array}{l}\text { Population and sample } \\
\text { described well }\end{array}$ & Unclear risk & $\begin{array}{l}\text { Characteristics of the population were generally described well - but ambigu- } \\
\text { ities between papers that ostensibly describe the same study population sur- } \\
\text { round the numbers involved }\end{array}$ \\
\hline
\end{tabular}

\begin{tabular}{|c|c|c|}
\hline Continuous evaluation & Low risk & $\begin{array}{l}\text { Data were collected at different time points, but only follow-up data were pre- } \\
\text { sented }\end{array}$ \\
\hline $\begin{array}{l}\text { Evaluation participation } \\
\text { equity and sampling }\end{array}$ & High risk & High level of non-participation is a matter of concern \\
\hline $\begin{array}{l}\text { Design and methods over- } \\
\text { all approach }\end{array}$ & Unclear risk & $\begin{array}{l}\text { Evaluation took into account multiple time points (all data were not necessari- } \\
\text { ly presented): however data from multiple stakeholders were not collected }\end{array}$ \\
\hline $\begin{array}{l}\text { Tools and methods of da- } \\
\text { ta collection reliable/cred- } \\
\text { ible }\end{array}$ & Low risk & Tools and methods used were reliable \\
\hline $\begin{array}{l}\text { Tools and methods of data } \\
\text { analysis reliable/credible }\end{array}$ & Low risk & $\begin{array}{l}\text { Analysis was straightforward, in part because of the relatively straightforward } \\
\text { research design }\end{array}$ \\
\hline $\begin{array}{l}\text { Performance bias/neutral- } \\
\text { ity/credibility/conforma- } \\
\text { bility }\end{array}$ & Low risk & One of the papers explicitly addressed negative cases \\
\hline $\begin{array}{l}\text { Reliability of findings and } \\
\text { recommendations }\end{array}$ & Unclear risk & $\begin{array}{l}\text { Taken together, the } 3 \text { papers associated with this study present an accurate } \\
\text { description of the processes undertaken, but numerous children did not par- } \\
\text { ticipate and no clear explanation for this was provided }\end{array}$ \\
\hline Transferability of findings & Unclear risk & $\begin{array}{l}\text { Study authors did not address transferability, but the data are rich enough for } \\
\text { exploration of contextual factors, etc. No explanation is provided as to why so } \\
\text { many children did not participate }\end{array}$ \\
\hline
\end{tabular}


Joseph 2010 (Continued)

Overall risk of bias of process evaluation

Methods

\section{Included as process evaluation}

Intervention study design: parallel-group randomised controlled trial

Unit of allocation: child

Process evaluation methods: survey based, including multi-variate analyses of outcomes

Setting: 6 high schools in Detroit, Michigan, USA
Age of children: mean age, 15.6 years
Child characteristics (BME/SES): $98 \%$ African American; $74 \%$ of children were in receipt of free or re-
duced price school meals
Asthma status: asthmatic only; severity unclear
Intervention recipients: children only
School type: high school
Intervention description: this is an adapted version of a tailored computer programme (Puff City) that
was tested in Joseph 2010. Puff City focusses on 3 behaviours: controller medication adherence, keep-
ing an inhaler nearby, and smoking reduction or cessation. This new intervention included new sub-
modules designed to target teens with characteristics shown to be associated with lack of behaviour
change in the previous trial, and who exhibited no change after 1 or more sessions. Students were pro-
vided 4 sessions in total

Control description: controls received 4 sessions of generic asthma education to match the experience of students in the treatment group

Theoretical framework: Puff City uses tailoring to apply behavioural theory. Also includes Health Belief Model, Attribution Theory, and motivational interviewing

\begin{tabular}{ll}
\hline Outcomes & Core processes evaluated (child level): attrition, dosage, adherence \\
\hline Notes & Process evaluation category: stand-alone and integrated (2 papers) \\
& Breadth and depth: breadth and depth \\
& Voice of children given prominence: featured but not sufficiently \\
& $\begin{array}{l}\text { Note: study is not included as an outcome evaluation because the comparison group received asthma } \\
\text { education (this study evaluated the added impact of providing tailored messaging) }\end{array}$ \\
& Funding source: National Institutes of Health; National Heart, Lung, and Blood Institute \\
\hline Risk of bias & Authors' judgement Support for judgement \\
\hline Bias & Unclear risk N/A \\
\hline $\begin{array}{l}\text { Random sequence genera- } \\
\text { tion (selection bias) }\end{array}$ &
\end{tabular}


Joseph 2013 (Continued)

\begin{tabular}{lll}
$\begin{array}{l}\text { Allocation concealment } \\
\text { (selection bias) }\end{array}$ & Unclear risk & \\
\hline $\begin{array}{l}\text { Blinding of participants } \\
\text { and personnel (perfor- }\end{array}$ & Unclear risk & N/A \\
mance bias) & \\
All outcomes &
\end{tabular}

\begin{tabular}{lll}
\hline Blinding of outcome as- & Unclear risk & N/A \\
sessment (detection bias) &
\end{tabular}

All outcomes

\begin{tabular}{|c|c|c|}
\hline $\begin{array}{l}\text { Incomplete outcome data } \\
\text { (attrition bias) } \\
\text { All outcomes }\end{array}$ & Unclear risk & $\mathrm{N} / \mathrm{A}$ \\
\hline $\begin{array}{l}\text { Selective reporting (re- } \\
\text { porting bias) }\end{array}$ & Unclear risk & $\mathrm{N} / \mathrm{A}$ \\
\hline Other bias & Unclear risk & $\mathrm{N} / \mathrm{A}$ \\
\hline $\begin{array}{l}\text { Transparent and clearly } \\
\text { stated aims }\end{array}$ & Low risk & Study aims were clearly stated \\
\hline $\begin{array}{l}\text { Explicit theories underpin- } \\
\text { ning and/or literature re- } \\
\text { view }\end{array}$ & Low risk & Behavioural theory informed the intervention \\
\hline $\begin{array}{l}\text { Transparent and clearly } \\
\text { stated methods and tools }\end{array}$ & High risk & Information collected from caregivers is unclear \\
\hline Selective reporting & High risk & $\begin{array}{l}\text { Data were collected from caregivers, but study authors did not state what } \\
\text { these data included }\end{array}$ \\
\hline Harmful effects & Low risk & Subgroup analyses of the impact of potential risk group were undertaken \\
\hline $\begin{array}{l}\text { Population and sample } \\
\text { described well }\end{array}$ & Unclear risk & More could have been done to describe caregivers \\
\hline Continuous evaluation & Low risk & Pre-post assignment data were collected \\
\hline $\begin{array}{l}\text { Evaluation participation } \\
\text { equity and sampling }\end{array}$ & Unclear risk & Data collected are unclear \\
\hline $\begin{array}{l}\text { Design and methods over- } \\
\text { all approach }\end{array}$ & Unclear risk & $\begin{array}{l}\text { Data were collected but were not presented; a clear outline of the research de- } \\
\text { sign was not presented }\end{array}$ \\
\hline $\begin{array}{l}\text { Tools and methods of da- } \\
\text { ta collection reliable/cred- } \\
\text { ible }\end{array}$ & High risk & Information collected from caregivers is unclear \\
\hline $\begin{array}{l}\text { Tools and methods of data } \\
\text { analysis reliable/credible }\end{array}$ & Low risk & Data presented were analysed in a straightforward way \\
\hline $\begin{array}{l}\text { Performance bias/neutral- } \\
\text { ity/credibility/conforma- } \\
\text { bility }\end{array}$ & Unclear risk & Steps taken to address this are unclear \\
\hline
\end{tabular}


Joseph 2013 (Continued)

Reliability of findings and High risk Very low proportion of eligible students took part, so reliability of study findrecommendations ings was compromised

Transferability of findings Unclear risk

Subgroup analyses were conducted; however study authors did not address transferability of findings, and the high level of non-response does impede data transferability

\begin{tabular}{|c|c|c|}
\hline $\begin{array}{l}\text { Overall risk of bias of } \\
\text { process evaluation }\end{array}$ & High risk & $\begin{array}{l}\text { Data collected were not presented clearly; high level of non-participation im- } \\
\text { pinges on ability to generalise, even to the population in question }\end{array}$ \\
\hline
\end{tabular}

Kintner 2009

Methods Included as outcome evaluation

Study design: clustered parallel-group design, with schools selected as the unit of randomisation

Setting: 5 schools in a south-central Michigan school district

Period: dates from recruitment to data collection spanned from September 2006 to June 2007

Participants Eligible sample frame: 85 pupils found to be eligible

Randomised: 66 pupils randomised at the school level: 38 to treatment group and 28 to control group

Completed (intervention): 59 pupils completed the trial (7 dropped out of the intervention group)

Inclusion criteria: student eligibility criteria included (a) diagnosis of asthma, (b) availability to participate in scheduled classes or make-up sessions, and (c) verbal and written assent to participate

Exclusion criteria: student's expressed unwillingness to participate, lack of consent from parent or legal guardian

\section{Baseline characteristics}

Age of children: 9 to 12 years of age, with mean age, approximately 10.5 years

Ethnicity: 32\% African American; 15\% mixed race; 11\% other; 3\% Hispanic

Socio-economic status: unclear: mean score $>50$ on Nam-Powers Socioeconomic Status Scale

Gender: overall, $52 \%$ of participants were male

Asthma status: unclear: mean score on the Severity of Illness Scale was $>5.8$ for intervention and control groups

Intervention: study evaluated the SHARP programme (Staying Healthy - Asthma Responsible and Prepared). Study authors describe that 'the SHARP programme was integrated into the schools as a teaching module. Students met for 50-minute sessions delivered once a week for 10 weeks from January through March. Students worked through the 100-page SHARP workbook, which was designed to be colourful, entertaining, educational, and developmentally appropriate, as well as diverse with regards to gender, race, and culture. The programme was incorporated into the existing curriculum as an elective course through inclusion of spelling words, math problems, reading and writing assignments, discussions, demonstrations, and hands-on learning activities from biology, psychology, and sociology. To support SHARP students, caregivers and others participated in a 3-hour information sharing programme

Control: not clearly stated (usual care)

Intensity: targeted asthmatic students received 10 sessions in total

Instructor: unclear (potentially teachers, although not clear) 
Theoretical framework: a lifespan development perspective guided this study and served as the framework for development of the Acceptance of Asthma Model. Cognitive, behavioural, and psychosocial needs of students with asthma were addressed to foster acceptance of asthma by increasing longterm responsibility for maintaining and promoting health, and for preventing complications

Parental engagement: caregivers were involved, but level of engagement is unclear

Child satisfaction: not reported

Timing of intervention in school day: integrated into class time

\begin{tabular}{ll}
\hline Outcomes & Extractable outcomes were collected for: \\
& Health-related quality of life (study authors described participation in activities as a measure of quality \\
of life) & Withdrawal \\
\hline Notes & $\begin{array}{l}\text { Considered as a process evaluation but did not include the core components of a process evaluation, } \\
\text { with process data collected via use of structured tools }\end{array}$ \\
& $\begin{array}{l}\text { Funding source: National Institutes of Health and Staying Healthy - Asthma Responsible and Pre- } \\
\text { pared, National Institute of Nursing Research (Primary); National Heart, Lung, and Blood Institute; Na- } \\
\text { tional Institute of Allergy \& Infectious Diseases; National Institute of Child Health \& Human Develop- } \\
\text { ment }\end{array}$
\end{tabular}

\section{Risk of bias}

\begin{tabular}{|c|c|c|}
\hline Bias & Authors' judgement & Support for judgement \\
\hline $\begin{array}{l}\text { Random sequence genera- } \\
\text { tion (selection bias) }\end{array}$ & Low risk & Randomisation was computer-generated following Time 1 data collection \\
\hline $\begin{array}{l}\text { Allocation concealment } \\
\text { (selection bias) }\end{array}$ & Unclear risk & Unclear; few schools were allocated \\
\hline $\begin{array}{l}\text { Blinding of participants } \\
\text { and personnel (perfor- } \\
\text { mance bias) } \\
\text { All outcomes }\end{array}$ & High risk & $\begin{array}{l}\text { Interveners and participants were not blinded to randomisation after schools } \\
\text { were designated to treatment and control groups }\end{array}$ \\
\hline $\begin{array}{l}\text { Blinding of outcome as- } \\
\text { sessment (detection bias) } \\
\text { All outcomes }\end{array}$ & Low risk & $\begin{array}{l}\text { Evaluators were instructed to not assume or ask the randomisation status of } \\
\text { participants. Participants were requested to not disclose randomisation status } \\
\text { to evaluators }\end{array}$ \\
\hline $\begin{array}{l}\text { Incomplete outcome data } \\
\text { (attrition bias) } \\
\text { All outcomes }\end{array}$ & Low risk & $\begin{array}{l}\text { Relatively low levels of attrition; baseline characteristics of those who dropped } \\
\text { out of the intervention group did not differ from characteristics of those who } \\
\text { completed post-intervention assessment }\end{array}$ \\
\hline $\begin{array}{l}\text { Selective reporting (re- } \\
\text { porting bias) }\end{array}$ & Unclear risk & $\begin{array}{l}\text { A large body of data was collected; data were aggregated into various scores, } \\
\text { hindering their interpretation and use within meta-analyses }\end{array}$ \\
\hline Other bias & Low risk & $\begin{array}{l}\text { Missingness - low risk - baseline characteristics of those who dropped out of } \\
\text { the intervention group did not differ from characteristics of those who com- } \\
\text { pleted post-intervention assessment } \\
\text { Baseline imbalance - low risk - baseline group imbalance was addressed by } \\
\text { adjusting for baseline values of outcomes in the analyses }\end{array}$ \\
\hline & & Risk of contamination - low - schools were the unit of randomisation \\
\hline
\end{tabular}


Kintner 2009 (Continued)

\begin{tabular}{|c|c|}
\hline $\begin{array}{l}\text { Transparent and clearly } \\
\text { stated aims }\end{array}$ & Unclear risk \\
\hline
\end{tabular}

\begin{tabular}{ll}
\hline Explicit theories underpin- & Unclear risk N/A \\
ning and/or literature re- &
\end{tabular}

view

\begin{tabular}{|c|c|c|}
\hline $\begin{array}{l}\text { Transparent and clearly } \\
\text { stated methods and tools }\end{array}$ & Unclear risk & $\mathrm{N} / \mathrm{A}$ \\
\hline Selective reporting & Unclear risk & $\mathrm{N} / \mathrm{A}$ \\
\hline Harmful effects & Unclear risk & $\mathrm{N} / \mathrm{A}$ \\
\hline $\begin{array}{l}\text { Population and sample } \\
\text { described well }\end{array}$ & Unclear risk & $\mathrm{N} / \mathrm{A}$ \\
\hline Continuous evaluation & Unclear risk & $\mathrm{N} / \mathrm{A}$ \\
\hline $\begin{array}{l}\text { Evaluation participation } \\
\text { equity and sampling }\end{array}$ & Unclear risk & $\mathrm{N} / \mathrm{A}$ \\
\hline $\begin{array}{l}\text { Design and methods over- } \\
\text { all approach }\end{array}$ & Unclear risk & $\mathrm{N} / \mathrm{A}$ \\
\hline $\begin{array}{l}\text { Tools and methods of da- } \\
\text { ta collection reliable/cred- } \\
\text { ible }\end{array}$ & Unclear risk & $\mathrm{N} / \mathrm{A}$ \\
\hline $\begin{array}{l}\text { Tools and methods of data } \\
\text { analysis reliable/credible }\end{array}$ & Unclear risk & $\mathrm{N} / \mathrm{A}$ \\
\hline $\begin{array}{l}\text { Performance bias/neutral- } \\
\text { ity/credibility/conforma- } \\
\text { bility }\end{array}$ & Unclear risk & $\mathrm{N} / \mathrm{A}$ \\
\hline $\begin{array}{l}\text { Reliability of findings and } \\
\text { recommendations }\end{array}$ & Unclear risk & $\mathrm{N} / \mathrm{A}$ \\
\hline Transferability of findings & Unclear risk & $\mathrm{N} / \mathrm{A}$ \\
\hline $\begin{array}{l}\text { Overall risk of bias of } \\
\text { process evaluation }\end{array}$ & Unclear risk & $\mathrm{N} / \mathrm{A}$ \\
\hline
\end{tabular}

Kintner 2012

\begin{tabular}{ll} 
Methods & Included as process evaluation \\
& Intervention study design: quasi-experimental, pre-post, no control \\
& Unit of allocation: N/A \\
& Process evaluation methods: descriptive/bivariate \\
\hline Participants & Country: USA \\
& Age of children: sixth and seventh grades \\
\hline
\end{tabular}


Kintner 2012 (Continued)

Child characteristics (BME/SES): 53.6\% African American; 35.7\% lower SES

Asthma status: asthmatic only

Intervention recipients: students, members of their social network
School type: high school

Intervention description: SHARP, community coalition component

Control description: N/A

Theoretical framework: asthma model and lifespan development perspective

\begin{tabular}{ll}
\hline Outcomes & Core processes evaluated (child level): dosage, adherence \\
\hline Notes & Process evaluation category: stand-alone \\
& Breadth and depth: breadth and depth \\
& Voice of children given prominence: sufficient coverage \\
& Funding source: not reported \\
\hline
\end{tabular}

\section{Risk of bias}

\begin{tabular}{|c|c|c|}
\hline Bias & Authors' judgement & Support for judgement \\
\hline $\begin{array}{l}\text { Random sequence genera- } \\
\text { tion (selection bias) }\end{array}$ & Unclear risk & $\mathrm{N} / \mathrm{A}$ \\
\hline $\begin{array}{l}\text { Allocation concealment } \\
\text { (selection bias) }\end{array}$ & Unclear risk & $\mathrm{N} / \mathrm{A}$ \\
\hline $\begin{array}{l}\text { Blinding of participants } \\
\text { and personnel (perfor- } \\
\text { mance bias) } \\
\text { All outcomes }\end{array}$ & Unclear risk & $\mathrm{N} / \mathrm{A}$ \\
\hline $\begin{array}{l}\text { Blinding of outcome as- } \\
\text { sessment (detection bias) } \\
\text { All outcomes }\end{array}$ & Unclear risk & $\mathrm{N} / \mathrm{A}$ \\
\hline $\begin{array}{l}\text { Incomplete outcome data } \\
\text { (attrition bias) } \\
\text { All outcomes }\end{array}$ & Unclear risk & $\mathrm{N} / \mathrm{A}$ \\
\hline $\begin{array}{l}\text { Selective reporting (re- } \\
\text { porting bias) }\end{array}$ & Unclear risk & $\mathrm{N} / \mathrm{A}$ \\
\hline Other bias & Unclear risk & $\mathrm{N} / \mathrm{A}$ \\
\hline $\begin{array}{l}\text { Transparent and clearly } \\
\text { stated aims }\end{array}$ & Low risk & Study aims were clearly stated \\
\hline
\end{tabular}

Explicit theories underpin- Low risk A theoretical framework was clearly presented ning and/or literature review 
Kintner 2012 (Continued)

\begin{tabular}{|c|c|c|}
\hline $\begin{array}{l}\text { Transparent and clearly } \\
\text { stated methods and tools }\end{array}$ & Low risk & Methods were well described \\
\hline Selective reporting & Unclear risk & Not all indicators were fully reported, for example, asthma severity \\
\hline Harmful effects & Unclear risk & These effects were not presented by study authors but can be inferred \\
\hline $\begin{array}{l}\text { Population and sample } \\
\text { described well }\end{array}$ & Low risk & Participant demographics were clearly described \\
\hline Continuous evaluation & Low risk & Pre-post assignment assessment of data was provided \\
\hline $\begin{array}{l}\text { Evaluation participation } \\
\text { equity and sampling }\end{array}$ & Low risk & All relevant stakeholders were apparently involved in the evaluation \\
\hline $\begin{array}{l}\text { Design and methods over- } \\
\text { all approach }\end{array}$ & Low risk & Data were collected from multiple sources and at multiple time points \\
\hline $\begin{array}{l}\text { Tools and methods of da- } \\
\text { ta collection reliable/cred- } \\
\text { ible }\end{array}$ & Low risk & Tools and methods were well described \\
\hline $\begin{array}{l}\text { Tools and methods of data } \\
\text { analysis reliable/credible }\end{array}$ & Unclear risk & Not everything was presented in full \\
\hline $\begin{array}{l}\text { Performance bias/neutral- } \\
\text { ity/credibility/conforma- } \\
\text { bility }\end{array}$ & Unclear risk & $\begin{array}{l}\text { These were not fully addressed; only a few steps appear to have been taken to } \\
\text { minimise the possibility of performance bias }\end{array}$ \\
\hline
\end{tabular}

\begin{tabular}{lll}
\hline $\begin{array}{l}\text { Reliability of findings and } \\
\text { recommendations }\end{array}$ & Low risk & How findings came about is clearly shown by study data \\
\hline Transferability of findings & Unclear risk & $\begin{array}{l}\text { Study authors did not focus on what might need to be changed to scale up. } \\
\text { Some measures (e.g. individual interviews with dyads) are not transferable, al- } \\
\text { though it is not clear if study authors shared this view }\end{array}$ \\
\hline
\end{tabular}

Overall risk of bias of Low risk This is a good example of a process evaluation study

process evaluation

Kouba 2012

\begin{tabular}{ll}
\hline Methods & Included as process evaluation \\
Intervention study design: quasi-experimental single-group study examining change pre-post inter- \\
vention \\
Unit of allocation: N/A \\
Process evaluation methods: descriptive and bivariate methods of analyses of survey-based data (as \\
well as administrative records) \\
Setting: 2 urban high schools in the USA \\
Age of children: ninth through 12 th grade; average age was 15.9 years \\
Child characteristics (BME/SES): $92 \%$ African American, $4 \%$ Hispanic, $4 \%$ mixed ethnicity. Combined \\
median family income ranged from USD30,000 to USD39,000
\end{tabular}


Kouba 2012 (Continued)

Asthma status: asthmatic only; $66 \%$ of children were deemed to have control of their asthma at baseline according to ACT tests

Intervention recipients: children only (targeted overweight/obese children)

Interventions

School type: 2 high schools

Intervention description: I Can Control Asthma and Nutrition Now (ICAN): the ICAN programme was developed as an adaptation of an existing intervention and is composed of 3 elements: (I) asthma education; (ii) coping skills training; and (iii) nurse practitioner-reinforcement visits. In this study, $60 \%$ of students were overweight or obese. Because of concerns about the increasing prevalence of both youth asthma and obesity, study authors added a nutrition component to the intervention, so that the intervention could address these comorbidities. The ICAN programme is thus composed of 4 elements: (I) asthma education, (ii) nutrition education synthesised with CST, targeting obesity prevention and management, (iii) reinforcement visits with a registered nurse (RN) and a dietetic intern, and (iv) a family information meeting

Control description: N/A

Theoretical framework: Orem's self-care deficit theory (SCDT)

Outcomes Core processes evaluated (child level): attrition, dosage

Notes Process evaluation category: integrated within outcome evaluation

Breadth and depth: depth - not breadth

Voice of children given prominence: voice and views of children not featured

Funding source: Loyola University Chicago Niehoff School of Nursing

\begin{tabular}{|c|c|c|}
\hline \multicolumn{3}{|l|}{ Risk of bias } \\
\hline Bias & Authors' judgement & Support for judgement \\
\hline $\begin{array}{l}\text { Random sequence genera- } \\
\text { tion (selection bias) }\end{array}$ & Unclear risk & $\mathrm{N} / \mathrm{A}$ \\
\hline $\begin{array}{l}\text { Allocation concealment } \\
\text { (selection bias) }\end{array}$ & Unclear risk & $\mathrm{N} / \mathrm{A}$ \\
\hline $\begin{array}{l}\text { Blinding of participants } \\
\text { and personnel (perfor- } \\
\text { mance bias) } \\
\text { All outcomes }\end{array}$ & Unclear risk & $\mathrm{N} / \mathrm{A}$ \\
\hline $\begin{array}{l}\text { Blinding of outcome as- } \\
\text { sessment (detection bias) } \\
\text { All outcomes }\end{array}$ & Unclear risk & $\mathrm{N} / \mathrm{A}$ \\
\hline $\begin{array}{l}\text { Incomplete outcome data } \\
\text { (attrition bias) } \\
\text { All outcomes }\end{array}$ & Unclear risk & $\mathrm{N} / \mathrm{A}$ \\
\hline $\begin{array}{l}\text { Selective reporting (re- } \\
\text { porting bias) }\end{array}$ & Unclear risk & $\mathrm{N} / \mathrm{A}$ \\
\hline Other bias & Unclear risk & $\mathrm{N} / \mathrm{A}$ \\
\hline
\end{tabular}


Kouba 2012 (Continued)

Transparent and clearly Low risk Study aims were clearly stated
stated aims

Explicit theories underpin- Low risk Orem's self-care deficit theory guided the intervention
ning and/or literature re-
view

\begin{tabular}{lll}
\hline $\begin{array}{l}\text { Transparent and clearly } \\
\text { stated methods and tools }\end{array}$ & Low risk & Methods and tools were fully described \\
\hline Selective reporting & Low risk & All planned outcomes were reported on \\
\hline Harmful effects & Unclear risk & $\begin{array}{l}\text { Generalisability was not considered. Some of the challenges of working with } \\
\text { obese kids who are not adherent were not reported }\end{array}$ \\
\hline
\end{tabular}

\begin{tabular}{lll}
\hline $\begin{array}{l}\text { Population and sample } \\
\text { described well }\end{array}$ & Low risk & Population demographics were clearly described \\
\hline Continuous evaluation & Low risk & Two follow-ups post intervention \\
\hline $\begin{array}{l}\text { Evaluation participation } \\
\text { equity and sampling }\end{array}$ & Unclear risk & No evidence of satisfaction evaluation and no child perspectives given \\
\hline
\end{tabular}

\begin{tabular}{|c|c|c|}
\hline $\begin{array}{l}\text { Design and methods over- } \\
\text { all approach }\end{array}$ & Unclear risk & Everything required for a good process evaluation was not captured \\
\hline $\begin{array}{l}\text { Tools and methods of da- } \\
\text { ta collection reliable/cred- } \\
\text { ible }\end{array}$ & Unclear risk & $\begin{array}{l}\text { Nothing suggests that this study was at high risk of bias; however no study } \\
\text { steps were described }\end{array}$ \\
\hline $\begin{array}{l}\text { Tools and methods of data } \\
\text { analysis reliable/credible }\end{array}$ & Low risk & All methods of data analysis are appropriate for the study \\
\hline $\begin{array}{l}\text { Performance bias/neutral- } \\
\text { ity/credibility/conforma- } \\
\text { bility }\end{array}$ & Unclear risk & $\begin{array}{l}\text { Not much evidence of this; however analysis was carried out by research asso- } \\
\text { ciates under the supervision of the statistician }\end{array}$ \\
\hline $\begin{array}{l}\text { Reliability of findings and } \\
\text { recommendations }\end{array}$ & Unclear risk & $\begin{array}{l}\text { This included a small sample, and the voice of children did not feature promi- } \\
\text { nently }\end{array}$ \\
\hline Transferability of findings & High risk & Small sample size limits the transferability of findings \\
\hline $\begin{array}{l}\text { Overall risk of bias of } \\
\text { process evaluation }\end{array}$ & Unclear risk & $\begin{array}{l}\text { As a process evaluation, this study was limited, and the breadth of the study in } \\
\text { including nutrition alongside asthma education was not evaluated }\end{array}$ \\
\hline
\end{tabular}

Langenfeld 2010

\begin{tabular}{ll}
\hline Methods & Included as process evaluation \\
& Intervention study design: quasi-experimental, pre-post, with no control \\
& Unit of allocation: N/A \\
& Process evaluation methods: bivariate analysis methods \\
\hline Participants & Country: USA
\end{tabular}


Age of children: students from grades 3, 4, and 5 were enrolled. All students were between 5 and 10 years old

Child characteristics (BME/SES): 63\% African American; large number of students were eligible for a free, or reduced price, lunch

Asthma status: asthmatic only

Intervention recipients: children and teachers

\begin{abstract}
Interventions
School type: 286 students from 12 elementary schools

Intervention description: children participated in the OAS programme, consisting of six 40-minute education sessions on asthma and asthma management. Children and staff also participated in the asthma programme, developed through case management strategies. This included 5 core components, including case management for specific children selected by the school nurse, and staff development and education about asthma
\end{abstract}

Control description: N/A

Theoretical framework: not reported

\begin{tabular}{ll}
\hline Outcomes & Core processes evaluated (child level): dosage \\
\hline Notes & Process evaluation category: stand-alone \\
& Breadth and depth: depth - not breadth \\
& Voice of children given prominence: not featured \\
& Funding source: not reported
\end{tabular}

\title{
Risk of bias
}

\begin{tabular}{lll}
\hline Bias & Authors' judgement & Support for judgement \\
\hline $\begin{array}{l}\text { Random sequence genera- } \\
\text { tion (selection bias) }\end{array}$ & Unclear risk & $\mathrm{N} / \mathrm{A}$ \\
\hline $\begin{array}{l}\text { Allocation concealment } \\
\text { (selection bias) }\end{array}$ & Unclear risk & $\mathrm{N} / \mathrm{A}$ \\
\hline
\end{tabular}

Blinding of participants Unclear risk N/A
and personnel (perfor-

mance bias)

All outcomes

\begin{tabular}{lll}
\hline Blinding of outcome as- & Unclear risk & N/A \\
sessment (detection bias) &
\end{tabular}

All outcomes

\begin{tabular}{l}
\hline Incomplete outcome data $\quad$ Unclear risk N/A \\
(attrition bias) \\
All outcomes
\end{tabular}

Selective reporting (re- $\quad$ Unclear risk $\quad$ N/A
porting bias)

Other bias Unclear risk N/A


Langenfeld 2010 (Continued)

Transparent and clearly Unclear risk Study aims can be inferred by the reader but were not clearly stated stated aims

Explicit theories underpin- High risk ning and/or literature review

\begin{tabular}{lll}
\hline $\begin{array}{l}\text { Transparent and clearly } \\
\text { stated methods and tools }\end{array}$ & Unclear risk & Methods described are relatively transparent, but some ambiguity remains \\
\hline Selective reporting & Unclear risk & $\begin{array}{l}\text { This paper did not claim to present outcomes but focused on whether the right } \\
\text { people received the right intervention }\end{array}$
\end{tabular}

\begin{tabular}{lll}
\hline Harmful effects & Unclear risk & $\begin{array}{l}\text { Harmful effects in terms of recruitment were definitely addressed, but other } \\
\text { harmful effects were not considered }\end{array}$ \\
\hline $\begin{array}{l}\text { Population and sample } \\
\text { described well }\end{array}$ & Unclear risk & $\begin{array}{l}\text { Study population and sample were generally well described, but school demo- } \\
\text { graphics were not reported }\end{array}$ \\
\hline $\begin{array}{l}\text { Continuous evaluation } \\
\text { Evaluation participation } \\
\text { equity and sampling }\end{array}$ & Low risk & Pre-post assessment was conducted \\
\hline
\end{tabular}

Design and methods over- Unclear risk

all approach

\begin{tabular}{lll}
\hline $\begin{array}{l}\text { Tools and methods of da- } \\
\text { ta collection reliable/cred- } \\
\text { ible }\end{array}$ & Low risk & Tools used for data collection were appropriate for the data \\
\hline $\begin{array}{l}\text { Tools and methods of data } \\
\text { analysis reliable/credible }\end{array}$ & Unclear risk & $\begin{array}{l}\text { No in-depth analysis was provided; insights gained from nurse interviews are } \\
\text { unclear }\end{array}$ \\
\hline $\begin{array}{l}\text { Performance bias/neutral- } \\
\text { ity/credibility/conforma- } \\
\text { bility }\end{array}$ & Unclear risk & $\begin{array}{l}\text { Premise of the paper involves thinking about the robustness of certain } \\
\text { processes, but it is unclear who conducted the analysis }\end{array}$ \\
\hline $\begin{array}{l}\text { Reliability of findings and } \\
\text { recommendations }\end{array}$ & Unclear risk & $\begin{array}{l}\text { This paper clearly presented one point, but obscurity surrounds nurses' rat- } \\
\text { ings }\end{array}$ \\
\hline $\begin{array}{l}\text { Transferability of findings } \\
\text { Overall risk of bias of }\end{array}$ & Low risk & Unclear risk \\
\begin{tabular}{l} 
process evaluation \\
\hline
\end{tabular} & $\begin{array}{l}\text { As a process evaluation, its low breadth introduces high risk of bias to this trial, } \\
\text { but in evaluating a single process, this is a really good trial }\end{array}$ \\
\hline
\end{tabular}

Lee 2011

Methods Included as process evaluation

Intervention study design: quasi-experimental single-group intervention examining change pre-post intervention

Unit of allocation: N/A 
Lee 2011 (Continued)

Process evaluation methods: qualitative and quantitative data collection; descriptive/bivariate, thematic/grounded theory, narrative data analysis

Setting: selected schools ( 67 schools) in Cleveland Metropolitan School District
Age of children: 8 to 11 years old
Child characteristics (BME/SES): no information
Asthma status: asthmatic only
Intervention recipients: children only
School type: primary/elementary
Intervention description: Open Airways for Schools (also testing the feasibility of undergraduate nurs-
ing students as instructors). The study author described that "the curriculum consists of six 40-minute
group lessons held during the school day. These lessons use group discussion, stories, role-playing, and
games to help the children understand more about asthma and to engage them more in the empow-
erment of managing their disease. The topics that are included in the program are basic information
about asthma, recognizing and managing asthma symptoms, using medication, avoiding asthma trig-
gers, getting enough exercise, and doing well in school. Each lesson focuses on one of the above topics
with a review of previous information for enforcement of the skills and knowledge learned. The overall
goals of the program are to (a) improve asthma self-management skills, (b) decrease asthma emergen-
cies, (c) raise awareness among parents/guardians, and (d) promote broader asthma management co-
ordination among physicians, parents, and schools"

Control description: N/A

Theoretical framework: functional context approach

\begin{tabular}{|c|c|c|}
\hline Outcomes & \multicolumn{2}{|c|}{ Core processes evaluated (child level): explored some indicators of adherence } \\
\hline \multirow[t]{4}{*}{ Notes } & \multicolumn{2}{|c|}{ Process evaluation category: integrated within outcome evaluation } \\
\hline & \multicolumn{2}{|c|}{ Breadth and depth: neither broad nor deep } \\
\hline & \multicolumn{2}{|c|}{ Voice of children given prominence: featured but not sufficiently } \\
\hline & \multicolumn{2}{|c|}{ Funding source: not reported } \\
\hline \multicolumn{3}{|l|}{ Risk of bias } \\
\hline Bias & Authors' judgement & Support for judgement \\
\hline $\begin{array}{l}\text { Random sequence genera- } \\
\text { tion (selection bias) }\end{array}$ & Unclear risk & $\mathrm{N} / \mathrm{A}$ \\
\hline $\begin{array}{l}\text { Allocation concealment } \\
\text { (selection bias) }\end{array}$ & Unclear risk & $\mathrm{N} / \mathrm{A}$ \\
\hline $\begin{array}{l}\text { Blinding of participants } \\
\text { and personnel (perfor- } \\
\text { mance bias) } \\
\text { All outcomes }\end{array}$ & Unclear risk & $\mathrm{N} / \mathrm{A}$ \\
\hline $\begin{array}{l}\text { Blinding of outcome as- } \\
\text { sessment (detection bias) } \\
\text { All outcomes }\end{array}$ & Unclear risk & $\mathrm{N} / \mathrm{A}$ \\
\hline
\end{tabular}


Lee 2011 (Continued)

Incomplete outcome data Unclear risk N/A
(attrition bias)
All outcomes

\begin{tabular}{lll}
\hline $\begin{array}{l}\text { Selective reporting (re- } \\
\text { porting bias) }\end{array}$ & Unclear risk \\
\hline Other bias & Unclear risk & $\mathrm{N} / \mathrm{A}$ \\
\hline $\begin{array}{l}\text { Transparent and clearly } \\
\text { stated aims }\end{array}$ & Low risk & Study aims were clearly stated \\
\hline
\end{tabular}

Explicit theories underpin- Low risk Functional context approach guided development of the intervention
ning and/or literature review

\begin{tabular}{|c|c|c|}
\hline $\begin{array}{l}\text { Transparent and clearly } \\
\text { stated methods and tools }\end{array}$ & Unclear risk & $\begin{array}{l}\text { In terms of the outcome evaluation and information collected from children, } \\
\text { lack of transparency surrounds the sample frame and tools }\end{array}$ \\
\hline Selective reporting & High risk & Many instruments were not presented \\
\hline Harmful effects & Unclear risk & $\begin{array}{l}\text { Some elements that could be negative were included, but nothing from the } \\
\text { children is included }\end{array}$ \\
\hline $\begin{array}{l}\text { Population and sample } \\
\text { described well }\end{array}$ & High risk & $\begin{array}{l}\text { Not much information was included on participants, and some details of } \\
\text { the children were not described. More information was provided about the } \\
\text { schools themselves }\end{array}$ \\
\hline Continuous evaluation & Unclear risk & Pre-post assessment, but post hoc only for nurses \\
\hline $\begin{array}{l}\text { Evaluation participation } \\
\text { equity and sampling }\end{array}$ & Unclear risk & Nothing from the teachers was reported \\
\hline $\begin{array}{l}\text { Design and methods over- } \\
\text { all approach }\end{array}$ & High risk & $\begin{array}{l}\text { Structured data about implementation - e.g. attrition, adherence - are insuffi- } \\
\text { cient }\end{array}$ \\
\hline $\begin{array}{l}\text { Tools and methods of da- } \\
\text { ta collection reliable/cred- } \\
\text { ible }\end{array}$ & High risk & $\begin{array}{l}\text { Lack of transparency is evident among the methods used to assess child out- } \\
\text { comes }\end{array}$ \\
\hline $\begin{array}{l}\text { Tools and methods of data } \\
\text { analysis reliable/credible }\end{array}$ & High risk & Quantitative data were not analysed fully - e.g. lack of subgroup analyses \\
\hline $\begin{array}{l}\text { Performance bias/neutral- } \\
\text { ity/credibility/conforma- } \\
\text { bility }\end{array}$ & High risk & How aspects around neutrality were addressed is unclear \\
\hline $\begin{array}{l}\text { Reliability of findings and } \\
\text { recommendations }\end{array}$ & High risk & $\begin{array}{l}\text { Study design did not support the research question - results from different in- } \\
\text { structors were not compared }\end{array}$ \\
\hline Transferability of findings & High risk & Generalisability was not explicitly considered in enough detail \\
\hline $\begin{array}{l}\text { Overall risk of bias of } \\
\text { process evaluation }\end{array}$ & High risk & $\begin{array}{l}\text { Study has several limitations, including reporting bias and lack of transparen- } \\
\text { cy }\end{array}$ \\
\hline
\end{tabular}




\section{Included as outcome evaluation and process evaluation}

Intervention study design: cluster parallel-group RCT with schools selected as unit of randomisation, based on a stratified design according to school characteristics

Setting: 14 elementary schools in Memphis, Tennessee, USA, school district participated in the study

Period: study was conducted over 2 school years between 1999 and 2001

Participants

Eligible sample frame: see below

Randomised (based on year 1): 14 schools randomised. In 8 treatment group schools, 115 students participated, and in 6 usual care schools, 128 students participated. This represented a consent rate of $48 \%$ for both groups of students, whose parents reported asthma on the student's registration form

Completed (intervention) (based on year 1): in the treatment group, 90 (78.3\%) parents completed both pre- and post-test surveys; in the usual care group, 72 (56.3\%) parents participated in post-test surveys

Inclusion criteria: children 6 to 10 years of age with a diagnosis of asthma reported on school health forms and whose parents provided consent (see above)

Exclusion criteria: not reported

\section{Baseline characteristics}

Age of children: 6 to 10 years of age; further breakdown not provided

Ethnicity (based on year 1): $97 \%$ of children in the treatment group and $99 \%$ of children in the control group were African American

Socio-economic status: (based on year 1): $81 \%$ of students in the treatment group and $85 \%$ in the control group were in receipt of TennCare health insurance (a state-specific version of Medicare)

Gender (based on year 1): treatment: 58\% male, $42 \%$ female; control: $57 \%$ male, $53 \%$ female

Asthma status: not reported directly

Intervention: the intervention consisted of the following: "(1) education (delivery of the Open Airways curriculum to students in a weekly group setting at school), (2) weekly monitoring of students' health status (following up on absences and symptoms with students, families, and teachers), and (3) coordination of care (contacting students, family members, school personnel, and medical providers to facilitate disease management and mitigate environmental triggers at school and at home)"

The intervention aimed to introduce the following principles of self-management: "(1) periodic physiologic assessment and monitoring of asthma symptoms, (2) appropriate use of medications, (3) patient education, and (4) control of factors contributing to asthma severity"

Control: usual care

Intensity: weekly group sessions and weekly individual sessions; "nurse case managers met with students weekly from October through May to teach and coach students on asthma knowledge and treatment techniques"

Instructor: school-based nurses

Theoretical framework: not directly reported

Parental engagement: difficulties reported; low levels of consent and high levels of attrition at posttest survey

Child satisfaction: not reported

Timing of intervention: during school day; exact time unclear 
Levy 2006 (Continued)

Outcomes

\section{Extractable outcomes were collected for:}

Exacerbations leading to hospital admission

Asthma symptoms leading to emergency hospital visits

Withdrawal

Core processes outcomes evaluated (child level): attrition
Notes for outcome evaluation: only data for year 1 used, as year 2 data not collected through randomised study design

\section{Additional measures of process evaluation quality}

Process evaluation category: integrated

Breadth and depth: breadth - not depth

Voice of children given prominence: not featured

Funding source: Tennessee Department of Health

\title{
Risk of bias
}

\begin{tabular}{lll}
\hline Bias & Authors' judgement & Support for judgement \\
\hline $\begin{array}{l}\text { Random sequence genera- } \\
\text { tion (selection bias) }\end{array}$ & Unclear risk & No information on method of randomisation was provided \\
\hline $\begin{array}{l}\text { Allocation concealment } \\
\text { (selection bias) }\end{array}$ & High risk & $\begin{array}{l}\text { Study author description suggests that allocation concealment was broken: } \\
\text { "although schools were matched on demographic variables, a greater number } \\
\text { of schools were randomised to intervention status than usual care because of } \\
\text { staffing considerations and the school district's request for intervention in as } \\
\text { many schools as possible" }\end{array}$ \\
\hline
\end{tabular}

Blinding of participants Low risk Some measures were taken to ensure blinding of personnel
and personnel (perfor-
mance bias)
All outcomes

\begin{tabular}{lll}
\hline $\begin{array}{l}\text { Blinding of outcome as- } \\
\text { sessment (detection bias) } \\
\text { All outcomes }\end{array}$ & Low risk & $\begin{array}{l}\text { Clear that blinding was implemented for several outcomes based on study au- } \\
\text { thor's description of staff being blinded to students' experimental condition in } \\
\text { dealing with abstracted medical data from computerised hospital records }\end{array}$ \\
\hline $\begin{array}{l}\text { Incomplete outcome data } \\
\text { (attrition bias) }\end{array}$ & High risk & $\begin{array}{l}\text { Study authors reported a differential loss to follow-up: "there was a 24\% loss } \\
\text { in follow-up with intervention parents and a 44\% dropout rate with usual care } \\
\text { parents" }\end{array}$
\end{tabular}

Selective reporting (re- High risk
porting bias)
High risk, as study authors tended to report in passing or incompletely out-
comes that were not significantly different porting bias)

Other bias Unclear risk

\begin{abstract}
Missingness - high risk - evidence shows substantial missing data, as study authors described that "in the usual care parent group, only 72 of the possible 128 parents participated in the posttest surveys, and considerable data were missing". How this affected outcomes is unclear

Baseline imbalance - low risk - no evidence shows a baseline imbalance in the characteristics of children
\end{abstract}


Levy 2006 (Continued)

Risk of contamination - low - randomisation took place by school, thereby lowering the risk of contamination

\begin{tabular}{|c|c|c|}
\hline $\begin{array}{l}\text { Transparent and clearly } \\
\text { stated aims }\end{array}$ & Unclear risk & Medium bias - aims were inferred \\
\hline $\begin{array}{l}\text { Explicit theories underpin- } \\
\text { ning and/or literature re- } \\
\text { view }\end{array}$ & High risk & No theory and not much literature were provided \\
\hline $\begin{array}{l}\text { Transparent and clearly } \\
\text { stated methods and tools }\end{array}$ & Unclear risk & These data were not clearly reported by study authors \\
\hline Selective reporting & High risk & Some outcomes were not fully reported (outcomes as opposed to processes) \\
\hline Harmful effects & Low risk & $\begin{array}{l}\text { Study authors did consider harmful effects and paid attention to harmful } \\
\text { processes or implementation problems }\end{array}$ \\
\hline $\begin{array}{l}\text { Population and sample } \\
\text { described well }\end{array}$ & Unclear risk & $\begin{array}{l}\text { Medium bias - some conflation between year } 1 \text { and year } 2 \text { populations is evi- } \\
\text { dent }\end{array}$ \\
\hline Continuous evaluation & Unclear risk & Medium bias - pre-post for most of the process evaluation \\
\hline $\begin{array}{l}\text { Evaluation participation } \\
\text { equity and sampling }\end{array}$ & High risk & No input from children or teachers and no satisfaction data were reported \\
\hline $\begin{array}{l}\text { Design and methods over- } \\
\text { all approach }\end{array}$ & Unclear risk & $\begin{array}{l}\text { Data were collected at multiple time points and from different sources; but } \\
\text { they were not very well reported }\end{array}$ \\
\hline $\begin{array}{l}\text { Tools and methods of da- } \\
\text { ta collection reliable/cred- } \\
\text { ible }\end{array}$ & Unclear risk & $\begin{array}{l}\text { Lack of transparency surrounds some aspects, so it is difficult to categorise } \\
\text { this risk of bias appropriately }\end{array}$ \\
\hline $\begin{array}{l}\text { Tools and methods of data } \\
\text { analysis reliable/credible }\end{array}$ & High risk & $\begin{array}{l}\text { Lack of disaggregated data and the bivariate nature of the data mean that this } \\
\text { study is likely at high risk of bias }\end{array}$ \\
\hline $\begin{array}{l}\text { Performance bias/neutral- } \\
\text { ity/credibility/conforma- } \\
\text { bility }\end{array}$ & Low risk & $\begin{array}{l}\text { Study authors attempted to introduce rigour and provided a full outline of the } \\
\text { caveats. Staff who were blinded to students' experimental condition abstract- } \\
\text { ed medical data from computerised hospital records }\end{array}$ \\
\hline $\begin{array}{l}\text { Reliability of findings and } \\
\text { recommendations }\end{array}$ & Low risk & How conclusions were reached based on the data is clear \\
\hline Transferability of findings & Low risk & Study findings are transferable to the wider population \\
\hline $\begin{array}{l}\text { Overall risk of bias of } \\
\text { process evaluation }\end{array}$ & Unclear risk & Bivariate nature of this study makes it have high risk of bias \\
\hline
\end{tabular}

Magzamen 2008

Methods Included as process evaluation

Intervention study design: quasi-experimental single-group intervention examining change pre-post intervention

Unit of allocation: N/A 
Magzamen 2008 (Continued)

Process evaluation methods: survey based using descriptive/bivariate analyses. Some regression analyses reported controlling for previous baseline observations

Setting: 15 schools in Oakland, California, USA
Age of children: 11 to 16 years of age (although more than $80 \%$ were 11 to 12 years old)
Child characteristics (BME/SES): ethnicity of children is unclear (although intervention took place in a
diverse catchment area). Socio-economic status of children unclear, although intervention took place
within a deprived school system
Asthma status: asthmatic only (diagnosed asthma)
Intervention recipients: children only
School type: junior/middle schools and high schools
Intervention description: study authors presented results from Kickin' Asthma, described as "a 4-
session curriculum developed jointly by experts, nurses and peer educators and delivered over a 3-
year period. Kickin' Asthma is similar structurally to Open Airways for Schools, a curriculum designed
for children at the elementary school level but with more advanced topics and learning modalities
more suitable for adolescents' level of cognition and awareness. The 4 Kickin' Asthma sessions were
each taught by a specialist nurse, about 50 minutes in length, and were spaced 1 week apart. The 4 ses-
sions covered (1) lung physiology and asthma basics; (2) triggers, symptoms, and warning signs; (3)
medication; and (4) emergencies, problem solving, and review. Each session has optional modules for
skits, games, videos, and role-playing scenarios and allowed certain modules to be taught by either the
health educator or peer educators. Customized letters were sent home to the parents or guardians of
all Kickin' Asthma participants that described the curriculum along with the specific health needs and
goals of each student as assessed by the nurse educator"

Control description: N/A (no control)

Theoretical framework: no information

\begin{tabular}{ll}
\hline Outcomes & Core processes evaluated (child level): attrition, dosage, adherence \\
\hline Notes & Process evaluation category: stand-alone and named section (2 papers) \\
Breadth and depth: depth - not breadth \\
Voice of children given prominence: not featured \\
Funding source: Centers for Disease Control and Prevention Controlling Asthma in American Cities \\
Project
\end{tabular}

\section{Risk of bias}

\begin{tabular}{lll}
\hline Bias & Authors' judgement & Support for judgement \\
\hline $\begin{array}{l}\text { Random sequence genera- } \\
\text { tion (selection bias) }\end{array}$ & Unclear risk & N/A \\
\hline $\begin{array}{l}\text { Allocation concealment } \\
\text { (selection bias) }\end{array}$ & Unclear risk & N/A \\
\hline $\begin{array}{l}\text { Blinding of participants } \\
\text { and personnel (perfor- } \\
\text { mance bias) }\end{array}$ & Unclear risk & N/A \\
All outcomes & \\
\hline
\end{tabular}

Blinding of outcome as-
sessment (detection bias) $\quad$ Unclear risk $\quad$ N/A


Magzamen 2008 (Continued)

All outcomes

\begin{tabular}{|c|c|c|}
\hline $\begin{array}{l}\text { Incomplete outcome data } \\
\text { (attrition bias) } \\
\text { All outcomes }\end{array}$ & Unclear risk & $\mathrm{N} / \mathrm{A}$ \\
\hline $\begin{array}{l}\text { Selective reporting (re- } \\
\text { porting bias) }\end{array}$ & Unclear risk & $\mathrm{N} / \mathrm{A}$ \\
\hline $\begin{array}{l}\text { Transparent and clearly } \\
\text { stated aims }\end{array}$ & Low risk & Study aims were clearly stated \\
\hline $\begin{array}{l}\text { Explicit theories underpin- } \\
\text { ning and/or literature re- } \\
\text { view }\end{array}$ & Unclear risk & $\begin{array}{l}\text { Some supporting literature was provided, but no theoretical framework was } \\
\text { stated }\end{array}$ \\
\hline $\begin{array}{l}\text { Transparent and clearly } \\
\text { stated methods and tools }\end{array}$ & Low risk & $\begin{array}{l}\text { Lots of details about the tools used and how the study was conducted were } \\
\text { provided }\end{array}$ \\
\hline Selective reporting & Unclear risk & Data were collected individually and were presented ecologically \\
\hline Harmful effects & Unclear risk & $\begin{array}{l}\text { Harmful impacts - particularly around equity and gathering a range of stake- } \\
\text { holder views - were not considered }\end{array}$ \\
\hline $\begin{array}{l}\text { Population and sample } \\
\text { described well }\end{array}$ & Unclear risk & Data on SES and ethnicity are missing \\
\hline Continuous evaluation & Unclear risk & $\begin{array}{l}\text { Data that support different cohorts were provided, but whether they were } \\
\text { analysed continuously (i.e. whether data were analysed and used to make any } \\
\text { necessary changes to the programme) remains unclear }\end{array}$ \\
\hline
\end{tabular}

\begin{tabular}{|c|c|c|}
\hline $\begin{array}{l}\text { Evaluation participation } \\
\text { equity and sampling }\end{array}$ & Unclear risk & $\begin{array}{l}\text { Equitable sampling was described, along with a low response rate; data were } \\
\text { not collected from all stakeholders involved }\end{array}$ \\
\hline
\end{tabular}

\begin{tabular}{|c|c|c|}
\hline $\begin{array}{l}\text { Design and methods over- } \\
\text { all approach }\end{array}$ & Unclear risk & $\begin{array}{l}\text { Only one source of evidence was provided; however this is disaggregated } \\
\text { across different cohorts }\end{array}$ \\
\hline $\begin{array}{l}\text { Tools and methods of da- } \\
\text { ta collection reliable/cred- } \\
\text { ible }\end{array}$ & High risk & $\begin{array}{l}\text { Very low response rates suggest that methods of data collection used are un- } \\
\text { reliable }\end{array}$ \\
\hline $\begin{array}{l}\text { Tools and methods of data } \\
\text { analysis reliable/credible }\end{array}$ & Unclear risk & $\begin{array}{l}\text { Two sets of analyses were conducted - ecological and individual. Insights } \\
\text { gained by using both sets remain unclear }\end{array}$ \\
\hline $\begin{array}{l}\text { Performance bias/neutral- } \\
\text { ity/credibility/conforma- } \\
\text { bility }\end{array}$ & Unclear risk & $\begin{array}{l}\text { Study authors did not describe steps taken to minimise performance bias. } \\
\text { Negative outcomes are discussed, as is delivery of the intervention by nurses } \\
\text { vs researchers. Confidentiality/anonymity is not discussed }\end{array}$ \\
\hline $\begin{array}{l}\text { Reliability of findings and } \\
\text { recommendations }\end{array}$ & High risk & Study authors reported low rates of response to the surveys used \\
\hline Transferability of findings & Unclear risk & $\begin{array}{l}\text { This was not assessed by study authors, but the information provided is rich } \\
\text { enough to assess transferability }\end{array}$ \\
\hline $\begin{array}{l}\text { Overall risk of bias of } \\
\text { process evaluation }\end{array}$ & Unclear risk & $\begin{array}{l}\text { As an impact study, issues are evident, but as a process evaluation, data pro- } \\
\text { vided are useful }\end{array}$ \\
\hline
\end{tabular}




\section{Included as outcome evaluation}

Study design: clustered parallel-group RCT with schools selected as the unit of randomisation

Setting: primary/junior schools in the south of England; 24 schools randomised (with one dropout); 12 treatment schools and 11 control schools included

Period: 2000 to 2001 . Outcomes were collected 6 months before and after the intervention

Eligible sample frame: 361 children and their parents were invited to participate

Randomised: 219 children and their parents agreed to participate. 113 pupils at 12 schools were in the control group; 106 pupils at 12 schools were in the intervention group

Completed (intervention): 1 school withdrew, resulting in 6 children withdrawing. 20 children withdrew because they moved out the area. Unclear whether these children came from the intervention group or the control group

Inclusion criteria: not reported

Exclusion criteria: not reported

Baseline characteristics

Age of children: 7 to 9 years of age

Ethnicity: not reported

Socio-economic status: $25 \%$ of pupils in the control group and $15 \%$ of those in the intervention group were socially deprived

Gender: 97 females and 122 males: 50 females and 56 males in the intervention group, 47 females and 66 males in the control group

Asthma status: asthmatic and non-asthmatic children were included, and selected outcomes for asthmatic children were presented separately

School type: 24 primary schools

Intervention description: intervention workshops focused on a description of the respiratory condition, consistent with the national science curriculum. Role-play between a teacher and a school nurse demonstrated what it was like to have asthma, and how one can help a friend who is coughing and struggling to breathe

Control description: children in the control group took part in a workshop about the respiratory system and how the body defends itself against infection; however asthma was not mentioned during the workshop

Theoretical framework: not reported

Intensity: one 45-minute workshop

Instructor: school nurse

Parental engagement: not reported

Child satisfaction: not reported

Timing of the intervention in the school day: not reported 
Core processes evaluated (child level): attrition, dosage, adherence

Notes Process evaluation category: integrated

Breadth and depth: depth - not breadth

Voice of children given prominence: featured but not sufficiently

Note: extractable outcomes were not collected for meta-analysis; this study described a low-intensity single-session intervention and was not included in QCAs

Funding source: National Health Service Research and Development Grant

\section{Risk of bias}

\begin{tabular}{lll}
\hline Bias & Authors' judgement & Support for judgement \\
\hline $\begin{array}{l}\text { Random sequence genera- } \\
\text { tion (selection bias) }\end{array}$ & Unclear risk & $\begin{array}{l}\text { This was not addressed by study authors, who described that "Pairs of schools } \\
\text { were matched on demographic characteristics and randomly assigned within } \\
\text { pairs" }\end{array}$ \\
\hline $\begin{array}{l}\text { Allocation concealment } \\
\text { (selection bias) }\end{array}$ & Unclear risk & $\begin{array}{l}\text { This was not addressed by study authors, who described that "Pairs of schools } \\
\text { were matched on demographic characteristics and randomly assigned within } \\
\text { pairs" }\end{array}$ \\
\hline $\begin{array}{l}\text { Blinding of participants } \\
\text { and personnel (perfor- } \\
\text { mance bias) }\end{array}$ & Unclear risk & This was not addressed by study authors \\
All outcomes &
\end{tabular}

\begin{tabular}{|c|c|c|}
\hline $\begin{array}{l}\text { Blinding of outcome as- } \\
\text { sessment (detection bias) }\end{array}$ & Unclear risk & This was not addressed by study authors \\
\hline
\end{tabular}

All outcomes

\section{Incomplete outcome data High risk}

(attrition bias)

All outcomes
Attrition was not disaggregated; 219 parents of children with asthma agreed their children could participate, of whom $193(88.1 \%)$ completed the study

\begin{tabular}{|c|c|c|}
\hline $\begin{array}{l}\text { Selective reporting (re- } \\
\text { porting bias) }\end{array}$ & High risk & $\begin{array}{l}\text { Full attendance data were not presented; full data for other outcomes also } \\
\text { were not presented }\end{array}$ \\
\hline
\end{tabular}

Other bias Unclear risk

Missingness - unclear risk - this is not fully described by study authors

Baseline imbalance - high risk - differences were apparent (e.g. the control group was twice as likely to report both parents smoking); these were accounted for only in some analyses

Risk of contamination - unclear - schools were the unit of randomisation

Transparent and clearly Low risk Study aims were clearly stated
stated aims

\begin{tabular}{ll}
\hline $\begin{array}{l}\text { Explicit theories underpin- } \\
\text { ning and/or literature re- }\end{array}$ & $\begin{array}{l}\text { Unclear risk } \\
\text { view }\end{array}$
\end{tabular}

\begin{tabular}{l}
$\begin{array}{l}\text { Transparent and clearly } \\
\text { stated methods and tools }\end{array} \quad$ Unclear risk Tools used are not clear, and it is not clear whether they were validated \\
\hline
\end{tabular}


McCann 2006 (Continued)

$\begin{array}{ll}\text { Selective reporting High risk } & \text { Although the direction of the result was presented in some cases, actual data } \\ \text { were not presented, meaning that they are not extractable }\end{array}$

\begin{tabular}{|c|c|c|}
\hline Harmful effects & Unclear risk & Some subgroup analyses were undertaken, but not always with justification \\
\hline $\begin{array}{l}\text { Population and sample } \\
\text { described well }\end{array}$ & Unclear risk & $\begin{array}{l}\text { Whether the population consists of all children or only asthmatic children is } \\
\text { not always clear }\end{array}$ \\
\hline Continuous evaluation & Low risk & Pre-post assessment was conducted \\
\hline $\begin{array}{l}\text { Evaluation participation } \\
\text { equity and sampling }\end{array}$ & Unclear risk & This was not clearly reported by study authors \\
\hline $\begin{array}{l}\text { Design and methods over- } \\
\text { all approach }\end{array}$ & Unclear risk & $\begin{array}{l}\text { Data were collected from multiple stakeholders, but they were not collected } \\
\text { continuously }\end{array}$ \\
\hline $\begin{array}{l}\text { Tools and methods of da- } \\
\text { ta collection reliable/cred- } \\
\text { ible }\end{array}$ & Unclear risk & Not much information about the questionnaires used was presented \\
\hline $\begin{array}{l}\text { Tools and methods of data } \\
\text { analysis reliable/credible }\end{array}$ & Unclear risk & $\begin{array}{l}\text { It appears that analysis of the data was conducted sensibly, but it is unclear } \\
\text { what happened in the light of results presented }\end{array}$ \\
\hline $\begin{array}{l}\text { Performance bias/neutral- } \\
\text { ity/credibility/conforma- } \\
\text { bility }\end{array}$ & Unclear risk & This was not addressed by study authors \\
\hline $\begin{array}{l}\text { Reliability of findings and } \\
\text { recommendations }\end{array}$ & High risk & $\begin{array}{l}\text { Data were not presented in full, and study authors reported statistical signifi- } \\
\text { cance, rather than presenting effect sizes }\end{array}$ \\
\hline Transferability of findings & Unclear risk & $\begin{array}{l}\text { This was not explicitly considered, but processes of implementation were de- } \\
\text { scribed in full (simple intervention in some ways) }\end{array}$ \\
\hline $\begin{array}{l}\text { Overall risk of bias of } \\
\text { process evaluation }\end{array}$ & Unclear risk & $\begin{array}{l}\text { Adherence and attrition data are not fully applicable in a single-session inter- } \\
\text { vention, and issues surround the disaggregation of outcome data between } \\
\text { asthmatic and non-asthmatic children }\end{array}$ \\
\hline
\end{tabular}

McGhan 2003

Methods Included as outcome evaluation

Study design: clustered parallel-group RCT, with schools selected as the unit of randomisation

Setting: 18 elementary schools in Edmonton, Canada

Period: dates on which study was conducted - intervention and subsequent data collection - are not clear (post-test data collection took place 9 months after the intervention)

Participants

Eligible sample frame: eligibility based on school and parent assent and asthma status - 162 children found to be eligible across 18 schools

Randomised: 162 pupils were randomised at the school level: 76 to the treatment group and 86 to the control group

Completed (intervention): 136 pupils completed the trial 
Inclusion criteria: Study authors stated: "the target population was children with asthma ages 7-12 years; however, other ages were included if the parent and child were interested in participating. Criteria for selection included: (1) a diagnosis of asthma by a physician, (2) informed consent from the parent/guardian, (3) ability to speak English, and (4) no previous participation in RAP"

Exclusion criteria: no additional exclusion criteria described

\section{Baseline characteristics}

Age of children: wide age range, with children 5 to 13 years old participating (most were 8 to 10 years of age)

Ethnicity: approximately $77.8 \%$ of children were white

Socio-economic status: unclear

Gender: approximately $59.2 \%$ of participants were male

Asthma status: approximately $66.6 \%$ of children were deemed to have mild asthma, and $6.3 \%$ were deemed to have severe asthma describe that "using the 30 page manual, the instructors taught six 60-minute sessions: (1) getting to know each other, goal setting, use of a peak flow meter, diary monitoring; (2) trigger identification, control and avoidance, basic pathophysiology; (3) medications and proper use of inhalers; (4) symptom recognition and action plan; (5) lifestyle, exercise, managing an asthma episode; and (6) sharing this information with teachers and parents. Parents and teachers in the intervention schools were invited to participate in a RAP parent/teacher asthma awareness event at the school"

Control: not clearly stated (usual care)

Intensity: asthmatic students received 6*60-minute lessons

Instructor: nursing and pharmacy students were asked to be RAP instructors

Theoretical framework: social cognitive theory

Parental engagement: it was intended that parents would be involved, although parent and teacher attendance ranged from $10 \%$ to $80 \%$

Child satisfaction: not reported

Timing of intervention in school day: not reported

Outcomes were extracted for:
Asthma symptoms leading to emergency hospital visits
Parent-reported absence from school
Unplanned GP or hospital visit due to asthma
Experience of daytime and night-time symptoms
Withdrawal

Notes

Considered for process evaluation: did not meet the definition of a process evaluation

Funding source: not reported

\section{Risk of bias}


McGhan 2003 (Continued)

Random sequence genera- Unclear risk tion (selection bias)

This is not addressed by study authors, who describe: "the study compared children with asthma in randomly assigned intervention schools with those in control schools"

$\begin{array}{ll}\begin{array}{l}\text { Allocation concealment } \\ \text { (selection bias) }\end{array} & \text { Unclear risk }\end{array} \begin{aligned} & \text { This is not addressed by study authors, who describe: "the study compared } \\ & \text { children with asthma in randomly assigned intervention schools with those } \\ & \text { in control schools" }\end{aligned}$

Blinding of participants Unclear risk Not addressed by study authors

and personnel (perfor-

mance bias)

All outcomes

Blinding of outcome as- $\quad$ Unclear risk Not addressed by study authors
sessment (detection bias)
All outcomes

\begin{tabular}{ll}
\hline $\begin{array}{l}\text { Incomplete outcome data } \\
\text { (attrition bias) }\end{array}$ & High risk \\
All outcomes & Twenty-six families (16\%) dropped out of the study; indications show that chil- \\
& who remained engaged: "the dropouts were significantly less likely to have re- \\
& ported seasonal asthma and unscheduled doctor visits in the last year"
\end{tabular}

Selective reporting (re- Unclear risk $\quad$ No evidence shows selective reporting
porting bias)

\begin{tabular}{ll}
\hline Other bias & $\begin{array}{l}\text { Missingness - unclear risk - information on missing data was not provided in } \\
\text { detail }\end{array}$ \\
$\begin{array}{l}\text { Baseline imbalance - high risk - the intervention group was more likely to have } \\
\text { received previous education on asthma, which is likely to have influenced re- } \\
\text { sponse to the intervention }\end{array}$ \\
Risk of contamination - low - unit of randomisation was schools
\end{tabular}

Transparent and clearly $\quad$ Unclear risk
stated aims

\begin{tabular}{lll}
\hline $\begin{array}{l}\text { Explicit theories underpin- } \\
\text { ning and/or literature re- } \\
\text { view }\end{array}$ & Unclear risk & \\
\hline $\begin{array}{l}\text { Transparent and clearly } \\
\text { stated methods and tools }\end{array}$ & Unclear risk & N/A \\
\hline Selective reporting & Unclear risk & N/A \\
\hline Harmful effects & Unclear risk & N/A \\
\hline $\begin{array}{l}\text { Population and sample } \\
\text { described well }\end{array}$ & Unclear risk & N/A \\
\hline $\begin{array}{l}\text { Continuous evaluation } \\
\text { Evaluation participation } \\
\text { equity and sampling }\end{array}$ & Unclear risk & N/A \\
\hline $\begin{array}{l}\text { Design and methods over- } \\
\text { all approach }\end{array}$ & Unclear risk & N/A \\
\hline
\end{tabular}

School-based self-management interventions for asthma in children and adolescents: a mixed methods systematic review (Review) 
McGhan 2003 (Continued)

Tools and methods of data collection reliable/cred-

ible

$\begin{aligned} & \text { Tools and methods of data } \\ & \text { analysis reliable/credible }\end{aligned}$
Unclear risk N/A

Performance bias/neutral- Unclear risk N/A
ity/credibility/conforma-
bility

Reliability of findings and Unclear risk N/A

recommendations

\begin{tabular}{lll}
\hline Transferability of findings & Unclear risk & N/A \\
\hline $\begin{array}{l}\text { Overall risk of bias of } \\
\text { process evaluation }\end{array}$ & Unclear risk & N/A \\
\hline
\end{tabular}

McGhan 2010

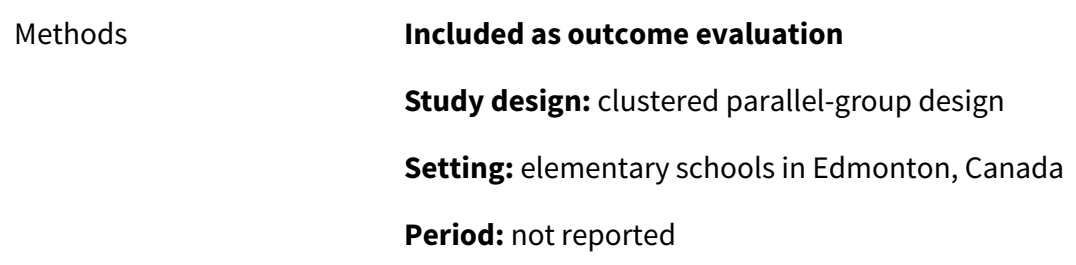

Participants

Eligible sample frame: all schools in Edmonton were eligible; an estimated 646 students from the 18 participating schools were eligible

Randomised: 162 pupils from 18 schools: 76 pupils in the intervention group and 86 in the control group

Completed (intervention): 136 pupils: 65 in the intervention group and 71 in the control group

Inclusion criteria: the target population was children 7 to 12 years of age with asthma. Pupils were eligible if they had received a diagnosis of asthma from their doctor and informed consent from their parent/guardian, were able to speak Eglish, and had not previously participated in Roaring Adventures of Puff (RAP)

Exclusion criteria: not reported

\section{Baseline characteristics}

Age of children: 5 to 10 years of age; $27.6 \%$ in the intervention group and $23.3 \%$ in the control group were 5 to 7 years old, and $61.8 \%$ in the intervention group and $57 \%$ in the control group were 8 to 10 years old

Ethnicity: $81.6 \%$ of children in the intervention group and $74.4 \%$ of children in the control group were Caucasian

Socio-economic status: not reported; however $26.8 \%$ of children in the intervention group and $36.4 \%$ in the control group had a father who achieved education grade 12 or less

Gender: males represented $55.3 \%$ of intervention students and $62.8 \%$ of control students 
McGhan 2010 (Continued)

Asthma status: mixed levels of severity: $62.7 \%$ of intervention children and $66.7 \%$ of control children had mild asthma; $29.3 \%$ of intervention children and $28.6 \%$ of control children had moderate asthma; $8 \%$ of intervention children and $4.8 \%$ of control children had severe asthma

Interventions

Intervention: parents and teachers in intervention schools were invited to participate in a RAP asthma awareness event at school. Parents and children in the intervention schools received information letters to share with their doctors, including suggested guidelines for a written action plan to be used at home and school. Doctors also received a summary letter from the RAP instructor at the end of the programme

Control: usual care

Intensity: six 60-minute sessions

Instructor: third year nursing and pharmacy students were RAP instructors under the guidance of their supervisor

Theoretical framework: social cognitive theory informed the intervention

Parental engagement: not reported

Child satisfaction: not reported

Timing of intervention in school day: not reported

\section{Outcomes}

\section{Extractable outcomes were collected for:}

Asthma symptoms leading to emergency hospital visit

Parent-reported absence from school

Unplanned visit to hospital or GP due to asthma symptoms

Experience of daytime and night-time symptoms

Withdrawal

\section{Notes}

Considered for process evaluation: did not meet the definition of a process evaluation

Funding source: Alberta Heritage Foundation for Medical Research - Health Research Fund

\section{Risk of bias}

\begin{tabular}{lll}
\hline Bias & Authors' judgement & Support for judgement \\
\hline $\begin{array}{l}\text { Random sequence genera- } \\
\text { tion (selection bias) }\end{array}$ & Low risk & $\begin{array}{l}\text { Schools were randomly assigned to RAP educational intervention or usual care } \\
\text { (control group) via a random numbers table }\end{array}$ \\
\hline $\begin{array}{l}\text { Allocation concealment } \\
\text { (selection bias) }\end{array}$ & Unclear risk & Selection of schools is unclear \\
\hline $\begin{array}{l}\text { Blinding of participants } \\
\begin{array}{l}\text { and personnel (perfor- } \\
\text { mance bias) } \\
\text { All outcomes }\end{array}\end{array}$ & Unclear risk & Not addressed by study authors \\
\hline
\end{tabular}

Blinding of outcome as- $\quad$ Unclear risk Not addressed by study authors
sessment (detection bias)
All outcomes

outcomes 
McGhan 2010 (Continued)

All outcomes

$\begin{array}{ll}\begin{array}{l}\text { Selective reporting (re- } \\ \text { porting bias) }\end{array} & \text { High risk }\end{array}$

\begin{tabular}{ll}
\hline Other bias & Missingness - unclear risk - no descriptions of missingness were provided \\
& Baseline imbalance - low risk - no differences between intervention and con- \\
& trol arms are apparent \\
& Risk of contamination - low - randomisation at the school level
\end{tabular}

\begin{tabular}{|c|c|c|}
\hline $\begin{array}{l}\text { Transparent and clearly } \\
\text { stated aims }\end{array}$ & Unclear risk & $\mathrm{N} / \mathrm{A}$ \\
\hline $\begin{array}{l}\text { Explicit theories underpin- } \\
\text { ning and/or literature re- } \\
\text { view }\end{array}$ & Unclear risk & $\mathrm{N} / \mathrm{A}$ \\
\hline $\begin{array}{l}\text { Transparent and clearly } \\
\text { stated methods and tools }\end{array}$ & Unclear risk & $\mathrm{N} / \mathrm{A}$ \\
\hline Selective reporting & Unclear risk & $\mathrm{N} / \mathrm{A}$ \\
\hline Harmful effects & Unclear risk & $\mathrm{N} / \mathrm{A}$ \\
\hline $\begin{array}{l}\text { Population and sample } \\
\text { described well }\end{array}$ & Unclear risk & $\mathrm{N} / \mathrm{A}$ \\
\hline Continuous evaluation & Unclear risk & $\mathrm{N} / \mathrm{A}$ \\
\hline $\begin{array}{l}\text { Evaluation participation } \\
\text { equity and sampling }\end{array}$ & Unclear risk & $\mathrm{N} / \mathrm{A}$ \\
\hline $\begin{array}{l}\text { Design and methods over- } \\
\text { all approach }\end{array}$ & Unclear risk & $\mathrm{N} / \mathrm{A}$ \\
\hline $\begin{array}{l}\text { Tools and methods of da- } \\
\text { ta collection reliable/cred- } \\
\text { ible }\end{array}$ & Unclear risk & $\mathrm{N} / \mathrm{A}$ \\
\hline $\begin{array}{l}\text { Tools and methods of data } \\
\text { analysis reliable/credible }\end{array}$ & Unclear risk & $\mathrm{N} / \mathrm{A}$ \\
\hline $\begin{array}{l}\text { Performance bias/neutral- } \\
\text { ity/credibility/conforma- } \\
\text { bility }\end{array}$ & Unclear risk & $\mathrm{N} / \mathrm{A}$ \\
\hline $\begin{array}{l}\text { Reliability of findings and } \\
\text { recommendations }\end{array}$ & Unclear risk & $\mathrm{N} / \mathrm{A}$ \\
\hline $\begin{array}{l}\text { Overall risk of bias of } \\
\text { process evaluation }\end{array}$ & Unclear risk & $\mathrm{N} / \mathrm{A}$ \\
\hline
\end{tabular}

\section{Mickel 2016}

Methods Included as process evaluation

School-based self-management interventions for asthma in children and adolescents: a mixed methods systematic review (Review) 
Intervention study design: quasi-experimental single-group intervention with change pre-post intervention examined

\section{Unit of allocation: N/A}

Process evaluation methods: descriptive/bivariate, descriptive qualitative analysis

Setting: 7 schools in an urban Midwest school district
Age of children: mean age, 9.3 years
Child characteristics (BME/SES): $63.6 \%$ of children were African American; $13.3 \%$ Hispanic; $20.2 \%$
white. Backgrounds of individual children were not presented, although schools were recruited from
districts in low-income areas
Asthma status: asthmatic and non-asthmatic children were included, with asthma diagnosed in ap-
proximately half of the 348 children
Intervention recipients: children only
School type: primary/elementary
Intervention description: study authors described an intervention (Iggy and the Inhalers (Iggy)). Ig-
gy is an asthma education video, poster, comic book, sticker, and trading card programme for children
between the ages of 7 and 12 years. Iggy education takes place over 1 session lasting approximately 30
minutes. Sessions include a brief welcome (2 minutes), the Iggy video (11 minutes), and interactive dis-
cussion based on posters ( 7 minutes). Each pre- and post-test worksheet takes approximately 5 min-
utes to complete. Children then take home trading cards, stickers, and comic books to share with their
parents

Control description: N/A

Theoretical framework: no information

\begin{tabular}{|c|c|c|}
\hline Outcomes & \multicolumn{2}{|c|}{ Core processes evaluated (child level): attrition (to post-test) } \\
\hline \multirow[t]{4}{*}{ Notes } & \multicolumn{2}{|c|}{ Process evaluation category: named section } \\
\hline & \multicolumn{2}{|c|}{ Breadth and depth: breadth and depth } \\
\hline & \multicolumn{2}{|c|}{ Voice of children given prominence: sufficient coverage } \\
\hline & \multicolumn{2}{|c|}{ Funding source: Wisconsin State Asthma Coalition mini-grant } \\
\hline \multicolumn{3}{|l|}{ Risk of bias } \\
\hline Bias & Authors' judgement & Support for judgement \\
\hline $\begin{array}{l}\text { Random sequence genera- } \\
\text { tion (selection bias) }\end{array}$ & Unclear risk & $\mathrm{N} / \mathrm{A}$ \\
\hline $\begin{array}{l}\text { Allocation concealment } \\
\text { (selection bias) }\end{array}$ & Unclear risk & $\mathrm{N} / \mathrm{A}$ \\
\hline $\begin{array}{l}\text { Blinding of participants } \\
\text { and personnel (perfor- } \\
\text { mance bias) } \\
\text { All outcomes }\end{array}$ & Unclear risk & $\mathrm{N} / \mathrm{A}$ \\
\hline
\end{tabular}

Blinding of outcome as- Unclear risk N/A sessment (detection bias) 
Mickel 2016 (Continued)

All outcomes

\begin{tabular}{|c|c|c|}
\hline $\begin{array}{l}\text { Incomplete outcome data } \\
\text { (attrition bias) } \\
\text { All outcomes }\end{array}$ & Unclear risk & $\mathrm{N} / \mathrm{A}$ \\
\hline $\begin{array}{l}\text { Selective reporting (re- } \\
\text { porting bias) }\end{array}$ & Unclear risk & $\mathrm{N} / \mathrm{A}$ \\
\hline Other bias & Unclear risk & $\mathrm{N} / \mathrm{A}$ \\
\hline $\begin{array}{l}\text { Transparent and clearly } \\
\text { stated aims }\end{array}$ & Low risk & Study aims were clearly stated \\
\hline $\begin{array}{l}\text { Explicit theories underpin- } \\
\text { ning and/or literature re- } \\
\text { view }\end{array}$ & High risk & Some literature was provided, but no theoretical framework was presented \\
\hline $\begin{array}{l}\text { Transparent and clearly } \\
\text { stated methods and tools }\end{array}$ & Low risk & Study approach was well described \\
\hline Selective reporting & Low risk & No evidence shows selective reporting, as negative comments were presented \\
\hline Harmful effects & Low risk & Negative responses from children were presented and discussed \\
\hline $\begin{array}{l}\text { Population and sample } \\
\text { described well }\end{array}$ & Low risk & Demographic information was provided \\
\hline Continuous evaluation & Low risk & Pre-post assessment was conducted \\
\hline $\begin{array}{l}\text { Evaluation participation } \\
\text { equity and sampling }\end{array}$ & Low risk & Parent, nurse, and child responses were collected \\
\hline $\begin{array}{l}\text { Design and methods over- } \\
\text { all approach }\end{array}$ & Low risk & Short intervention - data were collected at multiple time points \\
\hline $\begin{array}{l}\text { Tools and methods of da- } \\
\text { ta collection reliable/cred- } \\
\text { ible }\end{array}$ & Unclear risk & Description of qualitative data is limited \\
\hline $\begin{array}{l}\text { Tools and methods of data } \\
\text { analysis reliable/credible }\end{array}$ & Unclear risk & How themes were developed remains unclear \\
\hline $\begin{array}{l}\text { Performance bias/neutral- } \\
\text { ity/credibility/conforma- } \\
\text { bility }\end{array}$ & High risk & $\begin{array}{l}\text { These aspects were not considered, and programme staff collected qualitative } \\
\text { data }\end{array}$ \\
\hline $\begin{array}{l}\text { Reliability of findings and } \\
\text { recommendations }\end{array}$ & High risk & $\begin{array}{l}\text { Some of the claims around sustained impact were based on a 1-month post- } \\
\text { test }\end{array}$ \\
\hline Transferability of findings & Low risk & Enough data were provided for assessment of the relevance of challenges \\
\hline $\begin{array}{l}\text { Overall risk of bias of } \\
\text { process evaluation }\end{array}$ & Low risk & $\begin{array}{l}\text { Overall low risk, although some reservations around reporting of study design } \\
\text { and analysis of qualitative aspects remain }\end{array}$ \\
\hline
\end{tabular}


Monforte 2012

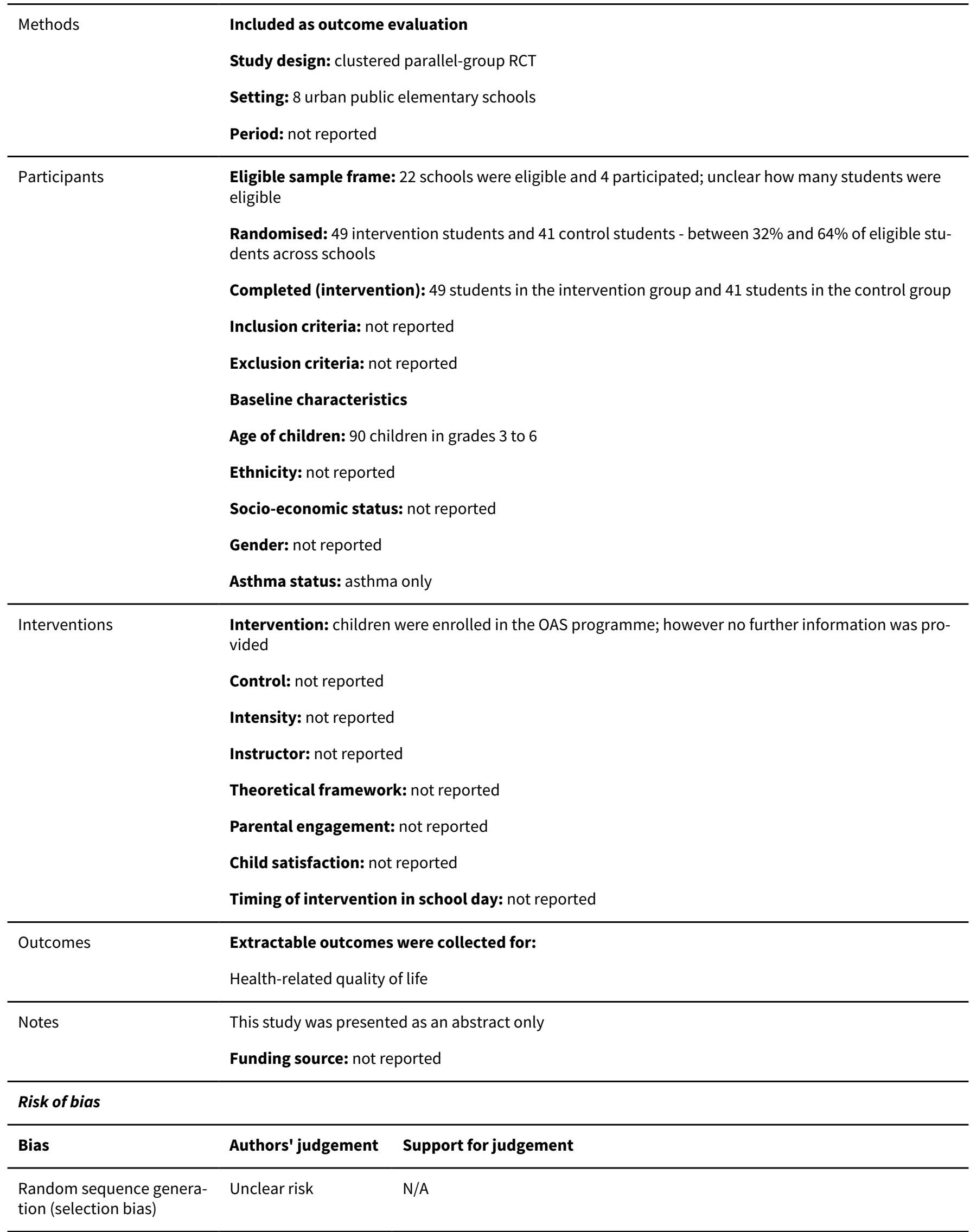


Monforte 2012 (Continued)

\begin{tabular}{lll}
$\begin{array}{l}\text { Allocation concealment } \\
\text { (selection bias) }\end{array}$ & Unclear risk & N/A \\
\hline $\begin{array}{l}\text { Blinding of participants } \\
\text { and personnel (perfor- } \\
\text { mance bias) }\end{array}$ & Unclear risk & N/A \\
All outcomes & &
\end{tabular}

All outcomes

$\begin{array}{lll}\text { Blinding of outcome as- } & \text { Unclear risk } & \text { N/A } \\ \text { sessment (detection bias) } & \end{array}$

All outcomes

\begin{tabular}{l}
\hline Incomplete outcome data Unclear risk N/A \\
(attrition bias)
\end{tabular}

All outcomes

\begin{tabular}{lll}
\hline $\begin{array}{l}\text { Selective reporting (re- } \\
\text { porting bias) }\end{array}$ & Unclear risk & N/A \\
\hline Other bias & Unclear risk & N/A \\
\hline $\begin{array}{l}\text { Transparent and clearly } \\
\text { stated aims }\end{array}$ & Unclear risk & N/A \\
\hline
\end{tabular}

Explicit theories underpin- Unclear risk N/A
ning and/or literature re-
view

\begin{tabular}{|c|c|c|}
\hline $\begin{array}{l}\text { Transparent and clearly } \\
\text { stated methods and tools }\end{array}$ & Unclear risk & $\mathrm{N} / \mathrm{A}$ \\
\hline Selective reporting & Unclear risk & $\mathrm{N} / \mathrm{A}$ \\
\hline Harmful effects & Unclear risk & $\mathrm{N} / \mathrm{A}$ \\
\hline $\begin{array}{l}\text { Population and sample } \\
\text { described well }\end{array}$ & Unclear risk & $\mathrm{N} / \mathrm{A}$ \\
\hline Continuous evaluation & Unclear risk & $\mathrm{N} / \mathrm{A}$ \\
\hline $\begin{array}{l}\text { Evaluation participation } \\
\text { equity and sampling }\end{array}$ & Unclear risk & $\mathrm{N} / \mathrm{A}$ \\
\hline $\begin{array}{l}\text { Design and methods over- } \\
\text { all approach }\end{array}$ & Unclear risk & $\mathrm{N} / \mathrm{A}$ \\
\hline $\begin{array}{l}\text { Tools and methods of da- } \\
\text { ta collection reliable/cred- } \\
\text { ible }\end{array}$ & Unclear risk & $\mathrm{N} / \mathrm{A}$ \\
\hline $\begin{array}{l}\text { Tools and methods of data } \\
\text { analysis reliable/credible }\end{array}$ & Unclear risk & $\mathrm{N} / \mathrm{A}$ \\
\hline $\begin{array}{l}\text { Performance bias/neutral- } \\
\text { ity/credibility/conforma- } \\
\text { bility }\end{array}$ & Unclear risk & $\mathrm{N} / \mathrm{A}$ \\
\hline
\end{tabular}


Monforte 2012 (Continued)

Reliability of findings and Unclear risk N/A
recommendations

\begin{tabular}{lll}
\hline Transferability of findings & Unclear risk & N/A \\
\hline $\begin{array}{l}\text { Overall risk of bias of } \\
\text { process evaluation }\end{array}$ & Unclear risk & N/A \\
\hline
\end{tabular}

Mosnaim 2011

$\begin{array}{ll}\text { Methods } & \text { Included as outcome evaluation } \\ \text { Study design: clustered parallel-group RCT } \\ \text { Setting: elementary schools in Chicago } \\ \text { Period: not reported }\end{array}$

\section{Participants}

\section{Eligible sample frame: not reported}

Randomised: 344 pupils within the youth group ( 275 in the intervention group and 69 in the control group) and 192 within the teen group (141 in the intervention group and 51 in the control group) participated. 25 youth classes (19 intervention groups and 6 control groups) and 16 teen classes (11 intervention group and 5 control groups) from 26 schools participated

Completed (intervention): not reported

Inclusion criteria: not reported

Exclusion criteria: not reported

\section{Baseline characteristics}

Age of children: youths and teens 5 to 15 years old. Median age was 10 for the youth group and 13 for the treatment group

Ethnicity: $65.5 \%$ in the youth intervention group were African American; $11.6 \%$ were Hispanic; and $22.3 \%$ were other. In the teen intervention group, $85.1 \%$ were African American, $7.1 \%$ were Hispanic, and $7.1 \%$ were other

Socio-economic status: not reported, but study author described participants as predominantly low income

Gender: females represented $43 \%$ of participants. In the youth intervention group, $41.5 \%$ were female; in the teen intervention group, $48.2 \%$ were female

Asthma status: not reported ter use, and use of an asthma action plan. The teen programme also addressed tobacco avoidance, asthma-related peer pressure, and asthma self-management skills

Control: usual care

Intensity: four 45-minute sessions conducted in school on 4 consecutive days

Instructor: certified asthma educators

Theoretical framework: not reported

Parental engagement: not reported 
Child satisfaction: not reported

Timing of intervention in school day: not explicitly reported, but sessions were scheduled at times with the least impact on instruction, as determined by each school

\begin{tabular}{ll}
\hline Outcomes & Extractable outcomes were collected for: None \\
\hline Notes & $\begin{array}{l}\text { Considered for process evaluation: study did not include the core components of a process evalua- } \\
\text { tion }\end{array}$
\end{tabular}

Funding source: Abbott Laboratories Unrestricted Grant

\section{Risk of bias}

\begin{tabular}{lll}
\hline Bias & Authors' judgement & Support for judgement \\
\hline $\begin{array}{l}\text { Random sequence genera- } \\
\text { tion (selection bias) }\end{array}$ & High risk & $\begin{array}{l}\text { Potential breach of randomisation schedule through systematic bias in selec- } \\
\text { tion of schools for the control group: "The allocation scheme first determined } \\
\text { whether an eligible school could accommodate the intervention schedule. } \\
\text { Those that could not were automatically assigned to the control group, where- } \\
\text { as those that could were subject to the 3:1 randomisation scheme" }\end{array}$ \\
\end{tabular}

$\begin{array}{ll}\begin{array}{l}\text { Allocation concealment } \\ \text { (selection bias) }\end{array} & \text { High risk } \\ & \begin{array}{l}\text { Potential breach of randomisation schedule through systematic bias in selec- } \\ \text { tion of schools for the control group: "The allocation scheme first determined } \\ \text { whether an eligible school could accommodate the intervention schedule. } \\ \text { Those that could not were automatically assigned to the control group, where- } \\ \text { as those that could were subject to the 3:1 randomisation scheme" }\end{array}\end{array}$

Blinding of participants Unclear risk Not addressed by study authors
and personnel (performance bias)

All outcomes

\begin{tabular}{lll}
\hline $\begin{array}{l}\text { Blinding of outcome as- } \\
\text { sessment (detection bias) } \\
\text { All outcomes }\end{array}$ & Unclear risk & Not addressed by study authors \\
\hline $\begin{array}{l}\text { Incomplete outcome data } \\
\text { (attrition bias) }\end{array}$ & Low risk & $\begin{array}{l}\text { Relatively low levels of missing data; study authors state that this had no im- } \\
\text { pact on the outcome: "Approximately 15\% of the participants overall were } \\
\text { mill outcomes }\end{array}$ \\
& $\begin{array}{l}\text { from our trained educators as to the source of the absenteeism, we concluded } \\
\text { that these missing data were missing at random and did not merit use of data } \\
\text { imputation methods" }\end{array}$ \\
\hline
\end{tabular}

\begin{tabular}{ll}
\hline $\begin{array}{l}\text { Selective reporting (re- } \\
\text { porting bias) }\end{array}$ & Low risk \\
& $\begin{array}{l}\text { No evidence of indicators measured but not reported. However, indicators } \\
\text { that were not included in our protocol were collected }\end{array}$
\end{tabular}

Other bias Unclear risk Missingness - unclear risk - missing data but no imputation strategy - study au-
thors stated that it was not necessary to implement imputation strategies

Baseline imbalance - unclear risk - some ethnic and gender differences at baseline but impact on outcomes unclear; no differences in asthma knowledge at baseline

Risk of contamination - low - unit of randomisation was the school, lowering the risk of contamination

$\begin{aligned} & \text { Transparent and clearly } \\ & \text { stated aims }\end{aligned}$
Unclear risk N/A


Mosnaim 2011 (Continued)

Explicit theories underpin- Unclear risk N/A
ning and/or literature re-

view

\begin{tabular}{|c|c|c|}
\hline $\begin{array}{l}\text { Transparent and clearly } \\
\text { stated methods and tools }\end{array}$ & Unclear risk & $\mathrm{N} / \mathrm{A}$ \\
\hline Selective reporting & Unclear risk & $\mathrm{N} / \mathrm{A}$ \\
\hline Harmful effects & Unclear risk & $\mathrm{N} / \mathrm{A}$ \\
\hline $\begin{array}{l}\text { Population and sample } \\
\text { described well }\end{array}$ & Unclear risk & $\mathrm{N} / \mathrm{A}$ \\
\hline Continuous evaluation & Unclear risk & $\mathrm{N} / \mathrm{A}$ \\
\hline $\begin{array}{l}\text { Evaluation participation } \\
\text { equity and sampling }\end{array}$ & Unclear risk & $\mathrm{N} / \mathrm{A}$ \\
\hline $\begin{array}{l}\text { Design and methods over- } \\
\text { all approach }\end{array}$ & Unclear risk & $\mathrm{N} / \mathrm{A}$ \\
\hline $\begin{array}{l}\text { Tools and methods of da- } \\
\text { ta collection reliable/cred- } \\
\text { ible }\end{array}$ & Unclear risk & $\mathrm{N} / \mathrm{A}$ \\
\hline $\begin{array}{l}\text { Tools and methods of data } \\
\text { analysis reliable/credible }\end{array}$ & Unclear risk & $\mathrm{N} / \mathrm{A}$ \\
\hline $\begin{array}{l}\text { Performance bias/neutral- } \\
\text { ity/credibility/conforma- } \\
\text { bility }\end{array}$ & Unclear risk & $\mathrm{N} / \mathrm{A}$ \\
\hline $\begin{array}{l}\text { Reliability of findings and } \\
\text { recommendations }\end{array}$ & Unclear risk & $\mathrm{N} / \mathrm{A}$ \\
\hline Transferability of findings & Unclear risk & $\mathrm{N} / \mathrm{A}$ \\
\hline $\begin{array}{l}\text { Overall risk of bias of } \\
\text { process evaluation }\end{array}$ & Unclear risk & $\mathrm{N} / \mathrm{A}$ \\
\hline
\end{tabular}

Mujuru 2011

Methods Included as process evaluation

Intervention study design: quasi-experimental design, pre-post follow-up, no control

Unit of allocation: N/A

Process evaluation methods: descriptive/bivariate analysis methods

\begin{tabular}{ll}
\hline Participants & Country: USA \\
& Age of children: 18 students in grades 3 to 5 \\
Child characteristics (BME/SES): $39 \%$ of students were in receipt of Medicaid. Ethnicity data were not \\
reported
\end{tabular}


Mujuru 2011 (Continued)

$$
\text { Asthma status: asthmatic only }
$$

Intervention recipients: children and parents

School type: 1 elementary school
Interventions

schools

Control description: N/A

Theoretical framework: not reported

\begin{tabular}{ll}
\hline Outcomes & Core processes evaluated (child level): attrition \\
\hline Notes & Process evaluation category: integrated \\
& Breadth and depth: breadth and depth \\
& Voice of children given prominence: featured but not sufficiently
\end{tabular}

Funding source: not reported

\section{Risk of bias}

\begin{tabular}{|c|c|c|}
\hline Bias & Authors' judgement & Support for judgement \\
\hline $\begin{array}{l}\text { Random sequence genera- } \\
\text { tion (selection bias) }\end{array}$ & Unclear risk & $\mathrm{N} / \mathrm{A}$ \\
\hline $\begin{array}{l}\text { Allocation concealment } \\
\text { (selection bias) }\end{array}$ & Unclear risk & $\mathrm{N} / \mathrm{A}$ \\
\hline $\begin{array}{l}\text { Blinding of participants } \\
\text { and personnel (perfor- } \\
\text { mance bias) } \\
\text { All outcomes }\end{array}$ & Unclear risk & $\mathrm{N} / \mathrm{A}$ \\
\hline $\begin{array}{l}\text { Blinding of outcome as- } \\
\text { sessment (detection bias) } \\
\text { All outcomes }\end{array}$ & Unclear risk & $\mathrm{N} / \mathrm{A}$ \\
\hline $\begin{array}{l}\text { Incomplete outcome data } \\
\text { (attrition bias) } \\
\text { All outcomes }\end{array}$ & Unclear risk & $\mathrm{N} / \mathrm{A}$ \\
\hline $\begin{array}{l}\text { Selective reporting (re- } \\
\text { porting bias) }\end{array}$ & Unclear risk & $\mathrm{N} / \mathrm{A}$ \\
\hline Other bias & Unclear risk & $\mathrm{N} / \mathrm{A}$ \\
\hline $\begin{array}{l}\text { Transparent and clearly } \\
\text { stated aims }\end{array}$ & Low risk & Study aims were clearly stated \\
\hline $\begin{array}{l}\text { Explicit theories underpin- } \\
\text { ning and/or literature re- } \\
\text { view }\end{array}$ & Unclear risk & A named theory is not present, but supporting literature was presented \\
\hline $\begin{array}{l}\text { Transparent and clearly } \\
\text { stated methods and tools }\end{array}$ & Low risk & Data collection tools were reasonably well described \\
\hline
\end{tabular}

School-based self-management interventions for asthma in children and adolescents: a mixed methods systematic review (Review) 
Mujuru 2011 (Continued)

\begin{tabular}{|c|c|c|}
\hline Selective reporting & Low risk & Negative aspects of the intervention were reported \\
\hline Harmful effects & Low risk & Low parental engagement and compliance were reported \\
\hline $\begin{array}{l}\text { Population and sample } \\
\text { described well }\end{array}$ & Unclear risk & Some expected fields, for example, ethnicity, were not reported \\
\hline Continuous evaluation & Low risk & $\begin{array}{l}\text { Pre-post assessment was conducted; however post follow-up engagement } \\
\text { was low }\end{array}$ \\
\hline $\begin{array}{l}\text { Evaluation participation } \\
\text { equity and sampling }\end{array}$ & Low risk & $\begin{array}{l}\text { Parents were involved, but little information was received from teachers or in- } \\
\text { structors }\end{array}$ \\
\hline $\begin{array}{l}\text { Design and methods over- } \\
\text { all approach }\end{array}$ & Low risk & The overall design and methods were well described and suitable for the study \\
\hline $\begin{array}{l}\text { Tools and methods of da- } \\
\text { ta collection reliable/cred- } \\
\text { ible }\end{array}$ & Low risk & Tools used for data collection were reported fully \\
\hline $\begin{array}{l}\text { Tools and methods of data } \\
\text { analysis reliable/credible }\end{array}$ & Unclear risk & $\begin{array}{l}\text { The validity of the parental survey is unclear. This survey contained a 32-item } \\
\text { questionnaire designed by investigators as based on a review of published } \\
\text { medical literature }\end{array}$ \\
\hline
\end{tabular}

Performance bias/neutral- Unclear risk

Some aspects were covered, but not all aspects were reported on ity/credibility/conformability

\begin{tabular}{ll}
\hline $\begin{array}{l}\text { Reliability of findings and Unclear risk } \\
\text { recommendations }\end{array}$ & $\begin{array}{l}\text { Some process outcomes might be generalisable, but study authors themselves } \\
\text { suggest that the "sample size was too small to generalise the results to a larger } \\
\text { population" }\end{array}$ \\
\hline
\end{tabular}

Transferability of findings Unclear risk Some process outcomes might be generalisable, but study authors themselves suggest that the "sample size was too small to generalise the results to a larger population"

\begin{tabular}{l}
$\begin{array}{l}\text { Overall risk of bias of } \\
\text { process evaluation }\end{array} \quad$ Low risk factors were considered high risk \\
\hline
\end{tabular}

\section{Patterson 2005}

Methods Included as outcome evaluation

Study design: clustered parallel-group design, with schools as the unit of randomisation

Setting: primary schools in Belfast, Northern Ireland

Period: participating schools entered the trial between September 2002 and September 2003. Preliminary assessment through to follow-up assessment took 31 weeks

Participants Eligible sample frame: 102 eligible children in intervention schools and 126 eligible children in control schools

Randomised: 84 eligible children in intervention schools and 92 eligible children in control schools

Completed (intervention): 99 children in intervention schools and 92 children in control schools 
Inclusion criteria: children were eligible if they were between 7 and 11 years of age and had received a diagnosis of asthma

Exclusion criteria: not reported

\section{Baseline characteristics}

Age of children: mean age, 9.01 years in the intervention group and 8.99 years in the control group

Ethnicity: not reported

Socio-economic status: $32 \%$ of children in the intervention group and $22 \%$ of children in the control group were eligible for free school meals

Gender: males represented $45 \%$ of the intervention group and $58 \%$ of the control group

Asthma status: not reported

Intervention: each session began with brief reinforcement of previous training and ended with session feedback. The SCAMP club workbook used during sessions was given to children at prize giving, along with the child-held asthma care pathway record and action plan

Control: control group received the same intervention after a 16-week interval

Intensity: weekly sessions for 8 weeks

Instructor: school nurse and a health visitor

Theoretical framework: study was informed by the Predisposing, Reinforcing, and Enabling Causes in Educational Diagnosis and Evaluation (PRECEDE) model

Parental engagement: not reported

Child satisfaction: not reported

Timing of intervention in school day: intervention was designed to be delivered at lunchtime

Extractable outcomes were
Days of restricted activity
Lung function
Health-related quality of life
Withdrawal

Funding source: South and East Belfast Health and Social Services Trust, Primary Care and Development Fund, Eastern Health and Social Services Board, Department of Child Health, Queen's University Belfast

\section{Risk of bias}

\begin{tabular}{lll}
\hline Bias & Authors' judgement & Support for judgement \\
\hline $\begin{array}{l}\text { Random sequence genera- } \\
\text { tion (selection bias) }\end{array}$ & Low risk & $\begin{array}{l}\text { In each pair, the toss of a coin was used to randomise schools to immediate or } \\
\text { delayed intervention }\end{array}$ \\
\hline $\begin{array}{l}\text { Allocation concealment } \\
\text { (selection bias) }\end{array}$ & Unclear risk & $\begin{array}{l}\text { Given that allocation was done within pairs of schools, allocation concealment } \\
\text { might have been breached }\end{array}$ \\
\hline
\end{tabular}


Patterson 2005 (Continued)

Blinding of participants Unclear risk Not addressed by study authors and personnel (performance bias)

All outcomes

\begin{tabular}{lll}
\hline $\begin{array}{l}\text { Blinding of outcome as- } \\
\text { sessment (detection bias) }\end{array}$ & Unclear risk & Not addressed by study authors \\
$\begin{array}{ll}\text { All outcomes } \\
\text { Incomplete outcome data }\end{array}$ & Low risk & Very low levels of attrition ( $2 / 83$ in the intervention group) \\
$\begin{array}{l}\text { (attrition bias) } \\
\text { All outcomes }\end{array}$ &
\end{tabular}

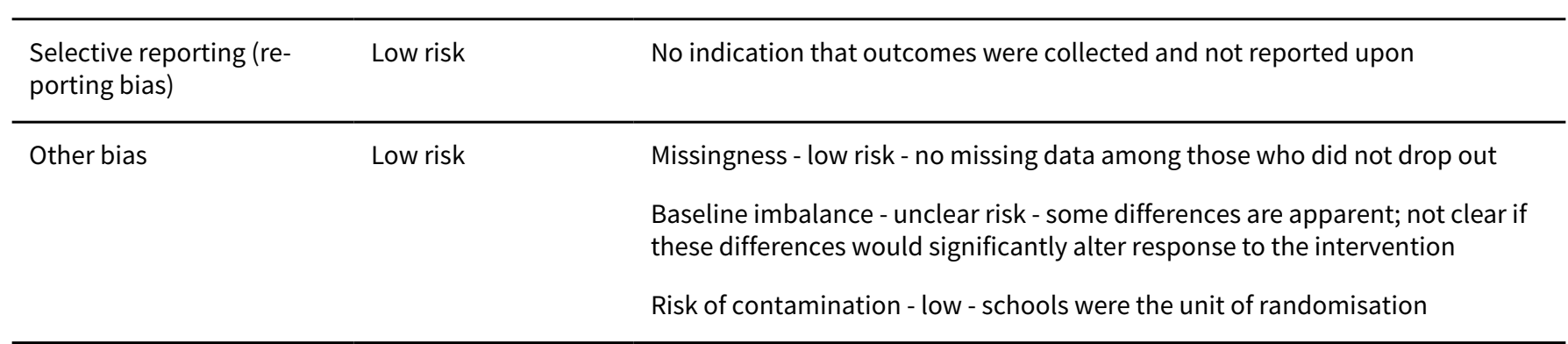

\begin{tabular}{lll}
\hline $\begin{array}{l}\text { Transparent and clearly } \\
\text { stated aims }\end{array}$ & Unclear risk & N/A
\end{tabular}

\begin{tabular}{|c|c|c|}
\hline $\begin{array}{l}\text { Explicit theories underpin- } \\
\text { ning and/or literature re- } \\
\text { view }\end{array}$ & Unclear risk & $\mathrm{N} / \mathrm{A}$ \\
\hline $\begin{array}{l}\text { Transparent and clearly } \\
\text { stated methods and tools }\end{array}$ & Unclear risk & $\mathrm{N} / \mathrm{A}$ \\
\hline Selective reporting & Unclear risk & $\mathrm{N} / \mathrm{A}$ \\
\hline Harmful effects & Unclear risk & $\mathrm{N} / \mathrm{A}$ \\
\hline $\begin{array}{l}\text { Population and sample } \\
\text { described well }\end{array}$ & Unclear risk & $\mathrm{N} / \mathrm{A}$ \\
\hline Continuous evaluation & Unclear risk & $\mathrm{N} / \mathrm{A}$ \\
\hline $\begin{array}{l}\text { Evaluation participation } \\
\text { equity and sampling }\end{array}$ & Unclear risk & $\mathrm{N} / \mathrm{A}$ \\
\hline $\begin{array}{l}\text { Design and methods over- } \\
\text { all approach }\end{array}$ & Unclear risk & $\mathrm{N} / \mathrm{A}$ \\
\hline $\begin{array}{l}\text { Tools and methods of da- } \\
\text { ta collection reliable/cred- } \\
\text { ible }\end{array}$ & Unclear risk & $\mathrm{N} / \mathrm{A}$ \\
\hline $\begin{array}{l}\text { Tools and methods of data } \\
\text { analysis reliable/credible }\end{array}$ & Unclear risk & $\mathrm{N} / \mathrm{A}$ \\
\hline $\begin{array}{l}\text { Performance bias/neutral- } \\
\text { ity/credibility/conforma- } \\
\text { bility }\end{array}$ & Unclear risk & $\mathrm{N} / \mathrm{A}$ \\
\hline
\end{tabular}


Patterson 2005 (Continued)

Reliability of findings and Unclear risk N/A
recommendations

\begin{tabular}{lll}
\hline Transferability of findings & Unclear risk & N/A \\
\hline $\begin{array}{l}\text { Overall risk of bias of } \\
\text { process evaluation }\end{array}$ & Unclear risk & N/A \\
\hline
\end{tabular}

\section{Persaud 1996}

Methods

\section{Included as outcome evaluation}

Study design: parallel-group design with children selected as the unit of randomisation

Setting: study was conducted in Galveston, Texas, USA, across children in 10 schools

Period: the intervention was conducted between September and December 1992
Eligible sample frame: 60 pupils found to be eligible; 43 were contacted and 36 agreed to participate

Randomised: 36 children were randomised, with 18 each selected into intervention and control arms

Completed (intervention): no students were described as having dropped out

Inclusion criteria: children 8 to 12 years of age with a diagnosis of asthma (several prior episodes of airway obstruction, clinical response to bronchodilator, and absence of other pulmonary disease)

Exclusion criteria: no additional exclusion criteria provided

\section{Baseline characteristics}

Age of children: mean age, 10.2 years

Ethnicity: $69 \%$ of children were African American

Socio-economic status: $69 \%$ of children were from families in receipt of Medicaid

Gender: $64 \%$ of children were male

Asthma status: $44 \%$ of children had mild asthma, $50 \%$ had moderately severe asthma, and $6 \%$ had severe asthma

Intervention: study authors described that "intervention subjects received individualized, weekly, 20min education sessions with the school nurse for 8 weeks. Each child had a personal peak flow meter in the school health office to use during teaching sessions. At each visit, the school nurse reviewed the asthma diary with the student and discussed progress, symptoms, and ability to take appropriate measures to control asthma. At each visit, the child demonstrated proper use of inhaled medications and the peak flow meter. The school nurses recorded each student's weekly progress on a checklist in the teaching manual"

Control: usual care

Intensity: target asthmatic students received 3 lessons from peer leaders (year 11 students)

Instructor: school nurses

Theoretical framework: no overarching theory named

Parental engagement: not reported

Child satisfaction: not reported 
Persaud 1996 (Continued)

Timing of intervention in school day: not reported

Notes

\section{Extractable outcomes were collected for:}

Emergency department visits

Absences from school

Considered for process evaluation: study did not include the core components of a process evaluation, and process data were collected with the use of structured tools

Funding source: not reported

\section{Risk of bias}

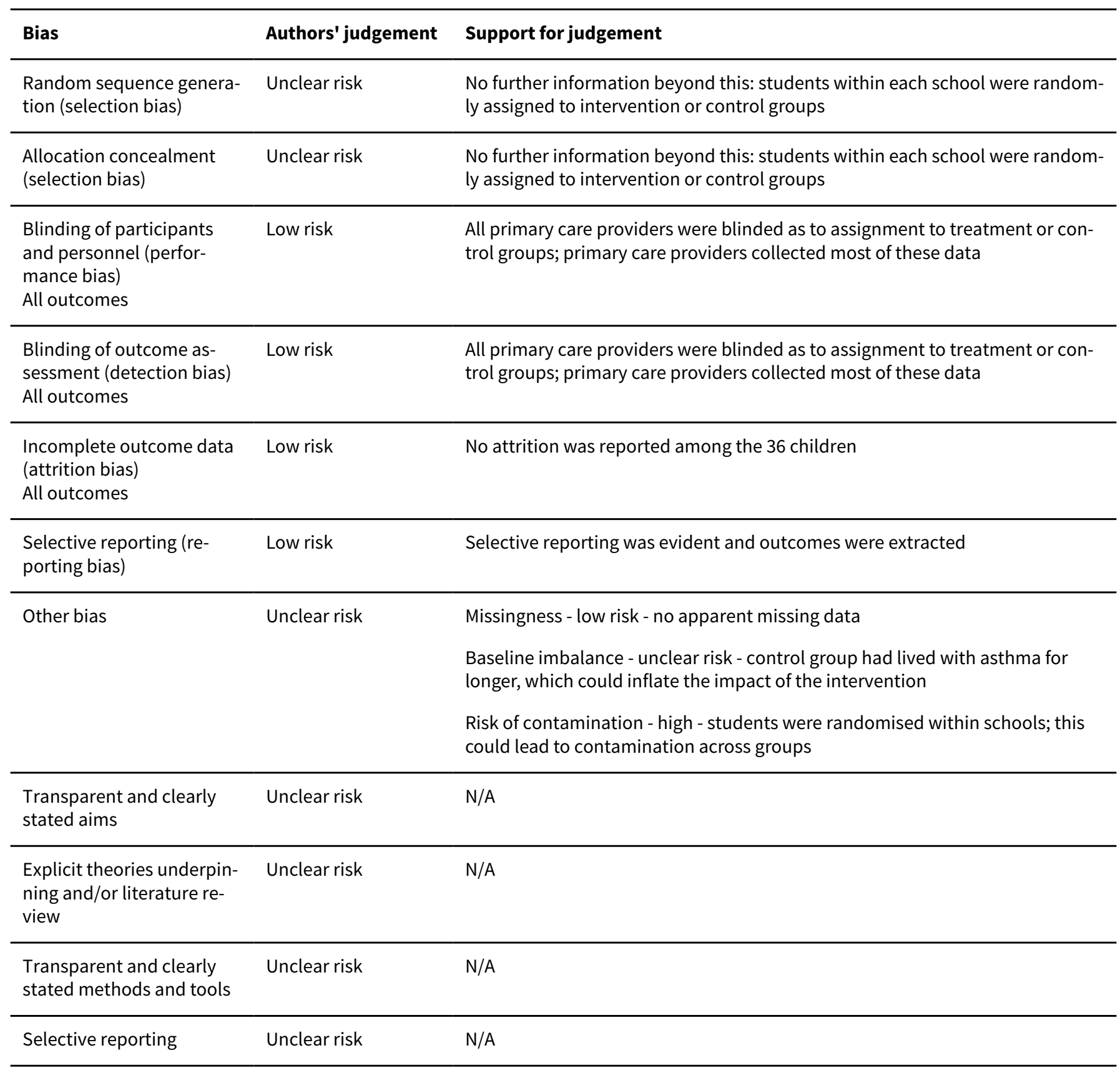


Persaud 1996 (Continued)

\begin{tabular}{|c|c|c|}
\hline Harmful effects & Unclear risk & $\mathrm{N} / \mathrm{A}$ \\
\hline $\begin{array}{l}\text { Population and sample } \\
\text { described well }\end{array}$ & Unclear risk & $\mathrm{N} / \mathrm{A}$ \\
\hline Continuous evaluation & Unclear risk & $\mathrm{N} / \mathrm{A}$ \\
\hline $\begin{array}{l}\text { Evaluation participation } \\
\text { equity and sampling }\end{array}$ & Unclear risk & $\mathrm{N} / \mathrm{A}$ \\
\hline $\begin{array}{l}\text { Design and methods over- } \\
\text { all approach }\end{array}$ & Unclear risk & $\mathrm{N} / \mathrm{A}$ \\
\hline $\begin{array}{l}\text { Tools and methods of da- } \\
\text { ta collection reliable/cred- } \\
\text { ible }\end{array}$ & Unclear risk & N/A \\
\hline $\begin{array}{l}\text { Tools and methods of data } \\
\text { analysis reliable/credible }\end{array}$ & Unclear risk & $\mathrm{N} / \mathrm{A}$ \\
\hline $\begin{array}{l}\text { Performance bias/neutral- } \\
\text { ity/credibility/conforma- } \\
\text { bility }\end{array}$ & Unclear risk & $\mathrm{N} / \mathrm{A}$ \\
\hline $\begin{array}{l}\text { Reliability of findings and } \\
\text { recommendations }\end{array}$ & Unclear risk & $\mathrm{N} / \mathrm{A}$ \\
\hline Transferability of findings & Unclear risk & $\mathrm{N} / \mathrm{A}$ \\
\hline $\begin{array}{l}\text { Overall risk of bias of } \\
\text { process evaluation }\end{array}$ & Unclear risk & $\mathrm{N} / \mathrm{A}$ \\
\hline
\end{tabular}

Pike 2011

Methods

\section{Included as process evaluation}

Intervention study design: quasi-experimental, pre-post, control groups

Unit of allocation: children in 15 classes were provided with the intervention (data available for 10), and 4 additional classrooms served as controls; 167 children were in the intervention group and 69 were in the control group

Process evaluation methods: survey data were collected with descriptive/bivariate analyses of data

Age of children: 9 to 11 years of age (based on grade)

Child characteristics (BME/SES): $81 \%$ of control group and $69 \%$ of intervention group were African American; $78 \%$ of intervention group and $86 \%$ of control group were receiving free school meals

Asthma status: asthmatic and non-asthmatic (mixed class; this is a core feature of the intervention so as not to disrupt normal school functioning)

Intervention recipients: children and teachers 
Pike 2011 (Continued)

Intervention description: a curriculum was developed for teachers that contained 15 lesson plans created or adapted from various existing sources and aligned with existing standards for communication arts, science, mathematics, and health. Intervention classroom teachers were asked to teach 7 of the 15 lesson plans, including 3 specific lesson plans chosen by the investigators (which included information on asthma basics, signs and symptoms, triggers, and use of a peak flow metre); the remaining 4 lesson plans were self-selected by the teacher

Control description: usual care/no additional asthma education

Theoretical framework: no information

Outcomes Core processes evaluated (child level): dosage

Notes Process evaluation category: stand-alone

Breadth and depth: depth - not breadth

Voice of children given prominence: not featured

Funding source: not reported

\section{Risk of bias}

\begin{tabular}{lll}
\hline Bias & Authors' judgement & Support for judgement \\
\hline $\begin{array}{l}\text { Random sequence genera- } \\
\text { tion (selection bias) }\end{array}$ & Unclear risk & N/A \\
\hline $\begin{array}{l}\text { Allocation concealment } \\
\text { (selection bias) }\end{array}$ & Unclear risk & N/A \\
\hline
\end{tabular}

Blinding of participants $\quad$ Unclear risk N/A
and personnel (perfor-
mance bias)
All outcomes

Blinding of outcome as- Unclear risk N/A

sessment (detection bias)

All outcomes

\begin{tabular}{l}
\hline Incomplete outcome data Unclear risk N/A \\
(attrition bias) \\
All outcomes
\end{tabular}

\begin{tabular}{lll}
\hline $\begin{array}{l}\text { Selective reporting (re- } \\
\text { porting bias) }\end{array}$ & Unclear risk & N/A \\
\hline Other bias & Unclear risk & N/A \\
\hline $\begin{array}{l}\text { Transparent and clearly } \\
\text { stated aims }\end{array}$ & Low risk & Study aims were clearly stated \\
\hline $\begin{array}{l}\text { Explicit theories underpin- } \\
\text { ning and/or literature re- } \\
\text { view }\end{array}$ & High risk & No theory was named and little literature was presented \\
\hline $\begin{array}{l}\text { Transparent and clearly } \\
\text { stated methods and tools }\end{array}$ & Unclear risk & $\begin{array}{l}\text { Some tools or aspects of tools were not clearly explained, for example, asthma } \\
\text { knowledge }\end{array}$ \\
\hline
\end{tabular}


Pike 2011 (Continued) $\begin{array}{ll}\text { Selective reporting } \quad \text { Unclear risk } & \begin{array}{l}\text { A full account of what was collected for assessment was not presented; some } \\ \text { aspects were not reported - e.g. the teacher focus group }\end{array}\end{array}$

\begin{tabular}{|c|c|c|}
\hline Harmful effects & Unclear risk & How study authors accounted for this remains unclear \\
\hline $\begin{array}{l}\text { Population and sample } \\
\text { described well }\end{array}$ & High risk & $\begin{array}{l}\text { Some demographic characteristics, particularly the asthma status of children, } \\
\text { were not explained well }\end{array}$ \\
\hline Continuous evaluation & Low risk & Pre-post assessment was included \\
\hline $\begin{array}{l}\text { Evaluation participation } \\
\text { equity and sampling }\end{array}$ & Unclear risk & Sample information was collected from several stakeholders \\
\hline $\begin{array}{l}\text { Design and methods over- } \\
\text { all approach }\end{array}$ & Low risk & Multiple sources of evidence were used \\
\hline $\begin{array}{l}\text { Tools and methods of da- } \\
\text { ta collection reliable/cred- } \\
\text { ible }\end{array}$ & High risk & How asthma knowledge was measured remains unclear \\
\hline $\begin{array}{l}\text { Tools and methods of data } \\
\text { analysis reliable/credible }\end{array}$ & High risk & No way to assess this without seeing a full output - e.g. of the focus group \\
\hline $\begin{array}{l}\text { Performance bias/neutral- } \\
\text { ity/credibility/conforma- } \\
\text { bility }\end{array}$ & Unclear risk & Some blinding was undertaken \\
\hline $\begin{array}{l}\text { Reliability of findings and } \\
\text { recommendations }\end{array}$ & Unclear risk & $\begin{array}{l}\text { Whether this was an effective intervention is not clear, as the information was } \\
\text { not presented fully }\end{array}$ \\
\hline Transferability of findings & Unclear risk & $\begin{array}{l}\text { Study authors explained how the curriculum was developed, so transferability } \\
\text { is low for part of this study - but not enough information was provided in other } \\
\text { sections }\end{array}$ \\
\hline $\begin{array}{l}\text { Overall risk of bias of } \\
\text { process evaluation }\end{array}$ & Unclear risk & $\begin{array}{l}\text { This is a good study of teachers, but study authors did not provide a lot of oth- } \\
\text { er information }\end{array}$ \\
\hline
\end{tabular}

Praena-Crespo 2010

Methods Included as outcome evaluation

Study design: clustered parallel-group RCT

Setting: 16 high schools

Period: baseline data were collected between November and December 2008

Eligible sample frame: 16 high schools ( 4090 children)
Randomised: 15 high schools ( 3827 children)
Completed (intervention): 15 high schools (3550 children)
Inclusion criteria: not reported
Exclusion criteria: not reported
Baseline characteristics


Praena-Crespo 2010 (Continued)

Age of children: 13 and 14 years of age

Ethnicity: not reported

Socio-economic status: not reported

Gender: mixed

Asthma status: asthmatic and non-asthmatic children

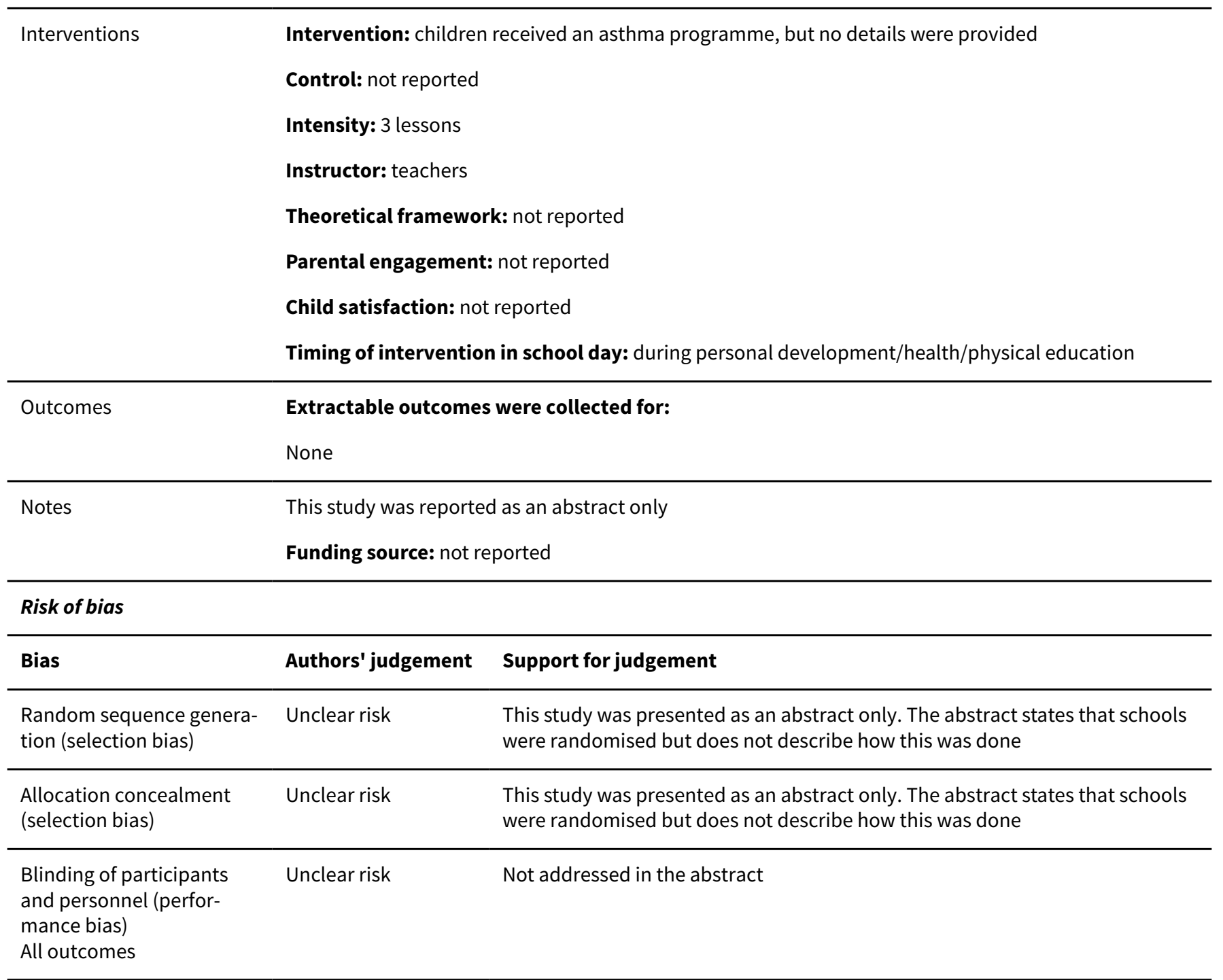

\begin{tabular}{lll}
\hline $\begin{array}{l}\text { Blinding of outcome as- } \\
\text { sessment (detection bias) } \\
\text { All outcomes }\end{array}$ & Unclear risk & Not addressed in the abstract \\
\hline $\begin{array}{l}\text { Incomplete outcome data } \\
\text { (attrition bias) } \\
\text { All outcomes }\end{array}$ & Unclear risk & $\begin{array}{l}\text { Questionnaires were returned by } 3827 / 4090 \text { students (279 with asthma) at } \\
\text { baseline and by } 3550 \text { at follow-up (261 with asthma) }\end{array}$ \\
\hline $\begin{array}{l}\text { Selective reporting (re- } \\
\text { porting bias) }\end{array}$ & Unclear risk & Not addressed in the abstract \\
\hline Other bias & Unclear risk & $\begin{array}{l}\text { Missing data - high risk - many forms were not returned and data were not dis- } \\
\text { aggregated }\end{array}$
\end{tabular}




\begin{tabular}{|c|c|c|}
\hline $\begin{array}{l}\text { Transparent and clearly } \\
\text { stated aims }\end{array}$ & Unclear risk & $\mathrm{N} / \mathrm{A}$ \\
\hline $\begin{array}{l}\text { Explicit theories underpin- } \\
\text { ning and/or literature re- } \\
\text { view }\end{array}$ & Unclear risk & $\mathrm{N} / \mathrm{A}$ \\
\hline $\begin{array}{l}\text { Transparent and clearly } \\
\text { stated methods and tools }\end{array}$ & Unclear risk & $\mathrm{N} / \mathrm{A}$ \\
\hline Selective reporting & Unclear risk & $\mathrm{N} / \mathrm{A}$ \\
\hline Harmful effects & Unclear risk & $\mathrm{N} / \mathrm{A}$ \\
\hline $\begin{array}{l}\text { Population and sample } \\
\text { described well }\end{array}$ & Unclear risk & $\mathrm{N} / \mathrm{A}$ \\
\hline Continuous evaluation & Unclear risk & $\mathrm{N} / \mathrm{A}$ \\
\hline $\begin{array}{l}\text { Evaluation participation } \\
\text { equity and sampling }\end{array}$ & Unclear risk & $\mathrm{N} / \mathrm{A}$ \\
\hline $\begin{array}{l}\text { Design and methods over- } \\
\text { all approach }\end{array}$ & Unclear risk & $\mathrm{N} / \mathrm{A}$ \\
\hline $\begin{array}{l}\text { Tools and methods of da- } \\
\text { ta collection reliable/cred- } \\
\text { ible }\end{array}$ & Unclear risk & $\mathrm{N} / \mathrm{A}$ \\
\hline $\begin{array}{l}\text { Tools and methods of data } \\
\text { analysis reliable/credible }\end{array}$ & Unclear risk & $\mathrm{N} / \mathrm{A}$ \\
\hline $\begin{array}{l}\text { Performance bias/neutral- } \\
\text { ity/credibility/conforma- } \\
\text { bility }\end{array}$ & Unclear risk & $\mathrm{N} / \mathrm{A}$ \\
\hline $\begin{array}{l}\text { Reliability of findings and } \\
\text { recommendations }\end{array}$ & Unclear risk & $\mathrm{N} / \mathrm{A}$ \\
\hline Transferability of findings & Unclear risk & $\mathrm{N} / \mathrm{A}$ \\
\hline $\begin{array}{l}\text { Overall risk of bias of } \\
\text { process evaluation }\end{array}$ & Unclear risk & $\mathrm{N} / \mathrm{A}$ \\
\hline
\end{tabular}

\section{Pulcini 2007}

\section{Methods}

\section{Included as outcome evaluation}

Study design: clustered parallel-group design, with schools as the unit of randomisation

Setting: middle schools in Massachusetts, USA 
Period: number of AAPs received was recorded by the school nurse in the fall of 2005 and was reported as a total number in early 2006 at the end of data collection

\section{Participants}

Eligible sample frame: not reported

Randomised: 40 students from 4 school districts - 20 students in each group

Completed (intervention): not reported

Inclusion criteria: children were eligible if they had received a diagnosis of asthma with medications ordered at school, had no current asthma action plan on file, were from English-speaking families, did not have any developmental disorders, and had a regular primary care provider or asthma specialist

Exclusion criteria: not reported

\section{Baseline characteristics}

Age of children: not reported, but children in grades 6 to 8 were recruited

Ethnicity: not reported

Socioeconomic status: not reported

Gender: not reported

Asthma status: not reported for measuring lung function. Peak flow was measured for 2 weeks and scores were recorded. All scores were sent to the physician along with a request for an asthma action plan

Control: school nurses in the control group continued to follow their standard procedure of requesting an AAP via the student's parents

Intensity: peak flow measured and recorded on a daily basis for 2 weeks

Instructor: school nurse

Theoretical framework: not reported

Parental engagement: not reported

Child satisfaction: not reported

Timing of intervention in school day: not reported

\begin{tabular}{|c|c|c|}
\hline Outcomes & \multicolumn{2}{|c|}{$\begin{array}{l}\text { Extractable outcomes were collected for: } \\
\text { None }\end{array}$} \\
\hline Notes & \multicolumn{2}{|c|}{$\begin{array}{l}\text { AAPs are important but are not a part of the outcomes in this review, so they cannot be extracted } \\
\text { Funding source: National Association of School Nurses Research Grant }\end{array}$} \\
\hline \multicolumn{3}{|l|}{ Risk of bias } \\
\hline Bias & Authors' judgement & Support for judgement \\
\hline $\begin{array}{l}\text { Random sequence genera- } \\
\text { tion (selection bias) }\end{array}$ & High risk & $\begin{array}{l}\text { Not specified, and low numbers randomised: each school district participating } \\
\text { in the study was required to have at least } 2 \text { middle schools, which were ran- } \\
\text { domly assigned to experimental or control groups }\end{array}$ \\
\hline
\end{tabular}


Pulcini 2007 (Continued)

$\begin{array}{ll}\begin{array}{l}\text { Allocation concealment } \\ \text { (selection bias) }\end{array} & \text { High risk } \\ & \begin{array}{l}\text { Not specified, and low numbers randomised: each school district participating } \\ \text { in the study was required to have at least } 2 \text { middle schools, which were ran- } \\ \text { domly assigned to experimental or control groups }\end{array}\end{array}$

\begin{tabular}{|c|c|c|}
\hline $\begin{array}{l}\text { Blinding of participants } \\
\text { and personnel (perfor- } \\
\text { mance bias) } \\
\text { All outcomes }\end{array}$ & Unclear risk & Not addressed by study authors \\
\hline $\begin{array}{l}\text { Blinding of outcome as- } \\
\text { sessment (detection bias) } \\
\text { All outcomes }\end{array}$ & Unclear risk & Not addressed by study authors \\
\hline $\begin{array}{l}\text { Incomplete outcome data } \\
\text { (attrition bias) } \\
\text { All outcomes }\end{array}$ & Unclear risk & Not addressed by study authors \\
\hline
\end{tabular}

Selective reporting (re- High risk $\quad$ Peak flow data were collected but were not published in full
porting bias)

\begin{tabular}{|c|c|c|}
\hline Other bias & Unclear risk & $\begin{array}{l}\text { Missingness - unclear risk - not all data were published } \\
\text { Baseline imbalance - unclear risk - not addressed in the study } \\
\text { Risk of contamination - low - allocation was done on a school basis }\end{array}$ \\
\hline $\begin{array}{l}\text { Transparent and clearly } \\
\text { stated aims }\end{array}$ & Unclear risk & $\mathrm{N} / \mathrm{A}$ \\
\hline $\begin{array}{l}\text { Explicit theories underpin- } \\
\text { ning and/or literature re- } \\
\text { view }\end{array}$ & Unclear risk & $\mathrm{N} / \mathrm{A}$ \\
\hline $\begin{array}{l}\text { Transparent and clearly } \\
\text { stated methods and tools }\end{array}$ & Unclear risk & $\mathrm{N} / \mathrm{A}$ \\
\hline Selective reporting & Unclear risk & $\mathrm{N} / \mathrm{A}$ \\
\hline Harmful effects & Unclear risk & $\mathrm{N} / \mathrm{A}$ \\
\hline $\begin{array}{l}\text { Population and sample } \\
\text { described well }\end{array}$ & Unclear risk & $\mathrm{N} / \mathrm{A}$ \\
\hline Continuous evaluation & Unclear risk & $\mathrm{N} / \mathrm{A}$ \\
\hline $\begin{array}{l}\text { Evaluation participation } \\
\text { equity and sampling }\end{array}$ & Unclear risk & $\mathrm{N} / \mathrm{A}$ \\
\hline $\begin{array}{l}\text { Design and methods over- } \\
\text { all approach }\end{array}$ & Unclear risk & $\mathrm{N} / \mathrm{A}$ \\
\hline $\begin{array}{l}\text { Tools and methods of da- } \\
\text { ta collection reliable/cred- } \\
\text { ible }\end{array}$ & Unclear risk & $\mathrm{N} / \mathrm{A}$ \\
\hline $\begin{array}{l}\text { Tools and methods of data } \\
\text { analysis reliable/credible }\end{array}$ & Unclear risk & $\mathrm{N} / \mathrm{A}$ \\
\hline
\end{tabular}


Pulcini 2007 (Continued)

Performance bias/neutral- Unclear risk N/A ity/credibility/conformability

\begin{tabular}{lll}
\hline $\begin{array}{l}\text { Reliability of findings and } \\
\text { recommendations }\end{array}$ & Unclear risk & N/A \\
\hline Transferability of findings & Unclear risk & N/A \\
\hline $\begin{array}{l}\text { Overall risk of bias of } \\
\text { process evaluation }\end{array}$ & Unclear risk & N/A \\
\hline
\end{tabular}

Richmond 2011

Included as process evaluation
Intervention study design: quasi-experimental, post follow-up only, no control
Unit of allocation: N/A
Process evaluation methods: descriptive/bivariate analysis methods

\section{Participants}

Country: USA

Age of children: elementary school age was reported, but no further details were given

Child characteristics (BME/SES): almost 100\% of students were African American; approximately $80 \%$ received free or reduced price lunch

Asthma status: asthmatic only

Intervention recipients: children only

School type: 14 elementary schools across 3 school districts
Interventions
aged to received an asthma action plan from their healthcare provider and to collect prescriptions from
the pharmacy. Students were also advised to give their action plans and medications to their school
nurse at the beginning of the school year

Control description: N/A

Theoretical framework: not reported

\begin{tabular}{ll}
\hline Outcomes & Core processes evaluated (child level): attrition, adherence \\
\hline Notes & Process evaluation category: stand-alone \\
& Breadth and depth: neither broad nor deep \\
& Voice of children given prominence: not featured \\
& Funding source: not reported \\
\hline Risk of bias & Authors' judgement Support for judgement \\
\hline Bias &
\end{tabular}


Richmond 2011 (Continued)

\begin{tabular}{lll}
$\begin{array}{l}\text { Random sequence genera- } \\
\text { tion (selection bias) }\end{array}$ & Unclear risk & N/A \\
\hline $\begin{array}{l}\text { Allocation concealment } \\
\text { (selection bias) }\end{array}$ & Unclear risk & N/A \\
\hline $\begin{array}{l}\text { Blinding of participants } \\
\text { and personnel (perfor- } \\
\text { mance bias) }\end{array}$ & Unclear risk & N/A \\
All outcomes & & \\
\hline
\end{tabular}

Blinding of outcome as-
sessment (detection bias) $\quad$ Unclear risk N/A

\begin{tabular}{ll}
\hline Incomplete outcome data Unclear risk & N/A \\
(attrition bias) &
\end{tabular}

All outcomes

\begin{tabular}{|c|c|c|}
\hline $\begin{array}{l}\text { Selective reporting (re- } \\
\text { porting bias) }\end{array}$ & Unclear risk & $\mathrm{N} / \mathrm{A}$ \\
\hline Other bias & Unclear risk & $\mathrm{N} / \mathrm{A}$ \\
\hline $\begin{array}{l}\text { Transparent and clearly } \\
\text { stated aims }\end{array}$ & Unclear risk & Study report shows some confusion over the purpose of the study \\
\hline $\begin{array}{l}\text { Explicit theories underpin- } \\
\text { ning and/or literature re- } \\
\text { view }\end{array}$ & High risk & No literature was provided; no named theory guided the intervention \\
\hline $\begin{array}{l}\text { Transparent and clearly } \\
\text { stated methods and tools }\end{array}$ & High risk & How the random sample was selected is not clear \\
\hline Selective reporting & High risk & Information discussed in the interviews is not clear - no schedule \\
\hline Harmful effects & Unclear risk & $\begin{array}{l}\text { This is inferred by the reader - impact on nurses if implemented across the } \\
\text { board }\end{array}$ \\
\hline $\begin{array}{l}\text { Population and sample } \\
\text { described well }\end{array}$ & High risk & $\begin{array}{l}\text { Age and gender were missing, as were the demographic characteristics of } \\
\text { asthmatic kids }\end{array}$ \\
\hline Continuous evaluation & High risk & $\begin{array}{l}\text { Post hoc evaluation only - particularly worrisome, as the intervention failed in } \\
\text { the first year but continued into the second year }\end{array}$ \\
\hline $\begin{array}{l}\text { Evaluation participation } \\
\text { equity and sampling }\end{array}$ & Unclear risk & $\begin{array}{l}\text { Parents were interviewed and some information was collected from school } \\
\text { nurses }\end{array}$ \\
\hline $\begin{array}{l}\text { Design and methods over- } \\
\text { all approach }\end{array}$ & Unclear risk & $\begin{array}{l}\text { Interviews with parents seem to be the most important element; these were } \\
\text { covered adequately }\end{array}$ \\
\hline $\begin{array}{l}\text { Tools and methods of da- } \\
\text { ta collection reliable/cred- } \\
\text { ible }\end{array}$ & High risk & Tools used were not presented (i.e. interview schedule) \\
\hline $\begin{array}{l}\text { Tools and methods of data } \\
\text { analysis reliable/credible }\end{array}$ & Unclear risk & Only descriptive/narrative analysis reported \\
\hline
\end{tabular}


Richmond 2011 (Continued)

Performance bias/neutral- Low risk Attention was given to negative cases and non-participation was investigated ity/credibility/conformability

\begin{tabular}{lll}
\hline $\begin{array}{l}\text { Reliability of findings and } \\
\text { recommendations }\end{array}$ & High risk & $\begin{array}{l}\text { Quantity of data collected was unclear; not easy to ascertain whether there } \\
\text { was more to be understood here }\end{array}$ \\
\hline Transferability of findings & Unclear risk & $\begin{array}{l}\text { Results show lack of understanding of the degree to which age and school fac- } \\
\text { tors played a part - much more remained to be said about this }\end{array}$ \\
\hline $\begin{array}{l}\text { Overall risk of bias of } \\
\text { process evaluation }\end{array}$ & Unclear risk & $\begin{array}{l}\text { Risk of bias not very high because of the nature of the messages and the sim- } \\
\text { ple methods employed; however reporting errors occurred }\end{array}$
\end{tabular}

Shah 2001

Included as outcome evaluation
Study design: clustered parallel-group design
Setting: high schools in Tamworth, rural New South Wales, Australia
Period: pupils were recruited in February 1998 and completed the study in October 1998 - 3 months af-
ter the intervention was completed

\section{Participants}

Eligible sample frame: 325 pupils

Randomised: 272 pupils: 148 in the control group and 124 in the intervention group

Completed (intervention): 251 pupils; 138 in the control group and 113 in the intervention group

Inclusion criteria: not reported

Exclusion criteria: not reported

Baseline characteristics

Age of children: 118 pupils in year $7 ; 133$ pupils in year 10

Ethnicity: not reported

Socio-economic status: not reported

Gender: $62 \%$ to $68 \%$ female in the intervention group; $44 \%$ to $48 \%$ female in the control group

Asthma status: $69 \%$ to $80 \%$ had received an asthma diagnosis

Intervention: the intervention involved a 3-step approach to educating and empowering students with asthma. In step 1, students learnt how to educate their peers about asthma and its management using games, videos, worksheets, and discussions as teaching tools. In step 2, peer leaders conducted three 45-mnute health lessons for year 10 classes at school. In step 3, year 10 students developed and presented to year 7 students key messages learnt in the lessons

Control: wait-list control group received usual care during data collection

Intensity: in step 1, volunteers from year 11 were trained as asthma peers during a 6-hour workshop. In step 2, three 45-minute sessions were taught. No information was recorded on length and intensity in step 3

Instructor: peers

Theoretical framework: not reported 
Shah 2001 (Continued)

Parental engagement: not reported

Child satisfaction: not reported

Timing of intervention in school day: not reported

\begin{tabular}{ll}
\hline Outcomes & Extractable outcomes were collected for: \\
& Experience of daytime and night-time symptoms \\
& Lung function \\
& Health-related quality of life \\
& Withdrawal
\end{tabular}

Notes School absence data were collected as median values but were not reported in full

Funding source: Commonwealth Department of Health and Aged Care and Asthma NSW

\section{Risk of bias}

\begin{tabular}{lll}
\hline Bias & Authors' judgement & Support for judgement \\
\hline $\begin{array}{l}\text { Random sequence genera- } \\
\text { tion (selection bias) }\end{array}$ & Low risk & $\begin{array}{l}\text { Concealed random allocation was performed by study author (who was not in- } \\
\text { volved in administration of the study), using a random number generator and } \\
\text { the closed envelope technique }\end{array}$ \\
\hline $\begin{array}{l}\text { Allocation concealment } \\
\text { (selection bias) }\end{array}$ & Low risk & $\begin{array}{l}\text { Concealed random allocation was performed by PGG (who was not involved in } \\
\text { administration of the study), using a random number generator and the closed } \\
\text { envelope technique }\end{array}$
\end{tabular}

Blinding of participants Unclear risk Not addressed by study authors

and personnel (perfor-

mance bias)

All outcomes

\begin{tabular}{ll}
\hline Blinding of outcome as- & Unclear risk Not addressed by study authors \\
sessment (detection bias) & \\
All outcomes &
\end{tabular}

\begin{tabular}{ll}
\hline $\begin{array}{l}\text { Incomplete outcome data } \\
\text { (attrition bias) }\end{array}$ & Low risk \\
All outcomes & Low levels of attrition: 272 participated in baseline testing; matched data at \\
\end{tabular}

\begin{tabular}{lll}
\hline $\begin{array}{l}\text { Selective reporting (re- } \\
\text { porting bias) }\end{array}$ & Unclear risk & $\begin{array}{l}\text { No direct evidence, although median number of days missed was collected } \\
\text { and could not be combined in the meta-analysis }\end{array}$ \\
\hline Other bias & $\begin{array}{l}\text { Missingness - low risk - missing data described as uncommon and occurred } \\
\text { owing to misclassification, students changing schools or being absent on the } \\
\text { day of testing, or failure to complete the questionnaire }\end{array}$ \\
& $\begin{array}{l}\text { Baseline imbalance - unclear risk - differences between groups, although it is } \\
\text { unclear if these are significant for the outcome }\end{array}$ \\
& $\begin{array}{l}\text { Risk of contamination - low - schools were the unit of randomisation, thereby } \\
\text { lowering risk of contamination }\end{array}$ \\
\hline $\begin{array}{l}\text { Transparent and clearly } \\
\text { stated aims }\end{array}$ & Unclear risk & N/A \\
\hline
\end{tabular}


Shah 2001 (Continued)

Explicit theories underpin- Unclear risk N/A ning and/or literature re-

view

\begin{tabular}{|c|c|c|}
\hline $\begin{array}{l}\text { Transparent and clearly } \\
\text { stated methods and tools }\end{array}$ & Unclear risk & $\mathrm{N} / \mathrm{A}$ \\
\hline Selective reporting & Unclear risk & $\mathrm{N} / \mathrm{A}$ \\
\hline Harmful effects & Unclear risk & $\mathrm{N} / \mathrm{A}$ \\
\hline $\begin{array}{l}\text { Population and sample } \\
\text { described well }\end{array}$ & Unclear risk & $\mathrm{N} / \mathrm{A}$ \\
\hline Continuous evaluation & Unclear risk & $\mathrm{N} / \mathrm{A}$ \\
\hline $\begin{array}{l}\text { Evaluation participation } \\
\text { equity and sampling }\end{array}$ & Unclear risk & N/A \\
\hline $\begin{array}{l}\text { Design and methods over- } \\
\text { all approach }\end{array}$ & Unclear risk & $\mathrm{N} / \mathrm{A}$ \\
\hline $\begin{array}{l}\text { Tools and methods of da- } \\
\text { ta collection reliable/cred- } \\
\text { ible }\end{array}$ & Unclear risk & $\mathrm{N} / \mathrm{A}$ \\
\hline $\begin{array}{l}\text { Tools and methods of data } \\
\text { analysis reliable/credible }\end{array}$ & Unclear risk & $\mathrm{N} / \mathrm{A}$ \\
\hline $\begin{array}{l}\text { Performance bias/neutral- } \\
\text { ity/credibility/conforma- } \\
\text { bility }\end{array}$ & Unclear risk & $\mathrm{N} / \mathrm{A}$ \\
\hline $\begin{array}{l}\text { Reliability of findings and } \\
\text { recommendations }\end{array}$ & Unclear risk & $\mathrm{N} / \mathrm{A}$ \\
\hline Transferability of findings & Unclear risk & $\mathrm{N} / \mathrm{A}$ \\
\hline $\begin{array}{l}\text { Overall risk of bias of } \\
\text { process evaluation }\end{array}$ & Unclear risk & $\mathrm{N} / \mathrm{A}$ \\
\hline
\end{tabular}

Spencer 2000

Methods Included as process evaluation

Intervention study design: quasi-experimental single-group intervention examining change pre-post intervention

Unit of allocation: N/A

Process evaluation methods: survey-based methods with descriptive/bivariate analyses of results

Participants

Setting: the study included 40 schools from 8 school districts throughout New York State, USA

Age of children: wide range of ages, with children 6 to 13 years old

Child characteristics (BME/SES): $36 \%$ of children were receiving free or reduced price lunch 
Asthma status: asthmatic only

Intervention recipients: children and parents

School type: primary/elementary
Interventions
Intervention description: Open Airways for Schools (OAS) described by study authors as consisting of
"six weekly (40-minute) hands-on sessions for the children, one or two sessions for the children's par-
ents, and a graduation ceremony for both parents and children. The children's portion of the program
covered such areas as: (I) basic information and feelings about asthma; (ii) recognizing and managing
asthma symptoms; (iii) solving problems with medicines and deciding how bad symptoms are; (iv) find-
ing and controlling asthma triggers; (v) getting enough exercise; and (vi) doing well at school. The par-
ents' program briefly covered content similar to the children's sessions. Parents also received letters
that familiarized them with the children's classroom content"

Control description: N/A

Theoretical framework: no information

\begin{tabular}{|c|c|c|}
\hline Outcomes & \multicolumn{2}{|c|}{ Core processes evaluated (child level): adherence } \\
\hline \multirow[t]{4}{*}{ Notes } & \multicolumn{2}{|c|}{ Process evaluation category: integrated } \\
\hline & \multicolumn{2}{|c|}{ Breadth and depth: neither broad nor deep } \\
\hline & \multicolumn{2}{|c|}{ Voice of children given prominence: not featured } \\
\hline & \multicolumn{2}{|c|}{ Funding source: not reported } \\
\hline \multicolumn{3}{|l|}{ Risk of bias } \\
\hline Bias & Authors' judgement & Support for judgement \\
\hline $\begin{array}{l}\text { Random sequence genera- } \\
\text { tion (selection bias) }\end{array}$ & Unclear risk & N/A \\
\hline $\begin{array}{l}\text { Allocation concealment } \\
\text { (selection bias) }\end{array}$ & Unclear risk & $\mathrm{N} / \mathrm{A}$ \\
\hline $\begin{array}{l}\text { Blinding of participants } \\
\text { and personnel (perfor- } \\
\text { mance bias) } \\
\text { All outcomes }\end{array}$ & Unclear risk & N/A \\
\hline $\begin{array}{l}\text { Blinding of outcome as- } \\
\text { sessment (detection bias) } \\
\text { All outcomes }\end{array}$ & Unclear risk & $\mathrm{N} / \mathrm{A}$ \\
\hline $\begin{array}{l}\text { Incomplete outcome data } \\
\text { (attrition bias) } \\
\text { All outcomes }\end{array}$ & Unclear risk & N/A \\
\hline $\begin{array}{l}\text { Selective reporting (re- } \\
\text { porting bias) }\end{array}$ & Unclear risk & N/A \\
\hline Other bias & Unclear risk & N/A \\
\hline $\begin{array}{l}\text { Transparent and clearly } \\
\text { stated aims }\end{array}$ & Unclear risk & Aims of OAS were clearly stated, but aims of the study were not explained \\
\hline
\end{tabular}


Spencer 2000 (Continued)

Explicit theories underpin- High risk No literature or theoretical framework was provided ning and/or literature re-

view

\begin{tabular}{|c|c|c|}
\hline $\begin{array}{l}\text { Transparent and clearly } \\
\text { stated methods and tools }\end{array}$ & Unclear risk & Not much detail provided on tools for parents and nurses \\
\hline Selective reporting & High risk & Not everything was reported; instruments were poorly reported \\
\hline Harmful effects & High risk & No evidence of provision for measuring harmful effects \\
\hline $\begin{array}{l}\text { Population and sample } \\
\text { described well }\end{array}$ & Unclear risk & No information about asthma severity \\
\hline Continuous evaluation & Low risk & Pre-post assessment was implemented \\
\hline $\begin{array}{l}\text { Evaluation participation } \\
\text { equity and sampling }\end{array}$ & Unclear risk & How almost half the sample of kids dropped out remains unclear \\
\hline $\begin{array}{l}\text { Design and methods over- } \\
\text { all approach }\end{array}$ & High risk & $\begin{array}{l}\text { Limited detail on design and methods was provided; problems with internal } \\
\text { validity were noted }\end{array}$ \\
\hline $\begin{array}{l}\text { Tools and methods of da- } \\
\text { ta collection reliable/cred- } \\
\text { ible }\end{array}$ & High risk & Not all tools were clearly described \\
\hline $\begin{array}{l}\text { Tools and methods of data } \\
\text { analysis reliable/credible }\end{array}$ & High risk & $\begin{array}{l}\text { No indication that clustering was accounted for; not enough information } \\
\text { (mean cluster size) was provided to estimate this }\end{array}$ \\
\hline $\begin{array}{l}\text { Performance bias/neutral- } \\
\text { ity/credibility/conforma- } \\
\text { bility }\end{array}$ & High risk & No attempt at blinding was presented \\
\hline $\begin{array}{l}\text { Reliability of findings and } \\
\text { recommendations }\end{array}$ & High risk & This study was not reported well enough to warrant that findings were reliable \\
\hline Transferability of findings & High risk & Details about context were lacking, making the findings difficult to transfer \\
\hline $\begin{array}{l}\text { Overall risk of bias of } \\
\text { process evaluation }\end{array}$ & High risk & Issues surround the tools and methods used to collect study data \\
\hline
\end{tabular}

\section{Splett 2006}

Methods Included as outcome evaluation and process evaluation

Study design: clustered parallel-group design, randomised at the school level

Setting: K-8 schools in Minneapolis, Minnesota, USA

Period: in 2000 and 2001, the HLAl was implemented in schools and was tested for effectiveness; in 2001 and 2002, the HLAl was expanded to all K-8 schools

Participants

Eligible sample frame: not reported; however 700 students with asthma were required in each group to detect a positive change in attendance

Randomised: 916 in intervention schools and 645 in control schools 
Inclusion criteria: not reported

Exclusion criteria: not reported

Baseline characteristics

Age of children: not reported

Ethnicity: 66\% were African American, $6 \%$ were Hispanic, 5\% were American-Indian, and 20\% were white

Socio-economic status: $73 \%$ of participants were eligible for free or reduced price lunches

Gender: males represented $58 \%$ of participants

Asthma status: not reported

Intervention: in participating schools, licensed school nurses, licensed practical nurses, and health service assistants received coaching and reinforcement by asthma resource nurses. Clinics also received training on NIH guidelines and guidance on implementing standards of care for asthma. Study authors reported: "staff followed 'Core Components of Asthma Management in the School Health Office' (Core Components), including case identification, nursing care procedures, care coordination, emergency care, and student education, to provide more systematic and consistent care to students with asthma and improve communication with school staff, parents, and health care providers", although further details of the student education component were not provided

Control: usual care

Intensity: not reported

Instructor: school nurse

Theoretical framework: not reported

Parental engagement: not reported

Child satisfaction: not reported

Timing of intervention in school day: not reported

\section{Outcomes $\quad$ Extractable outcomes were collected for:}

Absence from school

Unplanned visit to hospital or GP due to asthma symptoms

Core processes evaluated (child level): no information (other outcomes considered around sustainability)

\section{Notes}

This study conducted an ecological analysis

Process evaluation category: stand-alone, named section (2 papers)

Breadth and depth: neither broad nor deep

Voice of children given prominence: not featured

Funding source: Member Organisations of the Healthy Learners Board, Controlling Asthma in American Cities Grant

\section{Risk of bias}


Splett 2006 (Continued)

Bias Authors' judgement Support for judgement

Random sequence genera- Low risk tion (selection bias)

Allocation concealment Low risk
(selection bias)
(selection bias)

Blinding of participants Unclear risk
and personnel (perfor-
mance bias)
All outcomes

\begin{tabular}{lll}
\hline $\begin{array}{l}\text { Blinding of outcome as- } \\
\text { sessment (detection bias) } \\
\text { All outcomes }\end{array}$ & Unclear risk & No measures were described as implemented around blinding \\
\hline $\begin{array}{l}\text { Incomplete outcome data } \\
\text { (attrition bias) }\end{array}$ & Unclear risk & No direct reports describe attrition \\
All outcomes & \\
\hline
\end{tabular}

Study authors reported: "A random sequence of treatment codes, stratified by school system, was generated using the SAS System (Version 9.1, Cary, North Carolina) by the statistician"

\begin{tabular}{|c|c|c|}
\hline $\begin{array}{l}\text { Selective reporting (re- } \\
\text { porting bias) }\end{array}$ & Low risk & No evidence of selective reporting was found \\
\hline Other bias & Unclear risk & $\begin{array}{l}\text { Missingness - low risk - all those who were followed up submitted information } \\
\text { Baseline imbalance - low risk - no evidence of baseline imbalances between in- } \\
\text { tervention and control groups }\end{array}$ \\
\hline & & $\begin{array}{l}\text { Risk of contamination - high - children were the unit of randomisation; poten- } \\
\text { tial was present for children with different treatment allocations to share ma- } \\
\text { terials/information, etc }\end{array}$ \\
\hline
\end{tabular}

\begin{tabular}{ll}
\hline $\begin{array}{l}\text { Transparent and clearly } \\
\text { stated aims }\end{array}$ & Low risk \\
\end{tabular}

Explicit theories underpin- High risk No theoretical framework or supporting literature was provided
ning and/or literature review

\begin{tabular}{|c|c|c|}
\hline $\begin{array}{l}\text { Transparent and clearly } \\
\text { stated methods and tools }\end{array}$ & Low risk & Methods and tools were clearly stated \\
\hline Selective reporting & High risk & $\begin{array}{l}\text { A focus group is mentioned in the DuPlessis paper, but whether this occurrec } \\
\text { before or after or during the intervention is not clear. In addition, relevant re- } \\
\text { sults were not presented }\end{array}$ \\
\hline Harmful effects & High risk & Very broad study; harmful effects were not directly considered \\
\hline $\begin{array}{l}\text { Population and sample } \\
\text { described well }\end{array}$ & High risk & Some information is missing, including age of participants \\
\hline Continuous evaluation & Low risk & Process evaluation data were apparently collected throughout \\
\hline $\begin{array}{l}\text { Evaluation participation } \\
\text { equity and sampling }\end{array}$ & High risk & No information was collected from nurses \\
\hline
\end{tabular}


Splett 2006 (Continued)

Design and methods over- Unclear risk Some core elements are missing
all approach

\begin{tabular}{lll}
\hline $\begin{array}{l}\text { Tools and methods of da- } \\
\text { ta collection reliable/cred- } \\
\text { ible }\end{array}$ & Low risk & Based on administrative records - straightforward constructs \\
\hline $\begin{array}{l}\text { Tools and methods of data } \\
\text { analysis reliable/credible }\end{array}$ & Unclear risk & $\begin{array}{l}\text { Lack of age data makes it difficult to interpret some outcomes, although the } \\
\text { models include controls for age }\end{array}$ \\
\hline $\begin{array}{l}\text { Performance bias/neutral- } \\
\text { ity/credibility/conforma- } \\
\text { bility }\end{array}$ & Low risk & $\begin{array}{l}\text { As based on administrative records, little reason was provided to assign any- } \\
\text { thing but low risk of bias }\end{array}$ \\
\hline $\begin{array}{l}\text { Reliability of findings and } \\
\text { recommendations }\end{array}$ & Unclear risk & Reliability of findings was compromised by the K-12 age group \\
\hline $\begin{array}{l}\text { Transferability of findings } \\
\text { High risk }\end{array}$ & High risk & $\begin{array}{l}\text { Details are lacking, and standardisation introduced difficulties related to } \\
\text { transferability - particularly the wide age range }\end{array}$ \\
\hline $\begin{array}{l}\text { Overall risk of bias of } \\
\text { process evaluation }\end{array}$ & $\begin{array}{l}\text { Details around the ages of children and other key factors that could influence } \\
\text { outcomes are lacking }\end{array}$ \\
\hline
\end{tabular}

Srof 2012

Methods

\section{Included as outcome evaluation}

Study design: parallel-group design, randomised at the child level

Setting: high schools in midwestern America

Period: not reported, but post-test data were collected 6 weeks after the intervention was completed

Participants Eligible sample frame: 299 students with asthma across the 3 participating high schools

Randomised: 39 students: 21 in the intervention group and 18 in the control group

Completed (intervention): 39 students

Inclusion criteria: not reported

Exclusion criteria: not reported

Baseline characteristics

Age of children: 14 to 18 years of age; average age was 15.67 years

Ethnicity: not reported

Socio-economic status: not reported

Gender: the intervention group comprised 10 males and 11 females

Asthma status: not reported

Control: usual care 
Srof 2012 (Continued)

Intensity: sessions ranged from 40 minutes to 60 minutes in duration and were conducted over a period of 5 weeks

Instructor: principal investigator (PI)

Theoretical framework: Health Promotion Model (HPM) informed development of the intervention

Parental engagement: not reported

Child satisfaction: not reported

Timing of intervention in school day: sessions took place during the reserved study or resource period time block of the school day

\begin{tabular}{ll}
\hline Outcomes & Extractable outcomes were collected for: \\
& None \\
\hline Notes & Only some of the data in this study were reported, indicating evidence of possible selective reporting \\
& Considered for process evaluation but did not include expected required components \\
& Funding source: American Lung Association of Metropolitan Chicago, Nu Omicron Chapter of Sigma \\
& Theta Tau International, Pedipress Fulfillment Center, Respironics: HealthScan and Allergy Products
\end{tabular}

\section{Risk of bias}

\begin{tabular}{lll}
\hline Bias & Authors' judgement & Support for judgement \\
\hline $\begin{array}{l}\text { Random sequence genera- } \\
\text { tion (selection bias) }\end{array}$ & Low risk & $\begin{array}{l}\text { Names of participating students were placed in an envelope and were drawn } \\
\text { for random assignment to treatment and control groups within each school }\end{array}$ \\
\hline $\begin{array}{l}\text { Allocation concealment } \\
\text { (selection bias) }\end{array}$ & Unclear risk & $\begin{array}{l}\text { Unclear whether closed envelope technique was used and whether allocation } \\
\text { could be concealed }\end{array}$ \\
\hline $\begin{array}{l}\text { Blinding of participants } \\
\text { and personnel (perfor- } \\
\text { mance bias) } \\
\text { All outcomes }\end{array}$ & Unclear risk & Not addressed by study authors \\
\hline
\end{tabular}

Blinding of outcome as-
sessment (detection bias) $\quad$ High risk Lack of blinding was cited as a disadvantage of the study

All outcomes

\begin{tabular}{lll}
\hline $\begin{array}{l}\text { Incomplete outcome data } \\
\text { (attrition bias) } \\
\text { All outcomes }\end{array}$ & Unclear risk & Not addressed in full by study authors \\
\hline $\begin{array}{l}\text { Selective reporting (re- } \\
\text { porting bias) }\end{array}$ & High risk & $\begin{array}{l}\text { Evidence of some selective reporting; outcomes were not reported consistent- } \\
\text { ly throughout }\end{array}$ \\
\hline Other bias & Unclear risk & Missingness - unclear risk - not addressed in full by study authors \\
& $\begin{array}{l}\text { Baseline imbalance - low risk - no differences noted between groups on any of } \\
\text { the baseline variables, as would be expected following random assignment } \\
\text { Risk of contamination - high - children were randomised within schools, rais- } \\
\text { ing the risk of contamination }\end{array}$ \\
\hline $\begin{array}{l}\text { Transparent and clearly } \\
\text { stated aims }\end{array}$ & Unclear risk & N/A \\
\hline
\end{tabular}


Srof 2012 (Continued)

Explicit theories underpin- Unclear risk N/A ning and/or literature re-

view

\begin{tabular}{|c|c|c|}
\hline $\begin{array}{l}\text { Transparent and clearly } \\
\text { stated methods and tools }\end{array}$ & Unclear risk & $\mathrm{N} / \mathrm{A}$ \\
\hline Selective reporting & Unclear risk & $\mathrm{N} / \mathrm{A}$ \\
\hline Harmful effects & Unclear risk & $\mathrm{N} / \mathrm{A}$ \\
\hline $\begin{array}{l}\text { Population and sample } \\
\text { described well }\end{array}$ & Unclear risk & $\mathrm{N} / \mathrm{A}$ \\
\hline Continuous evaluation & Unclear risk & $\mathrm{N} / \mathrm{A}$ \\
\hline $\begin{array}{l}\text { Evaluation participation } \\
\text { equity and sampling }\end{array}$ & Unclear risk & N/A \\
\hline $\begin{array}{l}\text { Design and methods over- } \\
\text { all approach }\end{array}$ & Unclear risk & $\mathrm{N} / \mathrm{A}$ \\
\hline $\begin{array}{l}\text { Tools and methods of da- } \\
\text { ta collection reliable/cred- } \\
\text { ible }\end{array}$ & Unclear risk & $\mathrm{N} / \mathrm{A}$ \\
\hline $\begin{array}{l}\text { Tools and methods of data } \\
\text { analysis reliable/credible }\end{array}$ & Unclear risk & $\mathrm{N} / \mathrm{A}$ \\
\hline $\begin{array}{l}\text { Performance bias/neutral- } \\
\text { ity/credibility/conforma- } \\
\text { bility }\end{array}$ & Unclear risk & $\mathrm{N} / \mathrm{A}$ \\
\hline $\begin{array}{l}\text { Reliability of findings and } \\
\text { recommendations }\end{array}$ & Unclear risk & $\mathrm{N} / \mathrm{A}$ \\
\hline Transferability of findings & Unclear risk & $\mathrm{N} / \mathrm{A}$ \\
\hline $\begin{array}{l}\text { Overall risk of bias of } \\
\text { process evaluation }\end{array}$ & Unclear risk & $\mathrm{N} / \mathrm{A}$ \\
\hline
\end{tabular}

Terpstra 2012

Methods Included as process evaluation

Intervention study design: quasi-experimental design, pre-post follow-up, control group

Unit of allocation: school

Process evaluation methods: multi-variate analysis

Country: USA
Age of children: mean age, 12.52 in the intervention group and 12.10 in the control group
Child characteristics (BME/SES): $44 \%$ of intervention children and $56 \%$ of control group children were
Latino; average annual income was less than $\$ 20,000$


Terpstra 2012 (Continued)

$$
\text { Asthma status: asthmatic only }
$$

Intervention recipients: children and parents

School type: 2 middle schools

Intervention description: children received skills training addressing topics such as how to use a peak flow meter. These sessions took place over 6 weeks. Parents received a newsletter that was centred on an important theme identified during the research

Control description: equivalent intervention in which children received the intervention but parents did not receive the newsletter

Theoretical framework: social cognitive theory

\begin{tabular}{ll}
\hline Outcomes & Core processes evaluated (child level): attrition, dosage, adherence \\
\hline Notes & Process evaluation category: integrated \\
& Breadth and depth: neither broad nor deep \\
& Voice of children given prominence: not featured \\
& Funding source: American Lung Association
\end{tabular}

\section{Risk of bias}

\begin{tabular}{lll}
\hline Bias & Authors' judgement & Support for judgement \\
\hline $\begin{array}{l}\text { Random sequence genera- } \\
\text { tion (selection bias) }\end{array}$ & Unclear risk & $\mathrm{N} / \mathrm{A}$ \\
\hline
\end{tabular}

\begin{tabular}{lll}
\hline $\begin{array}{l}\text { Allocation concealment } \\
\text { (selection bias) }\end{array}$ & Unclear risk & $\mathrm{N} / \mathrm{A}$ \\
\hline $\begin{array}{l}\text { Blinding of participants } \\
\text { and personnel (perfor- }\end{array}$ & Unclear risk & $\mathrm{N} / \mathrm{A}$ \\
mance bias) & & \\
All outcomes & &
\end{tabular}

\begin{tabular}{lll}
\hline $\begin{array}{l}\text { Blinding of outcome as- } \\
\text { sessment (detection bias) } \\
\text { All outcomes }\end{array}$ & Unclear risk \\
\hline $\begin{array}{l}\text { Incomplete outcome data } \\
\text { (attrition bias) } \\
\text { All outcomes }\end{array}$ & Unclear risk & N/A \\
\hline $\begin{array}{l}\text { Selective reporting (re- } \\
\text { porting bias) }\end{array}$ & Unclear risk & N/A \\
\hline $\begin{array}{l}\text { Other bias } \\
\begin{array}{l}\text { Transparent and clearly } \\
\text { stated aims }\end{array}\end{array}$ & Unclear risk & N/A \\
\hline $\begin{array}{l}\text { Explicit theories underpin- } \\
\text { ning and/or literature re- } \\
\text { view }\end{array}$ & Unclear risk & $\begin{array}{l}\text { Some theory and supporting literature were provided, but why a newsletter } \\
\text { was used was not explained }\end{array}$ \\
\hline
\end{tabular}


Terpstra 2012 (Continued)

Transparent and clearly
stated methods and tools $\quad$ Low risk All tools were clearly described

\begin{tabular}{ll}
\hline Selective reporting & High risk \\
& This was not considered - particularly the absence of measures showing \\
& Also, as acknowledged by study authors, not only caregivers received the \\
& newsletter
\end{tabular}

\begin{tabular}{ll}
\hline Harmful effects $\quad$ Unclear risk & $\begin{array}{l}\text { This was not recorded, and the paper included no information on child out- } \\
\text { comes }\end{array}$
\end{tabular}

\begin{tabular}{|c|c|c|}
\hline $\begin{array}{l}\text { Population and sample } \\
\text { described well }\end{array}$ & Unclear risk & School characteristics were poorly described \\
\hline Continuous evaluation & Low risk & Pre-post assessment was carried out \\
\hline $\begin{array}{l}\text { Evaluation participation } \\
\text { equity and sampling }\end{array}$ & High risk & No child input was included \\
\hline $\begin{array}{l}\text { Design and methods over- } \\
\text { all approach }\end{array}$ & High risk & $\begin{array}{l}\text { Low breadth of scope was evident, for example, not all stakeholders and not } \\
\text { all outcomes were reported }\end{array}$ \\
\hline $\begin{array}{l}\text { Tools and methods of da- } \\
\text { ta collection reliable/cred- } \\
\text { ible }\end{array}$ & Low risk & Tools used for data collection were appropriate for the data \\
\hline $\begin{array}{l}\text { Tools and methods of data } \\
\text { analysis reliable/credible }\end{array}$ & Low risk & How conclusions were reached is clearly explained \\
\hline $\begin{array}{l}\text { Performance bias/neutral- } \\
\text { ity/credibility/conforma- } \\
\text { bility }\end{array}$ & Unclear risk & $\begin{array}{l}\text { Absence of 'other' stakeholders who received the newsletter might reduce } \\
\text { credibility; absence of any child outcomes certainly does reduce credibility }\end{array}$ \\
\hline $\begin{array}{l}\text { Reliability of findings and } \\
\text { recommendations }\end{array}$ & Unclear risk & Intervention did not work \\
\hline Transferability of findings & High risk & Information provided is not rich enough to support a similar study \\
\hline $\begin{array}{l}\text { Overall risk of bias of } \\
\text { process evaluation }\end{array}$ & High risk & Intervention was unsuccessful \\
\hline
\end{tabular}

Included as outcome evaluation
Study design: clustered parallel-group design with schools selected as the unit of randomisation
Setting: 4 inner city elementary schools in the USA
Period: dates on which study was conducted - intervention and subsequent data collection - are not
clear. Outcomes were collected from children immediately after they participated in the school-based
asthma intervention programme (2 weeks) and at 5 and 12 months

Participants

Eligible sample frame: 73 met inclusion criteria guidelines and were enrolled in the study

Randomised: 73 pupils were randomised at the school level, although distribution between groups is unclear 
Completed (intervention): a total of 52 students were included in the final analysis: 28 students in the treatment group and 24 in the control group

Inclusion criteria: children for whom a physician had diagnosed asthma, or who had demonstrated asthma-related symptoms and frequent asthma-related emergency department visits or hospital admissions

Exclusion criteria: no additional exclusion criteria

\section{Baseline characteristics}

Age of children: mean age of children in the treatment group was 10.2 years; mean age of children in the control group was 9.9 years

Ethnicity: all children were African American

Socio-economic status: unclear

Gender: the sample of completers was evenly split in terms of sex: 26 females and 26 males

Asthma status: children who received a diagnosis were 8 to 13 years old (mean age, 10 years of age)

Interventions
Intervention: 2-part intervention

First part consisted of Open Airways for School (OAS) as described by study authors: "the purposes of the Open Airways Program are to: (a) empower children with asthma by teaching them how to prevent asthma episodes and emergencies; and (b) to help schools control asthma by creating partnerships in asthma care with school personnel, school nurses, physicians, and families. The program consists of six 45-minute sessions offered once per week in which small groups of children learn new asthma management skills. The session topics include: (a) basic information about asthma; (b) how to recognize and respond to asthma symptoms; (c) using asthma medication and deciding when to seek help; (d) how to keep physically active; (e) identifying and controlling triggers to minimize asthma symptoms; and (f) handling problems related to asthma and school. The curriculum incorporates an interactive teaching approach utilizing group discussion, stories, games, and role-play to promote children's active involvement in the learning process"

Second part of the intervention consisted of nurse practitioner visits, which consisted of the following: " 5 monthly visits with the NP at the school-based health clinic. These follow-up visits were initiated after the students completed the asthma educational program. During the visits, the nurse assessed the student's asthma health, including auscultation of breath sounds, assessment of current medication use and availability, and history of symptoms, visits to the emergency department, and hospitalizations. Students were asked to demonstrate skills such as medication administration and peak flow meter techniques. The nurse included age-appropriate asthma education information (from the Open Airways curriculum) as deemed necessary to reinforce and/or increase asthma knowledge"

Control: wait-list control; the control group received the intervention after evaluation

Intensity: 6 group-based lessons plus individual nurse practitioner session

Instructor: principal Investigator for the first part, and nurse practitioner for the second part

Theoretical framework: Orem's Self-Care Deficit Theory of Nursing served as the guiding framework for this study

Parental engagement: not reported

Child satisfaction: not reported

Timing of intervention in school day: unclear

Outcomes

\section{Extractable outcomes were collected for:}

Emergency department visits 
Velsor-Friedrich 2005 (Continued)
Daytime and night-time symptoms
Lung function
Notes Considered as a process evaluation, but study did not seek to address process evaluation/implementa- tion research questions and did not include sufficient process data

Funding source: National Institute of Nursing Research, Loyola University Research Award, Respironics Corporation

\section{Risk of bias}

\begin{tabular}{|c|c|c|}
\hline Bias & Authors' judgement & Support for judgement \\
\hline $\begin{array}{l}\text { Random sequence genera- } \\
\text { tion (selection bias) }\end{array}$ & Unclear risk & $\begin{array}{l}\text { Unclear - not described in the study: the } 4 \text { schools were randomly assigned to } \\
\text { treatment or comparison groups }\end{array}$ \\
\hline $\begin{array}{l}\text { Allocation concealment } \\
\text { (selection bias) }\end{array}$ & High risk & $\begin{array}{l}\text { Not described in the study but potentially high, given the low number of ran- } \\
\text { domised schools and study authors' description of study design }\end{array}$ \\
\hline $\begin{array}{l}\text { Blinding of participants } \\
\text { and personnel (perfor- } \\
\text { mance bias) } \\
\text { All outcomes }\end{array}$ & Unclear risk & Not addressed by study authors \\
\hline $\begin{array}{l}\text { Blinding of outcome as- } \\
\text { sessment (detection bias) } \\
\text { All outcomes }\end{array}$ & Unclear risk & Not addressed by study authors \\
\hline $\begin{array}{l}\text { Incomplete outcome data } \\
\text { (attrition bias) } \\
\text { All outcomes }\end{array}$ & Low risk & Low risk posed by attrition - only 3 students dropped out \\
\hline $\begin{array}{l}\text { Selective reporting (re- } \\
\text { porting bias) }\end{array}$ & Unclear risk & Unclear - some data have high levels of variance \\
\hline Other bias & Unclear risk & $\begin{array}{l}\text { Missingness - unclear risk - not addressed by study authors } \\
\text { Baseline imbalance - low risk - not described by study authors as problematic } \\
\text { Risk of contamination - low - schools (low number) were the unit of randomi- } \\
\text { sation }\end{array}$ \\
\hline $\begin{array}{l}\text { Transparent and clearly } \\
\text { stated aims }\end{array}$ & Unclear risk & $\mathrm{N} / \mathrm{A}$ \\
\hline $\begin{array}{l}\text { Explicit theories underpin- } \\
\text { ning and/or literature re- } \\
\text { view }\end{array}$ & Unclear risk & $\mathrm{N} / \mathrm{A}$ \\
\hline $\begin{array}{l}\text { Transparent and clearly } \\
\text { stated methods and tools }\end{array}$ & Unclear risk & $\mathrm{N} / \mathrm{A}$ \\
\hline Selective reporting & Unclear risk & $\mathrm{N} / \mathrm{A}$ \\
\hline Harmful effects & Unclear risk & N/A \\
\hline $\begin{array}{l}\text { Population and sample } \\
\text { described well }\end{array}$ & Unclear risk & $\mathrm{N} / \mathrm{A}$ \\
\hline
\end{tabular}


Velsor-Friedrich 2005 (Continued)

\begin{tabular}{lll} 
Continuous evaluation & Unclear risk & N/A \\
\hline $\begin{array}{l}\text { Evaluation participation } \\
\text { equity and sampling }\end{array}$ & Unclear risk & N/A \\
\hline $\begin{array}{l}\text { Design and methods over- } \\
\text { all approach }\end{array}$ & Unclear risk & N/A \\
\hline $\begin{array}{l}\text { Tools and methods of da- } \\
\text { ta collection reliable/cred- } \\
\text { ible }\end{array}$ & Unclear risk & N/A \\
\hline $\begin{array}{l}\text { Tools and methods of data } \\
\text { analysis reliable/credible }\end{array}$ & Unclear risk & N/A \\
\hline $\begin{array}{l}\text { Performance bias/neutral- } \\
\text { ity/credibility/conforma- } \\
\text { bility }\end{array}$ & Unclear risk & N/A \\
\hline $\begin{array}{l}\text { Reliability of findings and } \\
\text { recommendations }\end{array}$ & Unclear risk & N/A \\
\hline $\begin{array}{l}\text { Transferability of findings } \\
\begin{array}{l}\text { Overall risk of bias of } \\
\text { process evaluation }\end{array}\end{array}$ & Unclear risk & N/A \\
\hline
\end{tabular}

AAP: Asthma Action Plan.

ACT: Asthma Control Test.

ASMA: Asthma Self-Management for Adolescents.

BME: black and minority ethnicity.

CNS: clinical nurse specialist.

CST: Corticosteroids.

ED: emergency department.

GP: general practitioner.

HLAI: Health Learners Asthma Initiative.

HPM: Health Promotion Model.

HRQoL: health-related quality of life.

ICAN: I Can Control Asthma and Nutrition Now.

ISACC: International Study of Asthma and Allergies in Childhood.

N/A: not applicable.

NAEPP: National Asthma Education and Prevention Program.

NHLBI: National Heart, Lung, and Blood Institute.

OAS: Open Airways for Schools.

PBP: Power Breathing Program.

$\mathrm{PD} / \mathrm{H} / \mathrm{PE}$ : personal development/health/physical education.

$\mathrm{PI}$ principal investigator.

PPF: pre-test/post-test/follow-up.

PRECEDE: Predisposing, Reinforcing, and Enabling Causes in Educational Diagnosis and Evaluation.

RAP: Roaring Adventures of Puff.

$\mathrm{RCT}$ : randomised controlled trial.

SCDT: self-care deficit theory.

SES: socio-economic status.

SHARP: Staying Healthy - Asthma Responsible \& Prepared.

Triple A: Adolescent Asthma Action.

\section{Characteristics of excluded studies [ordered by study ID]}




\begin{tabular}{|c|c|}
\hline Study & Reason for exclusion \\
\hline Akasawa 2016 & Considered for process evaluation: not published in the English language \\
\hline Al Aloola 2017 & $\begin{array}{l}\text { Considered for process evaluation: core processes not available; educational programme for teach- } \\
\text { ers - child data not collected }\end{array}$ \\
\hline Al-Sheyab 2015 & Considered for outcome evaluation: excluded on comparison (tested effects of TAJ-Plus vs TAJ) \\
\hline Alreshidi 2015 & $\begin{array}{l}\text { Considered for process evaluation: excluded as did not include implementation research ques- } \\
\text { tions. }\end{array}$ \\
\hline Anderson 2004 & $\begin{array}{l}\text { Considered for process evaluation: excluded as did not reflect a school setting - school specifically } \\
\text { designed for children with chronic disease }\end{array}$ \\
\hline Ando 2016 & Considered for process evaluation: core processes not evaluated \\
\hline Arnold 2012 & $\begin{array}{l}\text { Considered for process evaluation: did not include implementation research questions nor in- } \\
\text { depth process or contextual information (did not meet the criteria for a process evaluation) }\end{array}$ \\
\hline Arıkan-Ayyıldız 2016 & Considered for process evaluation: not school based; clinical settings \\
\hline \multirow[t]{2}{*}{ Augustin 2003} & $\begin{array}{l}\text { Considered for outcome evaluation: excluded on comparison: intervention group received weekly } \\
\text { workshops for } 6 \text { weeks, control group was given standard educational materials on asthma man- } \\
\text { agement }\end{array}$ \\
\hline & $\begin{array}{l}\text { Considered for process evaluation: did not include implementation research questions (did not } \\
\text { meet the criteria for a process evaluation) }\end{array}$ \\
\hline Becker 2003 & Considered for outcome evaluation: not school based \\
\hline Bignall 2015a & $\begin{array}{l}\text { Considered for outcome evaluation: excluded, as comparison received an intervention (interven- } \\
\text { tion group ( } 20 \text { minutes breathing retraining plus education) or control group ( } 20 \text { minutes standard } \\
\text { education)) }\end{array}$ \\
\hline
\end{tabular}
tion on implementation

\section{Bowen 2013}

Considered for outcome evaluation: not school based

Considered for process evaluation: did not contain core components of a process evaluation; did not include implementation research questions nor in-depth process or contextual information (did not meet the criteria for a process evaluation)

\begin{tabular}{ll}
\hline Brooten 2008 & $\begin{array}{l}\text { Considered for process evaluation: did not include implementation research questions nor in- } \\
\text { depth process or contextual information (did not meet the criteria for a process evaluation) }\end{array}$ \\
\hline Bruzzese 2001 & $\begin{array}{l}\text { Considered for process evaluation: did not include implementation research questions nor in- } \\
\text { depth process or contextual information (did not meet the criteria for a process evaluation) }\end{array}$ \\
\hline Bruzzese 2006 & $\begin{array}{l}\text { Considered for outcome evaluation: excluded, as focussed on family-level self-management, rather } \\
\text { than child-level self-management }\end{array}$ \\
\hline Bruzzese 2011a & $\begin{array}{l}\text { Considered for outcome evaluation: unclear whether asthmatic students (with diagnosed asthma) } \\
\text { were included. } \\
\text { Considered for process evaluation: in addition to the above, did not represent a study of implemen- } \\
\text { tation using recognised tools }\end{array}$ \\
\hline
\end{tabular}




\begin{tabular}{ll}
\hline Study & Reason for exclusion \\
\hline Burgess 2017 & Considered for process evaluation: did not address process questions \\
\hline Burkhart 2003 & $\begin{array}{l}\text { Considered for outcome evaluation: large number of children under } 5 \text { were included (mean age, }< \\
5)\end{array}$ \\
\hline Bush 2014 & $\begin{array}{l}\text { Considered for outcome evaluation: not an intervention study (observational design) } \\
\text { mentation formed a key part of the intervention }\end{array}$
\end{tabular}

Butz 2005 Considered for outcome evaluation: excluded on comparison: usual care not provided to comparison group

Considered for process evaluation: did not include implementation research questions nor indepth process or contextual information (did not meet the criteria for a process evaluation)

\begin{tabular}{|c|c|}
\hline Carpenter 2016b & Considered for process evaluation: not school-based; school not instrumental for delivery \\
\hline Cheung 2015 & $\begin{array}{l}\text { Considered for process evaluation: excluded, as provided a detailed description of planned inter- } \\
\text { vention but not of implementation }\end{array}$ \\
\hline Chini 2011 & Considered for process evaluation: did not contain core components of a process evaluation \\
\hline Christiansen 1997 & $\begin{array}{l}\text { Considered for process evaluation: did not include implementation research questions nor in- } \\
\text { depth process or contextual information (did not meet the criteria for a process evaluation) }\end{array}$ \\
\hline Clark 1986 & Considered for outcome evaluation: published before cutoff point \\
\hline Clark 2003 & Considered for outcome evaluation: duplicate (on manual screening) \\
\hline Coté 1997 & Considered for outcome evaluation: not school based \\
\hline De Godoi 2016 & Considered for process evaluation: not solely about asthma \\
\hline de Greef, 2017 & Considered for process evaluation: not an intervention study \\
\hline DePue 2007 & $\begin{array}{l}\text { Considered for process evaluation: limited process data were presented, although they were not } \\
\text { deemed to be collected via recognised tools nor reported by standardised means }\end{array}$ \\
\hline Eakin 2012 & Considered for outcome evaluation: large number of children under 5 years of age (mean age, <5) \\
\hline Evans 2001 & Considered as a process evaluation study and an outcome evaluation study \\
\hline Fernandes 2006 & $\begin{array}{l}\text { Considered for outcome evaluation: large number of participants outside the 5- to } 18 \text {-year-old tar- } \\
\text { get age range }\end{array}$ \\
\hline Francis 2001 & $\begin{array}{l}\text { Considered for process evaluation: not deemed to have included the core components of a process } \\
\text { evaluation via structured tools }\end{array}$ \\
\hline Gardida 2002 & Considered for outcome evaluation: not published in the English language \\
\hline Gerald 2016 & Considered for process evaluation: not an intervention study \\
\hline Gibson 1998 & $\begin{array}{l}\text { Considered for outcome evaluation: schools were not randomised, and inclusion of only } 2 \text { schools } \\
\text { means that intervention and randomisation effects would conflate if schools were randomised }\end{array}$ \\
\hline
\end{tabular}




\begin{tabular}{ll}
\hline Study & Reason for exclusion \\
\hline Crad 2009 & Considered for process evaluation: did not contain the core components of a process evaluation \\
\hline $\begin{array}{l}\text { Considered for process evaluation: not deemed to have included the core components of a process } \\
\text { evaluactured tools }\end{array}$ \\
\hline
\end{tabular}

Greenberg $2010 \quad \begin{aligned} & \text { Considered for process evaluation: focus of the study was long-term impact on student health, not } \\ & \text { implementation. Focus group data were collected, although these data were not presented }\end{aligned}$

\begin{tabular}{ll}
\hline Greer 2009 & Considered for process evaluation and outcome evaluation: focus on improving knowledge about \\
asthma among children without asthma
\end{tabular}

Considered for outcome evaluation: excluded on the basis of study design. Only 2 sites randomised - 1 school in each arm. Any intervention effect was conflated with school effect

Considered for process evaluation: was deemed to not address implementation research questions

\begin{tabular}{ll}
\hline Halterman 2004 & $\begin{array}{l}\text { Considered for outcome evaluation: excluded - deemed to not include a sufficient component of } \\
\text { self-management }\end{array}$ \\
\hline Halterman 2011 & $\begin{array}{l}\text { Considered for outcome evaluation: excluded - delivered in part at school and in part in the home } \\
\text { - included a substantial home component; not possible to disentangle which part may be driving } \\
\text { any change }\end{array}$
\end{tabular}

Halterman 2011a Considered for outcome evaluation: excluded, as comparison received asthma care
Considered for process evaluation: stand-alone process evaluation identified but focused on an al-
lied part of the trial that was not school based

Halterman 2012 Considered for outcome evaluation: excluded, as comparison received asthma care
Considered for process evaluation: deemed to not include the core components of a process evalu-
ation using structured tools

\begin{tabular}{ll}
\hline Hemate 2012 & Considered for process evaluation: did not contain the core components of a process evaluation \\
\hline Hill 1991 & $\begin{array}{l}\text { Considered for outcome evaluation: excluded, as the intervention did not foster self-management } \\
\text { skills }\end{array}$
\end{tabular}

Considered for outcome evaluation: excluded - study not designed as an RCT
Considered for process evaluation: deemed to not include the core components of a process evalu-
ation using structured tools

\begin{tabular}{ll}
\hline Horner 2003 & Considered for outcome evaluation: study design was non-experimental \\
\hline Hughes & $\begin{array}{l}\text { Considered for process evaluation: although some satisfaction data were collected, the study did } \\
\text { not include the core components of a process evaluation; process data were collected using struc- } \\
\text { tured tools }\end{array}$ \\
\hline Johnson 2016 & Considered for process evaluation: not school based; clinical settings only \\
\hline Jones 2005 & $\begin{array}{l}\text { Considered for process evaluation: school site was not judged to be instrumental for delivery of the } \\
\text { intervention; sites external to school were also used for intervention delivery }\end{array}$ \\
\hline Joseph 2004 & Considered for outcome evaluation: not school based \\
\hline Joseph 2007 & $\begin{array}{l}\text { Considered for outcome evaluation (along with linked papers): excluded, as comparison included } \\
\text { asthma education }\end{array}$
\end{tabular}




\begin{tabular}{l|l}
\hline Study & Reason for exclusion \\
\hline Note: included in process evaluation
\end{tabular}

Note: included in process evaluation

Joseph 2013a Considered for outcome evaluation (along with linked papers): excluded, as comparison included asthma education

Note: included in process evaluation

Kaufman 2011 Considered for process evaluation: study did not include the core components of a process evalua-
tion; process data were collected using structured tools

\begin{tabular}{ll}
\hline Kenny 2016 & Considered for process evaluation: not school based \\
\hline Khan 2014 & Considered for outcome evaluation: excluded, as not school based \\
\hline Khoshnavay 2013 & $\begin{array}{l}\text { Considered for process evaluation: received from study author; did not include core components of } \\
\text { a process evaluation; process data were collected using structured tools }\end{array}$
\end{tabular}

Kintner 2015 Considered for outcome evaluation: excluded on comparison as the control group received alternative asthma education

Considered for process evaluation: did not contain the core components of a process evaluation

Knight 2005 Considered for process evaluation: study did not include the core components of a process evalua-
tion; process data were collected using structured tools

Krishna $2006 \quad$ Considered for outcome evaluation: deemed as not school based

\begin{tabular}{|c|c|}
\hline Lakupoch 2017 & Considered for process evaluation: not school based \\
\hline Lewis 2005 & Considered for outcome evaluation: study as designed included no randomisation \\
\hline Li 2017 & Considered for process evaluation: did not address process evaluation research questions \\
\hline Liao 2006 & $\begin{array}{l}\text { Considered for process evaluation: study did not include the core components of a process evalua- } \\
\text { tion; process data were collected using structured tools; included home visit components }\end{array}$ \\
\hline Lin 2017 & Considered for process evaluation: school setting not central for delivery \\
\hline Lipman 2017 & Considered for process evaluation: did not address process evaluation research questions \\
\hline Loman 2017 & Considered for process evaluation: did not address process evaluation research questions centrally \\
\hline Louisias 2016 & Considered for process evaluation: did not address process questions \\
\hline Lu 2017 & Considered for process evaluation: did not include core components of process evaluation \\
\hline Lurie 2001 & $\begin{array}{l}\text { Considered for process evaluation: study did not include the core components of a process evalu- } \\
\text { ation; process data were collected using structured tools; some data on stakeholder perceptions } \\
\text { were collected, but study did not address implementation research questions }\end{array}$ \\
\hline Lwebuga-Mukasa 2002 & $\begin{array}{l}\text { Considered for process evaluation: study did not include the core components of a process evalua- } \\
\text { tion }\end{array}$ \\
\hline Maa 2010 & Considered for process evaluation: did not contain core components of a process evaluation \\
\hline MacPherson 2011 & Considered for process evaluation: did not contain core components of a process evaluation \\
\hline
\end{tabular}




\begin{tabular}{|c|c|}
\hline Study & Reason for exclusion \\
\hline Mangan 2006 & Considered for process evaluation: did not contain core components of a process evaluation \\
\hline Marabini 2002 & Considered for outcome evaluation: not focussed on children (mean age, approximately 50) \\
\hline McClure 2008 & $\begin{array}{l}\text { Considered for process evaluation: did not fall into the category of self-management (supported } \\
\text { management through observation) }\end{array}$ \\
\hline McElmurry 1999 & $\begin{array}{l}\text { Considered for process evaluation: study did not include the core components of a process evalua- } \\
\text { tion; process data were collected using structured tools; included home visit components }\end{array}$ \\
\hline McEwen 1998 & $\begin{array}{l}\text { Considered for process evaluation: study did not include the core components of a process evalua- } \\
\text { tion; process data were collected using structured tools; included home visit components }\end{array}$ \\
\hline McLaughlin 2006 & $\begin{array}{l}\text { Considered for process evaluation: study did not include the core components of a process evalua- } \\
\text { tion; process data were collected using structured tools }\end{array}$ \\
\hline Meng 2000 & $\begin{array}{l}\text { Considered for process evaluation: study did not include the core components of a process evalua- } \\
\text { tion; process data were collected using structured tools }\end{array}$ \\
\hline Meurer 1999 & $\begin{array}{l}\text { Considered for process evaluation: study did not include the core components of a process evalua- } \\
\text { tion; process data were collected using structured tools }\end{array}$ \\
\hline Millard 2003 & $\begin{array}{l}\text { Considered for outcome and process evaluation: not focussed on self-management; educational } \\
\text { activities were aimed at parents; study did not contain the core components of a process evalua- } \\
\text { tion }\end{array}$ \\
\hline Mitchell 2017 & Considered for process evaluation: core processes not available \\
\hline Morphew 2013 & Considered for process evaluation: presented an economic evaluation - not a process evaluation \\
\hline Morphew 2017 & Considered for process evaluation: core processes not available \\
\hline Morton 2017 & Considered for process evaluation: not reliant on schools for delivery \\
\hline Mosnaim 2017 & Considered for process evaluation: not an intervention study \\
\hline NCT00217776 & Considered for outcome evaluation: not an intervention study (trial protocol) \\
\hline Neuharth-Pritchett 2016 & $\begin{array}{l}\text { Considered for process evaluation: not focussed on children; focussed exclusively on training edu- } \\
\text { cators }\end{array}$ \\
\hline
\end{tabular}

Considered for process evaluation: study did not include the core components of a process evalua-
tion; process data were collected using structured tools

\begin{tabular}{ll}
\hline Otim 2015 & Considered for process evaluation: presented an economic evaluation - not a process evaluation \\
\hline Patel 2007 & $\begin{array}{l}\text { Considered for process evaluation: presented an outcome and economic evaluation - not a process } \\
\text { evaluation }\end{array}$
\end{tabular}

\begin{tabular}{ll}
\hline Peers 2017 & Considered for process evaluation: did not include core processes \\
\hline Pender-Phaneuf 2016 & $\begin{array}{l}\text { Considered for process evaluation: study did not include the core components of a process evalua- } \\
\text { tion; process data were collected using structured tools }\end{array}$ \\
\hline Perry 2000 & Considered for outcome evaluation: study not considered to be an RCT \\
\hline
\end{tabular}




\begin{tabular}{ll}
\hline Study & Reason for exclusion \\
\hline Petrie 2010 & Considered for process evaluation: study did not evaluate processes \\
\hline Quaranta 2012 & Considered for process evaluation: study did not report on implementation processes \\
\hline Quaranta 2015 & Considered for process evaluation: study did not involve an intervention \\
\hline Rasberry 2014 & Considered for process evaluation: study did not report on implementation processes \\
\hline Raun 2017 & Considered for process evaluation: correlational analysis \\
\hline Rhee 2012 & $\begin{array}{l}\text { Considered for process evaluation: presented an outcome and economic evaluation - not a process } \\
\text { evaluation }\end{array}$
\end{tabular}

Richterová $2016 \quad$ Considered for process evaluation: not published in the English language

Rodriguez-Martinez $2017 \quad$ Considered for process evaluation: focussed on an economic evaluation

Sabla 2017 Considered for process evaluation: did not contain core components of a process evaluation - focused on evaluating the validity of teaching materials

Salisbury $2002 \quad$ Considered for outcome evaluation: excluded, as comparison group received additional intervention beyond usual care

Considered for process evaluation: study did not include the core components of a process evaluation; process data were collected using structured tools

\begin{tabular}{ll}
\hline Scherer 2016 & Considered for process evaluation: not focussed on self-management among children \\
\hline Schlueter 2011 & Considered for process evaluation: study implementation focussed on parental smoke reduction \\
\hline Schneider 1997 & $\begin{array}{l}\text { Considered for process evaluation: study did not include the core components of a process eval- } \\
\text { uation; process data were collected using structured tools; some processes and context were de- } \\
\text { scribed but were not evaluated }\end{array}$
\end{tabular}

Schuller 2015

Considered for process evaluation: study did not include the core components of a process evaluation; process data were collected using structured tools; some processes and context were described but were not evaluated

Scott $2006 \quad \begin{aligned} & \text { Considered for process evaluation: did not allow for implementation processes to be evaluated; } \\ & \text { only } 6 \text { students were included, precluding assessment of core components of a school-based asth- } \\ & \text { ma intervention }\end{aligned}$

Scott 2008 Considered for process evaluation: study did not include the core components of a process evaluation; process data were collected using structured tools; unclear if school was not instrumental in delivery of the intervention

Scott 2011

Considered for process evaluation: study did not include the core components of a process evaluation; process data were collected using structured tools; unclear if school was not instrumental in delivery of the intervention

\begin{tabular}{ll}
\hline Shanovich 2009 & Considered for outcome evaluation: study was not judged to be an RCT \\
\hline Sharek 2002 & Considered for outcome evaluation: study was not school based \\
\hline Shaw 2005 & $\begin{array}{l}\text { Considered for process evaluation: study did not include the core components of a process evalua- } \\
\text { tion (reported that process evaluation was conducted but did not report the findings) }\end{array}$
\end{tabular}

School-based self-management interventions for asthma in children and adolescents: a mixed methods systematic review (Review) 


\begin{tabular}{ll}
\hline Study & Reason for exclusion \\
\hline Shaw 2016 & Considered for process evaluation: not school based \\
\hline Shegog 2001 & $\begin{array}{l}\text { Considered for outcome evaluation: delivery of the intervention not contingent on schools (not } \\
\text { school based) }\end{array}$ \\
\hline Shelef 2016 & Considered for process evaluation: described development of study protocol, not implementation \\
\hline Staudt 2015 & $\begin{array}{l}\text { Considered for process evaluation: study did not include the core components of a process evalua- } \\
\text { tion }\end{array}$ \\
\hline Suwannakeeree 2016 & $\begin{array}{l}\text { Considered for process evaluation: study did not include the core components of a process evalua- } \\
\text { tion; included diaries for symptom monitoring alone }\end{array}$ \\
\hline Szefler 2016 & \begin{tabular}{l} 
Considered for process evaluation: not an intervention study \\
\hline Szefler 2017
\end{tabular} \\
\hline Tate 2009 & $\begin{array}{l}\text { Considered for process evaluation: core aspects of the process evaluation were not addressed } \\
\text { tion research questions and did not include process data }\end{array}$ \\
\hline
\end{tabular}

Considered for outcome evaluation: excluded on comparison, as trial tested added impact on additional engagement with caregivers in an established intervention

Note: included as a process evaluation

\section{Thornton 2016 \\ Considered for process evaluation: school not instrumental for delivery; main components deliv- ered at home}

\begin{tabular}{ll} 
Urrutia-Pereira 2017 & Considered for process evaluation: core aspects of process evaluation not addressed \\
\hline Valery 2007 & Considered for outcome evaluation: intervention not school based \\
\hline Velsor-Friedrich 2004 & $\begin{array}{l}\text { Considered for outcome evaluation: no randomisation described (not an RCT) } \\
\text { Considered for process evaluation: study did not seek to address process evaluation/implementa- } \\
\text { tion research questions and did not include process data }\end{array}$
\end{tabular}

Velsor-Friedrich $2012 \quad$ Considered for outcome evaluation: excluded on comparison (study compared alternative asthma interventions)

Considered for process evaluation: study did not seek to address process evaluation/implementation research questions and did not include process data

\begin{tabular}{ll}
\hline Volerman 2017 & Considered for process evaluation: core aspects of process evaluation not addressed \\
\hline Walter 2016 & Considered for process evaluation: review in progress; not an intervention study \\
\hline Walton 2004 & $\begin{array}{l}\text { Considered for process evaluation: study did not seek to address process evaluation/implementa- } \\
\text { tion research questions and did not include sufficient process data }\end{array}$ \\
\hline Webber 2005 & $\begin{array}{l}\text { Considered for process evaluation: study did not seek to address process evaluation/implementa- } \\
\text { tion research questions and did not include sufficient process data }\end{array}$ \\
\hline Weng 2007 & Considered for outcome evaluation: study not deemed to be an RCT \\
\hline Wensley 2004 & Considered for outcome evaluation: not a school-based intervention
\end{tabular}




\begin{tabular}{|c|c|}
\hline Study & Reason for exclusion \\
\hline Whitman 1985 & Considered for outcome evaluation: published before cutoff date \\
\hline Willeboordse 2016 & Considered for process evaluation: school not instrumental in delivery \\
\hline Wilson 2008 & $\begin{array}{l}\text { Considered for process evaluation: did not contain the core components expected in a process } \\
\text { evaluation; focussed on implementation at a school district level rather than among students and } \\
\text { within schools }\end{array}$ \\
\hline Wyatt 2008 & $\begin{array}{l}\text { Considered for process evaluation: study did not seek to address process evaluation/implementa- } \\
\text { tion research questions and did not include sufficient process data }\end{array}$ \\
\hline Wyatt 2013 & $\begin{array}{l}\text { Considered for process evaluation: study provided in-depth description of the process of devel- } \\
\text { oping content but not implementation; study therefore did not seek to address process evalua- } \\
\text { tion/implementation research questions }\end{array}$ \\
\hline \multirow[t]{2}{*}{ Yawn 2000} & $\begin{array}{l}\text { Considered for outcome evaluation: not focussed on asthmatic children; study did not report on } \\
\text { outcomes for asthmatic children separately from non-asthmatic children }\end{array}$ \\
\hline & $\begin{array}{l}\text { Considered for process evaluation: study did not seek to address process evaluation/implementa- } \\
\text { tion research questions and did not include sufficient process data }\end{array}$ \\
\hline Yoshida 2011 & Considered for process evaluation: study was not an intervention study \\
\hline Young 2001 & $\begin{array}{l}\text { Considered for process evaluation: some implementation notes included, but study did not seek to } \\
\text { address process evaluation/implementation research questions using structured tools }\end{array}$ \\
\hline Zografos 2007 & $\begin{array}{l}\text { Considered for process evaluation: study did not seek to address process evaluation/implementa- } \\
\text { tion research questions using structured tools }\end{array}$ \\
\hline
\end{tabular}

RCT: randomised controlled trial.

TAJ: XXX.

Characteristics of studies awaiting assessment [ordered by study ID]

\section{Liptzin 2016a}

\begin{tabular}{ll}
\hline Methods & Pre-post study \\
\hline Participants & Children with asthma across a wide age range \\
\hline Interventions & $\begin{array}{l}\text { Step-Up Asthma Program, applying National Asthma Education and Prevention Program-National } \\
\text { Heart, Lung, and Blood Institute guidelines for evidence-based programmes for children with asth- } \\
\text { ma }\end{array}$ \\
\hline Outcomes & Number of asthma action plans; access to medication; asthma knowledge; asthma exacerbations \\
\hline Notes & Status as process evaluation to be classified \\
\hline
\end{tabular}

McCallum 2017

\begin{tabular}{ll}
\hline Methods & Pre-post study \\
\hline
\end{tabular}


McCallum 2017 (Continued)
Participants
Participants in high schools with large numbers of Indigenous Australian children; not all children were known to be asthmatic

Interventions

Peer-led, school-based educational programme called the Asthma and Smoking Prevention Project

(ASPP); split focus between asthma and smoking prevention

\begin{tabular}{ll}
\hline Outcomes & Lung function and wheezing; smoking status \\
\hline Notes & Status as process evaluation to be classified
\end{tabular}

Praena-Crespo 2017

\begin{tabular}{ll}
\hline Methods & Cluster randomised parallel-group trial \\
\hline Participants & Students engaging in physical activity lessons, including approximately $10 \%$ with asthma \\
\hline Interventions & Asthma, Sport, and Health (ASAH) programme taught by physical activity teachers \\
\hline Outcomes & Quality of life and asthma knowledge \\
\hline Notes & $\begin{array}{l}\text { Status as outcome evaluation to be classified; results for quality of life not expected to change con- } \\
\text { clusions }\end{array}$ \\
\hline
\end{tabular}

Reznik 2016

\begin{tabular}{ll}
\hline Methods & Cluster randomised parallel-group trial \\
\hline Participants & Children with asthma in primary schools \\
\hline Interventions & $\begin{array}{l}\text { School-wide asthma awareness event; facilitated collaboration with child's primary care provider, } \\
\text { classroom-based physical activity, and asthma education for families and school personnel }\end{array}$ \\
\hline Outcomes & Symptom-free days, medication adherence, and physical activity levels \\
\hline Notes & Status as outcome evaluation to be classified \\
\hline
\end{tabular}

Warren 2016

\begin{tabular}{ll}
\hline Methods & Pre-post evaluation design \\
\hline Participants & Children with asthma in high schools \\
\hline Interventions & $\begin{array}{l}\text { Student Asthma Research Team (START), engaged high school youth in a Photovoice investigation } \\
\text { of factors impacting asthma }\end{array}$ \\
\hline Outcomes & Asthma knowledge and lung function \\
\hline Notes & Status as process evaluation to be classified \\
\hline
\end{tabular}

ASAH: Asthma, Sport, and Health programme.

School-based self-management interventions for asthma in children and adolescents: a mixed methods systematic review (Review) 
ASPP: Asthma and Smoking Prevention Project.

START: Student Asthma Research Team.

Characteristics of ongoing studies [ordered by study ID]

Halterman 2017

Trial name or title

Development of School-Based Asthma Management Programs in Rochester, New York: Presented in Honor of Dr Robert Haggerty

\begin{tabular}{ll}
\hline Methods & Randomised trial \\
\hline Participants & Children with asthma \\
\hline Interventions & Telemedicine intervention \\
\hline Outcomes & Symptom days \\
\hline Starting date & Unclear \\
\hline Contact information & Jill Halterman; jill_halterman@urmc.rochester.edu \\
\hline
\end{tabular}

Notes

Lemanske 2016

Trial name or title School-Based Asthma Management Program (SAMPRO)

\begin{tabular}{ll}
\hline Methods & Study design unclear \\
\hline Participants & Children with asthma and numerous stakeholders \\
\hline Interventions & Multi-component intervention \\
\hline Outcomes & Unclear \\
\hline Starting date & Unclear
\end{tabular}

Contact information Robert F. Lemanske, Jr, MD; rfl@medicine.wisc.edu

Notes Description of establishment published in cited reference

\section{NCT03032744}

Trial name or title

Methods

Participants

Interventions

\section{Project IMPACT in Schools to Prevent Asthma Symptoms}

Randomised trial

60 students 6 to 16 years of age

Project IMPACT is a school-based health centre intervention programme that institutes guideline-based long-term asthma care and provides supervised administration with daily preventive asthma medications to improve asthma symptoms and lung function, reduce emergency visits, and decrease missed days of school among children from communities with health disparities 
NCT03032744 (Continued)

Outcomes

Asthma Symptoms; ACT score; Lung function; Missed days of school; Decrease in ED/urgent care visits; Hospitalisations; Oral/parenteral steroid use

\begin{tabular}{ll}
\hline Starting date & January 2017 \\
\hline Contact information & Lucy C Holmes, MD; lholmes@upa.chob.edu
\end{tabular}

Notes

\section{Perry 2015}

\begin{tabular}{ll}
\hline Trial name or title & Breath Connection \\
\hline Methods & Clustered parallel-group RCT \\
\hline Participants & Children 7 to 14 years of age, with a median age of 9.6 years \\
\hline Interventions & $\begin{array}{l}\text { Study used the Breath Connection programme to provide asthma education via telemedicine to } \\
\text { rural children with asthma, their caregivers, and school nurses }\end{array}$ \\
\hline Outcomes & Lung function; Use of reliever therapies \\
\hline Starting date & Unclear \\
\hline Contact information & $\begin{array}{l}\text { This study is available as an abstract only and describes ongoing recruitment; study author was } \\
\text { contacted for further information }\end{array}$ \\
\hline Notes &
\end{tabular}

\section{Phipatanakul 2017}

\begin{tabular}{ll}
\hline Trial name or title & The School Inner-City Asthma Intervention Study \\
\hline Methods & Randomised, blinded, sham-controlled intervention trial \\
\hline Participants & $\begin{array}{l}\text { Plan to enrol } 300 \text { students with asthma from multiple classrooms in } 40 \text { northeastern inner city ele- } \\
\text { mentary schools. }\end{array}$ \\
\hline Interventions & School environmental intervention \\
\hline Outcomes & Asthma symptoms \\
\hline Starting date & Unclear \\
\hline Contact information & Boston Children's Hospital \\
\hline Notes & \\
\hline
\end{tabular}

ACT: XXX.

ED: emergency department.

RCT: randomised controlled trial.

SAMPRO: School-Based Asthma Management Program.

School-based self-management interventions for asthma in children and adolescents: a mixed methods systematic review (Review)

Copyright @ 2019 The Cochrane Collaboration. Published by John Wiley \& Sons, Ltd. 
DATA AND ANALYSES

\section{Comparison 1. Effects of school-based asthma interventions vs usual care}

\begin{tabular}{|c|c|c|c|c|}
\hline Outcome or subgroup title & No. of studies & $\begin{array}{l}\text { No. of partici- } \\
\text { pants }\end{array}$ & Statistical method & Effect size \\
\hline $\begin{array}{l}1 \text { Exacerbations leading to hospitalisa- } \\
\text { tion }\end{array}$ & 6 & 1873 & $\begin{array}{l}\text { Std. Mean Difference (Ran- } \\
\text { dom, } 95 \% \mathrm{Cl} \text { ) }\end{array}$ & $-0.19[-0.35,-0.04]$ \\
\hline $\begin{array}{l}2 \text { Exacerbations leading to emergency } \\
\text { department (ED) visits }\end{array}$ & 13 & 3883 & $\begin{array}{l}\text { Odds Ratio (Random, 95\% } \\
\mathrm{Cl} \text { ) }\end{array}$ & $0.70[0.53,0.92]$ \\
\hline 3 Absence from school & 10 & 4609 & $\begin{array}{l}\text { Std. Mean Difference (Ran- } \\
\text { dom, } 95 \% \mathrm{Cl} \text { ) }\end{array}$ & $-0.07[-0.22,0.08]$ \\
\hline 4 Days of restricted activity & 3 & 1852 & $\begin{array}{l}\text { Std. Mean Difference (Ran- } \\
\text { dom, } 95 \% \mathrm{Cl} \text { ) }\end{array}$ & $-0.30[-0.41,-0.18]$ \\
\hline $\begin{array}{l}5 \text { Unplanned visit to hospital or GP due } \\
\text { to asthma symptoms }\end{array}$ & 5 & 3490 & $\begin{array}{l}\text { Odds Ratio (Random, 95\% } \\
\mathrm{Cl} \text { ) }\end{array}$ & $0.74[0.60,0.90]$ \\
\hline $\begin{array}{l}6 \text { Experience of daytime and night- } \\
\text { time symptoms - daytime symptoms }\end{array}$ & 5 & 1065 & $\begin{array}{l}\text { Std. Mean Difference (Ran- } \\
\text { dom, } 95 \% \mathrm{Cl} \text { ) }\end{array}$ & $-0.15[-0.33,0.02]$ \\
\hline $\begin{array}{l}7 \text { Experience of daytime and night- } \\
\text { time symptoms - night-time symptoms }\end{array}$ & 4 & 459 & $\begin{array}{l}\text { Std. Mean Difference (Ran- } \\
\text { dom, } 95 \% \mathrm{Cl} \text { ) }\end{array}$ & $-0.18[-0.52,0.15]$ \\
\hline $\begin{array}{l}8 \text { Use of reliever therapies, e.g. be- } \\
\mathrm{ta}_{2} \text {-agonists }\end{array}$ & 2 & 437 & $\begin{array}{l}\text { Odds Ratio (Random, 95\% } \\
\mathrm{Cl} \text { ) }\end{array}$ & $0.52[0.15,1.81]$ \\
\hline $\begin{array}{l}9 \text { Corticosteroid dosage and/or use of } \\
\text { add-on therapies (usage of) }\end{array}$ & 3 & 614 & $\begin{array}{l}\text { Odds Ratio (Random, 95\% } \\
\mathrm{Cl} \text { ) }\end{array}$ & $1.25[0.88,1.77]$ \\
\hline $\begin{array}{l}10 \text { Corticosteroid dosage and/or use of } \\
\text { add-on therapies (appropriate usage } \\
\text { of) }\end{array}$ & 2 & & $\begin{array}{l}\text { Std. Mean Difference (Ran- } \\
\text { dom, } 95 \% \mathrm{Cl} \text { ) }\end{array}$ & $\begin{array}{l}\text { Totals not select- } \\
\text { ed }\end{array}$ \\
\hline 11 Health-related quality of life (SMD) & 7 & 2587 & $\begin{array}{l}\text { Std. Mean Difference (Ran- } \\
\text { dom, } 95 \% \mathrm{Cl} \text { ) }\end{array}$ & $0.27[0.18,0.36]$ \\
\hline 12 Health-related quality of life (MD) & 8 & 2950 & $\begin{array}{l}\text { Mean Difference (IV, Ran- } \\
\text { dom, } 95 \% \mathrm{CI} \text { ) }\end{array}$ & $0.35[0.06,0.64]$ \\
\hline 13 Withdrawal from the study & 13 & 3442 & $\begin{array}{l}\text { Odds Ratio (Random, 95\% } \\
\mathrm{Cl} \text { ) }\end{array}$ & $1.14[0.92,1.43]$ \\
\hline
\end{tabular}


Analysis 1.1. Comparison 1 Effects of school-based asthma interventions vs usual care, Outcome 1 Exacerbations leading to hospitalisation.

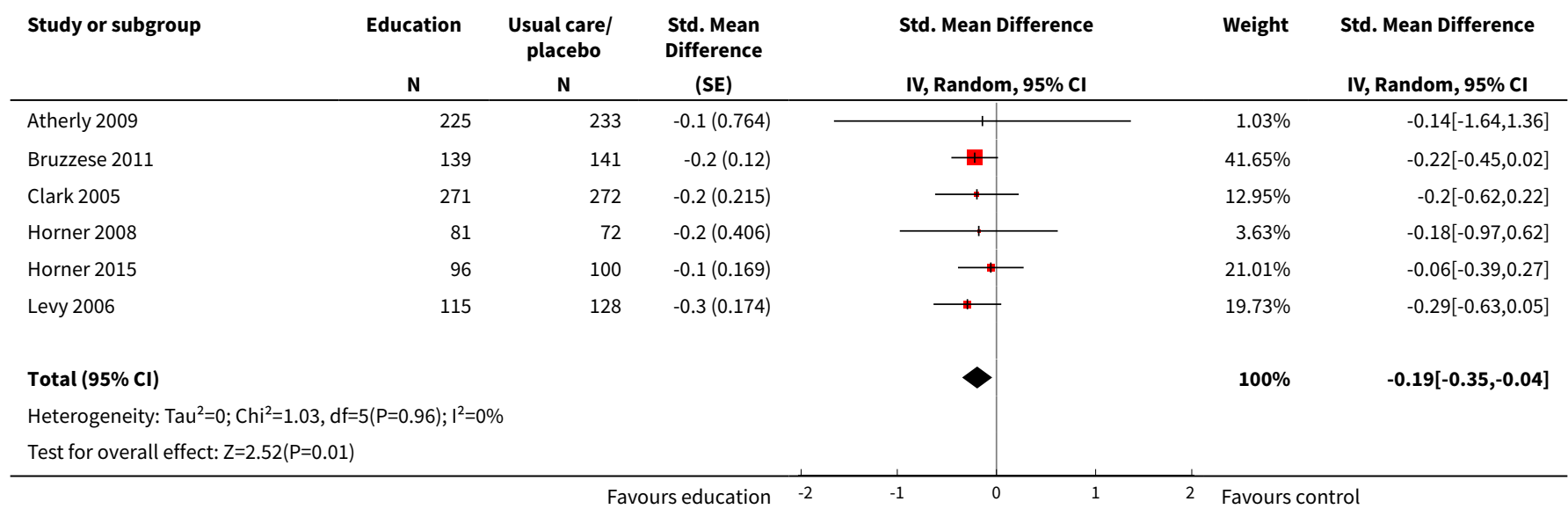

Analysis 1.2. Comparison 1 Effects of school-based asthma interventions vs usual care, Outcome 2 Exacerbations leading to emergency department (ED) visits.

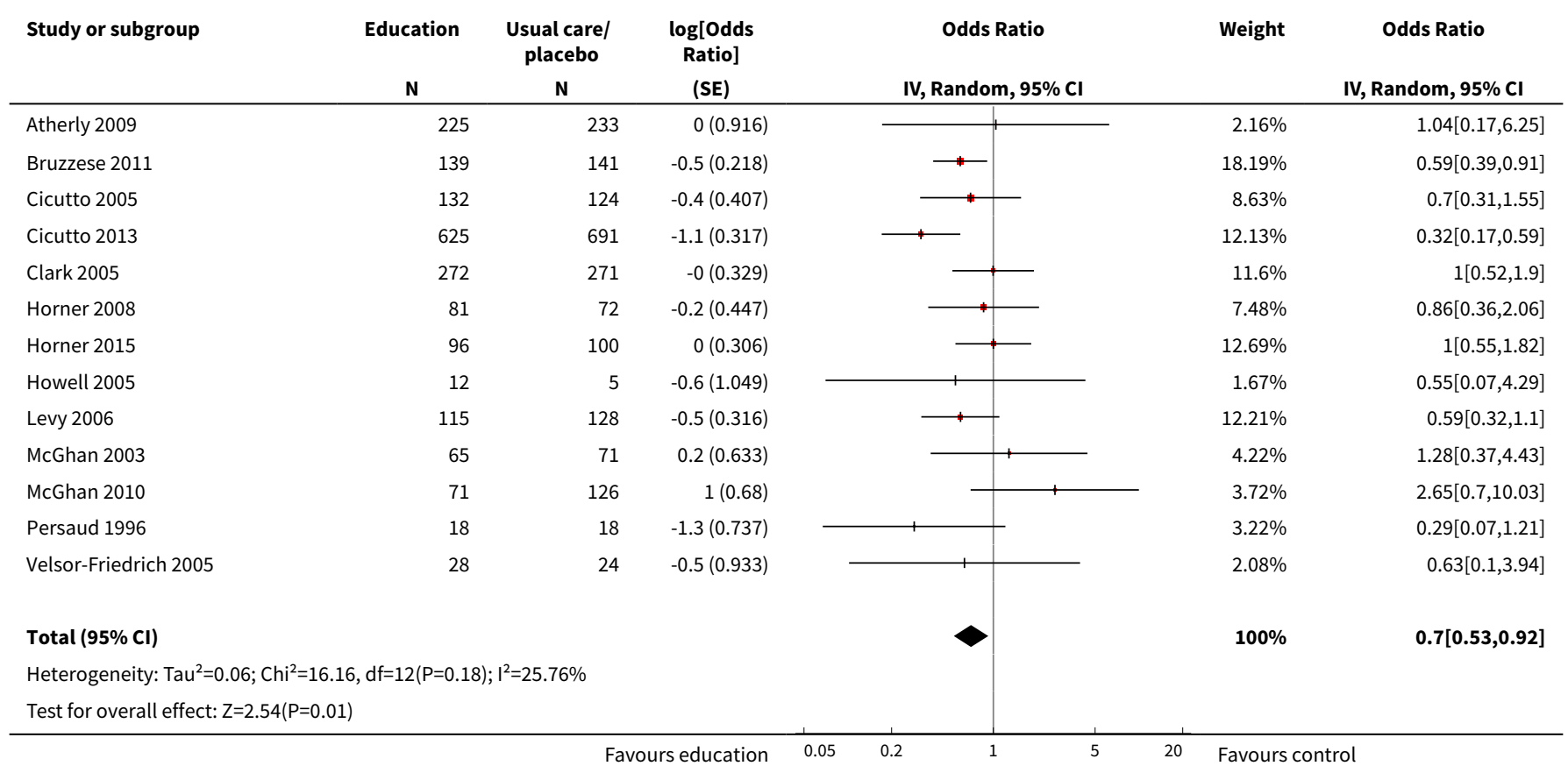

Analysis 1.3. Comparison 1 Effects of school-based asthma interventions vs usual care, Outcome 3 Absence from school.

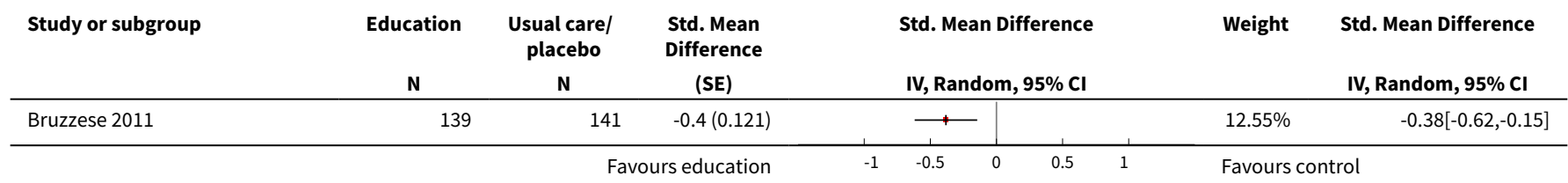




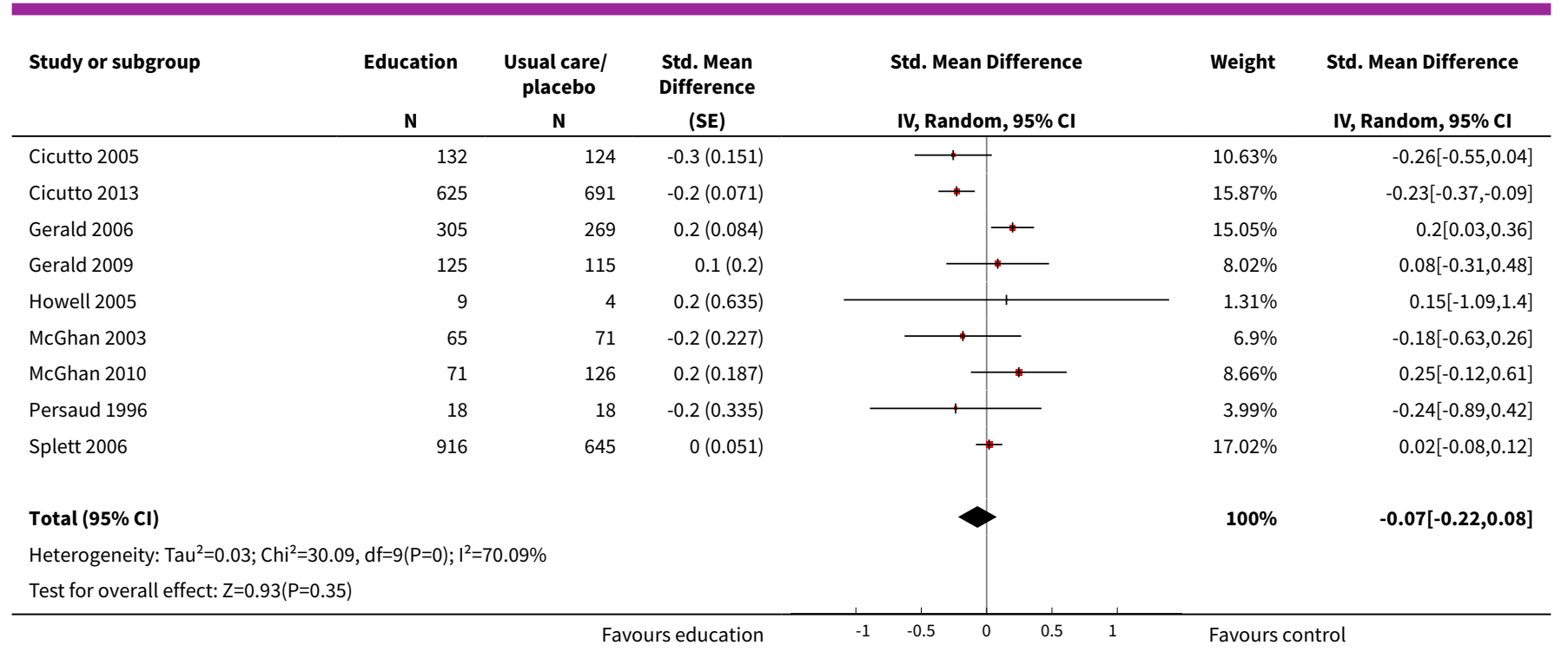

Analysis 1.4. Comparison 1 Effects of school-based asthma interventions vs usual care, Outcome 4 Days of restricted activity.

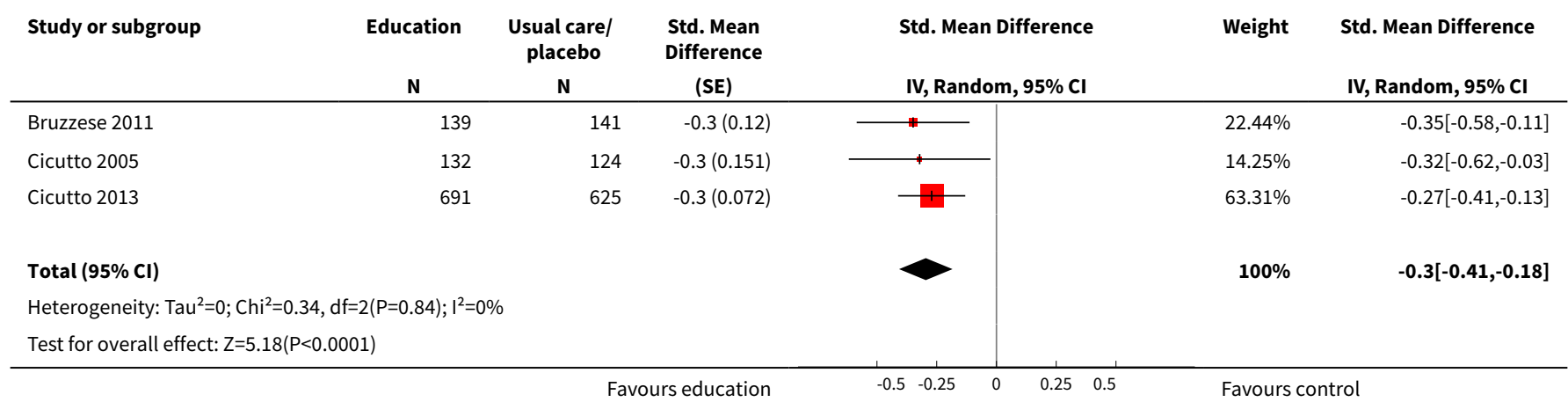

Analysis 1.5. Comparison 1 Effects of school-based asthma interventions vs usual care, Outcome 5 Unplanned visit to hospital or GP due to asthma symptoms.

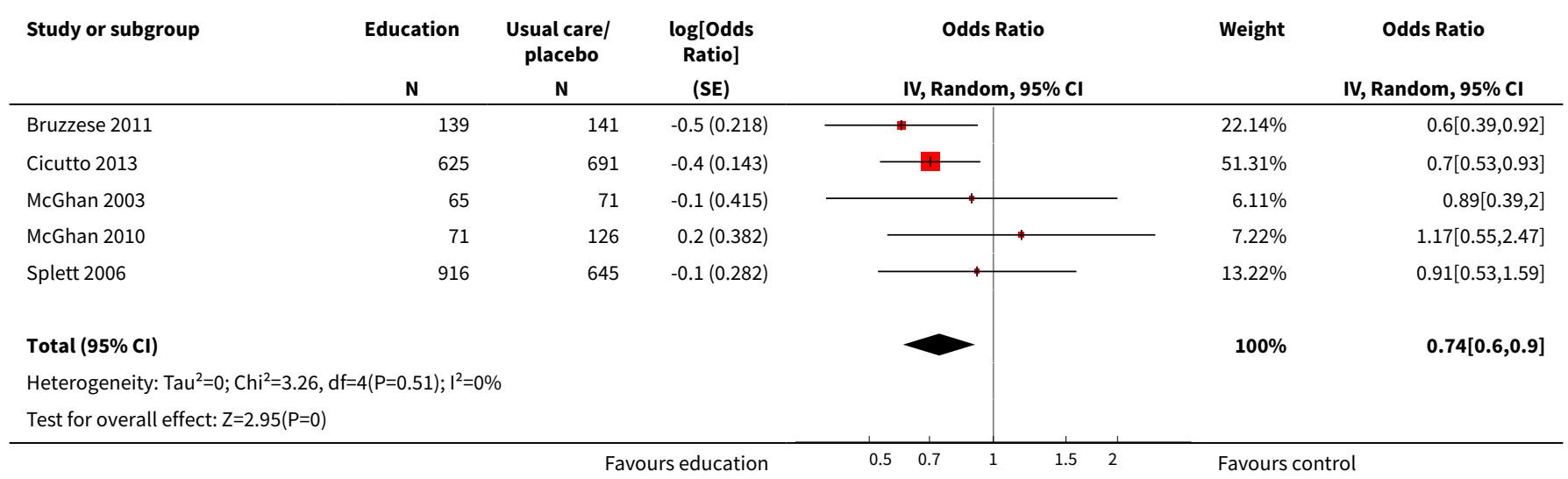


Analysis 1.6. Comparison 1 Effects of school-based asthma interventions vs usual care, Outcome 6 Experience of daytime and night-time symptoms - daytime symptoms.

\begin{tabular}{|c|c|c|c|c|c|c|}
\hline Study or subgroup & Education & $\begin{array}{c}\text { Usual care/ } \\
\text { placebo } \\
\mathbf{N} \\
\end{array}$ & $\begin{array}{c}\text { Std. Mean } \\
\text { Difference } \\
\text { (SE) } \\
\end{array}$ & IV, Random, 95\% CI & Weight & IV, Random, 95\% CI \\
\hline Atherly 2009 & 225 & 233 & $-0(0.168)$ & \pm & $26.95 \%$ & $-0.03[-0.36,0.3]$ \\
\hline Bruzzese 2008 & 12 & 11 & $-0.2(0.418)$ & & $4.36 \%$ & $-0.15[-0.97,0.67]$ \\
\hline Bruzzese 2011 & 139 & 142 & $-0.2(0.12)$ & & $53.1 \%$ & $-0.21[-0.44,0.03]$ \\
\hline Shah 2001 & 113 & 138 & $-0.2(0.269)$ & $\longrightarrow$ & $10.54 \%$ & $-0.24[-0.77,0.29]$ \\
\hline \multicolumn{4}{|l|}{ Total $(95 \% \mathrm{Cl})$} & & $100 \%$ & $-0.15[-0.33,0.02]$ \\
\hline \multicolumn{7}{|c|}{ Heterogeneity: $\mathrm{Tau}^{2}=0 ; \mathrm{Chi}^{2}=0.92, \mathrm{df}=4(\mathrm{P}=0.92) ; \mathrm{I}^{2}=0 \%$} \\
\hline \multicolumn{4}{|c|}{ Test for overall effect: $Z=1.77(P=0.08)$} & & & \\
\hline
\end{tabular}

Favours education

Favours control

\section{Analysis 1.7. Comparison 1 Effects of school-based asthma interventions vs usual care, Outcome 7 Experience of daytime and night-time symptoms - night-time symptoms.}

\begin{tabular}{|c|c|c|c|c|c|c|}
\hline Study or subgroup & Education & $\begin{array}{c}\text { Usual care/ } \\
\text { placebo } \\
\text { N }\end{array}$ & $\begin{array}{l}\text { Std. Mean } \\
\text { Difference } \\
\text { (SE) }\end{array}$ & $\begin{array}{l}\text { Std. Mean Difference } \\
\text { IV, Random, 95\% CI }\end{array}$ & Weight & IV, Random, 95\% CI \\
\hline Bruzzese 2008 & 12 & 11 & $-0.4(0.423)$ & $\rightarrow$ & $12.85 \%$ & $-0.43[-1.26,0.4]$ \\
\hline Bruzzese 2011 & 139 & 142 & $-0.4(0.121)$ & + & $48.26 \%$ & $-0.39[-0.62,-0.15]$ \\
\hline Howell 2005 & 12 & 7 & $0.3(0.521)$ & + & $9.11 \%$ & $0.25[-0.77,1.27]$ \\
\hline McGhan 2003 & 65 & 71 & $0.1(0.227)$ & $\#$ & $29.79 \%$ & $0.12[-0.33,0.56]$ \\
\hline Total $(95 \% \mathrm{CI})$ & & & & 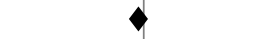 & $100 \%$ & $-0.18[-0.52,0.15]$ \\
\hline \multicolumn{7}{|c|}{ Heterogeneity: $\mathrm{Tau}^{2}=0.05 ; \mathrm{Chi}^{2}=5.01, \mathrm{df}=3(\mathrm{P}=0.17) ; \mathrm{I}^{2}=40.14 \%$} \\
\hline Test for overall effect & & & & & & \\
\hline
\end{tabular}

Analysis 1.8. Comparison 1 Effects of school-based asthma interventions vs usual care, Outcome 8 Use of reliever therapies, e.g. beta ${ }_{2}$-agonists.

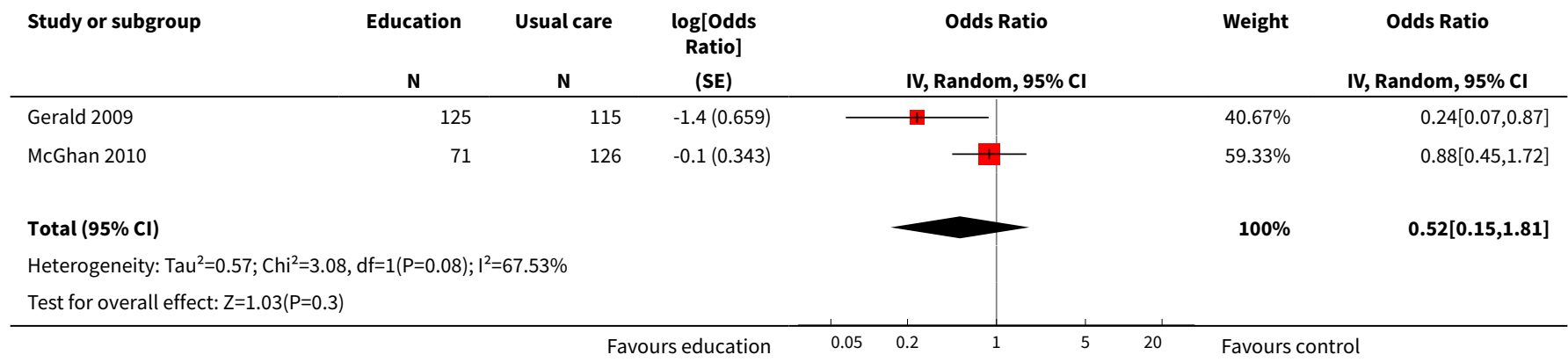


Analysis 1.9. Comparison 1 Effects of school-based asthma interventions vs usual care, Outcome 9 Corticosteroid dosage and/or use of add-on therapies (usage of).

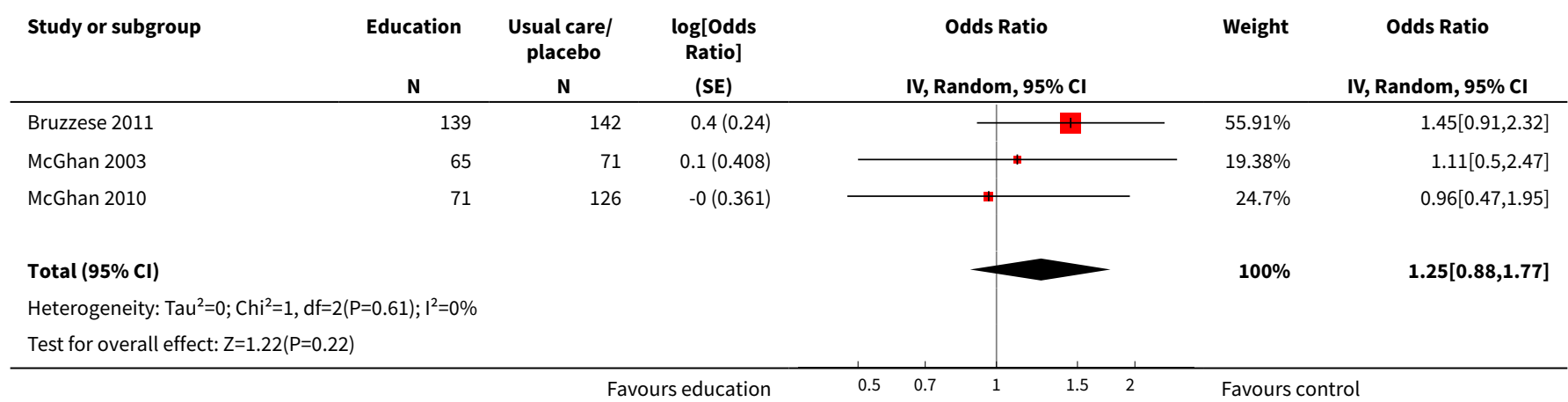

Analysis 1.10. Comparison 1 Effects of school-based asthma interventions vs usual care, Outcome 10 Corticosteroid dosage and/or use of add-on therapies (appropriate usage of).

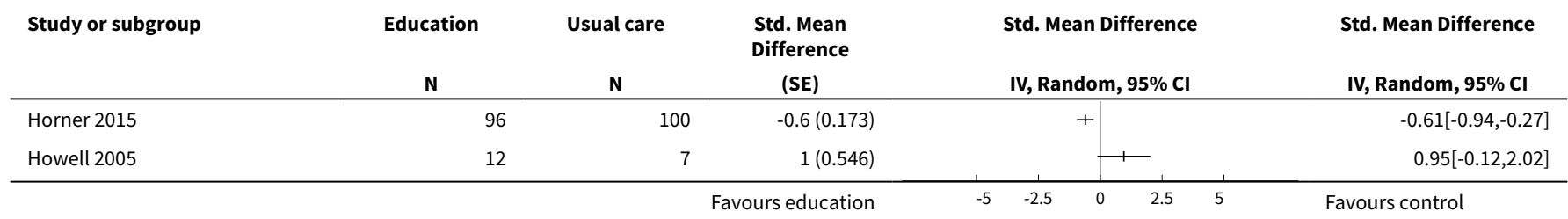

Analysis 1.11. Comparison 1 Effects of school-based asthma interventions vs usual care, Outcome 11 Health-related quality of life (SMD).

\begin{tabular}{|c|c|c|c|c|c|c|}
\hline \multirow{2}{*}{$\begin{array}{l}\text { Study or subgroup } \\
\text { Al-Sheyab } 2012\end{array}$} & $\begin{array}{l}\text { Education } \\
\mathbf{N} \\
\end{array}$ & $\begin{array}{c}\text { Usual care/ } \\
\text { placebo } \\
\mathbf{N} \\
\end{array}$ & $\begin{array}{c}\text { Std. Mean } \\
\text { Difference } \\
\text { (SE) }\end{array}$ & $\begin{array}{l}\text { Std. Mean Difference } \\
\text { IV, Random, } 95 \% \mathrm{Cl}\end{array}$ & Weight & $\begin{array}{l}\text { Std. Mean Difference } \\
\text { IV, Random, } 95 \% \mathrm{Cl}\end{array}$ \\
\hline & 126 & 118 & $0.3(0.129)$ & $\longrightarrow$ & $13.15 \%$ & $0.3[0.05,0.55]$ \\
\hline Cicutto 2005 & 132 & 124 & $0.4(0.157)$ & $\longrightarrow$ & $8.89 \%$ & $0.36[0.05,0.66]$ \\
\hline Cicutto 2013 & 625 & 691 & $0.3(0.064)$ & +1 & $53.02 \%$ & $0.31[0.18,0.43]$ \\
\hline Henry 2004 & 299 & 234 & $0.1(0.117)$ & $\longrightarrow$ & $16.02 \%$ & $0.13[-0.1,0.36]$ \\
\hline Howell 2005 & 16 & 8 & $0(0.484)$ & & $0.93 \%$ & $0.02[-0.93,0.97]$ \\
\hline Kintner 2009 & 34 & 27 & $0.6(0.334)$ & & $1.95 \%$ & $0.58[-0.07,1.24]$ \\
\hline Total $(95 \% \mathrm{Cl})$ & & & & & $100 \%$ & $0.27[0.18,0.36]$ \\
\hline \multicolumn{7}{|c|}{ Heterogeneity: $\mathrm{Tau}^{2}=0 ; \mathrm{Chi}^{2}=4.29, \mathrm{df}=6(\mathrm{P}=0.64) ; \mathrm{I}^{2}=0 \%$} \\
\hline Test for overall effect & & & & & & \\
\hline
\end{tabular}


Analysis 1.12. Comparison 1 Effects of school-based asthma interventions vs usual care, Outcome 12 Health-related quality of life (MD).

\begin{tabular}{|c|c|c|c|c|c|c|c|}
\hline \multirow{3}{*}{$\begin{array}{l}\text { Study or subgroup } \\
\text { Al-Sheyab } 2012\end{array}$} & \multicolumn{2}{|c|}{ Education } & \multicolumn{2}{|c|}{ Usual care/placebo } & \multirow{2}{*}{$\begin{array}{l}\text { Mean Difference } \\
\text { Random, } 95 \% \mathrm{Cl}\end{array}$} & \multirow[t]{2}{*}{ Weight } & \multirow{2}{*}{$\begin{array}{l}\text { Mean Difference } \\
\text { Random, } 95 \% \mathrm{Cl}\end{array}$} \\
\hline & $\mathbf{N}$ & Mean(SD) & $\mathbf{N}$ & Mean(SD) & & & \\
\hline & 126 & $5.4(1.6)$ & 118 & $4.1(1.5)$ & $\longrightarrow$ & $13.19 \%$ & $1.35[0.96,1.74]$ \\
\hline Cicutto 2005 & 132 & $5.5(2)$ & 124 & $5(2)$ & & $11.47 \%$ & $0.5[0,1]$ \\
\hline Cicutto 2013 & 625 & $5.8(1.6)$ & 691 & $5.4(1.9)$ & $\rightarrow$ & $16.15 \%$ & $0.4[0.21,0.59]$ \\
\hline Henry 2004 & 299 & $5.3(2.1)$ & 234 & $5.1(2.3)$ & $+\div$ & $13.26 \%$ & $0.16[-0.22,0.54]$ \\
\hline Horner 2008 & 81 & $1.7(0.8)$ & 72 & $1.7(0.8)$ & $\longrightarrow$ & $15.17 \%$ & $0.05[-0.21,0.31]$ \\
\hline Patterson 2005 & 81 & $0.3(1.2)$ & 92 & $0.2(1)$ & $\longrightarrow$ & $14.16 \%$ & $0.07[-0.26,0.4]$ \\
\hline Shah 2001 & 138 & $0.2(1.3)$ & 113 & $0.1(1.3)$ & 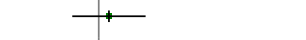 & $14.23 \%$ & $0.09[-0.23,0.41]$ \\
\hline Total $\star \star \star$ & 1498 & & 1452 & & & $100 \%$ & $0.35[0.06,0.64]$ \\
\hline \multicolumn{8}{|c|}{ Heterogeneity: $\operatorname{Tau}^{2}=0.12 ; \mathrm{Chi}^{2}=37.31, \mathrm{df}=7(\mathrm{P}<0.0001) ; \mathrm{I}^{2}=81.24 \%$} \\
\hline Test for overall effec & & & & & & & \\
\hline
\end{tabular}

Analysis 1.13. Comparison 1 Effects of school-based asthma interventions vs usual care, Outcome 13 Withdrawal from the study.

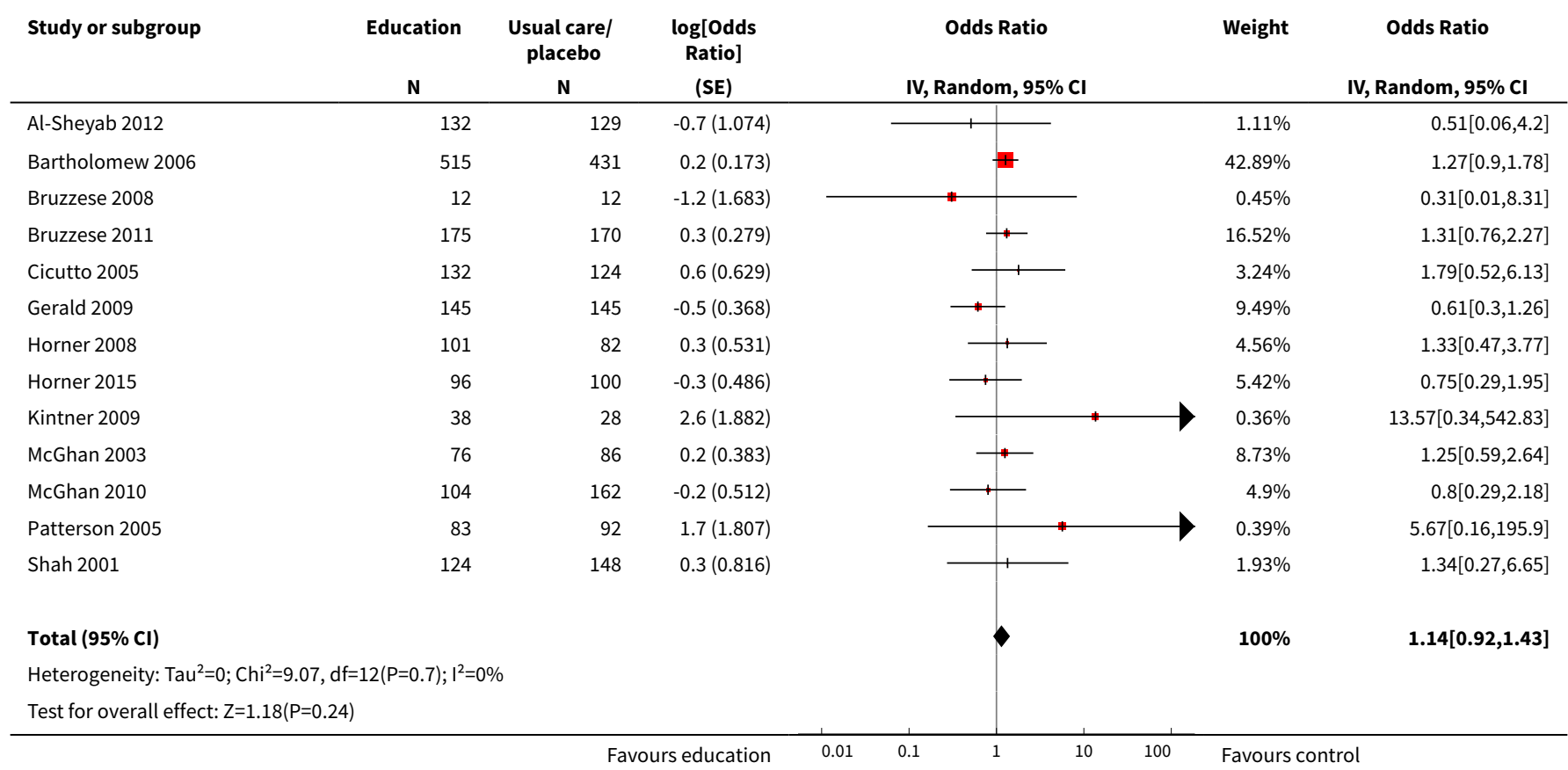


Comparison 2. Effects of school-based asthma interventions vs usual care subgrouped by school type

\begin{tabular}{|c|c|c|c|c|}
\hline Outcome or subgroup title & No. of studies & $\begin{array}{l}\text { No. of partici- } \\
\text { pants }\end{array}$ & Statistical method & Effect size \\
\hline $\begin{array}{l}1 \text { Exacerbations leading to } \\
\text { emergency department (ED) } \\
\text { visits }\end{array}$ & 13 & & Odds Ratio (Random, 95\% Cl) & $0.70[0.53,0.92]$ \\
\hline 1.1 Secondary/high school & 2 & & Odds Ratio (Random, 95\% Cl) & $0.61[0.40,0.92]$ \\
\hline 1.2 Primary/elementary school & 11 & & Odds Ratio (Random, 95\% Cl) & $0.73[0.52,1.02]$ \\
\hline 2 Absence from school & 10 & & $\begin{array}{l}\text { Std. Mean Difference (Random, } \\
95 \% \mathrm{Cl} \text { ) }\end{array}$ & $-0.07[-0.22,0.08]$ \\
\hline 2.1 Secondary/high school & 1 & & $\begin{array}{l}\text { Std. Mean Difference (Random, } \\
95 \% \mathrm{Cl} \text { ) }\end{array}$ & $-0.38[-0.62,-0.15]$ \\
\hline 2.2 Primary/elementary school & 7 & & $\begin{array}{l}\text { Std. Mean Difference (Random, } \\
95 \% \mathrm{Cl} \text { ) }\end{array}$ & $-0.05[-0.27,0.16]$ \\
\hline $\begin{array}{l}\text { 2.3 Primary/elementary and } \\
\text { middle schools }\end{array}$ & 1 & & $\begin{array}{l}\text { Std. Mean Difference (Random, } \\
95 \% \mathrm{Cl} \text { ) }\end{array}$ & $0.02[-0.08,0.12]$ \\
\hline 2.4 Middle school & 1 & & $\begin{array}{l}\text { Std. Mean Difference (Random, } \\
95 \% \mathrm{Cl} \text { ) }\end{array}$ & $0.08[-0.31,0.48]$ \\
\hline 3 Withdrawal from the study & 13 & & Odds Ratio (Random, 95\% Cl) & $1.14[0.92,1.43]$ \\
\hline 3.1 Secondary/high school & 3 & & Odds Ratio (Random, 95\% Cl) & $1.25[0.76,2.06]$ \\
\hline 3.2 Primary/elementary school & 8 & & Odds Ratio (Random, 95\% Cl) & $1.22[0.94,1.59]$ \\
\hline 3.3 Middle school & 2 & & Odds Ratio (Random, 95\% Cl) & $0.59[0.29,1.20]$ \\
\hline
\end{tabular}

\section{Analysis 2.1. Comparison 2 Effects of school-based asthma interventions vs usual care subgrouped by school type, Outcome 1 Exacerbations leading to emergency department (ED) visits.}

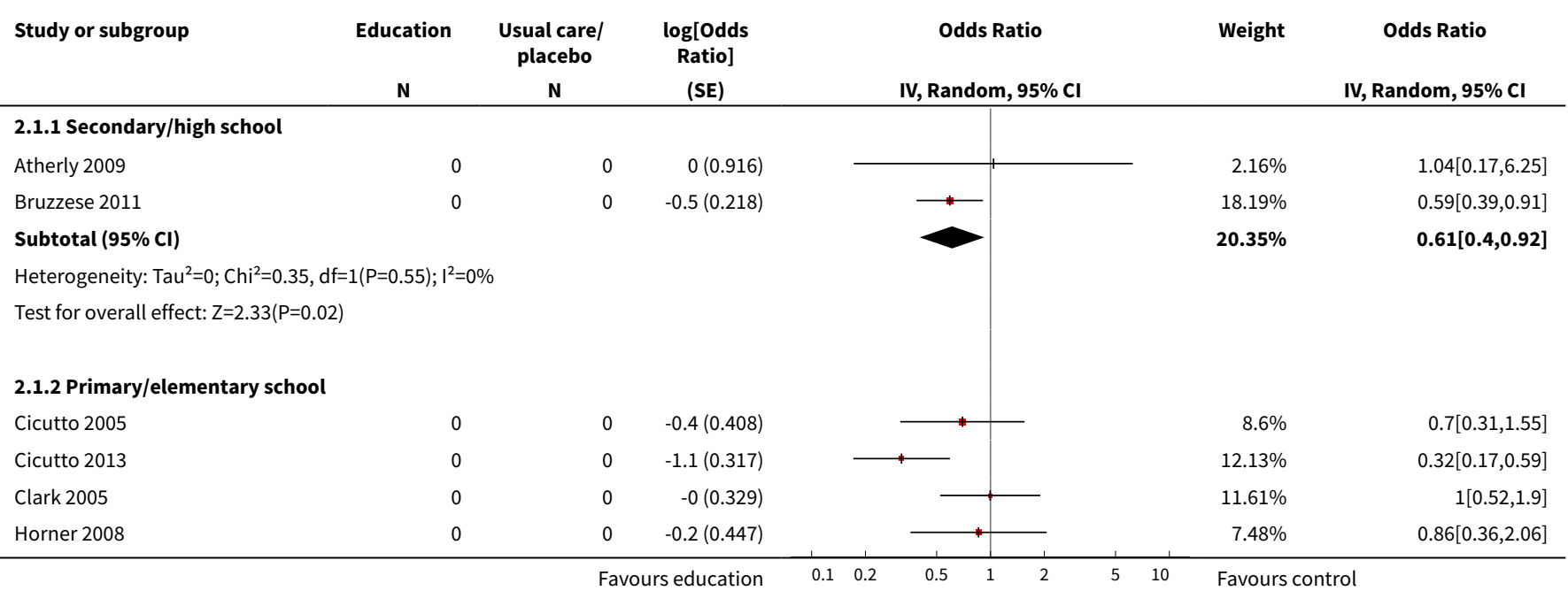




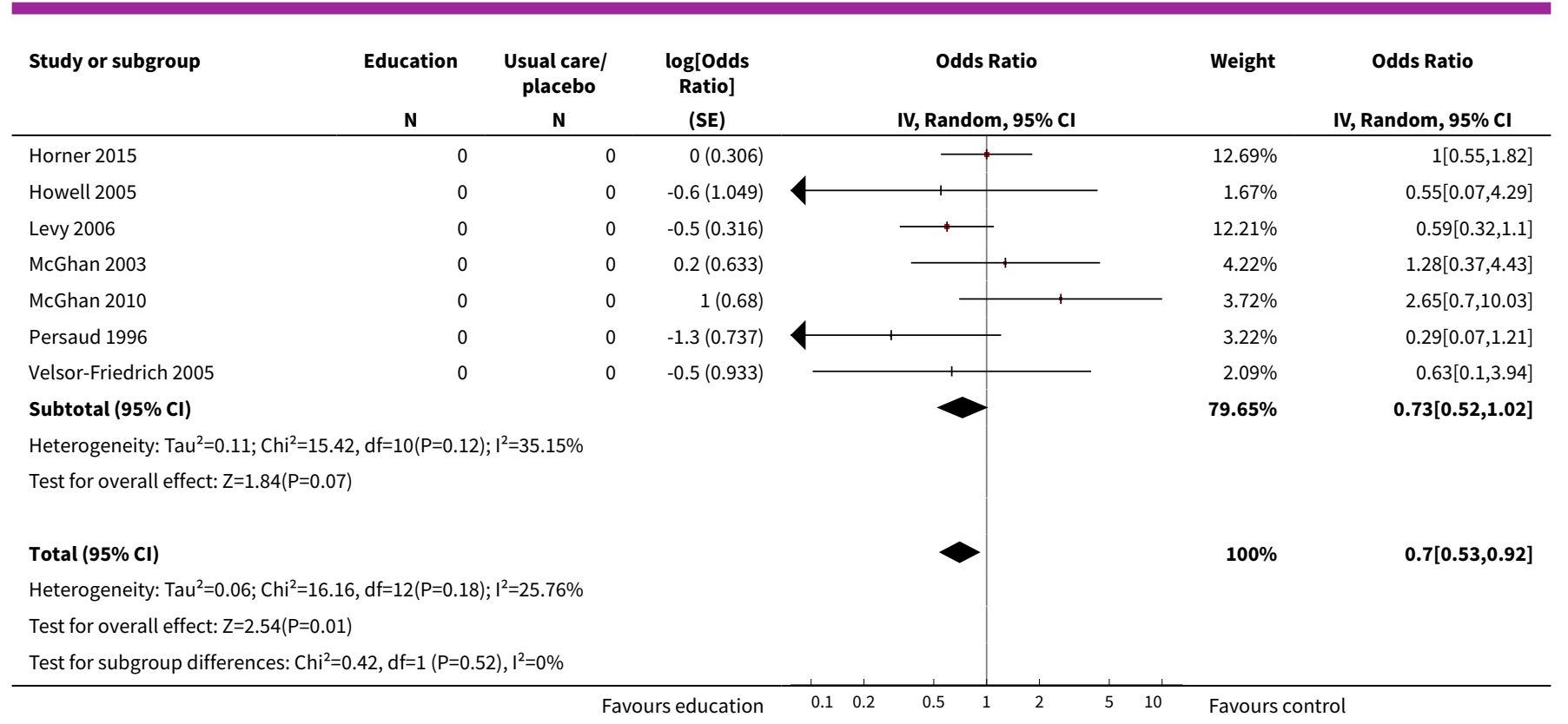

Analysis 2.2. Comparison 2 Effects of school-based asthma interventions vs usual care subgrouped by school type, Outcome 2 Absence from school.

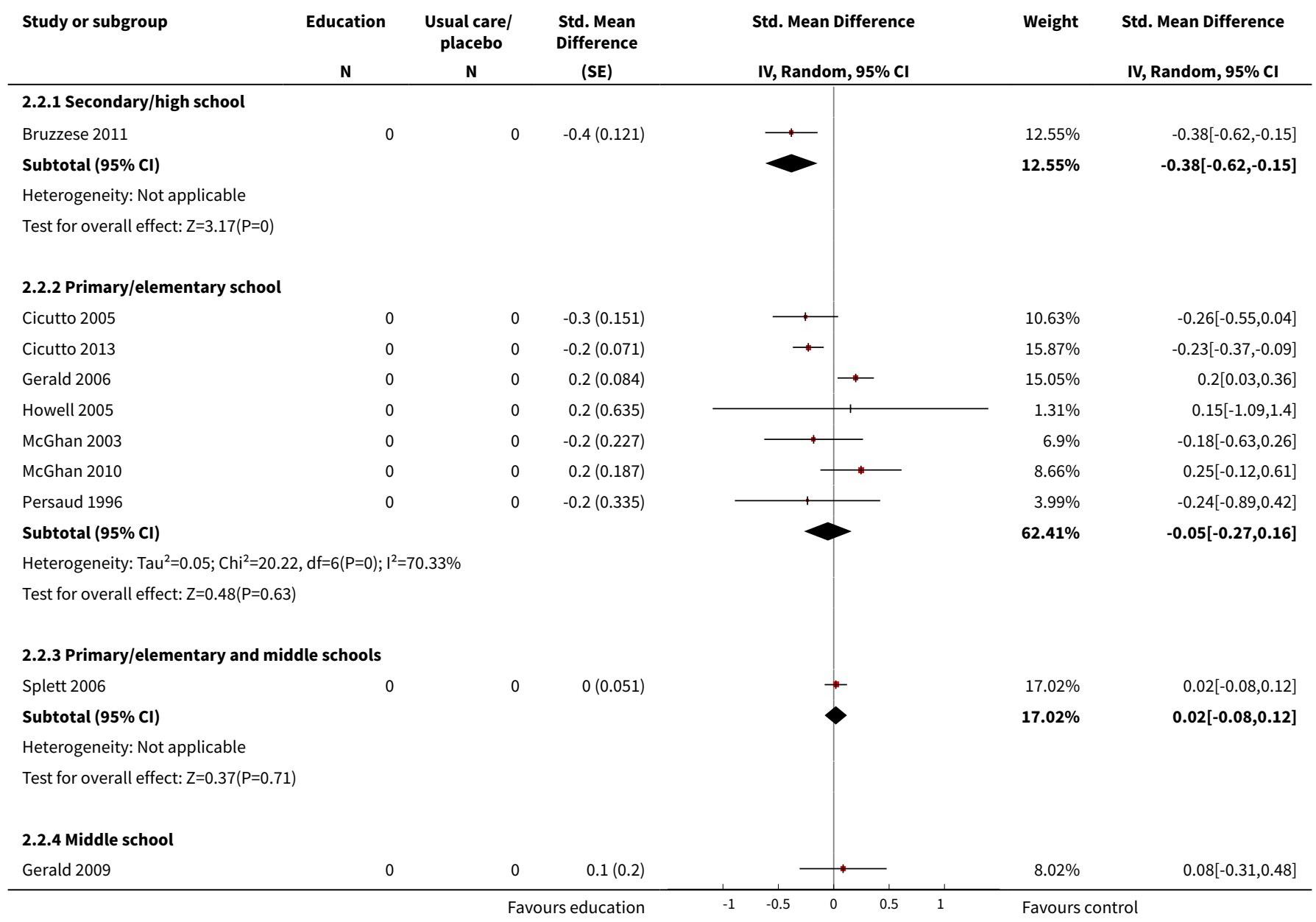




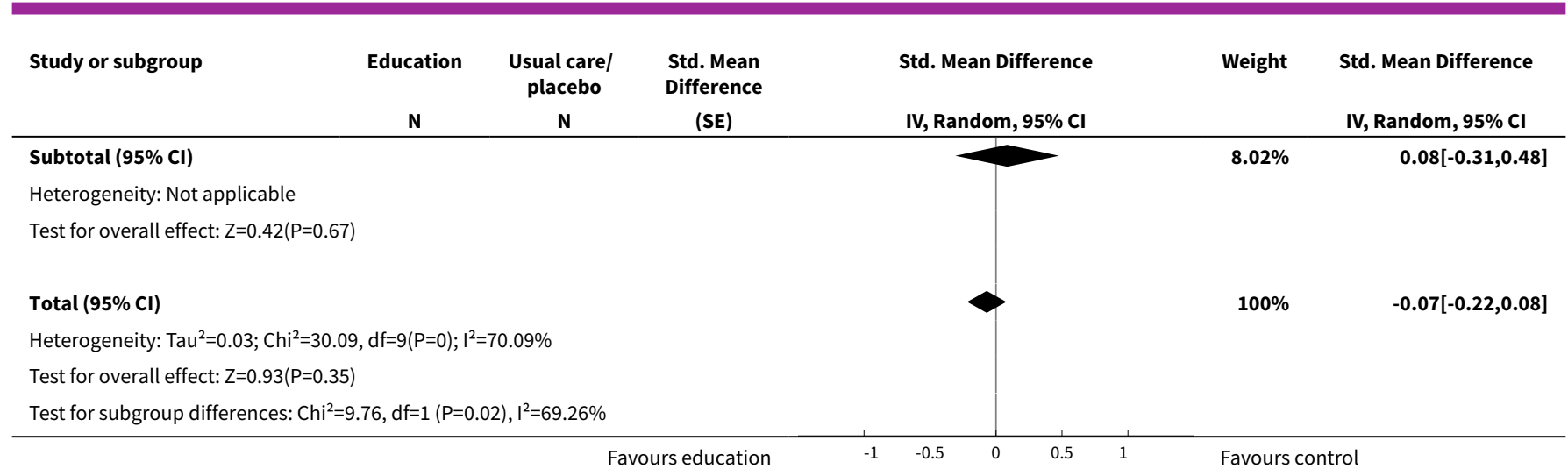

Analysis 2.3. Comparison 2 Effects of school-based asthma interventions vs usual care subgrouped by school type, Outcome 3 Withdrawal from the study.

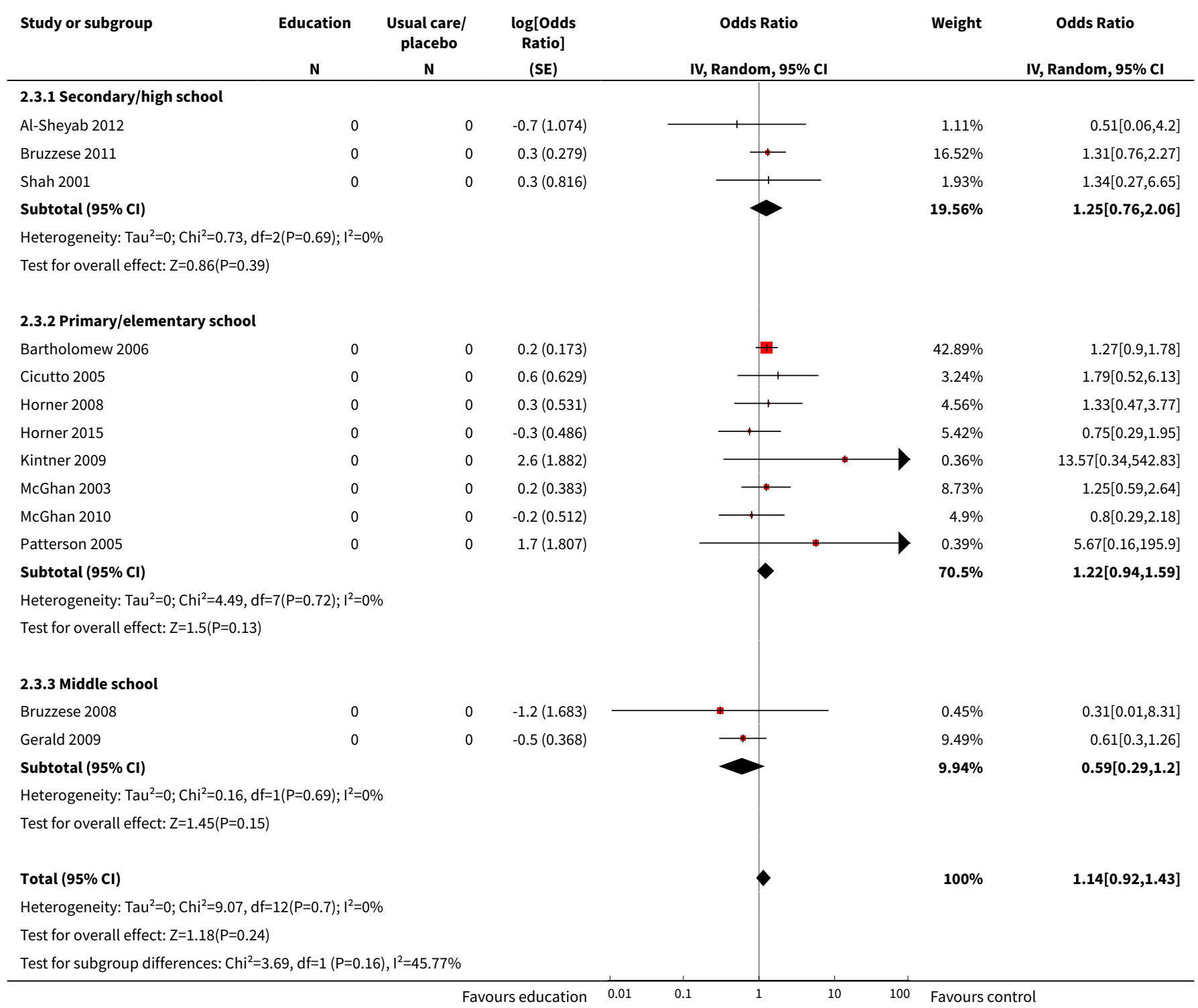


Comparison 3. Effects of school-based asthma interventions vs usual care subgrouped by age of children

\begin{tabular}{|c|c|c|c|c|}
\hline Outcome or subgroup title & No. of studies & $\begin{array}{l}\text { No. of partici- } \\
\text { pants }\end{array}$ & Statistical method & Effect size \\
\hline $\begin{array}{l}1 \text { Exacerbations leading to } \\
\text { emergency department (ED) } \\
\text { visits }\end{array}$ & 13 & & Odds Ratio (Random, 95\% Cl) & $0.70[0.53,0.92]$ \\
\hline 1.1 Aged 11 to 15,16 to 18 & 1 & & Odds Ratio (Random, 95\% Cl) & $0.59[0.39,0.91]$ \\
\hline 1.2 Aged 11 to 15 & 1 & & Odds Ratio (Random, 95\% Cl) & $1.04[0.17,6.25]$ \\
\hline 1.3 Aged 5 to 10,11 to 15 & 2 & & Odds Ratio (Random, 95\% Cl) & $0.64[0.15,2.76]$ \\
\hline 1.4 Aged 5 to 10 & 9 & & Odds Ratio (Random, 95\% Cl) & $0.74[0.51,1.06]$ \\
\hline 2 Absence from school & 10 & & $\begin{array}{l}\text { Std. Mean Difference (Random, } \\
95 \% \mathrm{Cl} \text { ) }\end{array}$ & $-0.07[-0.22,0.08]$ \\
\hline 2.1 Aged 11 to 15,16 to 18 & 1 & & $\begin{array}{l}\text { Std. Mean Difference (Random, } \\
95 \% \mathrm{Cl} \text { ) }\end{array}$ & $-0.38[-0.62,-0.15]$ \\
\hline 2.2 Aged 5 to 10,11 to 15 & 4 & & $\begin{array}{l}\text { Std. Mean Difference (Random, } \\
95 \% \mathrm{Cl} \text { ) }\end{array}$ & $0.03[-0.06,0.13]$ \\
\hline 2.3 Aged 5 to 10 & 5 & & $\begin{array}{l}\text { Std. Mean Difference (Random, } \\
95 \% \mathrm{Cl} \text { ) }\end{array}$ & $-0.09[-0.34,0.16]$ \\
\hline 3 Withdrawal from the study & 13 & & Odds Ratio (Random, 95\% Cl) & $1.14[0.92,1.43]$ \\
\hline 3.1 Aged 11 to 15,16 to 18 & 1 & & Odds Ratio (Random, 95\% Cl) & $1.31[0.76,2.27]$ \\
\hline 3.2 Aged 11 to 15 & 3 & & Odds Ratio (Random, 95\% Cl) & $0.82[0.25,2.67]$ \\
\hline 3.3 Aged 5 to 10,11 to 15 & 4 & & Odds Ratio (Random, 95\% Cl) & $1.08[0.48,2.43]$ \\
\hline 3.4 Aged 5 to 10 & 5 & & Odds Ratio (Random, 95\% Cl) & $1.19[0.90,1.58]$ \\
\hline
\end{tabular}

\section{Analysis 3.1. Comparison 3 Effects of school-based asthma interventions vs usual care subgrouped by age of children, Outcome 1 Exacerbations leading to emergency department (ED) visits.}

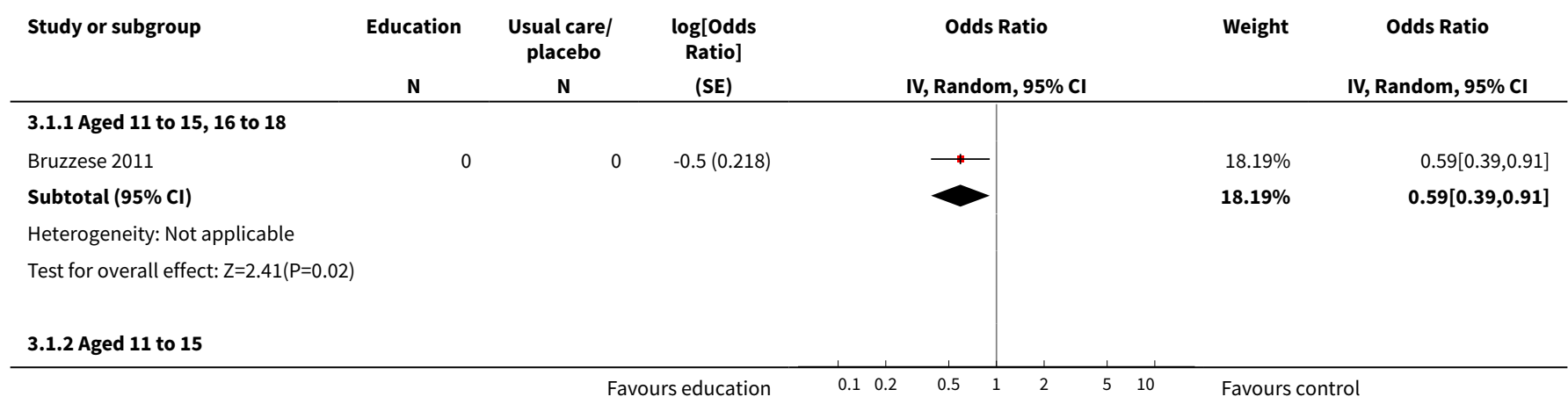




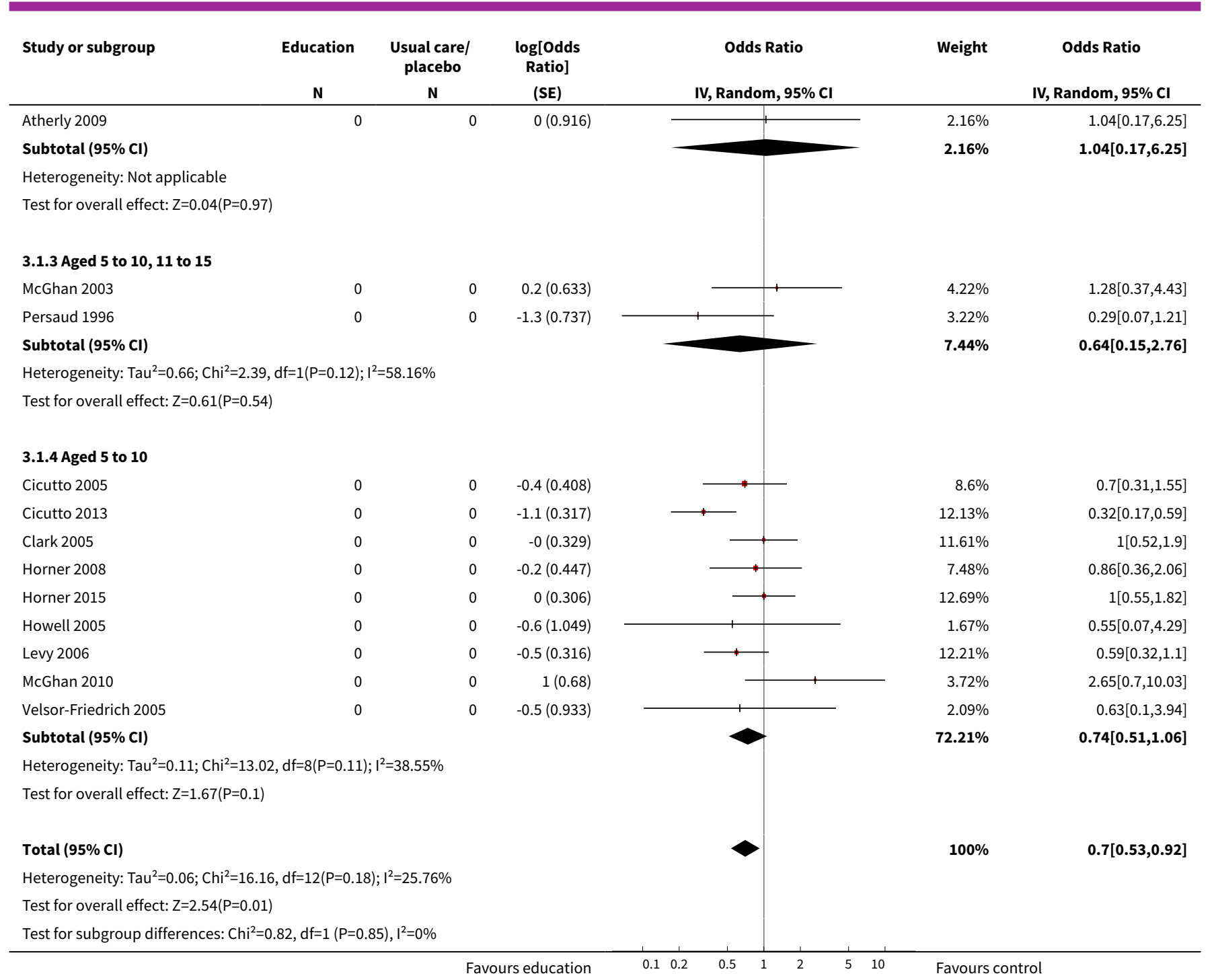

Analysis 3.2. Comparison 3 Effects of school-based asthma interventions vs usual care subgrouped by age of children, Outcome 2 Absence from school.

\begin{tabular}{|c|c|c|c|c|c|c|}
\hline Study or subgroup & Education & $\begin{array}{c}\text { Usual care/ } \\
\text { placebo } \\
\mathbf{N} \\
\end{array}$ & $\begin{array}{c}\text { Std. Mean } \\
\text { Difference } \\
(\mathrm{SE}) \\
\end{array}$ & $\begin{array}{l}\text { Std. Mean Difference } \\
\text { IV, Random, } 95 \% \mathrm{CI}\end{array}$ & Weight & $\begin{array}{l}\text { Std. Mean Difference } \\
\text { IV, Random, } 95 \% \mathrm{CI}\end{array}$ \\
\hline \multicolumn{7}{|c|}{ 3.2.1 Aged 11 to 15,16 to 18} \\
\hline Bruzzese 2011 & 0 & 0 & $-0.4(0.121)$ & $\longrightarrow$ & $12.54 \%$ & $-0.38[-0.62,-0.15]$ \\
\hline Subtotal $(95 \% \mathrm{CI})$ & & & & & $12.54 \%$ & $-0.38[-0.62,-0.15]$ \\
\hline \multicolumn{7}{|c|}{ Heterogeneity: Not applicable } \\
\hline \multicolumn{7}{|c|}{ 3.2.2 Aged 5 to 10,11 to 15} \\
\hline Gerald 2009 & 0 & 0 & $0.1(0.2)$ & + & $8.01 \%$ & $0.08[-0.31,0.48]$ \\
\hline McGhan 2010 & 0 & 0 & $0.2(0.187)$ & 4 & $8.65 \%$ & $0.25[-0.12,0.61]$ \\
\hline Persaud 1996 & 0 & 0 & $-0.2(0.335)$ & \begin{tabular}{r|r} 
\\
\end{tabular} & $3.98 \%$ & $-0.24[-0.89,0.42]$ \\
\hline Splett 2006 & 0 & 0 & $0(0.051)$ & 世 & $17 \%$ & $0.02[-0.08,0.12]$ \\
\hline
\end{tabular}




\begin{tabular}{|c|c|c|c|c|c|c|}
\hline Study or subgroup & Education & $\begin{array}{c}\text { Usual care/ } \\
\text { placebo } \\
\mathbf{N}\end{array}$ & $\begin{array}{l}\text { Std. Mean } \\
\text { Difference } \\
\quad(\mathrm{SE})\end{array}$ & $\begin{array}{l}\text { Std. Mean Difference } \\
\text { IV, Random, } 95 \% \mathrm{CI}\end{array}$ & Weight & $\begin{array}{l}\text { Std. Mean Difference } \\
\text { IV, Random, 95\% Cl }\end{array}$ \\
\hline Subtotal $(95 \% \mathrm{Cl})$ & & & & & $37.64 \%$ & $0.03[-0.06,0.13]$ \\
\hline \multicolumn{7}{|c|}{ Heterogeneity: $\mathrm{Tau}^{2}=0 ; \mathrm{Chi}^{2}=2.09, \mathrm{df}=3(\mathrm{P}=0.55) ; \mathrm{I}^{2}=0 \%$} \\
\hline \multicolumn{7}{|c|}{ Test for overall effect: $Z=0.68(P=0.5)$} \\
\hline \multicolumn{7}{|l|}{ 3.2.3 Aged 5 to 10} \\
\hline Cicutto 2005 & 0 & 0 & $-0.3(0.151)$ & $\longrightarrow$ & $10.62 \%$ & $-0.26[-0.55,0.04]$ \\
\hline Cicutto 2013 & 0 & 0 & $-0.2(0.071)$ & $\rightarrow$ & $15.85 \%$ & $-0.23[-0.37,-0.09]$ \\
\hline Gerald 2006 & 0 & 0 & $0.2(0.084)$ & - & $15.03 \%$ & $0.2[0.03,0.36]$ \\
\hline Howell 2005 & 0 & 0 & $0.2(0.602)$ & & $1.45 \%$ & $0.15[-1.03,1.33]$ \\
\hline McGhan 2003 & 0 & 0 & $-0.2(0.227)$ & 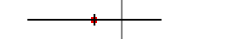 & $6.89 \%$ & $-0.18[-0.63,0.26]$ \\
\hline Subtotal $(95 \% \mathrm{Cl})$ & & & & & $49.83 \%$ & $-0.09[-0.34,0.16]$ \\
\hline \multicolumn{7}{|c|}{ Heterogeneity: Tau $^{2}=0.05 ; \mathrm{Chi}^{2}=17.15, \mathrm{df}=4(\mathrm{P}=0) ; \mathrm{I}^{2}=76.68 \%$} \\
\hline \multicolumn{7}{|c|}{ Test for overall effect: $Z=0.71(P=0.48)$} \\
\hline Total $(95 \% \mathrm{Cl})$ & & & & & $100 \%$ & $-0.07[-0.22,0.08]$ \\
\hline \multicolumn{7}{|c|}{ Heterogeneity: $\mathrm{Tau}^{2}=0.03 ; \mathrm{Chi}^{2}=30.11, \mathrm{df}=9(\mathrm{P}=0) ; \mathrm{I}^{2}=70.1 \%$} \\
\hline \multicolumn{7}{|c|}{ Test for overall effect: $Z=0.93(P=0.35)$} \\
\hline \multicolumn{7}{|c|}{ Test for subgroup differences: $\mathrm{Chi}^{2}=10.45, \mathrm{df}=1(\mathrm{P}=0.01), \mathrm{I}^{2}=80.87 \%$} \\
\hline
\end{tabular}

Analysis 3.3. Comparison 3 Effects of school-based asthma interventions vs usual care subgrouped by age of children, Outcome 3 Withdrawal from the study.

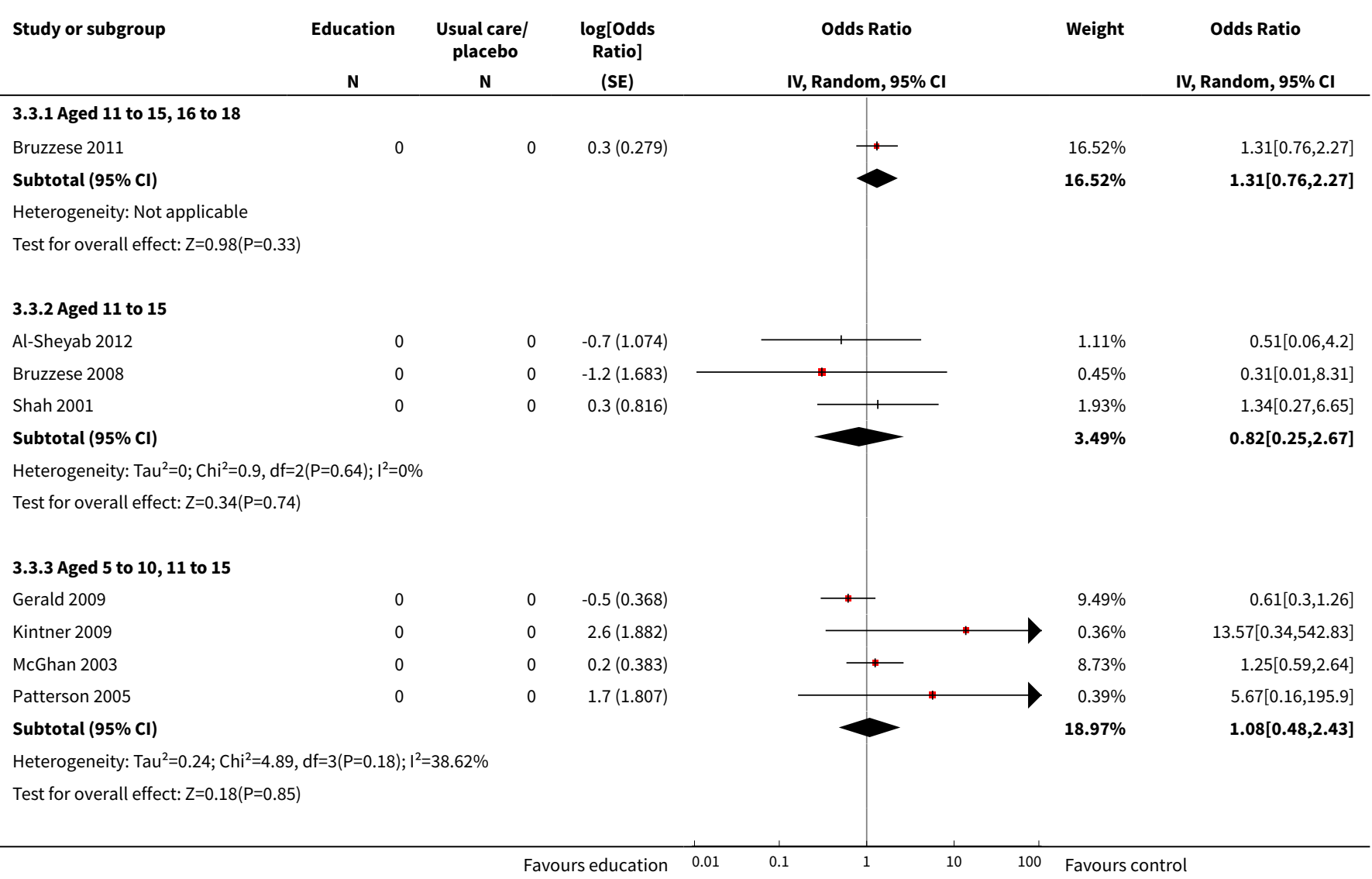




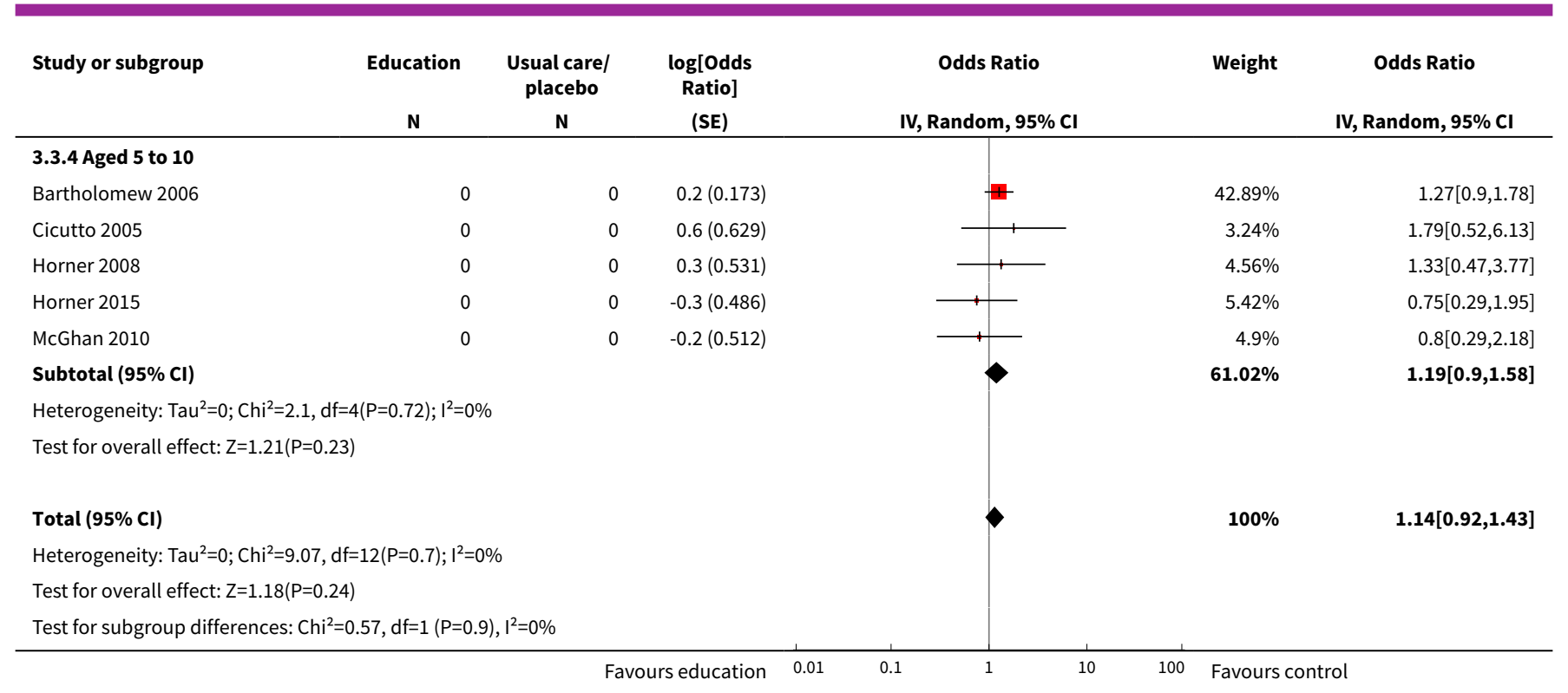

\section{Comparison 4. Effects of school-based asthma interventions vs usual care subgrouped by child socio-economic status (SES)}

\begin{tabular}{|c|c|c|c|c|}
\hline Outcome or subgroup title & No. of studies & $\begin{array}{l}\text { No. of partici- } \\
\text { pants }\end{array}$ & Statistical method & Effect size \\
\hline $\begin{array}{l}1 \text { Exacerbations leading to } \\
\text { emergency department (ED) } \\
\text { visits }\end{array}$ & 13 & & Odds Ratio (Random, 95\% Cl) & $0.70[0.53,0.92]$ \\
\hline 1.1 Low SES over $50 \%$ & 2 & & Odds Ratio (Random, 95\% Cl) & $0.53[0.30,0.94]$ \\
\hline 1.2 Low SES over $25 \%$ & 3 & & Odds Ratio (Random, 95\% Cl) & $0.69[0.28,1.69]$ \\
\hline 1.3 Unclear or not low SES & 8 & & Odds Ratio (Random, 95\% Cl) & $0.76[0.57,1.01]$ \\
\hline 2 Absence from school & 10 & & $\begin{array}{l}\text { Std. Mean Difference (Random, } \\
95 \% \mathrm{Cl} \text { ) }\end{array}$ & $-0.07[-0.22,0.08]$ \\
\hline 2.1 Low SES over $50 \%$ & 2 & & $\begin{array}{l}\text { Std. Mean Difference (Random, } \\
95 \% \mathrm{Cl} \text { ) }\end{array}$ & $0.01[-0.09,0.11]$ \\
\hline 2.2 Low SES over $25 \%$ & 2 & & $\begin{array}{l}\text { Std. Mean Difference (Random, } \\
95 \% \mathrm{Cl} \text { ) }\end{array}$ & $-0.23[-0.36,-0.09]$ \\
\hline 2.3 Unclear or not low SES & 6 & & $\begin{array}{l}\text { Std. Mean Difference (Random, } \\
95 \% \mathrm{Cl} \text { ) }\end{array}$ & $-0.02[-0.28,0.24]$ \\
\hline 3 Withdrawal from the study & 13 & & Odds Ratio (Random, 95\% Cl) & $1.14[0.92,1.43]$ \\
\hline 3.1 Low SES over $50 \%$ & 1 & & Odds Ratio (Random, 95\% Cl) & $1.27[0.90,1.78]$ \\
\hline 3.2 Low SES over $25 \%$ & 4 & & Odds Ratio (Random, 95\% Cl) & $1.16[0.61,2.23]$ \\
\hline 3.3 Unclear or not low SES & 8 & & Odds Ratio (Random, 95\% Cl) & $1.03[0.73,1.45]$ \\
\hline
\end{tabular}


Analysis 4.1. Comparison 4 Effects of school-based asthma interventions vs usual care subgrouped by child socio-economic status (SES), Outcome 1 Exacerbations leading to emergency department (ED) visits.

\begin{tabular}{|c|c|c|c|c|c|c|}
\hline Study or subgroup & $\begin{array}{c}\text { Education } \\
\mathrm{N} \\
\end{array}$ & $\begin{array}{c}\text { Control } \\
\mathrm{N} \\
\end{array}$ & \multirow[t]{2}{*}{$\begin{array}{c}\text { log[Odds } \\
\text { Ratio] } \\
\text { (SE) } \\
\end{array}$} & IV, Random, 95\% CI & Weight & $\begin{array}{c}\text { Odds Ratio } \\
\text { IV, Random, } 95 \% \text { CI }\end{array}$ \\
\hline \multicolumn{6}{|c|}{ 4.1.1 Low SES over $50 \%$} & \\
\hline Levy 2006 & 0 & 0 & $-0.5(0.316)$ & $\longrightarrow$ & $12.21 \%$ & $0.59[0.32,1.1]$ \\
\hline Persaud 1996 & 0 & 0 & $-1.3(0.737)$ & & $3.22 \%$ & $0.29[0.07,1.21]$ \\
\hline Subtotal $(95 \% \mathrm{Cl})$ & & & & & $15.43 \%$ & $0.53[0.3,0.94]$ \\
\hline \multicolumn{7}{|c|}{ Heterogeneity: $\operatorname{Tau}^{2}=0 ; \mathrm{Chi}^{2}=0.84, \mathrm{df}=1(\mathrm{P}=0.36) ; \mathrm{I}^{2}=0 \%$} \\
\hline \multicolumn{7}{|c|}{ Test for overall effect: $Z=2.18(P=0.03)$} \\
\hline \multicolumn{7}{|c|}{ 4.1.2 Low SES over $25 \%$} \\
\hline Cicutto 2013 & 0 & 0 & $-1.1(0.317)$ & 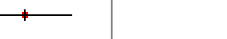 & $12.13 \%$ & $0.32[0.17,0.59]$ \\
\hline Horner 2015 & 0 & 0 & $0(0.306)$ & + & $12.69 \%$ & $1[0.55,1.82]$ \\
\hline McGhan 2003 & 0 & 0 & $0.2(0.633)$ & & $4.22 \%$ & $1.28[0.37,4.43]$ \\
\hline \multicolumn{7}{|c|}{ Heterogeneity: $\mathrm{Tau}^{2}=0.45 ; \mathrm{Chi}^{2}=8.17, \mathrm{df}=2(\mathrm{P}=0.02) ; \mathrm{I}^{2}=75.53 \%$} \\
\hline \multicolumn{7}{|c|}{ Test for overall effect: $Z=0.81(P=0.42)$} \\
\hline \multicolumn{7}{|c|}{ 4.1.3 Unclear or not low SES } \\
\hline Atherly 2009 & 0 & 0 & $0(0.916)$ & & $2.16 \%$ & $1.04[0.17,6.25]$ \\
\hline Bruzzese 2011 & 0 & 0 & $-0.5(0.218)$ & & $18.19 \%$ & $0.59[0.39,0.91]$ \\
\hline Cicutto 2005 & 0 & 0 & $-0.4(0.408)$ & 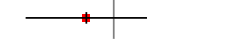 & $8.6 \%$ & $0.7[0.31,1.55]$ \\
\hline Clark 2005 & 0 & 0 & $-0(0.329)$ & & $11.61 \%$ & $1[0.52,1.9]$ \\
\hline Horner 2008 & 0 & 0 & $-0.2(0.447)$ & & $7.48 \%$ & $0.86[0.36,2.06]$ \\
\hline Howell 2005 & 0 & 0 & $-0.6(1.049)$ & & $1.67 \%$ & $0.55[0.07,4.29]$ \\
\hline McGhan 2010 & 0 & 0 & $1(0.68)$ & t & $3.72 \%$ & $2.65[0.7,10.03]$ \\
\hline Velsor-Friedrich 2005 & 0 & 0 & $-0.5(0.933)$ & & $2.09 \%$ & $0.63[0.1,3.94]$ \\
\hline Subtotal $(95 \% \mathrm{Cl})$ & & & & & $55.52 \%$ & $0.76[0.57,1.01]$ \\
\hline Total $(95 \% \mathrm{Cl})$ & & & & & $100 \%$ & $0.7[0.53,0.92]$ \\
\hline \multicolumn{7}{|c|}{ Heterogeneity: $\mathrm{Tau}^{2}=0.06 ; \mathrm{Chi}^{2}=16.16, \mathrm{df}=12(\mathrm{P}=0.18) ; \mathrm{I}^{2}=25.76 \%$} \\
\hline \multicolumn{7}{|c|}{ Test for overall effect: $Z=2.54(P=0.01)$} \\
\hline Test for subgroup diffe & $2, \mathrm{df}=1(\mathrm{P}=0.5$ & $=0 \%$ & & & & \\
\hline
\end{tabular}

Analysis 4.2. Comparison 4 Effects of school-based asthma interventions vs usual care subgrouped by child socio-economic status (SES), Outcome 2 Absence from school.

\begin{tabular}{|c|c|c|c|c|c|c|}
\hline Study or subgroup & Education & Control & $\begin{array}{l}\text { Std. Mean } \\
\text { Difference } \\
\text { (SE) }\end{array}$ & IV, Random, 95\% CI & Weight & $\begin{array}{l}\text { Std. Mean Difference } \\
\text { IV, Random, } 95 \% \mathrm{CI}\end{array}$ \\
\hline \multicolumn{7}{|l|}{ 4.2.1 Low SES over $50 \%$} \\
\hline Persaud 1996 & 0 & 0 & $-0.2(0.335)$ & & $3.99 \%$ & $-0.24[-0.89,0.42]$ \\
\hline Splett 2006 & 0 & 0 & $0(0.051)$ & * & $17.02 \%$ & $0.02[-0.08,0.12]$ \\
\hline Subtotal $(95 \% \mathrm{Cl})$ & & & & & $21.01 \%$ & $0.01[-0.09,0.11]$ \\
\hline
\end{tabular}




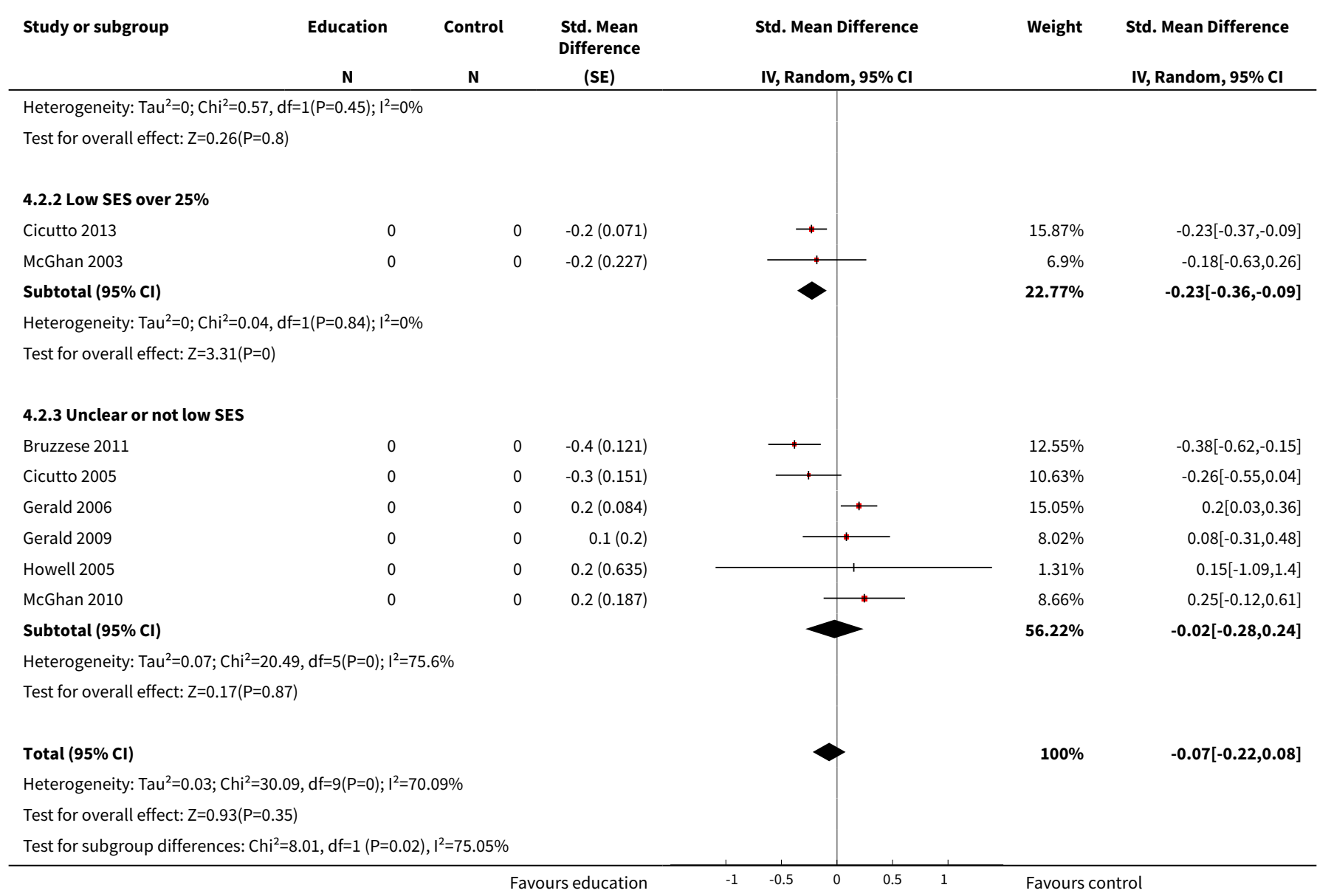

\section{Analysis 4.3. Comparison 4 Effects of school-based asthma interventions vs usual care subgrouped by child socio-economic status (SES), Outcome 3 Withdrawal from the study.}

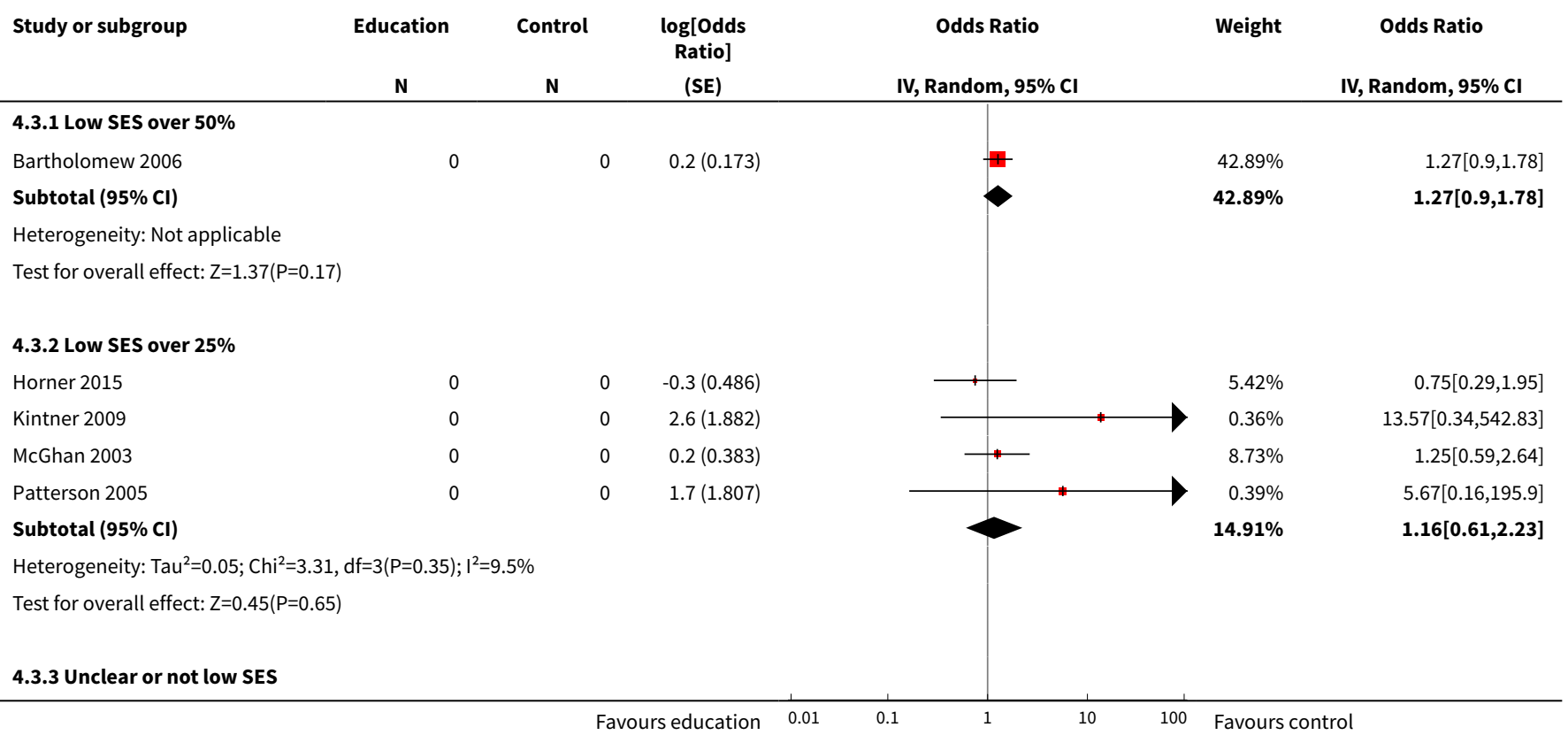

School-based self-management interventions for asthma in children and adolescents: a mixed methods systematic review (Review) 


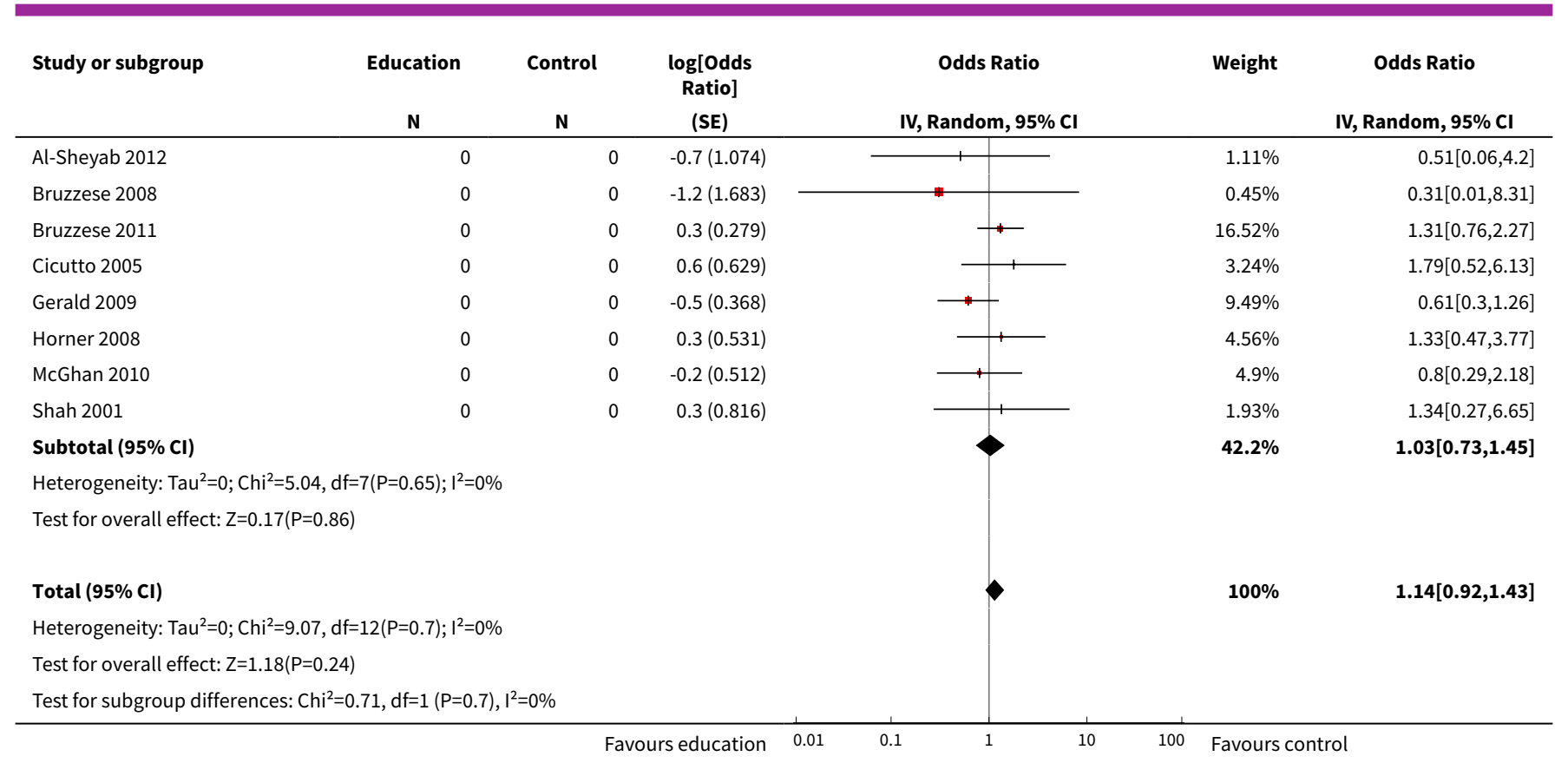

Comparison 5. Effects of school-based asthma interventions vs usual care subgrouped by involvement of school staff in direct delivery of self-management skills to children

\begin{tabular}{|c|c|c|c|c|}
\hline Outcome or subgroup title & No. of studies & $\begin{array}{l}\text { No. of partici- } \\
\text { pants }\end{array}$ & Statistical method & Effect size \\
\hline $\begin{array}{l}1 \text { Exacerbations leading to emergency } \\
\text { department }(E D) \text { visits }\end{array}$ & 13 & & $\begin{array}{l}\text { Odds Ratio (Random, 95\% } \\
\mathrm{Cl} \text { ) }\end{array}$ & $0.70[0.53,0.92]$ \\
\hline $\begin{array}{l}1.1 \text { Teachers involved in delivery (with } \\
\text { or without school nurses) }\end{array}$ & 2 & & $\begin{array}{l}\text { Odds Ratio (Random, 95\% } \\
\mathrm{Cl} \text { ) }\end{array}$ & $1.00[0.55,1.83]$ \\
\hline $\begin{array}{l}1.2 \text { School nurses alone involved in de- } \\
\text { livery }\end{array}$ & 1 & & $\begin{array}{l}\text { Odds Ratio (Random, 95\% } \\
\mathrm{Cl} \text { ) }\end{array}$ & $0.29[0.07,1.21]$ \\
\hline $\begin{array}{l}1.3 \text { Existing school staff not involved in } \\
\text { delivery }\end{array}$ & 10 & & $\begin{array}{l}\text { Odds Ratio (Random, 95\% } \\
\text { Cl) }\end{array}$ & $0.69[0.51,0.94]$ \\
\hline 2 Absence from school & 10 & & $\begin{array}{l}\text { Std. Mean Difference (Ran- } \\
\text { dom, } 95 \% \mathrm{Cl} \text { ) }\end{array}$ & $-0.07[-0.22,0.08]$ \\
\hline $\begin{array}{l}2.1 \text { School nurses or teachers involved } \\
\text { in delivery }\end{array}$ & 3 & & $\begin{array}{l}\text { Std. Mean Difference (Ran- } \\
\text { dom, } 95 \% \mathrm{Cl} \text { ) }\end{array}$ & $0.08[-0.08,0.24]$ \\
\hline $\begin{array}{l}\text { 2.2 Existing school staff not involved in } \\
\text { delivery }\end{array}$ & 7 & & $\begin{array}{l}\text { Std. Mean Difference (Ran- } \\
\text { dom, } 95 \% \mathrm{Cl} \text { ) }\end{array}$ & $-0.16[-0.32,-0.00]$ \\
\hline 3 Withdrawal from the study & 13 & & $\begin{array}{l}\text { Odds Ratio (Random, 95\% } \\
\mathrm{Cl} \text { ) }\end{array}$ & $1.14[0.92,1.43]$ \\
\hline 3.1 School nurses involved in delivery & 1 & & $\begin{array}{l}\text { Odds Ratio (Random, 95\% } \\
\text { Cl) }\end{array}$ & $5.67[0.16,195.90]$ \\
\hline
\end{tabular}




\begin{tabular}{lllll}
\hline Outcome or subgroup title & No. of studies & $\begin{array}{l}\text { No. of partici- } \\
\text { pants }\end{array}$ & Statistical method & Effect size \\
\hline $\begin{array}{l}\text { 3.2 Existing school staff not involved in } \\
\text { delivery }\end{array}$ & 12 & & $\begin{array}{l}\text { Odds Ratio (Random, 95\% } \\
\text { Cl) }\end{array}$ & $1.14[0.91,1.42]$ \\
\hline
\end{tabular}

\section{Analysis 5.1. Comparison 5 Effects of school-based asthma interventions vs usual care subgrouped by involvement of school staff in direct delivery of self-management skills to children, Outcome 1 Exacerbations leading to emergency department (ED) visits.}

\begin{tabular}{|c|c|c|c|c|c|c|c|}
\hline Study or subgroup & Education & $\begin{array}{c}\text { Usual care/ } \\
\text { placebo } \\
\mathbf{N}\end{array}$ & $\begin{array}{c}\text { log[Odds } \\
\text { Ratio] } \\
\text { (SE) }\end{array}$ & & IV, Random, 95\% CI & Weight & $\begin{array}{c}\text { Odds Ratio } \\
\text { IV, Random, } 95 \% \mathrm{Cl}\end{array}$ \\
\hline \multicolumn{8}{|c|}{ 5.1.1 Teachers involved in delivery (with or without school nurses) } \\
\hline Atherly 2009 & 0 & 0 & $0(0.916)$ & & & $2.16 \%$ & $1.04[0.17,6.25]$ \\
\hline Clark 2005 & 0 & 0 & $-0(0.329)$ & & 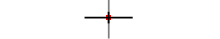 & $11.61 \%$ & $1[0.52,1.9]$ \\
\hline Subtotal $(95 \% \mathrm{Cl})$ & & & & & & $13.77 \%$ & $1[0.55,1.83]$ \\
\hline \multicolumn{8}{|c|}{ Test for overall effect: $Z=0(P=1)$} \\
\hline \multicolumn{8}{|c|}{ 5.1.2 School nurses alone involved in delivery } \\
\hline Persaud 1996 & 0 & 0 & $-1.3(0.737)$ & & & $3.22 \%$ & $0.29[0.07,1.21]$ \\
\hline Subtotal $(95 \% \mathrm{Cl})$ & & & & & & $3.22 \%$ & $0.29[0.07,1.21]$ \\
\hline \multicolumn{8}{|c|}{ Heterogeneity: Not applicable } \\
\hline \multicolumn{8}{|c|}{ 5.1.3 Existing school staff not involved in delivery } \\
\hline Bruzzese 2011 & 0 & 0 & $-0.5(0.218)$ & & $*$ & $18.19 \%$ & $0.59[0.39,0.91]$ \\
\hline Cicutto 2005 & 0 & 0 & $-0.4(0.408)$ & & $\rightarrow$ & $8.6 \%$ & $0.7[0.31,1.55]$ \\
\hline Cicutto 2013 & 0 & 0 & $-1.1(0.317)$ & & $\longrightarrow$ & $12.13 \%$ & $0.32[0.17,0.59]$ \\
\hline Horner 2008 & 0 & 0 & $-0.2(0.447)$ & & $\longrightarrow$ & $7.48 \%$ & $0.86[0.36,2.06]$ \\
\hline Horner 2015 & 0 & 0 & $0(0.306)$ & & 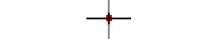 & $12.69 \%$ & $1[0.55,1.82]$ \\
\hline Howell 2005 & 0 & 0 & $-0.6(1.049)$ & & + & $1.67 \%$ & $0.55[0.07,4.29]$ \\
\hline Levy 2006 & 0 & 0 & $-0.5(0.316)$ & & $\rightarrow$ & $12.21 \%$ & $0.59[0.32,1.1]$ \\
\hline McGhan 2003 & 0 & 0 & $0.2(0.633)$ & & $\longrightarrow$ & $4.22 \%$ & $1.28[0.37,4.43]$ \\
\hline McGhan 2010 & 0 & 0 & $1(0.68)$ & & & $3.72 \%$ & $2.65[0.7,10.03]$ \\
\hline Velsor-Friedrich 2005 & 0 & 0 & $-0.5(0.933)$ & & +1 & $2.09 \%$ & $0.63[0.1,3.94]$ \\
\hline Subtotal $(95 \% \mathrm{CI})$ & & & & & 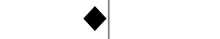 & $83.01 \%$ & $0.69[0.51,0.94]$ \\
\hline Total $(95 \% \mathrm{Cl})$ & & & & & $\nabla$ & $100 \%$ & $0.7[0.53,0.92]$ \\
\hline \multicolumn{8}{|c|}{ Heterogeneity: $\mathrm{Tau}^{2}=0.06 ; \mathrm{Chi}^{2}=16.16, \mathrm{df}=12(\mathrm{P}=0.18) ; \mathrm{I}^{2}=25.76 \%$} \\
\hline \multicolumn{8}{|c|}{ Test for overall effect: $Z=2.54(P=0.01)$} \\
\hline Test for subgroup diffe & $.78, d f=1(P=0$ & ), $I^{2}=27.94 \%$ & & & & & \\
\hline
\end{tabular}


Analysis 5.2. Comparison 5 Effects of school-based asthma interventions vs usual care subgrouped by involvement of school staff in direct delivery of self-management skills to children, Outcome 2 Absence from school.

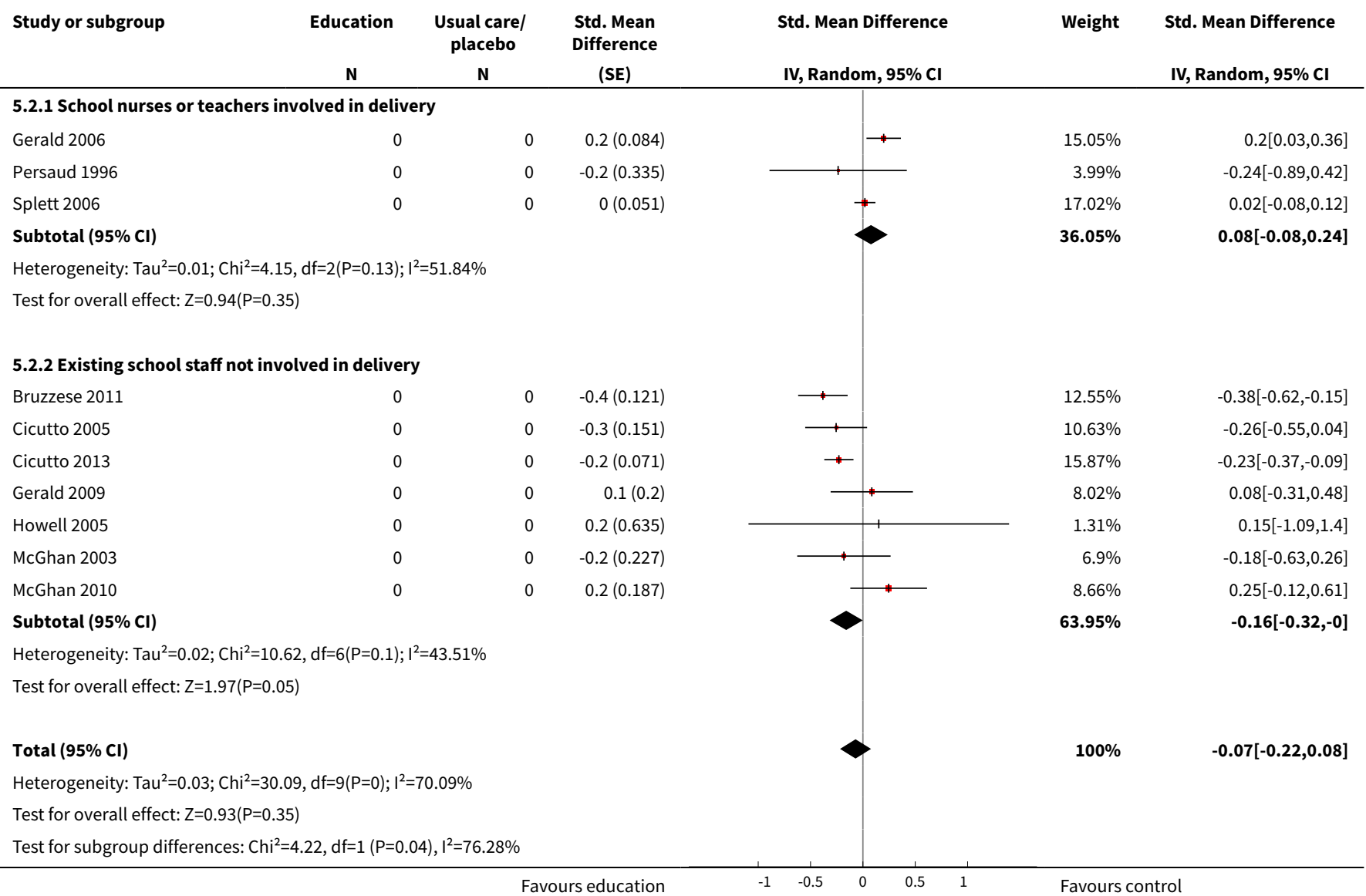

Analysis 5.3. Comparison 5 Effects of school-based asthma interventions vs usual care subgrouped by involvement of school staff in direct delivery of self-management skills to children, Outcome 3 Withdrawal from the study.

\begin{tabular}{|c|c|c|c|c|c|c|}
\hline Study or subgroup & Education & $\begin{array}{c}\text { Usual care/ } \\
\text { placebo } \\
\mathbf{N} \\
\end{array}$ & $\begin{array}{c}\text { log[Odds } \\
\text { Ratio] } \\
\text { (SE) }\end{array}$ & IV, Random, 95\% CI & Weight & $\begin{array}{c}\text { Odds Ratio } \\
\text { IV, Random, } 95 \% \text { CI }\end{array}$ \\
\hline \multicolumn{4}{|c|}{ 5.3.1 School nurses involved in delivery } & & & \\
\hline Patterson 2005 & 0 & 0 & $1.7(1.807)$ & 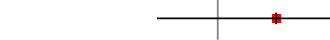 & $0.39 \%$ & $5.67[0.16,195.9]$ \\
\hline \multicolumn{4}{|l|}{ Subtotal $(95 \% \mathrm{CI})$} & & $0.39 \%$ & $5.67[0.16,195.9]$ \\
\hline \multicolumn{7}{|c|}{ Heterogeneity: Not applicable } \\
\hline \multicolumn{7}{|c|}{ 5.3.2 Existing school staff not involved in delivery } \\
\hline Al-Sheyab 2012 & 0 & 0 & $-0.7(1.074)$ & & $1.11 \%$ & $0.51[0.06,4.2]$ \\
\hline Bartholomew 2006 & 0 & 0 & $0.2(0.173)$ & \# & $42.89 \%$ & $1.27[0.9,1.78]$ \\
\hline Bruzzese 2008 & 0 & 0 & $-1.2(1.683)$ & *- & $0.45 \%$ & $0.31[0.01,8.31]$ \\
\hline Bruzzese 2011 & 0 & 0 & $0.3(0.279)$ & +- & $16.52 \%$ & $1.31[0.76,2.27]$ \\
\hline Gerald 2009 & 0 & 0 & $-0.5(0.368)$ & $\rightarrow$ & $9.49 \%$ & $0.61[0.3,1.26]$ \\
\hline Horner 2008 & 0 & 0 & $0.3(0.531)$ & 1 & $4.56 \%$ & $1.33[0.47,3.77]$ \\
\hline
\end{tabular}




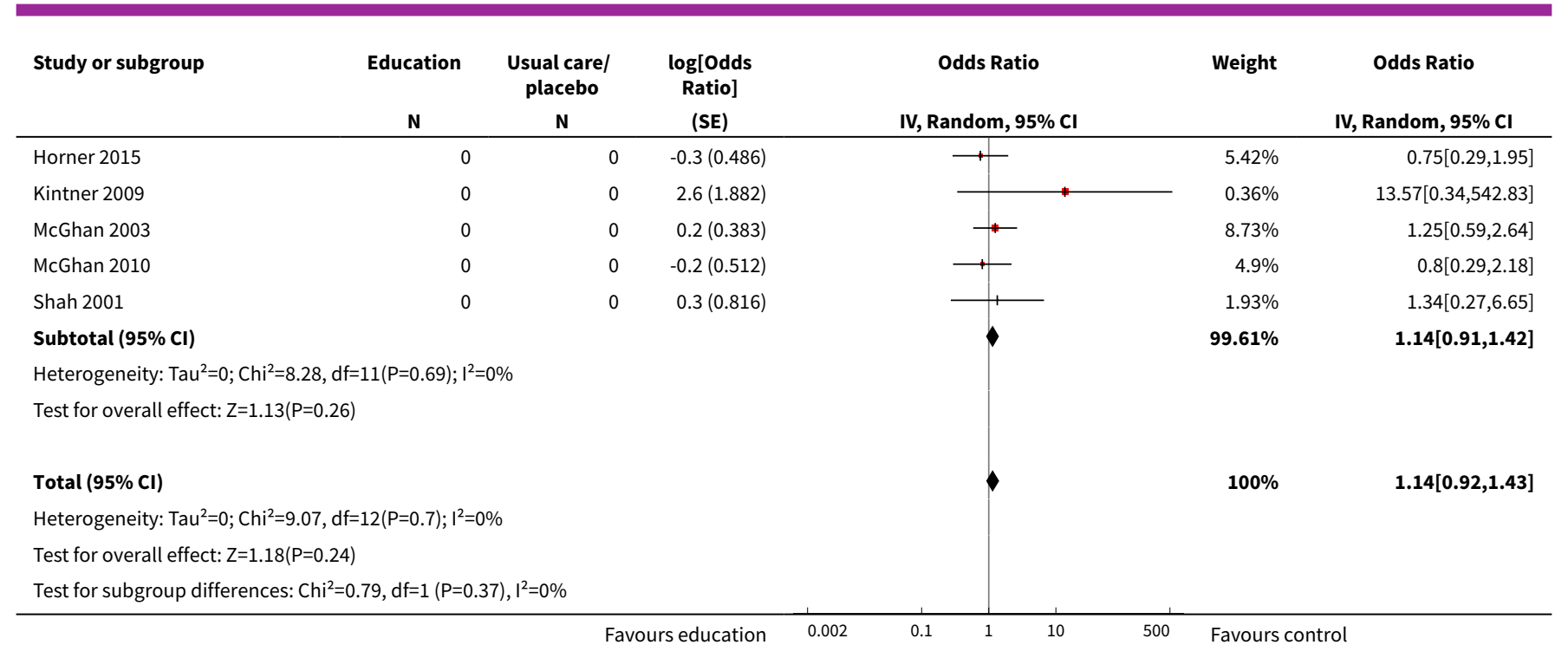

\section{Comparison 6. Effects of school-based asthma interventions vs usual care subgrouped by explicit use of theory}

\begin{tabular}{|c|c|c|c|c|}
\hline Outcome or subgroup title & No. of studies & $\begin{array}{l}\text { No. of partici- } \\
\text { pants }\end{array}$ & Statistical method & Effect size \\
\hline $\begin{array}{l}1 \text { Exacerbations leading to emer- } \\
\text { gency department (ED) visits }\end{array}$ & 13 & & Odds Ratio (Random, 95\% Cl) & $0.70[0.53,0.92]$ \\
\hline $\begin{array}{l}\text { 1.1 Theoretical framework utilised } \\
\text { explicitly }\end{array}$ & 10 & & Odds Ratio (Random, 95\% Cl) & $0.75[0.54,1.04]$ \\
\hline 1.2 Use of theory not explicit & 3 & & Odds Ratio (Random, 95\% Cl) & $0.56[0.33,0.97]$ \\
\hline 2 Absence from school & 10 & & $\begin{array}{l}\text { Std. Mean Difference (Ran- } \\
\text { dom, } 95 \% \mathrm{Cl} \text { ) }\end{array}$ & $-0.07[-0.22,0.08]$ \\
\hline $\begin{array}{l}2.1 \text { Theoretical framework utilised } \\
\text { explicitly }\end{array}$ & 6 & & $\begin{array}{l}\text { Std. Mean Difference (Ran- } \\
\text { dom, } 95 \% \mathrm{Cl} \text { ) }\end{array}$ & $-0.19[-0.35,-0.03]$ \\
\hline 2.2 Use of theory not explicit & 4 & & $\begin{array}{l}\text { Std. Mean Difference (Ran- } \\
\text { dom, } 95 \% \mathrm{Cl} \text { ) }\end{array}$ & $0.08[-0.05,0.20]$ \\
\hline 3 Withdrawal from the study & 13 & & Odds Ratio (Random, 95\% Cl) & $1.14[0.92,1.43]$ \\
\hline $\begin{array}{l}3.1 \text { Theoretical framework utilised } \\
\text { explicitly }\end{array}$ & 12 & & Odds Ratio (Random, 95\% Cl) & $1.22[0.97,1.54]$ \\
\hline 3.2 Use of theory not explicit & 1 & & Odds Ratio (Random, 95\% Cl) & $0.61[0.30,1.26]$ \\
\hline
\end{tabular}


Analysis 6.1. Comparison 6 Effects of school-based asthma interventions vs usual care subgrouped by explicit use of theory, Outcome 1 Exacerbations leading to emergency department (ED) visits.

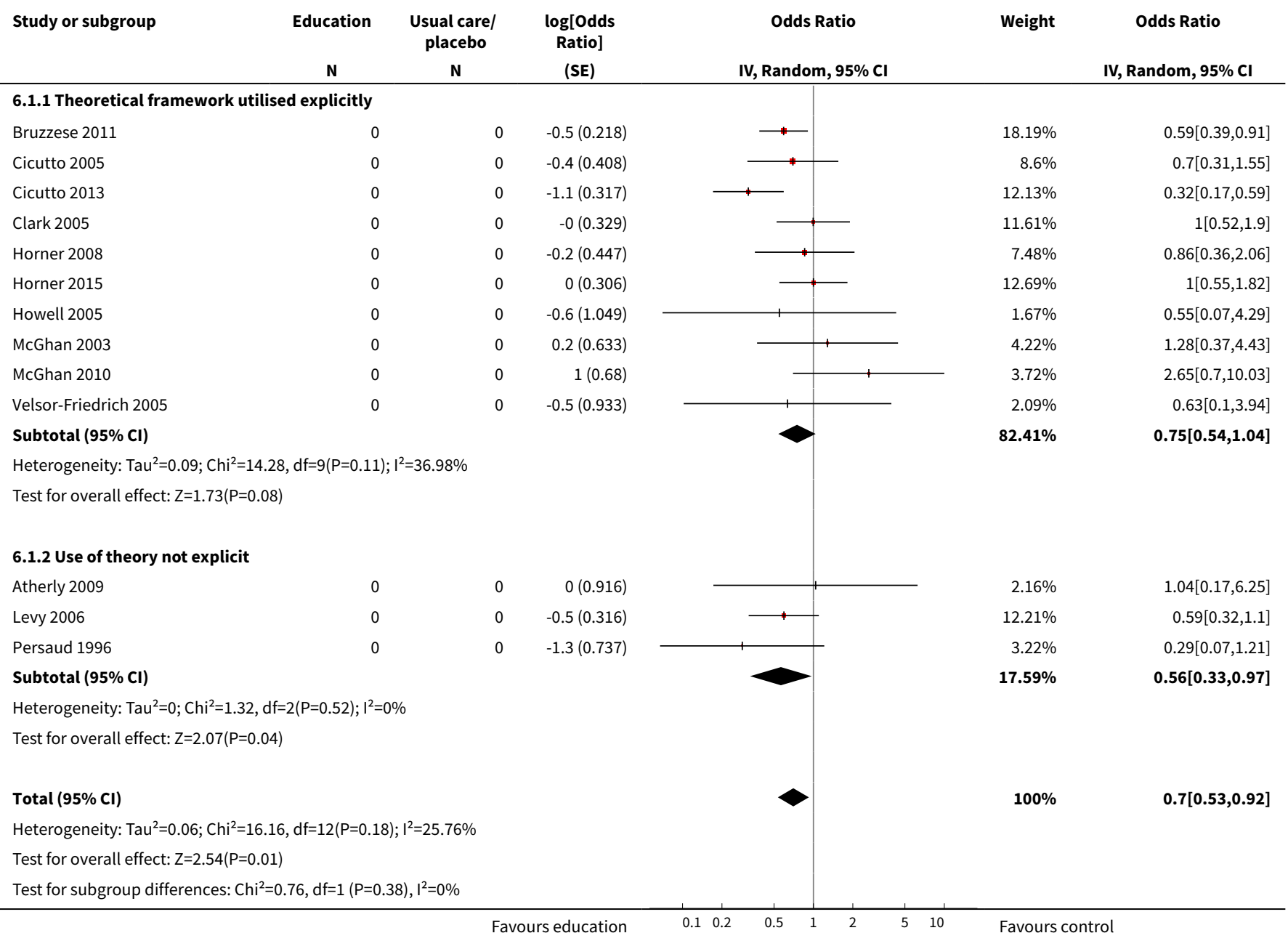

Analysis 6.2. Comparison 6 Effects of school-based asthma interventions vs usual care subgrouped by explicit use of theory, Outcome 2 Absence from school.

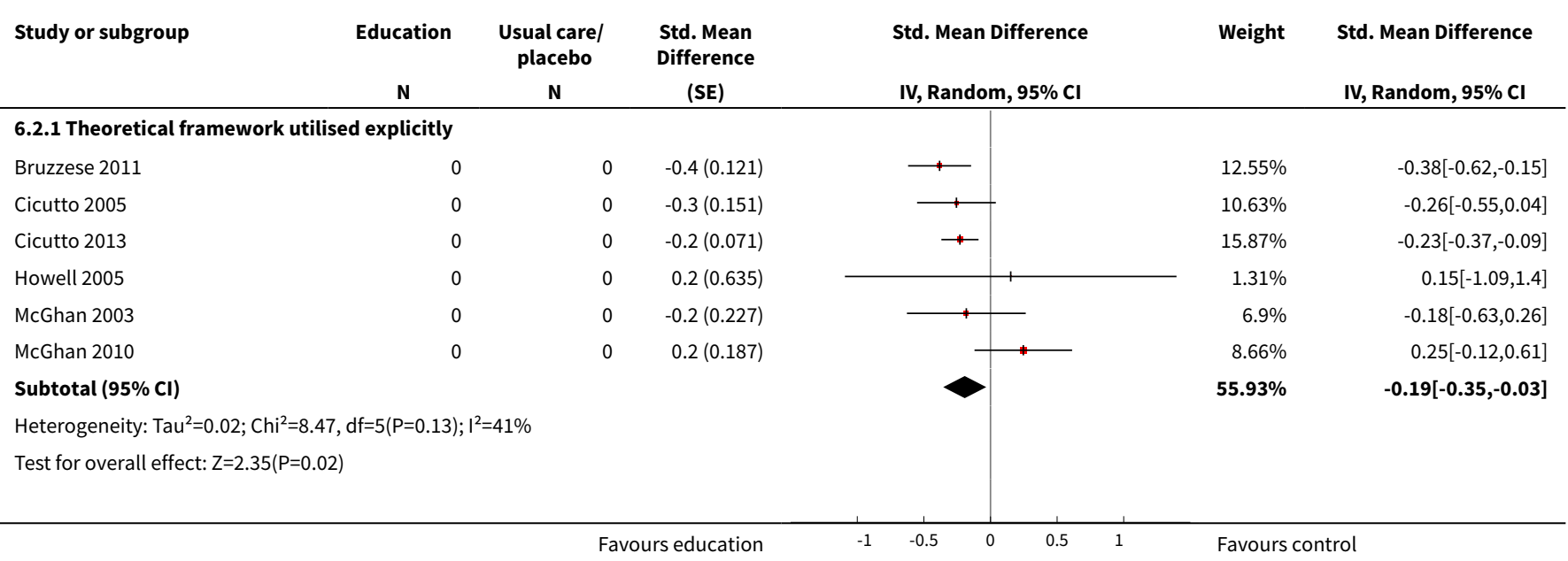




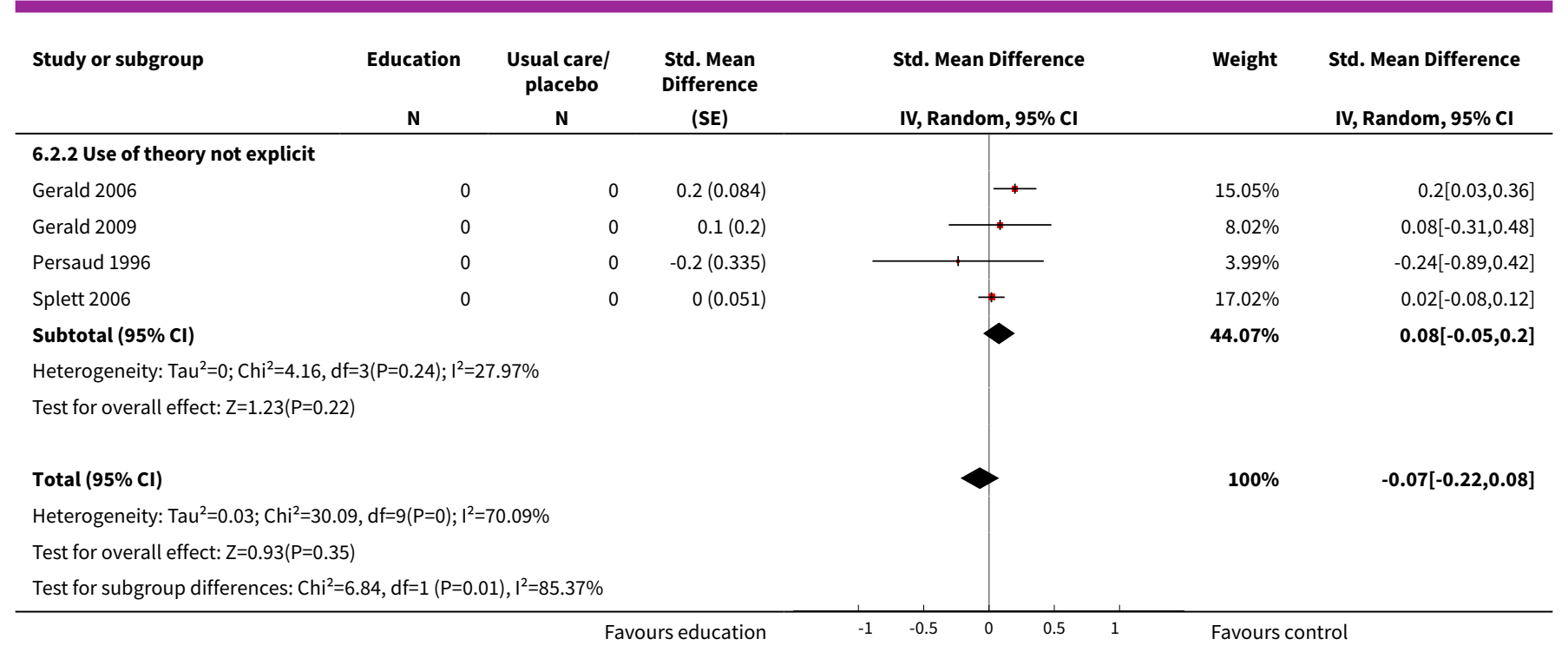

Analysis 6.3. Comparison 6 Effects of school-based asthma interventions vs usual care subgrouped by explicit use of theory, Outcome 3 Withdrawal from the study.

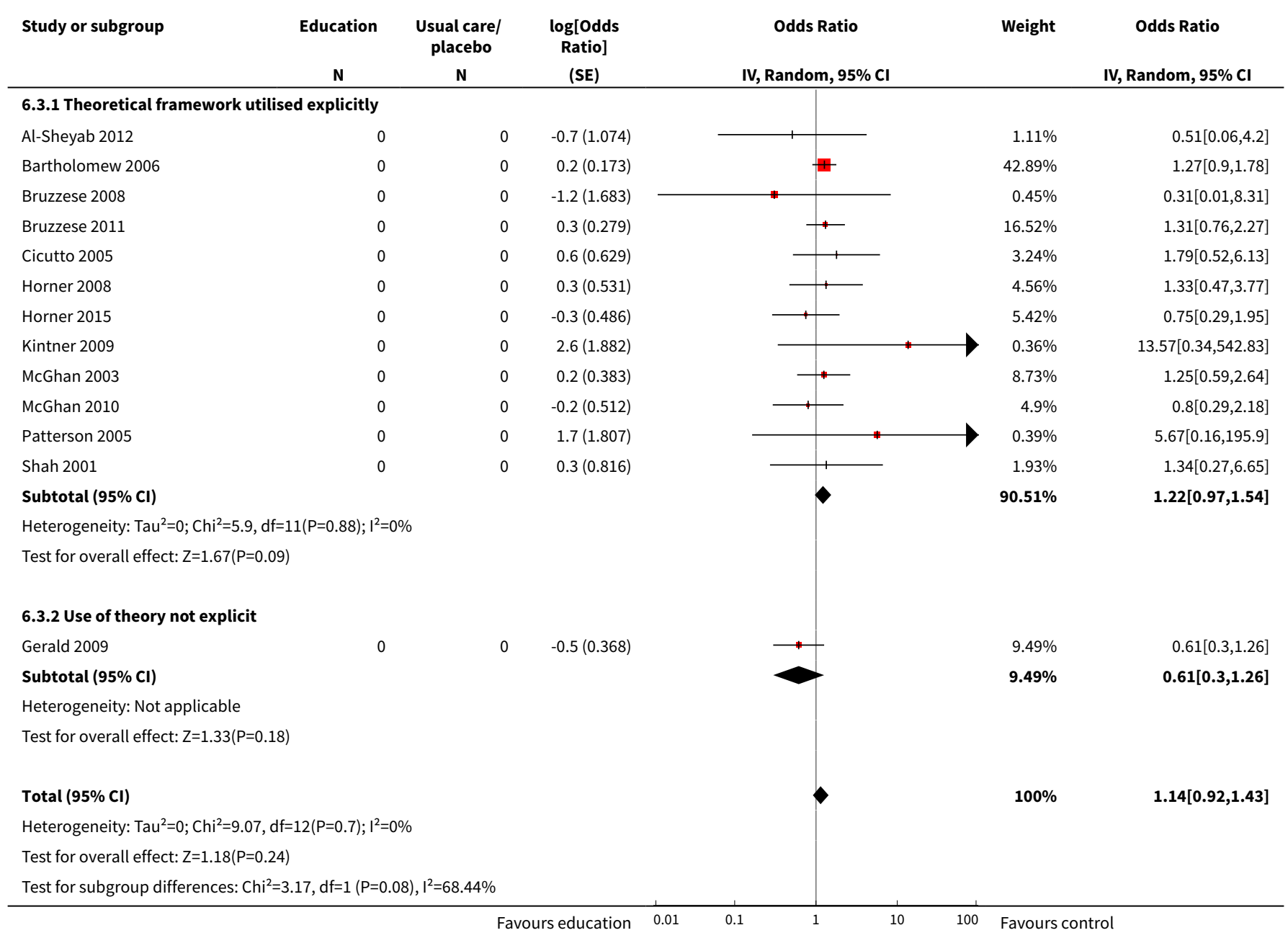


Comparison 7. Effects of school-based asthma interventions vs usual care subgrouped by whether design included active inclusion or participation of parents

\begin{tabular}{|c|c|c|c|c|}
\hline Outcome or subgroup title & No. of studies & $\begin{array}{l}\text { No. of partici- } \\
\text { pants }\end{array}$ & Statistical method & Effect size \\
\hline $\begin{array}{l}1 \text { Exacerbations leading to } \\
\text { emergency department (ED) vis- } \\
\text { its }\end{array}$ & 13 & & Odds Ratio (Random, 95\% Cl) & $0.70[0.53,0.92]$ \\
\hline 1.1 Parents actively included & 8 & & Odds Ratio (Random, 95\% Cl) & $0.82[0.53,1.25]$ \\
\hline 1.2 Not included/unclear & 5 & & Odds Ratio (Random, 95\% Cl) & $0.58[0.42,0.81]$ \\
\hline 2 Absence from school & 10 & & $\begin{array}{l}\text { Std. Mean Difference (Random, } \\
95 \% \mathrm{Cl} \text { ) }\end{array}$ & $-0.07[-0.22,0.08]$ \\
\hline 2.1 Parents actively included & 7 & & $\begin{array}{l}\text { Std. Mean Difference (Random, } \\
95 \% \mathrm{Cl} \text { ) }\end{array}$ & $-0.02[-0.23,0.18]$ \\
\hline 2.2 Not included/unclear & 3 & & $\begin{array}{l}\text { Std. Mean Difference (Random, } \\
95 \% \mathrm{Cl} \text { ) }\end{array}$ & $-0.18[-0.50,0.15]$ \\
\hline 3 Withdrawal from the study & 13 & & Odds Ratio (Random, 95\% Cl) & $1.14[0.92,1.43]$ \\
\hline 3.1 Parents actively included & 9 & & Odds Ratio (Random, 95\% Cl) & $1.21[0.93,1.58]$ \\
\hline 3.2 Not included/unclear & 4 & & Odds Ratio (Random, 95\% Cl) & $0.97[0.62,1.53]$ \\
\hline
\end{tabular}

Analysis 7.1. Comparison 7 Effects of school-based asthma interventions vs usual care subgrouped by whether design included active inclusion or participation of parents, Outcome 1 Exacerbations leading to emergency department (ED) visits.

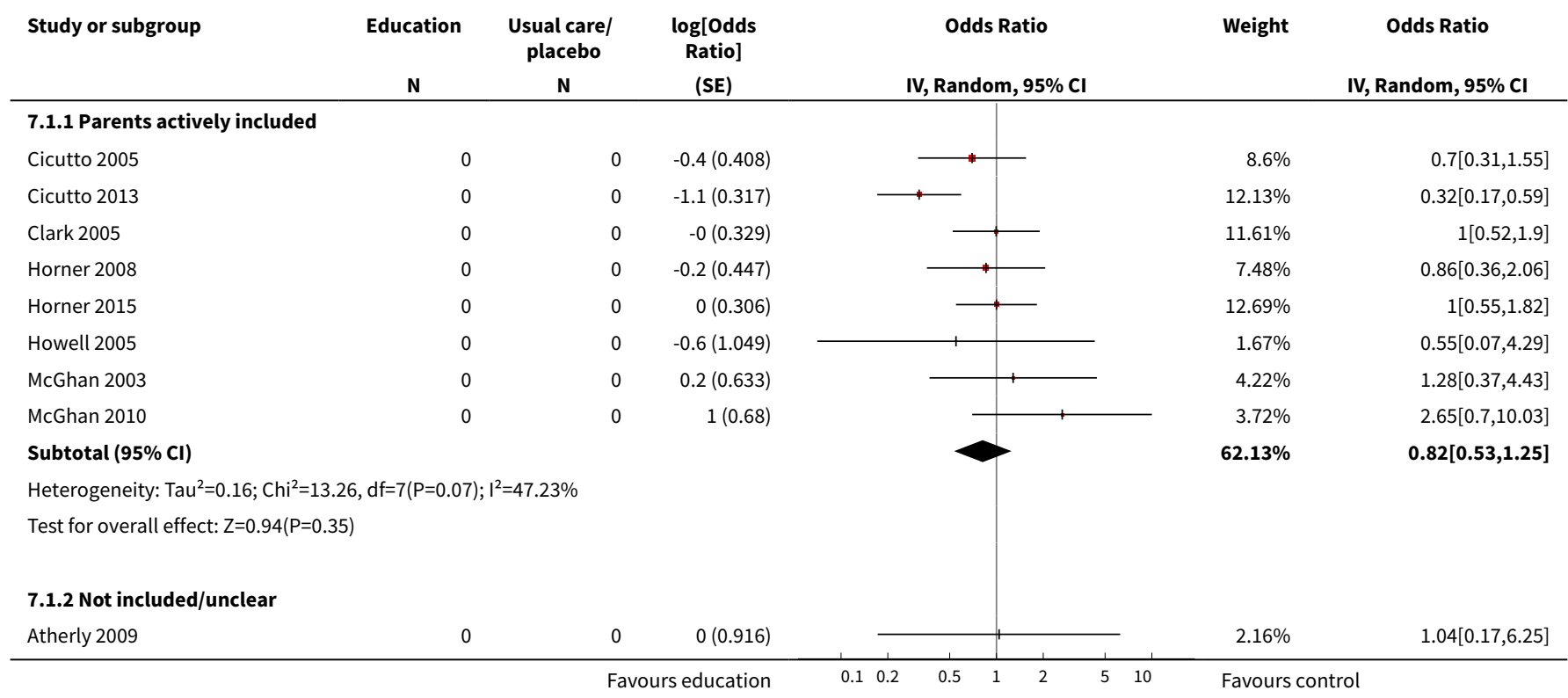




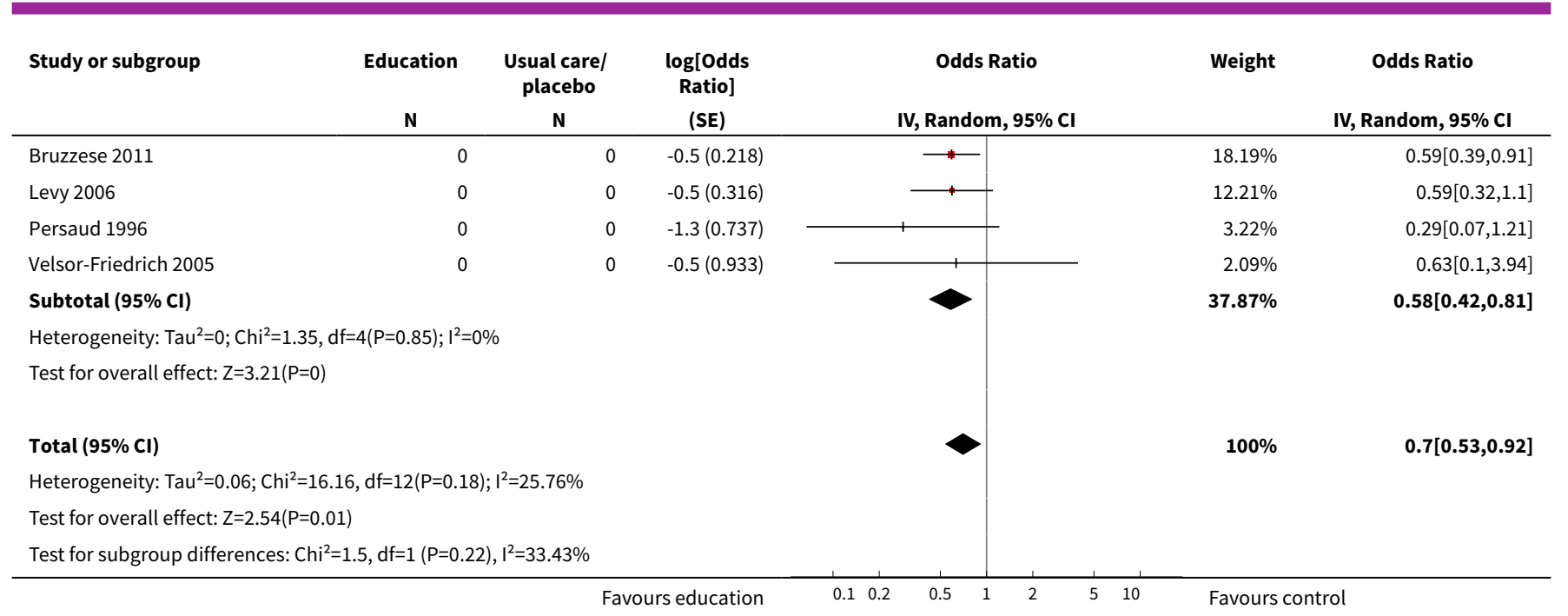

\section{Analysis 7.2. Comparison 7 Effects of school-based asthma interventions vs usual care subgrouped by whether design included active inclusion or participation of parents, Outcome 2 Absence from school.}

\begin{tabular}{|c|c|c|c|c|c|c|}
\hline Study or subgroup & $\begin{array}{c}\text { Education } \\
\mathbf{N} \\
\end{array}$ & $\begin{array}{c}\text { Usual care/ } \\
\text { placebo }\end{array}$ & $\begin{array}{l}\text { Std. Mean } \\
\text { Difference } \\
\text { (SE) } \\
\end{array}$ & $\begin{array}{l}\text { Std. Mean Difference } \\
\text { IV, Random, 95\% CI }\end{array}$ & Weight & $\begin{array}{l}\text { Std. Mean Difference } \\
\text { IV, Random, } 95 \% \mathrm{CI}\end{array}$ \\
\hline \multicolumn{4}{|c|}{ 7.2.1 Parents actively included } & & & \\
\hline Cicutto 2005 & 0 & 0 & $-0.3(0.151)$ & $\longrightarrow$ & $10.63 \%$ & $-0.26[-0.55,0.04]$ \\
\hline Cicutto 2013 & 0 & 0 & $-0.2(0.071)$ & $\rightarrow$ & $15.87 \%$ & $-0.23[-0.37,-0.09]$ \\
\hline Gerald 2006 & 0 & 0 & $0.2(0.084)$ & $\longrightarrow$ & $15.05 \%$ & $0.2[0.03,0.36]$ \\
\hline Howell 2005 & 0 & 0 & $0.2(0.635)$ & & $1.31 \%$ & $0.15[-1.09,1.4]$ \\
\hline McGhan 2003 & 0 & 0 & $-0.2(0.227)$ & - & $6.9 \%$ & $-0.18[-0.63,0.26]$ \\
\hline McGhan 2010 & 0 & 0 & $0.2(0.187)$ & 4 & $8.66 \%$ & $0.25[-0.12,0.61]$ \\
\hline Subtotal $(95 \% \mathrm{Cl})$ & & & & & $66.44 \%$ & $-0.02[-0.23,0.18]$ \\
\hline \multicolumn{7}{|c|}{ Heterogeneity: Tau $^{2}=0.04 ; \mathrm{Chi}^{2}=20.41, \mathrm{df}=6(\mathrm{P}=0) ; \mathrm{I}^{2}=70.6 \%$} \\
\hline \multicolumn{7}{|c|}{ Test for overall effect: $Z=0.21(P=0.83)$} \\
\hline \multicolumn{7}{|c|}{ 7.2.2 Not included/unclear } \\
\hline Bruzzese 2011 & 0 & 0 & $-0.4(0.121)$ & $\longrightarrow$ & $12.55 \%$ & $-0.38[-0.62,-0.15]$ \\
\hline Persaud 1996 & 0 & 0 & $-0.2(0.335)$ & & $3.99 \%$ & $-0.24[-0.89,0.42]$ \\
\hline Splett 2006 & 0 & 0 & $0(0.051)$ & - & $17.02 \%$ & $0.02[-0.08,0.12]$ \\
\hline Subtotal $(95 \% \mathrm{Cl})$ & & & & & $33.56 \%$ & $-0.18[-0.5,0.15]$ \\
\hline \multicolumn{7}{|c|}{ Heterogeneity: $\mathrm{Tau}^{2}=0.06 ; \mathrm{Chi}^{2}=9.68, \mathrm{df}=2(\mathrm{P}=0.01) ; \mathrm{I}^{2}=79.35 \%$} \\
\hline \multicolumn{7}{|c|}{ Test for overall effect: $Z=1.05(P=0.29)$} \\
\hline Total $(95 \% \mathrm{Cl})$ & & & & & $100 \%$ & $-0.07[-0.22,0.08]$ \\
\hline \multicolumn{7}{|c|}{ Heterogeneity: $\mathrm{Tau}^{2}=0.03 ; \mathrm{Chi}^{2}=30.09, \mathrm{df}=9(\mathrm{P}=0) ; \mathrm{I}^{2}=70.09 \%$} \\
\hline \multicolumn{7}{|c|}{ Test for overall effect: $\mathrm{Z}=0.93(\mathrm{P}=0.35)$} \\
\hline Test for subgroup dif & $61, \mathrm{df}=1(\mathrm{P}=0$. & ), $I^{2}=0 \%$ & & & & \\
\hline
\end{tabular}


Analysis 7.3. Comparison 7 Effects of school-based asthma interventions vs usual care subgrouped by whether design included active inclusion or participation of parents, Outcome 3 Withdrawal from the study.

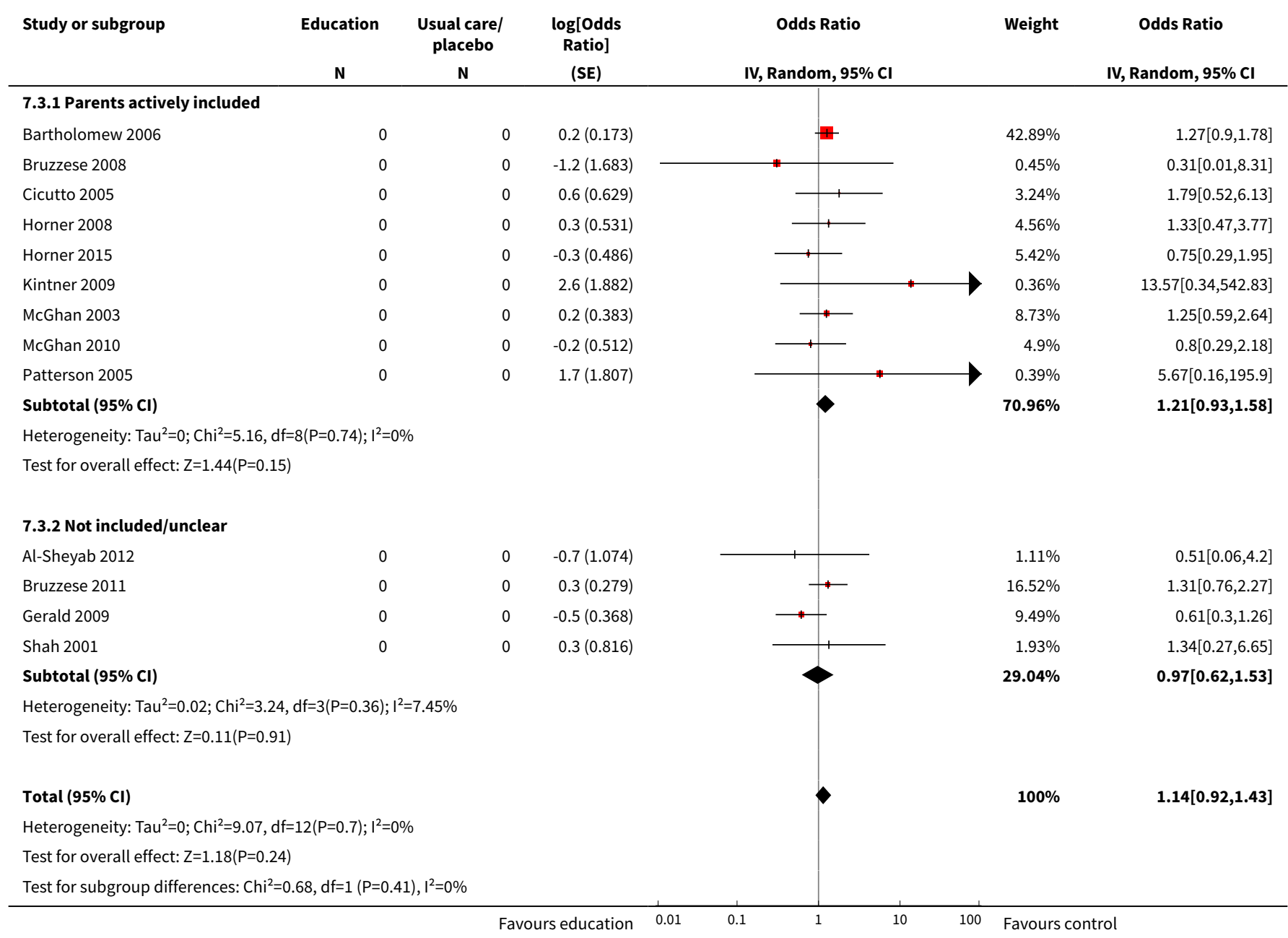

\section{Comparison 8. Effects of school-based asthma interventions vs usual care subgrouped by timing of intervention}

\begin{tabular}{|c|c|c|c|c|}
\hline Outcome or subgroup title & No. of studies & $\begin{array}{l}\text { No. of partici- } \\
\text { pants }\end{array}$ & Statistical method & Effect size \\
\hline $\begin{array}{l}1 \text { Exacerbations leading to emergency de- } \\
\text { partment (ED) visits }\end{array}$ & 13 & & $\begin{array}{l}\text { Odds Ratio (Random, } \\
95 \% \mathrm{Cl} \text { ) }\end{array}$ & $0.70[0.53,0.92]$ \\
\hline $\begin{array}{l}1.1 \text { Intervention mainly delivered during } \\
\text { students' free time }\end{array}$ & 5 & & $\begin{array}{l}\text { Odds Ratio (Random, } \\
95 \% \mathrm{Cl} \text { ) }\end{array}$ & $0.71[0.45,1.13]$ \\
\hline $\begin{array}{l}1.2 \text { Intervention took place during school } \\
\text { day (exact time unclear or variable) }\end{array}$ & 8 & & $\begin{array}{l}\text { Odds Ratio (Random, } \\
95 \% \mathrm{Cl} \text { ) }\end{array}$ & $0.67[0.48,0.92]$ \\
\hline 2 Absence from school & 10 & & $\begin{array}{l}\text { Std. Mean Difference } \\
\text { (Random, 95\% Cl) }\end{array}$ & $-0.07[-0.22,0.08]$ \\
\hline $\begin{array}{l}2.1 \text { Intervention mainly delivered during } \\
\text { students' free time }\end{array}$ & 2 & & $\begin{array}{l}\text { Std. Mean Difference } \\
\text { (Random, } 95 \% \mathrm{Cl} \text { ) }\end{array}$ & $-0.23[-0.36,-0.11]$ \\
\hline
\end{tabular}

School-based self-management interventions for asthma in children and adolescents: a mixed methods systematic review (Review) 


\begin{tabular}{|c|c|c|c|c|}
\hline Outcome or subgroup title & No. of studies & $\begin{array}{l}\text { No. of partici- } \\
\text { pants }\end{array}$ & Statistical method & Effect size \\
\hline $\begin{array}{l}2.2 \text { Intervention took place during school } \\
\text { day (exact time unclear or variable) }\end{array}$ & 8 & & $\begin{array}{l}\text { Std. Mean Difference } \\
\text { (Random, 95\% Cl) }\end{array}$ & $-0.01[-0.17,0.16]$ \\
\hline 3 Withdrawal from the study & 13 & & $\begin{array}{l}\text { Odds Ratio (Random, } \\
95 \% \mathrm{Cl} \text { ) }\end{array}$ & $1.14[0.92,1.43]$ \\
\hline $\begin{array}{l}3.1 \text { Intervention took place during class } \\
\text { time }\end{array}$ & 1 & & $\begin{array}{l}\text { Odds Ratio (Random, } \\
95 \% \mathrm{Cl} \text { ) }\end{array}$ & $\begin{array}{l}13.57[0.34 \\
542.83]\end{array}$ \\
\hline $\begin{array}{l}3.2 \text { Intervention mainly delivered during } \\
\text { students' free time }\end{array}$ & 4 & & $\begin{array}{l}\text { Odds Ratio (Random, } \\
95 \% \mathrm{Cl} \text { ) }\end{array}$ & $1.19[0.65,2.16]$ \\
\hline $\begin{array}{l}3.3 \text { Intervention took place during school } \\
\text { day (exact time unclear or variable) }\end{array}$ & 8 & & $\begin{array}{l}\text { Odds Ratio (Random, } \\
95 \% \mathrm{Cl} \text { ) }\end{array}$ & $1.13[0.89,1.43]$ \\
\hline
\end{tabular}

\section{Analysis 8.1. Comparison 8 Effects of school-based asthma interventions vs usual care subgrouped by timing of intervention, Outcome 1 Exacerbations leading to emergency department (ED) visits.}

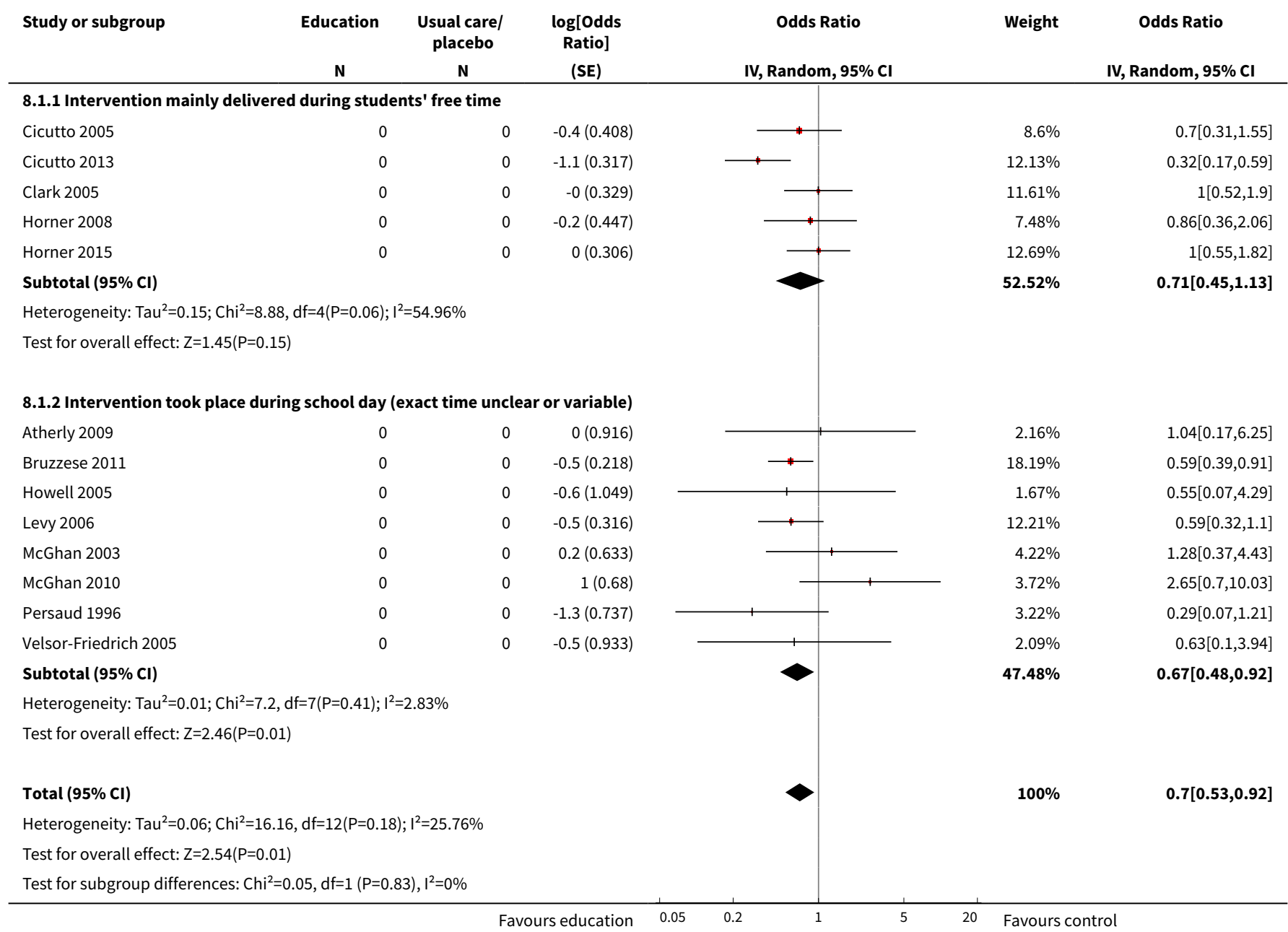




\section{Analysis 8.2. Comparison 8 Effects of school-based asthma interventions vs usual care subgrouped by timing of intervention, Outcome 2 Absence from school.}

\begin{tabular}{|c|c|c|c|}
\hline Study or subgroup & $\begin{array}{c}\text { Education } \\
\mathrm{N} \\
\end{array}$ & $\begin{array}{c}\text { Usual care/ } \\
\text { placebo } \\
\mathbf{N} \\
\end{array}$ & $\begin{array}{c}\text { Std. Mean } \\
\text { Difference } \\
\text { (SE) }\end{array}$ \\
\hline \multicolumn{4}{|c|}{ 8.2.1 Intervention mainly delivered during students' free time } \\
\hline Cicutto 2005 & 0 & 0 & $-0.3(0.151)$ \\
\hline Cicutto 2013 & 0 & 0 & $-0.2(0.071)$ \\
\hline \multicolumn{4}{|l|}{ Subtotal $(95 \% \mathrm{Cl})$} \\
\hline \multicolumn{4}{|c|}{ Heterogeneity: $\mathrm{Tau}^{2}=0 ; \mathrm{Chi}^{2}=0.02, \mathrm{df}=1(\mathrm{P}=0.87) ; \mathrm{I}^{2}=0 \%$} \\
\hline \multicolumn{4}{|c|}{ Test for overall effect: $Z=3.64(P=0)$} \\
\hline \multicolumn{4}{|c|}{ 8.2.2 Intervention took place during school day (exact time unclear or variable) } \\
\hline Bruzzese 2011 & 0 & 0 & $-0.4(0.121)$ \\
\hline Gerald 2006 & 0 & 0 & $0.2(0.084)$ \\
\hline Gerald 2009 & 0 & 0 & $0.1(0.2)$ \\
\hline Howell 2005 & 0 & 0 & $0.2(0.635)$ \\
\hline McGhan 2003 & 0 & 0 & $-0.2(0.227)$ \\
\hline McGhan 2010 & 0 & 0 & $0.2(0.187)$ \\
\hline Persaud 1996 & 0 & 0 & $-0.2(0.335)$ \\
\hline Splett 2006 & 0 & 0 & $0(0.051)$ \\
\hline
\end{tabular}

Std. Mean Difference

IV, Random, $95 \% \mathrm{CI}$
Weight Std. Mean Difference

IV, Random, 95\% CI

\section{Subtotal $(95 \% \mathrm{CI})$}

Heterogeneity: $\mathrm{Tau}^{2}=0.03 ; \mathrm{Chi}^{2}=18.64, \mathrm{df}=7(\mathrm{P}=0.01) ; \mathrm{I}^{2}=62.44 \%$

Test for overall effect: $Z=0.1(P=0.92)$

Total $(95 \% \mathrm{Cl})$

Heterogeneity: $\mathrm{Tau}^{2}=0.03 ; \mathrm{Chi}^{2}=30.09, \mathrm{df}=9(\mathrm{P}=0) ; \mathrm{I}^{2}=70.09 \%$

Test for overall effect: $\mathrm{Z}=0.93(\mathrm{P}=0.35)$

Test for subgroup differences: $\mathrm{Chi}^{2}=4.49, \mathrm{df}=1(\mathrm{P}=0.03), \mathrm{I}^{2}=77.73 \%$

$10.63 \%$

$15.87 \%$

$26.5 \%$
$-0.26[-0.55,0.04]$

$-0.23[-0.37,-0.09]$

$-0.23[-0.36,-0.11]$
$-0.38[-0.62,-0.15]$
$0.2[0.03,0.36]$
$0.08[-0.31,0.48]$
$0.15[-1.09,1.4]$
$-0.18[-0.63,0.26]$
$0.25[-0.12,0.61]$
$-0.24[-0.89,0.42]$
$0.02[-0.08,0.12]$
$-\mathbf{0 . 0 1}[-0.17,0.16]$

$100 \%$

$-0.07[-0.22,0.08]$

Analysis 8.3. Comparison 8 Effects of school-based asthma interventions vs usual care subgrouped by timing of intervention, Outcome 3 Withdrawal from the study.

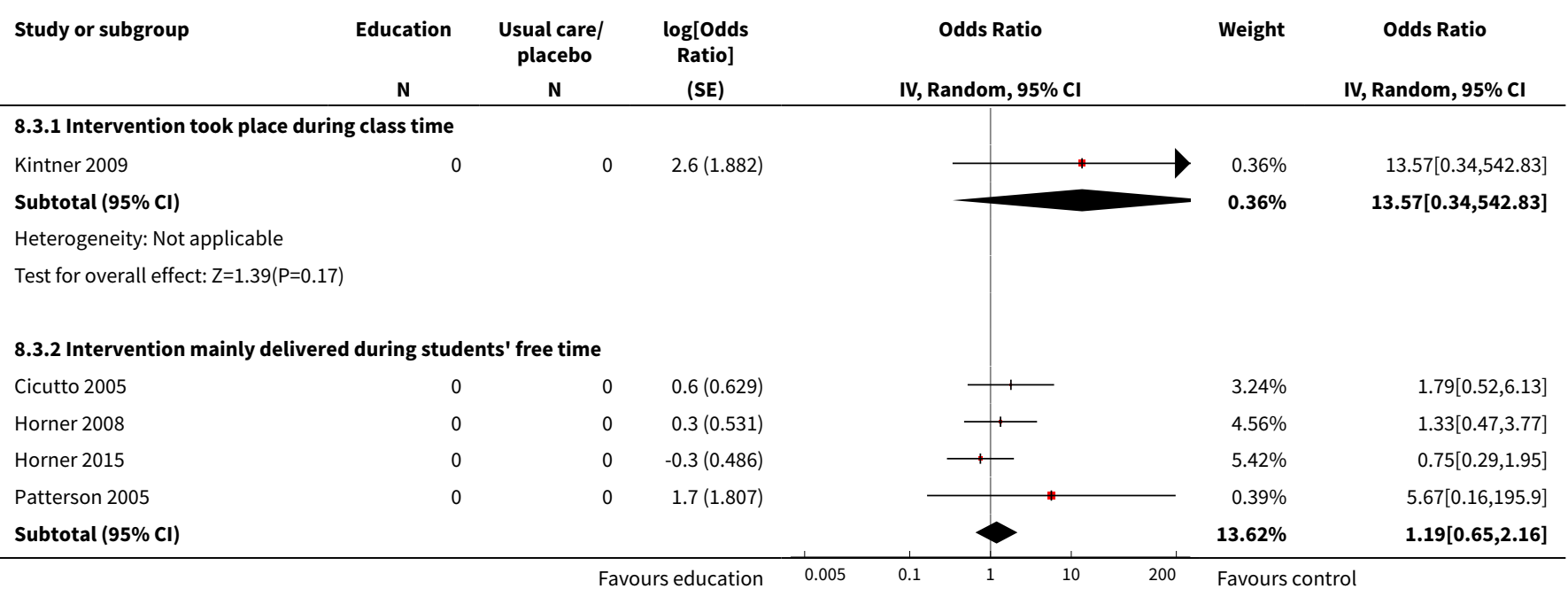




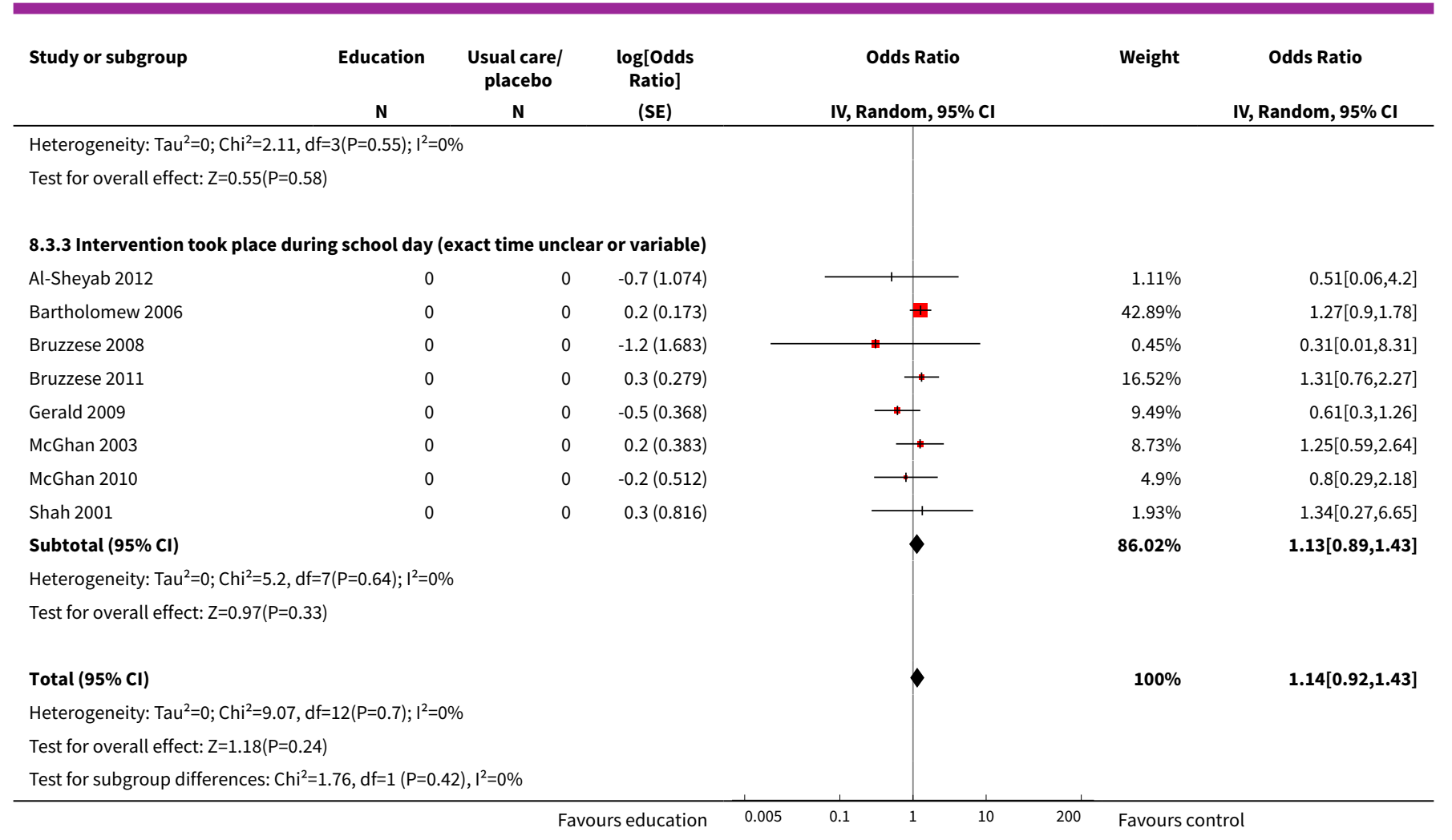

\section{Comparison 9. Effects of school-based asthma interventions vs usual care subgrouped by configuration of conditions}

\begin{tabular}{|c|c|c|c|c|}
\hline Outcome or subgroup title & No. of studies & $\begin{array}{l}\text { No. of partici- } \\
\text { pants }\end{array}$ & Statistical method & Effect size \\
\hline $\begin{array}{l}1 \text { Exacerbations leading to emergency } \\
\text { department (ED) visits }\end{array}$ & 13 & & $\begin{array}{l}\text { Odds Ratio (Random, 95\% } \\
\mathrm{Cl} \text { ) }\end{array}$ & $0.70[0.53,0.92]$ \\
\hline $\begin{array}{l}\text { 1.1 PI - theory, not in own time, no sub- } \\
\text { stantial school nurse involvement }\end{array}$ & 5 & & $\begin{array}{l}\text { Odds Ratio (Random, 95\% } \\
\mathrm{Cl} \text { ) }\end{array}$ & $0.85[0.47,1.52]$ \\
\hline $\begin{array}{l}1.2 \mathrm{PI} 2 \text { - theory, not individual, substan- } \\
\text { tial school nurse involvement }\end{array}$ & 0 & & $\begin{array}{l}\text { Odds Ratio (Random, 95\% } \\
\mathrm{Cl} \text { ) }\end{array}$ & $0.0[0.0,0.0]$ \\
\hline 1.3 Other configuration & 8 & & $\begin{array}{l}\text { Odds Ratio (Random, 95\% } \\
\mathrm{Cl} \text { ) }\end{array}$ & $0.67[0.47,0.94]$ \\
\hline 2 Absence from school & 10 & & $\begin{array}{l}\text { Std. Mean Difference (Ran- } \\
\text { dom, } 95 \% \mathrm{Cl} \text { ) }\end{array}$ & $-0.07[-0.22,0.08]$ \\
\hline $\begin{array}{l}\text { 2.1 PI1 - theory, not in own time, no sub- } \\
\text { stantial school nurse involvement }\end{array}$ & 4 & & $\begin{array}{l}\text { Std. Mean Difference (Ran- } \\
\text { dom, } 95 \% \mathrm{Cl} \text { ) }\end{array}$ & $-0.10[-0.46,0.25]$ \\
\hline $\begin{array}{l}2.2 \mathrm{PI} 2 \text { - theory, not individual, substan- } \\
\text { tial school nurse involvement }\end{array}$ & 0 & & $\begin{array}{l}\text { Std. Mean Difference (Ran- } \\
\text { dom, } 95 \% \mathrm{Cl} \text { ) }\end{array}$ & $0.0[0.0,0.0]$ \\
\hline 2.3 Other configuration & 6 & & $\begin{array}{l}\text { Std. Mean Difference (Ran- } \\
\text { dom, } 95 \% \mathrm{Cl} \text { ) }\end{array}$ & $-0.05[-0.21,0.12]$ \\
\hline
\end{tabular}




\begin{tabular}{|c|c|c|c|c|}
\hline Outcome or subgroup title & No. of studies & $\begin{array}{l}\text { No. of partici- } \\
\text { pants }\end{array}$ & Statistical method & Effect size \\
\hline 3 Withdrawal from the study & 13 & & $\begin{array}{l}\text { Odds Ratio (Random, 95\% } \\
\mathrm{Cl} \text { ) }\end{array}$ & $1.14[0.92,1.43]$ \\
\hline $\begin{array}{l}\text { 3.1 PI1 - theory, not in own time, no sub- } \\
\text { stantial school nurse involvement }\end{array}$ & 4 & & $\begin{array}{l}\text { Odds Ratio (Random, 95\% } \\
\text { Cl) }\end{array}$ & $0.88[0.55,1.40]$ \\
\hline $\begin{array}{l}\text { 3.2 PI2 - theory, not individual, substan- } \\
\text { tial school nurse involvement }\end{array}$ & 1 & & $\begin{array}{l}\text { Odds Ratio (Random, 95\% } \\
\text { Cl) }\end{array}$ & $5.67[0.16,195.90]$ \\
\hline 3.3 Other configuration & 8 & & $\begin{array}{l}\text { Odds Ratio (Random, 95\% } \\
\mathrm{Cl} \text { ) }\end{array}$ & $1.23[0.95,1.58]$ \\
\hline
\end{tabular}

\section{Analysis 9.1. Comparison 9 Effects of school-based asthma interventions vs usual care subgrouped by configuration of conditions, Outcome 1 Exacerbations leading to emergency department (ED) visits.}

\begin{tabular}{lccc} 
Study or subgroup & Education & $\begin{array}{c}\text { Usual care/ } \\
\text { placebo }\end{array}$ & $\begin{array}{c}\text { log[Odds } \\
\text { Ratio] } \\
\text { (SE) }\end{array}$ \\
\hline 9.1.1 PI1 - theory, not in own time, no substantial school nurse involvement \\
Bruzzese 2011 & 0 & 0 & $-0.5(0.218)$ \\
Howell 2005 & 0 & 0 & $-0.6(1.049)$ \\
McGhan 2003 & 0 & 0 & $0.2(0.633)$ \\
McGhan 2010 & 0 & 0 & $1(0.68)$ \\
Velsor-Friedrich 2005 & 0 & 0 & $-0.5(0.933)$
\end{tabular}

Subtotal $(95 \% \mathrm{Cl})$

Heterogeneity: $\mathrm{Tau}^{2}=0.12 ; \mathrm{Chi}^{2}=5.4, \mathrm{df}=4(\mathrm{P}=0.25) ; \mathrm{I}^{2}=25.88 \%$

Test for overall effect: $\mathrm{Z}=0.56(\mathrm{P}=0.57)$

9.1.2 PI2 - theory, not individual, substantial school nurse involvement

Subtotal $(95 \% \mathrm{CI})$

Heterogeneity: Not applicable

Test for overall effect: Not applicable

9.1.3 Other configuration

Atherly 2009

Cicutto 2005

0 (0.916)

Cicutto 2005

$-0.4(0.408)$

Clark 2005

$-1.1(0.317)$

Horner 2008

$-0(0.329)$

$-0.2(0.447)$

0 (0.306)

Horner 2015

$-0.5(0.316)$

Levy 2006

Persaud 1996

$-1.3(0.737)$

Subtotal $(95 \% \mathrm{Cl})$

Heterogeneity: $\mathrm{Tau}^{2}=0.08 ; \mathrm{Chi}^{2}=10.69, \mathrm{df}=7(\mathrm{P}=0.15) ; \mathrm{I}^{2}=34.5 \%$

Test for overall effect: $\mathrm{Z}=2.31(\mathrm{P}=0.02)$ 


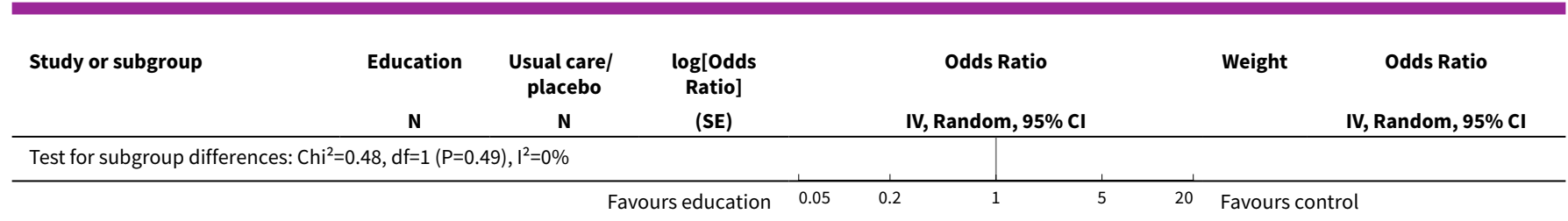

Analysis 9.2. Comparison 9 Effects of school-based asthma interventions vs usual care subgrouped by configuration of conditions, Outcome 2 Absence from school.

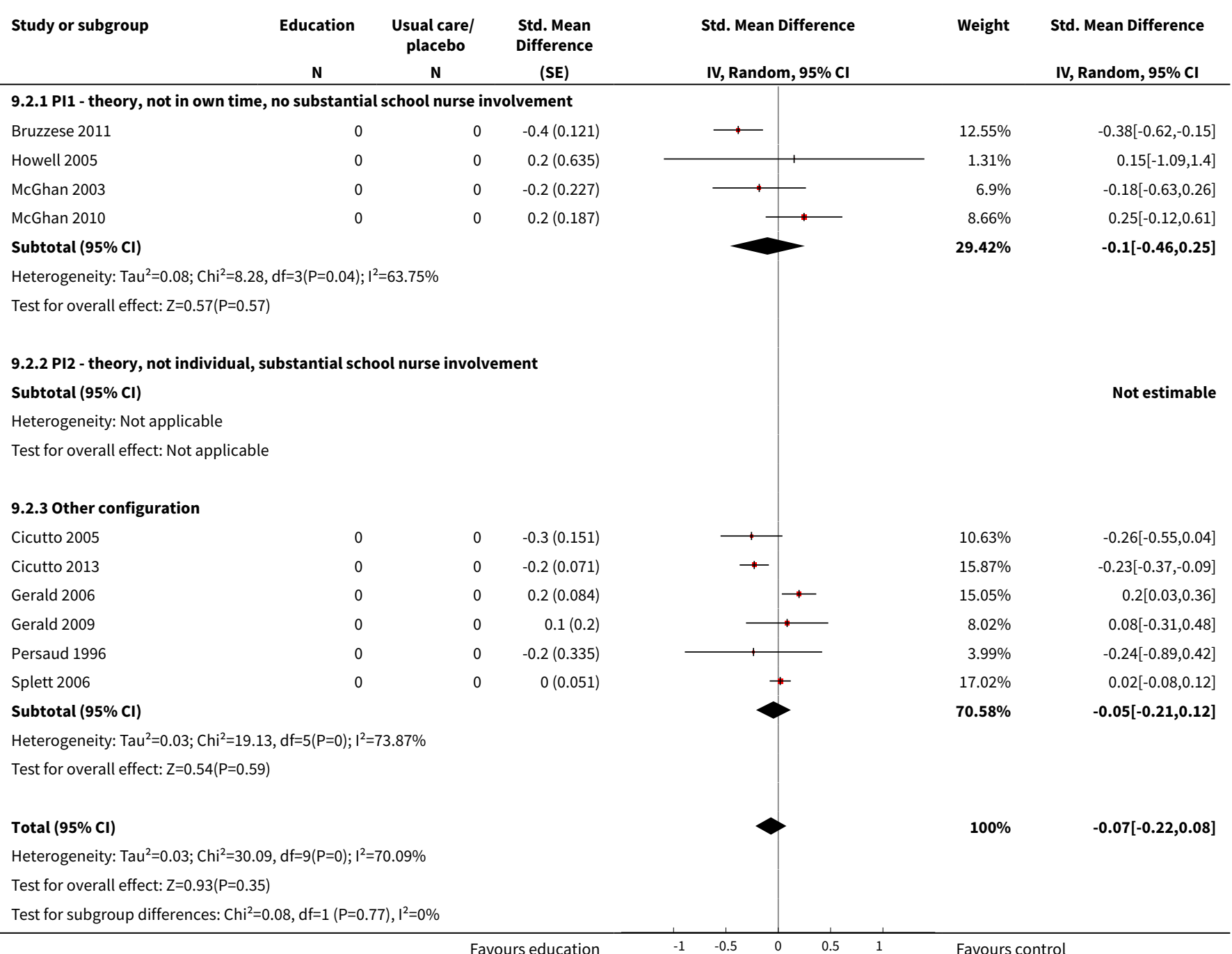

Favours control 
Analysis 9.3. Comparison 9 Effects of school-based asthma interventions vs usual care subgrouped by configuration of conditions, Outcome 3 Withdrawal from the study.

\begin{tabular}{|c|c|c|c|c|c|c|}
\hline Study or subgroup & Education & $\begin{array}{c}\text { Usual care/ } \\
\text { placebo } \\
\mathbf{N} \\
\end{array}$ & $\begin{array}{c}\text { log[Odds } \\
\text { Ratio] } \\
\text { (SE) } \\
\end{array}$ & $\begin{array}{c}\text { Odds Ratio } \\
\text { IV, Random, } 95 \% \mathrm{CI}\end{array}$ & Weight & $\begin{array}{c}\text { Odds Ratio } \\
\text { IV, Random, } 95 \% \mathrm{Cl}\end{array}$ \\
\hline \multicolumn{7}{|c|}{ 9.3.1 PI1 - theory, not in own time, no substantial school nurse involvement } \\
\hline Cicutto 2005 & 0 & 0 & $0.6(0.629)$ & + & $3.24 \%$ & $1.79[0.52,6.13]$ \\
\hline Gerald 2009 & 0 & 0 & $-0.5(0.368)$ & 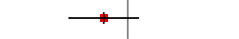 & $9.49 \%$ & $0.61[0.3,1.26]$ \\
\hline Horner 2008 & 0 & 0 & $0.3(0.531)$ & $1+$ & $4.56 \%$ & $1.33[0.47,3.77]$ \\
\hline Subtotal $(95 \% \mathrm{Cl})$ & & & & & $22.71 \%$ & $0.88[0.55,1.4]$ \\
\hline \multicolumn{7}{|c|}{ Heterogeneity: $\mathrm{Tau}^{2}=0 ; \mathrm{Chi}^{2}=2.95, \mathrm{df}=3(\mathrm{P}=0.4) ; \mathrm{I}^{2}=0 \%$} \\
\hline \multicolumn{7}{|c|}{ Test for overall effect: $\mathrm{Z}=0.56(\mathrm{P}=0.58)$} \\
\hline \multicolumn{7}{|c|}{ 9.3.2 PI2 - theory, not individual, substantial school nurse involvement } \\
\hline Patterson 2005 & 0 & 0 & $1.7(1.807)$ & & $0.39 \%$ & $5.67[0.16,195.9]$ \\
\hline \multicolumn{7}{|c|}{ Heterogeneity: Not applicable } \\
\hline \multicolumn{7}{|c|}{ Test for overall effect: $Z=0.96(P=0.34)$} \\
\hline \multicolumn{7}{|c|}{ 9.3.3 Other configuration } \\
\hline Al-Sheyab 2012 & 0 & 0 & $-0.7(1.074)$ & & $1.11 \%$ & $0.51[0.06,4.2]$ \\
\hline Bartholomew 2006 & 0 & 0 & $0.2(0.173)$ & \# & $42.89 \%$ & $1.27[0.9,1.78]$ \\
\hline Bruzzese 2008 & 0 & 0 & $-1.2(1.683)$ & " & $0.45 \%$ & $0.31[0.01,8.31]$ \\
\hline Bruzzese 2011 & 0 & 0 & $0.3(0.279)$ & + & $16.52 \%$ & $1.31[0.76,2.27]$ \\
\hline Kintner 2009 & 0 & 0 & $2.6(1.882)$ & & $0.36 \%$ & $13.57[0.34,542.83]$ \\
\hline McGhan 2003 & 0 & 0 & $0.2(0.383)$ & 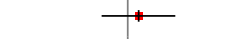 & $8.73 \%$ & $1.25[0.59,2.64]$ \\
\hline McGhan 2010 & 0 & 0 & $-0.2(0.512)$ & + & $4.9 \%$ & $0.8[0.29,2.18]$ \\
\hline Shah 2001 & 0 & 0 & $0.3(0.816)$ & + & $1.93 \%$ & $1.34[0.27,6.65]$ \\
\hline Subtotal $(95 \% \mathrm{Cl})$ & & & & $\gamma$ & $76.89 \%$ & $1.23[0.95,1.58]$ \\
\hline Total $(95 \% \mathrm{Cl})$ & & & & 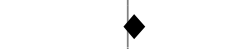 & $100 \%$ & $1.14[0.92,1.43]$ \\
\hline \multicolumn{7}{|c|}{ Heterogeneity: $\mathrm{Tau}^{2}=0 ; \mathrm{Chi}^{2}=9.07, \mathrm{df}=12(\mathrm{P}=0.7) ; \mathrm{I}^{2}=0 \%$} \\
\hline \multicolumn{7}{|c|}{ Test for overall effect: $Z=1.18(P=0.24)$} \\
\hline Test for subgroup dif & $34, \mathrm{df}=1(\mathrm{P}=0$ & $I^{2}=14.47 \%$ & & & & \\
\hline
\end{tabular}

Comparison 10. Effects of school-based asthma interventions vs usual care subgrouped by number of consistent conditions (use of theory, parental involvement, not in own time)

\begin{tabular}{lllll}
\hline Outcome or subgroup title & No. of studies & $\begin{array}{l}\text { No. of partici- } \\
\text { pants }\end{array}$ & Statistical method & Effect size \\
\hline $\begin{array}{l}1 \text { Exacerbations leading } \\
\text { to emergency department } \\
\text { (ED) visits }\end{array}$ & 13 & Odds Ratio (Random, 95\% Cl) & $0.70[0.53,0.92]$ \\
\hline 1.1 No conditions & 0 & Odds Ratio (Random, 95\% Cl) & $0.0[0.0,0.0]$ \\
\hline 1.2 One condition & 3 & Odds Ratio (Random, 95\% Cl) & $0.56[0.33,0.97]$ \\
\hline
\end{tabular}




\begin{tabular}{|c|c|c|c|c|}
\hline Outcome or subgroup title & No. of studies & $\begin{array}{l}\text { No. of partici- } \\
\text { pants }\end{array}$ & Statistical method & Effect size \\
\hline 1.3 Two conditions & 7 & & Odds Ratio (Random, 95\% Cl) & $0.67[0.49,0.94]$ \\
\hline 1.4 Three conditions & 3 & & Odds Ratio (Random, 95\% Cl) & $1.48[0.65,3.40]$ \\
\hline 2 Absence from school & 10 & & $\begin{array}{l}\text { Std. Mean Difference (Random, } \\
95 \% \mathrm{Cl} \text { ) }\end{array}$ & $-0.07[-0.22,0.08]$ \\
\hline 2.1 No conditions & 0 & & $\begin{array}{l}\text { Std. Mean Difference (Random, } \\
95 \% \mathrm{Cl} \text { ) }\end{array}$ & $0.0[0.0,0.0]$ \\
\hline 2.2 One condition & 3 & & $\begin{array}{l}\text { Std. Mean Difference (Random, } \\
95 \% \mathrm{Cl} \text { ) }\end{array}$ & $0.02[-0.08,0.11]$ \\
\hline 2.3 Two conditions & 4 & & $\begin{array}{l}\text { Std. Mean Difference (Random, } \\
95 \% \mathrm{Cl} \text { ) }\end{array}$ & $-0.16[-0.43,0.11]$ \\
\hline 2.4 Three conditions & 3 & & $\begin{array}{l}\text { Std. Mean Difference (Random, } \\
95 \% \mathrm{Cl} \text { ) }\end{array}$ & $0.07[-0.22,0.37]$ \\
\hline 3 Withdrawal from the study & 13 & & Odds Ratio (Random, 95\% Cl) & $1.14[0.92,1.43]$ \\
\hline 3.1 No conditions & 0 & & Odds Ratio (Random, 95\% Cl) & $0.0[0.0,0.0]$ \\
\hline 3.2 One condition & 1 & & Odds Ratio (Random, 95\% Cl) & $0.61[0.30,1.26]$ \\
\hline 3.3 Two conditions & 7 & & Odds Ratio (Random, 95\% Cl) & $1.22[0.83,1.80]$ \\
\hline 3.4 Three conditions & 5 & & Odds Ratio (Random, 95\% Cl) & $1.22[0.91,1.64]$ \\
\hline
\end{tabular}

\section{Analysis 10.1. Comparison 10 Effects of school-based asthma interventions vs usual care subgrouped by number of consistent conditions (use of theory, parental involvement, not in own time), Outcome 1 Exacerbations leading to emergency department (ED) visits.}

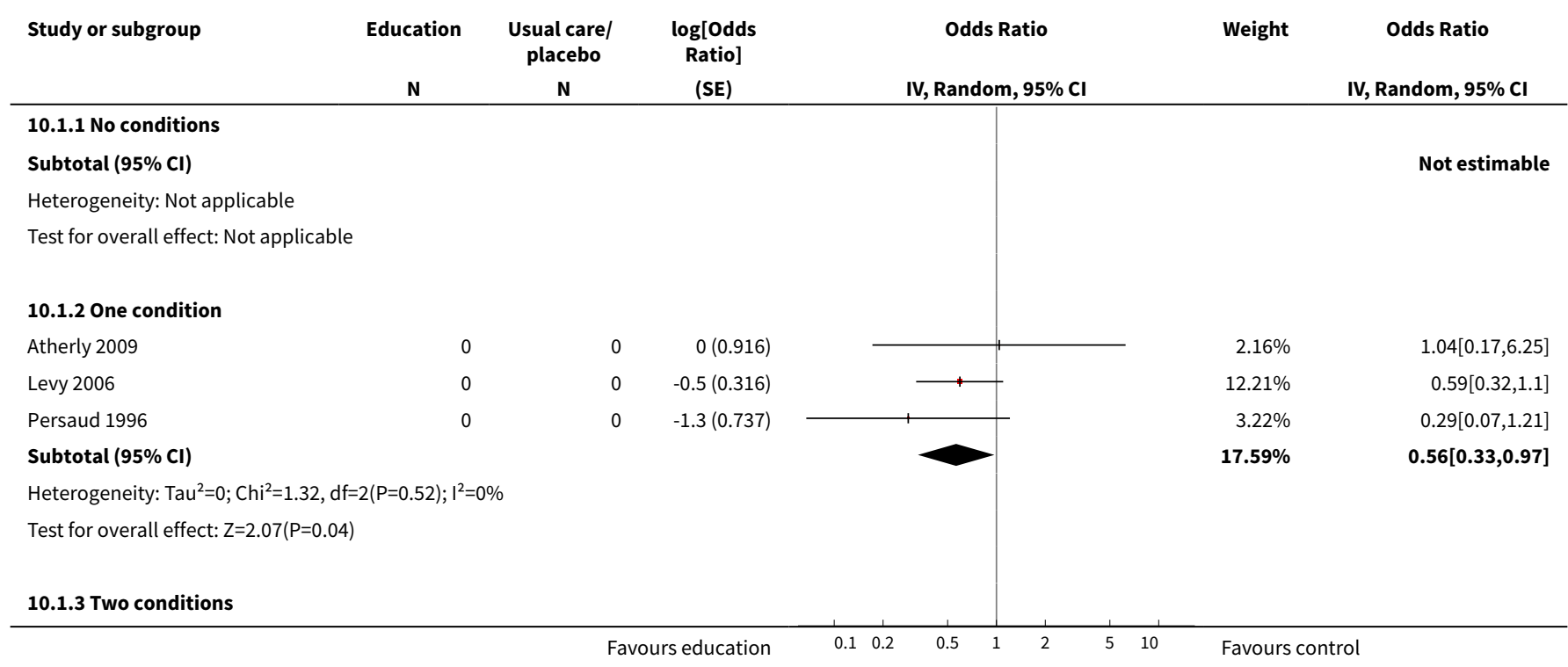




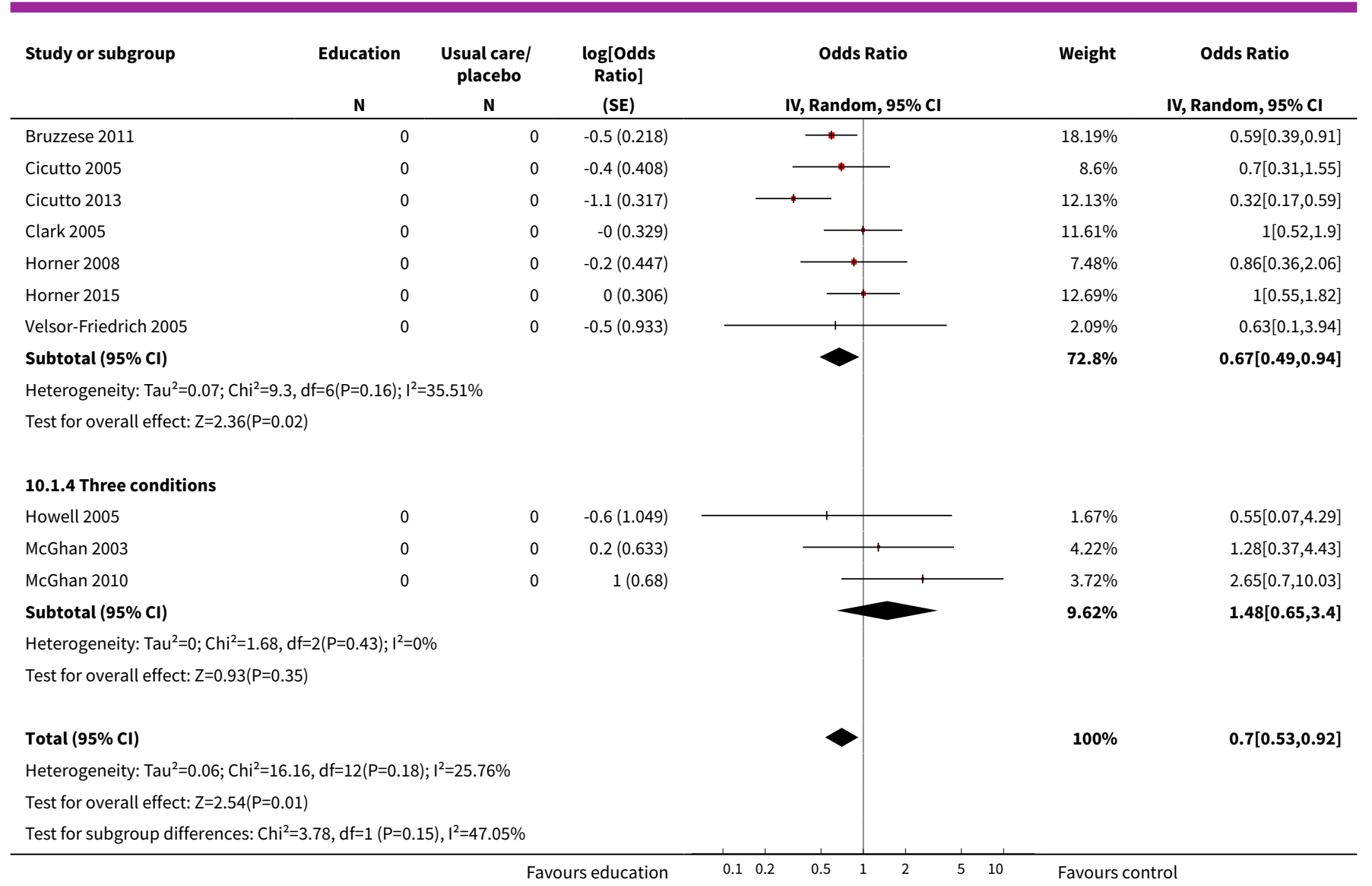

Analysis 10.2. Comparison 10 Effects of school-based asthma interventions vs usual care subgrouped by number of consistent conditions (use of theory, parental involvement, not in own time), Outcome 2 Absence from school.

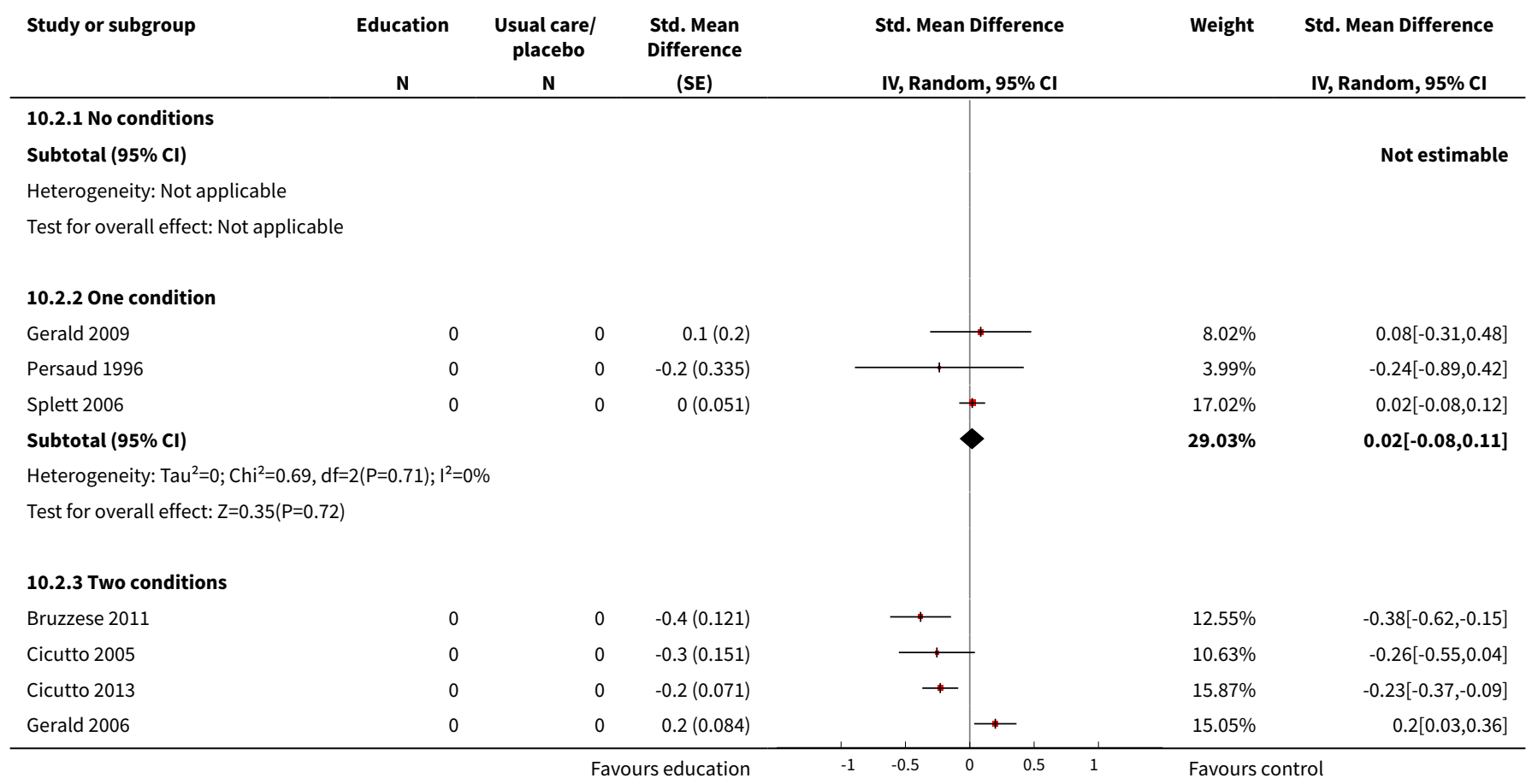




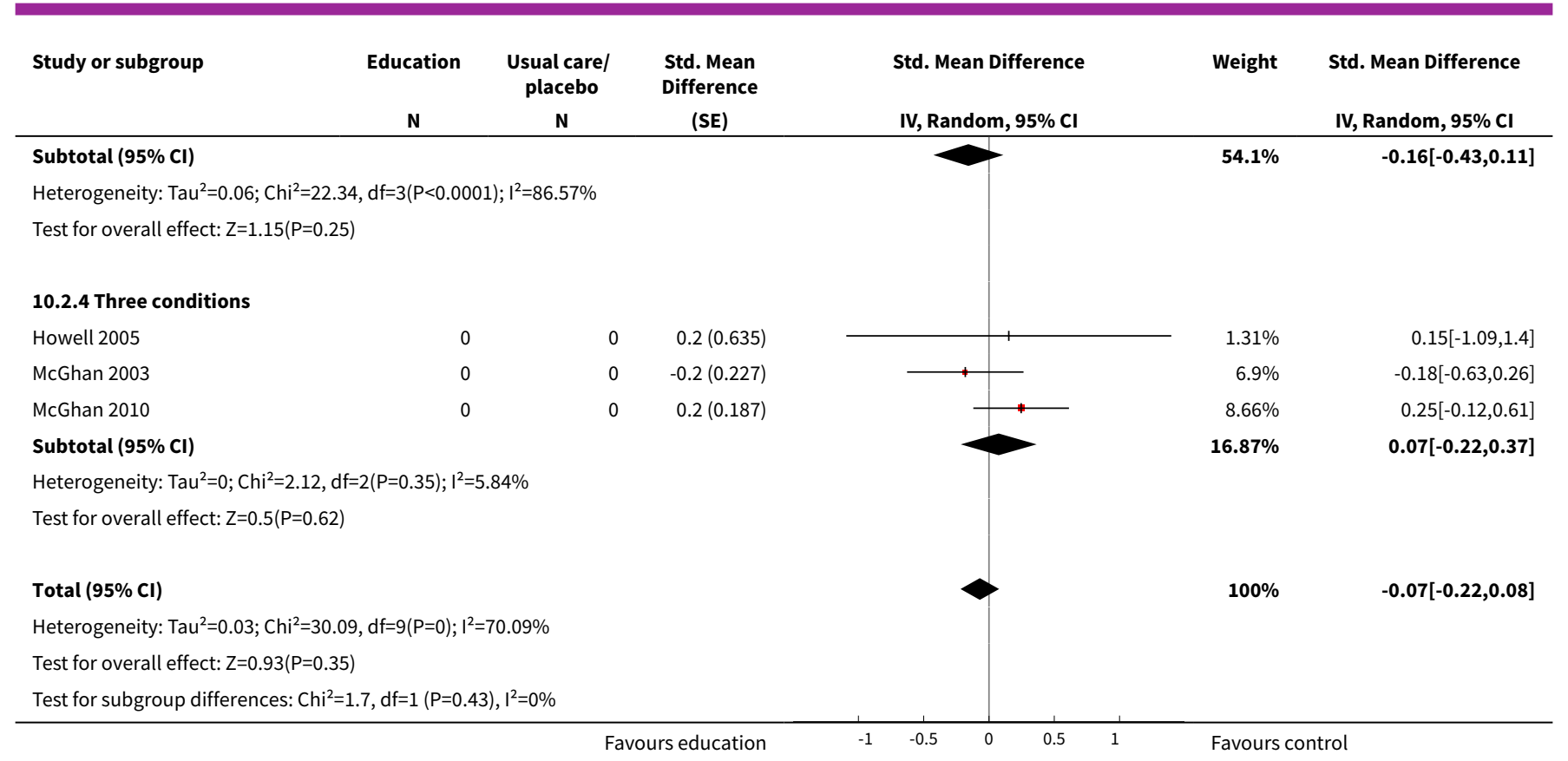

Analysis 10.3. Comparison 10 Effects of school-based asthma interventions vs usual care subgrouped by number of consistent conditions (use of theory, parental involvement, not in own time), Outcome 3 Withdrawal from the study.

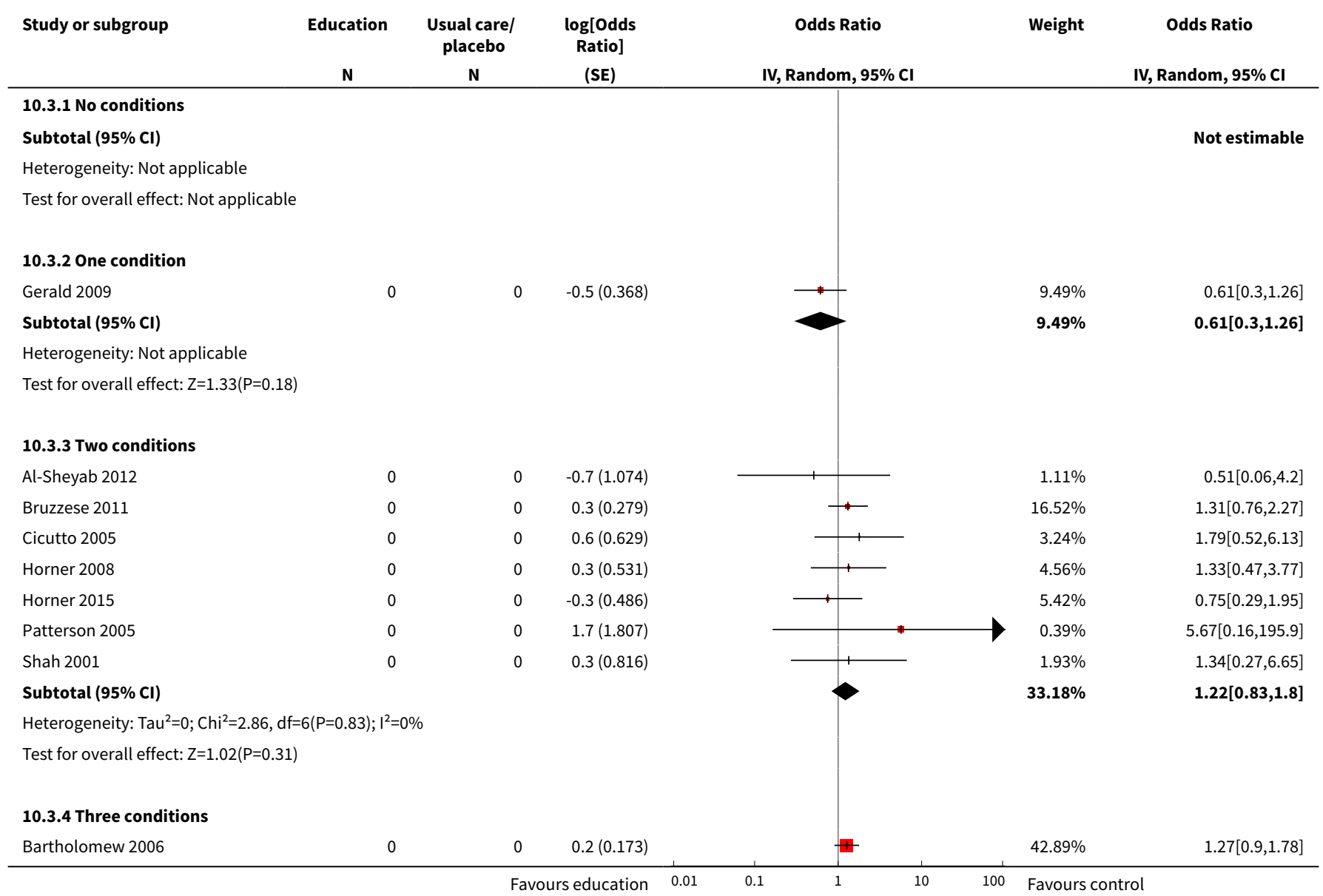




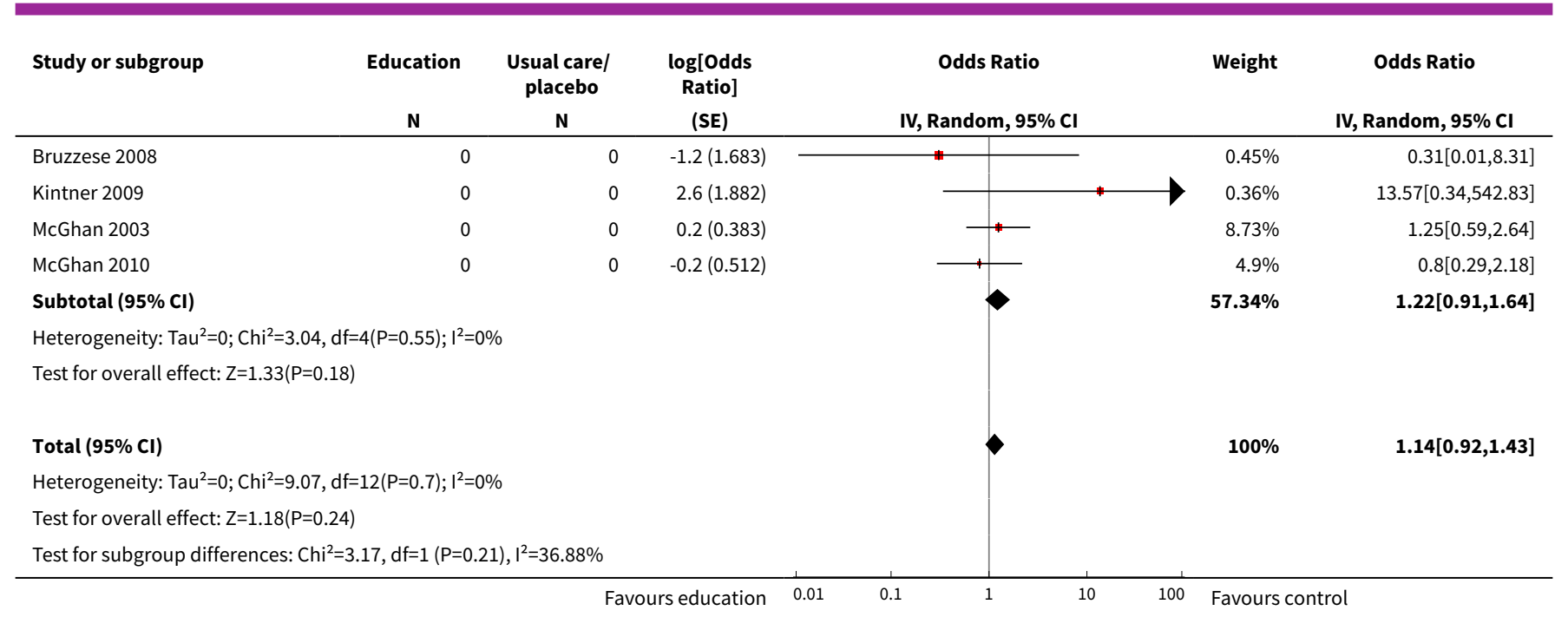

Comparison 11. Adjunct analyses - impact of Implementation on selected outcomes

\begin{tabular}{|c|c|c|c|c|}
\hline Outcome or subgroup title & No. of studies & $\begin{array}{l}\text { No. of partici- } \\
\text { pants }\end{array}$ & Statistical method & Effect size \\
\hline $\begin{array}{l}1 \text { Exacerbations leading to emer- } \\
\text { gency department (ED) visits }\end{array}$ & 7 & & $\begin{array}{l}\text { Std. Mean Difference (Random, } \\
95 \% \mathrm{Cl} \text { ) }\end{array}$ & $-0.21[-0.37,-0.04]$ \\
\hline 1.1 Successful implementation & 4 & & $\begin{array}{l}\text { Std. Mean Difference (Random, } \\
95 \% \mathrm{Cl} \text { ) }\end{array}$ & $-0.26[-0.48,-0.04]$ \\
\hline $\begin{array}{l}\text { 1.2 Potential issues in adherence, } \\
\text { attrition, or dosage }\end{array}$ & 3 & & $\begin{array}{l}\text { Std. Mean Difference (Random, } \\
95 \% \mathrm{Cl} \text { ) }\end{array}$ & $-0.09[-0.28,0.10]$ \\
\hline 2 Absence from school & 7 & & $\begin{array}{l}\text { Std. Mean Difference (Random, } \\
95 \% \mathrm{Cl} \text { ) }\end{array}$ & $-0.12[-0.28,0.04]$ \\
\hline 2.1 Successful implementation & 3 & & $\begin{array}{l}\text { Std. Mean Difference (Random, } \\
95 \% \mathrm{Cl} \text { ) }\end{array}$ & $-0.28[-0.39,-0.18]$ \\
\hline $\begin{array}{l}\text { 2.2 Potential issues in adherence, } \\
\text { attrition, or dosage }\end{array}$ & 4 & & $\begin{array}{l}\text { Std. Mean Difference (Random, } \\
95 \% \mathrm{Cl} \text { ) }\end{array}$ & $0.04[-0.09,0.18]$ \\
\hline
\end{tabular}

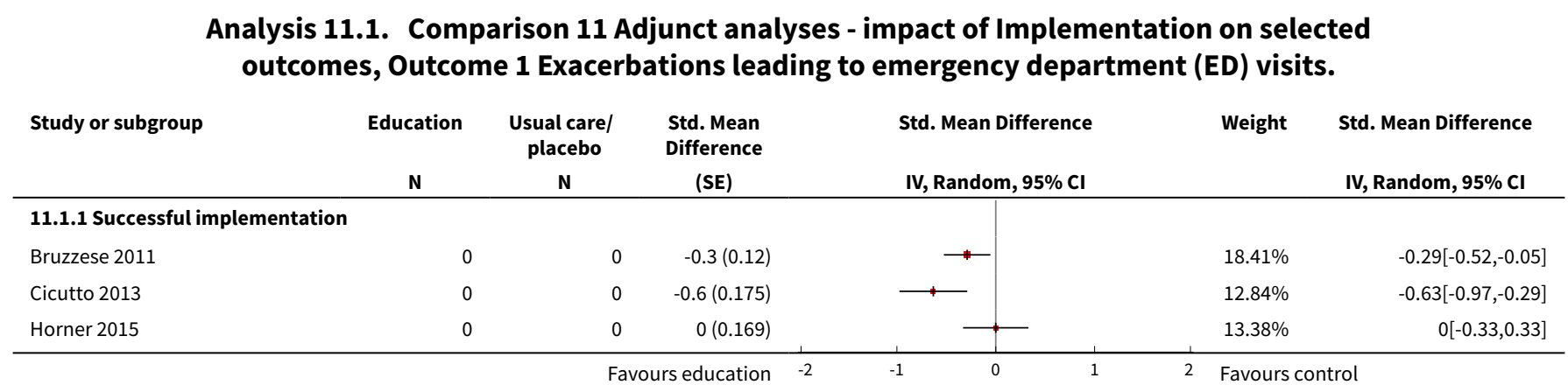




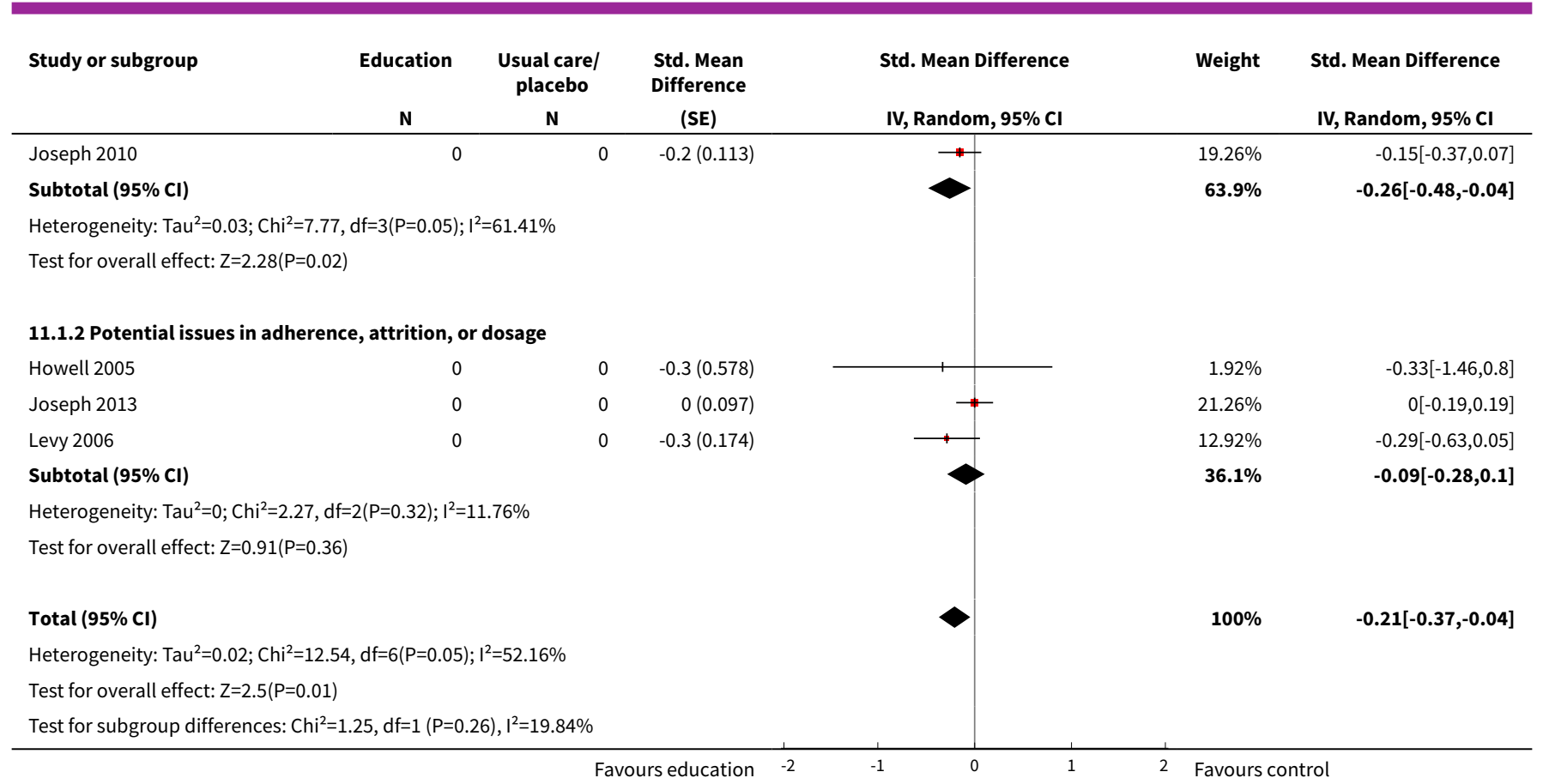

Analysis 11.2. Comparison 11 Adjunct analyses - impact of Implementation on selected outcomes, Outcome 2 Absence from school.

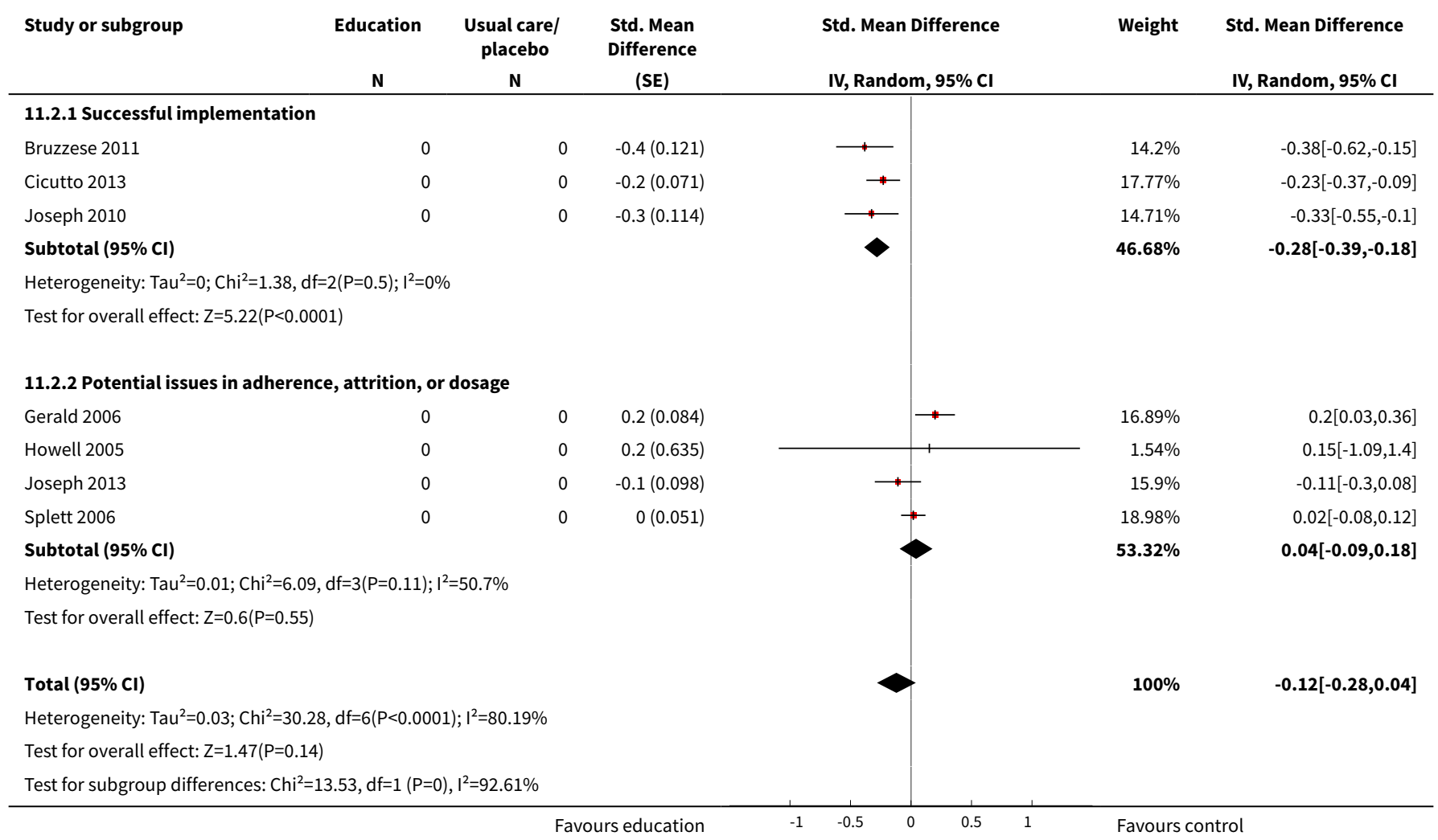


Comparison 12. Effects of school-based asthma interventions vs usual care, including disaggregated effect sizes

\begin{tabular}{|c|c|c|c|c|}
\hline Outcome or subgroup title & No. of studies & $\begin{array}{l}\text { No. of partici- } \\
\text { pants }\end{array}$ & Statistical method & Effect size \\
\hline $\begin{array}{l}1 \text { Exacerbations leading to hospitalisation - } \\
\text { standardised mean difference }\end{array}$ & 3 & 719 & $\begin{array}{l}\text { Std. Mean Difference } \\
\text { (Random, 95\% Cl) }\end{array}$ & $\begin{array}{l}-0.20[-0.36 \\
-0.03]\end{array}$ \\
\hline $\begin{array}{l}2 \text { Exacerbations leading to hospitalisation - } \\
\text { odds ratio }\end{array}$ & 3 & 1154 & $\begin{array}{l}\text { Odds Ratio (Random, } \\
95 \% \mathrm{Cl} \text { ) }\end{array}$ & $0.71[0.37,1.36]$ \\
\hline $\begin{array}{l}3 \text { Exacerbations leading to hospitalisation - } \\
\text { harmonised effect sizes }\end{array}$ & 6 & 1873 & $\begin{array}{l}\text { Std. Mean Difference } \\
\text { (Random, 95\% Cl) }\end{array}$ & $\begin{array}{l}-0.19[-0.35 \\
-0.04]\end{array}$ \\
\hline $\begin{array}{l}4 \text { Exacerbations leading to emergency de- } \\
\text { partment (ED) visits - standardised mean } \\
\text { difference }\end{array}$ & 4 & 736 & $\begin{array}{l}\text { Std. Mean Difference } \\
\text { (Random, 95\% Cl) }\end{array}$ & $\begin{array}{l}-0.22[-0.38 \\
-0.05]\end{array}$ \\
\hline $\begin{array}{l}5 \text { Exacerbations leading to emergency de- } \\
\text { partment (ED) visits - odds ratio }\end{array}$ & 9 & 3147 & $\begin{array}{l}\text { Odds Ratio (Random, } \\
95 \% \mathrm{Cl} \text { ) }\end{array}$ & $0.74[0.47,1.16]$ \\
\hline $\begin{array}{l}6 \text { Exacerbations leading to emergency de- } \\
\text { partment (ED) visits - harmonised effect } \\
\text { sizes }\end{array}$ & 13 & 3883 & $\begin{array}{l}\text { Odds Ratio (Random, } \\
95 \% \mathrm{Cl} \text { ) }\end{array}$ & $0.70[0.53,0.92]$ \\
\hline $\begin{array}{l}7 \text { Absence from school - standardised mean } \\
\text { difference }\end{array}$ & 6 & 2720 & $\begin{array}{l}\text { Std. Mean Difference } \\
\text { (Random, 95\% Cl) }\end{array}$ & $-0.10[-0.30,0.11]$ \\
\hline 8 Absence from school - odds ratio & 4 & 1889 & $\begin{array}{l}\text { Odds Ratio (Random, } \\
95 \% \mathrm{Cl} \text { ) }\end{array}$ & $0.91[0.59,1.42]$ \\
\hline $\begin{array}{l}9 \text { Absence from school - harmonised effect } \\
\text { sizes }\end{array}$ & 10 & 4609 & $\begin{array}{l}\text { Std. Mean Difference } \\
\text { (Random, 95\% Cl) }\end{array}$ & $-0.08[-0.22,0.07]$ \\
\hline $\begin{array}{l}10 \text { Days of restricted activity - standardised } \\
\text { mean difference }\end{array}$ & 2 & 536 & $\begin{array}{l}\text { Std. Mean Difference } \\
\text { (Random, 95\% Cl) }\end{array}$ & $\begin{array}{l}-0.34[-0.52 \\
-0.15]\end{array}$ \\
\hline 11 Days of restricted activity - odds ratio & 1 & & $\begin{array}{l}\text { Odds Ratio (Random, } \\
95 \% \mathrm{Cl} \text { ) }\end{array}$ & Subtotals only \\
\hline $\begin{array}{l}12 \text { Days of restricted activity - harmonised } \\
\text { effect sizes }\end{array}$ & 3 & 1852 & $\begin{array}{l}\text { Std. Mean Difference } \\
\text { (Random, 95\% Cl) }\end{array}$ & $\begin{array}{l}-0.30[-0.41 \\
-0.18]\end{array}$ \\
\hline $\begin{array}{l}13 \text { Experience of daytime and night-time } \\
\text { symptoms - daytime symptoms - standard- } \\
\text { ised mean difference }\end{array}$ & 3 & 762 & $\begin{array}{l}\text { Std. Mean Difference } \\
\text { (Random, 95\% Cl) }\end{array}$ & $-0.15[-0.33,0.04]$ \\
\hline $\begin{array}{l}14 \text { Experience of daytime and night-time } \\
\text { symptoms - daytime symptoms - odds ratio }\end{array}$ & 2 & 303 & $\begin{array}{l}\text { Odds Ratio (Random, } \\
95 \% \mathrm{Cl} \text { ) }\end{array}$ & $0.71[0.32,1.55]$ \\
\hline $\begin{array}{l}15 \text { Experience of daytime and night-time } \\
\text { symptoms - daytime symptoms - har- } \\
\text { monised effect sizes }\end{array}$ & 5 & 1065 & $\begin{array}{l}\text { Std. Mean Difference } \\
\text { (Random, 95\% Cl) }\end{array}$ & $-0.15[-0.32,0.02]$ \\
\hline $\begin{array}{l}16 \text { Experience of daytime and night-time } \\
\text { symptoms - night-time symptoms - stan- } \\
\text { dardised mean difference }\end{array}$ & 3 & 323 & $\begin{array}{l}\text { Std. Mean Difference } \\
\text { (Random, 95\% Cl) }\end{array}$ & $\begin{array}{l}-0.36[-0.58 \\
-0.14]\end{array}$ \\
\hline
\end{tabular}




\begin{tabular}{|c|c|c|c|c|}
\hline Outcome or subgroup title & No. of studies & $\begin{array}{l}\text { No. of partici- } \\
\text { pants }\end{array}$ & Statistical method & Effect size \\
\hline $\begin{array}{l}17 \text { Experience of daytime and night-time } \\
\text { symptoms - night-time symptoms - odds ra- } \\
\text { tio }\end{array}$ & 1 & 136 & $\begin{array}{l}\text { Odds Ratio (Random, } \\
95 \% \mathrm{Cl} \text { ) }\end{array}$ & $1.24[0.56,2.72]$ \\
\hline $\begin{array}{l}18 \text { Experience of daytime and night-time } \\
\text { symptoms - night-time symptoms - har- } \\
\text { monised effect sizes }\end{array}$ & 4 & & $\begin{array}{l}\text { Std. Mean Difference } \\
\text { (Random, 95\% Cl) }\end{array}$ & $-0.18[-0.52,0.15]$ \\
\hline $\begin{array}{l}19 \text { Use of reliever therapies, e.g. beta }{ }_{2} \text {-ago- } \\
\text { nists - odds ratio }\end{array}$ & 2 & 437 & $\begin{array}{l}\text { Odds Ratio (Random, } \\
95 \% \mathrm{Cl} \text { ) }\end{array}$ & $0.52[0.15,1.81]$ \\
\hline $\begin{array}{l}20 \text { Corticosteroid dosage and/or use of add- } \\
\text { on therapies (usage of) }\end{array}$ & 3 & 614 & $\begin{array}{l}\text { Odds Ratio (Random, } \\
95 \% \mathrm{Cl} \text { ) }\end{array}$ & $1.25[0.88,1.79]$ \\
\hline $\begin{array}{l}21 \text { Corticosteroid dosage and/or use of add- } \\
\text { on therapies (appropriate usage of) }\end{array}$ & 2 & & $\begin{array}{l}\text { Std. Mean Difference } \\
\text { (Random, 95\% Cl) }\end{array}$ & $\begin{array}{l}\text { Totals not select- } \\
\text { ed }\end{array}$ \\
\hline $\begin{array}{l}22 \text { Health-related quality of life - standard- } \\
\text { ised mean difference }\end{array}$ & 7 & 2502 & $\begin{array}{l}\text { Std. Mean Difference } \\
\text { (Random, } 95 \% \mathrm{Cl} \text { ) }\end{array}$ & $0.27[0.18,0.36]$ \\
\hline 23 Health-related quality of life (MD) & 8 & 2950 & $\begin{array}{l}\text { Mean Difference (IV, } \\
\text { Random, } 95 \% \mathrm{CI} \text { ) }\end{array}$ & $0.35[0.06,0.64]$ \\
\hline $\begin{array}{l}24 \text { Unplanned visit to hospital or GP due to } \\
\text { asthma symptoms - standardised mean dif- } \\
\text { ference }\end{array}$ & 1 & 280 & $\begin{array}{l}\text { Std. Mean Difference } \\
\text { (Random, 95\% Cl) }\end{array}$ & $\begin{array}{l}-0.28[-0.52 \\
-0.05]\end{array}$ \\
\hline $\begin{array}{l}25 \text { Unplanned visit to hospital or GP due to } \\
\text { asthma symptoms - odds ratio }\end{array}$ & 4 & 1316 & $\begin{array}{l}\text { Odds Ratio (Random, } \\
95 \% \mathrm{Cl} \text { ) }\end{array}$ & $0.78[0.62,0.98]$ \\
\hline $\begin{array}{l}26 \text { Unplanned visit to hospital or GP due to } \\
\text { asthma symptoms - harmonised effect sizes }\end{array}$ & 5 & 1596 & $\begin{array}{l}\text { Odds Ratio (Random, } \\
95 \% \mathrm{Cl} \text { ) }\end{array}$ & $0.74[0.60,0.90]$ \\
\hline 27 Withdrawal from the study & 13 & 3442 & $\begin{array}{l}\text { Odds Ratio (Random, } \\
95 \% \mathrm{Cl} \text { ) }\end{array}$ & $1.14[0.92,1.43]$ \\
\hline
\end{tabular}

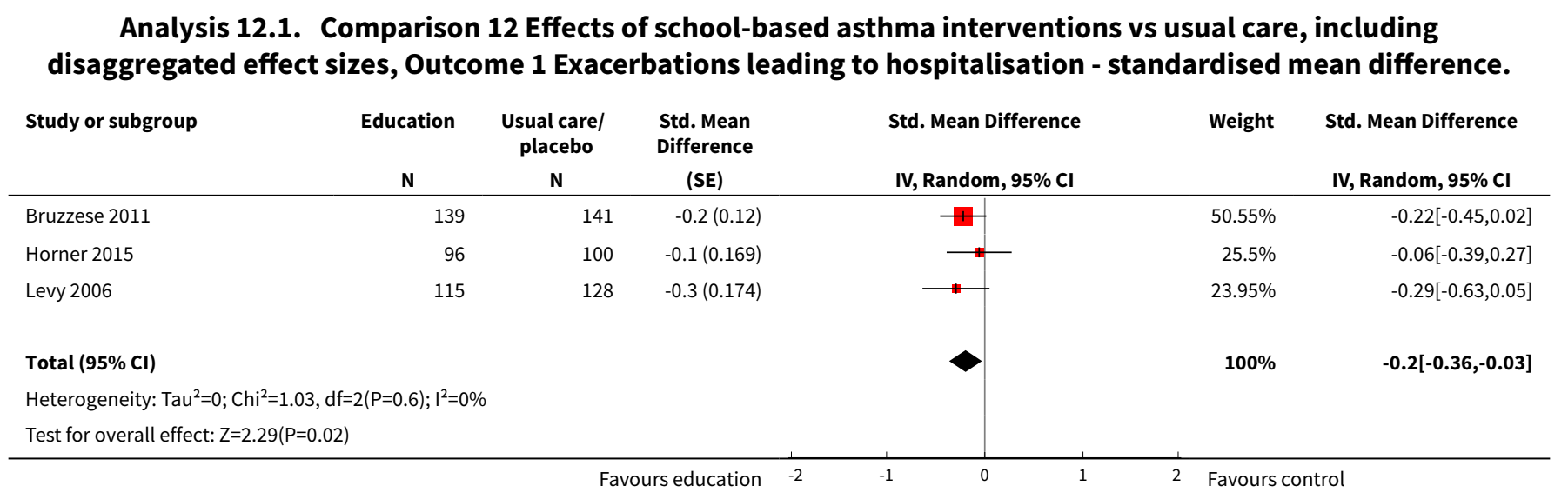


Analysis 12.2. Comparison 12 Effects of school-based asthma interventions vs usual care, including disaggregated effect sizes, Outcome 2 Exacerbations leading to hospitalisation - odds ratio.

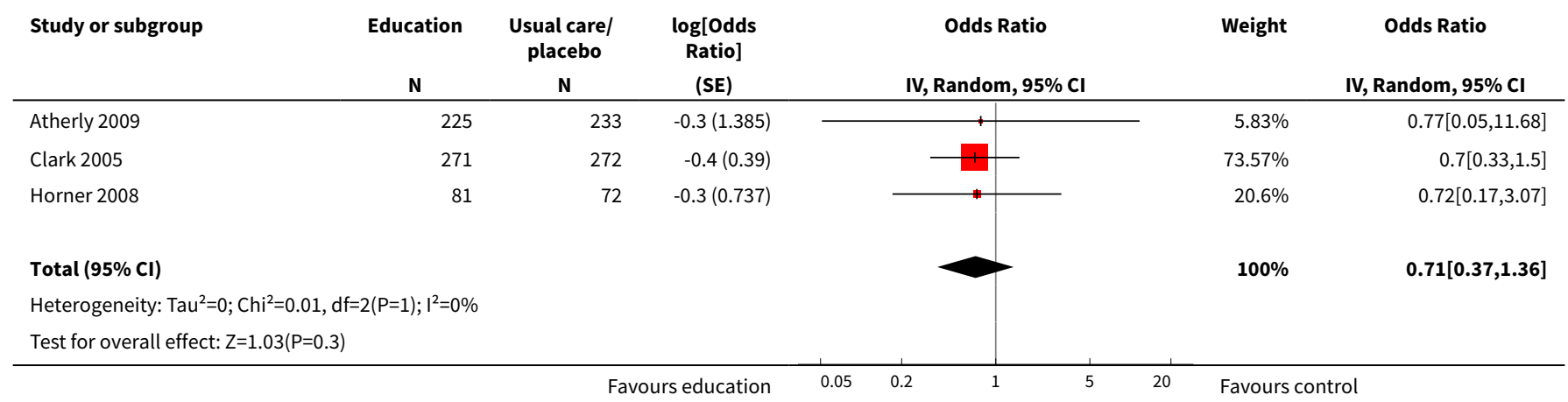

Analysis 12.3. Comparison 12 Effects of school-based asthma interventions vs usual care, including disaggregated effect sizes, Outcome 3 Exacerbations leading to hospitalisation - harmonised effect sizes.

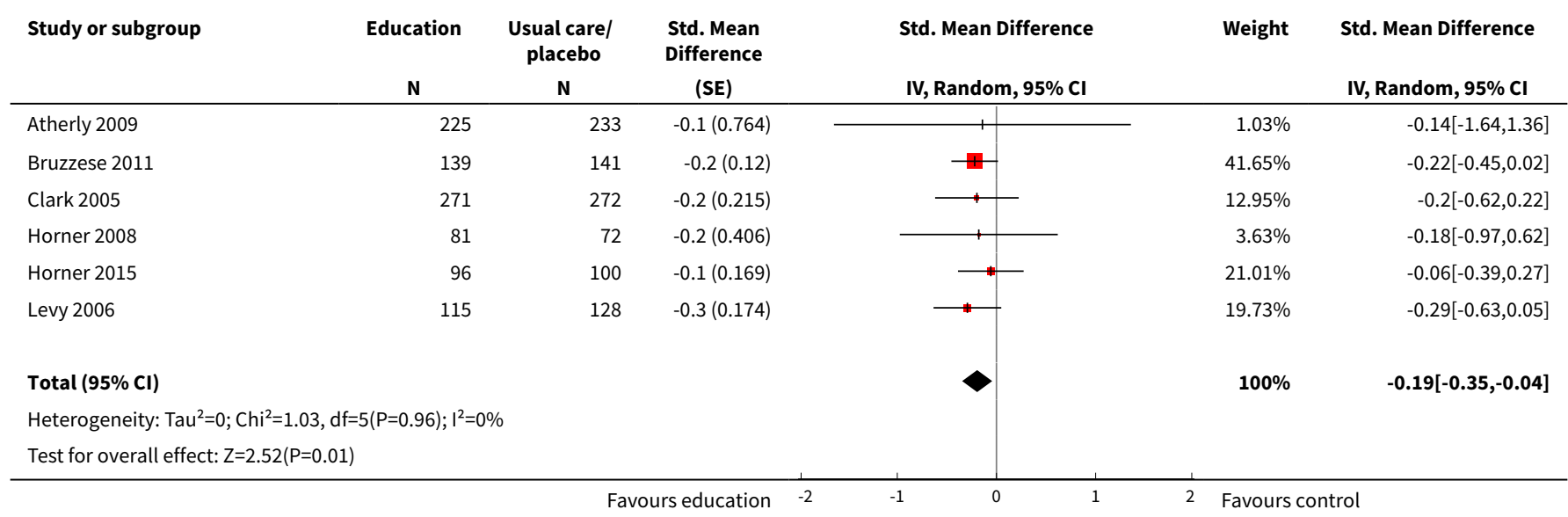

Analysis 12.4. Comparison 12 Effects of school-based asthma interventions vs usual care, including disaggregated effect sizes, Outcome 4 Exacerbations leading to emergency department (ED) visits - standardised mean difference.

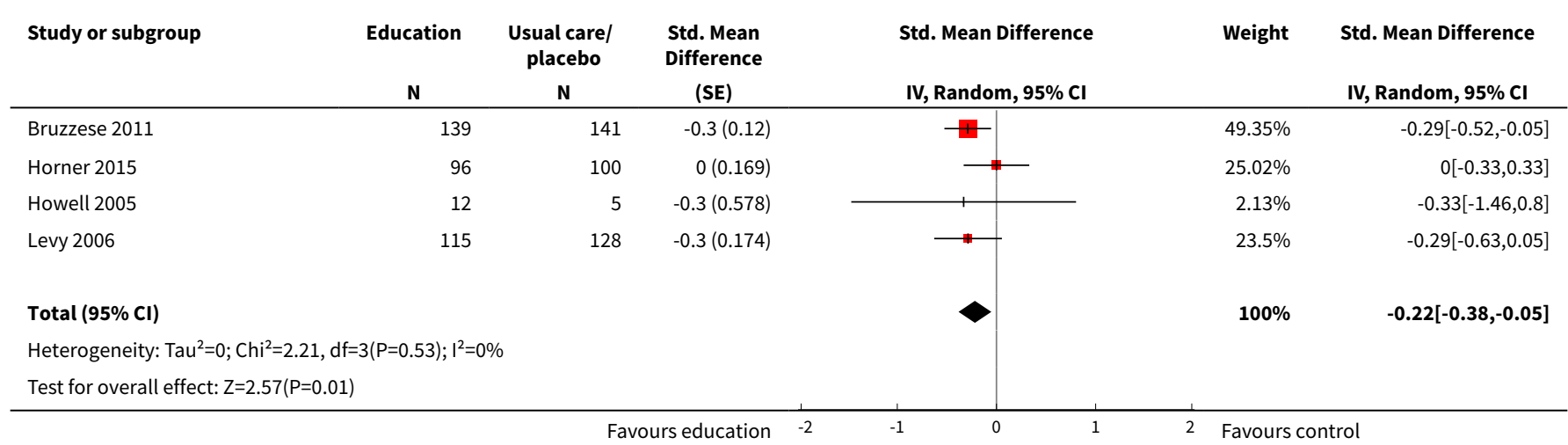


Analysis 12.5. Comparison 12 Effects of school-based asthma interventions vs usual care, including disaggregated effect sizes, Outcome 5 Exacerbations leading to emergency department (ED) visits - odds ratio.

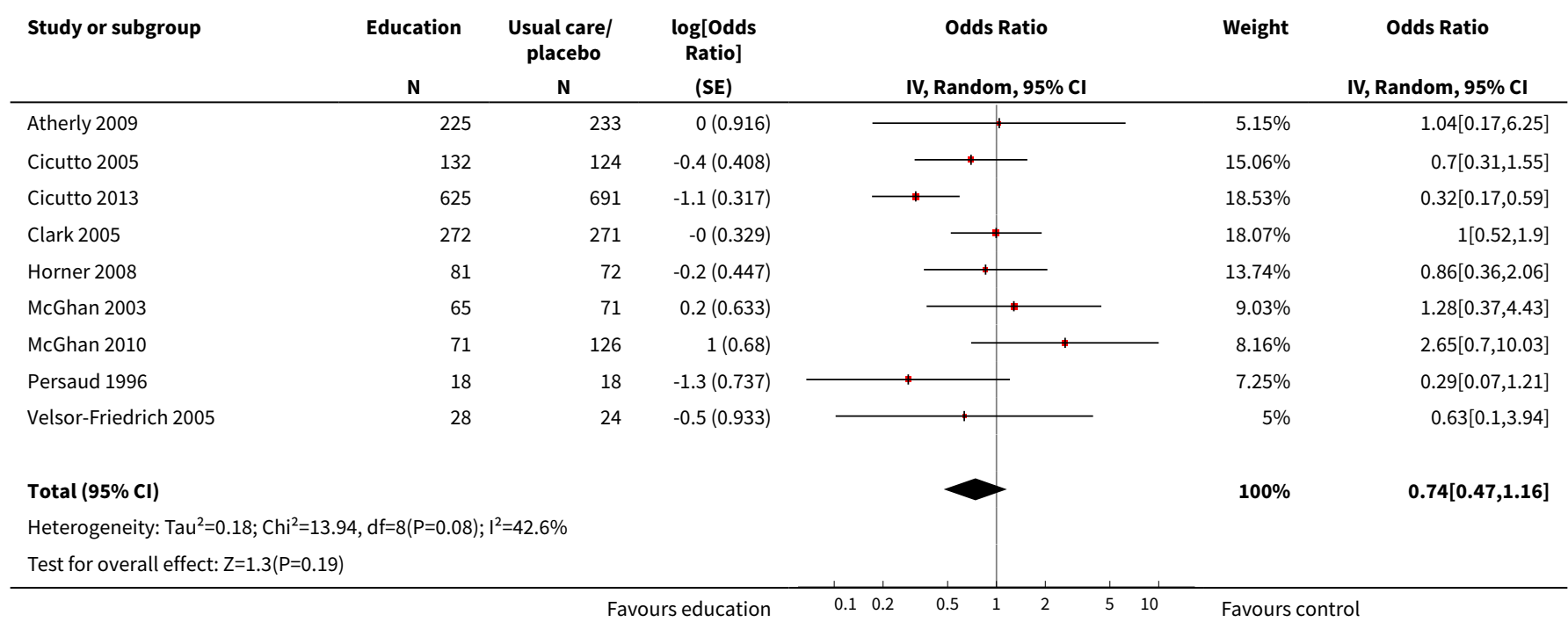

Analysis 12.6. Comparison 12 Effects of school-based asthma interventions vs usual care, including disaggregated effect sizes, Outcome 6 Exacerbations leading to emergency department (ED) visits - harmonised effect sizes.

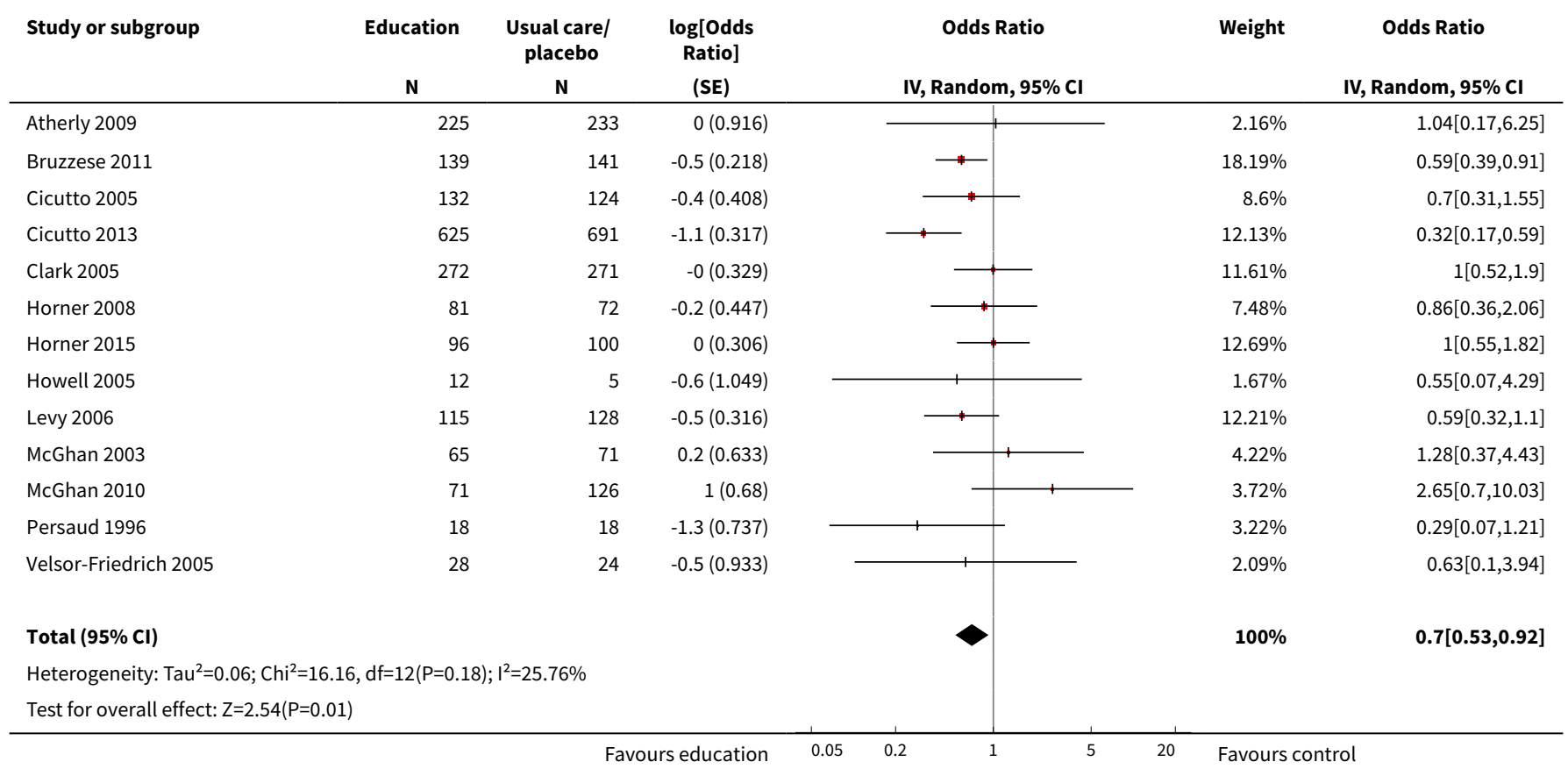


Analysis 12.7. Comparison 12 Effects of school-based asthma interventions vs usual care, including disaggregated effect sizes, Outcome 7 Absence from school - standardised mean difference.

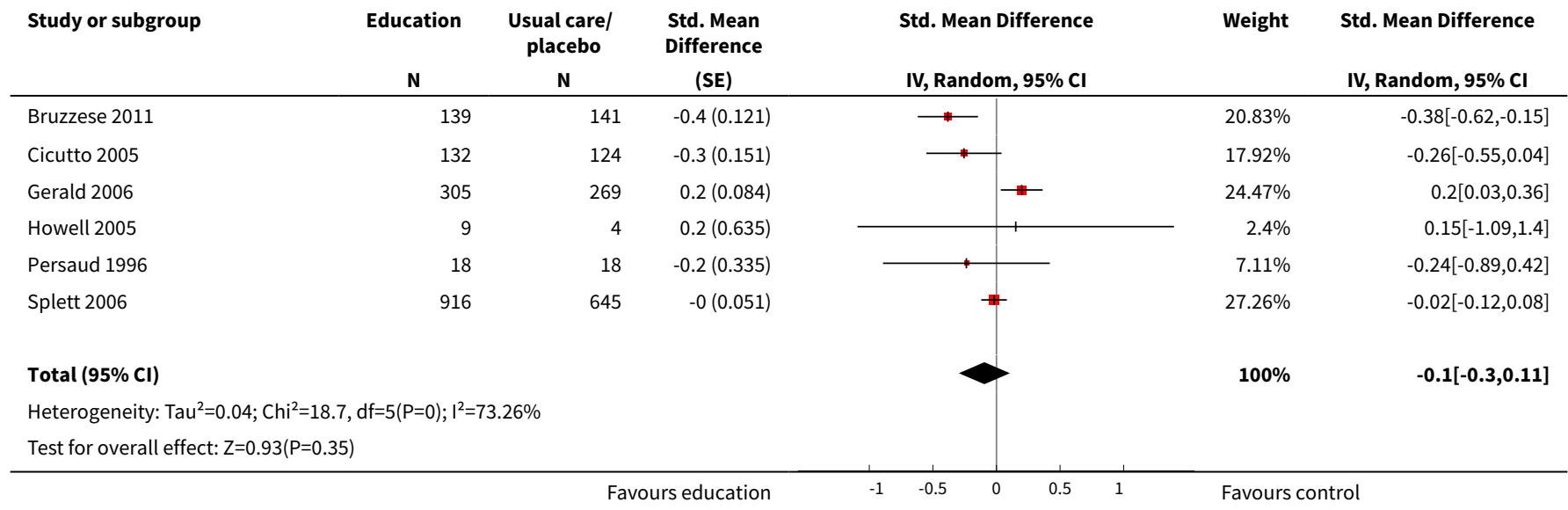

Analysis 12.8. Comparison 12 Effects of school-based asthma interventions vs usual care, including disaggregated effect sizes, Outcome 8 Absence from school - odds ratio.

\begin{tabular}{|c|c|c|c|c|c|c|c|}
\hline \multirow[t]{2}{*}{ Study or subgroup } & \multirow{2}{*}{$\begin{array}{c}\text { Education } \\
\mathrm{N} \\
\end{array}$} & \multirow{2}{*}{$\begin{array}{c}\text { Usual care/ } \\
\text { placebo } \\
\mathbf{N}\end{array}$} & \multirow{2}{*}{$\begin{array}{l}\text { log[Odds } \\
\text { Ratio] } \\
\text { (SE) }\end{array}$} & \multicolumn{2}{|c|}{ Odds Ratio } & \multirow[t]{2}{*}{ Weight } & \multirow{2}{*}{$\begin{array}{c}\text { Odds Ratio } \\
\text { IV, Random, } 95 \% \mathrm{CI}\end{array}$} \\
\hline & & & & \multicolumn{2}{|c|}{ IV, Random, 95\% CI } & & \\
\hline Cicutto 2013 & 625 & 691 & $-0.4(0.129)$ & $\longrightarrow$ & & $38.96 \%$ & $0.66[0.51,0.85]$ \\
\hline Gerald 2009 & 125 & 115 & $0.2(0.364)$ & & " & $20.56 \%$ & $1.17[0.57,2.38]$ \\
\hline McGhan 2003 & 65 & 71 & $-0.3(0.402)$ & & & $18.35 \%$ & $0.72[0.33,1.58]$ \\
\hline McGhan 2010 & 71 & 126 & $0.4(0.339)$ & & & $22.13 \%$ & $1.56[0.8,3.04]$ \\
\hline Total $(95 \% \mathrm{Cl})$ & & & & & & $100 \%$ & $0.91[0.59,1.42]$ \\
\hline \multicolumn{8}{|c|}{ Heterogeneity: Tau $^{2}=0.11 ; \mathrm{Chi}^{2}=7.13, \mathrm{df}=3(\mathrm{P}=0.07) ; \mathrm{I}^{2}=57.93 \%$} \\
\hline \multicolumn{8}{|c|}{ Test for overall effect: $Z=0.41(P=0.68)$} \\
\hline
\end{tabular}

Analysis 12.9. Comparison 12 Effects of school-based asthma interventions vs usual care, including disaggregated effect sizes, Outcome 9 Absence from school - harmonised effect sizes.

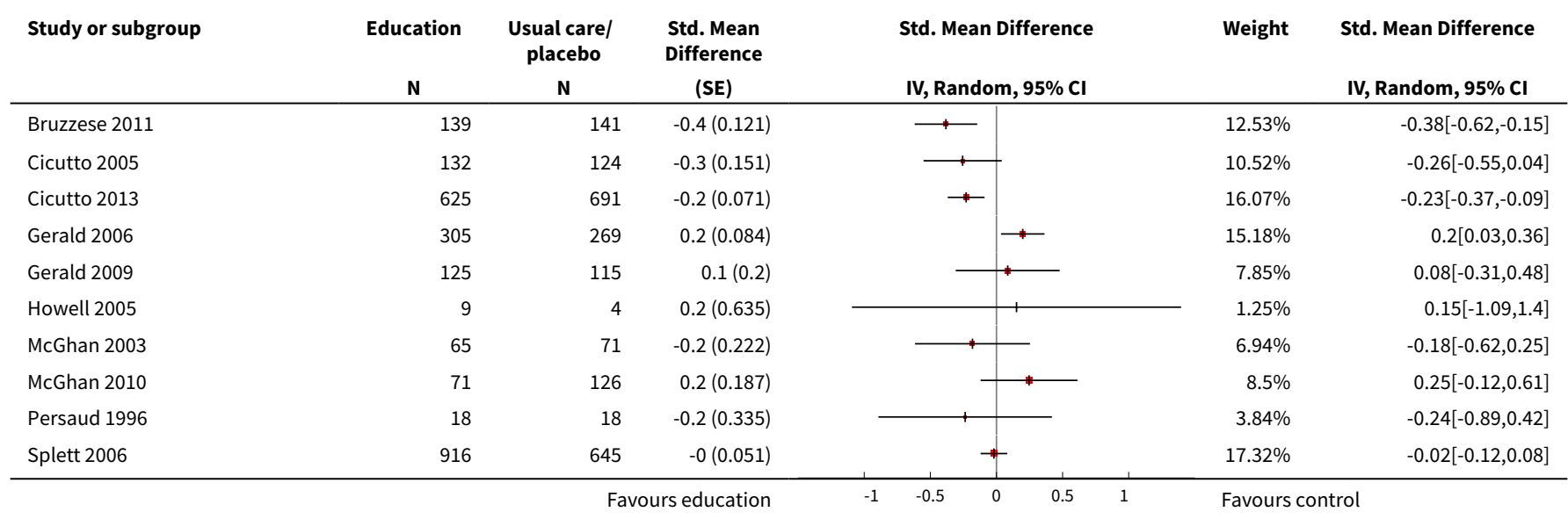




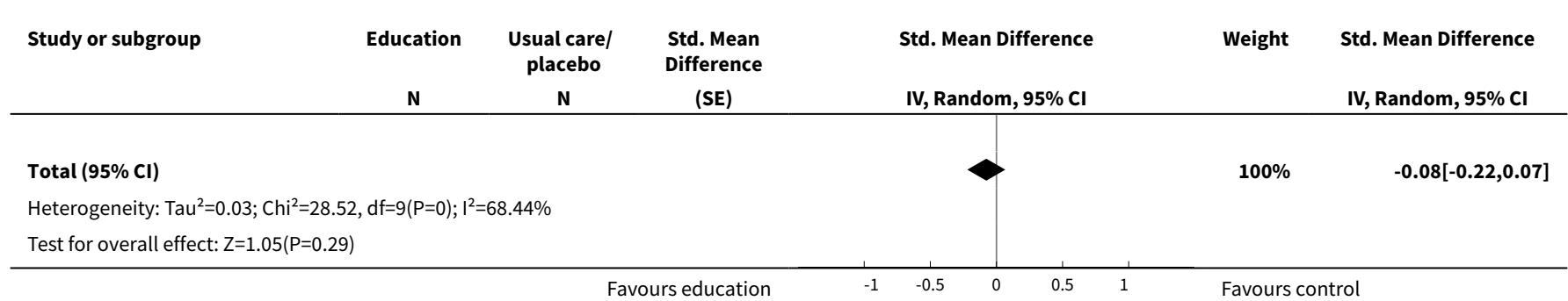

Analysis 12.10. Comparison 12 Effects of school-based asthma interventions vs usual care, including disaggregated effect sizes, Outcome 10 Days of restricted activity - standardised mean difference.

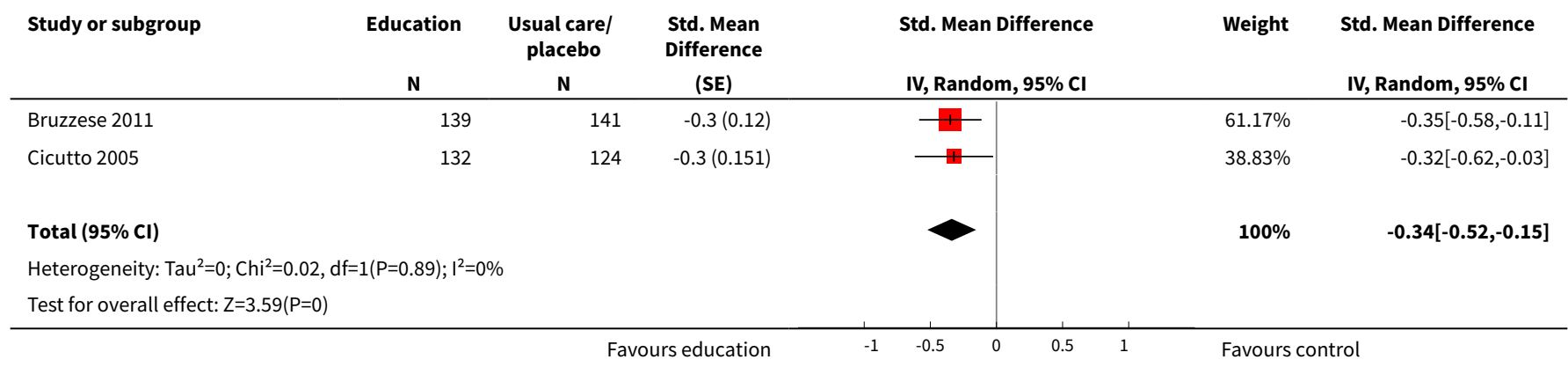

Analysis 12.11. Comparison 12 Effects of school-based asthma interventions vs usual care, including disaggregated effect sizes, Outcome 11 Days of restricted activity - odds ratio.

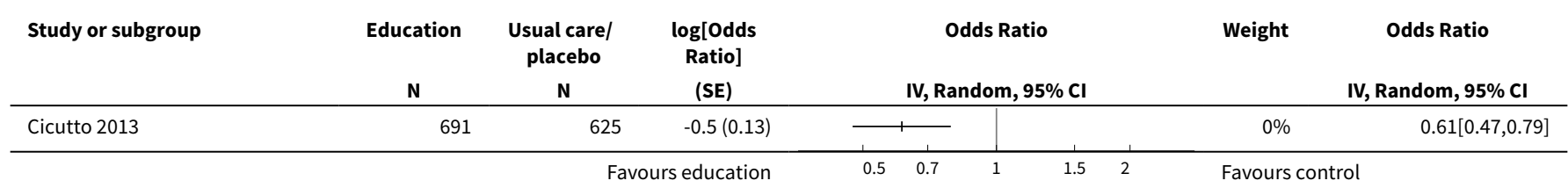

Analysis 12.12. Comparison 12 Effects of school-based asthma interventions vs usual care, including disaggregated effect sizes, Outcome 12 Days of restricted activity - harmonised effect sizes.

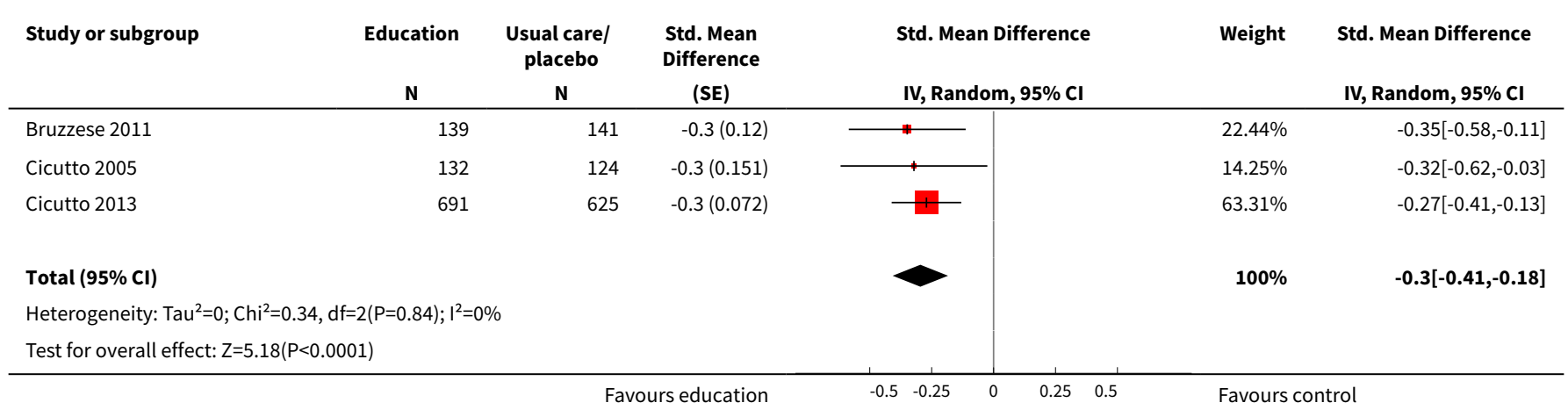


Analysis 12.13. Comparison 12 Effects of school-based asthma interventions vs usual care, including disaggregated effect sizes, Outcome 13 Experience of daytime and night-time symptoms - daytime symptoms - standardised mean difference.

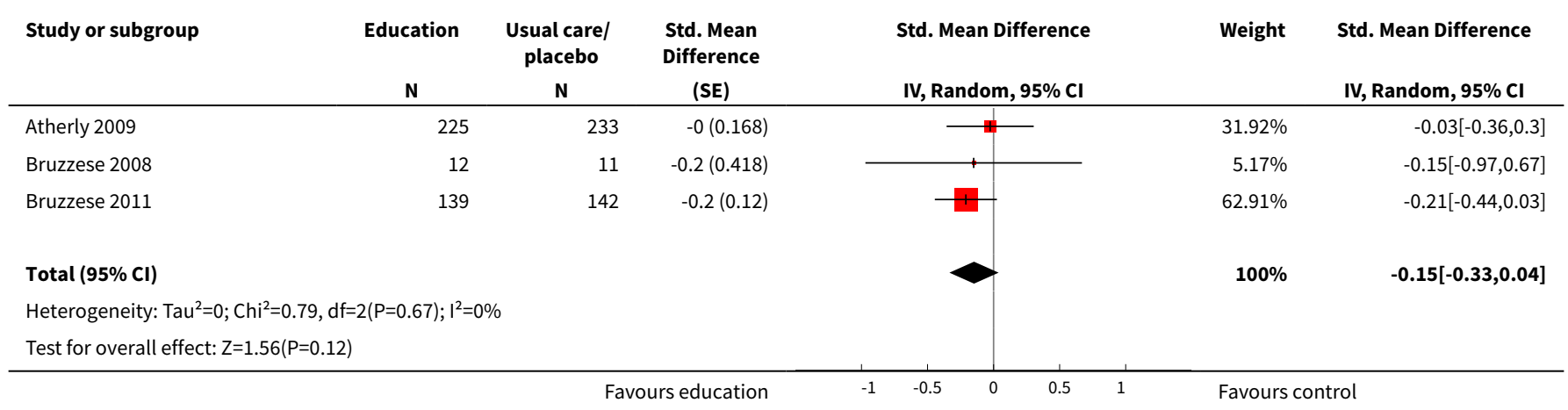

Analysis 12.14. Comparison 12 Effects of school-based asthma interventions vs usual care, including disaggregated effect sizes, Outcome 14 Experience of daytime and night-time symptoms - daytime symptoms - odds ratio.

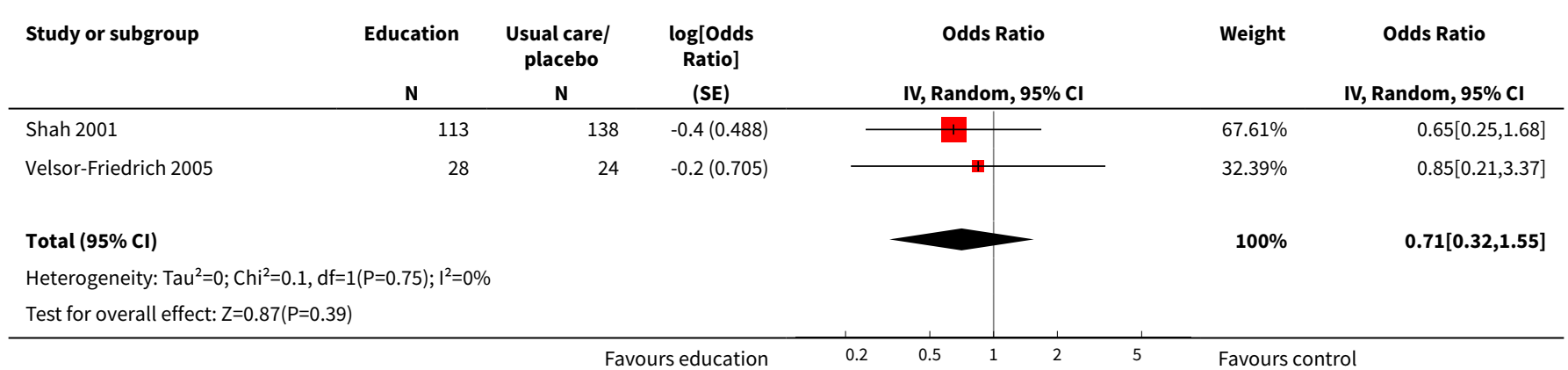

Analysis 12.15. Comparison 12 Effects of school-based asthma interventions vs usual care, including disaggregated effect sizes, Outcome 15 Experience of daytime and night-time symptoms - daytime symptoms - harmonised effect sizes.

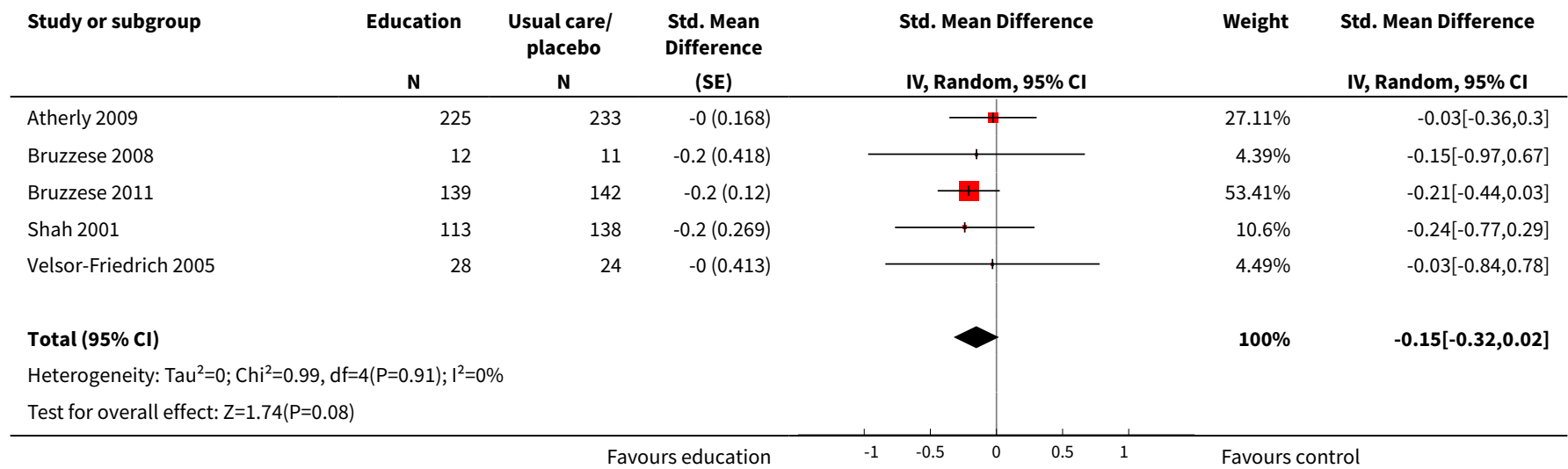


Analysis 12.16. Comparison 12 Effects of school-based asthma interventions vs usual care, including disaggregated effect sizes, Outcome 16 Experience of daytime and night-time symptoms - night-time symptoms - standardised mean difference.

\begin{tabular}{|c|c|c|c|c|c|c|}
\hline Study or subgroup & Education & $\begin{array}{c}\text { Usual care/ } \\
\text { placebo } \\
\mathbf{N} \\
\end{array}$ & $\begin{array}{c}\text { Std. Mean } \\
\text { Difference } \\
\text { (SE) }\end{array}$ & IV, Random, 95\% CI & Weight & IV, Random, 95\% CI \\
\hline Bruzzese 2008 & 12 & 11 & $-0.4(0.423)$ & + & $7.16 \%$ & $-0.43[-1.26,0.4]$ \\
\hline Bruzzese 2011 & 139 & 142 & $-0.4(0.121)$ & & $88.11 \%$ & $-0.39[-0.62,-0.15]$ \\
\hline Howell 2005 & 12 & 7 & $0.3(0.521)$ & 1 & $4.73 \%$ & $0.25[-0.77,1.27]$ \\
\hline \multicolumn{7}{|c|}{ Heterogeneity: $\mathrm{Tau}^{2}=0 ; \mathrm{Chi}^{2}=1.47, \mathrm{df}=2(\mathrm{P}=0.48) ; \mathrm{I}^{2}=0 \%$} \\
\hline Test for overall effect & & & & & & \\
\hline
\end{tabular}

Analysis 12.17. Comparison 12 Effects of school-based asthma interventions vs usual care, including disaggregated effect sizes, Outcome 17 Experience of daytime and night-time symptoms - night-time symptoms - odds ratio.

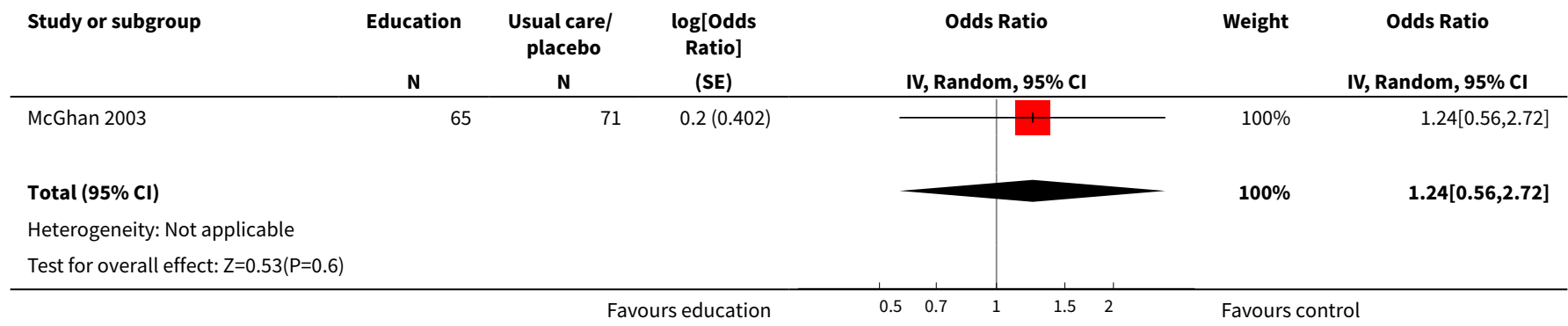

Analysis 12.18. Comparison 12 Effects of school-based asthma interventions vs usual care, including disaggregated effect sizes, Outcome 18 Experience of daytime and night-time symptoms - night-time symptoms - harmonised effect sizes.

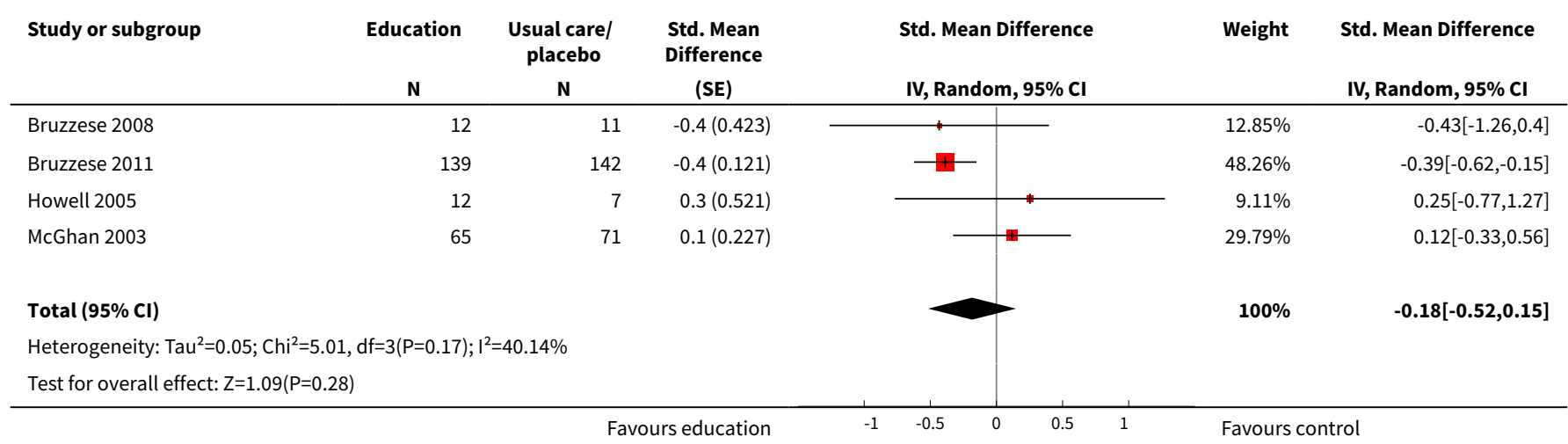


Analysis 12.19. Comparison 12 Effects of school-based asthma interventions vs usual care, including disaggregated effect sizes, Outcome 19 Use of reliever therapies, e.g. beta ${ }_{2}$-agonists - odds ratio.

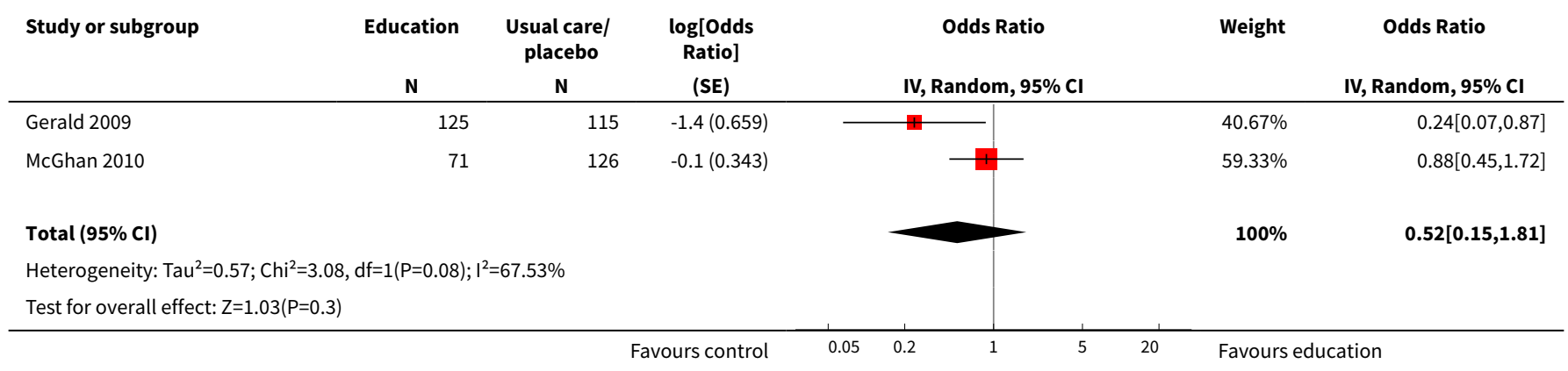

Analysis 12.20. Comparison 12 Effects of school-based asthma interventions vs usual care, including disaggregated effect sizes, Outcome 20 Corticosteroid dosage and/or use of add-on therapies (usage of).

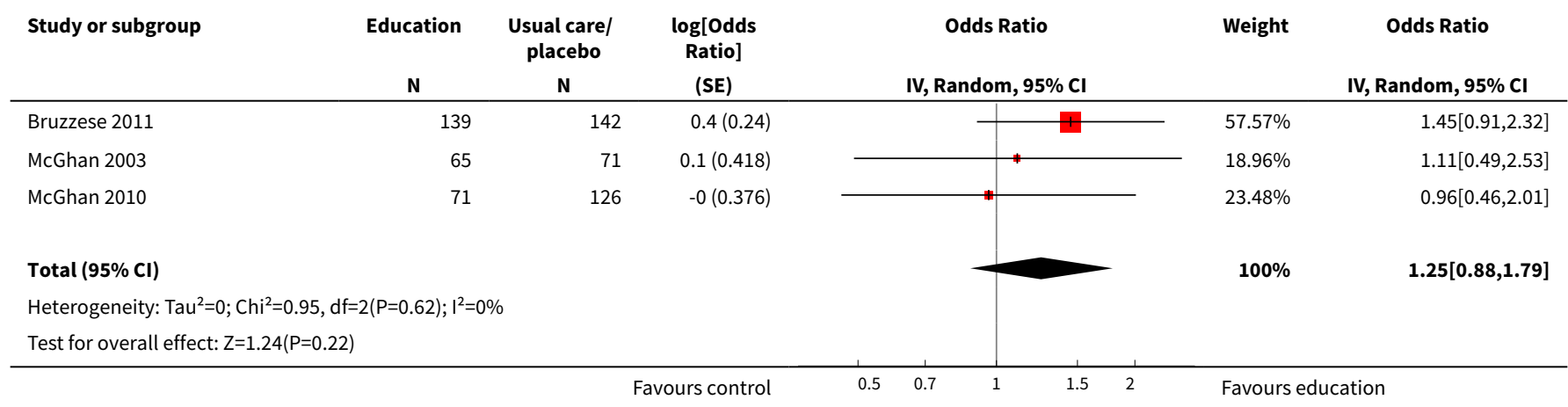

Analysis 12.21. Comparison 12 Effects of school-based asthma interventions vs usual care, including disaggregated effect sizes, Outcome 21 Corticosteroid dosage and/or use of add-on therapies (appropriate usage of).

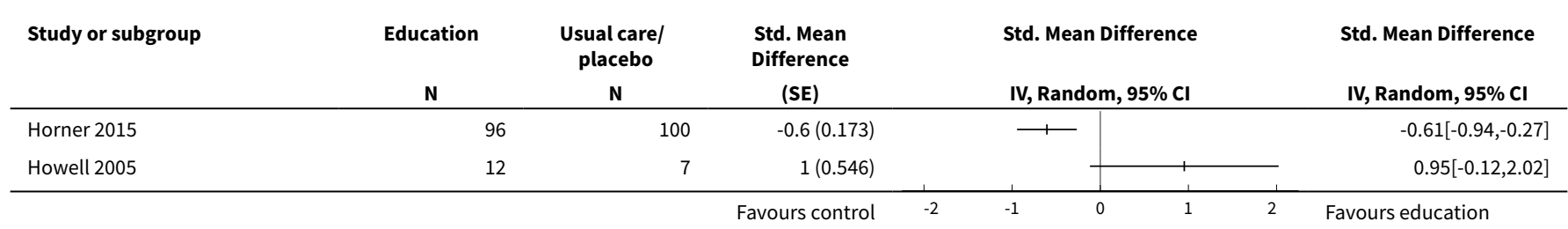

Analysis 12.22. Comparison 12 Effects of school-based asthma interventions vs usual care, including disaggregated effect sizes, Outcome 22 Health-related quality of life - standardised mean difference.

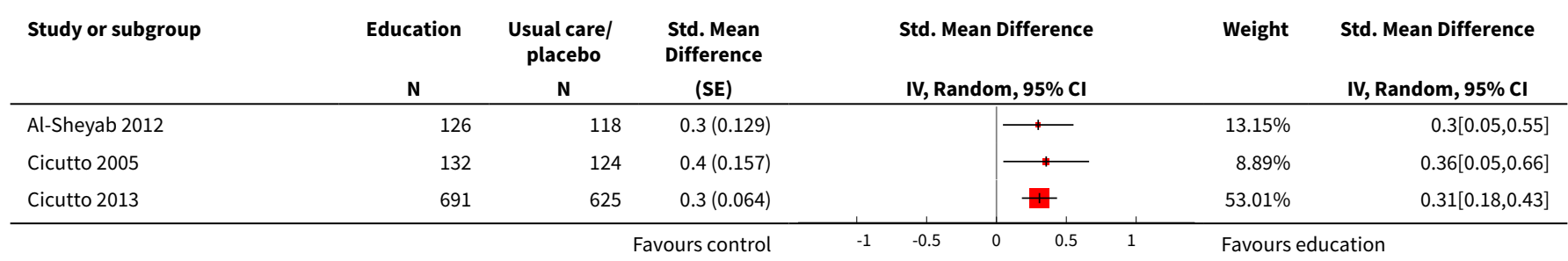




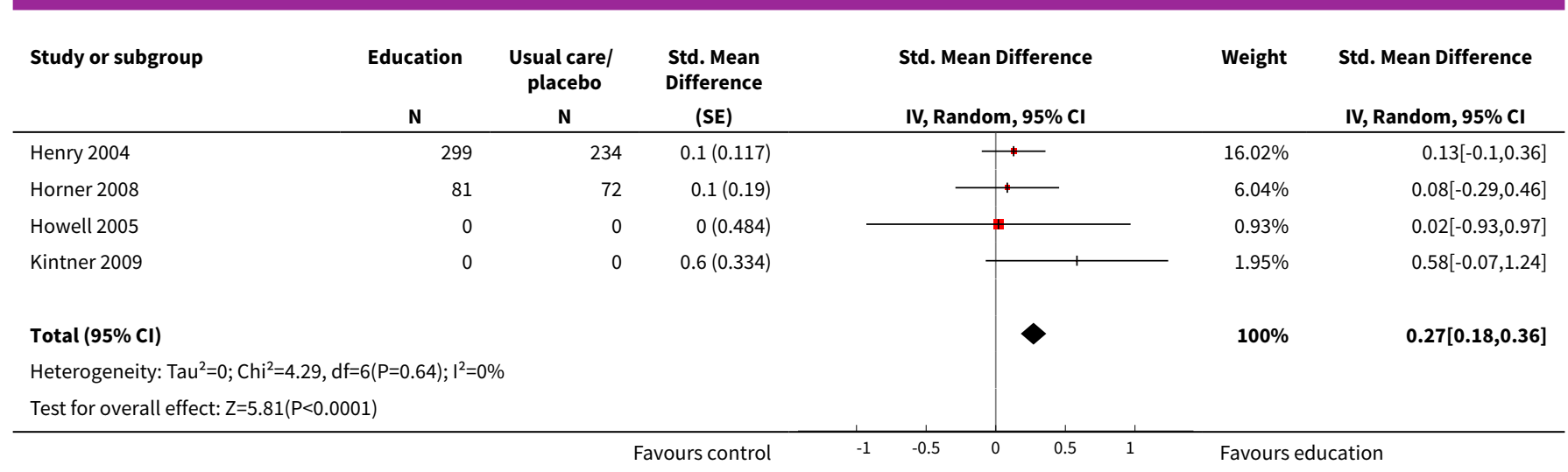

Analysis 12.23. Comparison 12 Effects of school-based asthma interventions vs usual care, including disaggregated effect sizes, Outcome 23 Health-related quality of life (MD).

\begin{tabular}{|c|c|c|c|c|c|c|c|c|}
\hline \multirow{3}{*}{$\begin{array}{l}\text { Study or subgroup } \\
\text { Al-Sheyab } 2012\end{array}$} & \multicolumn{2}{|c|}{ Education } & \multicolumn{2}{|c|}{ Usual care/placebo } & \multirow{2}{*}{\multicolumn{2}{|c|}{$\begin{array}{l}\text { Mean Difference } \\
\text { Random, } 95 \% \mathrm{CI}\end{array}$}} & \multirow[t]{2}{*}{ Weight } & \multirow{2}{*}{$\begin{array}{l}\text { Mean Difference } \\
\text { Random, } 95 \% \mathrm{Cl}\end{array}$} \\
\hline & $\mathbf{N}$ & Mean(SD) & $\mathbf{N}$ & Mean(SD) & & & & \\
\hline & 126 & $5.4(1.6)$ & 118 & $4.1(1.5)$ & & $\longrightarrow$ & $13.19 \%$ & $1.35[0.96,1.74]$ \\
\hline Cicutto 2005 & 132 & $5.5(2)$ & 124 & $5(2)$ & & & $11.47 \%$ & $0.5[0,1]$ \\
\hline Cicutto 2013 & 625 & $5.8(1.6)$ & 691 & $5.4(1.9)$ & & $\rightarrow$ & $16.15 \%$ & $0.4[0.21,0.59]$ \\
\hline Henry 2004 & 299 & $5.3(2.1)$ & 234 & $5.1(2.3)$ & & + & $13.26 \%$ & $0.16[-0.22,0.54]$ \\
\hline Horner 2008 & 81 & $1.7(0.8)$ & 72 & $1.7(0.8)$ & & $\rightarrow$ & $15.17 \%$ & $0.05[-0.21,0.31]$ \\
\hline Howell 2005 & 16 & $5(1.7)$ & 8 & $4.9(2.2)$ & & & $2.38 \%$ & $0.03[-1.71,1.77]$ \\
\hline Patterson 2005 & 81 & $0.3(1.2)$ & 92 & $0.2(1)$ & & + & $14.16 \%$ & $0.07[-0.26,0.4]$ \\
\hline Shah 2001 & 138 & $0.2(1.3)$ & 113 & $0.1(1.3)$ & & + & $14.23 \%$ & $0.09[-0.23,0.41]$ \\
\hline Total $\star \star \star$ & 1498 & & 1452 & & & & $100 \%$ & $0.35[0.06,0.64]$ \\
\hline \multicolumn{9}{|c|}{ Heterogeneity: Tau $^{2}=0.12 ; \mathrm{Chi}^{2}=37.31, \mathrm{df}=7(\mathrm{P}<0.0001) ; \mathrm{I}^{2}=81.24 \%$} \\
\hline \multicolumn{5}{|c|}{ Test for overall effect: $Z=2.4(P=0.02)$} & & & & \\
\hline
\end{tabular}

Analysis 12.24. Comparison 12 Effects of school-based asthma interventions vs usual care, including disaggregated effect sizes, Outcome 24 Unplanned visit to hospital or GP due to asthma symptoms - standardised mean difference.

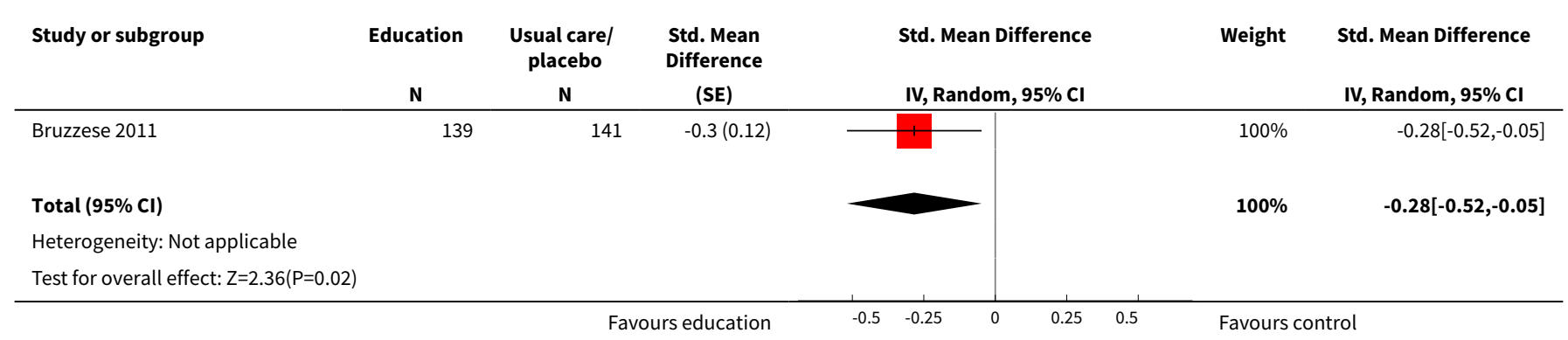


Analysis 12.25. Comparison 12 Effects of school-based asthma interventions vs usual care, including disaggregated effect sizes, Outcome 25 Unplanned visit to hospital or GP due to asthma symptoms - odds ratio.

\begin{tabular}{|c|c|c|c|c|c|c|}
\hline \multirow[t]{2}{*}{ Study or subgroup } & Education & $\begin{array}{l}\text { Usual care/ } \\
\text { placebo }\end{array}$ & \multirow[t]{2}{*}{$\begin{array}{c}\text { log[Odds } \\
\text { Ratio] } \\
\text { (SE) }\end{array}$} & \multirow{2}{*}{$\begin{array}{c}\text { Odds Ratio } \\
\text { IV, Random, } 95 \% \mathrm{CI}\end{array}$} & Weight & Ddds Ratio \\
\hline & $\mathbf{N}$ & N & & & \multicolumn{2}{|c|}{ IV, Random, $95 \% \mathrm{CI}$} \\
\hline Cicutto 2013 & 691 & 625 & $-0.4(0.143)$ & 1 & $65.9 \%$ & $0.7[0.53,0.93]$ \\
\hline McGhan 2003 & 0 & 0 & $-0.1(0.415)$ & 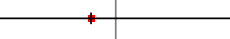 & $7.85 \%$ & $0.89[0.39,2]$ \\
\hline McGhan 2010 & 0 & 0 & $0.2(0.382)$ & & $9.27 \%$ & $1.17[0.55,2.47]$ \\
\hline Splett 2006 & 0 & 0 & $-0.1(0.282)$ & $*$ & $16.98 \%$ & $0.91[0.53,1.59]$ \\
\hline Total $(95 \% \mathrm{Cl})$ & & & & & $100 \%$ & $0.78[0.62,0.98]$ \\
\hline \multicolumn{7}{|c|}{ Heterogeneity: $\operatorname{Tau}^{2}=0 ; \mathrm{Chi}^{2}=2.06, \mathrm{df}=3(\mathrm{P}=0.56) ; \mathrm{I}^{2}=0 \%$} \\
\hline Test for overall effect & & & & & & \\
\hline
\end{tabular}

Analysis 12.26. Comparison 12 Effects of school-based asthma interventions vs usual care, including disaggregated effect sizes, Outcome 26 Unplanned visit to hospital or GP due to asthma symptoms - harmonised effect sizes.

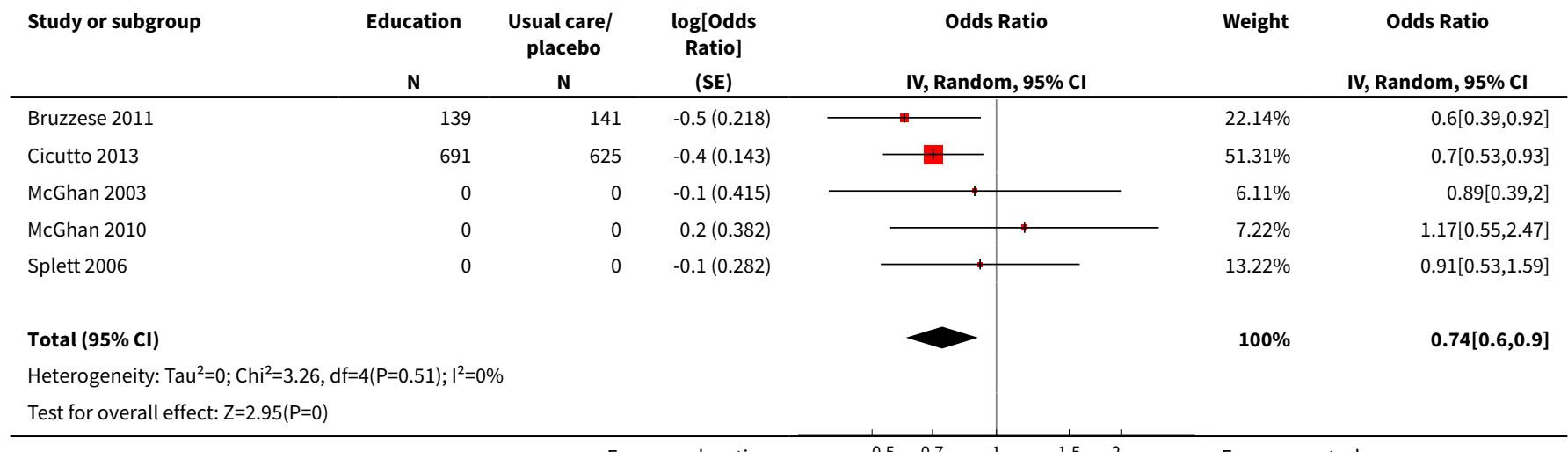

Analysis 12.27. Comparison 12 Effects of school-based asthma interventions vs usual care, including disaggregated effect sizes, Outcome 27 Withdrawal from the study.

\begin{tabular}{|c|c|c|c|c|c|c|c|}
\hline \multirow[t]{2}{*}{ Study or subgroup } & \multirow{2}{*}{$\begin{array}{c}\text { Education } \\
\mathrm{N} \\
\end{array}$} & \multirow{2}{*}{$\begin{array}{c}\text { Usual care/ } \\
\text { placebo } \\
\text { N }\end{array}$} & \multirow{2}{*}{$\begin{array}{c}\text { log[Odds } \\
\text { Ratio] } \\
\text { (SE) }\end{array}$} & \multicolumn{2}{|r|}{ Odds Ratio } & \multirow[t]{2}{*}{ Weight } & \multirow{2}{*}{$\begin{array}{c}\text { Odds Ratio } \\
\text { IV, Random, } 95 \% \mathrm{CI}\end{array}$} \\
\hline & & & & & IV, Random, $95 \% \mathrm{Cl}$ & & \\
\hline Al-Sheyab 2012 & 132 & 129 & $-0.7(1.074)$ & & \begin{tabular}{l|l}
1 & \\
1
\end{tabular} & $1.11 \%$ & $0.51[0.06,4.2]$ \\
\hline Bartholomew 2006 & 515 & 431 & $0.2(0.173)$ & & \# & $42.89 \%$ & $1.27[0.9,1.78]$ \\
\hline Bruzzese 2008 & 12 & 12 & $-1.2(1.683)$ & & 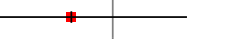 & $0.45 \%$ & $0.31[0.01,8.31]$ \\
\hline Bruzzese 2011 & 175 & 170 & $0.3(0.279)$ & & + & $16.52 \%$ & $1.31[0.76,2.27]$ \\
\hline Gerald 2009 & 145 & 145 & $-0.5(0.368)$ & & $\rightarrow$ & $9.49 \%$ & $0.61[0.3,1.26]$ \\
\hline Horner 2008 & 101 & 82 & $0.3(0.531)$ & & 1 & $4.56 \%$ & $1.33[0.47,3.77]$ \\
\hline Horner 2015 & 96 & 100 & $-0.3(0.486)$ & & $\longrightarrow$ & $5.42 \%$ & $0.75[0.29,1.95]$ \\
\hline Kintner 2009 & 38 & 28 & $2.6(1.882)$ & & $\rightarrow$ & $0.36 \%$ & $13.57[0.34,542.83]$ \\
\hline McGhan 2003 & 76 & 86 & $0.2(0.383)$ & & *- & $8.73 \%$ & $1.25[0.59,2.64]$ \\
\hline McGhan 2010 & 104 & 162 & $-0.2(0.512)$ & & 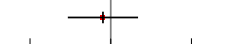 & $4.9 \%$ & $0.8[0.29,2.18]$ \\
\hline
\end{tabular}




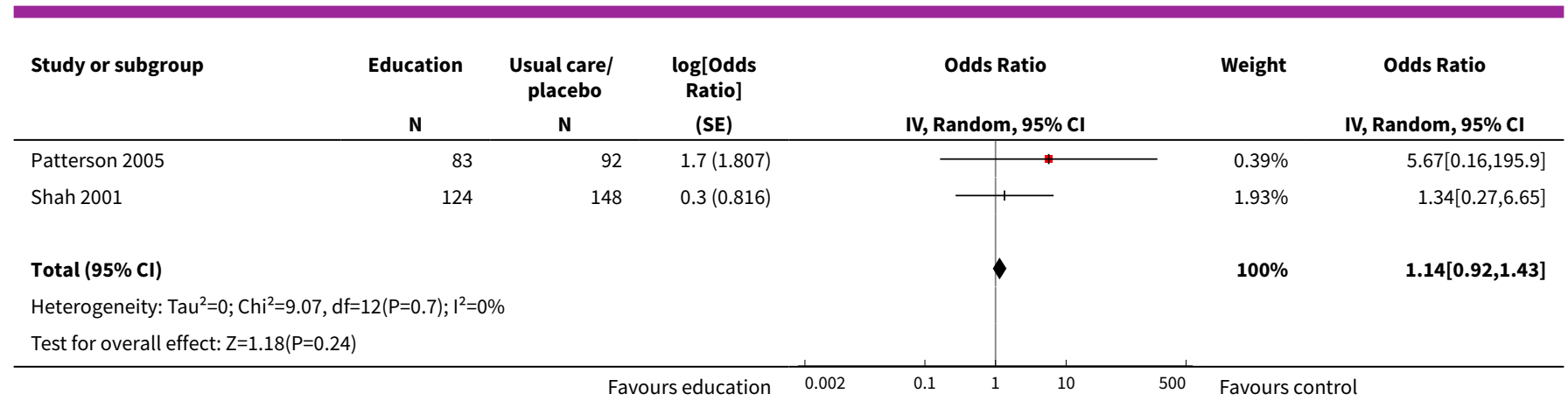

\section{ADDITIONAL TABLES}

Table 1. Detailed coding framework for conditions and outcomes

\begin{tabular}{|c|c|c|c|}
\hline & Field & $\begin{array}{l}\text { Instructions for extrac- } \\
\text { tors }\end{array}$ & Coding values and method \\
\hline & \multicolumn{3}{|c|}{ Setting and participants } \\
\hline 1 & $\begin{array}{l}\text { Number of chil- } \\
\text { dren }\end{array}$ & $\begin{array}{l}\text { Recorded total number } \\
\text { of children involved in in- } \\
\text { tervention }\end{array}$ & $\begin{array}{l}\text { Transformational assignment implemented to condition, } \\
\text { reflecting whether it was a 'large intervention'. Interven- } \\
\text { tions with } 15 \text { or fewer children }=0 \text {; interventions with } 90 \\
\text { children }=0.5 \text {; interventions with } 300 \text { or more children }=1 \text {. } \\
\text { Other values fell between } 0 \text { and } 1\end{array}$ \\
\hline 2 & Multiple settings & $\begin{array}{l}\text { Evidence if delivered at } \\
\text { more than } 1 \text { school }\end{array}$ & Direct assignment: yes (mentioned) $=1 ;$ no evidence $=0$ \\
\hline 3 & Single sex school & $\begin{array}{l}\text { Evidence if delivered at a } \\
\text { single sex school }\end{array}$ & Direct assignment: yes (mentioned) $=1 ;$ no evidence $=0$ \\
\hline 4 & Type of school & $\begin{array}{l}\text { High school; primary/ele- } \\
\text { mentary; junior/middle; } \\
\text { other } \\
\text { Variable transformed to } \\
\text { reflect whether the inter- } \\
\text { vention took place at a } \\
\text { high school }\end{array}$ & $\begin{array}{l}\text { Direct assignment: high school }=1 ; \text { middle } / \text { junior }=0.66 \text {; } \\
\text { elementary } / \text { primary }=0.33 ; \text { missing }=0.5 ; \text { mixture of high } \\
\text { schools and middle schools }=0.75\end{array}$ \\
\hline 5 & $\begin{array}{l}\text { Ethnicity of chil- } \\
\text { dren }\end{array}$ & $\begin{array}{l}\text { Whether minority eth- } \\
\text { nic children were target- } \\
\text { ed/represented. Actu- } \\
\text { al proportions recorded } \\
\text { where possible }\end{array}$ & $\begin{array}{l}\text { Transformational assignment } \\
\text { Interventions with } 25 \% \text { or fewer children from ethnic mi- } \\
\text { nority = } 0 \text {; interventions with } 33.3 \% \text { of children from eth- } \\
\text { nic minority }=0.5 \text {; interventions with } 50 \% \text { or more chil- } \\
\text { dren from ethnic minority = } 1 \\
\text { When value is missing (and no qualitative statement sup- } \\
\text { ports assumption of targeting), assume that this is 'proba- } \\
\text { bly not' - i.e. probably not targeted - input value of } 0.25\end{array}$ \\
\hline 6 & $\begin{array}{l}\text { Socio-economic } \\
\text { status of children }\end{array}$ & $\begin{array}{l}\text { Whether children from } \\
\text { lower socio-econom- } \\
\text { ic groups were target- } \\
\text { ed/represented }\end{array}$ & $\begin{array}{l}\text { Transformational assignment } \\
\text { Interventions with } 25 \% \text { or fewer children from low so- } \\
\text { cio-economic groups }=0 \text {; interventions with } 33.3 \% \text { of chil- } \\
\text { dren from low socio-economic groups }=0.5 \text {; interven- }\end{array}$ \\
\hline
\end{tabular}


Table 1. Detailed coding framework for conditions and outcomes

Actual proportions recorded where possible

Indicators included parents with low levels of education; low household income; receipt of free school meals tions with $50 \%$ or more children from low socio-economic groups $=1$

Where value is missing (and no qualitative statement supports assumption of targeting), assume that this is 'probably not' - i.e. probably not targeted - input value of 0.25

\begin{tabular}{|c|c|c|c|}
\hline 7 & Child age & $\begin{array}{l}\text { Age groups/classes tar- } \\
\text { geted: ages } 5 \text { to } 10\end{array}$ & Direct assignment: yes (mentioned) $=1 ;$ no evidence $=0$ \\
\hline 8 & & $\begin{array}{l}\text { Age groups/classes tar- } \\
\text { geted: ages } 11 \text { to } 14\end{array}$ & Direct assignment: yes (mentioned) $=1 ;$ no evidence $=0$ \\
\hline 9 & & $\begin{array}{l}\text { Age groups/classes tar- } \\
\text { geted: ages } 15 \text { to } 18\end{array}$ & Direct assignment: yes (mentioned) $=1 ;$ no evidence $=0$ \\
\hline 10 & Direct recipients & $\begin{array}{l}\text { Children directed recipi- } \\
\text { ents }\end{array}$ & Direct assignment: yes (mentioned) $=1 ;$ no evidence $=0$ \\
\hline 11 & & $\begin{array}{l}\text { Teachers directed recipi- } \\
\text { ents }\end{array}$ & Direct assignment: yes (mentioned) $=1 ;$ no evidence $=0$ \\
\hline 12 & & $\begin{array}{l}\text { Parents directed recipi- } \\
\text { ents }\end{array}$ & Direct assignment: yes (mentioned) $=1 ;$ no evidence $=0$ \\
\hline 13 & & $\begin{array}{l}\text { School nurses directed } \\
\text { recipients }\end{array}$ & Direct assignment: yes (mentioned) $=1 ;$ no evidence $=0$ \\
\hline
\end{tabular}

\section{Programme design}

\begin{tabular}{ll}
\hline Theory driven & Did the study name a $\quad$ Direct assignment: yes (mentioned) = 1; no evidence = 0 \\
& theoretical framework \\
& that underpins the inter- \\
& vention design or deliv- \\
& ery style?
\end{tabular}

$15 \quad \begin{aligned} & \text { Intensity of the pro- } \\ & \text { gramme }\end{aligned}$

Coded initially as follows: high intensity $=6+$ sessions (group and individual); medium intensity $=3$ to 5 sessions; low intensity/no evidence of $\mathrm{med} / \mathrm{high}=1$ to 2 sessions; unclear. Variable transformed to reflect whether the intervention was of high intensity
Personalisation/tai- Did the programme inloring clude individual sessions or use personalisation in any way to alter curriculum to individual students' needs?
Direct assignment: high intensity $=1$, medium intensity $=$ 0.66 ; low intensity $=0.33$. When no evidence on intensity of intervention was included ( 1 study = (Richmond 2011)), this was coded as 0.33 (no evidence of high intensity) interpreted as no evidence of high intensity; for Splett (Splett 2006), such is the degree of personalisation/tailoring that 0.5 was selected as the intensity - each individual session was personalised and lengthy

Direct assignment: yes, all sessions implemented were personalised/tailored $=1$; some sessions were personalised/tailored $=0.66$; personalisation/tailored sessions account for only a minor component $=0.5$; no evidence, only generic group sessions implemented $=0$

Note that this was personalised by or individual sessions were held with an instructor (included guided online ses- 
Table 1. Detailed coding framework for conditions and outcomes sions); self-study components including homework were not included here

$$
17
$$

Timing of the intervention
Did the intervention interfere with the child's own time (during lunch or after school)?
Direct assignment: yes, all sessions $\mathrm{did}=1$; yes, but not all sessions $=0.75$; missing data $=0.5$; described as not interfering with child's own time $=0$

Direct assignment: yes, all sessions did $=1$; yes, but not all sessions $=0.75$; missing data $=0.5$; described as not interfering with child's lessons/other education $=0$

terfere wi lessons/other education?

Described whether trialists were also providing a control for the main in-

Direct assignment: yes, an equivalent control $=1$; yes, but not an equivalent $=0.66$; no control described $=0$ tervention (intended to capture complexity of running an intervention and a control)

\begin{tabular}{|c|c|c|c|}
\hline 20 & $\begin{array}{l}\text { Instructor or facili- } \\
\text { tator }\end{array}$ & Teacher & $\begin{array}{l}\text { Direct assignment: yes, main instructor }=1 \text {; secondary in- } \\
\text { structor or facilitator }=0.66 \text {; not mentioned as an instruc- } \\
\text { tor } / \text { facilitator }=0\end{array}$ \\
\hline 21 & & Peer & $\begin{array}{l}\text { Direct assignment: yes, main instructor }=1 \text {; secondary in- } \\
\text { structor or facilitator }=0.66 \text {; not mentioned as an instruc- } \\
\text { tor/facilitator }=0\end{array}$ \\
\hline 22 & & School nurse & $\begin{array}{l}\text { Direct assignment: yes, main instructor }=1 \text {; secondary in- } \\
\text { structor or facilitator }=0.66 \text {; not mentioned as an instruc- } \\
\text { tor/facilitator }=0\end{array}$ \\
\hline 23 & & $\begin{array}{l}\text { Self-directed/child-di- } \\
\text { rected }\end{array}$ & $\begin{array}{l}\text { Direct assignment: yes, main instructor }=1 \text {; secondary in- } \\
\text { structor or facilitator }=0.66 ; \text { not mentioned as an instruc- } \\
\text { tor/facilitator }=0\end{array}$ \\
\hline 24 & & Parent & $\begin{array}{l}\text { Direct assignment: yes, main instructor }=1 \text {; secondary in- } \\
\text { structor or facilitator }=0.66 \text {; not mentioned as an instruc- } \\
\text { tor/facilitator }=0\end{array}$ \\
\hline 25 & & Other & $\begin{array}{l}\text { Direct assignment: yes, main instructor }=1 \text {; secondary in- } \\
\text { structor or facilitator }=0.66 \text {; not mentioned as an instruc- } \\
\text { tor/facilitator }=0\end{array}$ \\
\hline
\end{tabular}

Programme content

\begin{tabular}{|c|c|c|c|}
\hline 26 & Curriculum & $\begin{array}{l}\text { Lung physiology/asthma } \\
\text { biology }\end{array}$ & Direct assignment: yes (mentioned) $=1 ;$ no evidence $=0$ \\
\hline 27 & & $\begin{array}{l}\text { Asthma accep- } \\
\text { tance/asthma into iden- } \\
\text { tity }\end{array}$ & Direct assignment: yes (mentioned) $=1 ;$ no evidence $=0$ \\
\hline 28 & & $\begin{array}{l}\text { Symptom monitoring } \\
\text { and correct medication } \\
\text { use }\end{array}$ & Direct assignment: yes (mentioned) $=1 ;$ no evidence $=0$ \\
\hline 30 & & Avoiding triggers & Direct assignment: yes (mentioned) $=1 ;$ no evidence $=0$ \\
\hline
\end{tabular}

School-based self-management interventions for asthma in children and adolescents: a mixed methods systematic review (Review) 


\section{Table 1. Detailed coding framework for conditions and outcomes (Continued)}

\begin{tabular}{|c|c|c|}
\hline 31 & $\begin{array}{l}\text { General health including } \\
\text { exercise }\end{array}$ & Direct assignment: yes (mentioned) $=1 ;$ no evidence $=0$ \\
\hline 32 & $\begin{array}{l}\text { Strengthening alliances } \\
\text { including asthma action } \\
\text { plans with primary care } \\
\text { providers }\end{array}$ & Direct assignment: yes (mentioned) $=1 ;$ no evidence $=0$ \\
\hline 33 & $\begin{array}{l}\text { Specific focus on smok- } \\
\text { ing }\end{array}$ & Direct assignment: yes (mentioned) $=1 ;$ no evidence $=0$ \\
\hline 34 & $\begin{array}{l}\text { Personalised/tailored (in- } \\
\text { dividualised) }\end{array}$ & Direct assignment: yes (mentioned) $=1 ;$ no evidence $=0$ \\
\hline 35 & School performance & Direct assignment: yes (mentioned) $=1 ;$ no evidence $=0$ \\
\hline 36 & Emergencies & Direct assignment: yes (mentioned) $=1 ;$ no evidence $=0$ \\
\hline 37 & Unknown & Direct assignment: yes (mentioned) $=1 ;$ no evidence $=0$ \\
\hline 38 & $\begin{array}{l}\text { Specific focus on breath- } \\
\text { ing/relaxation tech- } \\
\text { niques }\end{array}$ & Direct assignment: yes (mentioned) $=1 ;$ no evidence $=0$ \\
\hline
\end{tabular}

\begin{tabular}{|c|c|c|c|}
\hline 39 & Learning styles & $\begin{array}{l}\text { Problem-solving compo- } \\
\text { nent }\end{array}$ & Direct assignment: yes (mentioned) $=1 ;$ no evidence $=0$ \\
\hline 40 & & $\begin{array}{l}\text { Self-directed (including } \\
\text { homework) component }\end{array}$ & Direct assignment: yes (mentioned) $=1 ;$ no evidence $=0$ \\
\hline 41 & & Peer delivery component & Direct assignment: yes (mentioned) $=1 ;$ no evidence $=0$ \\
\hline 42 & & $\begin{array}{l}\text { Interactive (non-didactic) } \\
\text { components }\end{array}$ & Direct assignment: yes (mentioned) $=1 ;$ no evidence $=0$ \\
\hline 43 & & Didactic components & Direct assignment: yes (mentioned) $=1 ;$ no evidence $=0$ \\
\hline 44 & & Other style/unclear & Direct assignment: yes (mentioned) $=1 ;$ no evidence $=0$ \\
\hline 45 & $\begin{array}{l}\text { Programme ethos/ } \\
\text { aims }\end{array}$ & $\begin{array}{l}\text { Emphasis on social bene- } \\
\text { fit }\end{array}$ & Direct assignment: yes (mentioned) $=1 ;$ no evidence $=0$ \\
\hline 46 & & $\begin{array}{l}\text { Emphasis on improving } \\
\text { well-being }\end{array}$ & Direct assignment: yes (mentioned) $=1 ;$ no evidence $=0$ \\
\hline 47 & & Emphasis on having fun & Direct assignment: yes (mentioned) $=1 ;$ no evidence $=0$ \\
\hline 48 & & $\begin{array}{l}\text { Emphasis on fostering in- } \\
\text { dependence/personal re- } \\
\text { sponsibility }\end{array}$ & Direct assignment: yes (mentioned) $=1 ;$ no evidence $=0$ \\
\hline 49 & & $\begin{array}{l}\text { Emphasis on developing } \\
\text { children's knowledge }\end{array}$ & Direct assignment: yes (mentioned) $=1 ;$ no evidence $=0$ \\
\hline 50 & & $\begin{array}{l}\text { Emphasis on collabora- } \\
\text { tion }\end{array}$ & Direct assignment: yes (mentioned) $=1 ;$ no evidence $=0$ \\
\hline
\end{tabular}




\section{Table 1. Detailed coding framework for conditions and outcomes (Continued)}

\begin{tabular}{llll}
51 & & $\begin{array}{l}\text { Emphasis on tailoring for } \\
\text { specific group needs }\end{array}$ & Direct assignment: yes (mentioned) $=1 ;$ no evidence $=0$ \\
\hline 52 & $\begin{array}{l}\text { Emphasis on breathing } \\
\text { technique }\end{array}$ & Direct assignment: yes (mentioned) $=1 ;$ no evidence $=0$ \\
\hline 53 & Unclear & Direct assignment: yes (mentioned) $=1 ;$ no evidence $=0$ \\
\hline 54 & $\begin{array}{l}\text { Additional compo- } \\
\text { nents - school asth- } \\
\text { ma policy }\end{array}$ & $\begin{array}{l}\text { Additional support pro- } \\
\text { vided for developing } \\
\text { school policy }\end{array}$ & Direct assignment: yes (mentioned) $=1 ;$ no evidence $=0$ \\
\hline 55 & & $\begin{array}{l}\text { School asthma policy de- } \\
\text { veloped organically }\end{array}$ & Direct assignment: yes (mentioned) $=1 ;$ no evidence $=0$
\end{tabular}

\begin{tabular}{|c|c|c|c|}
\hline & \multicolumn{3}{|c|}{ Additional processes undertaken - planned and unplanned } \\
\hline 56 & $\begin{array}{l}\text { Recruitment meth- } \\
\text { ods - school }\end{array}$ & $\begin{array}{l}\text { Ad hoc/convenience } \\
\text { sample of schools }\end{array}$ & Direct assignment: yes (mentioned) $=1 ;$ no evidence $=0$ \\
\hline 57 & & $\begin{array}{l}\text { Census of school district } \\
\text { (all schools invited and } \\
\text { potentially eligible) }\end{array}$ & Direct assignment: yes (mentioned) $=1 ;$ no evidence $=0$ \\
\hline 58 & & $\begin{array}{l}\text { Unspecified methods of } \\
\text { school recruitment }\end{array}$ & Direct assignment: yes (mentioned) $=1 ;$ no evidence $=0$ \\
\hline 59 & $\begin{array}{l}\text { Additional process- } \\
\text { es to improve/at- } \\
\text { tenuate attri- } \\
\text { tion/enrolment }\end{array}$ & $\begin{array}{l}\text { Marketing materials sent } \\
\text { to parents }\end{array}$ & Direct assignment: yes (mentioned) $=1 ;$ no evidence $=0$ \\
\hline 60 & & $\begin{array}{l}\text { Low motivation of stu- } \\
\text { dents acknowledged and } \\
\text { addressed }\end{array}$ & $\begin{array}{l}\text { Direct assignment: yes (mentioned) }=1 \text {; no evidence }=0 \\
\text { Note that } 1 \text { study received a value of } 0.75 \text {, as low motiva- } \\
\text { tion was acknowledged but was not explicitly described } \\
\text { as being addressed (Magzamen 2008) }\end{array}$ \\
\hline 61 & & $\begin{array}{l}\text { Incentives used (child or } \\
\text { parent) }\end{array}$ & $\begin{array}{l}\text { Direct assignment: yes (mentioned) }=1 \text {; no evidence }=0 \\
\text { Incentives for teachers and no evidence for chil- } \\
\text { dren/teachers coded as } 0.5\end{array}$ \\
\hline 62 & & $\begin{array}{l}\text { Make-up/catch-up ses- } \\
\text { sions provided }\end{array}$ & Direct assignment: yes (mentioned) $=1 ;$ no evidence $=0$ \\
\hline 63 & & $\begin{array}{l}\text { Reminders sent to par- } \\
\text { ents/children }\end{array}$ & Direct assignment: yes (mentioned) $=1 ;$ no evidence $=0$ \\
\hline 64 & $\begin{array}{l}\text { Relationships/en- } \\
\text { gagement }\end{array}$ & $\begin{array}{l}\text { Did teachers engage or } \\
\text { participate in the way } \\
\text { they were expected to? }\end{array}$ & $\begin{array}{l}\text { Direct assignment: yes, good reported throughout }=1 \text {; } \\
\text { yes, some weaker evidence of good relationships or evi- } \\
\text { dence that relationships improved during the course of } \\
\text { the intervention }=0.75 \text {; missing, not applicable, or unde- } \\
\text { termined }=0.5 ; \text { no, some weaker evidence of poorer rela- } \\
\text { tionships or evidence that relationships deteriorated dur- } \\
\text { ing the course of the intervention }=0.25 \text {; evidence of poor } \\
\text { relationships throughout }=0\end{array}$ \\
\hline
\end{tabular}


Table 1. Detailed coding framework for conditions and outcomes (Continued)

65
Did parents engage or participate in the way they were expected to?
Direct assignment: yes, good reported throughout $=1$; yes, some weaker evidence of good relationships or evidence that relationships improved during the course of the intervention $=0.75$; missing, not applicable, or undetermined $=0.5$; no, some weaker evidence of poorer relationships or evidence that relationships deteriorated during the course of the intervention $=0.25$; evidence of poor relationships throughout $=0$

One study described good levels of engagement, but review authors assigned value of 0.25 as a third of parents did not engage as expected (Kintner 2012); similar rationale for Mujuru 2011

\section{Process outcomes}

$68 \quad$ Child satisfaction

Did school nurses engage or participate in the way they were expected to?
Direct assignment: yes, good reported throughout $=1$; yes, some weaker evidence of good relationships or evidence that relationships improved during the course of the intervention $=0.75$; missing, not applicable, or undetermined $=0.5$; no, some weaker evidence of poorer relationships or evidence that relationships deteriorated during the course of the intervention $=0.25$; evidence of poor relationships throughout $=0$
Did other relevant stakeholders engage or participate in the way they were expected to?
Direct assignment: yes, good reported throughout $=1$; yes, some weaker evidence of good relationships or evidence that relationships improved during the course of the intervention $=0.75$; missing, not applicable, or undetermined $=0.5$; no, some weaker evidence of poorer relationships or evidence that relationships deteriorated during the course of the intervention $=0.25$; evidence of poor relationships throughout $=0$

Put in level of satisfac-
tion (\%) or record qualitative statement on child satisfaction with the intervention experience. Indicators of satisfaction include children reporting that they enjoyed the intervention; whether the children would recommend the intervention to others; whether children found the intervention helpful. Knowledge development should not be included here

\section{Elements of direct and transformational assignment in- cluded here}

[First] Direct assignment: where there is a qualitative statement indicating positive agreement, assign value of 0.66; where a qualitative statement indicating negative agreement, assign value of 0.33 ; where no child satisfaction data were collected or data were missing, assign value of 0.5

[Second; including of direct above] Transformational assignment implemented to condition reflecting whether children were satisfied. Interventions with $25 \%$ or fewer children satisfied $=0$; interventions with $50 \%$ of children satisfied $=0.5$; missing data coded as 0.5 ; interventions with $75 \%$ or more children satisfied

See text for further justification on use of the $75 \%$ threshold

Elements of direct and transformational assignment here. Note thresholds were higher than for satisfaction, as fewer data were missing

[First] Direct assignment: where there is a qualitative statement indicating high level of completion, assign value of 0.83 ; where a qualitative statement indicating prob- 
Table 1. Detailed coding framework for conditions and outcomes (Continued)

lematic completion, assign value of 0.66 . Where data are missing, assign value of 0.75

[Second; including of direct above] Transformational assignment implemented to condition reflecting level of completion. Interventions with $66 \%$ or fewer children completing the intervention $=0$; interventions with $75 \%$ of children completing the intervention $=0.5$; interventions with $83 \%$ or more children completing the intervention = 1. Missing data coded as 0.5

See text for further justification on the use of thresholds

Child dosage level

Did the children receive the intended dosage of the intervention? Put in level of dosage (\%) or record qualitative statement on child dosage.

Elements of direct and transformational assignment here. Note thresholds are higher than for satisfaction, as fewer data are missing

[First] Direct assignment: where there is a qualitative statement indicating high level of dosage, assign value of 0.83 ; where a qualitative statement indicating problematic dosage, assign value of 0.66 . Where data are missing, assign value of 0.75

[Second; including of direct above] Transformational assignment implemented to condition reflecting level of dosage. Interventions with $66 \%$ or fewer children receiving the full dosage $=0$; interventions with $75 \%$ of children receiving the full dosage $=0.5$; interventions with $83 \%$ or more of children receiving the full dosage $=1$. Missing data coded as 0.5

See text for further justification on the use of thresholds

\section{1}

Child adherence
Did the children adhere to the intervention instructions, e.g. students being compliant with paperwork; completing homework; going to visit PCPs as instructed, etc. Put in level of adherence (\%) or record qualitative statement on child dosage
Elements of direct and transformational assignment here. Note thresholds are higher than for satisfaction as fewer data are missing

[First] Direct assignment: where there is a qualitative statement indicating high level of adherence, assign value of 0.83 ; where a qualitative statement indicating problematic adherence, assign value of 0.66 . Where data are missing, assign value of 0.75

[Second; including of direct above] Transformational assignment implemented to condition reflecting level of adherence. Interventions with $66 \%$ or fewer children adherent $=0$; interventions with $75 \%$ of children adherent $=0.5$; interventions with $83 \%$ or more children adherent $=1$. Missing data coded as 0.5

See text for further justification on the use of thresholds

$72 \quad$ Consolidated
process variable
Summation of attrition, adherence, and dosage scores as a marker of implementation success
Transformational assignment

Score of $0=0$ implementation not successful; score of 1.5 $=$ mid point between successful and unsuccessful implementation; score of 3 = full implementation success 
Table 2. Original and reduced conditions for curriculum content, delivery style, and programme emphasis

\section{Curriculum - original conditions}

I. Lung physiology

ii. Asthma acceptance

iii. Symptom monitoring and treatment

iv. Trigger avoidance

v. General health

vi. Forming alliances

vii. Smoking

viii. Tailored/personalised

ix. School performance

$x$. Emergencies

xi. Unknown content

\section{Curriculum - reduced conditions $a$}
I. Symptom monitoring and alliances
ii. Lung physiology and general health
iii. Symptom monitoring and trigger avoidance
iv. Other various foci
v. Unknown

\begin{tabular}{ll}
\hline I. Problem-solving & I. Interactive focused style \\
$\begin{array}{ll}\text { ii. Self-direct } & \text { ii. Diverse style } \\
\text { iii. Peer delivery } & \text { iii. Unknown style } \\
\text { iv. Interactive } & \\
\text { v. Didactic } & \\
\text { vi. No information/other focus } & \end{array}$
\end{tabular}

\section{Intervention emphasis - original conditions}

Intervention emphasis - reduced conditionsc

\begin{tabular}{ll}
\hline I. Emphasis on social benefit & I. Emphasis on tailoring/personalisation \\
$\begin{array}{l}\text { ii. Emphasis on well-being } \\
\text { iii. Emphasis on having fun }\end{array}$ & ii. Emphasis on personal responsibility \\
iv. Emphasis on personal responsibility & iii. Diffuse emphasis/other \\
v. Emphasis on children's knowledge & \\
vi. Emphasis on collaboration \\
vii. Emphasis on tailoring/personalisation \\
viii. Emphasis unclear
\end{tabular}

aPseudo-F index $=5.66$.

bPseudo-F index $=8.36$.

cPseudo-F index $=6.50$. 
Table 3. Data table for QCA model 1 - setting and participants

\begin{tabular}{|c|c|c|c|c|c|c|}
\hline & $\begin{array}{l}\text { Successful in- } \\
\text { tervention }\end{array}$ & $\begin{array}{l}\text { School-based } \\
\text { health centre }\end{array}$ & $\begin{array}{l}\text { High } \\
\text { school }\end{array}$ & $\begin{array}{l}\text { Parents di- } \\
\text { rectly in- } \\
\text { volved }\end{array}$ & $\begin{array}{l}\text { Teachers } \\
\text { received } \\
\text { training }\end{array}$ & $\begin{array}{l}\text { School } \\
\text { nurses } \\
\text { or oth- } \\
\text { er stake- } \\
\text { holders } \\
\text { received } \\
\text { training }\end{array}$ \\
\hline Joseph 2010 & 0.52 & 0.55 & 1 & 0 & 0 & 0 \\
\hline Kouba 2012 & 0.33 & 0.33 & 1 & 1 & 0 & 0 \\
\hline Dore-Stites 2007 & 0.67 & 0.66 & 0 & 1 & 0 & 0 \\
\hline Joseph 2013 & 1.00 & 0.55 & 1 & 0 & 0 & 0 \\
\hline Mujuru 2011 & 0.67 & 0.66 & 0 & 0 & 1 & 0 \\
\hline Henry 2004 & 0.83 & 0.33 & 1 & 0 & 1 & 0 \\
\hline Pike 2011 & 0.67 & 0.33 & 0 & 0 & 1 & 0 \\
\hline Spencer 2000 & 0.33 & 0.66 & 0 & 1 & 0 & 0 \\
\hline Engelke 2013 & 0.50 & 0.66 & 0.5 & 1 & 1 & 1 \\
\hline Splett 2006 & 0.50 & 1.00 & 0.5 & 0 & 1 & 1 \\
\hline Kintner 2012 & 0.83 & 0.66 & 1 & 1 & 0 & 1 \\
\hline Berg 2004 & 0.83 & 0.66 & 1 & 0 & 0 & 0 \\
\hline Howell 2005 & 0.33 & 0.75 & 0 & 1 & 0 & 0 \\
\hline Gerald 2006 & 0.33 & 0.55 & 0 & 0 & 0 & 0 \\
\hline Langenfeld 2010 & 0.33 & 0.66 & 0 & 0 & 1 & 0 \\
\hline Al-Sheyab 2012 & 0.83 & 0.33 & 1 & 0 & 0 & 0 \\
\hline Levy 2006 & 0.52 & 0.33 & 0 & 0 & 1 & 0 \\
\hline Terpstra 2012 & 1.00 & 0.66 & 0.66 & 1 & 0 & 0 \\
\hline Horner 2015 & 0.67 & 0.66 & 0 & 0 & 0 & 0 \\
\hline Bruzzese 2008 & 0.94 & 0.66 & 0.66 & 1 & 0 & 0 \\
\hline Lee 2011 & 0.50 & 0.66 & 0 & 0 & 0 & 0 \\
\hline Bruzzese 2004 & 0.33 & 0.55 & 1 & 0 & 0 & 1 \\
\hline Cicutto 2013 & 0.67 & 0.33 & 0 & 0 & 0 & 1 \\
\hline Brasler 2006 & 0.00 & 0.66 & 0.66 & 1 & 0 & 0 \\
\hline
\end{tabular}


Table 3. Data table for QCA model 1 - setting and participants (Continued)

\begin{tabular}{lllllll} 
Crane 2014 & 0.50 & 0.33 & 0 & 0 & 0 \\
\hline Bruzzese 2011 & 0.88 & 0.55 & 1 & 0 & 0 & 1 \\
\hline Magzamen 2008 & 0.19 & 0.55 & 0.75 & 0 & 1 \\
\hline
\end{tabular}

QCA: qualitative comparative analysis.

Table 4. Data table for QCA model 2 - recruitment and retention processes

\begin{tabular}{|c|c|c|c|c|c|}
\hline & $\begin{array}{l}\text { Successful inter- } \\
\text { vention }\end{array}$ & $\begin{array}{l}\text { Provision of } \\
\text { additional } \\
\text { marketing } \\
\text { materials }\end{array}$ & $\begin{array}{l}\text { Provision of } \\
\text { incentives }\end{array}$ & $\begin{array}{l}\text { Make-up ses- } \\
\text { sions provid- } \\
\text { ed }\end{array}$ & $\begin{array}{l}\text { Reminders } \\
\text { provided for } \\
\text { attendance } \\
\text { at activity }\end{array}$ \\
\hline Joseph 2010 & 0.52 & 1 & 1 & 0 & 0 \\
\hline Kouba 2012 & 0.33 & 1 & 0 & 1 & 0 \\
\hline Dore-Stites 2007 & 0.67 & 1 & 1 & 0 & 0 \\
\hline Joseph 2013 & 1.00 & 1 & 1 & 0 & 0 \\
\hline Mujuru 2011 & 0.67 & 0 & 0 & 0 & 1 \\
\hline Henry 2004 & 0.83 & 0 & 0 & 0 & 0 \\
\hline Pike 2011 & 0.67 & 0 & 0.5 & 0 & 0 \\
\hline Spencer 2000 & 0.33 & 1 & 0 & 0 & 0 \\
\hline Engelke 2013 & 0.50 & 0 & 0 & 0 & 0 \\
\hline Splett 2006 & 0.50 & 0 & 0 & 0 & 0 \\
\hline Kintner 2012 & 0.83 & 1 & 1 & 1 & 0 \\
\hline Berg 2004 & 0.83 & 0 & 1 & 0 & 0 \\
\hline Howell 2005 & 0.33 & 0 & 1 & 1 & 1 \\
\hline Gerald 2006 & 0.33 & 0 & 0 & 0 & 0 \\
\hline Langenfeld 2010 & 0.33 & 0 & 1 & 0 & 0 \\
\hline Al-Sheyab 2012 & 0.83 & 0 & 0 & 0 & 0 \\
\hline Levy 2006 & 0.52 & 0 & 0 & 0 & 0 \\
\hline Terpstra 2012 & 1.00 & 1 & 1 & 1 & 1 \\
\hline Horner 2015 & 0.67 & 0 & 0 & 0 & 0 \\
\hline Bruzzese 2008 & 0.94 & 0 & 0 & 1 & 0 \\
\hline
\end{tabular}


Table 4. Data table for QCA model 2 - recruitment and retention processes (Continued)

\begin{tabular}{llllll} 
Lee 2011 & 0.50 & 0 & 0.75 & 0 & 0 \\
\hline Bruzzese 2004 & 0.33 & 0 & 1 & 1 & 0 \\
\hline Cicutto 2013 & 0.67 & 0 & 0 & 1 & 0 \\
\hline Brasler 2006 & 0.00 & 1 & 0 & 1 & 0 \\
\hline Crane 2014 & 0.50 & 0 & 0 & 0 \\
\hline Bruzzese 2011 & 0.88 & 0 & 1 & 0 \\
\hline Magzamen 2008 & 0.19 & 1 & 1
\end{tabular}

QCA: qualitative comparative analysis.

Table 5. Data table for QCA model 4 - modifiable design features

\begin{tabular}{|c|c|c|c|c|c|c|}
\hline & $\begin{array}{l}\text { Successful in- } \\
\text { tervention }\end{array}$ & $\begin{array}{l}\text { Theory dri- } \\
\text { ven }\end{array}$ & $\begin{array}{l}\text { Person- } \\
\text { alised or } \\
\text { individual } \\
\text { sessions }\end{array}$ & $\begin{array}{l}\text { Interven- } \\
\text { tion takes } \\
\text { place dur- } \\
\text { ing lesson } \\
\text { time }\end{array}$ & $\begin{array}{l}\text { Intervention } \\
\text { takes place } \\
\text { during stu- } \\
\text { dents' own } \\
\text { free time }\end{array}$ & $\begin{array}{l}\text { School } \\
\text { nurse in- } \\
\text { volved in } \\
\text { delivery of } \\
\text { the inter- } \\
\text { vention }\end{array}$ \\
\hline Joseph 2010 & 0.52 & 1 & 1 & 1 & 0.33 & 0 \\
\hline Kouba 2012 & 0.33 & 1 & 1 & 0 & 1 & 0 \\
\hline Dore-Stites 2007 & 0.67 & 1 & 0 & 0.33 & 0.33 & 0.66 \\
\hline Joseph 2013 & 1.00 & 1 & 1 & 0.75 & 0.75 & 0 \\
\hline Mujuru 2011 & 0.67 & 0 & 0 & 1 & 0 & 0 \\
\hline Henry 2004 & 0.83 & 0 & 0 & 1 & 0 & 0 \\
\hline Pike 2011 & 0.67 & 0 & 0 & 1 & 0 & 0 \\
\hline Spencer 2000 & 0.33 & 0 & 1 & 0.33 & 0.33 & 0.66 \\
\hline Engelke 2013 & 0.50 & 0 & 0.66 & 0.33 & 0.33 & 1 \\
\hline Splett 2006 & 0.50 & 0 & 1 & 0.33 & 0.33 & 1 \\
\hline Kintner 2012 & 0.83 & 1 & 0 & 1 & 1 & 0.66 \\
\hline Berg 2004 & 0.83 & 1 & 0.66 & 0.33 & 0.33 & 0.66 \\
\hline Howell 2005 & 0.33 & 1 & 1 & 0.33 & 0.33 & 0.66 \\
\hline Gerald 2006 & 0.33 & 0 & 0 & 1 & 0.33 & 0 \\
\hline Langenfeld 2010 & 0.33 & 0 & 1 & 0.33 & 0.33 & 1 \\
\hline
\end{tabular}


Table 5. Data table for QCA model 4 - modifiable design features (Continued)

\begin{tabular}{|c|c|c|c|c|c|c|}
\hline Al-Sheyab 2012 & 0.83 & 1 & 0 & 0.33 & 0.33 & 0 \\
\hline Levy 2006 & 0.52 & 0 & 0.66 & 0.33 & 0.33 & 1 \\
\hline Terpstra 2012 & 1.00 & 1 & 0 & 0 & 1 & 0.66 \\
\hline Horner 2015 & 0.67 & 1 & 0 & 0 & 1 & 0 \\
\hline Bruzzese 2008 & 0.94 & 1 & 0 & 0.33 & 0.33 & 0.66 \\
\hline Lee 2011 & 0.50 & 1 & 0 & 1 & 0 & 0.66 \\
\hline Bruzzese 2004 & 0.33 & 1 & 1 & 0.75 & 0.75 & 0 \\
\hline Cicutto 2013 & 0.67 & 1 & 0 & 0 & 1 & 0 \\
\hline Brasler 2006 & 0.00 & 0 & 0 & 0.75 & 0.75 & 0.66 \\
\hline Crane 2014 & 0.50 & 1 & 0 & 0 & 1 & 0.66 \\
\hline Bruzzese 2011 & 0.88 & 1 & 1 & 0.33 & 0.33 & 0 \\
\hline Magzamen 2008 & 0.19 & 0 & 0 & 0 & 1 & 1 \\
\hline
\end{tabular}

QCA: qualitative comparative analysis.

Table 6. Data table for QCA model 5 - stakeholder involvement and engagement

\begin{tabular}{|c|c|c|c|c|c|c|}
\hline & $\begin{array}{l}\text { Successful in- } \\
\text { tervention }\end{array}$ & $\begin{array}{l}\text { School } \\
\text { asthma } \\
\text { policy }\end{array}$ & $\begin{array}{l}\text { Good re- } \\
\text { lation- } \\
\text { ships/en- } \\
\text { gagement } \\
\text { with par- } \\
\text { ents }\end{array}$ & $\begin{array}{l}\text { Good re- } \\
\text { lation- } \\
\text { ships/en- } \\
\text { gage- } \\
\text { ment with } \\
\text { school } \\
\text { nurses }\end{array}$ & $\begin{array}{l}\text { Child Sat- } \\
\text { isfaction }\end{array}$ & $\begin{array}{l}\text { School } \\
\text { asthma } \\
\text { policy }\end{array}$ \\
\hline Joseph 2010 & 0.52 & 0 & 0 & 0 & 0 & 0 \\
\hline Kouba 2012 & 0.33 & 0 & 0 & 0 & 0 & 0 \\
\hline Dore-Stites 2007 & 0.67 & 0 & 0.75 & 1 & 1 & 0 \\
\hline Joseph 2013 & 1.00 & 0 & 1 & 0 & 0 & 0 \\
\hline Mujuru 2011 & 0.67 & 0 & 0.25 & 0 & 0 & 0 \\
\hline Henry 2004 & 0.83 & 1 & 0 & 0 & 0 & 1 \\
\hline Pike 2011 & 0.67 & 0 & 0 & 0 & 0 & 0 \\
\hline Spencer 2000 & 0.33 & 0 & 1 & 1 & 0 & 0 \\
\hline Engelke 2013 & 0.50 & 1 & 1 & 0 & 0 & 1 \\
\hline Splett 2006 & 0.50 & 1 & 0 & 1 & 0 & 1 \\
\hline
\end{tabular}


Table 6. Data table for QCA model 5 - stakeholder involvement and engagement (Continued)

\begin{tabular}{|c|c|c|c|c|c|c|}
\hline Kintner 2012 & 0.83 & 0 & 0.25 & 0 & 1 & 0 \\
\hline Berg 2004 & 0.83 & 0 & 0 & 0 & 1 & 0 \\
\hline Howell 2005 & 0.33 & 0 & 0.75 & 0.75 & 0.633333 & 0 \\
\hline Gerald 2006 & 0.33 & 0 & 0 & 0 & 0 & 0 \\
\hline Langenfeld 2010 & 0.33 & 1 & 0 & 1 & 0 & 1 \\
\hline Al-Sheyab 2012 & 0.83 & 0 & 0 & 0 & 0.633333 & 0 \\
\hline Levy 2006 & 0.52 & 1 & 0 & 0 & 0 & 1 \\
\hline Terpstra 2012 & 1.00 & 0 & 0.25 & 0 & 0 & 0 \\
\hline Horner 2015 & 0.67 & 0 & 0 & 0 & 0 & 0 \\
\hline Bruzzese 2008 & 0.94 & 0 & 1 & 0 & 1 & 0 \\
\hline Lee 2011 & 0.50 & 0 & 0 & 0 & 0 & 0 \\
\hline Bruzzese 2004 & 0.33 & 0 & 0 & 0 & 0.633333 & 0 \\
\hline Cicutto 2013 & 0.67 & 1 & 0 & 0 & 0 & 1 \\
\hline Brasler 2006 & 0.00 & 1 & 0 & 1 & 0.633333 & 1 \\
\hline Crane 2014 & 0.50 & 0 & 0 & 1 & 0 & 0 \\
\hline Bruzzese 2011 & 0.88 & 0 & 0 & 0 & 0 & 0 \\
\hline Magzamen 2008 & 0.19 & 0 & 0 & 0 & 0 & 0 \\
\hline
\end{tabular}

QCA: qualitative comparative analysis.

Table 7. Included process evaluation studies: methodological characteristics and processes described

\begin{tabular}{llll}
\hline Study & Type of study & Approach & Process evaluation elements \\
\hline Al-Sheyab 2012a & Feasibility study & Qualitative & Thematic analyses of student perceptions \\
\hline Berg 2004 & $\begin{array}{l}\text { Outcome and process } \\
\text { evaluation }\end{array}$ & $\begin{array}{l}\text { Qualitative and } \\
\text { quantitative }\end{array}$ & Thematic analyses of student perceptions \\
\hline Bignall 2015 & Feasibility study & $\begin{array}{l}\text { Qualitative and } \\
\text { quantitative }\end{array}$ & Thematic analyses of student perceptions \\
\hline Brasler 2006 & Feasibility/case study & $\begin{array}{l}\text { Quantitative data } \\
\text { and trialist reports }\end{array}$ & Implementation challenges and facilitators identified \\
\hline Bruzzese 2004 & Feasibility study & $\begin{array}{l}\text { Qualitative and } \\
\text { quantitative }\end{array}$ & $\begin{array}{l}\text { Section evaluating intervention reach, dosage, and student } \\
\text { satisfaction }\end{array}$ \\
\hline
\end{tabular}


Table 7. Included process evaluation studies: methodological characteristics and processes described

$\begin{array}{ll}\text { Bruzzese } 2011 \quad \text { Outcome evalua- } & \begin{array}{l}\text { Ountitative } \\ \text { tion with section on } \\ \text { process evaluation }\end{array}\end{array}$

\begin{tabular}{llll}
\hline Bruzzese 2008 & Feasibility study & $\begin{array}{l}\text { Qualitative and } \\
\text { quantitative }\end{array}$ & $\begin{array}{l}\text { Stand-alone section on process evaluation results assessing } \\
\text { implementation and student perceptions }\end{array}$ \\
\hline Carpenter 2016 & $\begin{array}{l}\text { Outcome and process } \\
\text { evaluation }\end{array}$ & $\begin{array}{l}\text { Qualitative and } \\
\text { quantitative }\end{array}$ & Thematic analyses of student perceptions \\
\hline Cicutto 2013 & $\begin{array}{l}\text { Outcome and process } \\
\text { evaluation }\end{array}$ & $\begin{array}{l}\text { (Mainly) Quantita- } \\
\text { tive }\end{array}$ & $\begin{array}{l}\text { In addition to information on other processes of interest, } \\
\text { provided a description of wider school support through poli- } \\
\text { cy changes (process of interest included in the logic model) }\end{array}$ \\
\hline Crane 2014 & Feasibility study & Quantitative & $\begin{array}{l}\text { Study was included as it represented an implementation } \\
\text { study (through focus on the impact of changing dosage } \\
\text { schedule) }\end{array}$ \\
\hline
\end{tabular}

\begin{tabular}{lll}
\hline Dore-Stites $2007 \quad$ Feasibility study Quantitative & $\begin{array}{l}\text { In addition to information on other processes of interest, } \\
\text { provided information on student satisfaction }\end{array}$
\end{tabular}

\begin{tabular}{|c|c|c|c|}
\hline Engelke 2013 & Feasibility study & Quantitative & Detailed process/implementation information was provided \\
\hline Gerald 2006 & $\begin{array}{l}\text { Outcome and process } \\
\text { evaluation }\end{array}$ & $\begin{array}{l}\text { (Mainly) Quantita- } \\
\text { tive }\end{array}$ & $\begin{array}{l}\text { In addition to information on other processes of interest, } \\
\text { provided a description of implementation challenges }\end{array}$ \\
\hline Henry 2004 & $\begin{array}{l}\text { Outcome and process } \\
\text { evaluation }\end{array}$ & $\begin{array}{l}\text { (Mainly) Quantita- } \\
\text { tive }\end{array}$ & $\begin{array}{l}\text { In addition to information on other processes of interest, } \\
\text { provided a description of wider school support through pol- } \\
\text { icy changes (process of interest in the logic model) and as- } \\
\text { sessment of sustainability }\end{array}$ \\
\hline Horner 2015 & $\begin{array}{l}\text { Outcome evaluation } \\
\text { with process evalua- } \\
\text { tion information }\end{array}$ & Quantitative & $\begin{array}{l}\text { Included detailed information on attrition and cost-effective- } \\
\text { ness }\end{array}$ \\
\hline Howell 2005 & $\begin{array}{l}\text { Outcome and process } \\
\text { evaluation }\end{array}$ & Quantitative & $\begin{array}{l}\text { In addition to information on other processes of interest, } \\
\text { provided information on student satisfaction }\end{array}$ \\
\hline Jackson 2006 & $\begin{array}{l}\text { Outcome evaluation } \\
\text { with process evalua- } \\
\text { tion information }\end{array}$ & Quantitative & $\begin{array}{l}\text { In addition to information on other processes of interest, } \\
\text { provided information on student satisfaction }\end{array}$ \\
\hline Joseph 2010 & $\begin{array}{l}\text { Outcome and process } \\
\text { evaluation }\end{array}$ & Quantitative & $\begin{array}{l}\text { In addition to information on other processes of interest, } \\
\text { provided detailed information on non-adherence }\end{array}$ \\
\hline Joseph 2013 & $\begin{array}{l}\text { Outcome and process } \\
\text { evaluation }\end{array}$ & Quantitative & $\begin{array}{l}\text { Included detailed studies of non-adherence and relationship } \\
\text { with student characteristics }\end{array}$ \\
\hline Kintner 2012 & Feasibility study & Quantitative & $\begin{array}{l}\text { In addition to information on other processes of interest, } \\
\text { provided information on student satisfaction }\end{array}$ \\
\hline Kouba 2012 & $\begin{array}{l}\text { Outcome evaluation } \\
\text { with process evalua- } \\
\text { tion information }\end{array}$ & Quantitative & $\begin{array}{l}\text { In addition to information on other processes of interest, } \\
\text { provided detailed information on dosage (and dose-re- } \\
\text { sponse) }\end{array}$ \\
\hline
\end{tabular}


Table 7. Included process evaluation studies: methodological characteristics and processes described

(Continued)

$\begin{array}{lll}\text { Langenfeld } 2010 \quad \text { Implementation study Quantitative } & \begin{array}{l}\text { In addition to information on other processes of interest, } \\ \text { provided detailed information on dosage (and dose-re- } \\ \text { sponse) }\end{array}\end{array}$

\begin{tabular}{llll}
\hline Lee 2011 & Implementation study & Qualitative & $\begin{array}{l}\text { In addition to information on other processes of interest, } \\
\text { provided detailed information on instructor experiences }\end{array}$ \\
\hline Levy 2006 & $\begin{array}{l}\text { Outcome evaluation } \\
\text { with process evalua- } \\
\text { tion information }\end{array}$ & Quantitative & $\begin{array}{l}\text { In addition to information on other processes of interest, } \\
\text { provided information on parental adherence to intervention } \\
\text { protocol }\end{array}$ \\
\hline
\end{tabular}

\begin{tabular}{|c|c|c|c|}
\hline Magzamen 2008 & $\begin{array}{l}\text { Outcome evaluation } \\
\text { with process evalua- } \\
\text { tion information }\end{array}$ & Quantitative & $\begin{array}{l}\text { In addition to information on other processes of interest, } \\
\text { provided information on attrition }\end{array}$ \\
\hline McCann 2006 & $\begin{array}{l}\text { Outcome evaluation } \\
\text { with process evalua- } \\
\text { tion information }\end{array}$ & Quantitative & $\begin{array}{l}\text { In addition to information on other processes of interest, } \\
\text { provided information on teacher adherence/school level } \\
\text { commitment }\end{array}$ \\
\hline Mickel 2016 & $\begin{array}{l}\text { Outcome and process } \\
\text { evaluation }\end{array}$ & $\begin{array}{l}\text { Qualitative and } \\
\text { quantitative }\end{array}$ & Thematic analyses of student perceptions \\
\hline Mujuru 2011 & $\begin{array}{l}\text { Outcome and process } \\
\text { evaluation }\end{array}$ & $\begin{array}{l}\text { (Mainly) Quantita- } \\
\text { tive }\end{array}$ & $\begin{array}{l}\text { In addition to information on other processes of interest, } \\
\text { provided a description of parental satisfaction }\end{array}$ \\
\hline Pike 2011 & $\begin{array}{l}\text { Outcome and process } \\
\text { evaluation }\end{array}$ & $\begin{array}{l}\text { (Mainly) Quantita- } \\
\text { tive }\end{array}$ & $\begin{array}{l}\text { In addition to information on other processes of interest, } \\
\text { provided information on teacher adherence/school level } \\
\text { commitment }\end{array}$ \\
\hline Richmond 2011 & $\begin{array}{l}\text { Outcome and process } \\
\text { evaluation }\end{array}$ & $\begin{array}{l}\text { (Mainly) Quantita- } \\
\text { tive }\end{array}$ & Included detailed information on adherence and awareness \\
\hline Spencer 2000 & $\begin{array}{l}\text { Outcome and process } \\
\text { evaluation }\end{array}$ & Quantitative & $\begin{array}{l}\text { In addition to information on other processes of interest, } \\
\text { provided information on instructor satisfaction and school } \\
\text { level commitment }\end{array}$ \\
\hline Splett 2006 & $\begin{array}{l}\text { Outcome and process } \\
\text { evaluation }\end{array}$ & Quantitative & $\begin{array}{l}\text { In addition to information on other processes of interest, } \\
\text { provided information on adherence and school level com- } \\
\text { mitment }\end{array}$ \\
\hline Terpstra 2012 & $\begin{array}{l}\text { Outcome and process } \\
\text { evaluation }\end{array}$ & Quantitative & $\begin{array}{l}\text { In addition to information on other processes of interest, } \\
\text { represented an implementation study by including a focus } \\
\text { on the impact of parental involvement/increasing parental } \\
\text { awareness }\end{array}$ \\
\hline
\end{tabular}

Table 8. Process evaluation studies - summary of intervention characteristics

\begin{tabular}{|c|c|c|c|c|c|c|}
\hline & $\begin{array}{l}\text { Named } \\
\text { theoretical } \\
\text { framework }\end{array}$ & Aim & Intervention type & Control & Intensity & $\begin{array}{l}\text { Included in } \\
\text { QCA }\end{array}$ \\
\hline $\begin{array}{l}\text { Al-Sheyab } \\
2012 a\end{array}$ & $\begin{array}{l}\text { Develop- } \\
\text { mental } \\
\text { stages (not } \\
\text { named) }\end{array}$ & $\begin{array}{l}\text { To assess feasibility in the } \\
\text { Jordanian context of a } \\
\text { peer-led, school-based } \\
\text { asthma education pro- } \\
\text { gramme }\end{array}$ & $\begin{array}{l}\text { Triple A. Children received } \\
\text { education through interac- } \\
\text { tive teaching and learning } \\
\text { activities }\end{array}$ & $\mathrm{N} / \mathrm{A}$ & $\begin{array}{l}14 \text { hours } \\
\text { over } 6 \text { days }\end{array}$ & $\begin{array}{l}\text { Setting } \\
\text { and partic- } \\
\text { ipants; fur- } \\
\text { ther modifi- } \\
\text { able design }\end{array}$ \\
\hline
\end{tabular}




\begin{tabular}{lll}
\hline Berg 2004 & $\begin{array}{l}\text { Social } \\
\text { learning } \\
\text { theory }\end{array}$ & $\begin{array}{l}\text { To evaluate effects of the } \\
\text { Power Breathing pro- } \\
\text { gramme and individual } \\
\text { coaching sessions on asth- } \\
\text { ma knowledge and func- } \\
\text { tional health status }\end{array}$ \\
\end{tabular}

Power Breathing. Children

received education in a

group session on asthma

management

tional health status
N/A

2 weeks

Stakeholder involvement and engagement

Bignall Non
2015

To test the feasibility and
preliminary efficacy of
a school-based RCT on
breathing retraining for
asthma outcomes and
anxiety symptoms

Single workshop for children. Children received information on relaxation/breathing techniques

$\begin{array}{ll}30 \text { minutes } & 2 \text { face-to- } \\ \text { of standard } & \text { face visits } \\ \text { asthma ed- } & 1 \text { month } \\ \text { ucation } & \text { apart }\end{array}$

None asthma ed- 1 month

\begin{tabular}{|c|c|c|}
\hline $\begin{array}{l}\text { Brasler } \\
2006\end{array}$ & None & $\begin{array}{l}\text { To provide adolescents } \\
\text { with knowledge and } \\
\text { skills to take control of } \\
\text { their asthma; to enhance } \\
\text { knowledge and skills of } \\
\text { school staff, health profes- } \\
\text { sionals, and parents }\end{array}$ \\
\hline
\end{tabular}

$\begin{array}{llll}\text { Power Breathing. Children } & \text { N/A } & 3 \times 90- & \text { None } \\ \text { received basic asthma ed- } & & \text { minute } & \\ \text { ucation and addressed so- } & & \text { or } 6 \times 45- & \\ \text { cial/lifestyle concerns } & & \text { minute ses- } & \\ & & \text { sions } & \end{array}$

\begin{tabular}{lll}
\hline Bruzzese & Self-regula- & To help students weave \\
2004 & tion theory & $\begin{array}{l}\text { asthma and management } \\
\text { strategies into their self- } \\
\text { identity }\end{array}$
\end{tabular}

$\begin{array}{llll}\begin{array}{l}\text { ASMA. Students were taught } \\ \text { how to manage their asth- }\end{array} & \text { Usual care } & \begin{array}{l}3 \text { work- } \\ \text { shops 2 or }\end{array} & \begin{array}{l}\text { Stakehold- } \\ \text { er involve- }\end{array} \\ \text { ma to prevent symptoms } & 3 \text { weeks } & \text { ment and } \\ \text { and reduced quality of life. } & \text { apart for } 8 & \text { engage- } \\ \text { Continued medical edu- } & \text { weeks } & \text { ment }\end{array}$

cation was also offered to medical providers

\begin{tabular}{lll}
\hline Bruzzese & Social cog- & To test the efficacy of AS- \\
2011 & nitive theo- & MA \\
& ry
\end{tabular}

ASMA; academic detailing.
Students attended work-
shops to empower them to
manage their asthma. Par-
ents received training on
how to support their child's
need to manage his or her
asthma

\begin{tabular}{|c|c|c|}
\hline Usual care & $\begin{array}{l}\text { 8-week pro- } \\
\text { gramme/3x } \\
45-\text {-minute } \\
\text { sessions } \\
\text { and in- } \\
\text { dividual } \\
\text { coaching } \\
\text { sessions } \\
\text { once a } \\
\text { week for } 5 \\
\text { weeks }\end{array}$ & $\begin{array}{l}\text { Further } \\
\text { modifiable } \\
\text { design fea- } \\
\text { tures }\end{array}$ \\
\hline Usual care & $\begin{array}{l}6 \times 75- \\
\text { minute } \\
\text { group ses- } \\
\text { sions once } \\
\text { a week for } \\
6 \text { weeks; } \\
\text { caregiv- } \\
\text { er } 5 \times 90- \\
\text { minute ses- }\end{array}$ & $\begin{array}{l}\text { Setting } \\
\text { and partic- } \\
\text { ipants; fur- } \\
\text { ther modifi- } \\
\text { able design } \\
\text { features; } \\
\text { stakehold- } \\
\text { er involve- } \\
\text { ment and }\end{array}$ \\
\hline
\end{tabular}

\begin{tabular}{|c|c|c|c|c|c|c|}
\hline $\begin{array}{l}\text { Bruzzese } \\
2008\end{array}$ & $\begin{array}{l}\text { Social cog- } \\
\text { nitive the- } \\
\text { ory; cogni- } \\
\text { tive-behav- } \\
\text { ioural ther- } \\
\text { apy }\end{array}$ & $\begin{array}{l}\text { To test the feasibility and } \\
\text { short-term outcomes of } \\
\text { asthma: it's a family affair! }\end{array}$ & $\begin{array}{l}\text { OAS and ASMA; caregiver } \\
\text { education. Intervention stu- } \\
\text { dents received education } \\
\text { about asthma, based on ex- } \\
\text { isting materials, from cop- } \\
\text { ing with asthma at home } \\
\text { and at school; OAS and AS- } \\
\text { MA }\end{array}$ & Usual care & $\begin{array}{l}6 \times 75- \\
\text { minute } \\
\text { group ses- } \\
\text { sions once } \\
\text { a week for } \\
6 \text { weeks; } \\
\text { caregiv- } \\
\text { er } 5 \times 90- \\
\text { minute ses- }\end{array}$ & $\begin{array}{l}\text { Setting } \\
\text { and partic- } \\
\text { ipants; fur- } \\
\text { ther modifi- } \\
\text { able design } \\
\text { features; } \\
\text { stakehold- } \\
\text { er involve- } \\
\text { ment and }\end{array}$ \\
\hline
\end{tabular}


Table 8. Process evaluation studies - summary of intervention characteristics

\begin{tabular}{|c|c|c|c|c|c|c|}
\hline & & & & & $\begin{array}{l}\text { sions once } \\
\text { a week }\end{array}$ & $\begin{array}{l}\text { engage- } \\
\text { ment }\end{array}$ \\
\hline $\begin{array}{l}\text { Carpenter } \\
2016\end{array}$ & None & $\begin{array}{l}\text { To test whether a tailored } \\
\text { inhaler technique video } \\
\text { intervention could be fea- } \\
\text { sibly implemented by } \\
\text { school nurses; to improve } \\
\text { the inhaler technique of } \\
\text { children with asthma }\end{array}$ & $\begin{array}{l}\text { Multiple sessions for chil- } \\
\text { dren. Children watched a } \\
\text { tailored video and demon- } \\
\text { strated their inhaler tech- } \\
\text { nique before and after }\end{array}$ & $\mathrm{N} / \mathrm{A}$ & $\begin{array}{l}6 \text { weeks or } \\
\text { less }\end{array}$ & None \\
\hline
\end{tabular}

\begin{tabular}{lll}
\hline Cicutto & Social cog- & $\begin{array}{l}\text { To prepare and support } \\
\text { nitive theo- } \\
\text { children with asthma to } \\
\text { be successful managers } \\
\text { of their asthma, thereby } \\
\text { reducing school absen- } \\
\text { teeism, interrupted activi- } \\
\end{array}$ \\
& &
\end{tabular}

\begin{abstract}
Roaring Adventures of Puff. Workshops included goalsetting and self-monitoring, trigger identification, control and avoidance, basic pathophysiology, medication use, symptom recognition, and the asthma action plan, using interactive techniques
\end{abstract}

Usual care Unclear

Setting and participants

\begin{tabular}{|c|c|c|c|c|c|c|}
\hline Crane 2014 & $\begin{array}{l}\text { Education- } \\
\text { al theory of } \\
\text { Jean Piaget }\end{array}$ & $\begin{array}{l}\text { To pilot a shorter, con- } \\
\text { densed OAS education } \\
\text { programme as an alterna- } \\
\text { tive, yet still effective, de- } \\
\text { livery approach compared } \\
\text { to the lengthier original } \\
\text { programme }\end{array}$ & $\begin{array}{l}\text { OAS. Children received edu- } \\
\text { cation from OAS }\end{array}$ & $\begin{array}{l}\text { Non-equiv- } \\
\text { alent inter- } \\
\text { vention }\end{array}$ & 10 weeks & $\begin{array}{l}\text { Setting } \\
\text { and partic- } \\
\text { ipants; fur- } \\
\text { ther modifi- } \\
\text { able design } \\
\text { features }\end{array}$ \\
\hline $\begin{array}{l}\text { Dore-Stites } \\
2007\end{array}$ & None & Unclear & $\begin{array}{l}\text { OAS; Quest for the Code. } \\
\text { Children received a com- } \\
\text { puter game, home activi- } \\
\text { ties, and caregiver informa- } \\
\text { tion }\end{array}$ & $\mathrm{N} / \mathrm{A}$ & $\begin{array}{l}20 \text { minutes } \\
\text { a week for } 8 \\
\text { to } 9 \text { weeks }\end{array}$ & $\begin{array}{l}\text { Further } \\
\text { modifiable } \\
\text { design fea- } \\
\text { tures }\end{array}$ \\
\hline $\begin{array}{l}\text { Engelke } \\
2013\end{array}$ & $\begin{array}{l}\text { Case man- } \\
\text { agement } \\
\text { theory }\end{array}$ & $\begin{array}{l}\text { To identify the process of } \\
\text { case management used by } \\
\text { school nurses, and when } \\
\text { they provide case man- } \\
\text { agement to students with } \\
\text { asthma. The second aim } \\
\text { was to identify the impact } \\
\text { of case management on } \\
\text { parent perception of how } \\
\text { well the child manages ill- } \\
\text { ness; parent perception of } \\
\text { how well the child keeps } \\
\text { up with school work; qual- } \\
\text { ity of life and academic } \\
\text { achievement of children }\end{array}$ & $\begin{array}{l}\text { Case management; nurse } \\
\text { meetings; multiple sessions } \\
\text { for children; multiple ses- } \\
\text { sions for staff. Children re- } \\
\text { ceived education and coun- } \\
\text { selling, and parent/family } \\
\text { education was delivered, } \\
\text { as well as education and } \\
\text { healthcare co-ordination for } \\
\text { teachers/staff }\end{array}$ & $\mathrm{N} / \mathrm{A}$ & Unclear & None \\
\hline Gerald 2006 & None & $\begin{array}{l}\text { To evaluate a comprehen- } \\
\text { sive school-based asthma } \\
\text { management programme } \\
\text { in an inner city, largely } \\
\text { African American school } \\
\text { system }\end{array}$ & $\begin{array}{l}\text { OAS. The intervention in- } \\
\text { cluded } 3 \text { educational pro- } \\
\text { grammes and medical man- } \\
\text { agement for children, as } \\
\text { well as education for school } \\
\text { staff }\end{array}$ & Usual care & Unclear & None \\
\hline
\end{tabular}


Table 8. Process evaluation studies - summary of intervention characteristics

\begin{tabular}{|c|c|c|}
\hline Henry 2004 & Unclear & $\begin{array}{l}\text { To determine whether an } \\
\text { asthma education pro- } \\
\text { gramme in schools would } \\
\text { have a direct impact on } \\
\text { student knowledge and } \\
\text { attitudes toward asthma } \\
\text { and quality of life of stu- } \\
\text { dents with asthma; an in- } \\
\text { direct impact on teacher } \\
\text { knowledge and attitudes } \\
\text { on asthma and on school } \\
\text { policies about asthma; } \\
\text { and a sustainable pro- } \\
\text { gramme after resources }\end{array}$ \\
\hline
\end{tabular}

Asthma education. A pack-

age about asthma was

Usual care Unclear

Setting

taught within the PD/H/PE

and partici-

(Personal Development,

pants

Health and Physical Educa-

tion) strand of the school

curriculum

\begin{tabular}{lll}
\hline Horner & Bruhn's & To test effects of 2 modes \\
theoreti- & of delivering an asthma \\
& cal model & educational intervention \\
& of asthma \\
self-man- & on health outcomes and \\
agement & \\
& & \\
\hline
\end{tabular}

\begin{tabular}{|c|c|c|c|c|c|c|}
\hline $\begin{array}{l}\text { Howell } \\
2005\end{array}$ & $\begin{array}{l}\text { Learning } \\
\text { theory and } \\
\text { behaviour }\end{array}$ & $\begin{array}{l}\text { To examine whether it } \\
\text { was feasible to implement } \\
\text { an interactive computer }\end{array}$ & $\begin{array}{l}\text { Quest for the Code. Com- } \\
\text { puter game }\end{array}$ & Usual care & $\begin{array}{l}4 \times 30- \\
\text { minute ses- } \\
\text { sions }\end{array}$ & None \\
\hline
\end{tabular}

modifica- game at school health cen-

\begin{tabular}{|c|c|c|}
\hline $\begin{array}{l}\text { 7-topic curriculum. The in- } \\
\text { tervention was designed for } \\
\text { children in rural areas and } \\
\text { included asthma informa- } \\
\text { tion }\end{array}$ & $\begin{array}{l}\text { In-school } \\
\text { asthma } \\
\text { classes }\end{array}$ & $\begin{array}{l}16 \times 15- \\
\text { minute ses- } \\
\text { sions for } 5 \\
\text { weeks }\end{array}$ \\
\hline $\begin{array}{l}\text { Quest for the Code. Com- } \\
\text { puter game }\end{array}$ & Usual care & $\begin{array}{l}4 \times 30- \\
\text { minute ses- } \\
\text { sions }\end{array}$ \\
\hline
\end{tabular}

tion tres. Second, to examine

whether exposure to the game was effective in increasing asthma knowledge, reducing asthma symptoms, and reducing unnecessary healthcare use compared with no exposure to the game

\begin{tabular}{|c|c|c|c|c|c|c|}
\hline $\begin{array}{l}\text { Jackson } \\
2006\end{array}$ & None & $\begin{array}{l}\text { To evaluate knowledge } \\
\text { and attitude outcomes of } \\
\text { an educational asthma } \\
\text { programme for third grade } \\
\text { children with and without } \\
\text { asthma }\end{array}$ & $\begin{array}{l}\text { Single sessions for chil- } \\
\text { dren. Children completed } \\
\text { an educational programme. } \\
\text { Teachers were also encour- } \\
\text { aged to attend }\end{array}$ & $\mathrm{N} / \mathrm{A}$ & $\begin{array}{l}3 \text { classes } \\
\text { per session } \\
\text { for } 11 \text { ses- } \\
\text { sions }\end{array}$ & None \\
\hline $\begin{array}{l}\text { Joseph } \\
2010\end{array}$ & None & $\begin{array}{l}\text { To develop and evaluate a } \\
\text { multi-media, web-based } \\
\text { asthma management pro- } \\
\text { gramme }\end{array}$ & $\begin{array}{l}\text { Puff City. A web-based pro- } \\
\text { gramme was delivered to } \\
\text { children to focus on adher- } \\
\text { ence, inhaler availability, } \\
\text { and smoking cessation/re- } \\
\text { duction }\end{array}$ & $\begin{array}{l}\text { Generic } \\
\text { asthma } \\
\text { websites }\end{array}$ & Unclear & $\begin{array}{l}\text { Further } \\
\text { modifi- } \\
\text { able design } \\
\text { features; } \\
\text { stakehold- } \\
\text { er involve- } \\
\text { ment and } \\
\text { engage- } \\
\text { ment }\end{array}$ \\
\hline $\begin{array}{l}\text { Joseph } \\
2013\end{array}$ & $\begin{array}{l}\text { Behaviour- } \\
\text { al theory }\end{array}$ & $\begin{array}{l}\text { To evaluate a school- } \\
\text { based RCT to evaluate Puff } \\
\text { City }\end{array}$ & $\begin{array}{l}\text { Adapted version of the Puff } \\
\text { City computer programme }\end{array}$ & $\begin{array}{l}\text { Generic } \\
\text { asthma ed- } \\
\text { ucation }\end{array}$ & $\begin{array}{l}4 \times 15- \\
\text { minute ses- } \\
\text { sions }\end{array}$ & None \\
\hline
\end{tabular}


Table 8. Process evaluation studies - summary of intervention characteristics

$\begin{array}{lll}\text { Kintner } & \text { Lifespan } & \begin{array}{l}\text { To evaluate the feasibility } \\ \text { of the SHARP programme }\end{array} \\ & \begin{array}{l}\text { develop- } \\ \text { ment per- } \\ \text { spective }\end{array} & \begin{array}{l}\text { for students, their fami- } \\ \text { ly, school personnel, and } \\ \text { community partners }\end{array}\end{array}$

SHARP; Community Coalition component

N/A
Once a Setting week for 10 and particweeks plus ipants; fura 3-hour ther modificommunity able design component features; stakeholder involvement and engagement

\begin{tabular}{lll}
\hline Kouba 2012 & $\begin{array}{l}\text { Orem's self- } \\
\text { care deficit } \\
\text { theory }\end{array}$ & $\begin{array}{l}\text { To determine the effec- } \\
\text { tiveness of the ICAN pro- } \\
\text { gramme for nutrition } \\
\text { knowledge and dietary be- } \\
\text { haviours }\end{array}$
\end{tabular}

Single workshop for staff;

N/A

8 weeks

None multiple sessions for children; Quest for the Code; Fight Asthma Now; additional nurse meetings; combined education

\begin{tabular}{|c|c|c|c|c|c|c|}
\hline $\begin{array}{l}\text { Langenfeld } \\
2010\end{array}$ & None & Unclear & $\begin{array}{l}\text { OAS; case management; } \\
\text { stand-alone respiratory } \\
\text { therapy. Children received } \\
\text { the OAS curriculum and } \\
\text { case management asthma } \\
\text { strategies developed with } \\
\text { teachers }\end{array}$ & $\mathrm{N} / \mathrm{A}$ & $\begin{array}{l}6 \times 40- \\
\text { minute ses- } \\
\text { sions for } 1 \\
\text { school year }\end{array}$ & None \\
\hline Lee 2011 & $\begin{array}{l}\text { The func- } \\
\text { tional con- } \\
\text { text ap- } \\
\text { proach }\end{array}$ & $\begin{array}{l}\text { To evaluate the effective- } \\
\text { ness and feasibility of us- } \\
\text { ing undergraduate nurs- } \\
\text { ing students as facilitators } \\
\text { to deliver an asthma man- } \\
\text { agement programme }\end{array}$ & $\begin{array}{l}\text { OAS. Children received the } \\
\text { OAS curriculum }\end{array}$ & $\mathrm{N} / \mathrm{A}$ & Unclear & $\begin{array}{l}\text { Further } \\
\text { modifiable } \\
\text { design fea- } \\
\text { tures }\end{array}$ \\
\hline Levy 2006 & None & $\begin{array}{l}\text { To evaluate the effective- } \\
\text { ness of a school-based } \\
\text { nurse case management } \\
\text { approach to asthma in } \\
\text { students with poor control }\end{array}$ & $\begin{array}{l}\text { OAS; monitoring of stu- } \\
\text { dents; health status. Stu- } \\
\text { dents received OAS educa- } \\
\text { tion and weekly monitoring } \\
\text { of their health status }\end{array}$ & Usual care & $\begin{array}{l}1 \text { school } \\
\text { term }\end{array}$ & None \\
\hline $\begin{array}{l}\text { Magzamen } \\
2008\end{array}$ & None & $\begin{array}{l}\text { To evaluate the implemen- } \\
\text { tation of Kickin' Asthma }\end{array}$ & $\begin{array}{l}\text { Multiple sessions for chil- } \\
\text { dren; Kickin' Asthma. Ed- } \\
\text { ucational sessions, simi- } \\
\text { lar to the OAS curriculum. } \\
\text { Customised letters were al- } \\
\text { so sent home to describe } \\
\text { health needs and goals for } \\
\text { each child }\end{array}$ & $\mathrm{N} / \mathrm{A}$ & 3 months & None \\
\hline $\begin{array}{l}\text { McCann } \\
2006\end{array}$ & None & $\begin{array}{l}\text { To assess whether a } \\
\text { school-based intervention } \\
\text { would produce clinical } \\
\text { and psychological benefits } \\
\text { for children with asthma }\end{array}$ & $\begin{array}{l}\text { Education; role-play. The in- } \\
\text { tervention focused on de- } \\
\text { scribing the respiratory con- } \\
\text { dition through a role-play }\end{array}$ & $\begin{array}{l}\text { Respiratory } \\
\text { education }\end{array}$ & $\begin{array}{l}45-\text { minute } \\
\text { session }\end{array}$ & None \\
\hline Mickel 2016 & None & $\begin{array}{l}\text { To provide Iggy education } \\
\text { to more than } 75 \% \text { of chil- } \\
\text { dren with asthma; To in- } \\
\text { crease asthma knowledge; }\end{array}$ & $\begin{array}{l}\text { Iggy and the Inhalers inter- } \\
\text { vention. Children received } \\
\text { an asthma education video, } \\
\text { poster, comic book, stick- }\end{array}$ & $\mathrm{N} / \mathrm{A}$ & Unclear & None \\
\hline
\end{tabular}


Table 8. Process evaluation studies - summary of intervention characteristics (Continued)

increase families' awareness of asthma; and cultivate collaboration between school nurses and asthma providers

$\begin{array}{ll}\text { Mujuru None } & \text { To demonstrate the fea- } \\ & \text { sibility of a school-based } \\ & \text { asthma education pro- } \\ & \text { gramme for students and } \\ & \text { to evaluate parents' per- } \\ & \text { spectives on the interven- } \\ & \text { tion }\end{array}$

Pike 2011 None $\quad$ To assess student asthma
knowledge gain, teacher acceptance, and grade appropriateness after an intervention

\author{
er, and t
gramme
}

$\begin{array}{lll}\text { OAS. Children received the N/A } & \text { 40-minute None } \\ \text { OAS curriculum } & \text { session } \\ & \text { once a } \\ & \text { week for 2 } \\ & \text { months }\end{array}$

Multiple sessions for children; integrated into the curriculum. Teachers taught

$\begin{array}{ll}\text { Usual care } & 7 \text { lesson } \\ \text { plans }\end{array}$
Setting and participants lessons with information about asthma

\begin{tabular}{ll}
\hline Richmond None & $\begin{array}{l}\text { To increase the number } \\
\text { of current provider-writ- } \\
\text { ten asthma action plans } \\
\text { submitted to the school } \\
\text { nurse at the beginning of } \\
\text { the school year }\end{array}$
\end{tabular}

\begin{tabular}{|c|c|c|}
\hline $\begin{array}{l}\text { Spencer } \\
2000\end{array}$ & None & $\begin{array}{l}\text { To evaluate the OAS pro- } \\
\text { gramme for children }\end{array}$ \\
\hline
\end{tabular}

Breathe Your Best. Students were encouraged to receive

N/A Unclear None an asthma action plan from their doctor and to collect their prescriptions

$\begin{array}{lll}\text { OAS. Children received the N/A } & \begin{array}{l}6 \times 40- \\ \text { minute ses- } \\ \text { OAS curriculum }\end{array} & \text { None } \\ & \text { sions }\end{array}$

\begin{tabular}{ll}
\hline Splett $2006 \quad$ None $\quad \begin{array}{l}\text { To evaluate the effective- } \\
\text { ness and sustainability of } \\
\text { the Healthy Learners Asth- } \\
\text { ma Initiative }\end{array}$
\end{tabular}

\begin{tabular}{|c|c|c|}
\hline $\begin{array}{l}\text { Children received training } \\
\text { on asthma self-manage- } \\
\text { ment. Licensed nurses and } \\
\text { healthcare assistants re- } \\
\text { ceived coaching and rein- }\end{array}$ & Usual care & $\begin{array}{l}\text { Varied ac- } \\
\text { cording } \\
\text { to asthma } \\
\text { severity } \\
\text { and need }\end{array}$ \\
\hline
\end{tabular}
forcement from asthma resource nurses

\begin{tabular}{|c|c|c|c|c|c|c|}
\hline $\begin{array}{l}\text { Terpstra } \\
2012\end{array}$ & $\begin{array}{l}\text { Social cog- } \\
\text { nitive theo- } \\
\text { ry }\end{array}$ & $\begin{array}{l}\text { To test a version of an in- } \\
\text { tervention with a caregiver } \\
\text { newsletter vs no newslet- } \\
\text { ter }\end{array}$ & $\begin{array}{l}\text { Multiple sessions for chil- } \\
\text { dren; materials for parents. } \\
\text { Children received skills } \\
\text { training on how to use a } \\
\text { peak flow meter. Parents re- } \\
\text { ceived a newsletter about } \\
\text { an important theme from } \\
\text { the research }\end{array}$ & $\begin{array}{l}\text { Interven- } \\
\text { tion or in- } \\
\text { terven- } \\
\text { tion with a } \\
\text { newsletter }\end{array}$ & $\begin{array}{l}\text { 6-week } \\
\text { training }\end{array}$ & $\begin{array}{l}\text { Setting } \\
\text { and partic- } \\
\text { ipants; fur- } \\
\text { ther modifi- } \\
\text { able design } \\
\text { features }\end{array}$ \\
\hline
\end{tabular}

ASMA: Asthma Self-Management for Adolescents.

ICAN: I Can Control Asthma and Nutrition Now.

N/A: not applicable.

OAS: Open Airways for Schools.

RCT: randomised controlled trial.

SHARP: Staying Healthy-Asthma Responsible \& Prepared.

Triple A: Adolescent Asthma Action. 


\begin{tabular}{|c|c|c|c|c|c|c|c|c|}
\hline & Study design & $\begin{array}{l}\text { Number of } \\
\text { children }\end{array}$ & Country & $\begin{array}{l}\text { Type of } \\
\text { School }\end{array}$ & Recipients & $\begin{array}{l}\text { Age of } \\
\text { children } \\
\text { (years) }\end{array}$ & $\begin{array}{l}\text { Representation of children } \\
\text { from BME backgrounds }\end{array}$ & $\begin{array}{l}\text { Representation of } \\
\text { children from low } \\
\text { SES backgrounds }\end{array}$ \\
\hline $\begin{array}{l}\text { Al-Sheyab } \\
\text { 2012a }\end{array}$ & Case study & 31 & Jordan & High & Children & 11 to 18 & Unclear & Unclear \\
\hline Berg 2004 & $\begin{array}{l}\text { Quasi-experi- } \\
\text { mental }\end{array}$ & 13 & USA & High & Children & 15 to 18 & 46.2\% African American & Unclear \\
\hline Bignall 2015 & $\begin{array}{l}\text { Parallel-group } \\
\text { RCT }\end{array}$ & 33 & USA & High & Children & 11 to 18 & $\begin{array}{l}100 \% \text { Black or African Ameri- } \\
\text { can }\end{array}$ & Unclear \\
\hline Brasler 2006 & Case study & 342 & USA & Junior/middle & $\begin{array}{l}\text { Children; teach- } \\
\text { ers; parents }\end{array}$ & 11 to 14 & Unclear & Unclear \\
\hline $\begin{array}{l}\text { Bruzzese } \\
2004\end{array}$ & $\begin{array}{l}\text { Parallel-group } \\
\text { RCT }\end{array}$ & 45 & USA & High & Children & 11 to 18 & Unclear & Unclear \\
\hline $\begin{array}{l}\text { Bruzzese } \\
2011\end{array}$ & $\begin{array}{l}\text { Parallel-group } \\
\text { RCT }\end{array}$ & 345 & USA & High & Children & 11 to 18 & $\begin{array}{l}45.5 \% \text { Hispanic; } 37.7 \% \text { African } \\
\text { American; } 11.6 \% \text { mixed; } 5.2 \% \\
\text { other }\end{array}$ & $\begin{array}{l}75 \% \text { free school } \\
\text { meals }\end{array}$ \\
\hline $\begin{array}{l}\text { Bruzzese } \\
2008\end{array}$ & $\begin{array}{l}\text { Parallel-group } \\
\text { RCT }\end{array}$ & 24 & USA & Junior/middle & $\begin{array}{l}\text { Children; par- } \\
\text { ents }\end{array}$ & 11 to 14 & $\begin{array}{l}41 \% \text { Hispanic; } 17 \% \text { White; } 8 \% \\
\text { African American; } 34 \% \text { other }\end{array}$ & $\begin{array}{l}8 \% \text { unemployed; } \\
21 \% \text { part-time em- } \\
\text { ployment; } 71 \% \text { full- } \\
\text { time employment }\end{array}$ \\
\hline $\begin{array}{l}\text { Carpenter } \\
2016\end{array}$ & $\begin{array}{l}\text { Quasi-experi- } \\
\text { mental }\end{array}$ & 25 & USA & $\begin{array}{l}\text { All school } \\
\text { types }\end{array}$ & $\begin{array}{l}\text { Children; nurs- } \\
\text { es }\end{array}$ & Unclear & $\begin{array}{l}72 \% \text { White; } 12 \% \text { Hispanic; } 8 \% \\
\text { African American; } 8 \% \text { Black }\end{array}$ & Unclear \\
\hline Cicutto 2013 & Cluster RCT & 1316 & Canada & $\begin{array}{l}\text { Primary/ele- } \\
\text { mentary }\end{array}$ & $\begin{array}{l}\text { Children; } \\
\text { school board; } \\
\text { head teacher; } \\
\text { teachers; peers }\end{array}$ & 5 to 10 & Unclear & $\begin{array}{l}25 \% \text { to } 50 \% \text { de- } \\
\text { prived }\end{array}$ \\
\hline Crane 2014 & $\begin{array}{l}\text { Quasi-experi- } \\
\text { mental }\end{array}$ & 45 & USA & $\begin{array}{l}\text { Primary/ele- } \\
\text { mentary }\end{array}$ & Children & 5 to 10 & Unclear & Unclear \\
\hline $\begin{array}{l}\text { Dore-Stites } \\
2007\end{array}$ & $\begin{array}{l}\text { Quasi-experi- } \\
\text { mental }\end{array}$ & 32 & USA & $\begin{array}{l}\text { Primary/ele- } \\
\text { mentary }\end{array}$ & $\begin{array}{l}\text { Children; par- } \\
\text { ents }\end{array}$ & 5 to 10 & $\begin{array}{l}39 \% \text { African American; } 28.6 \% \\
\text { Caucasian; } 14.3 \% \text { Hispanic; } \\
\text { 18\% biracial }\end{array}$ & $\begin{array}{l}34.6 \%<\$ 20,000 \\
53.8 \% \$ 21,000 \text { to } \\
\$ 40,000\end{array}$ \\
\hline
\end{tabular}




\begin{tabular}{|c|c|c|c|c|c|c|c|c|}
\hline $\begin{array}{l}\text { Engelke } \\
2013\end{array}$ & $\begin{array}{l}\text { Quasi-experi- } \\
\text { mental }\end{array}$ & 143 & USA & $\begin{array}{l}\text { All school } \\
\text { types }\end{array}$ & $\begin{array}{l}\text { Children; teach- } \\
\text { ers; parents; } \\
\text { nurses }\end{array}$ & Unclear & $\begin{array}{l}40.6 \% \text { Caucasian; } 37.8 \% \\
\text { African American; } 7 \% \text { Latino; } \\
14 \% \text { other }\end{array}$ & $63.6 \%$ on Medicaid \\
\hline Gerald 2006 & Cluster RCT & 736 & USA & $\begin{array}{l}\text { Primary/ele- } \\
\text { mentary }\end{array}$ & $\begin{array}{l}\text { Children; teach- } \\
\text { ers }\end{array}$ & 5 to 10 & 97\% African American & Unclear \\
\hline Henry 2004 & Cluster RCT & 4161 & Australia & High & $\begin{array}{l}\text { Children; teach- } \\
\text { ers }\end{array}$ & 11 to 14 & Predominantly Caucasian & Unclear \\
\hline Horner 2015 & Cluster RCT & 292 & USA & $\begin{array}{l}\text { Primary/ele- } \\
\text { mentary }\end{array}$ & Children & 5 to 10 & $\begin{array}{l}21.2 \% \text { African American; } 25 \% \\
\text { Spanish speaking }\end{array}$ & $30.7 \%$ low SES \\
\hline Howell 2005 & Cluster RCT & 24 & USA & $\begin{array}{l}\text { Primary/ele- } \\
\text { mentary }\end{array}$ & $\begin{array}{l}\text { Children; par- } \\
\text { ents }\end{array}$ & 5 to 10 & 75\% African American & Unclear \\
\hline $\begin{array}{l}\text { Jackson } \\
2006\end{array}$ & $\begin{array}{l}\text { Quasi-experi- } \\
\text { mental }\end{array}$ & 943 & USA & $\begin{array}{l}\text { Primary/ele- } \\
\text { mentary }\end{array}$ & Children & 5 to 10 & Unclear & Unclear \\
\hline Joseph 2010 & $\begin{array}{l}\text { Parallel-group } \\
\text { RCT }\end{array}$ & 314 & USA & High & Children & 11 to 18 & Unclear & $\begin{array}{l}52 \% \text { eligible for } \\
\text { free school meals }\end{array}$ \\
\hline Joseph 2013 & $\begin{array}{l}\text { Parallel-group } \\
\text { RCT }\end{array}$ & 422 & USA & High & Children & 11 to 18 & 98\% African American & $73 \%$ on Medicaid \\
\hline Kintner 2012 & $\begin{array}{l}\text { Quasi-experi- } \\
\text { mental }\end{array}$ & 28 & USA & High & $\begin{array}{l}\text { Children; peers; } \\
\text { families; teach- } \\
\text { ers }\end{array}$ & 11 to 14 & $\begin{array}{l}\text { 53.6\% African American; } \\
\text { 32.1\% White; 3.6\% American; } \\
\text { 10.7\% biracial }\end{array}$ & $\begin{array}{l}35.7 \% \text { low SES; } \\
42.9 \% \text { low middle } \\
\text { SES; } 17.8 \% \text { upper } \\
\text { middle SES; } 3.6 \% \\
\text { high SES }\end{array}$ \\
\hline Kouba 2012 & $\begin{array}{l}\text { Quasi-experi- } \\
\text { mental }\end{array}$ & 25 & USA & High & Children & 11 to 18 & $\begin{array}{l}\text { 92\% African American; } 4 \% \\
\text { Hispanic; } 4 \% \text { mixed }\end{array}$ & $\begin{array}{l}25 \% \text { to } 50 \% \text { de- } \\
\text { prived }\end{array}$ \\
\hline $\begin{array}{l}\text { Langenfeld } \\
2010\end{array}$ & $\begin{array}{l}\text { Quasi-experi- } \\
\text { mental }\end{array}$ & 286 & USA & $\begin{array}{l}\text { Primary/ele- } \\
\text { mentary }\end{array}$ & $\begin{array}{l}\text { Children; teach- } \\
\text { ers }\end{array}$ & 5 to 10 & $\begin{array}{l}63 \% \text { African American; } 23.9 \% \\
\text { Hispanic; } 6.4 \% \text { White; } 2.6 \% \\
\text { Asian }\end{array}$ & $\begin{array}{l}\text { High percentage on } \\
\text { free school meals }\end{array}$ \\
\hline Lee 2011 & $\begin{array}{l}\text { Quasi-experi- } \\
\text { mental }\end{array}$ & 827 & USA & $\begin{array}{l}\text { Primary/ele- } \\
\text { mentary }\end{array}$ & Children & 5 to 10 & Unclear & Unclear \\
\hline Levy 2006 & Cluster RCT & 243 & USA & $\begin{array}{l}\text { Primary/ele- } \\
\text { mentary }\end{array}$ & $\begin{array}{l}\text { Children; teach- } \\
\text { ers }\end{array}$ & 5 to 10 & 97\% African American & $80 \%$ on Medicaid \\
\hline
\end{tabular}




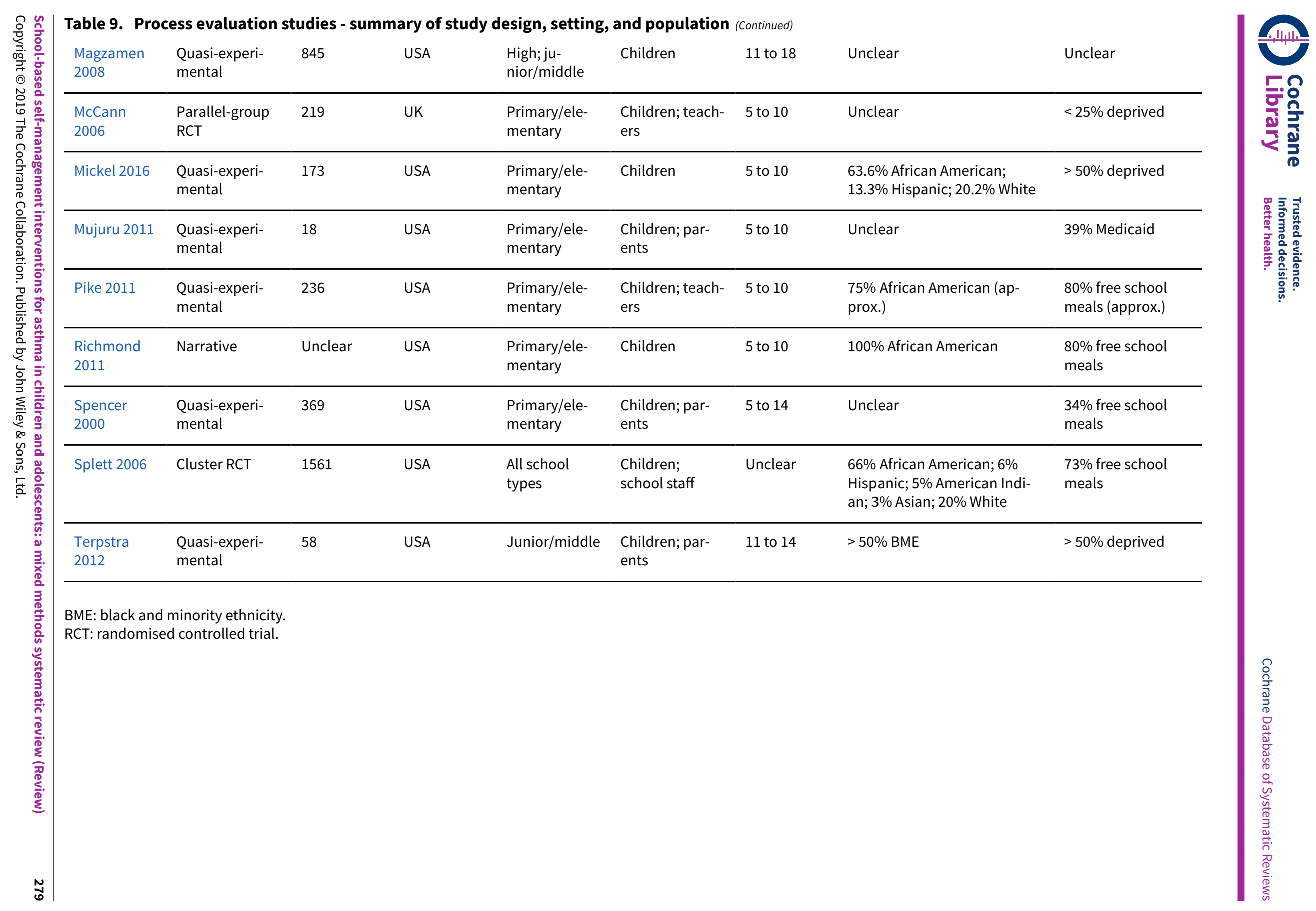




\section{Table 10. Outcome evaluation studies not included in the analyses}

\begin{tabular}{|c|c|}
\hline Study included as outcome & Reason data not included in quantitative analysis \\
\hline Bruzzese 2004 & Feasibility study uses randomised controlled trial (RCT) design with no quantitative data presented \\
\hline Bruzzese 2010 & Abstract only located and outcomes were not presented in an extractable format \\
\hline Clark 2004 & Published effect sizes that were extractable but of a different effect size from other studies \\
\hline Clark 2010 & No outcome measured in the study matched the review protocol \\
\hline McCann 2006 & $\begin{array}{l}\text { Outcomes were not presented in an extractable format (disaggregated data for asthmatic children } \\
\text { unavailable) }\end{array}$ \\
\hline Monforte 2012 & Abstract only located and outcomes were not presented in an extractable format \\
\hline Mosnaim 2011 & No outcome measured in the study matched the review protocol \\
\hline Praena-Crespo 2010 & Abstract only located and outcomes were not presented in an extractable format \\
\hline Pulcini 2007 & No outcome measured in the study matched the review protocol \\
\hline Srof 2012 & $\begin{array}{l}\text { Outcomes were not presented in an extractable format (data on overall quality of life were not pre- } \\
\text { sented in full; only subdomains of quality of life are available) }\end{array}$ \\
\hline
\end{tabular}




\begin{tabular}{|c|c|c|c|c|c|c|c|c|}
\hline & Study design & $\begin{array}{l}\text { Number of } \\
\text { children }\end{array}$ & Country & Type of school & Recipients & $\begin{array}{l}\text { Age of chil- } \\
\text { dren } \\
\text { (years) }\end{array}$ & $\begin{array}{l}\text { Representa- } \\
\text { tion of children } \\
\text { from BME back- } \\
\text { grounds }\end{array}$ & $\begin{array}{l}\text { Representation } \\
\text { of children from } \\
\text { low SES back- } \\
\text { grounds }\end{array}$ \\
\hline $\begin{array}{l}\text { Al-Sheyab } \\
2012\end{array}$ & Clustered parallel RCT & 261 & Jordan & $\begin{array}{l}4 \text { public high } \\
\text { schools }\end{array}$ & Children & 11 to 15 & Unclear & Unclear \\
\hline Atherly 2009 & Clustered parallel RCT & 524 & USA & $\begin{array}{l}\text { Junior and high } \\
\text { schools }\end{array}$ & Children & 11 to 15 & Unclear & Unclear \\
\hline $\begin{array}{l}\text { Bartholomew } \\
2006\end{array}$ & Clustered parallel RCT & 948 & USA & Elementary schools & $\begin{array}{l}\text { Children; care } \\
\text { providers; } \\
\text { parents/car- } \\
\text { ers }\end{array}$ & 5 to 10 & $\begin{array}{l}\text { 45\% African } \\
\text { American; 51\% } \\
\text { Hispanic; 3\% } \\
\text { Caucasian }\end{array}$ & $\begin{array}{l}\text { Deprived individ- } \\
\text { uals }>50 \%\end{array}$ \\
\hline $\begin{array}{l}\text { Bruzzese } \\
2004\end{array}$ & $\mathrm{RCT}$ & 45 & USA & $\begin{array}{l}2 \text { public high } \\
\text { schools }\end{array}$ & Children & Unclear & Unclear & Unclear \\
\hline $\begin{array}{l}\text { Bruzzese } \\
2008\end{array}$ & Clustered parallel RCT & 24 & USA & 1 middle school & $\begin{array}{l}\text { Children; } \\
\text { caregivers }\end{array}$ & 11 to 15 & $\begin{array}{l}41 \% \text { Hispan- } \\
\text { ic; } 17 \% \text { African } \\
\text { American }\end{array}$ & $\begin{array}{l}71 \% \text { parents full- } \\
\text { time employment }\end{array}$ \\
\hline $\begin{array}{l}\text { Bruzzese } \\
2010\end{array}$ & Clustered parallel RCT & Unclear & USA & 25 public schools & $\begin{array}{l}\text { Children; } \\
\text { caregivers }\end{array}$ & $\begin{array}{l}\text { Mean age, } \\
12.8\end{array}$ & Unclear & Unclear \\
\hline $\begin{array}{l}\text { Bruzzese } \\
2011\end{array}$ & Clustered parallel RCT & 340 & USA & 5 high schools & Children & $\begin{array}{l}\text { Mean age, } \\
15\end{array}$ & $>80 \% \mathrm{BME}$ & Unclear \\
\hline Cicutto 2005 & Clustered parallel RCT & 256 & Canada & $\begin{array}{l}26 \text { elementary } \\
\text { schools }\end{array}$ & Children & 5 to 10 & Unclear & $\begin{array}{l}\text { Average house- } \\
\text { hold income } \\
\$ 53,000\end{array}$ \\
\hline Cicutto 2013 & Clustered RCT & 1316 & Canada & $\begin{array}{l}170 \text { primary/ele- } \\
\text { mentary schools }\end{array}$ & $\begin{array}{l}\text { Children; fam- } \\
\text { ilies }\end{array}$ & 5 to 10 & Unclear & $\begin{array}{l}\text { Deprived individ- } \\
\text { uals } 25 \% \text { to } 50 \%\end{array}$ \\
\hline Clark 2004 & Clustered parallel RCT & 835 & USA & $\begin{array}{l}14 \text { public high } \\
\text { schools }\end{array}$ & $\begin{array}{l}\text { Children; par- } \\
\text { ents/carers; } \\
\text { classmates; } \\
\text { school per- } \\
\text { sonnel }\end{array}$ & 5 to 10 & $\begin{array}{l}98 \% \text { African } \\
\text { American }\end{array}$ & $\begin{array}{l}45 \% \text { annual in- } \\
\text { come }<\$ 15,000\end{array}$ \\
\hline
\end{tabular}




\begin{tabular}{|c|c|c|c|c|c|c|c|c|}
\hline Clark 2005 & Clustered parallel RCT & 639 & China & $\begin{array}{l}21 \text { elementary } \\
\text { schools }\end{array}$ & Children & 7 to 11 & Unclear & Unclear \\
\hline Clark 2010 & Clustered parallel RCT & 1292 & USA & 19 middle schools & Children & $\begin{array}{l}\text { Mean age, } \\
11.6\end{array}$ & $\begin{array}{l}93 \% \text { African } \\
\text { American }\end{array}$ & $\begin{array}{l}48 \% \text { annual in- } \\
\text { come }<\$ 15,000\end{array}$ \\
\hline Gerald 2006 & Parallel-group RCT & 736 & USA & $\begin{array}{l}54 \text { elementary } \\
\text { schools }\end{array}$ & Children & $\begin{array}{l}\text { Mean age, } \\
11\end{array}$ & 97\% Black & Unclear \\
\hline Gerald 2009 & Parallel-group RCT & 290 & USA & Unclear & Children & 5 to 10 & 91\% Black & Unclear \\
\hline Henry 2004 & Clustered parallel RCT & Unclear & Australia & Secondary schools & Children & 11 to 15 & $<50 \% \mathrm{BME}$ & Unclear \\
\hline Horner 2008 & Clustered parallel RCT & 183 & USA & $\begin{array}{l}18 \text { elementary } \\
\text { schools }\end{array}$ & Children & 5 to 10 & $\begin{array}{l}\text { 47\% Hispanic; } \\
30 \% \text { White; } 22 \% \\
\text { African American }\end{array}$ & Unclear \\
\hline Horner 2015 & Clustered parallel RCT & 196 & USA & $\begin{array}{l}3 \text { elementary } \\
\text { schools }\end{array}$ & Children & 5 to 10 & $>50 \%$ BME & $\begin{array}{l}\text { Deprived individ- } \\
\text { uals } 25 \% \text { to } 50 \%\end{array}$ \\
\hline Howell 2005 & Clustered parallel RCT & 25 & USA & $\begin{array}{l}4 \text { elementary } \\
\text { schools }\end{array}$ & $\begin{array}{l}\text { Children; fam- } \\
\text { ilies }\end{array}$ & 5 to 10 & $\begin{array}{l}75 \% \text { African } \\
\text { American }\end{array}$ & Unclear \\
\hline Kintner 2009 & Clustered parallel RCT & 59 & USA & 5 schools & Children & 9 to 12 & $\begin{array}{l}30 \% \text { Black; } 36 \% \\
\text { White; } 18 \% \text { bira- } \\
\text { cial }\end{array}$ & $\begin{array}{l}\text { Deprived individ- } \\
\text { uals } 25 \% \text { to } 50 \%\end{array}$ \\
\hline Levy 2006 & Clustered parallel RCT & 243 & USA & $\begin{array}{l}14 \text { elementary } \\
\text { schools }\end{array}$ & Children & 5 to 10 & $\begin{array}{l}98 \% \text { African } \\
\text { American }\end{array}$ & $85 \%$ TennCare \\
\hline $\begin{array}{l}\text { McCann } \\
2006\end{array}$ & Clustered parallel RCT & 229 & England & $\begin{array}{l}24 \text { primary/junior } \\
\text { schools }\end{array}$ & $\begin{array}{l}\text { Children; par- } \\
\text { ents }\end{array}$ & 5 to 10 & Unclear & $\begin{array}{l}\text { Deprived individ- } \\
\text { uals }<25 \%\end{array}$ \\
\hline $\begin{array}{l}\text { McGhan } \\
2003\end{array}$ & Clustered parallel RCT & 162 & Canada & $\begin{array}{l}18 \text { elementary } \\
\text { schools }\end{array}$ & Children & 5 to 10 & $<50 \%$ BME & $\begin{array}{l}\text { Deprived individ- } \\
\text { uals } 25 \% \text { to } 50 \%\end{array}$ \\
\hline $\begin{array}{l}\text { McGhan } \\
2010\end{array}$ & Clustered parallel RCT & 206 & Canada & Elementary schools & $\begin{array}{l}\text { Children; par- } \\
\text { ents/carers; } \\
\text { teachers }\end{array}$ & $\begin{array}{l}\text { Mean age, } \\
8.6\end{array}$ & Unclear & Unclear \\
\hline $\begin{array}{l}\text { Monforte } \\
2012\end{array}$ & Clustered parallel RCT & Unclear & USA & $\begin{array}{l}8 \text { elementary } \\
\text { schools }\end{array}$ & Children & 5 to 10 & Unclear & Unclear \\
\hline
\end{tabular}




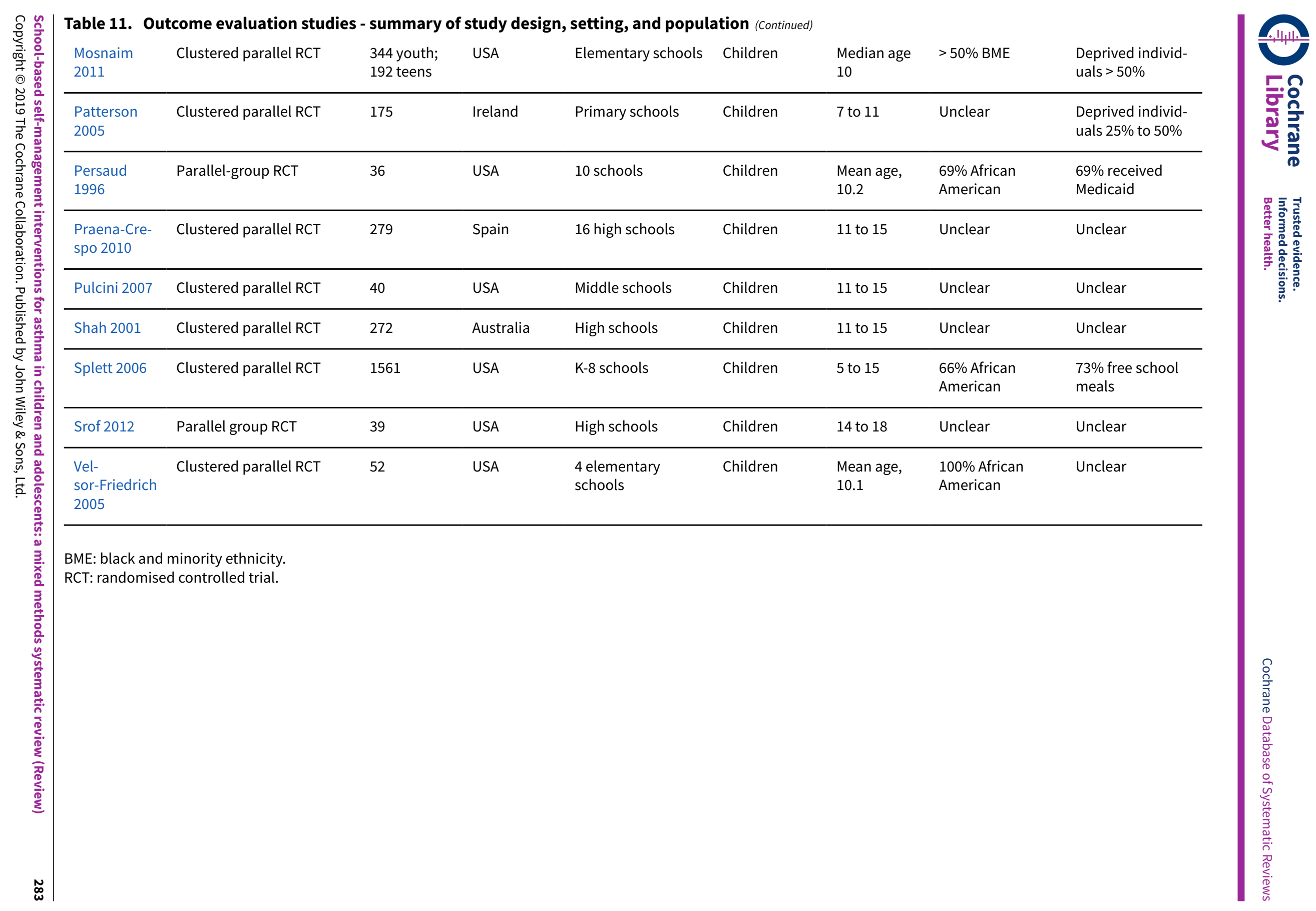


Table 12. Outcome evaluation studies - summary of intervention characteristics

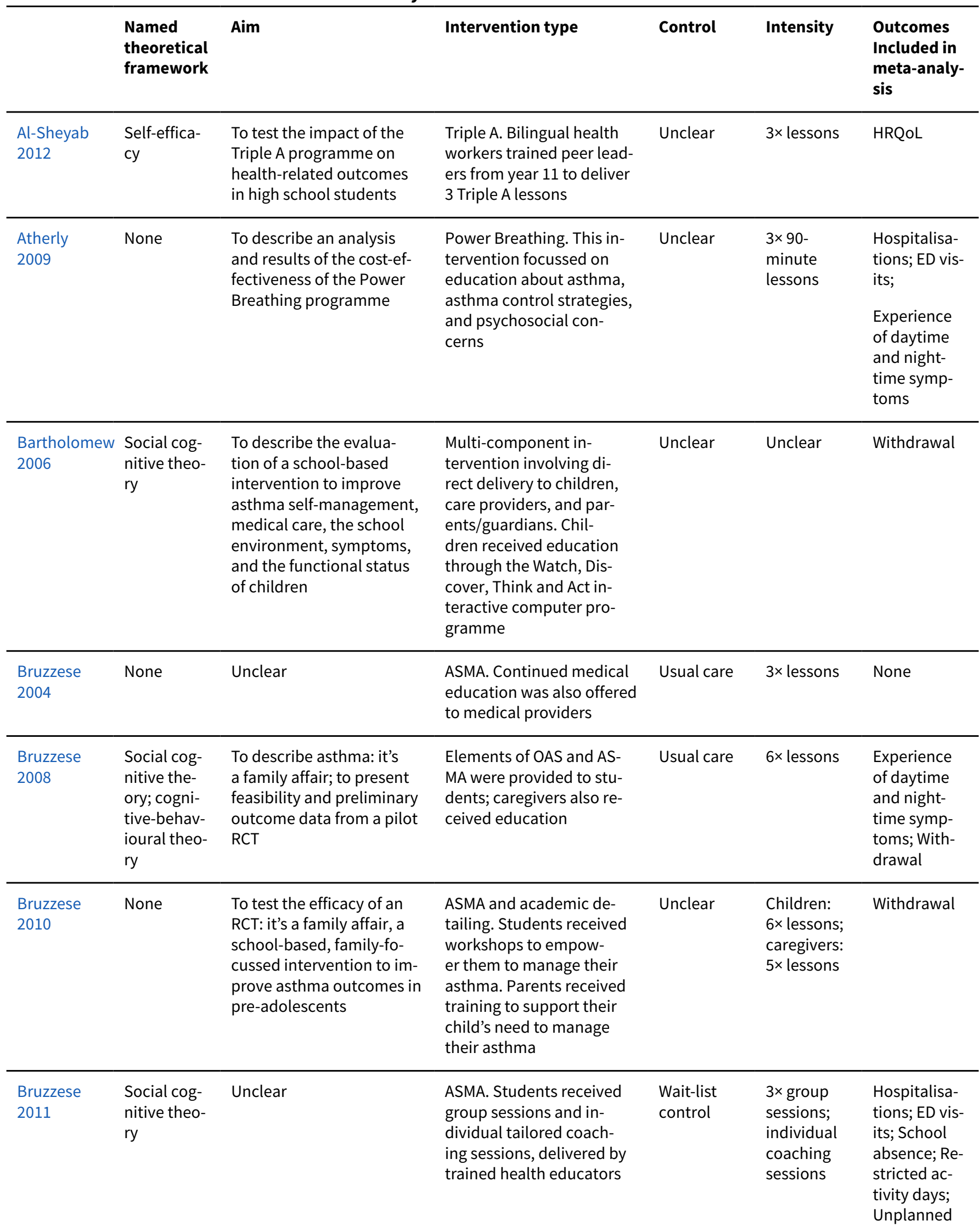


Table 12. Outcome evaluation studies - summary of intervention characteristics (Continued)

GP or hospi-

tal visits; Ex-

perience of

daytime and

night-time

symptoms;

Use of corti-

costeroids;

Withdrawal

\begin{tabular}{|c|c|c|c|c|c|c|}
\hline $\begin{array}{l}\text { Cicutto } \\
2005\end{array}$ & $\begin{array}{l}\text { Social cog- } \\
\text { nitive theo- } \\
\text { ry; self-reg- } \\
\text { ulation the- } \\
\text { ory }\end{array}$ & $\begin{array}{l}\text { To evaluate an asthma ed- } \\
\text { ucation programme for } \\
\text { children with asthma }\end{array}$ & $\begin{array}{l}\text { Roaring Adventures of } \\
\text { Puff. Children received } \\
\text { group sessions on asthma } \\
\text { and goal-setting }\end{array}$ & Usual care & $6 \times$ lessons & $\begin{array}{l}\text { Hospitalisa- } \\
\text { tions; ED vis- } \\
\text { its; School } \\
\text { absence; Re- } \\
\text { stricted ac- } \\
\text { tivity days }\end{array}$ \\
\hline $\begin{array}{l}\text { Cicutto } \\
2013\end{array}$ & $\begin{array}{l}\text { Social cog- } \\
\text { nitive theo- } \\
\text { ry }\end{array}$ & $\begin{array}{l}\text { To implement an elemen- } \\
\text { tary school-based asthma } \\
\text { self-management educa- } \\
\text { tion programme for chil- } \\
\text { dren with asthma; to work } \\
\text { with schools to create an } \\
\text { asthma-friendly support- } \\
\text { ive school environment; to } \\
\text { evaluate the programme }\end{array}$ & $\begin{array}{l}\text { Roaring Adventures of } \\
\text { Puff. Children received } \\
\text { group sessions on asthma } \\
\text { and goal-setting }\end{array}$ & Usual care & $6 \times$ lessons & $\begin{array}{l}\text { ED visits; } \\
\text { School ab- } \\
\text { sence; Re- } \\
\text { stricted ac- } \\
\text { tivity days; } \\
\text { Unplanned } \\
\text { GP or hos- } \\
\text { pital vis- } \\
\text { it; HRQoL; } \\
\text { Withdrawal }\end{array}$ \\
\hline Clark 2004 & None & $\begin{array}{l}\text { To assess the impact of a } \\
\text { comprehensive school- } \\
\text { based asthma programme }\end{array}$ & $\begin{array}{l}\text { OAS; control strategies for } \\
\text { schools }\end{array}$ & $\begin{array}{l}\text { Wait-list } \\
\text { control }\end{array}$ & $\begin{array}{l}6 \times \text { lessons } \\
\text { and } 2 \times \\
\text { classroom } \\
\text { sessions }\end{array}$ & $\begin{array}{l}\text { School ab- } \\
\text { sence }\end{array}$ \\
\hline Clark 2005 & $\begin{array}{l}\text { Social cog- } \\
\text { nitive theo- } \\
\text { ry }\end{array}$ & $\begin{array}{l}\text { To assess effectiveness } \\
\text { in children in China of an } \\
\text { asthma education pro- } \\
\text { gramme adapted from a } \\
\text { model developed in the } \\
\text { USA }\end{array}$ & $\begin{array}{l}\text { OAS; intervention directed } \\
\text { at children only }\end{array}$ & Unclear & $5 \times$ lessons & $\begin{array}{l}\text { Hospitalisa- } \\
\text { tions; ED vis- } \\
\text { its }\end{array}$ \\
\hline
\end{tabular}

\begin{tabular}{|c|c|c|c|c|c|c|}
\hline Clark 2010 & None & $\begin{array}{l}\text { To assess self-manage- } \\
\text { ment and self-manage- } \\
\text { ment plus peer involve- } \\
\text { ment }\end{array}$ & $\begin{array}{l}\text { OAS; peer component. In } \\
\text { the first treatment arm, an } \\
\text { adapted form of OAS was } \\
\text { delivered to children. In } \\
\text { the second treatment arm, } \\
\text { a peer education compo- } \\
\text { nent was added }\end{array}$ & Usual care & $6 \times$ lessons & $\begin{array}{l}\text { Experience } \\
\text { of daytime } \\
\text { and night- } \\
\text { time symp- } \\
\text { toms }\end{array}$ \\
\hline Gerald 2006 & None & Unclear & $\begin{array}{l}\text { OAS. The intervention in- } \\
\text { cluded educational pro- } \\
\text { grammes and medical } \\
\text { management for children, } \\
\text { as well as education for } \\
\text { school staff }\end{array}$ & Usual care & $6 \times$ lessons & $\begin{array}{l}\text { Hospitalisa- } \\
\text { tions; ED vis- } \\
\text { its; School } \\
\text { absence }\end{array}$ \\
\hline Gerald 2009 & None & $\begin{array}{l}\text { To determine the effec- } \\
\text { tiveness of school-based } \\
\text { supervised asthma ther- } \\
\text { apy in improving asthma } \\
\text { control }\end{array}$ & $\begin{array}{l}\text { Children received asthma } \\
\text { education, including a dis- } \\
\text { cussion of trigger avoid- } \\
\text { ance (not manualised) }\end{array}$ & Usual care & $\begin{array}{l}1 \times \text { lesson; } \\
\text { multiple } \\
\text { supervi- } \\
\text { sions }\end{array}$ & $\begin{array}{l}\text { School ab- } \\
\text { sence; Lung } \\
\text { function; Use } \\
\text { of reliever }\end{array}$ \\
\hline
\end{tabular}

School-based self-management interventions for asthma in children and adolescents: a mixed methods systematic review (Review) 
Table 12. Outcome evaluation studies - summary of intervention characteristics (Continued)

therapies;

Withdrawal

\begin{tabular}{|c|c|c|}
\hline Henry 2004 & None & $\begin{array}{l}\text { To determine whether an } \\
\text { asthma education pro- } \\
\text { gramme in schools would } \\
\text { have a direct impact on } \\
\text { student knowledge and at- } \\
\text { titudes on asthma and an } \\
\text { indirect impact on teacher } \\
\text { knowledge and attitudes }\end{array}$ \\
\hline
\end{tabular}

\begin{tabular}{lll}
\hline Horner & Asthma & $\begin{array}{l}\text { To examine changes in rur- } \\
\text { al children's asthma self- } \\
\text { health ed- } \\
\text { ucation } \\
\text { model }\end{array}$ \\
& $\begin{array}{l}\text { management after they re- } \\
\text { ceived classes, but before } \\
\text { they received the family } \\
\text { education session }\end{array}$
\end{tabular}

Asthma education. A pack- Usual care $3 \times$ lessons HRQoL
age about asthma was taught within the PD/H/PE strand of the school curriculum

\begin{tabular}{|c|c|c|}
\hline $\begin{array}{l}\text { Horner } \\
2015\end{array}$ & $\begin{array}{l}\text { Bruhn's } \\
\text { theoreti- } \\
\text { cal model } \\
\text { of asthma } \\
\text { self-man- } \\
\text { agement }\end{array}$ & $\begin{array}{l}\text { To test effects of } 2 \text { modes } \\
\text { of delivering an asthma } \\
\text { educational intervention } \\
\text { on health outcomes and } \\
\text { asthma self-management } \\
\text { in school-aged children } \\
\text { living in rural areas }\end{array}$ \\
\hline
\end{tabular}

7-topic curriculum. The intervention was designed for children in rural areas and included asthma information

Asthma self-management. The curriculum included a 7-step asthma self-management plan

Health promotion ed-

$16 \times$ lessons ucation

Hospitalisations; Withdrawal Health pro$16 \times$ lessons motion edHospitaliucation sations; ED visits; Withdrawal

\begin{tabular}{ll}
\hline Howell & Social \\
2005 & learning \\
& theory
\end{tabular}

\title{
To examine the feasibili-
} ty of an interactive computer game in schoolbased health centres; to test whether exposure to the game was effective in improving knowledge and reducing symptoms and healthcare use

\author{
Quest for the Code com- \\ puter game. The caregiver \\ also participated in med- \\ ication interviews and re- \\ ceived a home visit
}

Usual care $\begin{aligned} & \text { 30-minute } \\ & \text { session }\end{aligned}$
Usual care $10 \times$ lessons
ED visits; Ex- perience of daytime and night-time symptoms; HRQoL; School ab- sence; Cor- ticosteroid dosage
HRQoL; Withdrawal

\begin{tabular}{lll}
\hline Kintner & $\begin{array}{l}\text { Lifespan } \\
\text { develop- } \\
\text { ment per- } \\
\text { spective }\end{array}$ & $\begin{array}{l}\text { To evaluate the prelimi- } \\
\text { nary efficacy of SHARP }\end{array}$ \\
&
\end{tabular}
SHARP. Students worked through the SHARP cur- riculum. Caregivers also received a 3-hour informa- tion sharing programme

\begin{tabular}{lll}
\hline Levy 2006 None $\quad \begin{array}{ll}\text { To evaluate the effective- } \\
\text { ness of a school-based } \\
\text { nurse case management } \\
\text { approach to asthma in } \\
\text { students with poor control }\end{array}$ & $\begin{array}{l}\text { OAS; monitoring of stu- } \\
\text { dents; health status. Stu- } \\
\text { dents received OAS educa- } \\
\text { tion and weekly monitor- } \\
\text { ing of their health status }\end{array}$ \\
\end{tabular}

\begin{tabular}{lll} 
Usual care & $\begin{array}{l}\text { Weekly } \\
\text { group ses- } \\
\text { sions and } \\
\text { weekly in- } \\
\text { dividual } \\
\text { sessions }\end{array}$ & $\begin{array}{l}\text { Hospitali- } \\
\text { sations; ED } \\
\text { visits; With- } \\
\text { drawal }\end{array}$ \\
\hline $\begin{array}{l}\text { Education } \\
\text { about the } \\
\text { respiratory } \\
\text { system }\end{array}$ & $\begin{array}{l}1 \times \text { work- } \\
\text { shop }\end{array}$ & None \\
\hline
\end{tabular}

\begin{tabular}{|c|c|c|c|c|c|c|}
\hline $\begin{array}{l}\text { McCann } \\
2006\end{array}$ & None & $\begin{array}{l}\text { To assess whether schools } \\
\text { are an appropriate con- } \\
\text { text for an intervention de- } \\
\text { signed to produce clinical } \\
\text { and psychological benefits } \\
\text { for children with asthma }\end{array}$ & $\begin{array}{l}\text { Education; role-play. The } \\
\text { intervention focussed on } \\
\text { describing the respiratory } \\
\text { condition through a role- } \\
\text { play }\end{array}$ & $\begin{array}{l}\text { Education } \\
\text { about the } \\
\text { respiratory } \\
\text { system }\end{array}$ & $\begin{array}{l}1 \times \text { work- } \\
\text { shop }\end{array}$ & None \\
\hline
\end{tabular}
respirator 
Table 12. Outcome evaluation studies - summary of intervention characteristics (Continued)

$\begin{array}{llll}\text { McGhan } & \text { Social cog- } & \text { To determine whether } & \text { Roaring Adventures of } \\ \text { nitive theo- } & \text { an interactive childhood } & \text { Puff. Children received } \\ \text { asthma education pro- } & \text { education on asthma in } \\ & & \begin{array}{l}\text { gramme improved asth- } \\ \text { ma management behav- } \\ \text { iours, health status, and } \\ \text { quality of life in elemen- } \\ \text { tary school children }\end{array} & \begin{array}{l}\text { a group setting. Parents } \\ \text { and teachers were invited } \\ \text { to participate in a school- } \\ \end{array} \\ & & \text { based asthma awareness } \\ & & \text { event }\end{array}$

\section{Usual care}

$6 \times$ lessons

ED visits; School absence; Unplanned GP or hospital visit; Experience of daytime and night-time symptoms; Withdrawal

\begin{tabular}{lll}
\hline McGhan & Social cog- & To assess the feasibility \\
2010 & nitive theo- & and impact of the Roar- \\
ry; self-reg- & ing Adventures of Puff pro- \\
& ulation the- & gramme \\
& ory &
\end{tabular}

Roaring Adventures of Puff delivered to children. Parents and teachers participated in an asthma awareness event.

Usual care $\quad 6 \times$ lessons

ED visits; School absence; Unplanned GP or hospital visit; Experience of daytime and night-time symptoms; Withdrawal

$\begin{array}{lllll}\begin{array}{l}\text { Monforte None } \\ 2012\end{array} & \begin{array}{l}\text { To evaluate the implemen- } \\ \text { tation of OAS }\end{array} & \begin{array}{l}\text { OAS. No further informa- } \\ \text { tion was given }\end{array} & \text { Unclear } & \text { Unclear }\end{array}$

\begin{tabular}{lll}
\hline Mosnaim None & To assess the impact of the & One-to-one training on \\
& Fight Asthma Now educa- & spacer technique, peak \\
& tional programme among & flow meter use, and use \\
2 populations of predomi- & of an asthma action plan. \\
& nantly low-income minori- & Teens also received educa- \\
ty students & tion on tobacco avoidance \\
& and peer pressure
\end{tabular}

\begin{tabular}{lll}
\hline Patterson & PRECEDE & $\begin{array}{l}\text { To evaluate the effective- } \\
\text { model }\end{array}$ \\
& & ness of a programme of \\
& asthma clubs in improving \\
& quality of life for primary \\
& school children with asth- \\
& ma
\end{tabular}

$\begin{array}{llll}\text { SCAMP. Children used a } & \text { Wait-list } & 8 \times \text { sessions } & \text { Restricted } \\ \text { workbook during sessions } & \text { control } & & \text { activity days; } \\ \text { to learn about asthma } & & \text { Lung func- } \\ & & \text { tion; HRQoL; } \\ & & \text { Withdrawal }\end{array}$

\begin{tabular}{|c|c|c|c|c|c|c|}
\hline $\begin{array}{l}\text { Persaud } \\
1996\end{array}$ & None & $\begin{array}{l}\text { To assess the effective- } \\
\text { ness of an intervention } \\
\text { on knowledge, locus of } \\
\text { control, attitudes towards } \\
\text { asthma, functional status, } \\
\text { school attendance, and ED } \\
\text { visits }\end{array}$ & $\begin{array}{l}\text { Individualised education } \\
\text { sessions. Children had a } \\
\text { personal peak flow meter } \\
\text { in the school health office. } \\
\text { The school nurse also re- } \\
\text { viewed the student asth- } \\
\text { ma diary and discussed } \\
\text { this with them }\end{array}$ & Usual care & $\begin{array}{l}3 \times \text { lessons } \\
\text { and weekly } \\
\text { education } \\
\text { sessions }\end{array}$ & $\begin{array}{l}\text { ED visits; } \\
\text { School ab- } \\
\text { sence }\end{array}$ \\
\hline $\begin{array}{l}\text { Prae- } \\
\text { na-Crespo } \\
2010\end{array}$ & None & $\begin{array}{l}\text { To verify whether an asth- } \\
\text { ma education program } \\
\text { in schools would have di- } \\
\text { rect benefit for student } \\
\text { knowledge and attitudes } \\
\text { towards asthma and qual- } \\
\text { ity of life for students with } \\
\text { asthma }\end{array}$ & $\begin{array}{l}\text { Asthma programme. No } \\
\text { further information was } \\
\text { given (abstract only) }\end{array}$ & Unclear & $3 \times$ lessons & None \\
\hline
\end{tabular}

School-based self-management interventions for asthma in children and adolescents: a mixed methods systematic review (Review) 287 
Table 12. Outcome evaluation studies - summary of intervention characteristics (Continued)

\begin{tabular}{|c|c|c|c|c|c|}
\hline $\begin{array}{l}\text { Pulcini } \\
2007\end{array}$ & None & $\begin{array}{l}\text { To determine the effec- } \\
\text { tiveness of an intervention } \\
\text { to increase the number of } \\
\text { AAPs in schools }\end{array}$ & $\begin{array}{l}\text { Peak flow education. Chil- } \\
\text { dren were given a peak } \\
\text { flow meter and were edu- } \\
\text { cated on the correct tech- } \\
\text { nique to measure lung } \\
\text { function }\end{array}$ & Unclear & $\begin{array}{l}\text { Daily for } 2 \\
\text { weeks }\end{array}$ \\
\hline
\end{tabular}

\begin{tabular}{|c|c|c|}
\hline Shah 2001 & None & $\begin{array}{l}\text { To determine the effects } \\
\text { of a peer-led programme } \\
\text { for asthma education on } \\
\text { quality of life and related } \\
\text { morbidity in adolescents } \\
\text { with asthma }\end{array}$ \\
\hline
\end{tabular}

Triple-A: asthma education and empowerment. Students learnt how to educate their peers about asthma. Peers also led 3 health lessons for classes in school

\begin{tabular}{|c|c|}
\hline $\begin{array}{l}\text { Wait-list } \\
\text { control }\end{array}$ & $3 \times$ sessions \\
\hline
\end{tabular}

School absence; Unplanned GP or hospital visit sences, hospitalisations, and ED visits

\begin{tabular}{ll}
\hline Splett $2006 \quad$ None $\quad$ & To improve asthma man- \\
& agement among school \\
& children and reduce asth- \\
& ma-related school ab- \\
& sences, hospitalisations, \\
& and ED visits
\end{tabular}

Children received training

Usual care Unclear on managing their asthma. Licensed nurses and healthcare assistants received coaching and reinforcement from asthma resource nurses

\begin{tabular}{|c|c|c|c|c|c|c|}
\hline Srof 2012 & $\begin{array}{l}\text { Health pro- } \\
\text { motion } \\
\text { model }\end{array}$ & $\begin{array}{l}\text { To determine effects of } \\
\text { coping skills on asthma } \\
\text { self-efficacy, social sup- } \\
\text { port, quality of life, and } \\
\text { peak flow among adoles- } \\
\text { cents }\end{array}$ & $\begin{array}{l}\text { Asthma diary; } 5 \times \text { coping } \\
\text { skills sessions. Students } \\
\text { received coping skills } \\
\text { training and completed di- } \\
\text { ary entries }\end{array}$ & Usual care & $\begin{array}{l}\text { Sessions } \\
\text { over } 5 \\
\text { weeks }\end{array}$ & None \\
\hline $\begin{array}{l}\text { Vel- } \\
\text { sor-Friedrich } \\
2005\end{array}$ & $\begin{array}{l}\text { Self-care } \\
\text { deficit the- } \\
\text { ory }\end{array}$ & $\begin{array}{l}\text { To test a 2-part interven- } \\
\text { tion on selected psychoso- } \\
\text { cial and health outcomes } \\
\text { for children with asthma }\end{array}$ & $\begin{array}{l}\text { OAS; nurse practitioner } \\
\text { visits. Children received } \\
\text { the OAS education curricu- } \\
\text { lum and nurse practition- } \\
\text { er visits to assess asthma } \\
\text { health and further educa- } \\
\text { tion }\end{array}$ & Usual care & $\begin{array}{l}6 \times \text { group } \\
\text { sessions; } \\
\text { individual } \\
\text { nurse ses- } \\
\text { sions }\end{array}$ & $\begin{array}{l}\text { ED visits; Ex- } \\
\text { perience of } \\
\text { daytime and } \\
\text { night-time } \\
\text { symptoms; } \\
\text { Lung func- } \\
\text { tion }\end{array}$ \\
\hline
\end{tabular}

AAP: XXX.

ASMA: Asthma Self-Management for Adolescents.

ED: emergency department.

GP: general practitioner.

HRQoL: health-related quality of life.

ICAN: I Can Control Asthma and Nutrition Now.

OAS: Open Airways for Schools.

$\mathrm{PD} / \mathrm{H} / \mathrm{PE}$ : personal development/health/physical education.

PRECEDE: Predisposing, Reinforcing, and Enabling Causes in Educational Diagnosis and Evaluation.

RCT: randomised controlled trial.

SCAMP: School Care and Asthma Management Project.

SHARP: Staying Healthy-Asthma Responsible \& Prepared.

Triple A: Adolescent Asthma Action. 


\begin{tabular}{|c|c|c|c|c|c|c|c|}
\hline Study & Indicator & $\begin{array}{l}\text { Collection/reporting } \\
\text { point }\end{array}$ & $\begin{array}{l}\text { Mean clus- } \\
\text { ter size (if ap- } \\
\text { plicable) }\end{array}$ & $\begin{array}{l}\text { Intracluster } \\
\text { correlation } \\
\text { coefficient } \\
\text { applied (if } \\
\text { applicable) }\end{array}$ & $\begin{array}{l}\text { Data trans- } \\
\text { formation }\end{array}$ & $\begin{array}{l}\text { Original ef- } \\
\text { fect size and } \\
\text { standard er- } \\
\text { ror (with ad- } \\
\text { justment for } \\
\text { clustering if } \\
\text { applicable) }\end{array}$ & $\begin{array}{l}\text { Final or trans- } \\
\text { formed effect size } \\
\text { and standard er- } \\
\text { ror (with adjust- } \\
\text { ment for cluster- } \\
\text { ing if applicable) }\end{array}$ \\
\hline \multicolumn{8}{|c|}{ Hospitalisations } \\
\hline Atherly 2009 & $\begin{array}{l}\text { Instances of hospitalisation in pre- } \\
\text { vious } 4 \text { weeks }\end{array}$ & $\begin{array}{l}\text { Post intervention (3- } \\
\text { month follow-up) }\end{array}$ & 45.8 & 0.05 & $\begin{array}{l}\text { Yes - trans- } \\
\text { formed from } \\
\text { odds ratio to } \\
\text { SMD }\end{array}$ & $\begin{array}{l}\text { OR }(0.7736) \\
\text { SE (InOR) } \\
(1.385)\end{array}$ & $\begin{array}{l}\text { SMD (-0.141); SE } \\
(0.764)\end{array}$ \\
\hline Bruzzese 2011 & $\begin{array}{l}\text { Hospitalisations in the past } 2 \\
\text { months }\end{array}$ & $\begin{array}{l}\text { Post intervention (12- } \\
\text { month follow-up) }\end{array}$ & $\mathrm{N} / \mathrm{A}$ & $\mathrm{N} / \mathrm{A}$ & No & $\mathrm{N} / \mathrm{A}$ & $\begin{array}{l}\text { SMD (-0.219); SE } \\
(0.120)\end{array}$ \\
\hline Clark 2005 & Hospitalisations & $\begin{array}{l}\text { Post intervention (12- } \\
\text { month follow-up) }\end{array}$ & $\begin{array}{l}\text { Deemed } \\
\text { that analysis } \\
\text { methods ac- } \\
\text { counted for } \\
\text { clustering }\end{array}$ & $\begin{array}{l}\text { Deemed } \\
\text { that analysis } \\
\text { methods ac- } \\
\text { counted for } \\
\text { clustering }\end{array}$ & $\begin{array}{l}\text { Yes - trans- } \\
\text { formed from } \\
\text { odds ratio to } \\
\text { SMD }\end{array}$ & $\begin{array}{l}\text { OR (1.43); SE } \\
\text { (estimated } \\
\text { from P value } \\
(\ln O R)) 0.39\end{array}$ & $\begin{array}{l}\text { SMD (-0.197); SE } \\
(0.215)\end{array}$ \\
\hline Gerald 2006 & $\begin{array}{l}\text { Median hospitalisations (not com- } \\
\text { bined) }\end{array}$ & $\mathrm{N} / \mathrm{A}$ & $\mathrm{N} / \mathrm{A}$ & $\mathrm{N} / \mathrm{A}$ & $\mathrm{N} / \mathrm{A}$ & $\mathrm{N} / \mathrm{A}$ & $\mathrm{N} / \mathrm{A}$ \\
\hline Horner 2008 & $\begin{array}{l}\text { Any hospital stays in the past } 12 \\
\text { months (based on parents report- } \\
\text { ing any stay) }\end{array}$ & $\begin{array}{l}\text { Post intervention ( } 7 \text { - } \\
\text { month follow-up) }\end{array}$ & $\begin{array}{l}10.1 \text { (reported } \\
\text { by study au- } \\
\text { thors) }\end{array}$ & 0.05 & $\begin{array}{l}\text { Yes - trans- } \\
\text { formed from } \\
\text { odds ratio to } \\
\text { SMD }\end{array}$ & $\begin{array}{l}\mathrm{OR}(0.882) ; \mathrm{SE} \\
(\operatorname{lnOR})(0.791)\end{array}$ & $\begin{array}{l}\operatorname{SMD}(-0.069) ; \mathrm{SE} \\
(0.436)\end{array}$ \\
\hline Horner 2015 & $\begin{array}{l}\text { Mean number of hospitalisations } \\
\text { since the previous data collection } \\
\text { (at } 8 \text { months) }\end{array}$ & $\begin{array}{l}\text { Post intervention (12- } \\
\text { month follow-up) }\end{array}$ & 8.9 (approx.) & 0.05 & No & N/A & $\begin{array}{l}\text { SMD }(-0.057) ; \text { SE } \\
(0.169)\end{array}$ \\
\hline Levy 2006 & Mean hospital days & $\begin{array}{l}\text { Post test (at interven- } \\
\text { tion end) }\end{array}$ & 17.36 & 0.05 & No & N/A & $\begin{array}{l}\text { SMD (-0.293); SE } \\
(0.174)\end{array}$ \\
\hline \multicolumn{8}{|c|}{ Emergency department visits } \\
\hline Atherly 2009 & $\begin{array}{l}\text { Instances of ED visits in previous } 4 \\
\text { weeks }\end{array}$ & $\begin{array}{l}\text { Post intervention (3- } \\
\text { month follow-up) }\end{array}$ & 45.8 & 0.05 & No & N/A & $\begin{array}{l}\text { OR (1.036); SE } \\
(\operatorname{lnOR})(0.916)\end{array}$ \\
\hline
\end{tabular}




\begin{tabular}{|c|c|c|c|c|c|c|c|}
\hline Bruzzese 2011 & Mean ED visits in the past 2 months & $\begin{array}{l}\text { Post intervention (12- } \\
\text { month follow-up) }\end{array}$ & $\mathrm{N} / \mathrm{A}$ & $\mathrm{N} / \mathrm{A}$ & $\begin{array}{l}\text { Yes - trans- } \\
\text { formed from } \\
\text { SMD to OR }\end{array}$ & $\begin{array}{l}\text { SMD (-0.289); } \\
\text { SE }(0.120)\end{array}$ & $\begin{array}{l}\text { OR (0.592); SE } \\
\text { (InOR) (0.218) }\end{array}$ \\
\hline Cicutto 2005 & ED visits in the past year & $\begin{array}{l}\text { Post intervention (12- } \\
\text { month follow-up) }\end{array}$ & 9.85 & 0.05 & No & N/A & $\begin{array}{l}\text { OR (0.697); SE } \\
(\operatorname{lnOR})(0.407)\end{array}$ \\
\hline Cicutto 2013 & $\begin{array}{l}\text { ED visits in the past year (reports } \\
\text { of) }\end{array}$ & $\begin{array}{l}\text { Post intervention (12- } \\
\text { month follow-up) }\end{array}$ & 7.7 & 0.05 & No & N/A & $\begin{array}{l}\text { OR (0.318); SE } \\
\text { (InOR) (0.317) }\end{array}$ \\
\hline Clark 2005 & ED visits & $\begin{array}{l}\text { Post intervention (12- } \\
\text { month follow-up) }\end{array}$ & $\begin{array}{l}\text { Deemed } \\
\text { that analysis } \\
\text { methods ac- } \\
\text { counted for } \\
\text { clustering }\end{array}$ & $\begin{array}{l}\text { Deemed } \\
\text { that analysis } \\
\text { methods ac- } \\
\text { counted for } \\
\text { clustering }\end{array}$ & $\begin{array}{l}\text { No, but see } \\
\text { notes }\end{array}$ & N/A & $\begin{array}{l}\text { OR (1.002)*; SE (es- } \\
\text { timated from P val- } \\
\text { ue (InOR)) } 0.072 \\
{ }^{\star} \text { Note that the OR } \\
\text { was reported as } \\
1.00 \text { in the paper } \\
\text { with a P value of } \\
0.98 . \text { So informa- } \\
\text { tion could be used } \\
\text { and an SE extract- } \\
\text { ed, a small correc- } \\
\text { tion to an OR of } \\
1.002 \text { was applied }\end{array}$ \\
\hline Gerald 2006 & Median ED visits (not combined) & $\mathrm{N} / \mathrm{A}$ & $\mathrm{N} / \mathrm{A}$ & $\mathrm{N} / \mathrm{A}$ & $\mathrm{N} / \mathrm{A}$ & N/A & $\mathrm{N} / \mathrm{A}$ \\
\hline Horner 2008 & $\begin{array}{l}\text { Any ED visits in the past } 12 \text { months } \\
\text { (based on parents reporting any } \\
\text { stay) }\end{array}$ & $\begin{array}{l}\text { Post intervention (7- } \\
\text { month follow-up) }\end{array}$ & $\begin{array}{l}10.1 \text { (reported } \\
\text { by study au- } \\
\text { thors) }\end{array}$ & 0.05 & No & N/A & $\begin{array}{l}\text { OR (0.857); SE } \\
(\operatorname{lnOR})(0.461)\end{array}$ \\
\hline Horner 2015 & $\begin{array}{l}\text { Mean number of ED visits since } \\
\text { the previous data collection ( } 8 \\
\text { months) }\end{array}$ & $\begin{array}{l}\text { Post intervention (12- } \\
\text { month follow-up) }\end{array}$ & 8.9 (approx.) & 0.05 & $\begin{array}{l}\text { Yes - trans- } \\
\text { formed from } \\
\text { SMD to OR }\end{array}$ & $\begin{array}{l}\operatorname{SMD}(0) ; \mathrm{SE} \\
(0.169)\end{array}$ & $\begin{array}{l}\text { OR (1.00); SE } \\
(0.306)\end{array}$ \\
\hline Howell 2005 & $\begin{array}{l}\text { Mean number of ED visits in the } \\
\text { past } 6 \text { weeks }\end{array}$ & $\begin{array}{l}\text { Post intervention (3- } \\
\text { month follow-up) }\end{array}$ & 4.25 & 0.05 & $\begin{array}{l}\text { Yes - trans- } \\
\text { formed from } \\
\text { SMD to OR }\end{array}$ & $\begin{array}{l}\text { SMD }(-0.331) \\
\text { SE }(0.578)\end{array}$ & $\begin{array}{l}\text { OR (0.549); SE } \\
(1.049)\end{array}$ \\
\hline Levy 2006 & $\begin{array}{l}\text { Mean urgent care or emergency } \\
\text { visits }\end{array}$ & $\begin{array}{l}\text { Post test (at interven- } \\
\text { tion end) }\end{array}$ & 17.36 & 0.05 & $\begin{array}{l}\text { Yes - trans- } \\
\text { formed from } \\
\text { SMD to OR }\end{array}$ & $\begin{array}{l}\text { SMD }(-0.286) \\
\text { SE }(0.174)\end{array}$ & $\begin{array}{l}\text { OR (0.595); SE } \\
(0.318)\end{array}$ \\
\hline
\end{tabular}




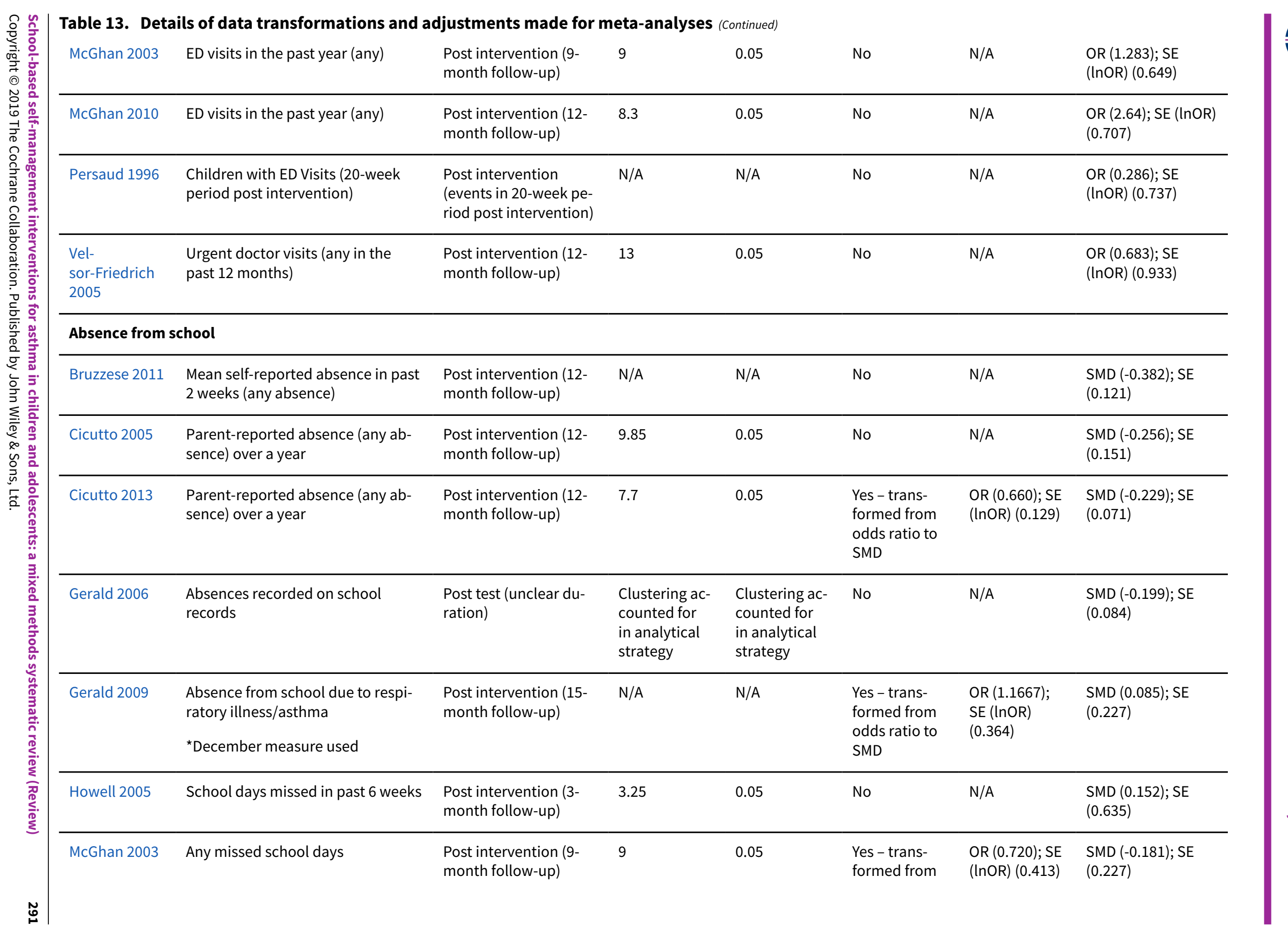




\begin{tabular}{|c|c|c|c|c|c|c|c|}
\hline & & & & & $\begin{array}{l}\text { odds ratio to } \\
\text { SMD }\end{array}$ & & \\
\hline McGhan 2010 & $\begin{array}{l}\text { (No) Missed school days (any) over } \\
\text { past } 12 \text { months }\end{array}$ & $\begin{array}{l}\text { Post intervention (12- } \\
\text { month follow-up) }\end{array}$ & 8.3 & 0.05 & $\begin{array}{l}\text { Yes - trans- } \\
\text { formed from } \\
\text { odds ratio to } \\
\text { SMD }\end{array}$ & $\begin{array}{l}\text { OR (0.640); SE } \\
(\operatorname{lnOR})(0.353)\end{array}$ & $\begin{array}{l}\text { SMD (0.246); SE } \\
(0.195) \\
\text { (note: inverse tak- } \\
\text { en as the interven- } \\
\text { tion favours con- } \\
\text { trol) }\end{array}$ \\
\hline Persaud 1996 & $\begin{array}{l}\text { Mean school days of absence } \\
\text { based on school records }\end{array}$ & $\begin{array}{l}\text { Post intervention (im- } \\
\text { mediately afterwards) }\end{array}$ & $\mathrm{N} / \mathrm{A}$ & $\mathrm{N} / \mathrm{A}$ & No & N/A & $\begin{array}{l}\operatorname{SMD}(-0.236) ; \mathrm{SE} \\
(0.335)\end{array}$ \\
\hline Splett 2006 & Mean percentage of days attended & $\begin{array}{l}\text { Post intervention (12- } \\
\text { month follow-up) }\end{array}$ & $\begin{array}{l}\text { Deemed } \\
\text { that analysis } \\
\text { methods ac- } \\
\text { counted for } \\
\text { clustering }\end{array}$ & $\begin{array}{l}\text { Deemed } \\
\text { that analysis } \\
\text { methods ac- } \\
\text { counted for } \\
\text { clustering }\end{array}$ & No & N/A & $\begin{array}{l}\text { SMD (0.019); SE } \\
(0.051)\end{array}$ \\
\hline \multicolumn{8}{|c|}{ Days of restricted activity } \\
\hline Bruzzese 2011 & $\begin{array}{l}\text { Mean self-reported days of restrict- } \\
\text { ed activity in past } 2 \text { weeks }\end{array}$ & $\begin{array}{l}\text { Post intervention (12- } \\
\text { month follow-up) }\end{array}$ & $\mathrm{N} / \mathrm{A}$ & $\mathrm{N} / \mathrm{A}$ & No & N/A & $\begin{array}{l}\text { SMD }(-0.349) ; \text { SE } \\
(0.120)\end{array}$ \\
\hline Cicutto 2005 & $\begin{array}{l}\text { Days of limited activity due to asth- } \\
\text { ma }\end{array}$ & $\begin{array}{l}\text { Post intervention (12- } \\
\text { month follow-up) }\end{array}$ & 9.85 & 0.05 & No & N/A & $\begin{array}{l}\operatorname{SMD}(-0.318) ; \mathrm{SE} \\
(0.151)\end{array}$ \\
\hline Cicutto 2013 & $\begin{array}{l}\text { Percentage of students reporting } \\
\text { days of restricted activity }\end{array}$ & $\begin{array}{l}\text { Post intervention (12- } \\
\text { month follow-up) }\end{array}$ & 7.7 & 0.05 & $\begin{array}{l}\text { Yes - trans- } \\
\text { formed from } \\
\text { odds ratio to } \\
\text { SMD }\end{array}$ & $\begin{array}{l}\mathrm{OR}(0.612) ; \mathrm{SE} \\
(\operatorname{lnOR})(0.130)\end{array}$ & $\begin{array}{l}\operatorname{SMD}(-0.271) ; \mathrm{SE} \\
(0.072)\end{array}$ \\
\hline \multicolumn{8}{|c|}{ Unplanned visits to medical providers } \\
\hline Bruzzese 2011 & $\begin{array}{l}\text { Mean acute care visits in the past } 2 \\
\text { months }\end{array}$ & $\begin{array}{l}\text { Post intervention (12- } \\
\text { month follow-up) }\end{array}$ & $\mathrm{N} / \mathrm{A}$ & $\mathrm{N} / \mathrm{A}$ & $\begin{array}{l}\text { Yes - trans- } \\
\text { formed from } \\
\text { SMD to OR }\end{array}$ & $\begin{array}{l}\text { SMD }(-0.283) \\
\text { SE }(0.120)\end{array}$ & $\begin{array}{l}\text { OR (0.598); SE } \\
(0.217)\end{array}$ \\
\hline Cicutto 2013 & $\begin{array}{l}\text { Unscheduled care in the past year } \\
\text { (reports of) }\end{array}$ & $\begin{array}{l}\text { Post intervention (12- } \\
\text { month follow-up) }\end{array}$ & 7.7 & 0.05 & No & $\begin{array}{l}\mathrm{OR}(0.703) ; \mathrm{SE} \\
(\operatorname{InOR})(0.143)\end{array}$ & $\begin{array}{l}\operatorname{SMD}(-0.194) ; \mathrm{SE} \\
(0.079)\end{array}$ \\
\hline McGhan 2003 & Any unscheduled doctor visits & $\begin{array}{l}\text { Post intervention (9- } \\
\text { month follow-up) }\end{array}$ & 9 & 0.05 & No & $\begin{array}{l}\mathrm{OR}(0.886) ; \mathrm{SE} \\
(\operatorname{lnOR})(0.426)\end{array}$ & $\begin{array}{l}\operatorname{SMD}(-0.067) ; \mathrm{SE} \\
(0.235)\end{array}$ \\
\hline
\end{tabular}




\begin{tabular}{|c|c|c|c|c|c|c|c|}
\hline McGhan 2010 & $\begin{array}{l}\text { Unscheduled GP visits (any) over } \\
\text { past } 12 \text { months }\end{array}$ & $\begin{array}{l}\text { Post intervention (12- } \\
\text { month follow-up) }\end{array}$ & 8.3 & 0.05 & No & $\begin{array}{l}\text { OR (1.169); SE } \\
\text { (InOR) (0.397) }\end{array}$ & $\begin{array}{l}\text { SMD (0.086); SE } \\
(0.219)\end{array}$ \\
\hline Splett 2006 & $\begin{array}{l}\text { Episodic asthma visits to school } \\
\text { health office (over } 6 \text { months fol- } \\
\text { lowing start of intervention) }\end{array}$ & $\begin{array}{l}\text { Over } 6 \text { months follow- } \\
\text { ing start of interven- } \\
\text { tion }\end{array}$ & 97.6 & 0.05 & No & $\begin{array}{l}\text { OR (0.913); SE } \\
(\operatorname{lnOR})(0.282)\end{array}$ & $\begin{array}{l}\text { SMD (-0.046); SE } \\
(0.156)\end{array}$ \\
\hline \multicolumn{8}{|c|}{ Daytime symptoms } \\
\hline Atherly 2009 & $\begin{array}{l}\text { Mean number of days with asthma } \\
\text { symptoms }\end{array}$ & $\begin{array}{l}\text { Post intervention (3- } \\
\text { month follow-up) }\end{array}$ & 45.8 & 0.05 & No & N/A & $\begin{array}{l}\text { SMD (-0.026); SE } \\
(0.168)\end{array}$ \\
\hline Bruzzese 2008 & $\begin{array}{l}\text { Mean days last } 2 \text { weeks with asth- } \\
\text { ma symptoms }\end{array}$ & $\begin{array}{l}\text { Post intervention ( } 2- \\
\text { month follow-up) }\end{array}$ & $\mathrm{N} / \mathrm{A}$ & $\mathrm{N} / \mathrm{A}$ & No & N/A & $\begin{array}{l}\text { SMD (-0.151); SE } \\
(0.418)\end{array}$ \\
\hline Bruzzese 2011 & $\begin{array}{l}\text { Mean days last } 2 \text { weeks with asth- } \\
\text { ma symptoms }\end{array}$ & $\begin{array}{l}\text { Post intervention (12- } \\
\text { month follow-up) }\end{array}$ & $\mathrm{N} / \mathrm{A}$ & $\mathrm{N} / \mathrm{A}$ & No & N/A & $\begin{array}{l}\text { SMD }(-0.210) ; \text { SE } \\
(0.120)\end{array}$ \\
\hline Shah 2001 & $\begin{array}{l}\text { Number of students reporting at- } \\
\text { tacks in school at follow-up }\end{array}$ & $\begin{array}{l}\text { Post intervention (6- } \\
\text { month follow-up) }\end{array}$ & 41.8 & 0.05 & $\begin{array}{l}\text { Yes - trans- } \\
\text { formed from } \\
\text { odds ratio to } \\
\text { SMD }\end{array}$ & $\begin{array}{l}\text { OR (0.647); SE } \\
\text { (InOR) (0.488) }\end{array}$ & $\begin{array}{l}\text { SMD }(-0.240) ; \text { SE } \\
(0.269)\end{array}$ \\
\hline $\begin{array}{l}\text { Vel- } \\
\text { sor-Friedrich } \\
2005\end{array}$ & Symptom days in past 2 weeks & $\begin{array}{l}\text { Post intervention (12- } \\
\text { month follow-up) }\end{array}$ & 13 & 0.05 & $\begin{array}{l}\text { Yes - trans- } \\
\text { formed from } \\
\text { odds ratio to } \\
\text { SMD }\end{array}$ & $\begin{array}{l}\text { OR (0.846); SE } \\
\text { (InOR) (0.705) }\end{array}$ & $\begin{array}{l}\text { SMD }(-0.030) ; \text { SE } \\
(0.413)\end{array}$ \\
\hline \multicolumn{8}{|c|}{ Night-time symptoms } \\
\hline Bruzzese 2008 & $\begin{array}{l}\text { Mean nights woken last } 2 \text { weeks } \\
\text { with asthma symptoms }\end{array}$ & $\begin{array}{l}\text { Post intervention ( } 2 \text { - } \\
\text { month follow-up) }\end{array}$ & $\mathrm{N} / \mathrm{A}$ & $\mathrm{N} / \mathrm{A}$ & No & N/A & $\begin{array}{l}\operatorname{SMD}(-0.433) ; \mathrm{SE} \\
(0.423)\end{array}$ \\
\hline Bruzzese 2011 & $\begin{array}{l}\text { Mean self-reported night-time } \\
\text { awakenings in past } 2 \text { weeks }\end{array}$ & $\begin{array}{l}\text { Post intervention (12- } \\
\text { month follow-up) }\end{array}$ & $\mathrm{N} / \mathrm{A}$ & $\mathrm{N} / \mathrm{A}$ & No & N/A & $\begin{array}{l}\text { SMD }(-0.388) ; \text { SE } \\
(0.121)\end{array}$ \\
\hline Howell 2005 & $\begin{array}{l}\text { Mean number of night-time awak- } \\
\text { enings in past } 6 \text { weeks }\end{array}$ & $\begin{array}{l}\text { Post intervention (3- } \\
\text { month follow-up) }\end{array}$ & 4.25 & 0.05 & No & $\mathrm{N} / \mathrm{A}$ & $\begin{array}{l}\operatorname{SMD}(0.253) ; \mathrm{SE} \\
(0.478)\end{array}$ \\
\hline McGhan 2003 & $\begin{array}{l}\text { Waking up in past } 2 \text { weeks twice or } \\
\text { more }\end{array}$ & $\begin{array}{l}\text { Post intervention (9- } \\
\text { month follow-up) }\end{array}$ & 9 & 0.05 & $\begin{array}{l}\text { Yes - trans- } \\
\text { formed from } \\
\text { odds ratio to } \\
\text { SMD }\end{array}$ & $\begin{array}{l}\text { OR (1.237); SE } \\
\text { (InOR) (0.412) }\end{array}$ & $\begin{array}{l}\text { SMD }(0.117) ; \text { SE } \\
(0.227)\end{array}$ \\
\hline
\end{tabular}




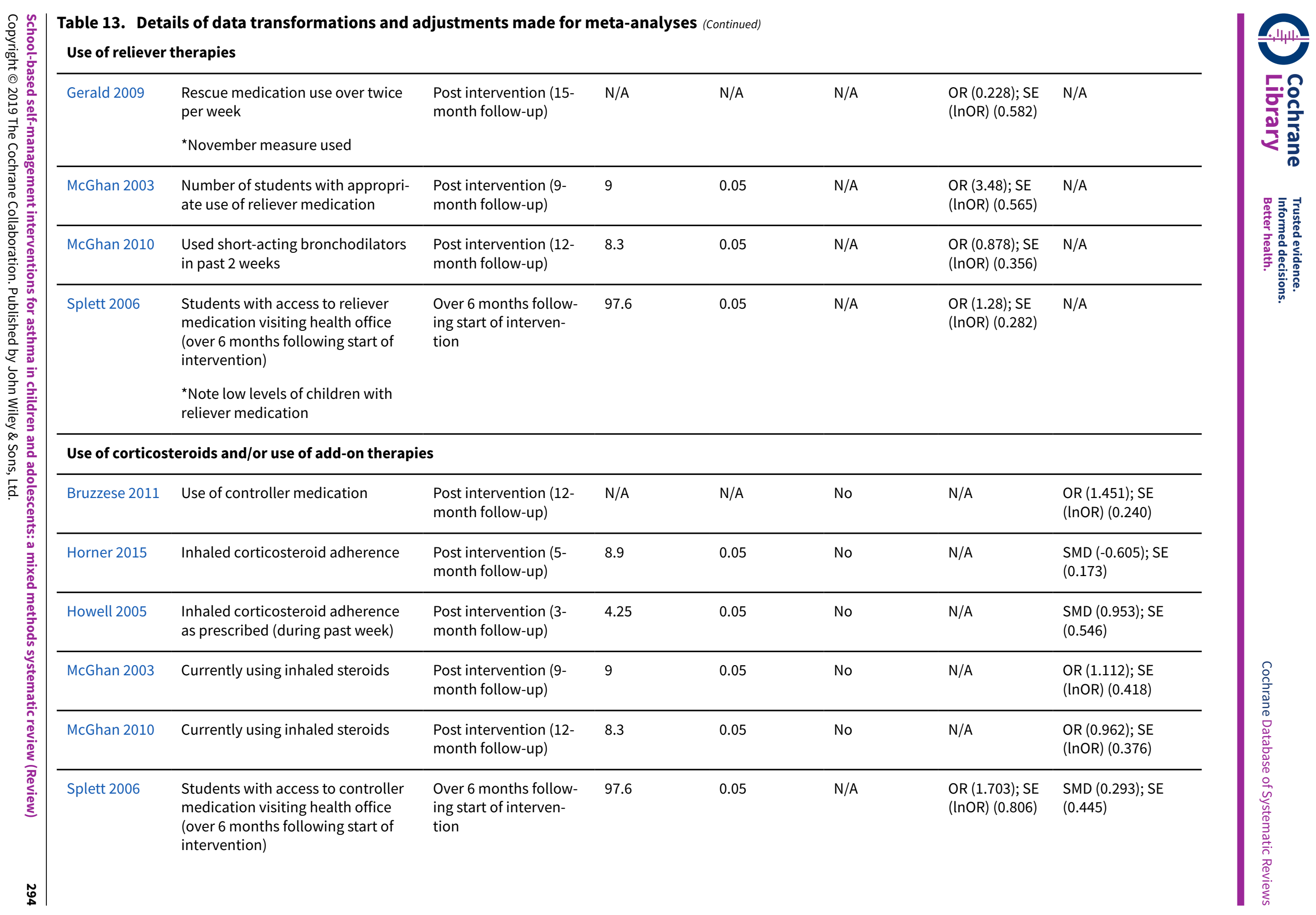




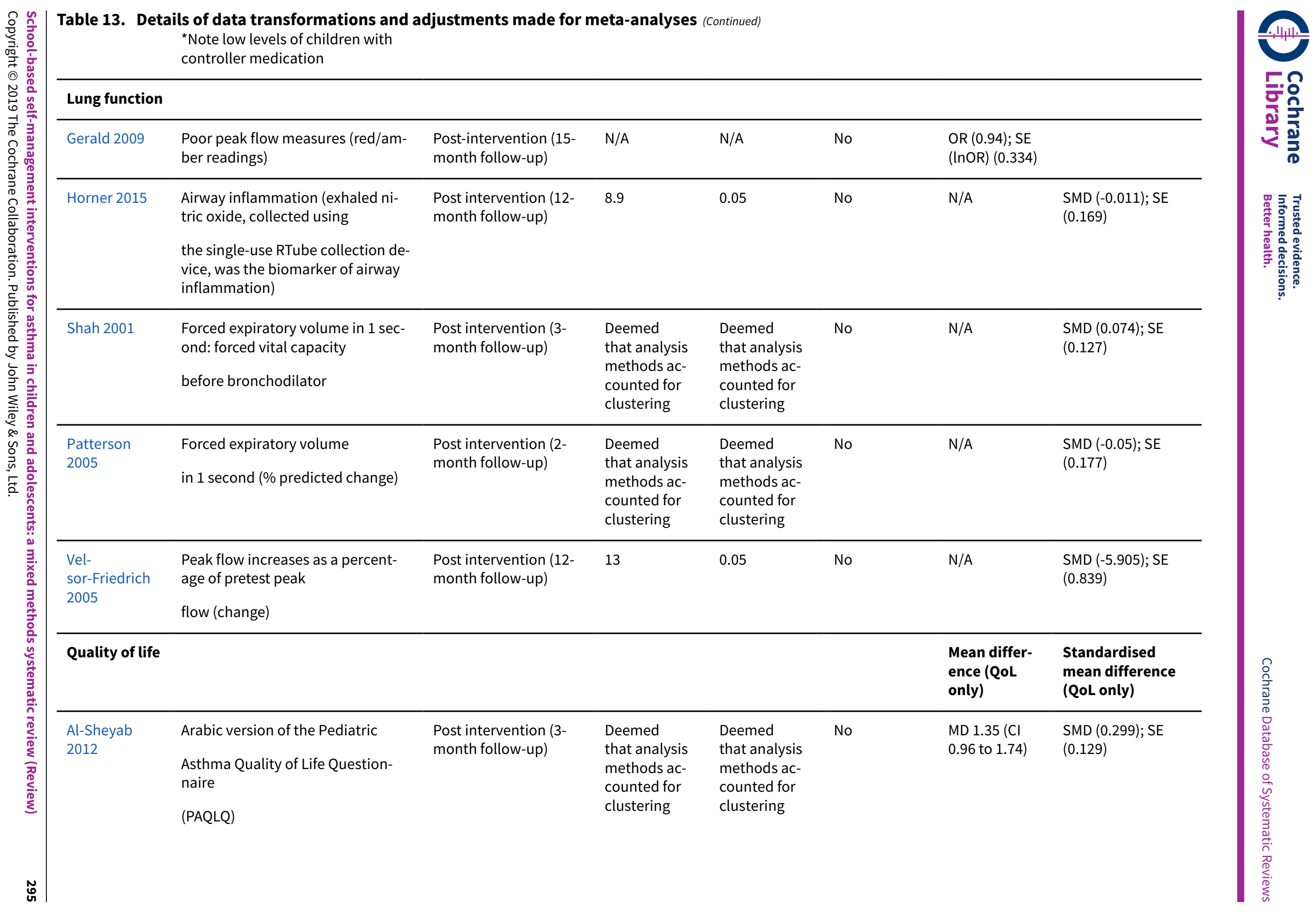




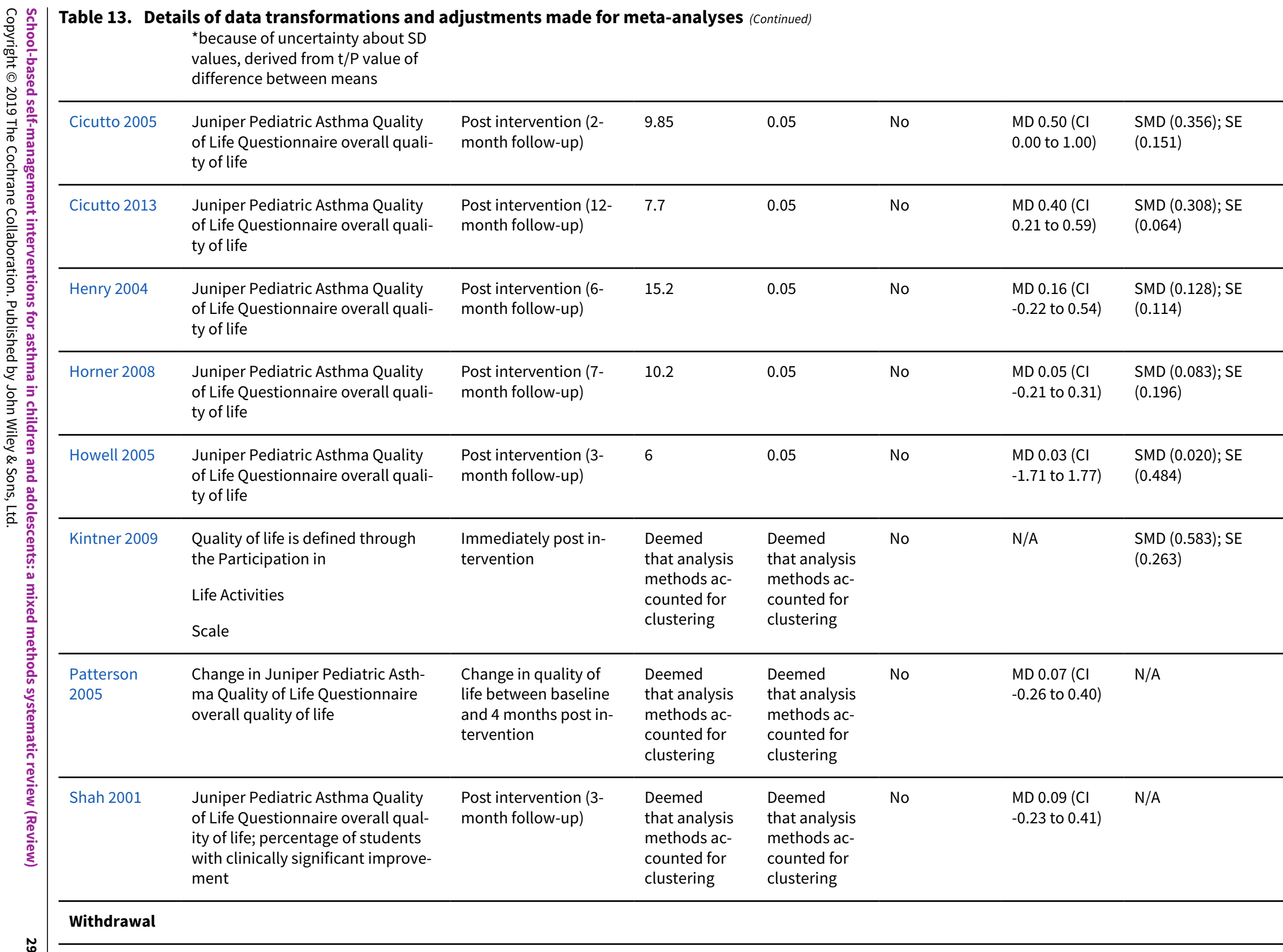




\begin{tabular}{|c|c|c|c|c|c|c|c|}
\hline $\begin{array}{l}\text { Al-Sheyab } \\
2012\end{array}$ & $\begin{array}{l}\text { Withdrew between baseline and } \\
\text { outcome collection }\end{array}$ & $\begin{array}{l}\text { Post intervention (3- } \\
\text { month follow-up) }\end{array}$ & 65.25 & 0.05 & No & N/A & $\begin{array}{l}\mathrm{OR}(0.511) ; \mathrm{SE} \\
(\operatorname{lnOR})(1.074)\end{array}$ \\
\hline $\begin{array}{l}\text { Bartholomew } \\
2006\end{array}$ & $\begin{array}{l}\text { Lost to follow-up at post-test mea- } \\
\text { sure }\end{array}$ & $\begin{array}{l}\text { Post intervention (du- } \\
\text { ration unclear) }\end{array}$ & 11.2 & 0.05 & No & N/A & $\begin{array}{l}\text { OR (0.237); SE } \\
(\operatorname{lnOR})(0.145)\end{array}$ \\
\hline Bruzzese 2008 & $\begin{array}{l}\text { Withdrew between baseline and } \\
\text { outcome collection }\end{array}$ & $\begin{array}{l}\text { Immediate post inter- } \\
\text { vention }\end{array}$ & $\mathrm{N} / \mathrm{A}$ & $\mathrm{N} / \mathrm{A}$ & No & $\mathrm{N} / \mathrm{A}$ & $\begin{array}{l}\text { OR (0.307); SE } \\
\text { (InOR) (1.683) }\end{array}$ \\
\hline Bruzzese 2011 & $\begin{array}{l}\text { Withdrew between baseline and } \\
\text { outcome collection }\end{array}$ & $\begin{array}{l}\text { Post intervention (12- } \\
\text { month follow-up) }\end{array}$ & $\mathrm{N} / \mathrm{A}$ & $\mathrm{N} / \mathrm{A}$ & No & $\mathrm{N} / \mathrm{A}$ & $\begin{array}{l}\text { OR (1.313); SE } \\
\text { (InOR) (0.279) }\end{array}$ \\
\hline Cicutto 2005 & $\begin{array}{l}\text { Withdrew between baseline and } \\
\text { outcome collection }\end{array}$ & $\begin{array}{l}\text { Post intervention (6- } \\
\text { month follow-up) }\end{array}$ & 9.85 & 0.05 & No & $\mathrm{N} / \mathrm{A}$ & $\begin{array}{l}\text { OR (1.788); SE } \\
\text { (InOR) (0.629) }\end{array}$ \\
\hline Gerald 2009 & $\begin{array}{l}\text { Withdrew between baseline and } \\
\text { outcome collection }\end{array}$ & $\begin{array}{l}\text { Post intervention (6- } \\
\text { month follow-up) }\end{array}$ & $\mathrm{N} / \mathrm{A}$ & $\mathrm{N} / \mathrm{A}$ & No & N/A & $\begin{array}{l}\text { OR (1.788); SE } \\
(\operatorname{lnOR})(0.613)\end{array}$ \\
\hline Horner 2008 & $\begin{array}{l}\text { Withdrew between baseline and } \\
\text { outcome collection }\end{array}$ & $\begin{array}{l}\text { Post intervention (7- } \\
\text { month follow-up) }\end{array}$ & 10.2 & 0.05 & No & N/A & $\begin{array}{l}\mathrm{OR}(1.333) ; \mathrm{SE} \\
(\operatorname{InOR})(0.531)\end{array}$ \\
\hline Horner 2015 & $\begin{array}{l}\text { Failed to complete final data col- } \\
\text { lection }\end{array}$ & $\begin{array}{l}\text { Post intervention (12- } \\
\text { month follow-up) }\end{array}$ & 8.9 & 0.05 & No & N/A & $\begin{array}{l}\text { OR (0.75); SE (InOR) } \\
(0.486)\end{array}$ \\
\hline Kintner 2009 & $\begin{array}{l}\text { Withdrew during intervention and } \\
\text { between end of intervention and } \\
\text { follow-up }\end{array}$ & $\begin{array}{l}\text { Post intervention (12- } \\
\text { month follow-up) }\end{array}$ & 13.2 & 0.05 & No & N/A & $\begin{array}{l}\text { OR (30.176); SE } \\
\text { (InOR) (1.860) }\end{array}$ \\
\hline Levy 2006 & $\begin{array}{l}\text { Failure to complete outcome eval- } \\
\text { uation }\end{array}$ & $\begin{array}{l}\text { Post intervention (12- } \\
\text { month follow-up) }\end{array}$ & 17.36 & 0.05 & No & $\mathrm{N} / \mathrm{A}$ & $\begin{array}{l}\text { OR }(0.357) ; \mathrm{SE} \\
(\operatorname{lnOR})(0.3881)\end{array}$ \\
\hline McGhan 2003 & $\begin{array}{l}\text { Withdrew between baseline and } \\
\text { outcome collection }\end{array}$ & $\begin{array}{l}\text { Post intervention (9- } \\
\text { month follow-up) }\end{array}$ & 9 & 0.05 & No & N/A & $\begin{array}{l}\mathrm{OR}(1.147) ; \mathrm{SE} \\
(\operatorname{lnOR})(0.5381)\end{array}$ \\
\hline McGhan 2010 & $\begin{array}{l}\text { Withdrew between baseline and in- } \\
\text { terim outcome collection }\end{array}$ & $\begin{array}{l}\text { Post intervention (6- } \\
\text { month follow-up) }\end{array}$ & 8.3 & 0.05 & No & N/A & $\begin{array}{l}\text { OR (1.007); SE } \\
\text { (InOR) (0.387) }\end{array}$ \\
\hline $\begin{array}{l}\text { Patterson } \\
2005\end{array}$ & Withdrew during intervention & $\begin{array}{l}\text { Post intervention - im- } \\
\text { mediately following in- } \\
\text { tervention }\end{array}$ & 7.95 & 0.05 & No & $\mathrm{N} / \mathrm{A}$ & $\begin{array}{l}\text { OR (5.675); SE } \\
\text { (InOR) (1.087) }\end{array}$ \\
\hline Shah 2001 & $\begin{array}{l}\text { Withdrew between baseline and } \\
\text { outcome collection }\end{array}$ & $\begin{array}{l}\text { Post intervention (3- } \\
\text { month follow-up) }\end{array}$ & 45.3 & 0.05 & No & N/A & $\begin{array}{l}\text { OR (1.343); SE } \\
(\operatorname{lnOR})(0.475)\end{array}$ \\
\hline
\end{tabular}



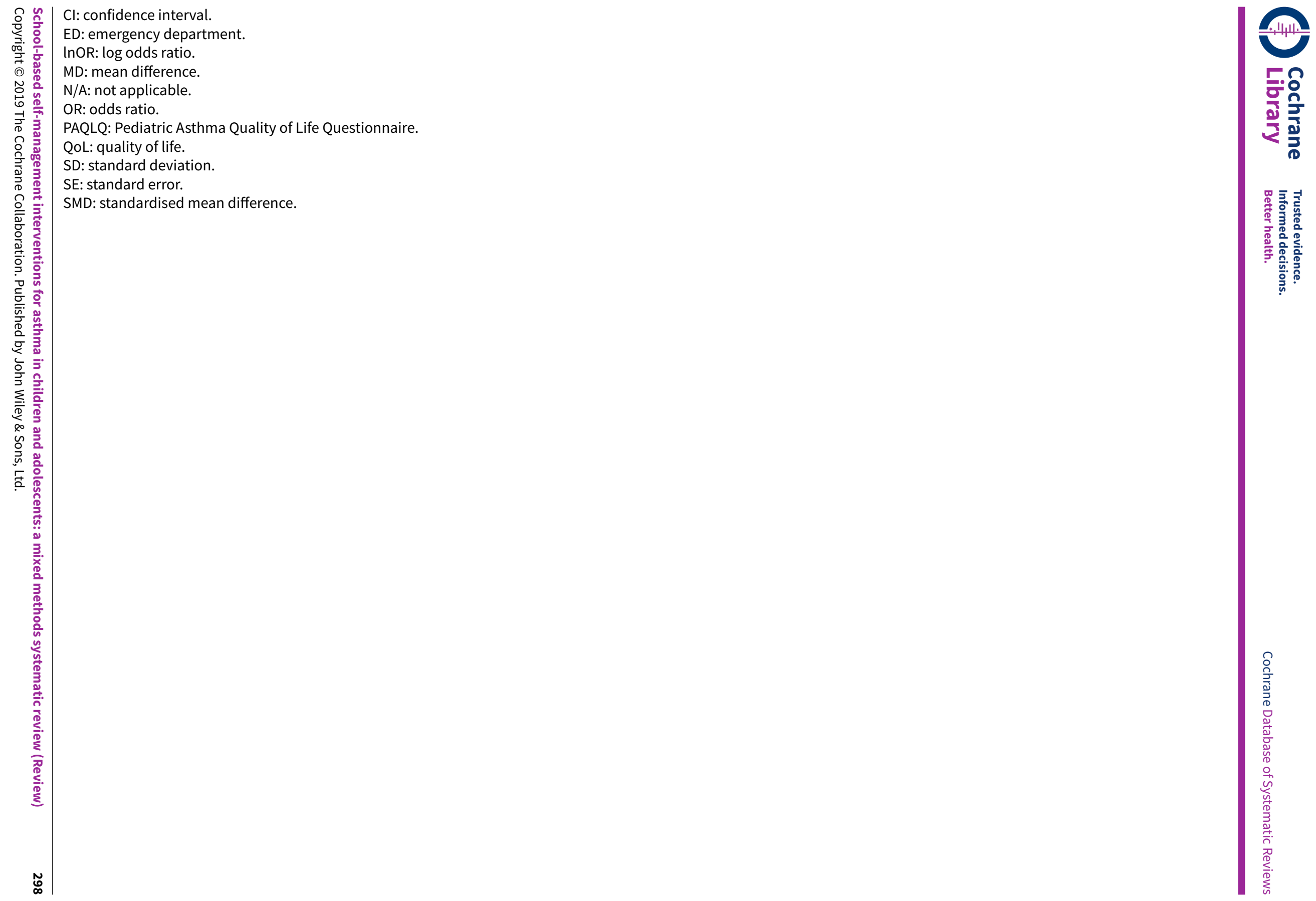
Table 14. Summary of interventions, conditions entered, and model results

$\begin{array}{ll}\text { Domain (model) Conditions entered } & \text { Sufficient configura- } \\ & \text { tions identified that } \\ \text { trigger successful im- } & \text { plementation }\end{array}$

1. Setting and participant features

School health centre; high school; parents direct intervention recipients; teachers direct intervention recipients; school nurses/others direct intervention recipients
Yes Additional marketing materials; provision of incentives; provision of catch-up No

\section{sessions; provision of reminders \\ 2. Recruitment and re- tention processes}

\section{Curriculum, peda-} gogy, and intervention emphasis

\section{Modifiable interven- tion processes}

Focus on establishing alliances with care providers; focus on asthma symptom recognition and management; tailored content; emphasis on personal responsibility; interactive pedagogical style; diverse pedagogical style plementation

\begin{tabular}{lll}
$\begin{array}{l}\text { 5. Stakeholder engage- } \\
\text { ment }\end{array}$ & $\begin{array}{l}\text { School asthma policy; child satisfaction; teachers engaged/relationships de- } \\
\text { veloped; parents engaged/relationships developed; school nurses engaged/re- } \\
\text { lationships developed }\end{array}$ & Yes \\
\hline 6. Consolidated model & $\begin{array}{l}\text { Theory driven; run in students' free time; child satisfaction; parents en- } \\
\text { gaged/relationships developed; high school }\end{array}$ & Yes
\end{tabular}

Theory driven; run in class time; run in students' free time; school nurse key Yes role in delivery or teaching; personalised or individual 1-to-1 instruction gaged/relationships developed; high school

Table 15. Data table for QCA model 6 - consolidated model

\begin{tabular}{|c|c|c|c|c|c|c|}
\hline & $\begin{array}{l}\text { Successful in- } \\
\text { tervention }\end{array}$ & $\begin{array}{l}\text { High } \\
\text { school }\end{array}$ & $\begin{array}{l}\text { Child satis- } \\
\text { faction }\end{array}$ & $\begin{array}{l}\text { Theory dri- } \\
\text { ven }\end{array}$ & $\begin{array}{l}\text { Intervention } \\
\text { takes place } \\
\text { during stu- } \\
\text { dents' own } \\
\text { free time }\end{array}$ & $\begin{array}{l}\text { Good re- } \\
\text { lation- } \\
\text { ships/en- } \\
\text { gagement } \\
\text { with par- } \\
\text { ents }\end{array}$ \\
\hline Joseph 2010 & 0.52 & 1 & 0 & 1 & 0.33 & 0 \\
\hline Kouba 2012 & 0.33 & 1 & 0 & 1 & 1 & 0 \\
\hline Dore-Stites 2007 & 0.67 & 0 & 1 & 1 & 0.33 & 0.75 \\
\hline Joseph 2013 & 1.00 & 1 & 0 & 1 & 0.75 & 1 \\
\hline Mujuru 2011 & 0.67 & 0 & 0 & 0 & 0 & 0.25 \\
\hline Henry 2004 & 0.83 & 1 & 0 & 0 & 0 & 0 \\
\hline Pike 2011 & 0.67 & 0 & 0 & 0 & 0 & 0 \\
\hline Spencer 2000 & 0.33 & 0 & 0 & 0 & 0.33 & 1 \\
\hline Engelke 2013 & 0.50 & 0.5 & 0 & 0 & 0.33 & 1 \\
\hline Splett 2006 & 0.50 & 0.5 & 0 & 0 & 0.33 & 0 \\
\hline
\end{tabular}

School-based self-management interventions for asthma in children and adolescents: a mixed methods systematic review (Review) 
Table 15. Data table for QCA model 6 - consolidated model (Continued)

\begin{tabular}{|c|c|c|c|c|c|c|}
\hline Kintner 2012 & 0.83 & 1 & 1 & 1 & 1 & 0.25 \\
\hline Berg 2004 & 0.83 & 1 & 1 & 1 & 0.33 & 0 \\
\hline Howell 2005 & 0.33 & 0 & 0.633333 & 1 & 0.33 & 0.75 \\
\hline Gerald 2006 & 0.33 & 0 & 0 & 0 & 0.33 & 0 \\
\hline Langenfeld 2010 & 0.33 & 0 & 0 & 0 & 0.33 & 0 \\
\hline Al-Sheyab 2012 & 0.83 & 1 & 0.633333 & 1 & 0.33 & 0 \\
\hline Levy 2006 & 0.52 & 0 & 0 & 0 & 0.33 & 0 \\
\hline Terpstra 2012 & 1.00 & 0.66 & 0 & 1 & 1 & 0.25 \\
\hline Horner 2015 & 0.67 & 0 & 0 & 1 & 1 & 0 \\
\hline Bruzzese 2008 & 0.94 & 0.66 & 1 & 1 & 0.33 & 1 \\
\hline Lee 2011 & 0.50 & 0 & 0 & 1 & 0 & 0 \\
\hline Bruzzese 2004 & 0.33 & 1 & 0.633333 & 1 & 0.75 & 0 \\
\hline Cicutto 2013 & 0.67 & 0 & 0 & 1 & 1 & 0 \\
\hline Brasler 2006 & 0.00 & 0.66 & 0.633333 & 0 & 0.75 & 0 \\
\hline Crane 2014 & 0.50 & 0 & 0 & 1 & 1 & 0 \\
\hline Bruzzese 2011 & 0.88 & 1 & 0 & 1 & 0.33 & 0 \\
\hline Magzamen 2008 & 0.19 & 0.75 & 0 & 0 & 1 & 0 \\
\hline
\end{tabular}

QCA: qualitative comparative analysis. 


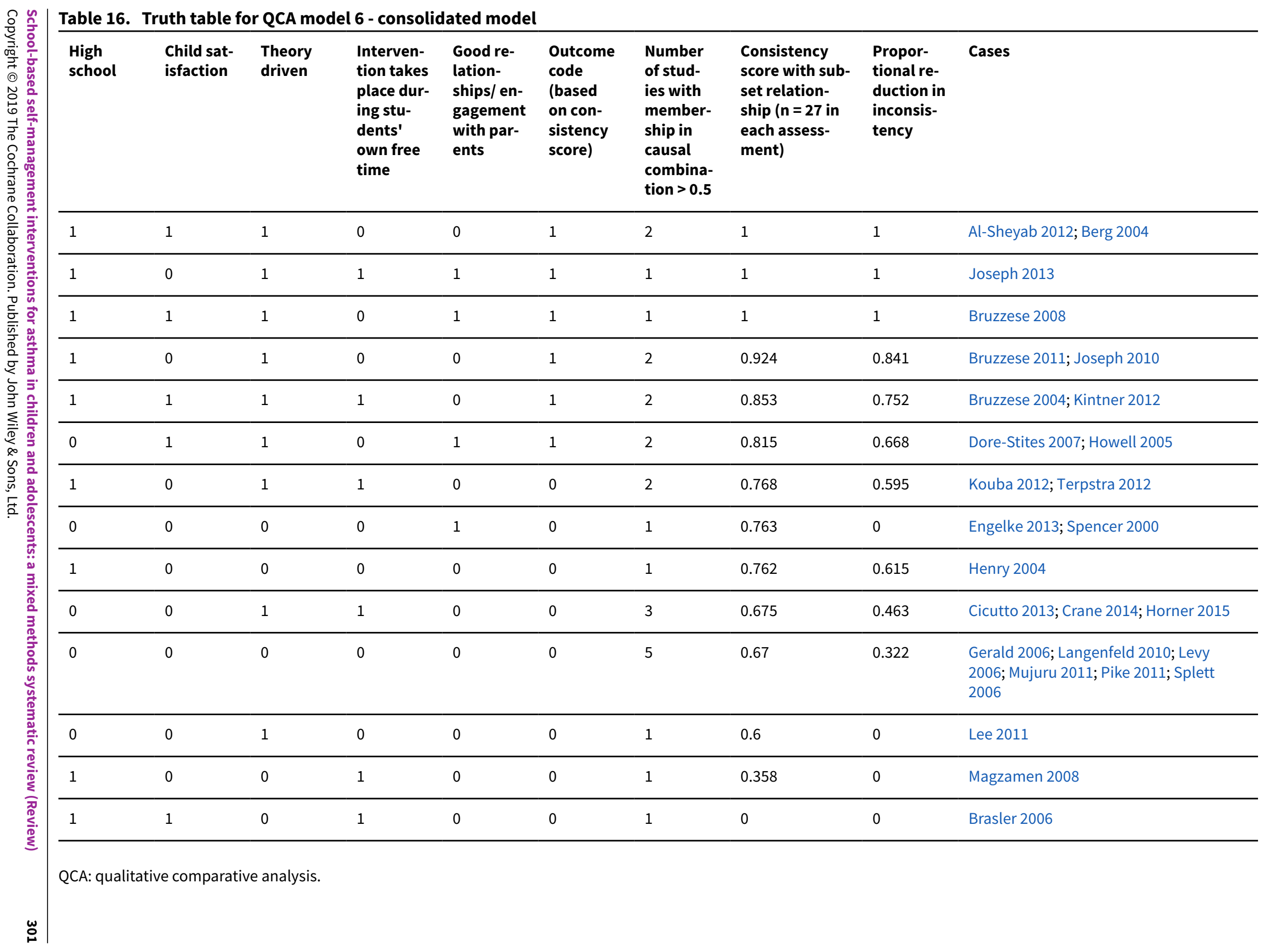


Table 17. Complex solution for QCA model 6 - consolidated model

\begin{tabular}{|c|c|c|c|c|c|c|}
\hline & & $\begin{array}{l}\text { Consisten- } \\
\text { cy score with } \\
\text { subset rela- } \\
\text { tionship (n = } \\
27 \text { in each as- } \\
\text { sessment) }\end{array}$ & $\begin{array}{l}\text { Propor- } \\
\text { tional re- } \\
\text { duction in } \\
\text { inconsis- } \\
\text { tency }\end{array}$ & $\begin{array}{l}\text { Raw cover- } \\
\text { age }\end{array}$ & $\begin{array}{l}\text { Unique } \\
\text { coverage }\end{array}$ & Cases \\
\hline 1 & $\begin{array}{l}\text { CHILDSAT*THEORY- } \\
\text { DRIVENruninstudent- } \\
\text { time*GOODRELPAR }\end{array}$ & 0.846 & 0.756 & 0.106 & 0.106 & $\begin{array}{l}\text { Bruzzese 2008; Dore- } \\
\text { Stites 2007; Howell } \\
2005\end{array}$ \\
\hline 2 & $\begin{array}{l}\text { HIGHS- } \\
\text { CHOOL }^{\star} \text { CHILDSAT }^{\star} \text { THEORY- } \\
\text { DRIVEN }^{\star} \text { goodrelpar }\end{array}$ & 0.845 & 0.786 & 0.162 & 0.063 & $\begin{array}{l}\text { Al-Sheyab 2012; Berg } \\
\text { 2004; Bruzzese 2004; } \\
\text { Kintner } 2012\end{array}$ \\
\hline 3 & $\begin{array}{l}\text { HIGHSCHOOL *THEORY- } \\
\text { DRIVENruninstudent- } \\
\text { time*goodrelpar }\end{array}$ & 0.949 & 0.914 & 0.177 & 0.078 & $\begin{array}{l}\text { Al-Sheyab 2012; Berg } \\
\text { 2004; Bruzzese 2011; } \\
\text { Joseph } 2010\end{array}$ \\
\hline \multirow[t]{2}{*}{4} & $\begin{array}{l}\text { HIGHSCHOOL* }{ }^{*} \text { ildsat*THE- } \\
\text { ORYDRIVEN*RUNINSTU- } \\
\text { DENTTIME*GOODRELPAR }\end{array}$ & 1 & 1 & 0.064 & 0.064 & Joseph 2013 \\
\hline & M1 & 0.875 & 0.823 & 0.41 & & \\
\hline
\end{tabular}

QCA: qualitative comparative analysis.

[Notation: Upper case $=$ condition is present; Lower case $=$ condition is absent; ${ }^{*}=$ logical and; + logical or; Key: HIGHSCHOOL $=$ High School (lower case not in high school); THEORYDRIVEN = Authors explicitly named theory or presented conceptual model for intervention; RUNINSTUDENTTIME = Substantial component run in students' own time (e.g. lunchtime); GOODRELPAR = Good level of reported in engagement and/or developing relationships with parents; CHILDSAT = Children reported as satisfied; SUCCESSFULIMPLEMENTATION = Implementation of intervention successful]

Table 18. Intermediate solution for QCA model 6 - consolidated model

\begin{tabular}{|c|c|c|c|c|c|c|}
\hline & & $\begin{array}{l}\text { Consisten- } \\
\text { cy score with } \\
\text { subset rela- } \\
\text { tionship (n= } \\
27 \text { in each as- } \\
\text { sessment) }\end{array}$ & $\begin{array}{l}\text { Propor- } \\
\text { tional re- } \\
\text { duction in } \\
\text { inconsis- } \\
\text { tency }\end{array}$ & $\begin{array}{l}\text { Raw cover- } \\
\text { age }\end{array}$ & $\begin{array}{l}\text { Unique } \\
\text { coverage }\end{array}$ & Cases \\
\hline 1 & $\begin{array}{l}\text { HIGHS- } \\
\mathrm{CHOOL}^{\star} \mathrm{CHILDSAT}{ }^{\star} \mathrm{THE}- \\
\text { ORYDRIVEN }\end{array}$ & 0.839 & 0.791 & 0.21 & 0.053 & $\begin{array}{l}\text { Al-Sheyab 2012; Berg 2004; } \\
\text { Bruzzese 2004; Bruzzese } \\
\text { 2008; Kintner } 2012\end{array}$ \\
\hline 2 & $\begin{array}{l}\text { HIGHSCHOOL*THEO- } \\
\text { RYDRIVENGOODREL- } \\
\text { PAR }\end{array}$ & 1 & 1 & 0.138 & 0.064 & Bruzzese 2008; Joseph 2013 \\
\hline 3 & $\begin{array}{l}\text { HIGHSCHOOL }{ }^{\star} \text { THEO- } \\
\text { RYDRIVENruninstu- } \\
\text { denttime }\end{array}$ & 0.961 & 0.942 & 0.235 & 0.078 & $\begin{array}{l}\text { Al-Sheyab 2012; Berg 2004; } \\
\text { Bruzzese 2008; Bruzzese } \\
\text { 2011; Joseph } 2013\end{array}$ \\
\hline 4 & $\begin{array}{l}\text { CHILDSAT*THEORY- } \\
\text { DRIVENruninstudent- } \\
\text { time*GOODRELGPAR }\end{array}$ & 0.846 & 0.756 & 0.106 & 0.064 & $\begin{array}{l}\text { Bruzzese 2008; Dore-Stites } \\
\text { 2007; Howell } 2005\end{array}$ \\
\hline
\end{tabular}


Table 18. Intermediate solution for QCA model 6 - consolidated model (Continued)
M1
0.862
0.81
0.432

QCA: qualitative comparative analysis.

[Notation: Upper case = condition is present; Lower case = condition is absent; ${ }^{*}=$ logical and; + logical or; Key: HIGHSCHOOL = High School (lower case not in high school); THEORYDRIVEN = Authors explicitly named theory or presented conceptual model for intervention; RUNINSTUDENTTIME = Substantial component run in students' own time (e.g. lunchtime); GOODRELPAR = Good level of reported in engagement and/or developing relationships with parents; CHILDSATB = Children reported as satisfied; SUCCESSFULIMPLEMENTATION = Implementation of intervention successful]

Table 19. Summary of results from consolidated model

\begin{tabular}{|c|c|c|c|c|c|c|}
\hline Consolidated model & Theory driven & $\begin{array}{l}\text { Run in chil- } \\
\text { dren's free } \\
\text { time }\end{array}$ & $\begin{array}{l}\text { Child satis- } \\
\text { faction }\end{array}$ & $\begin{array}{l}\text { Parents engaged/re- } \\
\text { lationships devel- } \\
\text { oped }\end{array}$ & $\begin{array}{l}\text { High } \\
\text { school }\end{array}$ & $\begin{array}{l}\text { Successful } \\
\text { interven- } \\
\text { tion }\end{array}$ \\
\hline Pathway 1 & Present & - & Present & - & Present & Yes \\
\hline Pathway 2 & Present & - & - & Present & Present & Yes \\
\hline Pathway 3 & Present & Absent & - & - & Present & Yes \\
\hline Pathway 4 & Present & Absent & Present & Present & - & Yes \\
\hline
\end{tabular}

Absent: absence of condition is essential in triggering success.

Present: presence of condition is essential in triggering success.

- (symbol): presence or absence of condition is not essential in triggering success. 


\begin{tabular}{|c|c|c|c|c|c|c|c|}
\hline $\begin{array}{l}\text { Model } 1 \text {. Setting and partici- } \\
\text { pant features }\end{array}$ & $\begin{array}{l}\text { School health } \\
\text { centre }\end{array}$ & High school & $\begin{array}{l}\text { Parents direct } \\
\text { intervention re- } \\
\text { cipients }\end{array}$ & $\begin{array}{l}\text { Teachers direct in- } \\
\text { tervention recipi- } \\
\text { ents }\end{array}$ & $\begin{array}{l}\text { School nurs- } \\
\text { es/others direct } \\
\text { intervention re- } \\
\text { cipients }\end{array}$ & & $\begin{array}{l}\text { Successful in- } \\
\text { tervention }\end{array}$ \\
\hline Pathway 1 & Present & Present & Present & Absent & - & & Yes \\
\hline Pathway 2 & Absent & Present & Absent & - & - & & Yes \\
\hline Pathway 3 & Absent & - & Absent & Absent & Absent & & Yes \\
\hline Pathway 4 & Present & Present & Present & - & Present & & Yes \\
\hline $\begin{array}{l}\text { Model } 2 . \text { Recruitment and } \\
\text { retention processes }\end{array}$ & $\begin{array}{l}\text { Additional mar- } \\
\text { keting materials }\end{array}$ & $\begin{array}{l}\text { Provision of } \\
\text { incentives }\end{array}$ & $\begin{array}{l}\text { Provision of } \\
\text { catch-up ses- } \\
\text { sions }\end{array}$ & $\begin{array}{l}\text { Provision of re- } \\
\text { minders }\end{array}$ & & & $\begin{array}{l}\text { Successful in- } \\
\text { tervention }\end{array}$ \\
\hline & - & - & - & - & - & & $\begin{array}{l}\text { No solution } \\
\text { found }\end{array}$ \\
\hline $\begin{array}{l}\text { Model 3. Curriculum, peda- } \\
\text { gogy, and intervention em- } \\
\text { phasis }\end{array}$ & $\begin{array}{l}\text { Focus on es- } \\
\text { tablishing al- } \\
\text { liances with care } \\
\text { providers }\end{array}$ & $\begin{array}{l}\text { Focus on } \\
\text { asthma } \\
\text { symptom } \\
\text { recognition } \\
\text { and manage- } \\
\text { ment }\end{array}$ & Tailored content & $\begin{array}{l}\text { Emphasis on per- } \\
\text { sonal responsibility }\end{array}$ & $\begin{array}{l}\text { Interactive ped- } \\
\text { agogical style }\end{array}$ & $\begin{array}{l}\text { Diverse ped- } \\
\text { agogical } \\
\text { style }\end{array}$ & $\begin{array}{l}\text { Successful in- } \\
\text { tervention }\end{array}$ \\
\hline & - & - & - & - & - & & $\begin{array}{l}\text { No solution } \\
\text { found }\end{array}$ \\
\hline $\begin{array}{l}\text { Model 4. Modifiable inter- } \\
\text { vention processes }\end{array}$ & Theory driven & $\begin{array}{l}\text { Run in class } \\
\text { time }\end{array}$ & $\begin{array}{l}\text { Run in students' } \\
\text { free time }\end{array}$ & $\begin{array}{l}\text { School nurse key } \\
\text { role in delivery or } \\
\text { teaching }\end{array}$ & $\begin{array}{l}\text { Personalised or } \\
\text { individual 1-to-1 } \\
\text { instruction }\end{array}$ & & $\begin{array}{l}\text { Successful in- } \\
\text { tervention }\end{array}$ \\
\hline Pathway 1 & Present & - & Absent & Absent & - & & Yes \\
\hline Pathway 2 & Present & - & - & Present & Absent & & Yes \\
\hline $\begin{array}{l}\text { Model 5. Stakeholder en- } \\
\text { gagement }\end{array}$ & $\begin{array}{l}\text { School asthma } \\
\text { policy }\end{array}$ & $\begin{array}{l}\text { Child satis- } \\
\text { faction }\end{array}$ & $\begin{array}{l}\text { Teachers en- } \\
\text { gaged/ relation- } \\
\text { ships developed }\end{array}$ & $\begin{array}{l}\text { Parents engaged/ } \\
\text { relationships devel- } \\
\text { oped }\end{array}$ & $\begin{array}{l}\text { School nurses } \\
\text { engaged/ rela- } \\
\text { tionships devel- } \\
\text { oped }\end{array}$ & & $\begin{array}{l}\text { Successful in- } \\
\text { tervention }\end{array}$ \\
\hline
\end{tabular}




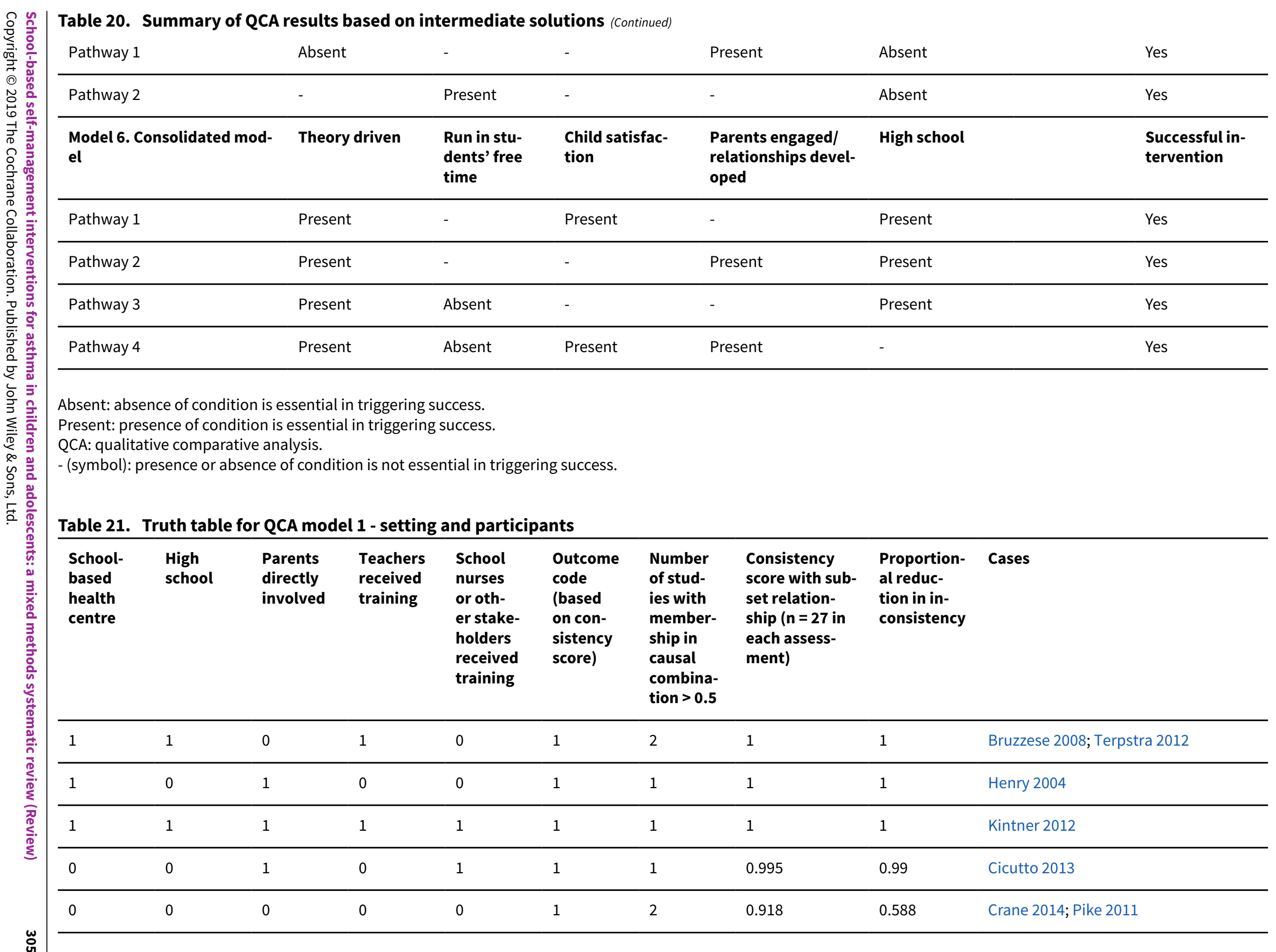




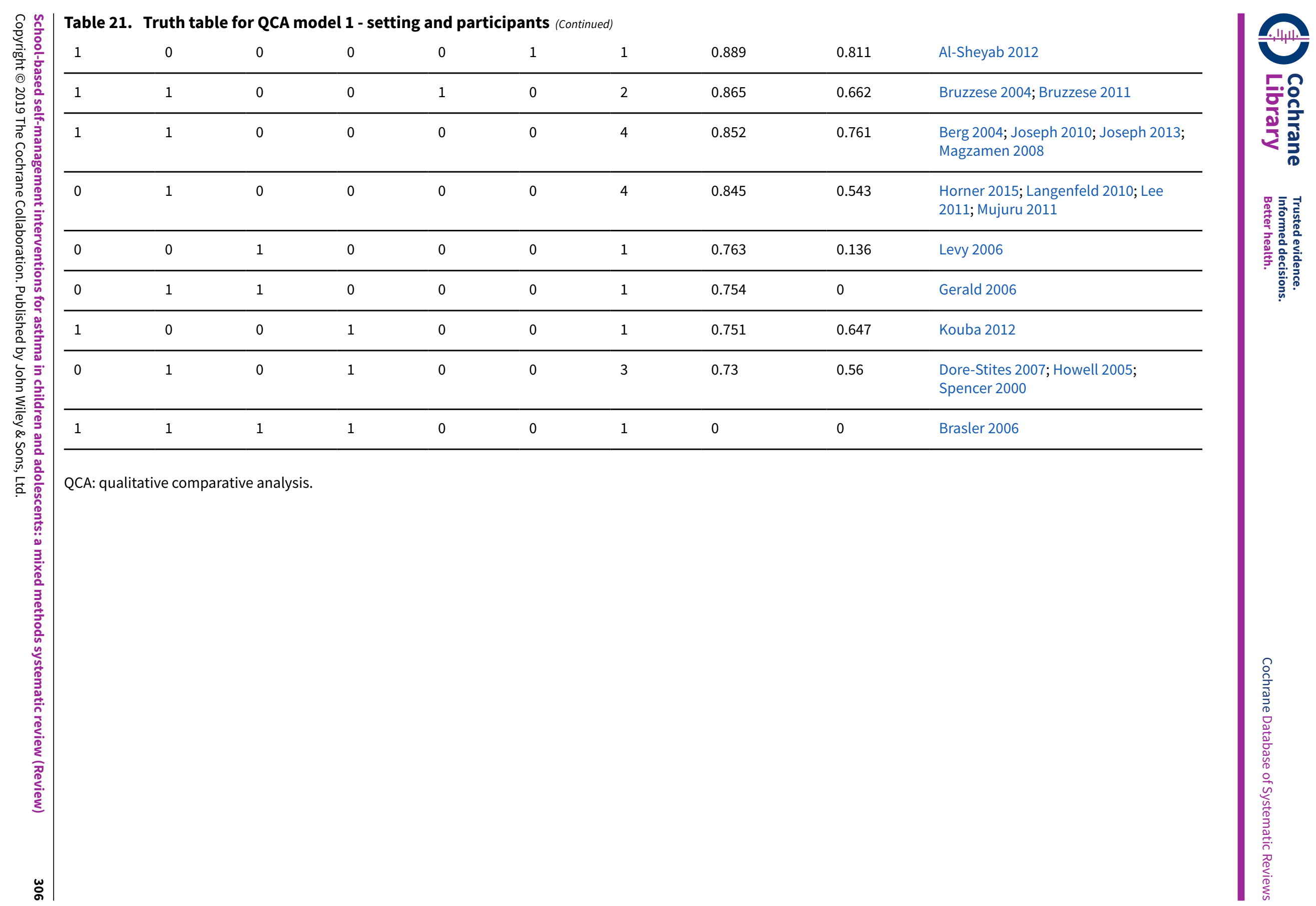


Table 22. Complex solution for QCA model 1 - setting and participants

\begin{tabular}{|c|c|c|c|c|c|c|}
\hline & & $\begin{array}{l}\text { Consistency } \\
\text { score with } \\
\text { subset rela- } \\
\text { tionship ( } \mathrm{n} \\
=27 \text { in each } \\
\text { assessment) }\end{array}$ & $\begin{array}{l}\text { Propor- } \\
\text { tional re- } \\
\text { duction in } \\
\text { inconsis- } \\
\text { tency }\end{array}$ & $\begin{array}{l}\text { Raw cover- } \\
\text { age }\end{array}$ & $\begin{array}{l}\text { Unique } \\
\text { coverage }\end{array}$ & Cases \\
\hline 1 & $\begin{array}{l}\text { HIGHSCHOOL*schoolbased- } \\
\text { health" parentdirect*anyothdir }\end{array}$ & 0.913 & 0.861 & 0.176 & 0.043 & $\begin{array}{l}\text { Al-Sheyab 2012; } \\
\text { Henry } 2004\end{array}$ \\
\hline 2 & $\begin{array}{l}\text { schoolbasedhealth*teacherdirec- } \\
\mathrm{t}^{\star} \text { parentdirect }{ }^{\star} \text { anyothdir }\end{array}$ & 0.913 & 0.769 & 0.294 & 0.16 & $\begin{array}{l}\text { Al-Sheyab 2012; } \\
\text { Crane 2014; } \\
\text { Pike } 2011\end{array}$ \\
\hline 3 & $\begin{array}{l}\text { highschool*schoolbased- } \\
\text { health*TEACHERDIRECT* parentdirec- } \\
\text { t*ANYOTHDIR }^{\star}\end{array}$ & 0.995 & 0.99 & 0.042 & 0.042 & Cicutto 2013 \\
\hline 4 & $\begin{array}{l}\text { HIGHSCHOOL }{ }^{\star} \text { SCHOOLBASED- } \\
\text { HEALTH*teacherdirect*PARENT- } \\
\text { DIRECT*anyothdir }\end{array}$ & 1 & 1 & 0.105 & 0.105 & $\begin{array}{l}\text { Bruzzese 2008; } \\
\text { Terpstra } 2012\end{array}$ \\
\hline \multirow[t]{2}{*}{5} & $\begin{array}{l}\text { HIGHSCHOOL }{ }^{*} \text { SCHOOLBASED- } \\
\text { HEALTH*TEACHERDIRECT*PARENT- } \\
\text { DIRECT*ANYOTHDIR }\end{array}$ & 1 & 1 & 0.074 & 0.074 & Kintner 2012 \\
\hline & M1 & 0.952 & 0.901 & 0.558 & & \\
\hline
\end{tabular}

QCA: qualitative comparative analysis.

[Notation: Upper case $=$ condition is present; Lower case $=$ condition is absent; ${ }^{*}=$ logical and; + logical or; Key: $\mathrm{HIGHSCHOOL}=\mathrm{High}$ School (lower case not in high school); SCHOOLBASEDHEALTH = School Based Health Centre; TEACHERDIRECT = Teachers received directly received component of intervention; PARENTDIRECT = Parents directly received component of intervention; ANYOTHDIR = School nurses or other stakeholders (apart from children) directly received component of intervention; SUCCESSFULIMPLEMENTATION = Implementation of intervention successful]

Table 23. Intermediate solution for QCA model 1 - setting and participants

\begin{tabular}{|c|c|c|c|c|c|c|}
\hline & & $\begin{array}{l}\text { Consisten- } \\
\text { cy score with } \\
\text { subset rela- } \\
\text { tionship ( } n= \\
27 \text { in each as- } \\
\text { sessment) }\end{array}$ & $\begin{array}{l}\text { Propor- } \\
\text { tional re- } \\
\text { duction in } \\
\text { inconsis- } \\
\text { tency }\end{array}$ & $\begin{array}{l}\text { Raw cover- } \\
\text { age }\end{array}$ & $\begin{array}{l}\text { Unique } \\
\text { coverage }\end{array}$ & Cases \\
\hline 1 & $\begin{array}{l}\text { HIGHSCHOOL }{ }^{*} \text { schoolbased- } \\
\text { health*parentdirect }\end{array}$ & 0.904 & 0.838 & 0.226 & 0.093 & $\begin{array}{l}\text { Al-Sheyab 2012; } \\
\text { Henry } 2004\end{array}$ \\
\hline 2 & $\begin{array}{l}\text { HIGHSCHOOL }{ }^{*} \text { SCHOOLBASED- } \\
\text { HEALTH*teacherdirect*PARENT- } \\
\text { DIRECT }\end{array}$ & 1 & 1 & 0.105 & 0.105 & $\begin{array}{l}\text { Bruzzese 2008; } \\
\text { Terpstra } 2012\end{array}$ \\
\hline 3 & $\begin{array}{l}\text { schoolbasedhealth*teacherdirec- } \\
\mathrm{t}^{\star} \text { parentdirect*anyothdir }\end{array}$ & 0.913 & 0.769 & 0.294 & 0.16 & $\begin{array}{l}\text { Crane 2014; } \\
\text { Pike } 2011\end{array}$ \\
\hline
\end{tabular}


Table 23. Intermediate solution for QCA model 1 - setting and participants (Continued)

\begin{tabular}{lllllll}
4 & $\begin{array}{l}\text { highschool*TEACHERDIREC- } \\
\text { T*ANYOTHDIR }\end{array}$ & 0.778 & 0.5 & 0.074 & 0.042 & Cicutto 2013 \\
\hline 5 & $\begin{array}{l}\text { HIGHSCHOOL }{ }^{*} \text { SCHOOLBASED- } \\
\text { HEALTH*PARENTDIREC- } \\
\text { T*ANYOTHDIR }\end{array}$ & 1 & 1 & 0.074 & 0.042 & Kintner 2012 \\
& Solution & 0.915 & 0.831 & 0.608 & \\
\hline
\end{tabular}

QCA: qualitative comparative analysis.

\section{Overall solution}

HIGHSCHOOL*schoolbasedhealth* parentdirect +

schoolbasedhealth*teacherdirect ${ }^{\star}$ parentdirect*anyothdir +

HIGHSCHOOL*SCHOOLBASEDHEALTH*teacherdirect*PARENTDIRECT +

(highschool ${ }^{\star}$ TEACHERDIRECT*ANYOTHDIR + HIGHSCHOOL*SCHOOLBASEDHEALTH*PARENTDIRECT*ANYOTHDIR)

$=>$ SUCCESSFULIMPLEMENTATION

[Notation: Upper case $=$ condition is present; Lower case $=$ condition is absent; ${ }^{*}=$ logical and; + logical or; Key: $\mathrm{HIGHSCHOOL}=\mathrm{High}$ School (lower case not in high school); SCHOOLBASEDHEALTH = School Based Health Centre; TEACHERDIRECT = Teachers received directly received component of intervention; PARENTDIRECT = Parents directly received component of intervention; ANYOTHDIR = School nurses or other stakeholders (apart from children) directly received component of intervention; SUCCESSFULIMPLEMENTATION = Implementation of intervention successful] 


\begin{tabular}{|c|c|c|c|c|c|c|c|}
\hline & $\begin{array}{l}\text { Successful inter- } \\
\text { vention }\end{array}$ & $\begin{array}{l}\text { Curriculum } \\
\text { reflected } \\
\text { forming al- } \\
\text { liances and } \\
\text { monitoring } \\
\text { symptoms }\end{array}$ & $\begin{array}{l}\text { Curricu- } \\
\text { lum reflect- } \\
\text { ed learning } \\
\text { about asth- } \\
\text { ma triggers } \\
\text { and moni- } \\
\text { toring symp- } \\
\text { toms }\end{array}$ & $\begin{array}{l}\text { Emphasised } \\
\text { the interven- } \\
\text { tion as being } \\
\text { tailored or } \\
\text { personalised }\end{array}$ & $\begin{array}{l}\text { Emphasised } \\
\text { developing } \\
\text { personal re- } \\
\text { sponsibility } \\
\text { as aim of the } \\
\text { intervention }\end{array}$ & $\begin{array}{l}\text { Pedagogical } \\
\text { style focused } \\
\text { on interac- } \\
\text { tive methods }\end{array}$ & $\begin{array}{l}\text { Diverse ped- } \\
\text { agogical } \\
\text { style em- } \\
\text { ployed }\end{array}$ \\
\hline Joseph 2010 & 0.52 & 0 & 1 & 1 & 0 & 0 & 0 \\
\hline Kouba 2012 & 0.33 & 0 & 0 & 0 & 1 & 0 & 0 \\
\hline Dore-Stites 2007 & 0.67 & 1 & 0 & 0 & 1 & 0 & 0 \\
\hline Joseph 2013 & 1.00 & 0 & 1 & 1 & 0 & 0 & 1 \\
\hline Mujuru 2011 & 0.67 & 0 & 1 & 0 & 0 & 0 & 0 \\
\hline Henry 2004 & 0.83 & 0 & 0 & 0 & 0 & 0 & 0 \\
\hline Pike 2011 & 0.67 & 0 & 1 & 0 & 0 & 0 & 0 \\
\hline Spencer 2000 & 0.33 & 0 & 0 & 0 & 0 & 1 & 0 \\
\hline Engelke 2013 & 0.50 & 0 & 0 & 0 & 0 & 0 & 1 \\
\hline Splett 2006 & 0.50 & 0 & 0 & 0 & 0 & 1 & 0 \\
\hline Kintner 2012 & 0.83 & 0 & 1 & 0 & 0 & 0 & 0 \\
\hline Berg 2004 & 0.83 & 0 & 1 & 1 & 0 & 0 & 0 \\
\hline Howell 2005 & 0.33 & 0 & 1 & 0 & 0 & 0 & 0 \\
\hline Gerald 2006 & 0.33 & 1 & 0 & 0 & 0 & 0 & 0 \\
\hline Cheung 2015 & 0.33 & 0 & 0 & 0 & 1 & 0 & 0 \\
\hline Al-Sheyab 2012 & 0.83 & 0 & 1 & 0 & 1 & 0 & 1 \\
\hline Levy 2006 & 0.52 & 0 & 0 & 0 & 0 & 0 & 1 \\
\hline
\end{tabular}




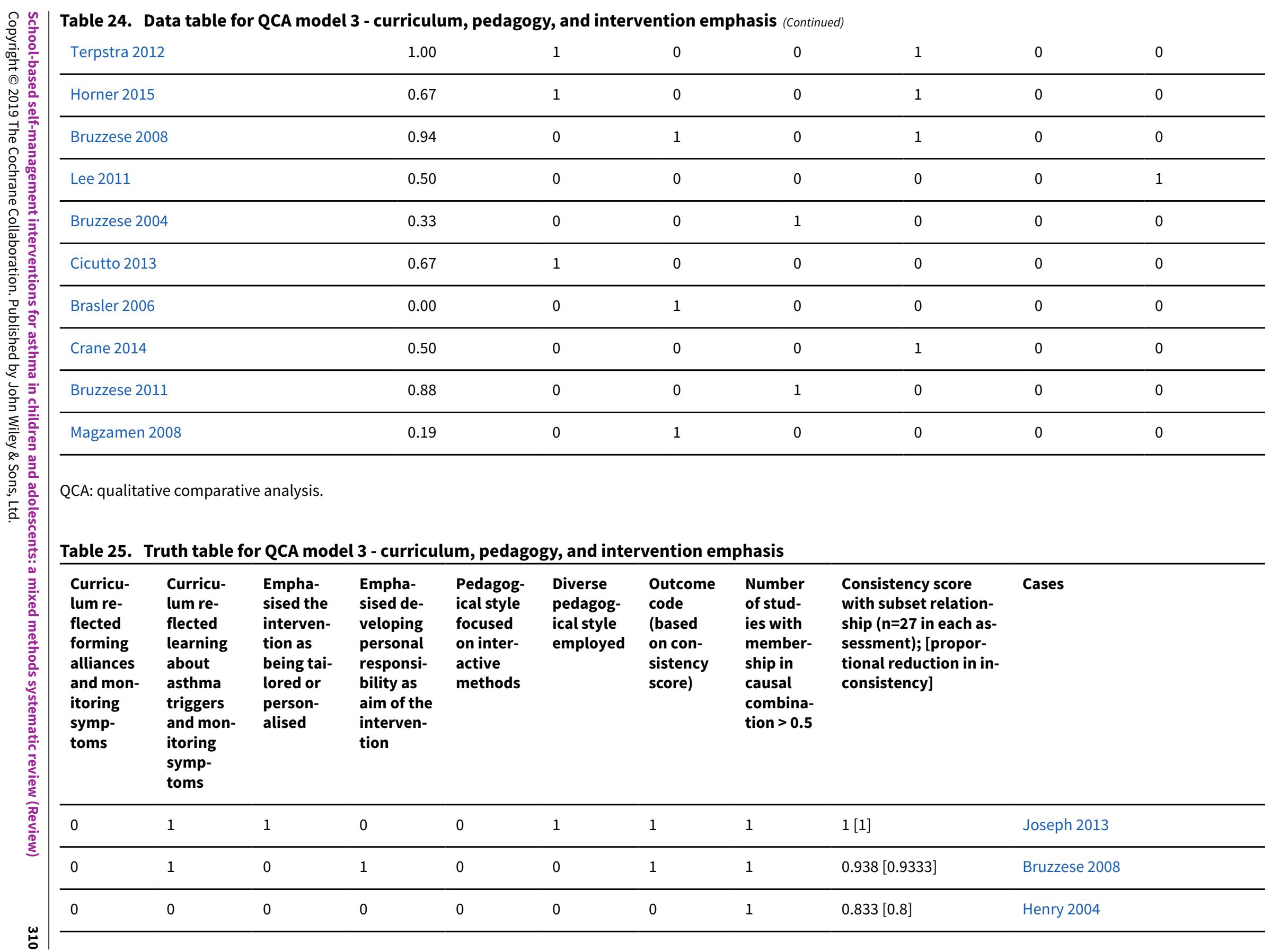




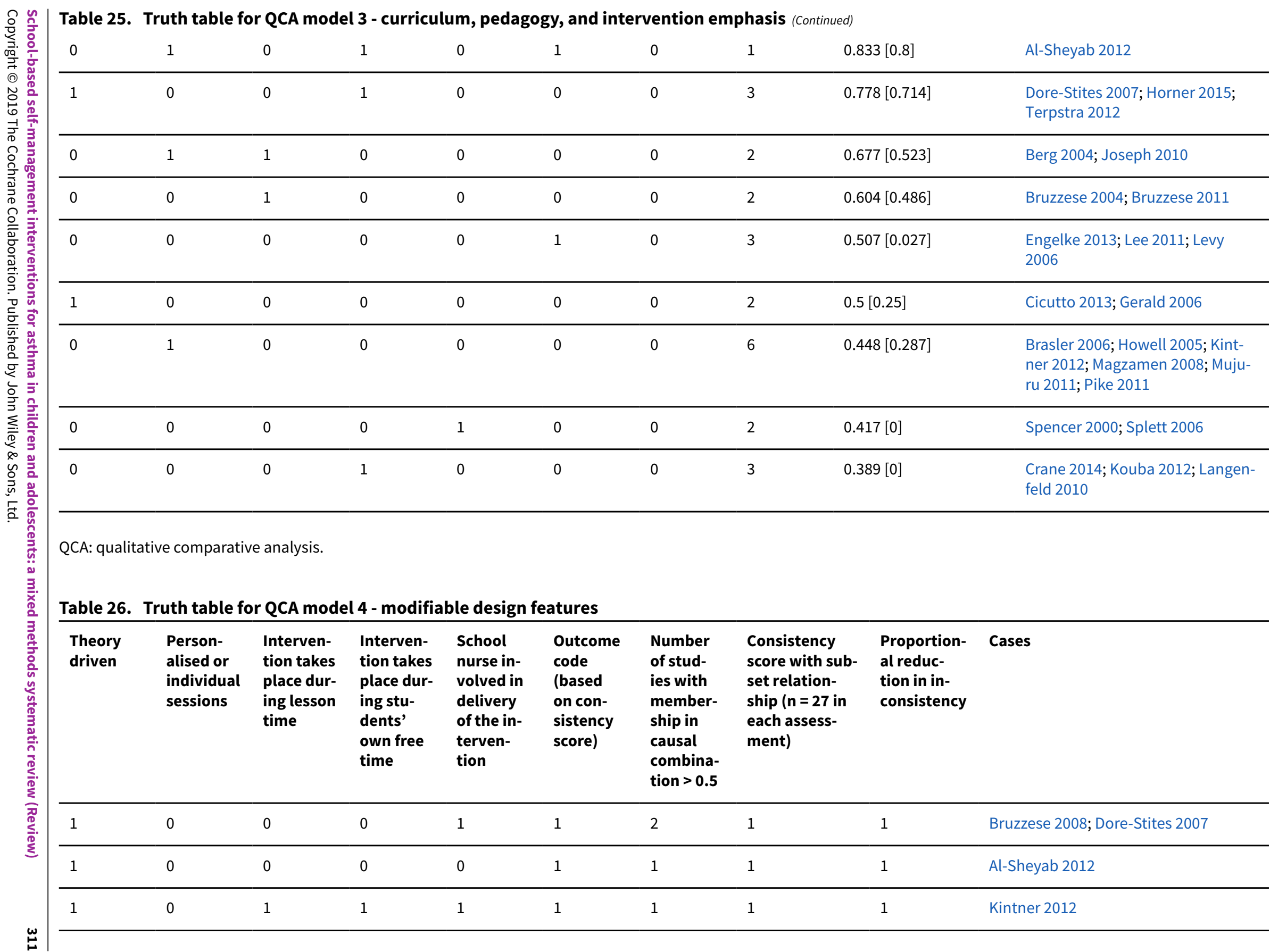




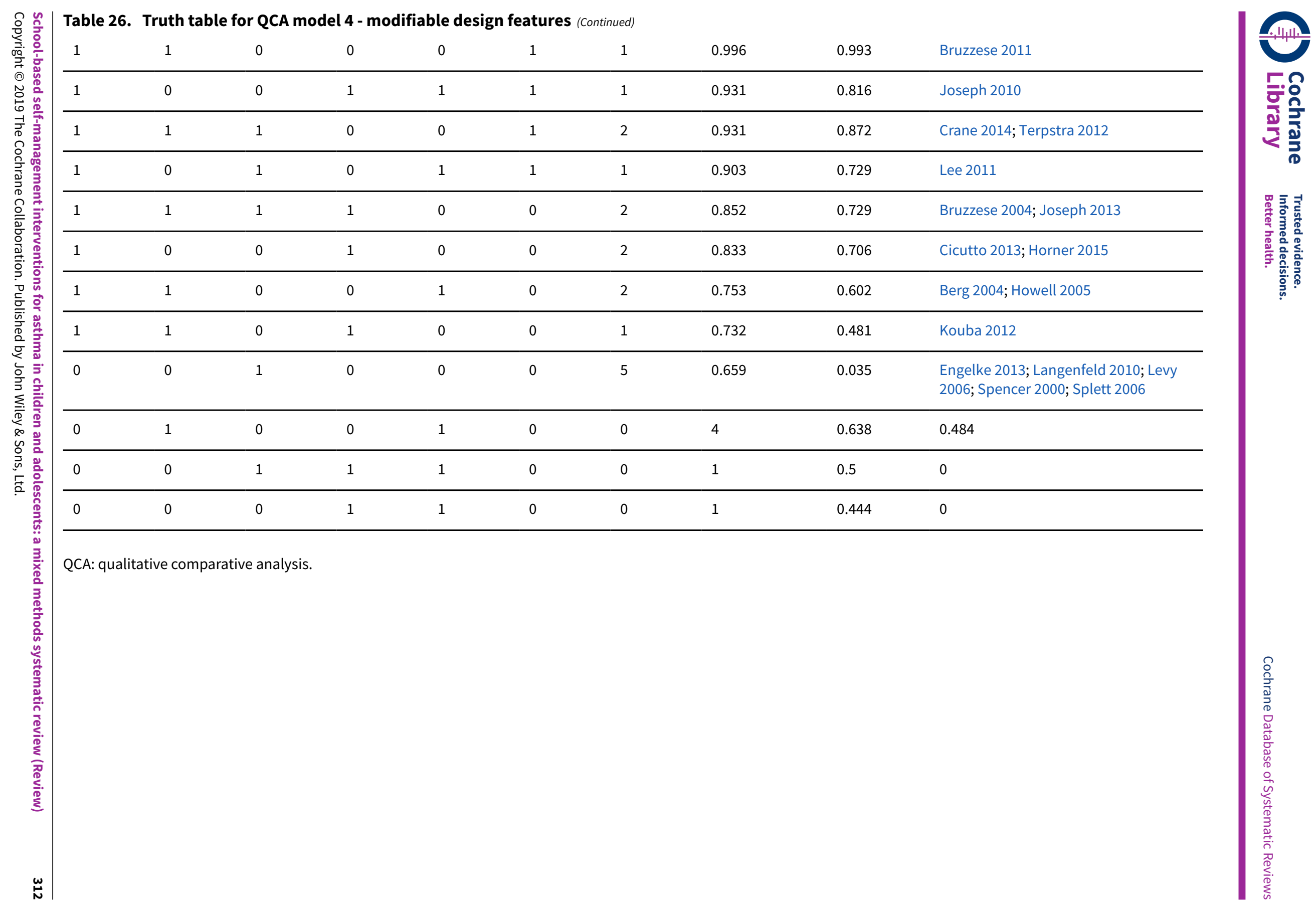


Table 27. Complex solution for QCA model 4 - modifiable design features

\begin{tabular}{|c|c|c|c|c|c|c|}
\hline & & $\begin{array}{l}\text { Consisten- } \\
\text { cy score with } \\
\text { subset rela- } \\
\text { tionship (n = } \\
27 \text { in each as- } \\
\text { sessment) }\end{array}$ & $\begin{array}{l}\text { Propor- } \\
\text { tional re- } \\
\text { duction in } \\
\text { inconsis- } \\
\text { tency }\end{array}$ & $\begin{array}{l}\text { Raw cover- } \\
\text { age }\end{array}$ & $\begin{array}{l}\text { Unique } \\
\text { coverage }\end{array}$ & Cases \\
\hline 1 & $\begin{array}{l}\text { THEORYDRIVEN* }{ }^{*} \text { person- } \\
\text { alorindividual }{ }^{\star} \text { SCHOOL- } \\
\text { NURSEINSTRUCT }\end{array}$ & 0.926 & 0.876 & 0.253 & 0.148 & $\begin{array}{l}\text { Bruzzese 2008; Crane } \\
\text { 2014; Dore-Stites 2007; } \\
\text { Kintner 2012; Lee 2011; } \\
\text { Terpstra 2012 }\end{array}$ \\
\hline 2 & $\begin{array}{l}\text { THEORYDRIVEN*PERSON- } \\
\text { ALORINDIVIDUAL*runinstu- } \\
\text { denttime* }{ }^{\star} \text { schoolnursein- } \\
\text { struct }\end{array}$ & 0.938 & 0.866 & 0.151 & 0.033 & $\begin{array}{l}\text { Bruzzese 2011; Joseph } \\
2013\end{array}$ \\
\hline \multirow[t]{2}{*}{3} & $\begin{array}{l}\text { THEORYDRIVEN* }{ }^{*} \text { person- } \\
\text { alorindividual }{ }^{*} \text { runinlesson- } \\
\mathrm{s}^{\star} \text { runinstudenttime }\end{array}$ & 0.999 & 0.998 & 0.149 & 0.001 & $\begin{array}{l}\text { Al-Sheyab 2012a; } \\
\text { Bruzzese 2008; Dore- } \\
\text { Stites } 2007\end{array}$ \\
\hline & M1 & 0.933 & 0.883 & 0.426 & & \\
\hline
\end{tabular}

QCA: qualitative comparative analysis.

[Notation: Upper case $=$ condition is present; Lower case $=$ condition is absent; ${ }^{*}=$ logical and; + logical or; Key: THEORYDRIVEN $=$ Authors explicitly named theory or presented conceptual model for intervention; SCHOOLNURSEINSTRUCT = Substantial component delivered by schools' nurse; PERSONALORINDIVIDUAL = Substantial components delivered that were individually personalised or delivered to individuals; RUNINSTUDENTTIME = Substantial component run in students' own time (e.g. lunchtime); RUNINLESSONS = Substantial component run during lesson time; SUCCESSFULIMPLEMENTATION = Implementation of intervention successful]

Table 28. Intermediate solution for QCA model 4 - further modifiable intervention design features

\begin{tabular}{|c|c|c|c|c|c|c|}
\hline & & $\begin{array}{l}\text { Consistency } \\
\text { score with sub- } \\
\text { set relationship } \\
\text { ( } n=27 \text { in each } \\
\text { assessment) }\end{array}$ & $\begin{array}{l}\text { Proportion- } \\
\text { al reduction } \\
\text { in inconsis- } \\
\text { tency }\end{array}$ & $\begin{array}{l}\text { Raw cover- } \\
\text { age }\end{array}$ & $\begin{array}{l}\text { Unique } \\
\text { coverage }\end{array}$ & Cases \\
\hline 1 & $\begin{array}{l}\text { THEORYDRIVEN* }{ }^{*} \text { per- } \\
\text { sonalorindivid- } \\
\text { ual*SCHOOL- } \\
\text { NURSEINSTRUCT }\end{array}$ & 0.926 & 0.876 & 0.253 & 0.167 & $\begin{array}{l}\text { Bruzzese 2008; Crane } \\
\text { 2014; Dore-Stites 2007; } \\
\text { Kintner 2012; Lee 2011; } \\
\text { Terpstra } 2012\end{array}$ \\
\hline \multirow[t]{2}{*}{2} & $\begin{array}{l}\text { THEORY- } \\
\text { DRIVENruninstu- } \\
\text { denttime*schoolnur- } \\
\text { seinstruct }\end{array}$ & 0.963 & 0.92 & 0.258 & 0.172 & $\begin{array}{l}\text { Al-Sheyab 2012; Bruzzese } \\
\text { 2011; Joseph } 2010\end{array}$ \\
\hline & M1 & 0.933 & 0.883 & 0.425 & & \\
\hline
\end{tabular}

QCA: qualitative comparative analysis.

[Notation: Upper case $=$ condition is present; Lower case $=$ condition is absent; ${ }^{*}=$ logical and; + logical or; Key: THEORYDRIVEN $=$ Authors explicitly named theory or presented conceptual model for intervention; SCHOOLNURSEINSTRUCT = Substantial component delivered by schools' nurse; PERSONALORINDIVIDUAL = Substantial components delivered that were individually personalised or delivered to individuals; RUNINSTUDENTTIME = Substantial component run in students' own time (e.g. lunchtime); RUNINLESSONS = Substantial component run during lesson time; SUCCESSFULIMPLEMENTATION = Implementation of intervention successful] 


\section{Overall solution}

THEORYDRIVEN*runinstudenttime*schoolnurseinstruct +

THEORYDRIVEN*personalorindividual*SCHOOLNURSEINSTRUCT => PROCOUTSUM 


\begin{tabular}{|c|c|c|c|c|c|c|c|c|c|}
\hline & $\begin{array}{l}\text { School } \\
\text { asthma } \\
\text { policy }\end{array}$ & $\begin{array}{l}\text { Good re- } \\
\text { lation- } \\
\text { ships/ en- } \\
\text { gagement } \\
\text { with par- } \\
\text { ents }\end{array}$ & $\begin{array}{l}\text { Good re- } \\
\text { lation- } \\
\text { ships/ } \\
\text { engage- } \\
\text { ment with } \\
\text { school } \\
\text { nurses }\end{array}$ & $\begin{array}{l}\text { Child sat- } \\
\text { isfaction }\end{array}$ & $\begin{array}{l}\text { Outcome } \\
\text { code } \\
\text { (based } \\
\text { on con- } \\
\text { sistency } \\
\text { score) }\end{array}$ & $\begin{array}{l}\text { Number } \\
\text { of stud- } \\
\text { ies with } \\
\text { member- } \\
\text { ship in } \\
\text { causal } \\
\text { combina- } \\
\text { tion }>0.5\end{array}$ & $\begin{array}{l}\text { Consisten- } \\
\text { cy score with } \\
\text { subset rela- } \\
\text { tionship ( } n= \\
27 \text { in each as- } \\
\text { sessment) }\end{array}$ & $\begin{array}{l}\text { Propor- } \\
\text { tional re- } \\
\text { duction in } \\
\text { inconsis- } \\
\text { tency }\end{array}$ & Cases \\
\hline 1 & 0 & 1 & 0 & 0 & 1 & 1 & 1 & 1 & Joseph 2013 \\
\hline 2 & 0 & 1 & 0 & 1 & 1 & 1 & 0.958 & 0.939 & Bruzzese 2008 \\
\hline 3 & 0 & 0 & 0 & 1 & 1 & 4 & 0.857 & 0.786 & $\begin{array}{l}\text { Al-Sheyab 2012; Berg 2004; Bruzzese 2004; } \\
\text { Kintner } 2012\end{array}$ \\
\hline 4 & 0 & 1 & 1 & 1 & 0 & 2 & 0.723 & 0.465 & Dore-Stites 2007; Howell 2005 \\
\hline 5 & 1 & 0 & 0 & 0 & 0 & 3 & 0.674 & 0.515 & Cicutto 2013; Henry 2004; Levy 2006 \\
\hline 6 & 0 & 0 & 0 & 0 & 0 & 10 & 0.615 & 0.405 & $\begin{array}{l}\text { Bruzzese 2011; Gerald 2006; Horner 2015; } \\
\text { Joseph 2010; Kouba 2012; Lee 2011; } \\
\text { Magzamen 2008; Mujuru 2011; Pike 2011; } \\
\text { Terpstra } 2012\end{array}$ \\
\hline 7 & 0 & 0 & 1 & 0 & 0 & 1 & 0.6 & 0 & Crane 2014 \\
\hline 8 & 1 & 1 & 0 & 0 & 0 & 1 & 0.5 & 0 & Engelke 2013 \\
\hline 9 & 0 & 1 & 1 & 0 & 0 & 1 & 0.488 & 0 & Spencer 2000 \\
\hline 10 & 1 & 0 & 1 & 0 & 0 & 2 & 0.352 & 0 & Langenfeld 2010; Splett 2006 \\
\hline 11 & 1 & 0 & 1 & 1 & 0 & 1 & 0 & 0 & Brasler 2006 \\
\hline
\end{tabular}

QCA: qualitative comparative analysis. 
Table 30. Complex solution for QCA model 5 - stakeholder involvement and engagement

\begin{tabular}{|c|c|c|c|c|c|c|}
\hline & & $\begin{array}{l}\text { Consistency score } \\
\text { with subset rela- } \\
\text { tionship ( } n=27 \text { in } \\
\text { each assessment) }\end{array}$ & $\begin{array}{l}\text { Proportional } \\
\text { reduction in } \\
\text { inconsistency }\end{array}$ & $\begin{array}{l}\text { Raw cover- } \\
\text { age }\end{array}$ & $\begin{array}{l}\text { Unique } \\
\text { coverage }\end{array}$ & Cases \\
\hline 1 & $\begin{array}{l}\text { anysch- } \\
\text { pol*goodrel- } \\
\text { nur*CHILDSAT }\end{array}$ & 0.846 & 0.794 & 0.243 & 0.152 & $\begin{array}{l}\text { Al-Sheyab 2012; Berg } \\
\text { 2004; Bruzzese 2004; } \\
\text { Bruzzese 2008; Kintner } \\
2012\end{array}$ \\
\hline \multirow[t]{2}{*}{2} & $\begin{array}{l}\text { anysch- } \\
\text { pol*GOODREL- } \\
\text { PAR }^{\star} \text { goodrelnur }\end{array}$ & 0.979 & 0.972 & 0.187 & 0.095 & $\begin{array}{l}\text { Bruzzese 2008; Joseph } \\
2013\end{array}$ \\
\hline & M1 & 0.884 & 0.849 & 0.339 & & \\
\hline
\end{tabular}

QCA: qualitative comparative analysis.

[Notation: Upper case = condition is present; Lower case $=$ condition is absent; ${ }^{*}=$ logical and; + logical or; Key: ANYSCHPOL $=$ School asthma policy; GOODRELNUR = Good level of engagement and/or developing relationships with school nurses; GOODRELPAR

= Good level of reported in engagement and/or developing relationships with parents; CHILDSAT = Children reported as satisfied; SUCCESSFULIMPLEMENTATION = Implementation of intervention successful]

Table 31. Intermediate solution for QCA model 5 - stakeholder involvement and engagement

\begin{tabular}{|c|c|c|c|c|c|c|}
\hline & & $\begin{array}{l}\text { Consistency score } \\
\text { with subset relation- } \\
\text { ship ( } n=27 \text { in each } \\
\text { assessment) }\end{array}$ & $\begin{array}{l}\text { Proportional } \\
\text { reduction in } \\
\text { inconsistency }\end{array}$ & $\begin{array}{l}\text { Raw cover- } \\
\text { age }\end{array}$ & $\begin{array}{l}\text { Unique } \\
\text { coverage }\end{array}$ & Cases \\
\hline 1 & $\begin{array}{l}\text { goodrel- } \\
\text { nur*CHILDSAT }\end{array}$ & 0.846 & 0.794 & 0.243 & 0.152 & $\begin{array}{l}\text { Al-Sheyab 2012; Berg 2004; } \\
\text { Bruzzese 2004; Bruzzese } \\
\text { 2008; Kintner } 2012\end{array}$ \\
\hline \multirow[t]{2}{*}{2} & $\begin{array}{l}\text { anysch- } \\
\text { pol*GOODREL- } \\
\text { GPAR* goodrel- } \\
\text { nur }\end{array}$ & 0.979 & 0.972 & 0.187 & 0.095 & $\begin{array}{l}\text { Bruzzese 2008; Joseph } \\
2010\end{array}$ \\
\hline & M1 & 0.884 & 0.849 & 0.339 & & \\
\hline
\end{tabular}

QCA: qualitative comparative analysis.

[Notation: Upper case $=$ condition is present; Lower case $=$ condition is absent; ${ }^{*}=$ logical and; + logical or; Key: ANYSCHPOL $=$ School asthma policy; GOODRELNUR = Good level of engagement and/or developing relationships with school nurses; GOODRELGPAR

= Good level of reported in engagement and/or developing relationships with parents; CHILDSAT = Children reported as satisfied; SUCCESSFULIMPLEMENTATION = Implementation of intervention successful]

\section{AP PE N DICES}

\section{Appendix 1. Sources and search methods for the Cochrane Airways Group Specialised Register (CAGR)}

Electronic searches: core databases 


\begin{tabular}{ll}
\hline Database & Frequency of search \\
\hline CENTRAL & Monthly \\
\hline MEDLINE (Ovid) & Weekly \\
\hline Embase (Ovid) & Weekly \\
\hline PSycINFO (Ovid) & Monthly \\
\hline CINAHL (EBSCO) & Monthly \\
\hline AMED (EBSCO) & Monthly \\
\hline
\end{tabular}

\section{Handsearches: core respiratory conference abstracts}

\begin{tabular}{ll}
\hline Conference & Years searched \\
\hline American Academy of Allergy, Asthma and Immunology (AAAAI) & 2001 onwards \\
\hline American Thoracic Society (ATS) & 2001 onwards \\
\hline Asia Pacific Society of Respirology (APSR) & 2004 onwards \\
\hline British Thoracic Society Winter Meeting (BTS) & 2000 onwards \\
\hline Chest Meeting & 2003 onwards \\
\hline European Respiratory Society (ERS) & $1992,1994,2000$ onwards \\
\hline International Primary Care Respiratory Group Congress (IPCRG) & 2002 onwards \\
\hline Thoracic Society of Australia and New Zealand (TSANZ) & 1999 onwards \\
\hline
\end{tabular}

\section{MEDLINE search strategy used to identify trials for the CAGR}

\section{Asthma search}

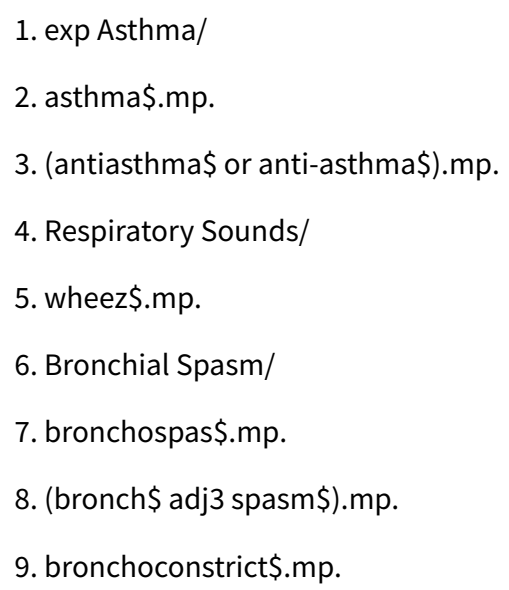


10. exp Bronchoconstriction/

11. (bronch\$ adj3 constrict\$).mp.

12. Bronchial Hyperreactivity/

13. Respiratory Hypersensitivity/

14. ((bronchial\$ or respiratory or airway\$ or lung\$) adj3 (hypersensitiv\$ or hyperreactiv\$ or allerg\$ or insufficiency)).mp.

15. ((dust or mite\$) adj3 (allerg\$ or hypersensitiv\$)).mp.

16. or/1-15

\section{Filter to identify RCTs}

1. exp "clinical trial [publication type]"/

2. (randomised or randomised).ab,ti.

3. placebo.ab,ti.

4. dt.fs.

5. randomly.ab,ti.

6. trial.ab,ti.

7. groups.ab,ti.

8. or/1-7

9. Animals/

10. Humans/

11.9 not $(9$ and 10$)$

12. 8 not 11

The MEDLINE strategy and RCT filter are adapted to identify trials in other electronic databases.

Appendix 2. Search strategy to identify relevant trials from the CAGR (via the Cochrane Register of Studies - CRS) \#1 AST:MISC1

\#2 MeSH DESCRIPTOR Asthma Explode All

\#3 asthma*:ti,ab

\#4 \#1 or \#2 or \#3

\#5 MeSH DESCRIPTOR Schools Explode All

\#6 MeSH DESCRIPTOR School Health Services

\#7 MeSH DESCRIPTOR School Nursing

\#8 school*:ti,ab,kw

\#9 academ*:ti,ab,kw

$\# 10$ colleg*:ti,ab,kw

\#11 lesson*:ti,ab,kw

\#12 pupil*:ti,ab,kw

$\# 13 \# 5$ or \#6 or \#7 or \#8 or \#9 or \#10 or \#11 or \#12

School-based self-management interventions for asthma in children and adolescents: a mixed methods systematic review (Review) 
\#14 MeSH DESCRIPTOR Self Care Explode All

\#15 MeSH DESCRIPTOR Health Education Explode All

\#16 MeSH DESCRIPTOR Case Management

\#17 MeSH DESCRIPTOR Patient Education as Topic

\#18 educat ${ }^{\star}: \mathrm{ti}, \mathrm{ab}, \mathrm{kw}$

\#19 manag*:ti,ab,kw

\#20 self-car*:ti,ab,kw

\#21 self NEXT car*:ti,ab,kw

\#22 train*:ti,ab,kw

\#23 instruct*:ti,ab,kw

\#24 teach*:ti,ab,kw

\#25 patient-cent*:ti,ab,kw

\#26 patient NEXT cent*:ti,ab,kw

\#27 MeSH DESCRIPTOR Patient-Centered Care

\#28 patient-focus*:ti,ab,kw

\#29 patient NEXT focus*:ti,ab,kw

\#30 coach*:ti,ab,kw

\#31 skill*:ti,ab,kw

\#32 knowledge NEXT develop*:ti,ab,kw

\#33 tutor*:ti,ab,kw

$\# 34$ \#14 or \#17 or \#18 or \#19 or \#20 or \#21 or \#22 or \#23 or \#24 or \#25 or \#26 or \#27 or \#28 or \#29 or \#30 or \#31 or \#32 or \#33

\#35 \#4 AND \#13 AND \#34

[Note: in search line \#1, MISC1 denotes the field in the record in which the reference has been coded for condition, in this case, asthma]

\section{Appendix 3. CENTRAL search strategy}

Ti ab kw combined rather than ti; ab for asthma

\begin{tabular}{ll}
\hline$\# 1$ & MESH DESCRIPTOR asthma EXPLODE ALL TREES \\
\hline$\# 2$ & asthma*:TI,AB,KY \\
\hline$\# 3$ & \#1 OR \#2 \\
\hline$\# 4$ & MESH DESCRIPTOR Schools EXPLODE ALL TREES \\
\hline$\# 5$ & MESH DESCRIPTOR School Health Services EXPLODE ALL TREES \\
\hline$\# 6$ & MESH DESCRIPTOR School Nursing EXPLODE ALL TREES \\
\hline$\# 7$ & school*:TI,AB,KY OR academ*:TI,AB,KY OR colleg*:TI,AB,KY OR lesson*:TI,AB,KY OR pupil*:TI,AB,KY \\
\hline
\end{tabular}

School-based self-management interventions for asthma in children and adolescents: a mixed methods systematic review (Review) 


\begin{tabular}{|c|c|}
\hline \#8 & \#4 OR \#5 OR \#6 OR \#7 \\
\hline \#9 & 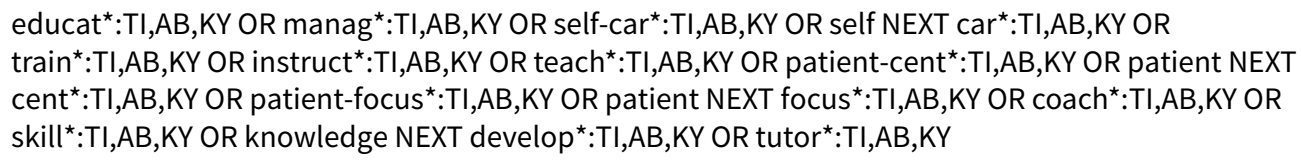 \\
\hline$\# 10$ & MESH DESCRIPTOR Self Care EXPLODE ALL TREES \\
\hline \#11 & MESH DESCRIPTOR Health Education EXPLODE ALL TREES \\
\hline \#12 & MESH DESCRIPTOR Case Management EXPLODE ALL TREES \\
\hline \#13 & MESH DESCRIPTOR Patient Education EXPLODE ALL TREES \\
\hline \#14 & MESH DESCRIPTOR Patient-Centred Care EXPLODE ALL TREES \\
\hline$\# 15$ & \#9 OR \#10 OR \#11 OR \#12 OR \#13 OR \#14 \\
\hline \#16 & \#3 AND \#8 AND \#15 \\
\hline
\end{tabular}

\section{Appendix 4. CT.gov search strategy}

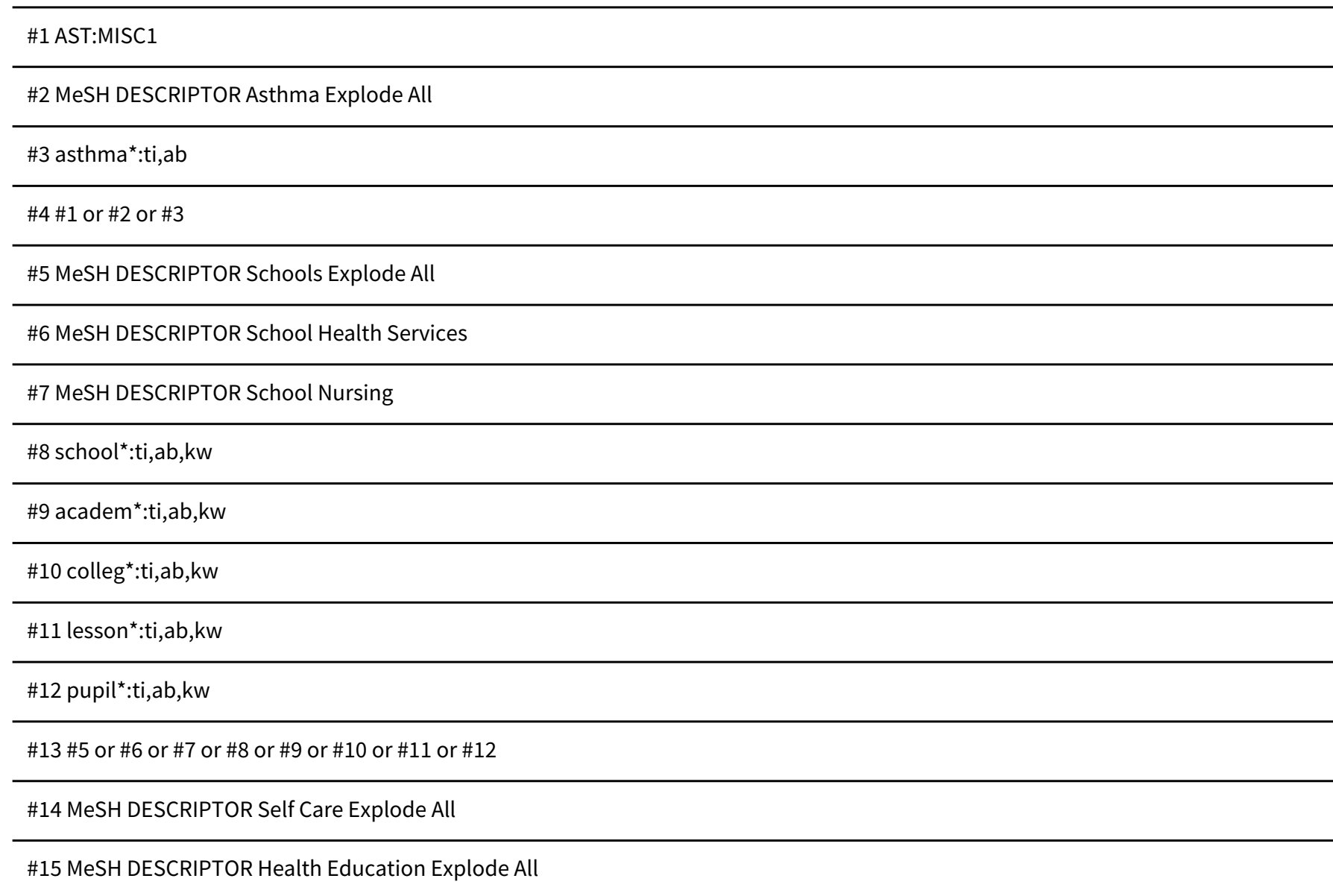


(Continued)

\#16 MeSH DESCRIPTOR Case Management

\#17 MeSH DESCRIPTOR Patient Education as Topic

\#18 educat ${ }^{\star}: \mathrm{ti}, \mathrm{ab}, \mathrm{kw}$

\#19 manag*:ti,ab,kw

\#20 self-car ${ }^{\star}:$ ti,ab,kw

\#21 self NEXT car*:ti,ab,kw

\#22 train*:ti,ab,kw

\#23 instruct*:ti,ab,kw

\#24 teach*:ti,ab,kw

\#25 patient-cent ${ }^{\star}$ :ti,ab,kw

\#26 patient NEXT cent*:ti,ab,kw

\#27 MeSH DESCRIPTOR Patient-Centered Care

\#28 patient-focus ${ }^{\star}$ :ti,ab,kw

\#29 patient NEXT focus*:ti,ab,kw

\#30 coach*:ti,ab,kw

\#31 skill*:ti,ab,kw

\#32 knowledge NEXT develop*:ti,ab,kw

\#33 tutor*:ti,ab,kw

$\# 34 \# 14$ or \#17 or \#18 or \#19 or \#20 or \#21 or \#22 or \#23 or \#24 or \#25 or \#26 or \#27 or \#28 or \#29 or \#30 or \#31 or \#32 or \#33

\#35 \#4 AND \#13 AND \#34

\section{Appendix 5. CINAHL search strategy}

\begin{tabular}{|c|c|}
\hline \#1 & asthma* \\
\hline \#2 & (MH "Asthma+") \\
\hline \#3 & 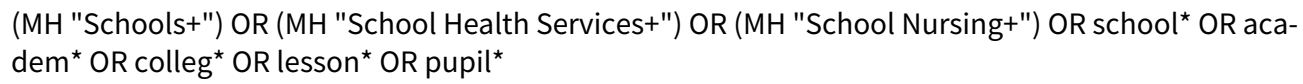 \\
\hline \#4 & $\begin{array}{l}\text { (MH "Self Care+") OR (MH "Health Education+") OR (MH "Case Management+") OR (MH "Patient Ed- } \\
\text { ucation+") OR educat }{ }^{\star} \text { OR manag }{ }^{\star} \text { OR self-car }{ }^{\star} \text { OR self n1 } \text { car }^{\star} \text { OR train* OR instruct* OR teach OR } \\
\text { patient-cent* }\end{array}$ \\
\hline
\end{tabular}




\begin{tabular}{ll}
\hline$\# 6$ & S4 OR S5 \\
\hline$\# 7$ & S1 OR S2 \\
\hline$\# 8$ & S3 AND S6 AND S7
\end{tabular}

\section{Appendix 6. AMED search strategy}

\begin{tabular}{|c|c|}
\hline \#1 & exp Asthma/ \\
\hline \#2 & exp Schools/ \\
\hline \#3 & asthma*.mp. [mp=title, other title, abstract, heading words] \\
\hline \#4 & 1 or 3 \\
\hline \#5 & exp School health services/ \\
\hline \#6 & exp School nursing/ \\
\hline \#7 & 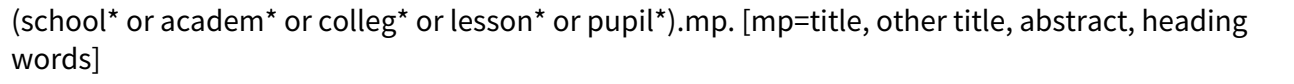 \\
\hline \#8 & 2 or 5 or 6 or 7 \\
\hline \#9 & $\begin{array}{l}\text { (educat }{ }^{\star} \text { or manag* or self-car }{ }^{\star} \text { or train* or instruct }{ }^{\star} \text { or teach }{ }^{\star} \text { or patient-cent }{ }^{\star} \text { or } \text { coach }^{\star} \text { or skill }{ }^{\star} \text { or } \\
\left.\text { tutor }{ }^{\star}\right) . m p .[\mathrm{mp}=\text { title, other title, abstract, heading words] }\end{array}$ \\
\hline \#10 & 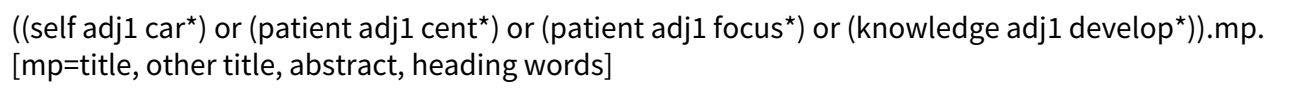 \\
\hline \#11 & exp Self care/ \\
\hline \#12 & exp Health education/ \\
\hline \#13 & exp Case management/ \\
\hline \#14 & exp Patient education/ \\
\hline \#15 & exp patient centred care/ \\
\hline \#16 & 9 or 10 or 11 or 12 or 13 or 14 or 15 \\
\hline \#17 & 4 and 8 and 16 \\
\hline \#18 & from 17 keep 1-100 \\
\hline \#19 & limit 18 to yr="1995 -Current" \\
\hline
\end{tabular}




\section{Appendix 7. Embase search strategy}

\begin{tabular}{|c|c|}
\hline$\# 1$ & 'Asthma' \\
\hline \#2 & 'Schools' \\
\hline \#3 & 'School Health Services' \\
\hline \#4 & 'School Nursing' \\
\hline \#5 & 'School' \\
\hline \#6 & Academy' \\
\hline$\# 7$ & 'Academic' \\
\hline \#8 & 'Academies' \\
\hline \#9 & 'college' \\
\hline \#10 & 'Colleges' \\
\hline \#11 & 'lesson' \\
\hline \#12 & 'Lessons' \\
\hline \#13 & 'pupil' \\
\hline \#14 & 'Pupils' \\
\hline \#15 & $\# 2$ or $\# 3$ or $\# 4$ or $\# 5$ or $\# 6$ or $\# 7$ or $\# 8$ or \#9 or $\# 10$ or \#11 or \#12 or \#13 or \#14 \\
\hline \#16 & 'Self Care' \\
\hline \#17 & 'Health Education' \\
\hline \#18 & 'Case Management' \\
\hline \#19 & 'Patient Education' \\
\hline \#20 & 'Educate' \\
\hline \#21 & 'Education' \\
\hline \#22 & 'Educator' \\
\hline \#23 & 'Manage' \\
\hline \#24 & 'Management' \\
\hline \#25 & 'self-care' \\
\hline \#26 & 'train' \\
\hline \#27 & 'Training' \\
\hline
\end{tabular}

School-based self-management interventions for asthma in children and adolescents: a mixed methods systematic review (Review) 


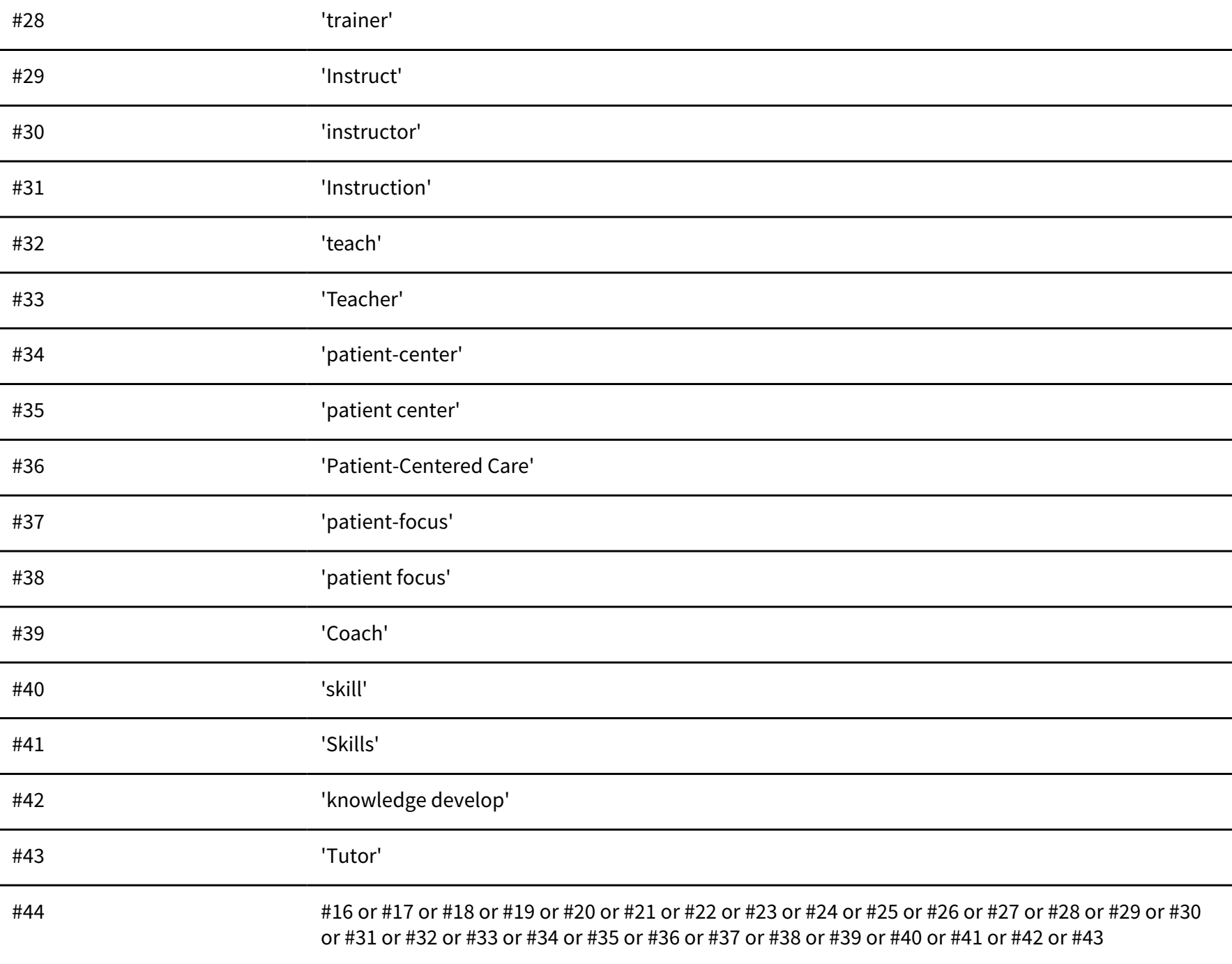

\section{Appendix 8. Additional information on the synthesis of process evaluation data and qualitative comparative analysis}

\section{Background and theoretical basis for qualitative comparative analysis (QCA)}

The QCA approach was developed in the political sciences during the 1980s by Charles Ragin (Ragin 2008), and in turn was based on mathematical developments in electrical engineering and analytical philosophy (Miech 2015; Thiem 2015). In its application within political sciences, QCA was utilised in comparing the characteristics of nations to enhance understanding of the conditions associated with different forms of governance and rule (Thomas 2014). Since then, its use has broadened, and it has been applied in as fields as diverse as ecology (e.g. Hellström 2001), education (Cooper 2005), and dentistry (Singh 2012). In each case, QCA was employed as a solution to the challenge of analysing data containing a small number of cases, each with an extensive array of conditions that may be necessary to trigger a given outcome. This 'small N-many variables' challenge is similar to that often faced by systematic review authors, and we followed the approach developed by Thomas and colleagues in employing QCA to understand conditions associated with our outcome of interest based on published data within primary studies (Thomas 2014). In this review, however, rather than attempting to understand how different configurations of conditions are associated with differentials in effect size (see further examples in Brunton 2014 and Brunton 2014a), we explored their association with different levels of implementation success.

QCA has its basis in set-theoretic logic, and it can be conceptualised as bridging the qualitative and quantitative divide, not only in terms of the types of data employed to undertake QCA but also in the research process and theoretical standpoints taken during stages of the QCA. Aspects of QCA that are aligned with a qualitative standpoint include the iterative process of case selection, reconceptualisation of conditions (or variables), and reconceptualisation of the outcome of interest that takes place during the model specification (Schneider 
2010); in this review, the approach adopted very much mirrors the hypothesis-generating role of qualitative research. In contrast, exploration of patterns in their alignment of conditions with the outcome of interest mirrors the quantitative practice of testing variables simultaneously in a regression framework (Schneider 2010). Unlike most quantitative research, QCA is based on set-theoretic principles, where the focus is on sets of conditions as units, rather than on the individual constituent components. This is aligned closely with the statements that social scientists routinely make about the nature of social phenomena that involve descriptions of groups as subsets of larger groups (Ragin 2008). Furthermore, the nature of these relationships is asymmetrical, unlike the symmetrical principles of statistical correlational research.

QCAs allow us to consider two aspects of set relationships. First, the number of those with an outcome who share a given condition, and second, the number with a given condition who share an outcome. The first aspect allows us to consider the degree to which a condition is a 'necessary' component of triggering the outcome (necessity); the second allows us to consider the extent to which a given condition is a sufficient condition for triggering the outcome (sufficiency), with particular application to exploring combinations of cases (Ragin 2008). It It is this second application that is of greatest interest, as it allows us to consider more complex configurations that may trigger an outcome. QCA allows for quantification of these relationships through exploration of different combinations of conditions that achieve an outcome. QCA was developed first by exploring conditions in binary form, although later extensions have allowed for fuzzy-set QCA that allows for ambiguity in both outcome and condition sets (Ragin 2009).

A set membership score for each case based on its characteristics is calculated from the data table, and these are analysed against outcome membership scores. Subset relationships are identified by observing when the membership scores in one set (i.e. a combination of conditions) are consistently less than those in another set (i.e. outcome) (see Ragin 2009). In line with the guidance set out by Ragin (Ragin 2009), and reflective of the size of the data set, we set a frequency threshold of one around the number of cases with a membership score greater than 0.5 in each combination (i.e. configuration of conditions and outcomes). QCA is reliant on Boolean algebra to reduce multiple configurations of conditions that lead to outcomes to their instrumental parts, to form a parsimonious solution. Although QCA is analogous in some ways to data reduction techniques employed in statistical analyses, the conditions tested in QCA analyses are included only on the basis of pre-existing theoretical knowledge of the analyst. In this case, our logic model, presented in Figure 1, helped to guide much of our thinking.

\section{Explanation and example of coding strategy}

We developed a strategy involving direct and indirect transformation in assembling our data for the QCA. For example, we developed a single variable to reflect whether an intervention took place within a high school. Those interventions that took place exclusively within high schools were assigned a value of 1 (fully within the set), and those that took place exclusively within elementary/primary schools were assigned a value of 0 . Further details of this example are found below in Table 1.

\section{Appendix Table 1. Example coding of direct assignment of values - whether the intervention took place in a high school}

\section{Condition $=$ high school}

\section{Directly assigned value}

High school(s)

1

High schools and junior/middle schools

Junior/middle school(s)

0.66

Missing information on age

0.5

Primary/elementary school(s)

0

When values were directly assigned in this way, no further calibration was required. In other cases, the assignment followed a combination of direct and transformational assignment. Direct assignment involves a researcher directly assigning values (usually based on categorical or binary source indicators); transformational assignment involves developing rules for how values that are more continuous in nature (and not necessarily bounded by 0 and 1 ) are coded between zero and one. Transformational assignment was conducted using R, as was most of the QCA synthesis, and a full explanation of transformational assignment and the underlying theoretical principles is provided in Thiem and Dusa (Thiem 2012). In all cases, transformational assignment was based on positive endpoint values, and involved setting thresholds indicating full exclusion from a set, a cross-over point (maximum ambiguity (0.5); which was also used in the case of missing data), and a threshold for full inclusion. An example is provided in Appendix Table 2 below for identification of whether an intervention could be considered a 'large' intervention based on the total number of students involved. 
Appendix Table 2. Example coding of transformational assignment of values - whether the intervention was a large intervention

\section{Condition $=$ large intervention}

Large interventions (whole school interventions taking place in large schools)

Moderately large interventions (approximately 3 classes in the intervention)

(maximum ambiguity)

Small interventions (less than a single class)

\section{Threshold values}

300

90

15

We extracted information supporting several conditions (over 90) for studies. We identified five key domains in which these belonged.

- Outcome: process outcomes.

- Condition group 1: setting and participants.

- Condition group 2: programme design.

- Condition group 3: programme content and style.

- Condition group 4: additional processes undertaken to facilitate implementation (planned and unplanned).

To limit the problem of limited diversity, when a large number of possible logical combinations are not supported by cases, we tested the relationship between each condition group and the outcome separately to identify the individual pathway recipes to successful implementation. We then consolidated the information to understand the instrumental components across all four groups and their membership in the outcome set. This approach is analogous to stepwise entry of antecedent variables into a regression model.

\section{Initial results from QCA modelling: a single domain}

\section{Model 1. Setting and participant characteristics}

We initially considered constructing sets based on a number of conditions (size of intervention, presence of existing health facilities in schools, high schools, black and minority ethnic students, low socio-economic status, whether teachers received additional training, whether school nurses or others received additional training, and whether parents received an intervention). However, given that we were working with 27 studies, we were concerned that limited diversity would be an issue and re-examined the theoretical justification for inclusion of each condition. In the case of the condition measuring the size of the intervention, we were concerned that this would reflect only a distinction between whether the intervention was a pilot/feasibility study or a full intervention and would not reflect modifiable 'process' and interaction with context per se. With regards to ethnicity and socio-economic status of children, we were concerned that this would be uninformative with regards to modifiable 'processes' and their interaction with context, and we did not include these conditions in the model. A data table and a truth table were constructed for the five remaining conditions (see Table 3 and Table 21).

The truth table showed six configurations that were indicative of a subset relationship with the outcome set and showed good distribution of configurations associated with the outcome and its negation. Several studies formed sets with high levels of consistency, and five showed proportional reduction in inconsistency (PRI) scores above 0.6. PRI is indicative of how distinct a subset configuration is of the outcome compared to negation of the outcome. No suggestions were provided as to an adequate threshold for PRI scores, although 0.6 falls between high and low values suggested elsewhere (Schwellnus 2013). We then proceeded to explore whether the truth table contained contradictory configurations. As we were conducting fsQCA, identification of contradictory configurations was less straightforward than would be the case for crisp-set QCA, and we explored the stability of rows supported by multiple cases for potential contradictory configurations, primarily by examining the original data in Table 3, although we found no evidence.

A complex solution was generated through Boolean minimisation (Table 22), suggesting five pathways towards generating the outcome of interest. We then incorporated information from logical remainders making explicit hypotheses that the presence of school-based health services (including school nurses), the involvement of parents, and the provision of additional training for teachers and other stakeholders would be beneficial to a successful intervention, but making no specific hypotheses about the impact of the intervention conducted in a high school. The intermediate solution gave four potential minimal sums (Table 23), each of which contained three essential prime implicants (three essential routes to the outcome) and two inessential prime implicants (interchangeable routes to the outcome needed to complete the minimal sum). Selection of the minimal solution was based on the most theoretically plausible; we also confirmed that no contradictory simplifying assumptions were made on the outcome and its negation. We explored whether any of the prime implicants selected were associated with negation of the outcome, finding little evidence based on consistency scores achieved for 'unsuccessful' 
interventions. The solution is displayed below (where upper case notation represents that the condition is present, and lower case represents that the condition is absent).

\section{Overall solution}

HIGHSCHOOL*schoolbasedhealth*parentdirect +

schoolbasedhealth*teacherdirect* parentdirect*anyothdir +

HIGHSCHOOL*SCHOOLBASEDHEALTH*teacherdirect*PARENTDIRECT +

(highschool*TEACHERDIRECT*ANYOTHDIR + HIGHSCHOOL*SCHOOLBASEDHEALTHPARENTDIRECT*ANYOTHDIR)

=> SUCCESSFULIMPLEMENTATION

[Notation: Upper case = condition is present; Lower case = condition is absent; * = logical and; + logical or; => leads to; Key: HIGHSCHOOL = High School (lower case not in high school); SCHOOLBASEDHEALTH = School Based Health Centre; TEACHERDIRECT = Teachers received directly received component of intervention; PARENTDIRECT = Parents directly received component of intervention; $A N Y O T H D I R=S c h o o l$ nurses or other stakeholders (apart from children) directly received component of intervention; SUCCESSFULIMPLEMENTATION = Implementation of intervention successful]

Our intermediate model achieved an overall coverage score of 0.61 and a consistency score of 0.951 , indicating that the solution accounted for most instances of the outcome and was highly sufficient in triggering the outcome (Table 23). The three essential prime implicants were identified, two of which suggested very different pathways to running a successful intervention in a high school. In the first pathway, supported by two studies (Al-Sheyab 2012a; Henry 2004), successful interventions were observed with no school-based health centre and no direct parental involvement. In contrast, evidence from Bruzzese 2008 and Terpstra 2012 suggested that successful interventions were observed with a school-based health centre and direct parental involvement (but no additional training for teachers). When we explored further contextual characteristics (not included within the model due to issues around limited diversity and difficulties in convergence with inclusion of these conditions), both studies indicating that support from a school-based health facility and direct involvement of parents were not essential for running a successful intervention took place in schools with low numbers of children from ethnic minorities or low socio-economic status backgrounds. In contrast, both studies suggesting that school-based health centres and involvement of parents were necessary conditions for a successful intervention took place in locations with large numbers of children from lower socio-economic status backgrounds and large numbers from an ethnic minority background (i.e. not the majority ethnic group in the country). This suggests that interventions taking place among larger numbers of marginalised children with asthma are successful support is received from existing school medical facilities or personnel, or when parents are directly involved; this support is not necessary when interventions take place among children who are not predominantly disadvantaged. This is reconfirmed by inclusion of a further (inessential) pathway suggesting that successful interventions in high schools are accompanied by direct parental involvement, additional training for school nurses and other stakeholders (not teachers), and current school-based health facilities (row 5; Table 23). Here the supporting case, represented by Kintner 2012, also took place within a location with a large number of children from low socio-economic status backgrounds and large numbers of children from ethnic minority backgrounds.

Two further prime implicants were identified. The first (inessential) implicant suggested that when interventions were implemented outside high schools, additional teacher training and training of other stakeholders were conditions that were sufficient to generate a successful outcome. A second (essential) primary implicant suggested that not having school-based health facilities, not having additional training for teachers or other stakeholders, and not having additional parental involvement were sufficient conditions to generate an outcome, regardless of whether the intervention took place in a high school. Both of these prime implicants were supported by studies that took place in primary/elementary schools, although additional systematic differences in context were not identifiable.

Such complexity in causal pathways is perhaps an artefact of the fuzzy QCA implemented here, in which we have focused on the setting and on actors involved in the intervention. Results suggest that even among this limited set of conditions, successful interventions with regards to implementation are triggered through a variety of seemingly contradictory pathways that may also reflect non-modifiable contextual characteristics. The evidence presented here suggests that when interventions take place in high schools (or junior schools) with large numbers of marginalised children with asthma, additional components involving parents or support from school-based health facilities are important conditions for ensuring successful implementation. These additional conditions are not necessary found in high schools with a less marginalised student body and may be detrimental to successful implementation. A similar difference was not immediately apparent for interventions taking place outside high schools.

\section{Model 2. Recruitment and retention processes}

We attempted to construct a truth table to explore a number of conditions based on recruitment and retention processes, eventually focusing on the use of incentives, marketing materials, reminders, and provision of make-up sessions. Nevertheless, we were unable to detect configurations that were potential subsets of the outcome based on the truth table output (not shown; see Table 4 for data). No configuration was identified as stable enough to support classification as a subset of a positive outcome value. We also tested these conditions against the negation of the outcome but again detected high levels of inconsistency. As such, we decided that this group 
of conditions did not form configurations that were subsets of successful interventions, and we did not consider them further in our consolidated model.

\section{Model 3. Curriculum, pedagogy, and intervention emphasis}

We constructed a model to explore the impact of conditions reflecting curriculum content, pedagogical style, and authors' descriptions of the emphasis of the intervention (see Table 24 for data). After several iterations, six conditions were entered into a model reflecting (I) whether the curriculum reflected forming alliances and monitoring symptoms, (ii) whether the curriculum reflected learning about asthma triggers and monitoring symptoms, (iii) whether study authors emphasised the intervention as tailored or personalised, (iv) whether study authors emphasised developing personal responsibility as an aim of the intervention, (v) whether the pedagogical style focused on interactive methods, and (vi) whether a diverse pedagogical style was employed. These conditions were selected on the basis of being the most theoretically informative (i.e. not simply a reflection of diffuse styles, e.g. other curriculum foci). A truth table was constructed but showed just two configurations that were sufficient to trigger a successful intervention and were supported by a single study each (Table 25). This low coverage of the outcome precluded further analysis. The same conditions were tested against the negation of the outcome, but no configuration displayed adequate levels of consistency.

\section{Model 4. Further modifiable intervention design features}

Conditions included in this model reflected modifiable design features of the intervention that were reported by study authors. The first condition reflected the extent to which study authors reported that their interventions were grounded in a named theoretical framework underpinning the intervention. Although it is likely that all interventions were grounded in theory to some extent, and a vast majority of studies presented supporting literature to justify delivery of the intervention, reporting a named theoretical framework in the process evaluation may signal that a theoretical framework continued to shape the study and was used as a reference point throughout the design of all stages of the trial. Two conditions reflected whether students' own time was interrupted (e.g. lunchtime, free periods), or whether their normal educational programme was interrupted by delivery of the intervention. We included a condition reflecting the extent to which the intervention was delivered or facilitated by a school nurse to capture the importance (or not) of having known medical personnel involved in the intervention as a condition for successful implementation. Finally, it was hypothesised that running personalised or individual sessions may impact the ability of trialists to deliver a successful intervention; negatively, this may impact on trialists' ability to balance individualised sessions across a larger cohort of students.

From the raw data (Table 5), configurations were created and were examined for their sufficiency in generating a successful intervention initially through construction of a truth table (Table 26). This initially showed six configurations that were associated with generating a successful intervention, all of which had been theory driven and included as a condition (although just one combination was supported by multiple cases). This table was then minimised, and a complex solution was generated (Table 27). However, a number of logical remainders were omitted from the derivation of this solution (17) and were used in developing a parsimonious solution (not shown) and an intermediate solution (Table 28). In developing the intermediate solutions, we hypothesised that conditions that reflected the presence of personalised and individual sessions, or that suggested that the intervention interrupted students' free time, would be negatively associated with successful implementation, and the presence of other conditions entered would impact positively.

\section{Overall solution}

THEORYDRIVEN*runinstudenttime*schoolnurseinstruct +

\section{THEORYDRIVEN* ${ }^{*}$ personalorindividual ${ }^{*}$ SCHOOLNURSEINSTRUCT $=>$ SUCCESSFULIMPLEMENTATION}

[Notation: Upper case = condition is present; Lower case = condition is absent; ${ }^{*}=$ logical and; + logical or; => leads to; Key: THEORYDRIVEN = Authors explicitly named theory or presented conceptual model for intervention; SCHOOLNURSEINSTRUCT = Substantial component delivered by schools' nurse; PERSONALORINDIVIDUAL = Substantial components delivered that were individually personalised or delivered to individuals; RUNINSTUDENTTIME = Substantial component run in students' own time (e.g. lunchtime); RUNINLESSONS = Substantial component run during lesson time; SUCCESSFULIMPLEMENTATION = Implementation of intervention successful]

The intermediate solution above was checked for the presence of contradictory simplifying assumptions (none were detected), and the sufficiency of the configurations was checked for negation of the outcome (no sufficient configurations were detected). The solution confirms the importance of being theory driven as a sufficient condition in generating the outcome and identifies two configurations that include this condition. Two pathways were identified: the first pathway (row 1) suggests that school nurse involvement is needed if the intervention does not involve personalised or individualised sessions. This suggests that when an intervention provides only groupbased or generic content, school nurse input is needed to ensure successful implementation. However, the second pathway suggests that when interventions are not provided during students' free time (and therefore implicitly take place during students' learning time), successful implementation is achieved when involvement of a school nurse is not observed. A factorised (simplified) version of the solution is displayed below: 
The factorised solution emphasised that being theory driven is a common condition for triggering a successful outcome within specific configurations. The presence of a school nurse in facilitating successful implementation is important in group or non-tailored interventions, although absence of the school nurse is important for interventions that may take place within lesson or assembly time. Although the model captures two configurations with high levels of sufficiency (both consistency scores and overall model score are over 0.9), modest coverage scores $(0.43$ for the model) suggest that there remain several other pathways in which the outcome is triggered that are not identified in this set of conditions (Table 28).

\section{Model 5. Stakeholder involvement and engagement}

We explored levels of stakeholder involvement and engagement (I) across the school level (through development of school policies for asthma), (ii) at the child level (by measuring child satisfaction), and (iii) at the level of other stakeholders by exploring teacher, parent, and school nurse engagement. These latter conditions reflected whether study authors reported instances of problematic or enthusiastic engagement. For the latter four conditions, a missing code was initially entered into models to reflect where the condition was irrelevant (the intervention did not include involvement of a given stakeholder) or where the information was not collected. High levels of missing data precluded construction of appropriate configurations of conditions, and missing data were later coded as 'zero', indicating that the condition was absent or unreported (this is reflected in the raw data table (Table 6); this also meant that negative instances of a condition were coded in the same way as missing data or 'not applicable', although no instances of negative child satisfaction were identified). We lowered the consistency threshold to 0.8 (which remained relatively high) to reflect the low frequency of occurrence of these conditions.

The initial truth table revealed substantial amounts of limited diversity, and no configurations predicting the outcome were supported by multiple cases (Table 29); therefore we removed teacher engagement due to its infrequent occurrence (low coverage as a condition). We then explored the revised truth table, identifying three configurations sufficient for the outcome and supported by five studies. The truth table also revealed that five configurations remained as logical remainders that were not supported by observations. We then implemented Boolean minimisation to generate a complex solution (Table 30), and we imposed specific directional hypotheses on child satisfaction and development of the school asthma policy (both expected to lead to positive outcomes) to create an intermediate solution (Table 31).

\section{Overall solution}

goodrelnur ${ }^{*}$ CHILDSAT +

anyschpol ${ }^{\star}$ GOODRELPAR ${ }^{\star}$ goodrelnur $=>$ SUCCESSFULIMPLEMENTATION

[Notation: Upper case = condition is present; Lower case = condition is absent; ${ }^{*}=$ logical and; + logical or; Key: ANYSCHPOL = School asthma policy; GOODRELNUR = Good level of engagement and/or developing relationships with school nurses; GOODRELPAR = Good level of reported in engagement and/or developing relationships with parents; CHILDSAT = Children reported as satisfied; SUCCESSFULIMPLEMENTATION = Implementation of intervention successful]

The intermediate solution indicated two essential prime implicants (pathways) sufficient to generate a positive outcome. One included child satisfaction as a sufficient condition, and one included reporting good levels of engagement with parents. Student satisfaction, although rarely measured, is a sufficient condition for generating a successful intervention. Similarly, parental engagement, even when parents were not necessarily directly involved in the intervention beyond providing consent for student participation, appeared to be a sufficient condition for successful implementation later. Both were sufficient only in the presence (or absence) of other conditions. Each implicant had high levels of consistency and high PRI levels, suggestive of sufficient configurations, although individual implicants exhibited low levels of coverage, and overall the solution had low levels of raw coverage (0.339, lying beneath thresholds suggested elsewhere (e.g. Ho et al., 2016)). This low coverage reflects the low frequency of reporting of included conditions by study authors. Although such low coverage would usually indicate that conditions lacked empirical importance, it is difficult to make this link with these data. What the evidence in model 5 does reinforce is the importance of measuring child satisfaction and parental engagement, as higher levels of both are found to trigger successful implementation. Despite this link, these conditions go unreported by trialists in almost half of studies, even within process evaluations (see data in Table 6).

\section{Model 6. Consolidated model}

We developed a consolidated model using evidence from models 1,4 , and 5 to understand some of the most important conditions to consider in designing an intervention. As we wanted to identify conditions that were empirically meaningful as well as sufficient, we selected conditions that were included in configurations with high consistency and coverage scores. To aid interpretability of the solutions, we focused on conditions with a consistent direction in a solution; this also reflected the type of data that we were working with that are dependent on trialists' reports, as opposed to standardised inventories of activities from across interventions.

We selected four conditions from model 4 (modifiable intervention design features) that reflected whether interventions were theorydriven, were delivered as personalised or individual sessions, or took place during students' own free time, and whether a school nurse was involved in delivery of the intervention. In addition, we included whether high levels of child satisfaction were reported (from model 5) and whether the intervention took place in a high school (model 1). The data table for this model is found in Table 15. The initial truth table showed six configurations of conditions spread across 10 studies that were classified as triggering a successful intervention (Table 16). Each of these configurations was theory-driven, and a complex solution was generated based on this initial truth table identifying four essential prime implicants sufficient for triggering successful implementation of an intervention (see Table 17). Logical remainders were 
an issue, with 18 configurations unsupported by cases, and parsimonious and intermediate solutions were generated using simplifying assumptions from these configurations. The intermediate solution, presented in Table 18, makes directional assumptions on four of the five conditions, with child satisfaction, parental engagement, and interventions being theory driven expected to lead to a positive outcome, while running an intervention during students' own time was expected to lead to a negative outcome.

\section{Overall solution}

HIGHSCHOOL ${ }^{\star} \mathrm{CHILDSAT}^{\star}$ THEORYDRIVEN +

HIGHSCHOOL*THEORYDRIVEN* GOODRELGPAR +

HIGHSCHOOL*THEORYDRIVEN*runinstudenttime +

CHILDSAT ${ }^{\star}$ THEORYDRIVEN $^{\star}$ runinstudenttime ${ }^{\star}$ GOODRELENGPAR $=>$ SUCCESSFULIMPLEMENTATION

[Notation: Upper case = condition is present; Lower case = condition is absent; ${ }^{*}=$ logical and; + logical or; Key: HIGHSCHOOL = High School (lower case not in high school); THEORYDRIVEN = Authors explicitly named theory or presented conceptual model for intervention; RUNINSTUDENTTIME = Substantial component run in students' own time (e.g. lunchtime); GOODRELPAR = Good level of reported in engagement and/or developing relationships with parents; CHILDSAT = Children reported as satisfied; SUCCESSFULIMPLEMENTATION = Implementation of intervention successful]

The solution emphasises the importance of being theory-driven across all settings. Three of the essential prime implicants were restricted in coverage to high schools. Here the evidence suggests that in addition to being theory-based, having good levels of engagement with parents, or having high levels of child satisfaction, or running the intervention outside the students' own time leads to a successfully implemented intervention. An essential prime implicant that is not restricted to high schools also reinforces these findings by showing that being theory-based, fostering high levels of student satisfaction, reporting good levels of parental engagement, and running an intervention outside students' own time are sufficient conditions for triggering a positive outcome. As with a large portion of the data included here, this solution has modest levels of coverage, accounting for almost half of the instances of the outcome, and indicating that there remain diverse pathways to running a successful intervention that have not been included within the QCA solution. A factorised representation of the minimal sum, as presented below, helps to simplify the solution.

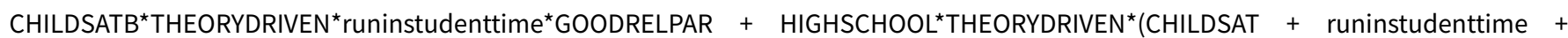
GOODRELPAR) $=>$ SUCCESSFULIMPLEMENTATION

[Notation: Upper case $=$ condition is present; Lower case $=$ condition is absent; ${ }^{*}=$ logical and; + logical or; Key: HIGHSCHOOL $=$ High School (lower case not in high school); THEORYDRIVEN = Authors explicitly named theory or presented conceptual model for intervention; RUNINSTUDENTTIME = Substantial component run in students' own time (e.g. lunchtime); GOODRELPAR = Good level of reported in engagement and/or developing relationships with parents; CHILDSATB = Children reported as satisfied; SUCCESSFULIMPLEMENTATION = Implementation of intervention successful]

We checked for the presence of contradictory configurations in developing the solution and examined whether any of the configurations described above also predicted negation of the outcome, but we found no evidence in either case. This confirms that these combinations did consistently lead to successful implementation of school-based asthma interventions, and were not associated with poor levels of implementation, but that they were not necessarily observed with a high degree of frequency.

\section{WHAT'S NEW}

\begin{tabular}{lll}
\hline Date & Event & Description \\
\hline 28 January 2019 & Amended & $\begin{array}{l}\text { Mistake in the plain language summary corrected. The total } \\
\text { number of included studies was 55, not 66. }\end{array}$ \\
\hline
\end{tabular}

\section{CONTRIBUTIONS OF AUTHORS}

\begin{tabular}{lc}
\hline Role & Author \\
\hline Drafting the protocol & $\mathrm{KH}, \mathrm{DK}, \mathrm{TL}, \mathrm{JG}, \mathrm{VM}, \mathrm{JT}$ \\
\hline Developing a search strategy & $\mathrm{KH}, \mathrm{DK}, \mathrm{TL}, \mathrm{JG}, \mathrm{VM}, \mathrm{JT}$
\end{tabular}


Searching for trials

\begin{tabular}{ll}
\hline Obtaining copies of trials & $\mathrm{KH}, \mathrm{DK}$ \\
\hline Providing subject expertise & $\mathrm{JG}$ \\
\hline Providing methodological expertise & $\mathrm{JT}, \mathrm{TL}$ \\
\hline Selecting which trials to include & $\mathrm{KH}, \mathrm{DK}, \mathrm{JG}, \mathrm{JT}$ \\
\hline Extracting data from trials & $\mathrm{KH}, \mathrm{DK}$ \\
\hline Entering data into RevMan & $\mathrm{KH}, \mathrm{DK}$ \\
\hline Carrying out the analysis & $\mathrm{KH}, \mathrm{DK}$ \\
\hline Interpreting the analysis & $\mathrm{KH}, \mathrm{DK}, \mathrm{TL}, \mathrm{JG}, \mathrm{JT}$ \\
\hline Drafting the final review & $\mathrm{KH}, \mathrm{DK}, \mathrm{TL}, \mathrm{JG}, \mathrm{JT}$
\end{tabular}

Dylan Kneale and Katherine Harris are the joint lead authors of this review.

\section{DECLARATIONS OF INTEREST}

A grant from NIHR North Thames CLAHRC is supporting some of this work, although it is not contributing to any individual time spent on the review (James Thomas).

This paper presents independent research and was funded by the National Institute for Health Research Collaboration for Leadership in Applied Health Research and Care (NIHR CLAHRC) North Thames at Bart's Health NHS Trust.

The views expressed are those of the review author(s) and are not necessarily those of the NHS, the NIHR, or the Department of Health.

\section{SOURCES OF SUPPORT}

\section{Internal sources}

- The review authors declare that no internal source of funding was received for this systematic review, UK.

\section{External sources}

- NIHR CLAHRC North Thames, UK.

Katherine Harris is in receipt of funding from the NIHR CLAHRC North Thames for her PhD.

\section{DIFFERENCES BETWEEN PROTOCOL AND REVIEW}

Our original protocol specified that we would locate process evaluation studies, although our inclusion criteria were expanded to include studies that evaluated the process of implementing interventions, regardless of whether they were stand-alone process evaluations. Although our original protocol did not specify that we would include only stand-alone process evaluations, this clarification does represent a difference between the language of protocol and the studies included in the review.

We extracted all data on school absences, and they were eligible for analysis, regardless of collection method, including those collected through administrative records. This is a deviation from the published protocol, which specified that parent-reported absences alone would be our outcome of interest.

We intended to conduct a sensitivity analysis of alternative estimated ICCs to studies for which these values are missing; however a suitable alternative ICC apart from the estimate used in analyses was not identified; thus we did not conduct additional sensitivity analyses. 
Sensitivity analyses were planned on the basis of country (OECD country vs other), although we did not carry out these analyses due to lack of variation according to this characteristic.

We had originally specified daytime and night-time symptoms as a single outcome, but we split this into two separate outcomes to maintain conceptual coherence. Similarly, we developed two models for corticosteroid usage in an attempt to ensure conceptual coherence.

Our protocol originally specified that thorough investigation of heterogeneity would take place only when heterogeneity as measured as $I^{2}$ exceeding $25 \%$. This was the case for ED visits and school absences; no other outcome met this threshold. However, we also conducted subgroup analyses of withdrawals, despite low heterogeneity, because of the conceptual similarity of this outcome and the implementation success that was the focus of the QCA.

Our protocol reported that we would include all outcomes in the 'Summary of findings' table (except withdrawals). In practice, we did not include lung function because of insufficient evidence to develop a meta-analysis. In addition, we did not include evidence of corticosteroid dosage because it is unclear whether the outcome reflected dosage as part of step-up or step-down therapy (see discussion).

Because our QCA involved examining dosage, attrition, and adherence as process outcomes, we deemed that these were not suitable for examining effects among studies of short duration. This meant we excluded six reports of interventions that involved one or two face-toface sessions (Bignall 2015; Carpenter 2016; Jackson 2006; McCann 2006; Mickel 2016; Richmond 2011), which we did not explicitly describe in the protocol.

\section{N DEX TERMS}

\section{Medical Subject Headings (MeSH)}

*School Health Services; Absenteeism; Anti-Asthmatic Agents [therapeutic use]; Asthma [*therapy]; Disease Progression; Emergency Service, Hospital [statistics \& numerical data]; Evaluation Studies as Topic; Health Services Needs and Demand [statistics \& numerical data]; Hospitalization [statistics \& numerical data]; Randomized Controlled Trials as Topic; Self-Management [ ${ }^{\star}$ methods]

\section{MeSH check words}

Adolescent; Child; Humans 